\title{
The Impact of Knowledge Mannagement Practices on Nuclear Power Plant Organization Performance
}

\author{
by \\ John F. P. de Grosbois \\ A thesis submitted to the Faculty of Graduate and Postdoctoral Affairs \\ in partial fulfillment of the requirements for the degree of \\ Doctor of Philosophy \\ in \\ Management \\ Carleton University \\ Ottawa, Ontario
}

(C) 2011

John F. P. de Grosbois 


$\begin{array}{ll}\begin{array}{l}\text { Library and Archives } \\ \text { Canada }\end{array} & \begin{array}{l}\text { Bibliotheque et } \\ \text { Archives Canada }\end{array} \\ \begin{array}{l}\text { Published Heritage } \\ \text { Branch }\end{array} & \begin{array}{l}\text { Direction du } \\ \text { Patrimoine de l'édition }\end{array} \\ 395 \text { Wellington Street } & \text { 395, rue Wellington } \\ \text { Ottawa ON K1A 0N4 } & \text { Ottawa ON K1A ON4 } \\ \text { Canada } & \text { Canada }\end{array}$

Your file Votre référence

ISBN: 978-0-494-81537-3

Our file Notre référence

ISBN: $978-0-494-81537-3$

NOTICE:

The author has granted a nonexclusive license allowing Library and Archives Canada to reproduce, publish, archive, preserve, conserve, communicate to the public by telecommunication or on the Internet, loan, distribute and sell theses worldwide, for commercial or noncommercial purposes, in microform, paper, electronic and/or any other formats.

The author retains copyright ownership and moral rights in this thesis. Neither the thesis nor substantial extracts from it may be printed or otherwise reproduced without the author's permission.
AVIS:

L'auteur a accordé une licence non exclusive permettant à la Bibliothèque et Archives Canada de reproduire, publier, archiver, sauvegarder, conserver, transmettre au public par télécommunication ou par l'Internet, prêter, distribuer et vendre des thèses partout dans le monde, à des fins commerciales ou autres, sur support microforme, papier, électronique et/ou autres formats.

L'auteur conserve la propriété du droit d'auteur et des droits moraux qui protège cette thèse. $\mathrm{Ni}$ la thèse ni des extraits substantiels de celle-ci ne doivent être imprimés ou autrement reproduits sans son autorisation.
In compliance with the Canadian Privacy Act some supporting forms may have been removed from this thesis.

While these forms may be included in the document page count, their removal does not represent any loss of content from the thesis.
Conformément à la loi canadienne sur la protection de la vie privée, quelques formulaires secondaires ont été enlevés de cette thèse.

Bien que ces formulaires aient inclus dans la pagination, il n'y aura aucun contenu manquant.

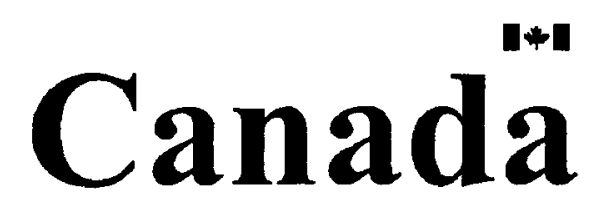




\section{ABSTRACT}

Effective knowledge management (KM) enhances a firm's capability to assimilate, create and exploit knowledge. $\mathrm{KM}$ is widely recognized in both the management literature and in practice as an important driver of long-term organizational performance and a necessary condition for sustained commercial success. Its role is particularly important in technology intensive industries such as the nuclear industry. Knowledge management practices, if effectively applied in nuclear power plants, have the potential to be an important enabler of operational and safety performance improvements, of reductions in operational and personnel risk, and of opportunities for plant design improvement. However, although a number of researchers have studied the relationship between KM practices and firm performance, little empirical evidence exists to support these claims in the context of the nuclear energy industry.

Knowledge management has only recently caught the attention of the nuclear industry. Various nuclear power plants (NPPs) around the globe have begun to recognize the strategic importance of KM initiatives in achieving sustained high levels of operational performance. Although these organizations have been involved in KM-related activities for some time, they typically have not been recognized as such. Thus they have not been viewed and managed from a KM perspective. Several NPPs have been early adopters of $\mathrm{KM}$ practices in the nuclear industry, and have been proactive in implementing company-wide KM programs. However, at other NPPs, the concepts and 
benefits of $\mathrm{KM}$ are only beginning to be understood and are not yet applied. The various approaches taken to $\mathrm{KM}$ and the relative benefits obtained from $\mathrm{KM}$ initiatives vary from station to station. Little is known as to how prevalent specific KM practices are and how effective they have proven to be. There is little prior research to guide NPP managers in the implementation of KM. Research is needed to determine what practices are effective and what benefits can be expected.

This thesis investigates the impact of KM practices on organizational effectiveness and operational performance in NPPs using quantitative research approaches (i.e. a large scale industry survey). It explores the effect of $\mathrm{KM}$ practices on the quality of knowledge processes and the resulting impact on organizational performance. Specific contextual aspects of the nuclear industry in general and of nuclear power plants in particular are identified and considered in the process. A theoretical framework is developed and subsequent hypothesis are derived and tested. Independent variable constructs include the level of support for KM practices and the effectiveness of technology support. Intermediate variable constructs considered are the quality of knowledge processes and the degree of supportive organizational culture. Dependent variables include organizational effectiveness and operational performance. Subconstructs and measures are developed and explained in detail. In conclusion, the goal of the research is to show the nature and extent of the influence of KM practices on NPP organizational performance, and the specific mechanisms by which this occurs. 


\section{ACKNOWLEIDGMENTS}

It is with sincere gratitude and great respect that I extend my thanks to my supervisor, Dr. Vinod Kumar for his guidance and encouragement. I am just one of a very large many who have benefited from his career long dedication and contribution to the field of management research. He has inspired us all and taught us much and we are grateful to have had the opportunity to learn from him.

Of course not enough can be said to express my gratitude to my very special and beloved wife, Danuta, who supported me throughout this endeavor. Appreciation must be expressed to my dearest daughters, Adalyn and Karina, who never cease to inspire and motivate me. I wish also to thank my Mother and Father who passed on to me their love of learning and provided a "supportive culture for knowledge acquisition".

I wish to thank Mr. Yanko Yanev, Mr. Andrey Kossilov and Mr. Zoltan Pasztory at the IAEA for their support and collaboration. Finally, I am grateful for the support of my long-time employer, Atomic Energy of Canada Ltd. (truly a knowledge-based and technology-driven enterprise), and more specifically, Carl Turner, Bob Speranzini, Ian Love, and Jerry Hopwood. 


\section{TABLE OF CONTENTS}

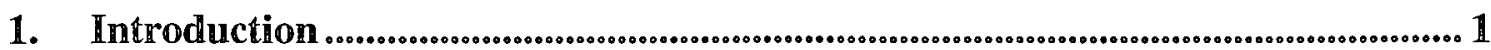

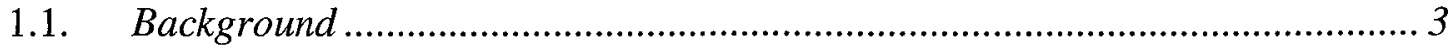

1.2. Research Objectives ......................................................................... 6

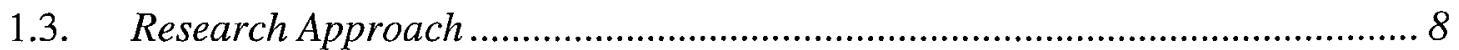

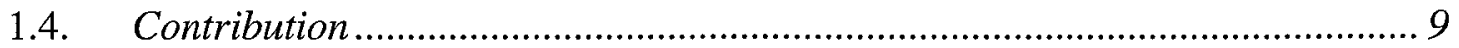

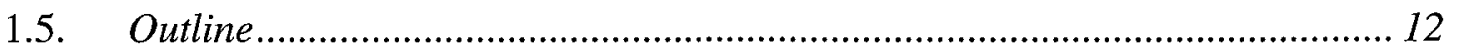

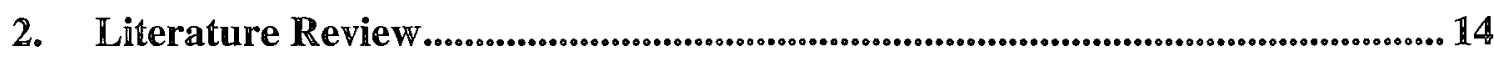

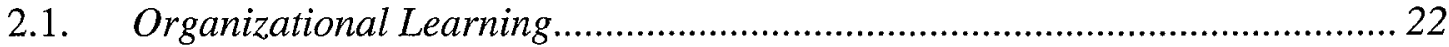

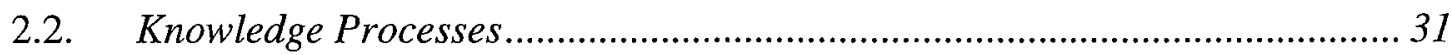

2.3. Knowledge Management Practices............................................................ 46

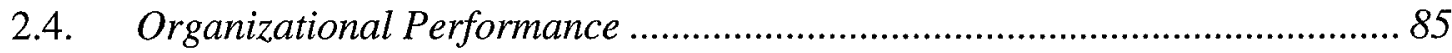

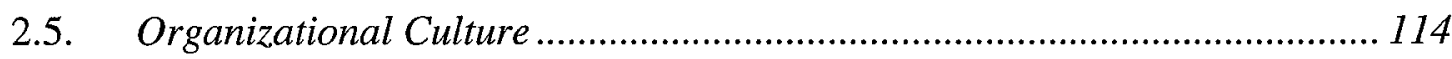

2.6. Information Systems and Information Technology Support......................... 122

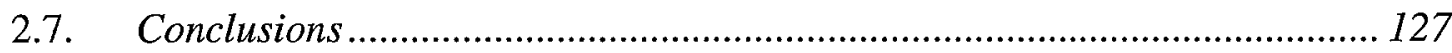

3. Theoretical Framework ...................................................................................... 129

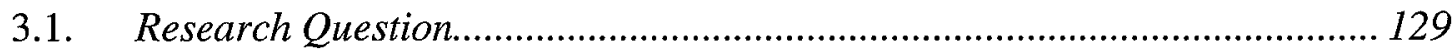

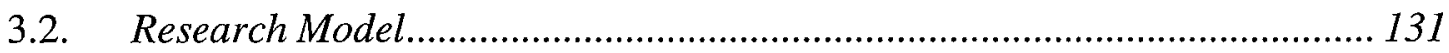

3.3. Operationalizing the Model Constructs ................................................. 156

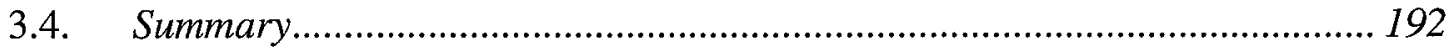

4. Research Methodology..................................................................................................... 193

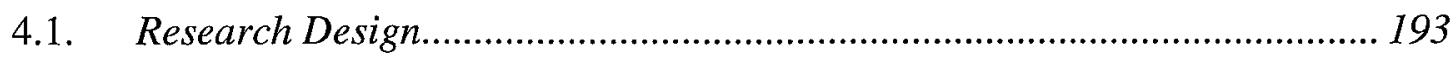

4.2. Survey Distribution List and Distribution Method....................................... 211

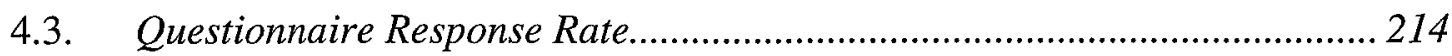

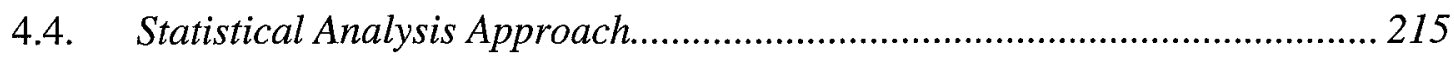

5. Descriptive Statistics ...................................................................................................... 219

5.1. Demographic Profile of Population (all stations)....................................... 219

5.2. Demographic Profile of Responding Stations ............................................... 222 


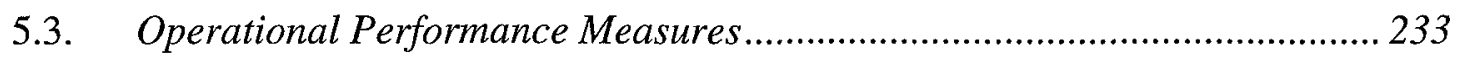

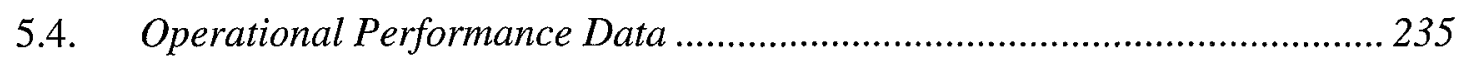

5.5. Descriptive Data for Indicator Measures ………....................................... 239

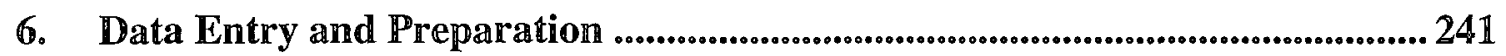

6.1. Coding of Multiple Unit Responses......................................................... 241

6.2. Data Quality and Screening ...................................................................... 244

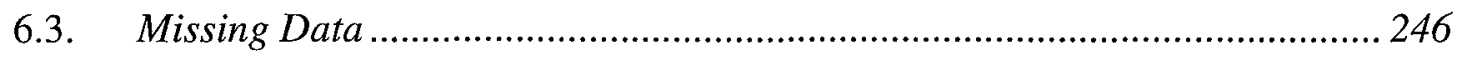

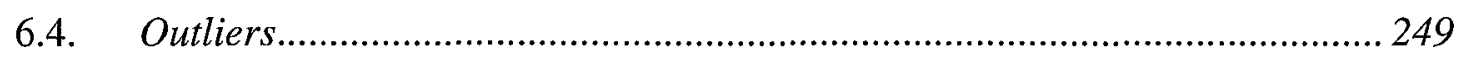

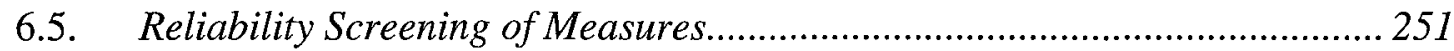

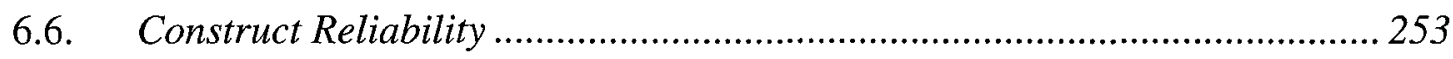

7. Linear Regression Assessments................................................................................... 265

7.1. Linking Support for KMPs and OTSs to Quality of KA Processes ............... 266

7.2. Linking Support for KMPs and OTSs to Quality of KS Processes................ 269

7.3. Linking Support for KMPs and OTSs to Quality of KR Processes ............... 270

7.4. Linking Support for KMPs and OTSs to Quality of KG Processes............... 272

7.5. Linking Support for KMPs and OTSs to Quality of KU Processes............... 273

7.6. Linking Support for KMPs and OTSs to SOC............................................ 275

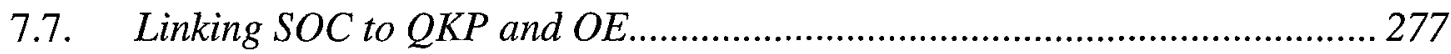

7.8. Role of SOC in Effects of Support for KMPS/OTSs on QKPs....................... 283

7.9. Inter-relationships Among QKP Constructs .............................................. 283

7.10. Linking KMPs and OTSs to Organizational Effectiveness (OE)................... 293

7.11. Linking Organizational Effectiveness to Operational Performance.............. 295

7.12. Effect of Plant Size, Units and Age on OE and OP ..................................... 299

7.13. Finite Population Considerations ................................................................. 300

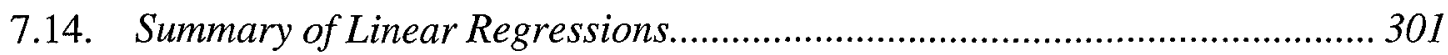

8. Path Analysis Assessment .............................................................................................. 302

8.1. Summary of Goodness of Fit (GOF) Statistics Used ....................................... 304

8.2. Hypothesis Testing via Stepwise Development of Path Models..................... 307

8.3. Path Model 1: Top-level Model with KMPs................................................ 308

8.4. Path Model 2: Top-level Model with OTS ………....................................... 313 
8.5. Path Model 3: Top-level Model with IST ................................................. 315

8.6. Path Model 4: Top-level Model with Advanced OSS Support ...................... 318

8.7. Path Model 5\&6: Top-level Model of Plant Age \& Units by Type ............... 320

8.8. Path Model 7\&8: Interaction Amongst QKP Constructs.............................. 329

8.9. Path Model 9: Interactions Between KMPs and QKPs .............................. 335

8.10. Path Model 10: Interactions of SOC with KMPs and QKP ...................... 343

8.11. Path Model 11: Full Model with OE Added .............................................. 347

8.12. Path Model 12: Full Model with OP Added .............................................. 352

8.13. Path Model 13: Competing Full Model with KR as Causal to KU............... 356

8.14. Path Model 14: Effect of Support for KMP High vs. KMP Low.................. 360

8.15. Path Model 15: Effect of SOC High vs. SOC Low....................................... 364

8.16. Path Model 16: Estimating Raw Operational Performance ......................... 370

9. Discussion and Research Implications ............................................................... 380

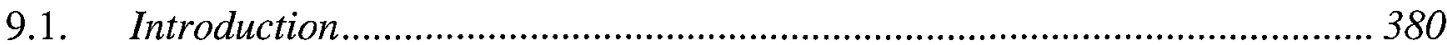

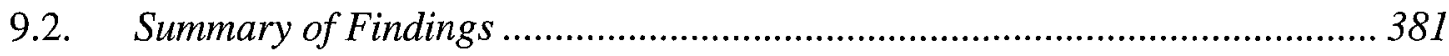

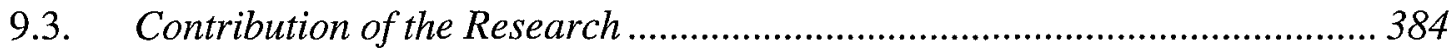

9.4. Relationship Between KMPs and QKP s................................................... 387

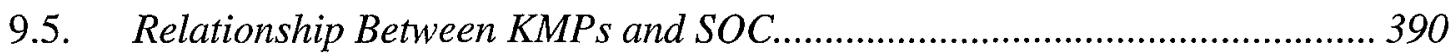

9.6. Inter-relationships Amongst the QKPs ........................................................... 390

9.7. Influence of QKPs on Organizational Effectiveness .......................................392

9.8. Effect of KMPs on Organizational Performance .......................................... 392

9.9. Supportive Organizational Culture as Moderator ............................................ 393

9.10. Findings related to Organizational Performance ........................................... 394

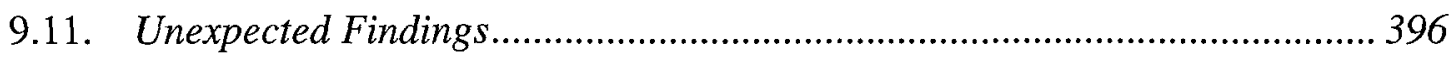

10. Conclusions ............................................................................................................................. 401

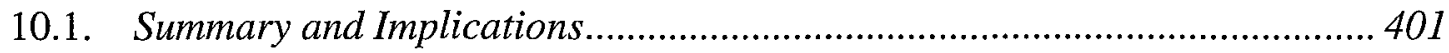

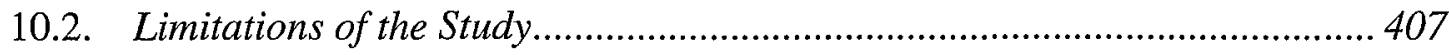

10.3. Recommendations for Future Research .................................................... 413

11. References ............................................................................................................................4 417 


\section{LIST OF TABLES}

Table 1. Summary of Definitions of Knowledge ...................................................... 16

Table 2. Definitions of Knowledge Management ……………………..................... 47

Table 3. Summary of KM Methods and Tools Found in the Literature..................... 52

Table 4. Measurement of Knowledge and Knowledge Management ......................... 64

Table 5. Issues Important to NPP Performance Improvement (IAEA, '99) ............ 105

Table 6. Summary of all NPPs by Country and Reactor Type.................................. 222

Table 7. Responding NPPs by $\%$ of population and country ................................... 224

Table 8. Responding NPPs by Country and Reactor Type ……….......................... 227

Table 9. Derivation of Operating Performance Indicators ...................................... 234

Table 10. Normalization bins for combined performance index................................ 237

Table 11. Responding NPP performance by plant type and units per site ................. 238

Table 12. Descriptive Statistics for Constructs ......................................................... 240

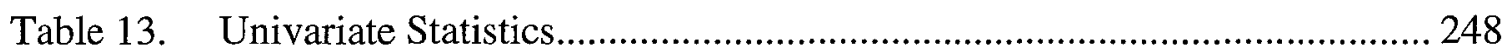

Table 14. Missing Value Analysis ..................................................................... 249

Table 15. Repeated Measure \%-Change Response ……………………………...... 252

Table 16. Inter-Item \& Item-to-Total Correlations for KMS ..................................... 253

Table 17. Inter-Item \& Item-to-Total Correlations for SOL ...................................... 254

Table 18. Inter-Item \& Item-to-Total Correlations for PMP..................................... 254

Table 19. Inter-Item \& Item-to-Total Correlations for IMP ………......................... 255

Table 20. Inter-Item \& Item-to-Total Correlations for OPM.................................... 256

Table 21. Inter-Item \& Item-to-Total Correlations for TRP ....................................... 256

Table 22. Inter-Item \& Item-to-Total Correlations for Reduced TRP ....................... 257

Table 23. Inter-Item \& Item-to-Total Correlations for HRP ……............................. 257

Table 24. Inter-Item \& Item-to-Total Correlations for IST........................................ 258

Table 25. Inter-Item \& Item-to-total Correlations for OSS...................................... 259

Table 26. Inter-Item \& Item-to-Total Correlations for KA ....................................... 259

Table 27. Inter-Item \& Item-to-Total Correlations for Reduced KA.......................... 260 
Table 28. Inter-Item \& Item-to-Total Correlations for KG ...................................... 260

Table 29. Inter-Item \& Item-to-Total Correlations for KS ....................................... 261

Table 30. Inter-Item \& Item-to-Total Correlations for KU ......................................... 261

Table 31. Inter-Item \& Item-to-Total Correlations for KR ...................................... 262

Table 32. Inter-Item \& Item-to-Total Correlations for SOC ..................................... 262

Table 33. Summary Statistics for OE Summated Scale .............................................. 263

Table 34. Squared Correlation Matrix \& Discriminant Validity................................. 264

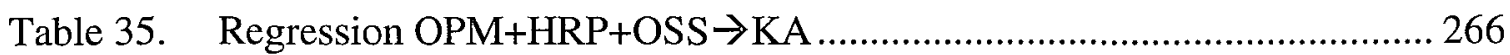

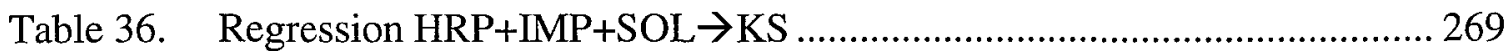

Table 37. Regression HRP+TRP+OPM $\rightarrow$ KR ………………………………….... 270

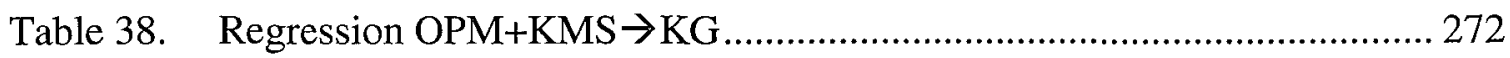

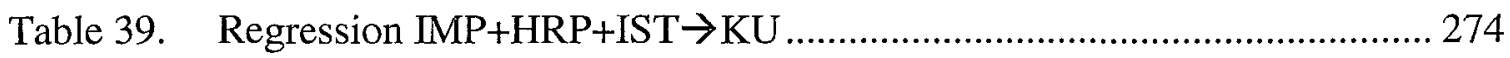

Table 40. Regression IMP+HRP+IST+SOL+KMS $\rightarrow$ SOC …………...................... 275

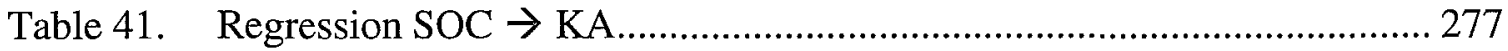

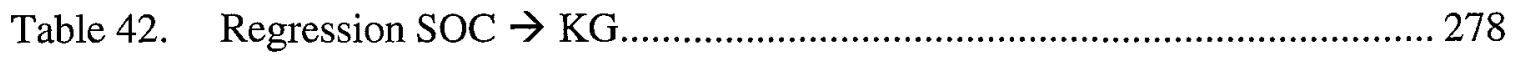

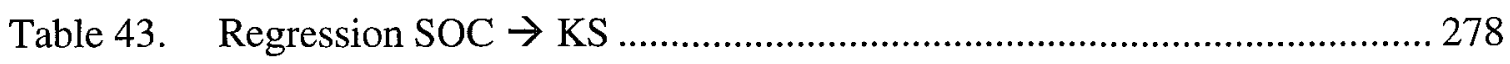

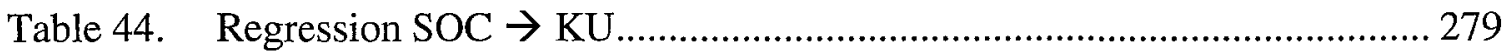

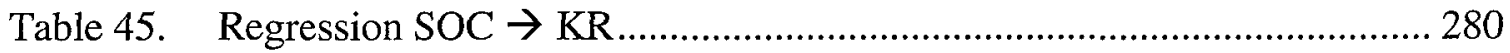

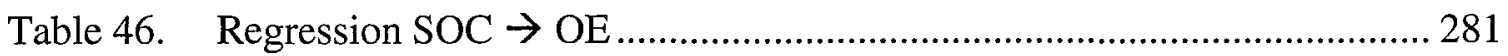

Table 47. Regression KG+KS+KU+KR<--->KA .................................................. 284

Table 48. Regression KG+KU+KR+KA<--->KS ............................................... 285

Table 49. Regression KG+KU+KA+KS<--->KR ................................................... 287

Table 50. Regression KU+KA+KS+KR<--->KG .................................................. 288

Table 51. Regression $\mathrm{KA}+\mathrm{KS}+\mathrm{KR}+\mathrm{KG}<--->\mathrm{KU}$................................................... 290

Table 52. Regression KA+KS+KR+KG+KU<--->OE .......................................... 291

Table 53. Regression KMS+PMP+IMP+IST $\rightarrow \mathrm{OE}$................................................. 293

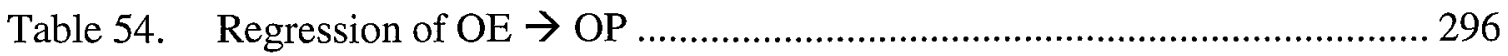

Table 55. Regression OE $\rightarrow$ OP_raw (CPerfINDEX) ……….................................... 296

Table 56. Regression Size + Units + Age $\rightarrow$ OE ..................................................... 299

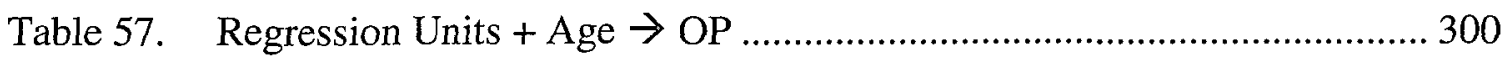

Table 58. Format for Reporting GOF (fictitious acceptable fit values) ...................... 307 
Table 59. Model 1 - Goodness of Fit Statistics …………...................................... 310

Table 60. Model 1 Regression Weights .................................................................. 310

Table 61. Model 1 Squared Multiple Correlations ..................................................... 310

Table 62. Model 1 Standardized Total Effects......................................................... 311

Table 63. Model 1 Standardized Direct Effects …………........................................... 311

Table 64. Model 1 Standardized Indirect Effects...................................................... 311

Table 65. Summary of Model 1 Findings ............................................................. 312

Table 66. Model Goodness of Fit Statistics for Path Model 2 ………...................... 313

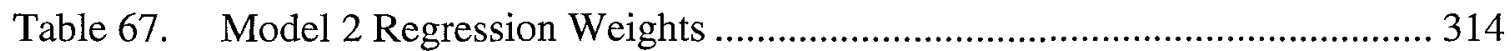

Table 68. Model 2 Squared Multiple Correlations....................................................... 314

Table 69. Model 2 Standardized Total Effects.......................................................... 314

Table 70. Model 2 Standardized Direct Effects ……………………........................ 314

Table 71. Model 2 Standardized Indirect Effects..................................................... 314

Table 72. Summary of Model 2 Findings ………….............................................. 315

Table 73. Model Goodness of Fit Statistics for Path Model 3 ................................... 316

Table 74. Model 3 Regression Weights ................................................................... 316

Table 75. Model 3 Standardized Regression Weights ............................................... 316

Table 76. Model 3 Squared Multiple Correlations.................................................... 316

Table 77. Model 3 Standardized Total Effects.......................................................... 317

Table 78. Model 3 Standardized Direct Effects ....................................................... 317

Table 79. Model 3 Standardized Indirect Effects...................................................... 317

Table 80. Summary of Model 3 Findings …………............................................. 318

Table 81. Model Goodness of Fit Statistics for Path Model 4 ................................... 318

Table 82. Model 4 Regression Weights ............................................................... 319

Table 83. Model 4 Standardized Regression Weights ............................................... 319

Table 84. Model 4 Squared Multiple Correlations..................................................... 319

Table 85. Model 4 Standardized Total Effects......................................................... 319

Table 86. Model 4 Standardized Direct Effects ..................................................... 319

Table 87. Model 4 Standardized Indirect Effects..................................................... 320

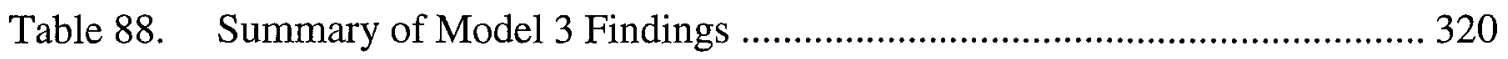

Table 89. GOF for Model 5 - Impact of Age and No. of Units ................................ 322 


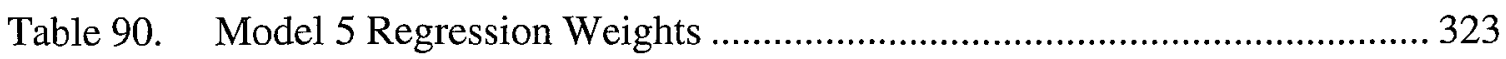

Table 91. Model 5 Squared Multiple Correlations...................................................... 323

Table 92. Model 5 Standardized Total Effects......................................................... 323

Table 93. Model 5 Standardized Direct Effects ...................................................... 323

Table 94. Model 5 Standardized Indirect Effects...................................................... 324

Table 95. GOF for Model 6a \& 6b (PWR vs. BWR) ............................................. 325

Table 96. Model 6a,b Comparison (assumes structural intercepts correct) ............... 326

Table 97. Model 6a,b Means (assumes structural intercepts equal)........................... 326

Table 98. Model 6a,b Regression Weights (structural intercepts equal)..................... 327

Table 99. Model 6a,b Squared Mult. Correlations (structural intercepts equal) ........ 327

Table 100. Model 6a,b Stand. Total Effects (structural intercepts equal)................ 327

Table 101. Model 6a,b Stand. Direct Effects (structural intercepts equal) .............. 327

Table 102. Model 6a,b Stand. Indirect Effects (structural intercepts equal)............ 327

Table 103. Summary of Model 5 and 6a,b Findings ............................................... 328

Table 104. Model GOF Statistics for Model 7 ………........................................ 330

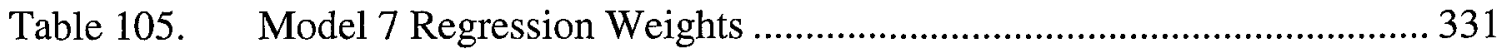

Table 106. Model 7 Squared Multiple Correlations.................................................... 331

Table 107. Model GOF Statistics for Model 7 ………......................................... 332

Table 108. Model 8 Regression Weights …………........................................... 333

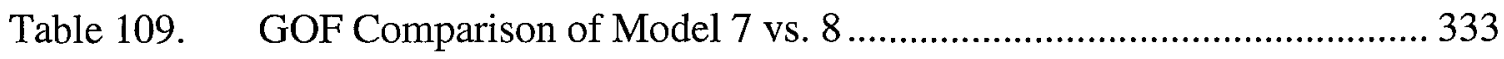

Table 110. Model 7 \& 8 Squared Multiple Correlations............................................ 334

Table 111. Summary of Model 7 Findings …………............................................. 334

Table 112. Model Goodness of Fit Statistics for Path Model 9a ............................ 338

Table 113. Model Goodness of Fit Statistics for Path Model 9a and 9b................. 340

Table 114. Model 9a,b Regression Weights.............................................................. 341

Table 115. Model 9 Squared Multiple Correlations................................................... 341

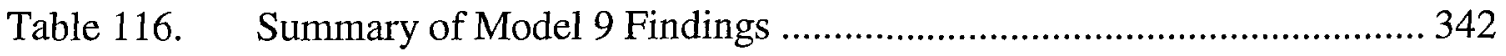

Table 117. Model GOF Statistics for Model 10 ................................................... 345

Table 118. Model 10 Regression Weights ............................................................... 346

Table 119. Model 10 - Squared Multiple Correlations ............................................. 346

Table 120. Summary of Model 10 Findings ………................................................ 347 


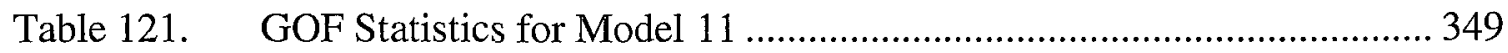

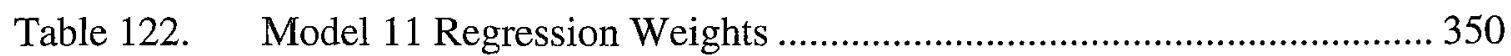

Table 123. Model 11 Squared Multiple Correlations............................................... 351

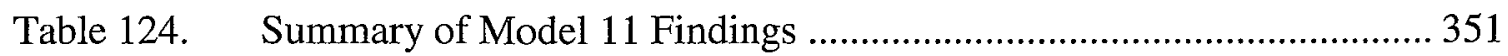

Table 125. Model GOF Statistics for Model 12 ………………………............... 353

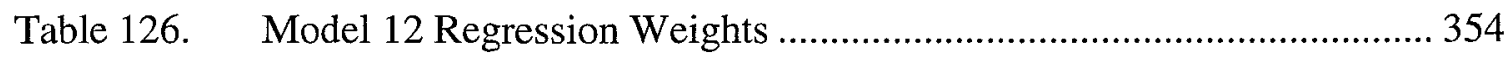

Table 127. Model 12 - KMP Means ................................................................... 355

Table 128. Model 12 Squared Multiple Correlations.............................................. 355

Table 129. Summary of Model 12 Findings …………………………………..... 356

Table 130. Model 12 Standardized Effects ....................................................... 356

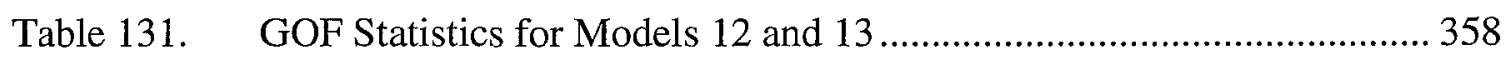

Table 132. Model 12 \& 13 Regression Weights .................................................... 358

Table 133. Model 12 \& 13 Squared Multiple Correlations........................................ 359

Table 134. Multi-Group Analysis of Models 14a,b (KMP High vs. Low) ............... 361

Table 135. Model 14a,b Comparison (assume structural intercepts correct) ........... 362

Table 136. Model 14a,b Means (structural intercepts equal) .................................... 362

Table 137. Model 14a,b Regression Weights (structural intercepts equal).............. 362

Table 138. Model 14a,b Squared Mult. Correlations (structural intercepts equal).. 363

Table 139. Model 14a,b Stand. Total Effects (structural intercepts equal).............. 363

Table 140. Model 14a,b Stand. Direct Effects (structural intercepts equal) ............ 363

Table 141. Model 14a,b Stand. Indirect Effects (structural intercepts equal).......... 363

Table 142. Multi-Group Analysis - Models 15a,b (SOC High vs. Low) ................. 366

Table 143. Model 15a,b Comparison (assuming structural weights correct)........... 367

Table 144. Model 15a,b Means for SOC High \& Low (structural weights equal) .. 367

Table 145. Model 15a,b Regression Weights (structural weights equal)................. 367

Table 146. Model 15a,b Squared Mult. Correlations (structural weights equal) ..... 368

Table 147. Model 15a,b Stand. Total Effects (structural weights equal for both) ... 368

Table 148. Model 15a,b Stand. Direct Effects (structural weights equal) ............... 368

Table 149. Model 15a,b Stand. Indirect Effects (structural intercepts equal).......... 368

Table 150. Model 16a - GOF Statistics.............................................................. 372

Table 151. Model 16a - Regression Weights ………………………………….... 372 


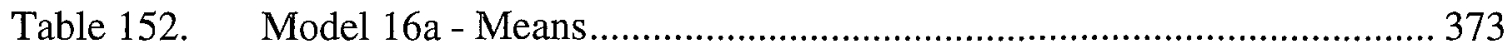

Table 153. Model 16a - Intercepts................................................................... 373

Table 154. Model 16a - Squared Multiple Correlations........................................... 373

Table 155. Total Effects - Model 16a (non-standardized values) …......................... 374

Table 156. Direct Effects - Model 16a (non-standardized values) .......................... 374

Table 157. Indirect Effects - Model 16a (non-standardized values) ......................... 374

Table 158. GOF Statistics for Model 16a,b.......................................................... 376

Table 159. Model 16b Regression Weights ........................................................... 376

Table 160. Model 16b Squared Multiple Correlations............................................. 377

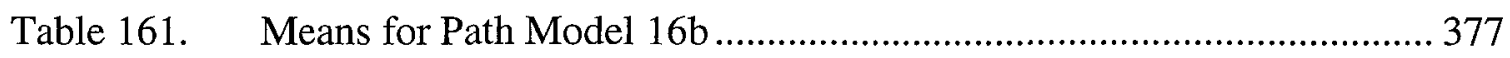

Table 162. Model 16b Intercepts for Operational Performance Factors ................... 378

Table 163. Total Effects - Model 16b (non-standardized values)............................. 379

Table 164. Direct Effects - Model 16b (non-standardized values) .......................... 379

Table 165. Indirect Effects - Model 16b (non-standardized values)........................ 379

Table 166. Summary of Findings by Hypothesis .................................................... 382

Table 167. Relationships Found But Not Hypothesized ........................................... 396

Table 168. Typical NPP Production Price and Value ............................................... 404

Table 169. Total Effect of KMPs/OTSs on Raw Performance Factors ................... 404

Table 170. Predicted Effect of Unit Scale Increase of KMPs/OTSs (MWh/d)....... 404

Table 171. Predicted Effect of Unit Scale Increase of KMPs/OTSs in M\$year ..... 404

Table 172. Prediced Production Benefit (ignoring PMP effect) ............................... 405

Table 173. Indep. Samples T-test - Responding vs. Non-Responding NPPs........... 411

Table 174. Responding vs. Non-Responding Group Statistics ................................. 411

Table 175. Summary of Participating Reactor Units .............................................. 537

Table 176. Index of Variable (and Construct) Names Used in Scatter-plots........... 543 


\section{LIST OF FIGURES}

Figure 1. Knowledge Viewed as a Resource, Process or Asset............................. 17

Figure 2. Knowledge Pyramid (Bollinger and Smith, 2001) .................................. 20

Figure 3. OL Key to Maintaining Knowledge-Base (Adapted from Senge, 1999) ..... 23

Figure 4. OL Processes (adapted from Koorneef, 2000) ........................................ 26

Figure 5. Knowledge Transfer Processes (Nonaka and Takeuchi, 1994) ................. 39

Figure 6. Summary of all NPPs by start-up date (WANO, 2010) ........................... 73

Figure 7. NPP as Knowledge-Driven Enterprise (adapted from Wiig, 1999) ............ 74

Figure 8. The NPP Integrated and Shared Knowledge Base ............................... 76

Figure 9. Viewing Work Processes from a KM Perspective ................................. 78

Figure 10. US NPP “Scram” Rates (Nuclear News, June 2009) ............................. 84

Figure 11. US Fleet Refueling Outage Days (NEI Website, 01/11)....................... 84

Figure 12. US Fleet Capacity Factor (NEI Website, Jan/11) .............................. 85

Figure 13. World Avg. Energy Availability Factors (IAEA, '99) .......................... 92

Figure 14. Avg. Planned \& Unplanned Energy Unavailability (IAEA, '99) ........... 94

Figure 15. Planned \& Unplanned EUF by Reactor Type (IAEA, '99)...................95

Figure 16. KM for Competitive Advantage (Nonaka and Takeuchi, '95)............... 97

Figure 17. Knowledge Asset Value Spiral (Carlucci1 \& Schiuma, '06)............... 100

Figure 18. KM Benefits Chain (adapted from Wiig, 1997).............................. 102

Figure 19. Nuclear Plant Life-cycle (Olmstead and Didsbury, 2002).................. 107

Figure 20. Alignment of Knowledge Processes (adapted from IAEA, '06b)......... 108

Figure 21. The Standard Nuclear Performance Model (SNPM) (NEI, 2003) ........ 110

Figure 22. IS/IT System Success Model (adapted, DeLone \& McLean, '92)........ 125

Figure 23. Synergy and Feedback Effect of IT/IS Support ................................. 126

Figure 24. The "Knowledge Management Performance Model" (KMPM) ........... 132

Figure 25. The NPP Knowledge Management Performance Model ..................... 138

Figure 26. Links to Supportive Organizational Culture .................................... 141

Figure 27. Framework for KM (Shankar and Gupta, 2005) ............................. 143

Figure 28. The KM/KMS Value Chain (Lee \& OuYang, ' 10 ) ........................... 145 
Figure 29. Expected Value-Chain Links Amongst the QKPs and with OE ............ 147

Figure 30. Hypothesized Links Between KMS and QKPs...................................... 148

Figure 31. Hypothesized Links Between SOL and QKPs.................................... 149

Figure 32. Hypothesized Links Between HRP and QKPs.................................... 149

Figure 33. Hypothesized Links Between TRP and QKPs ..................................... 150

Figure 34. Hypothesized Links Between IMP and QKPs ……………................. 151

Figure 35. Hypothesized Links Between IST and QKPs .................................... 151

Figure 36. Hypothesized Links Between OSS and QKPs ....................................... 152

Figure 37. Hypothesized Links Between PMP and QKPs ................................... 152

Figure 38. Hypothesized Links Between OPM and QKPs..................................... 153

Figure 39. Possible PMP and OPM Links to OE and OP.................................... 154

Figure 40. Hypothesized Links for KMPs and QKPs with SOC............................ 155

Figure 41. Primary Knowledge Process Sub-Constructs......................................... 165

Figure 42. Personalized IAEA E-mail to Station Managers ...................................... 213

Figure 43. Summary of all NPPs by reactor type ................................................. 220

Figure 44. Summary of all NPPs by output rating (MWe)................................. 220

Figure 45. Summary of all NPPs by start-up date ………………………………. 221

Figure 46. No. of Responding NPPs by country ………………………………... 223

Figure 47. Responding NPPs by Reactor Type and Country …………................. 223

Figure 48. Breakdown of responding NPPs by plant output (MWe) ...................... 225

Figure 49. Breakdown of responding NPPs by no of units at site........................... 225

Figure 50. No. of NPPs by Operator by Age (yrs from start-up) ............................ 226

Figure 51. No. of Responding NPPs by Operator and Output (MWe).................... 226

Figure 52. No. of Responding NPPs by Operator and Plant Type .......................... 227

Figure 53. No. of responding NPPs by operator.................................................. 228

Figure 54. No. stations by no. of employees ………………………………...... 228

Figure 55. No. of stations by FTE contractors in outage .......................................... 229

Figure 56. No. of stations by FTE contractors at power....................................... 229

Figure 57. No. Station by $\%$ of staff university grads .......................................... 230

Figure 58. No. of NPPs by COP total-score ..................................................... 231

Figure 59. No. of NPPs by Age (in years since start-up) ……............................... 231 
Figure 60. NPP Output (MWe) by Reactor Type ……....................................... 232

Figure 61. No. of NPPs by no. of responding managers ....................................... 233

Figure 62. Responding NPPs - Raw \& Indexed Op'l Performance Histograms..... 236

Figure 63. Responding NPPs by performance (normalized index) ......................... 237

Figure 64. Example of possible NPP fleet configuration ....................................... 242

Figure 65. Example of residuals plotted by response category ............................... 250

Figure 66. Scatterplot - residuals for OPM+HRP+OSS $\rightarrow$ KA.............................. 267

Figure 67. KMP and OTS Links to Quality of KA Processes .................................. 268

Figure 68. Residuals for HRP+IMP+SOL $\rightarrow$ KS .................................................. 269

Figure 69. KMP Links to Quality of KS Processes ................................................. 270

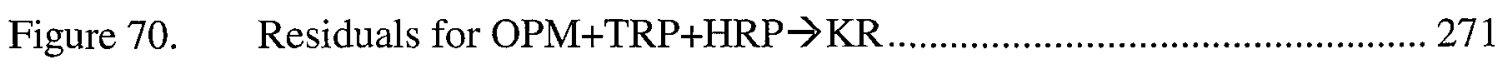

Figure 71. KMP Links to Quality of KR Processes .............................................. 272

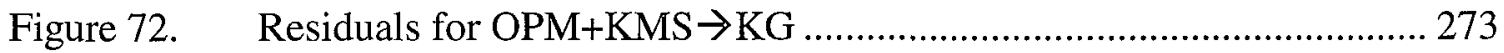

Figure 73. KMP Links to Quality of KG Processes ................................................ 273

Figure 74. Residuals for IMP+HRP+IST $\rightarrow$ KU ……......................................... 274

Figure 75. KMP and OTS Links to Quality of KU Processes................................. 275

Figure 76. Residuals for IMP+HRP+IST+SOL+KMS $\rightarrow$ SOC ................................ 276

Figure 77. KMP and OTS Links to Supportive Organizational Culture (SOC)..... 276

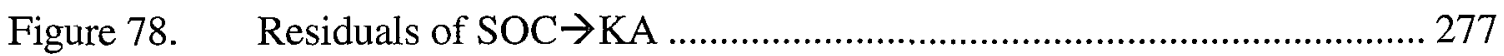

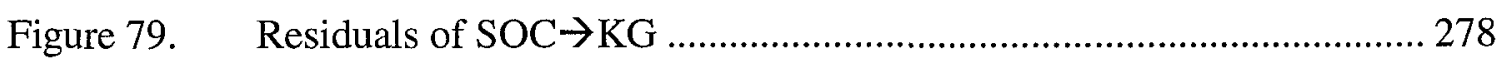

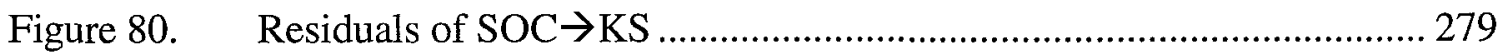

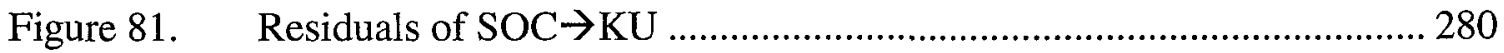

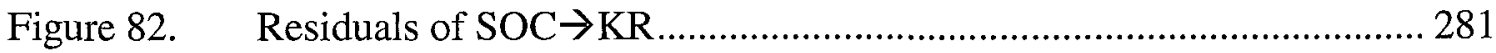

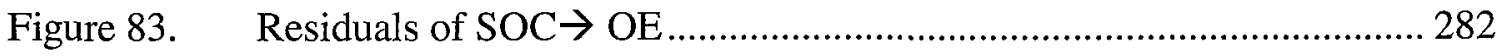

Figure 84. Supportive Organizational Culture Links to QKPs and OE................... 282

Figure 85. Residuals of $\mathrm{KG}+\mathrm{KS}<-->\mathrm{KA}$........................................................... 285

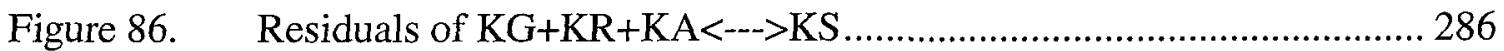

Figure 87. Residuals of KS+KU<--->KR ....................................................... 287

Figure 88. Residuals of $\mathrm{KA}+\mathrm{KS}+\mathrm{KU}<--->\mathrm{KG}$..................................................... 289

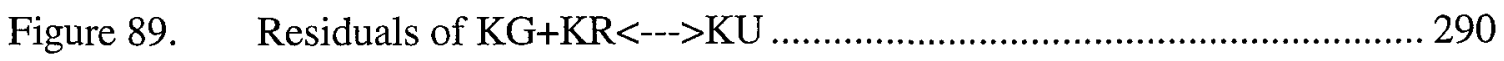

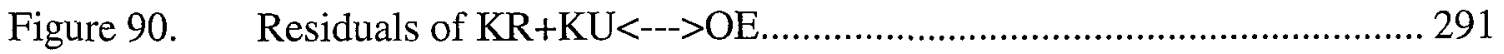


Figure 91. Prelim. Findings - Links Among QKPs and to OE ............................... 292

Figure 92. Residuals of KMS+PMP+IMP+IST $\rightarrow$ OE .............................................. 294

Figure 93. Possible KMP Links to Organizational Effectiveness ............................ 295

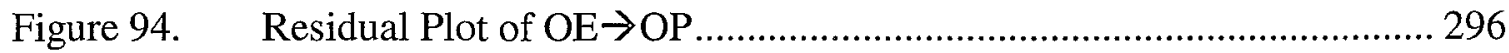

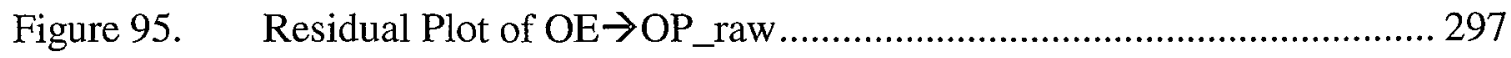

Figure 96. Mean OP_raw \& Mode OP vs. OE ...................................................... 298

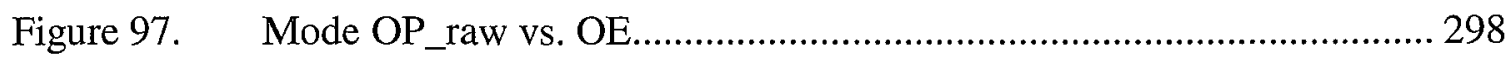

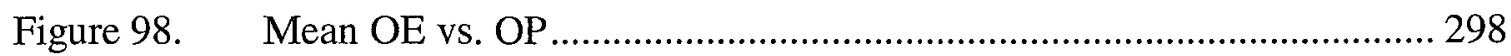

Figure 99. Path Diagram for Model 1................................................................. 309

Figure 100. Path Diagram for Model 2 ................................................................. 313

Figure 101. Path Diagram for Model 3............................................................. 316

Figure 102. Path Diagram for Model 4............................................................. 318

Figure 103. Model 5 - Impact of Age and No. of Units on OE \& OP ...................... 322

Figure 104. Model 6a,b - PWR vs. BWR Plants ..................................................... 325

Figure 105. Path Diagram for Model 7 .................................................................. 330

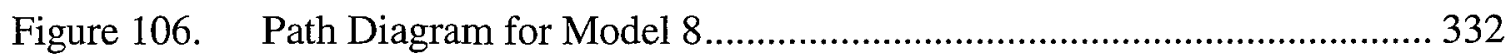

Figure 107. Path Diagram for Model 9a (with $\mathrm{OPM} \rightarrow \mathrm{KR}$ ) ……………………....... 338

Figure 108. Path Diagram for Model 9b (without OPM $\rightarrow \mathrm{KR}$ ) ................................. 340

Figure 109. Path Diagram for Model 10.................................................................. 344

Figure 110. Path Model 11 .............................................................................. 349

Figure 111. Path Diagram for Model 12 .............................................................. 353

Figure 112. Path Diagram for Model 13 ................................................................ 357

Figure 113. Model 14a: KMP High - Structural Weights \& Intercepts Equal ......... 360

Figure 114. Model 14b: KMP Low - Structural Weights \& Intercepts Equal........... 361

Figure 115. Model 15a: SOC High (structural weights assumed equal) …………... 365

Figure 116. Model 15b: SOC Low (structural weights assumed equal)....................366

Figure 117. Path Model 16a: Full Model with Raw Performance Measures ........... 371

Figure 118. Path Model 16b: Direct KMP \& OTS Effects on Raw OP Measures... 375

Figure 119. Core NPP Knowledge Process Model.................................................... 397

Figure 120. Operational Performance Indicators (Typical Values in \%) .................. 531 


\section{LIST OF PATH DIAGRAMS}

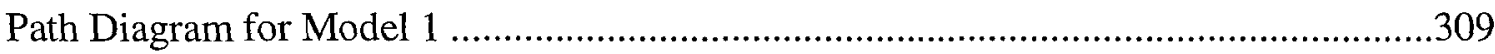

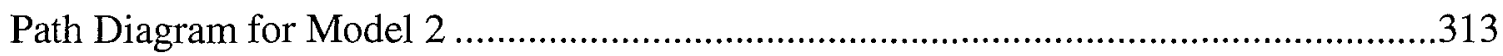

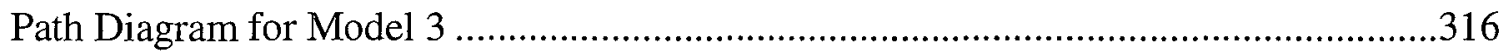

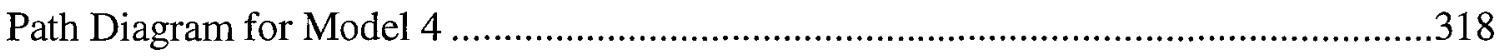

Path Diagram for Model 5 - Impact of Age and No. of Units on OE \& OP ..................322

Path Diagram for Model 6a and 6b - PWR vs. BWR Plants.......................................325

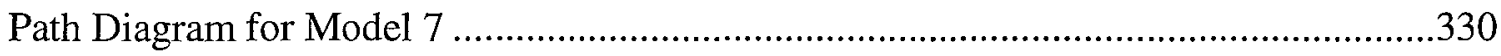

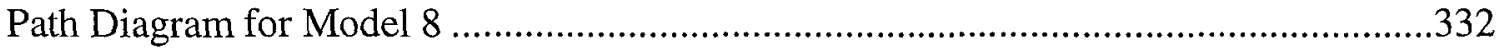

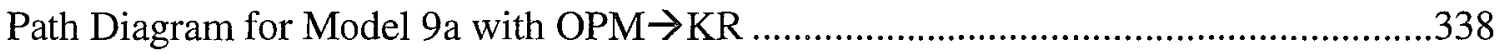

Path Diagram for Model 9b without OPM $\rightarrow$ KR .......................................................340

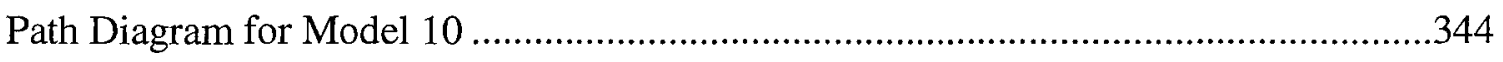

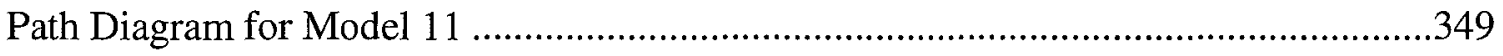

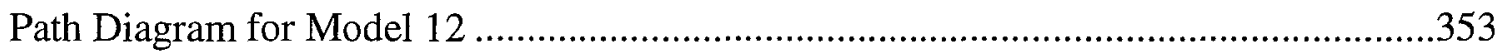

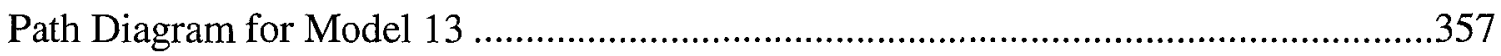

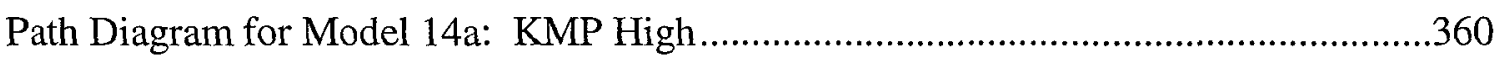

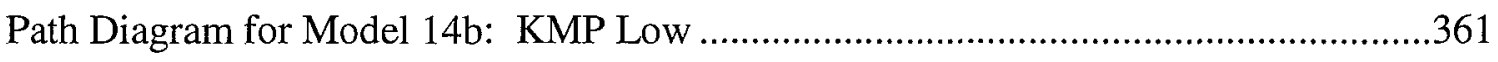

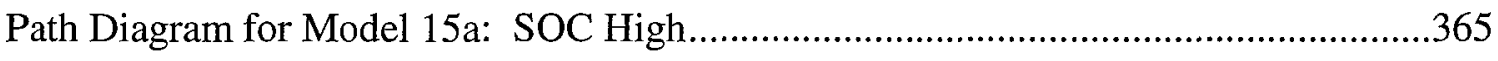

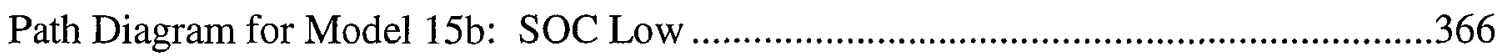

Path Diagram for Model 16a: Full Model with Raw Performance Measures ................371

Path Diagram for Model 16b: Direct KMP Effects on Raw Performance Measures .....375 


\section{LIST OF APPENDICES}

APPENDIX A. English Version of Survey Instrument ..............................................445

APPENDIX B. Russian Translation Survey Instrument ........................................... 460

APPENDIX C. Chinese Translation of Survey Instrument........................................ 479

APPENDIX D. French Translation of Survey Instrument....................................... 512

APPENDIX E. Operational Performance Indicators ............................................... 530

APPENDIX F. Summary of Stations Participating …………………………...... 537

APPENDIX G. Bivariate Scatterplots - All Measures ............................................. 543 


\section{Introduction}

It is argued that in today's business environment, traditional factors of production (land, labour, and capital) are insufficient to maintain competitive advantage (Allee, 1997). Several author's maintain that sustained business performance can only be achieved when knowledge (particularly core competency) is applied with a strategic business focus (Drucker, 1994; Klein and Prusak, 1994; Quinn, 1992). Quinn (1992) argues that the majority of organizations today are shifting focus to become repositories of knowledge and coordinators of knowledge-based activities. Managing and leveraging the contribution of human intellect to business activities and functions and converting this to useful products and services is seen to be a critical success factor (Quinn et al. 1996). However, although many researchers identify and claim a link between organizational knowledge and sustained business performance, other authors point out that empirical research to establish the linkage and the specific mechanisms by which it is achieved is clearly lacking (Feng, et al., 2004; Darrock, 2005; Carneiro, 2000).

In the specific context of the nuclear industry, nuclear power plants (NPPs) are strong examples of knowledge driven organizations operating in a technology intensive industry. NPPs and their associated supporting industrial infrastructure (e.g. nuclear suppliers, research and design organizations, nuclear regulators, technical support organizations, etc.) collectively incorporate advanced technical knowledge representing billions of dollars of research and development invested over decades. The ongoing successful operation and maintenance of this industrial infrastructure and its associated 
nuclear facilities demands the continuous availability of high levels of expertise in many disciplines ranging from (for example) material science, to physics, to engineering, to chemistry, to thermohydraulics, to safety analysis, to instrumentation and control. It also requires highly trained and skilled management, operations and maintenance staff. Maintaining this knowledge base is essential to the success and safety of the industry.

Over the past two decades, the nuclear industry has faced some unique challenges. The unfortunate and very serious accidents at Three Mile Island (1979), Chernobyl (1986), and more recently Fukushima (2011) drastically undermined public confidence in the industry. Such events create a political environment where further government investment in nuclear infrastructure is difficult to support. As a result, for the two decades following Three Mile Island and Chernobyl events, investment in nuclear energy declined globally. Until only recently, there was very little interest in, or prospect of, investment in new reactor builds, but this renewed interest may quickly wane in the wake of the Fukushima incident. Interest in and funding for university programs and postgraduate research in nuclear related disciplines had also dwindled dramatically in most countries through the 1990's (IAEA, 2004) and only since the turn of the century has there been a slight upturn. Over the same period, the average age of the workforce at most nuclear plants has been increasing and knowledge loss due to retirements has become a real challenge (IAEA, 2004). The demand for nuclear knowledge workers across the industry is becoming more acute due to the following two factors:

i.) the existing fleet of NPPs is reaching the end of the its design life (i.e. approximately 30 years on average) and additional specialized skills are 
needed for their refurbishment (i.e. for life-extension) and continued operation, and

ii.) there is an increase in new reactor construction and commissioning in some countries (e.g. China) to meet growing energy needs and this is creating demand for experienced nuclear workers.

Thus just as many experienced nuclear specialists are retiring across the industry globally, very few new graduates are available in most countries with specialized skills needed to replace them, and the demand for these specialized skills is increasing. The combined effect of these industry-wide circumstances have created heightened concern in NPP organizations. There is a recognized need to more carefully establish and maintain (i.e. actively manage) the availability of critical core knowledge and competencies, including specialized nuclear skills and the organizational experience base. This has given rise to increased awareness of the strategic importance of knowledge management in operating NPPs in general.

\subsection{Background}

Nuclear power plants provide about $17 \%$ of the world's electricity production (IAEA, 1999). Developed industrialized economies have grown to rely heavily on the continuous availability of a reliable supply of affordable electricity. The achievement of sustained high operational performance in terms of production reliability, safety, and cost is expected and required. This requires good management, reliable equipment, competent 
employees, and effective processes. However, NPPs are also large and complex sociotechnical systems. They must operate highly technical plant systems and equipment to very high performance standards, and in a cost-effective manner. Thus NPP's rely heavily on building and maintaining a strong organizational knowledge base to achieve these goals. This research investigates the role of KM in the NPP organizational context. This section provides a brief background on the subject of knowledge management and explains why it is an issue of much importance and concern in nuclear power plants. It also outlines the research objectives and approach and highlights the need for this work.

In knowledge-intensive organizations, the process of assimilation and institutionalization of expertise and know-how becomes a key challenge important to the success of the firm. With ever changing business needs and priorities, regular employee mobility and attrition, advances in technology and the challenges of plant life management, organizational knowledge must be developed and renewed on an ongoing basis. The more knowledge-intensive an organization is, the more its success will be dependent on employee learning and competence, and the effective utilization of organizational knowledge. Workers in knowledge intensive organizations can be seen as active participants in the ongoing process of interpreting or making sense of information and events and making decisions and applying resources to advance the firms goals (Daft and Weick, 1984).

Effective knowledge management enhances a firm's capability to assimilate, generate and exploit knowledge and is widely recognized, both in the management literature and 
practice, as an important driver of long-term performance and a necessary requirement for sustained commercial success, particularly in technology intensive industries (Brockman and Morgan, 2003; Thomas and Keithley, 2002; Nelson and Cooprider, 1996; Jie, 2001; Hall, 2002; De Gooijer, 2000). There is a strong interest (and belief) in the promise that knowledge management practices, if effectively applied to nuclear power plants, will result in performance improvement.

However, introducing and maintaining effective knowledge management in any knowledge-intensive organization is difficult (Chen Liu, 2003). A nuclear plant is no exception, and offers its own unique challenges. Nuclear utilities are making progress towards the implementation of knowledge management practices and systems (IAEA, 2006) but in general progress lags other industries and marginal benefits are being obtained (IAEA, 2004). Further, nuclear utilities are often not able to determine the effectiveness of KM practices that have been implemented, and in many cases are not able to properly identify key areas in need of improvement (IAEA, 1999).

The planning, implementation, and maintenance of an effective knowledge management system is challenging (Chen Liu, 2003). Nuclear facilities have unique organizational attributes and constraints that make the problem all the more difficult (IAEA, 2006). NPP managers need sound guidance and insights along with practical methods to take full advantage of $\mathrm{KM}$ principles in their organizations. Both academic literature and industry experience are weak in providing guidance in this industry (IAEA, 2006). This thesis explores two central questions of interest to managers that are yet unanswered. 
The first is "what effect does management support for KM practices have on NPP organizational effectiveness"? The second is "can a link be established between management support for KM practices and plant operational performance"? The following section elaborates further on these research objectives, presents an overview of the research approach, summarizes the expected contribution of the research, and provides an outline of the organization of the document.

\subsection{Research Objectives}

Effective knowledge management enhances an organization's capability to build on, maintain, and fully utilize its existing knowledge base (Sasson and Douglas, 2006; Starns and Odom, 2006). It is argued that the role of $\mathrm{KM}$ is particularly important in the technology-intensive nuclear industry (Beraha et al., 2005). Further, it has been suggested that knowledge management practices focused on building and maintaining human competencies in nuclear power plants enable operational and safety performance improvements, including reductions in operational and personnel safety risk (Bhattacharya, 2005, Gharib, 2005). For these reasons, various nuclear power plants (NPPs) around the globe have begun to recognize the strategic importance of KM initiatives in achieving overall operational performance.

Although awareness of the need to manage knowledge and ensure its effectiveness in the NPP organizational context varies from plant to plant, some NPPs have been early adopters of $\mathrm{KM}$ thinking and practice, and have been proactive in implementing $\mathrm{KM}$ initiatives (IAEA, 2006). However, at other NPPs the concepts and benefits of KM are 
only beginning to be understood and applied. The various approaches taken to KM and the relative benefits obtained from $\mathrm{KM}$ initiatives are expected to vary from station to station. More research is needed to fully understand what $\mathrm{KM}$ practices have successfully been applied in NPPs, the extent of their benefits, and in general, how management can benefit from KM.

Although a large number of qualitative and conceptual studies on the subject of KM and its impact on performance in organizations have been conducted (Ahn and Chang, 2004, Gallivan et al., 2003; Gorelick and Tantawy-Monsou, 2005; Hall, 2002; Hoffman et al., 2005; Kailing, 2003; Thomas and Keithley, 2002), very few empirical studies have been reported in the literature that directly explore the relationship (Syed-Ikhsan and Rowland, 2004; Keskin, 2005; Lee et al., 2005; Lewis, 2004). In general, the issue has not been extensively researched and is not well understood. Further, no empirical research appears to have been done linking KM to operational performance in the specific context of NPPs (Lowthert, 1996).

Several factors are thought to influence the effectiveness of $\mathrm{KM}$ initiatives in improving NPP performance. Organizational culture (including safety culture) is thought to be important (Jennex and Olfman, 2002). Other issues, such as the extent to which $\mathrm{KM}$ practices are aligned with and support operational best practices are also thought to be significant (Lowthert, 1996). For example, life-cycle equipment maintenance and configuration management of plant design basis information are both areas where $\mathrm{KM}$ 
initiatives are thought to have a positive impact (IAEA, 1999). However, there is a lack of empirical evidence to support these claims.

The objective of this research is to obtain a deeper understanding of KM in the NPP context and in specific to investigate and establish the relationships between support for $\mathrm{KM}$ practices and their overall impact on organizational performance. By gaining insights into what specific knowledge management strategies and tactics are effective, by what mechanisms (e.g., knowledge processes) this occurs, and what benefits are achieved, much-needed guidance can be provided to help NPP managers maximize the benefit from $\mathrm{KM}$ initiatives in future.

\subsection{Research Approach}

Bailey (1982, p54-55) outlines the classical approach to research as involving work at both the conceptual and the empirical level through three steps: step one is at the conceptual level and involves the development of concepts and construction of research propositions; step two bridges the gap between conceptual and empirical levels and involves the development of concept measures and specific hypotheses; and step three is at the empirical level and involves the actual administration of the measurements on a sample of the target population and the subsequent analysis of data to determine whether

the hypothesized relationships hold. This research is an applied research study and follows the classical approach as described by Bailey. It has been conducted in the form of a formal empirical explanatory study. 
A literature review has provided a theoretical basis for the construct and model development. Specific research questions, a theoretical framework and research model, and hypotheses are developed. Specific measures are derived from the literature as a basis for the survey instrument. Findings from the quantitative (empirical survey) research are interpreted in light of the literature review findings. Established statistical methods are employed to analyze the data. The research methodology, including design issues related to the survey instrument development, validity and reliability issues, sources of error, sampling frame, data collection procedures and statistical methodology are described in detail.

\subsection{Contribution}

Limited empirical research has been published on the determinants of NPP performance. A extensive literature search revealed no formal empirical research appears to have been conducted on the link between KM and NPP performance. NPPs are difficult organizations for researchers to obtain access to. Since the tragic September 11, 2001 terrorist attacks on the World Trade Center in New York, and the resulting heightened security measures that have been put in place at nuclear facilities, access to nuclear power plant organizations has become even more restricted. Despite the obvious interest from and need for NPP managers to understand and be able to influence and improve knowledge and learning processes in their organizations, a consistent theoretical basis and meaningful guidance for practitioners on the subject is lacking. Although a limited amount of informal and qualitative industry (practitioner) research has been 
carried out by organizations such as the IAEA, very few academic publications address this topic, and they are limited to case study research of individual NPP organizations or qualitative research on single dimensions of KM (e.g. tacit knowledge retention or knowledge sharing). This research advances understanding of the application and benefit of KM in NPPs in several ways:

- It provides a better understanding of what KM methods and practices are currently being applied in NPPs and to what extent these practices vary from plant to plant. There is little or no empirical research on this topic to date.

- It provides a better understanding of how mature KM practices in NPPs are. Answers to questions such as whether or not many NPPs have implemented KM at a strategic level and if and how successfully they have implemented specific $\mathrm{KM}$ practices have not been previously researched.

- It provides a better understanding of the perceived quality of knowledge processes in NPPs. An improved understanding of what specific aspects of knowledge processes (i.e., knowledge acquisition, knowledge generation, knowledge transfer, knowledge retention, and knowledge utilization) are seen to be strengths or weaknesses in NPPs, and whether or not any specific trends or patterns exist (e.g., is there a high degree of variability within the industry?).

- It provides a fuller understanding of the extent of use of information systems and technology (IST) in NPPs and its impact on the quality of knowledge processes. Although research work as been done on various aspects of this relationship, measures are not well developed in the literature and little (possibly no) prior 
research has been done to investigative this relationship in operating nuclear plant organizations.

- It provides a better understanding of the importance and influence of a supportive organizational culture and how it effects the impact of $\mathrm{KM}$ practices on knowledge processes and ultimately organizational performance. Prior research has been done on NPP organizational culture, but little of it has been empirically based, and it is primarily focused on safety culture. A fuller understanding of the linkages between $\mathrm{KM}$, organizational culture, and organization performance in NPPs is derived.

- It provides a better understanding of the key elements of NPP organizational performance. Empirical research on NPP performance has been limited to a handful of studies correlating only published production performance indicators to factors such as reactor age and plant type. Although data on key performance indicators (KPI's) exists from active NPP community of practice workgroups from countries such as in the U.S.A, it is not publicly available information. Further, only qualitative research appears to have been published to date on NPP organizational effectiveness. This research helps to fill an important void by contributing to the understanding of organizational performance in NPPs.

- It provides a deeper understanding of the link between $\mathrm{KM}$ practices and organizational performance in NPPs. Although there exists a large volume of qualitative research linking $\mathrm{KM}$ to organizational performance in the management literature in general, only a few empirical studies exist. Further, 
much of the current research has limited applicability to NPP organizations due to the distinct characteristics of NPPs. This research advances understanding of this relationship and provides deeper insights into how KM contributes to NPP performance.

- It provides a better understanding of the effect of the quality of knowledge processes in NPPs and their impact on organizational effectiveness. No prior research frameworks existed to explain NPP performance this perspective. This research builds on research from academia and industry to explain the mechanisms by which KM practices (aimed at improving knowledge processes) influence organizational effectiveness and overall operational performance.

- The measurement instruments developed for this research will contribute to the ongoing assessment of KM practices, knowledge processes, and NPP performance as both industry level benchmarking tools and as an organization level self-assessment tool. The potential use of the measurement tools over time for benchmarking can generate needed empirical data for a deeper understanding of $\mathrm{KM}$ across the industry.

- Finally, this research helps to identify key issues and questions in need of further research and contributes to both the theoretical and empirical basis for it.

\subsection{Outline}

The next chapter (Chapter 2) provides a summary of a review of current literature on the subject. Chapter 3 presents the theoretical framework for the research, describes the 
research model, presents various research hypotheses that are derived from it, and summarizes the development of the research constructs and their empirical measures. Chapter 4 explains the research methodology (both qualitative and quantitative), the sampling frame, the survey instrument development, the data collection approach, the response rate and the statistical and analytical methods employed. Chapter 5 provides descriptive statistics for the entire NPP population and the survey respondents. Chapter 6 summarizes the data entry and preparation process and methods including data quality and screening, assessment and handling of missing data, assessment and handling of outliers, reliability screening of measures, and construct reliability assessment. Chapter 7 describes the findings from linear regression assessments. Chapter 8 describes the findings from path analysis assessment. Chapter 9 discusses the finding and research implications. Chapter 10 provides a summary, discusses the limitations of the research, and identifies issues and provides recommendations for possible future research. 


\section{Literature Review}

This chapter presents an overview of some of the key literature related to the subject of knowledge management. It begins by reviewing the basic definitions of knowledge and knowledge processes, and goes on to explain relevant theories regarding organizational learning (OL) and its relationship to knowledge processes. Next, the specific literature on knowledge management is discussed. The various knowledge management perspectives are presented, including the resource-based and process-based views of the firm and the concept of intellectual capital. A discussion of KM practices and KM measurement approaches follows. Literature on organizational performance and the role $\mathrm{KM}$ plays in achieving it are then explored in detail. This provides the background for a review and discussion of findings in the literature that apply in the context of NPP management, and in particular to the issue of the role of $\mathrm{KM}$ in the achievement of operational performance. Finally, the nature and importance of organizational culture is investigated as a facilitator and enabler that moderates the effect that $\mathrm{KM}$ practices have on the quality of knowledge processes and the subsequent effects both have on organizational performance.

Several authors have pointed out the significance of a gradual shift in the management literature over recent decades from a view of firms achieving competitive advantage through optimization of production (and the production function) to a view of achieving competitive advantage based on differentiation of resources (such as their value or rareness, or how easily they might be imitated or substituted) (Wernerfelt, 1984; 
Barney, 1991, Grant, 1996). This trend was followed by an increasing shift in the literature on strategic management and organizational learning toward a view of knowledge as a distinct characteristic of a firm's core competence (Tippins and Sohi, 2003; Hamel and Prahalad, 1990). Thus it can be argued that knowledge management theory is a logical evolution of the resource-based view of organizations (i.e. with knowledge seen as a vital resource).

Knowledge management literature is closely related to and in part developed out of the literature on organizational learning, decision making, and sense making. Author's like Choo (1998) and Huber (1991) helped bridge a gap in the literature by offering integrative perspectives on knowledge processes as part of a larger organizational learning process. A knowledge-based view of organizations provides a different and value-added perspective over the conventional production-based view or the resourcebased view. Many authors taking the knowledge-based view see organizations as ultimately producers and users of knowledge (e.g., Grant, 1996; Nonaka et al., 1996; Foss, 1996). This perspective takes a system view and sees each firm as an independent knowing organization, with knowledge creation, maintenance, and application as the primary goals of the organizational system, and with knowledge as the critical resource (Choo, 1998). Before it is possible to investigate the contribution of $\mathrm{KM}$ to organizational performance, it is important to first clarify what is meant by knowledge and knowledge processes. 
There are numerous definitions of knowledge in the literature, some with quite different interpretations of what knowledge is. Table 1 provides a summary of some of these definitions and gives a flavour of their range and emphasis.

Table 1. Summary of Definitions of Knowledge

\begin{tabular}{|c|l|}
\hline Author(s) & \multicolumn{1}{|c|}{ Definition } \\
\hline Nonaka, 1994 & knowledge is a justified true belief... \\
\hline Liebeskind, 1996 & $\begin{array}{l}\text { knowledge is information whose validity has been established through } \\
\text { the tests of proof... }\end{array}$ \\
\hline Szulanski, 1996 & knowledge is what the firm knows in terms of best practices... \\
\hline Davenport and Prusak, 1998 & $\begin{array}{l}\text { knowledge is a mix of framed experience, important values, } \\
\text { contextual information, and expert insight that provides a framework } \\
\text { for evaluation and incorporation of new experiences and } \\
\text { information... }\end{array}$ \\
\hline Soliman and Youssef, 2003 & knowledge is information that is contextual, relevant and actionable \\
\hline Alavi and Leidner, 2001 & knowledge is information that is processed in the minds of individuals \\
\hline Allee, 1997 & $\begin{array}{l}\text { knowledge is a person's experience gained over time (it can be } \\
\text { communicated and shared) }\end{array}$ \\
\hline Brooking, 1996 & $\begin{array}{l}\text { knowledge is placing information in some type of context. It brings } \\
\text { information together and helps understand the know-why and know- } \\
\text { how of it }\end{array}$ \\
\hline Wiig, 1993 & $\begin{array}{l}\text { knowledge is a person's beliefs, perspectives, concepts, judgments, } \\
\text { and expectations, methodologies and know-how }\end{array}$ \\
\hline Bruner, 1973 & $\begin{array}{l}\text { knowledge is that which we come to believe and value based on } \\
\text { meaningfully organized information accumulated through experience, } \\
\text { communication or inference }\end{array}$ \\
\hline
\end{tabular}

There are also several different classifications found in the literature for types of knowledge. Knowledge can be viewed as a resource or a process (Assudani, 2005) or as an output or asset (Lev, 1997; Edvinsson and Malone, 1997; Sveiby, 1997; Stewart, 1997 Stewart, 1997; Sullivan, 2000) (see Figure 1). 


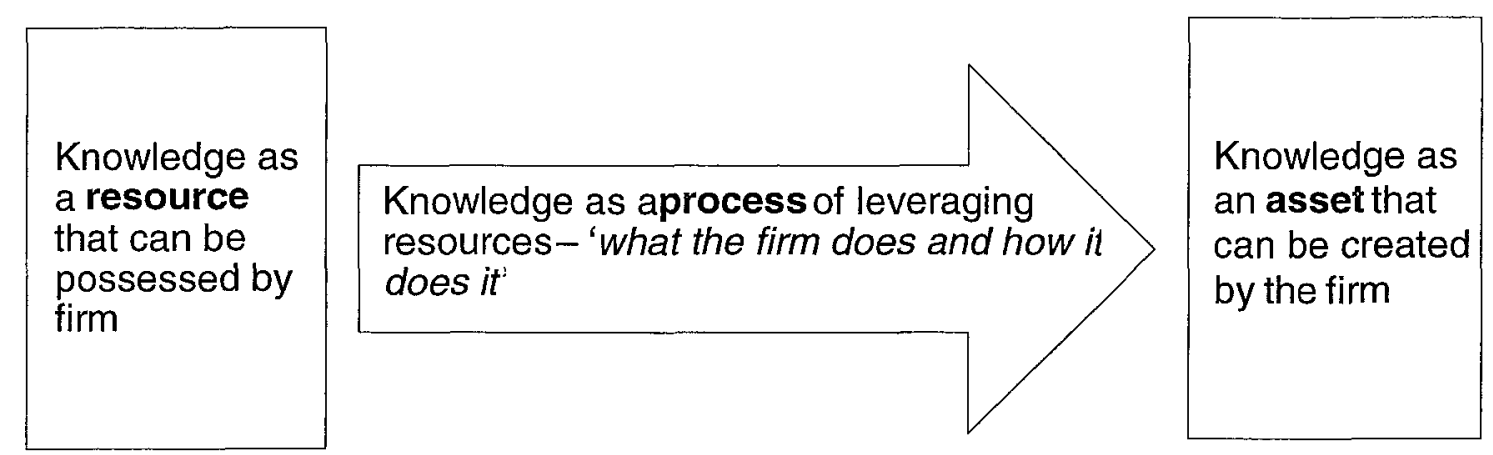

Figure 1. Knowledge Viewed as a Resource, Process or Asset

Knowledge can also be classified as: individual, group, or organization level knowledge (Hedlund, 1994; Nonaka, 1991); as tacit (i.e., internal know-how) or explicit (i.e., documented) knowledge (Nonaka and Takeuchi, 1995); as factual, conceptual, procedural, or metacognitive knowledge (i.e. knowledge about knowledge) (Anderson et al., 1998); or finally, as cognitive (know-what), skills-oriented (know-how) or understanding (know-why) (Quinn, Anderson, and Finkelstein, 1996).

The most frequent distinction of knowledge type found in the review of the literature was on the basis of tacit and explicit. Explicit knowledge is seen as knowledge that is easily expressed, captured, transferred, and codified such as organizational practices or processes that are captured as rules, instructions, routines, methods, or procedures (Polanyi, 1996; Nonaka and Takeuchi, 1995). Van Beveren (2002) refutes this view and argues that implicit knowledge does not exist and that knowledge exists only in the minds of individuals. His basic premise is that only information can be codified. Thus information is acquired and processed by the mind (i.e. interpreted) and stored in 
memory as new knowledge. Conversely, prior knowledge can be externalized by transferring it to information through language or demonstration to convey to others.

Grayson and O'Dell (1998) describe tacit knowledge as lessons learned, know-how, judgment, rules of thumb, and intuition. Wiig et al. (1977) point out that tacit knowledge accumulates over time through repeated experience and application. Polanyi (1967) defines tacit knowledge as knowledge that cannot be fully expressed by an expert and is difficult to transfer from one person to another except by similar experience. Lim (1999) characterized tacit knowledge as unarticulated knowledge that is difficult to share. Badaracco (1991) describes tacit knowledge as knowledge that is embedded in the heads of individuals and social groups. Finally, Zack (1999) describes tacit knowledge as subconsciously understood and applied, difficult to articulate, developed from direct experience and action, and usually shared through highly interactive conversation, storytelling, and shared experience. Thus tacit knowledge is much more difficult to express and share. It is based on experience, perception, personal sense making, and is context specific. It involves cognitive and technical components, and is one's representation and interpretation of experience (Nonaka, 1994). Although the definitions vary somewhat, there is general agreement (noting that exceptions exist) in the literature on the meaning of tacit knowledge.

Most authors emphasize the importance of distinguishing data and information from knowledge. Davenport and Prusak (1998) describe data (i.e. numbers, facts, images) as possessing no inherent meaning and of little inherent value unless it is transformed or 
processed into a form that is useful and meaningful to the user. They define information as data with relevance and purpose, however, point out that information is none-the-less not knowledge. McDermott (1999) defines six characteristics of knowledge that differentiate it from information: knowledge is a human act; knowledge is the residue of thinking; knowledge is created in the present moment; knowledge belongs to communities; knowledge circulates through communities in many ways; and new knowledge is created at the boundaries of old. Bollinger and Smith (2001) describe the pyramid model that explains how data is assimilated into information, information into knowledge, and ultimately knowledge into wisdom. The process also acts in reverse, as wisdom guides the application of knowledge, knowledge helps to interpret, filter, and make sense of information, and likewise information helps us to assimilate raw data and put it in context (see Figure 2). 


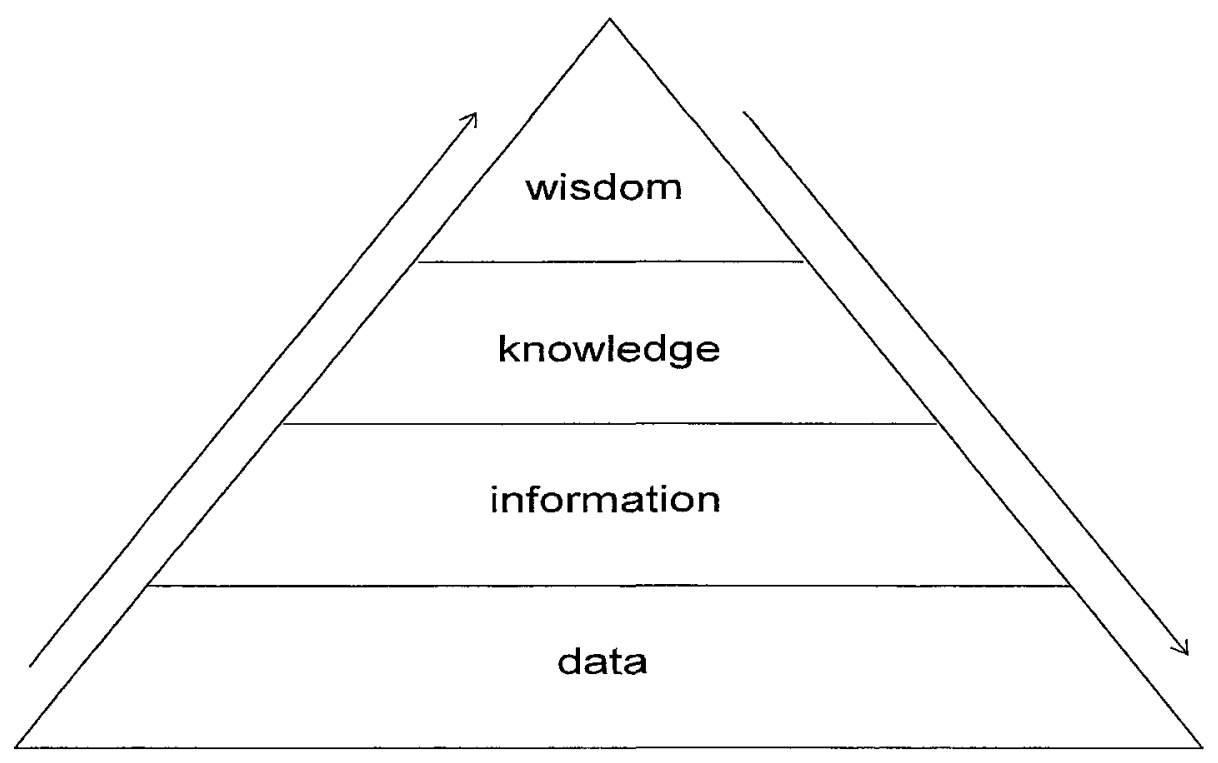

Figure 2. Knowledge Pyramid (Bollinger and Smith, 2001)

Spiegler (2000) provides a novel perspective in that he saw the difference between data, information and knowledge as only distinguished with respect to time (i.e. yesterday's data is today's information and tomorrow's knowledge). Zack (1999) defined information as something that results from placing data within a meaningful context. Machlup (1983) defines information as a component of knowledge, but not the whole of it. Marshal (1997) elaborates on this and states that information is transformed into knowledge when an individual acquires, interprets, and applies that information to a specific work function. He goes on to distinguish a person who simply acquires information, from a second person who acquires and interprets the same information and based on previous experience is able to apply it (e.g. to make better business decisions), and this again from a third person who may take the same information and apply it in entirely different ways than the second. Thus one person's information can become another's knowledge. Finally, information can be viewed as a symbolic 
representation of reality, and such symbolic representation is often undertaken to enable decisions to be made (Haines, 1978).

Much of the literature also distinguishes data, information, and knowledge on the basis of a learning process, whereby knowledge is the higher concept and end result. Different authors expressed the process in slightly differing ways:

- from data to information to knowledge (Alter, 1999; Davenport, 1997; Nonaka and Takeuchi, 1995);

- from data to information to knowledge to wisdom (Bollinger and Smith, 2001);

- from data to information to knowledge to expertise to capability (Beckman, 1997); and

- from data to information to knowledge to meaning to philosophy to wisdom (Allee, 1997).

The literature generally agrees on the many characteristics of knowledge: knowledge is contextual and it can be re-used (Allee, 1997); its benefits are obtained only if it is applied (Davenport and Prusak, 1998); its value may change over time (Davenport and Prusak, 1998); it has to be renewed and maintained (Dixon, 1999); it can be difficult to transfer, capture, distribute (Sveiby, 1997); it is developed through learning processes (Nonaka and Takeuchi, 1995); its acquisition depends on memory, past experience, expertise, knowledge transfer mechanisms, opportunities for learning (O'Dell et al., 1999); it facilitates effectiveness in problem solving and decision making (Davenport 
and Prusak, 1998); it enables "sense-making" abilities (Brooking et al., 1998); it enables higher learning of complex concepts and deep insights (i.e. it has accumulative benefits) (Soliman and Youssef, 2003); and finally, its creation and utilization can be enhanced with technology (Gallivan et al., 2003).

Knowledge in an organizational context is subject to a number of processes. Authors define and describe these processes in different ways but in most cases they include an equivalent of each of the following five key processes which are widely described in the literature: external knowledge adoption or acquisition processes, knowledge generation or creation processes, knowledge sharing or transfer processes, knowledge utilization and application processes, and knowledge retention or storage processes. The literature on organizational learning identifies these processes and helps to understand them in terms of how they contribute to organizational performance and competitive advantage. Therefore before discussing knowledge processes and knowledge management in more detail, the next section provides a brief summary of organizational learning literature and explains its contribution to the current research.

\subsection{Organizational Learning}

Organizational learning (OL) is a subject of immense interest in both industry and academia. In any knowledge intensive organization, the characteristics of individual knowledge and organizational knowledge are such that there are many ongoing threats to maintaining them. For example, with simply the passage of time, the context of knowledge may change due to changes in the external environment or advances in 
technology, and this may render knowledge less relevant. Alternatively, knowledge may be lost from retirements, lack of efforts to capture it, or even lack of use or application. Organizations need continuous learning processes to maintain their knowledge base and ensure excellence in decision making and capacity for effective action (see Figure 3).

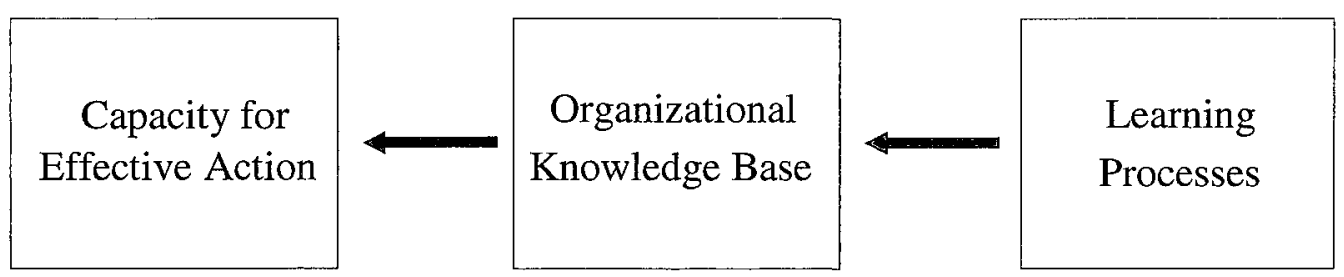

Figure 3. OL Key to Maintaining Knowledge-Base (Adapted from Senge, 1999)

There are many different definitions and perspectives of organizational learning (Garvin, 1993; Daft and Huber, 1987). Some of the more salient literature on the subject helps to explain the connection to and overlap with the knowledge management literature. Nonaka and Takeuchi (1995) describe organizational learning as the creation, absorption, and sharing of tacit knowledge and emphasize the role of human interaction. Klimechki and Lassleben (1998) present organizational learning as essentially a communication-based process that permits knowledge sharing, mutual interaction and influence, and adaptation to situational stimulus. Huber (1991) saw it as an information-processing process that includes acquiring, interpreting, disseminating, and storing of information and with organizational memory as the outcome or product. Levitt and March (1988) argued organizations learned by encoding inferences from history into routines that guide behaviour. Kim (1993) considered organizational learning as acquiring of know-how or skills that enable action and the know-why to 
understand and express the experience. Garvin (1993) describes the learning organization as skilled in creating, acquiring and transferring knowledge, and at modifying its behavior to reflect new knowledge and insights. He integrates many of these ideas by demonstrating that organizational learning proceeds through stages of cognitive learning, behavioural adjustment, and performance improvement. Argyris and Schon (1978) believed that organizational learning was a reactive process and the result of organizations adjusting to change in their business environments. Conversely, Dixon (1998) describes organizational learning as a proactive and intentional process to facilitate organizational transformation and change.

Cooks and Yanow (1993) believe organizational learning occurs only when learning is occurring collectively as a total organizational unit. This is quite at odds with the view of Argyrus and Schon (1996) who see individual learning as the construction and modification of personal beliefs, and as the primary contributor to organizational learning. March and Olsen (1975) tied both forms of learning together in a cyclic process of interactive learning and theorized that individual actions and beliefs lead to expressions and behaviors that result in organizational actions. These can be viewed as environmental responses that in turn, influence individual actions and learning. Levitt and March (1988) and Huber (1991) correctly point out however that organizational learning does not necessarily always lead to intelligent behavior or positive results and distinguish "productive" learning as that which enhances capability and has the potential to improve performance. 
Duncan and Weiss (1979) looked at organizational learning as a process whereby knowledge about action-response relationships and the effect of the environment on these relationships is developed. The outcome is the development of a knowledge base. The idea of an organizational knowledge base is that it connects individual knowledge to organizational knowledge and this occurs through a two-step learning process: first knowledge exchange and sharing occurs, then a process of knowledge integration with other related knowledge follows. Argyris and Schon (1978) expressed a somewhat analogous theory and proposed the concept of "double-loop learning" as a change of deeply rooted assumptions, as opposed to single-loop learning, as corrections or changes in the way people work without changing more deeply rooted assumptions or changing work routines. This is based on the assumption that learning occurs at various intellectual levels based on a process of detecting and correcting errors.

Koorneef (2000) also provides insights into continuous learning processes based on a double-loop learning model. His model (adapted, see Figure 4) illustrates how individual single-loop learning occurs as a matter of course as staff carry out daily duties, and adapt and typically modify their approaches and behaviours within the constraints of the work environment. This single-loop learning also occurs at an organizational level (within teams, departments, etc.) as norms and expectations develop, accepted practices evolve, and approaches that are an interpretation and adaptation of the so-called governing variables of the firm (i.e. the values, norms, and means including work procedures, official policies, standards, etc.) mature. More importantly, double-loop learning also occurs, and this takes the form of adaptation and 
adjustment of the governing variables to improve overall operations. Key agents (stakeholders or influencers) play a key role in the organizational learning processes.

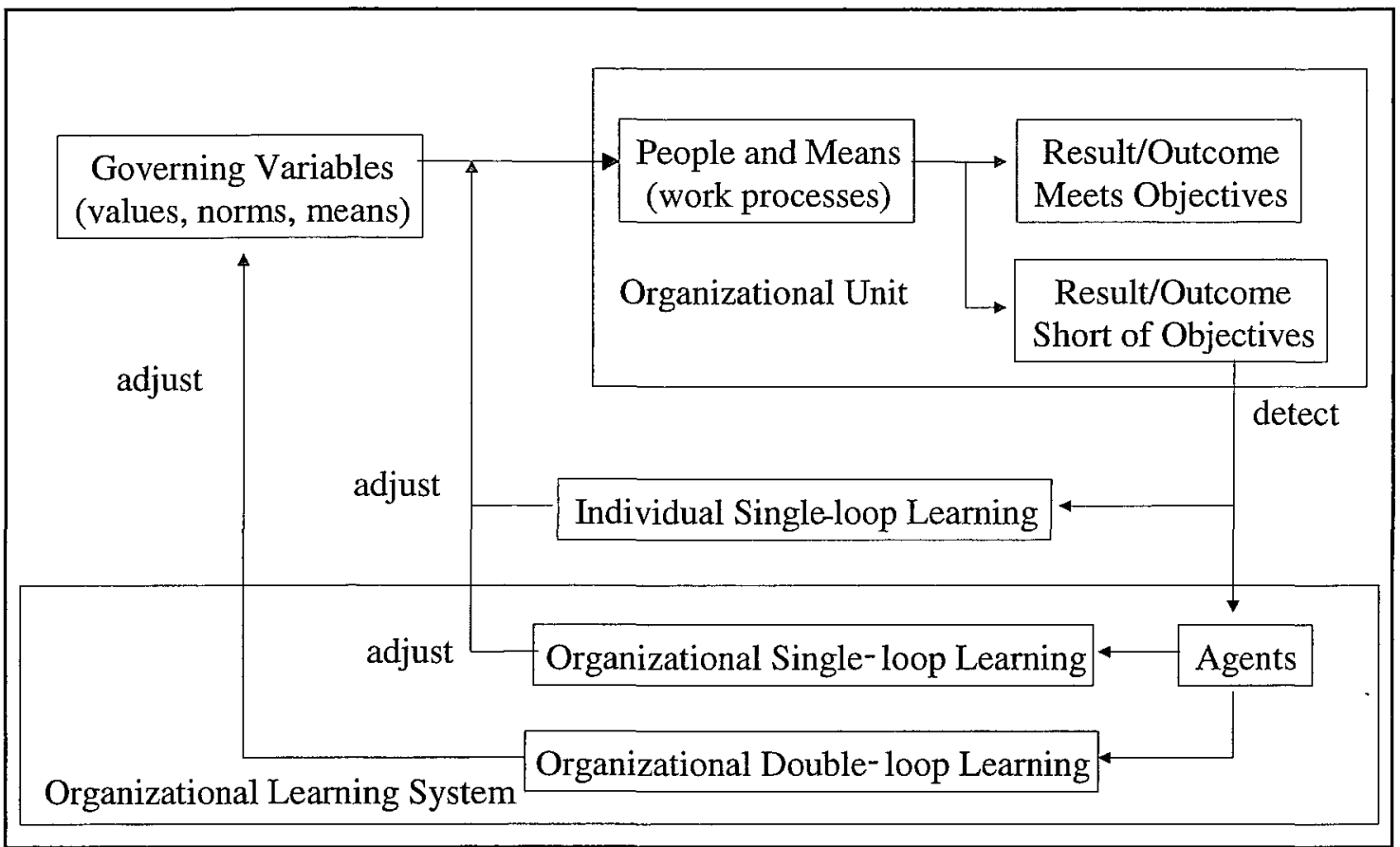

Figure 4. OL Processes (adapted from Koorneef, 2000)

Daft and Huber (1987) summarized two common views on organizational learning found in the literature: the interpretive view, which emphasizes the development of shared meanings and beliefs; and the systems-structural view emphasizing the acquisition and distribution of information. Shrivastava (1993) proposed four distinct and contrasting categories of organizational learning: adaptive learning; institutionalized experience effects; assumption sharing; and development of a knowledge base. Fiol and Lyles (1985) described four key factors influencing organizational learning: culture, strategy, environment, and structure. 
Organizational memory is seen as a key component of organizational learning in that organizations acquire, distribute and interpret information and store knowledge in organizational memory for future use (i.e. an outcome of knowledge processes) (Huber, 1991). This is consistent with the concept of the organizational knowledge base. Huber (1991) argues that several factors contribute to the quality of organizational memory: controlling the ongoing loss of the human components of organizational memory (i.e., due to attrition, lack of knowledge use, or lack of knowledge renewal); ensuring information is captured and recorded; ensuring effective distribution and interpretation of information; and ensuring the whereabouts of information is known. Thus Huber characterizes organizational memory and organizational learning as being facilitated through what many other authors define as knowledge processes.

The overlap between organizational learning literature and knowledge management literature can further be seen in the perspective of organizational memory provided by Walsh and Ungson (1991). They take an integrated view of organizational memory and see it as composed of the structure of its retention facility, the information contained in it, the processes of information acquisition and retrieval, and its consequential effects. They view organizational memory as stored information from an organization's history that can be brought to bear on present decisions. Redding and Catalanello (1994) point out the overlap in the literature between knowledge management and organizational learning and that it is difficult to distinguish clearly between the concepts. Willmann (1991) believed that organizational learning is a key aspect of an effective knowledge 
management system and it enables an organization to embody, adjust and implement changing and new technology.

Literature on organizational learning, cognitive theory, and the processes of team learning has focused on aspects of knowledge acquisition, transfer, retention and organizational learning culture (Ke and Wei, 2006; Kiehl, 2004; Kock, 1996; Levinthal and Claus, 2005; Liu, 2003; Loermans, 2003; Lopez et al., 2004; Marsick and Watkins, 2003). In summary, there are a range of theories and perspectives on organizational learning but clearly the concepts in this body of literature support the notion that organizational learning processes are associated with the acquisition, generation, maintenance, storage, sharing, and application of knowledge. In the knowledge management literature, these are referred to as "knowledge processes".

Several author's have established the link between organizational learning and knowledge processes. For example, research by Pham and Swierczek (2006) assessed learning activity within the firm in terms of knowledge acquisition, knowledge sharing, and knowledge utilization. Knowledge acquisition was measured by the extent to which: new knowledge was created or acquired by individuals; employees had improved their professional knowledge; and employees reflect on their work and learn from experience. Knowledge sharing was viewed in terms of how quickly new skills or knowledge could be disseminated in the firm, how much and often knowledge was shared among the professionals, and how much the staff learned from these exchanges. 
Knowledge utilization considered how much professional staff applied new approaches in their jobs, and how often they changed their design methods or work process.

Firestone and McElroy (2004) further introduce the concept of knowledge claim evaluation (KCE) as an important aspect of knowledge processing and organizational learning. They argue operational business processes are performed by agents using previous knowledge found in the distributed organizational knowledge base (DOKB) (i.e. their own mental knowledge and linguistic knowledge in organizational repositories) and situational knowledge (the result of ongoing single-loop learning to make decisions). When knowledge gaps occur, a knowledge production process is initiated in a search for answers, or more specifically, a double-loop learning process is started. Information is acquired, individual and group level learning may occur, new knowledge claims are put forward, and the knowledge claim evaluation process transpires. Knowledge integration occurs once new or revised knowledge claims emerge, and this involves four sub-processes: knowledge and information broadcasting, searching/retrieving, knowledge sharing, and teaching. The various outcomes of knowledge processes (i.e. both production and integration phases) are the inputs to the DOKB. The ongoing utilization of the DOKB by agents for decisions and actions for operational business processes gives rise to the knowledge life-cycle.

Their research helps to explain the integration of organizational learning theory into the theoretical framework of KM. Organizational learning is incorporated within the knowledge lifecycle and knowledge processing elements and this $\mathrm{KM}$ framework go 
beyond KM theories and models that focus only on knowledge integration processes. Of particular relevance to this research is the distinction made between operational business processes, knowledge processes, and processes (or practices) for managing knowledge processes (i.e. KM), and the concept of the DOKB and the recognition that knowledge (i.e. organizational learning) processes build and maintain the DOKB. Firestone and McElroy's model, while useful, is conceptual only, and lacked a welldeveloped set of measures and empirical validation. Further, their model did not address the influence of organizational culture and its impact on factors that influence human behavior (and learning) such as motivation, commitment, trust, leadership, empowerment, accountability and common vision.

The OL literature continues to evolve but it is clear that there is a strong overlap with knowledge management literature. The OL literature and perspective helps to clarify and establishes the importance of the link between $\mathrm{KM}$ and organizational learning. Much of the organizational learning literature tends to take one of two perspectives: either a process view to organizational learning (i.e. with the goal of identifying and understanding the organizational learning process mechanisms); or the view of the learning organization as an idealized end state to be achieved. The former view is more consistent with the concept of knowledge processes in KM literature. Clearly organizations can improve performance and build competitive advantage through increased knowledge, and the accumulation, retention and use of knowledge in organizations is subject to effective knowledge processes. These processes will be explored further in the next section. 


\subsection{Knowledge Processes}

Many authors in the management literature were found to have explored the important contribution of knowledge processes to innovation (Jie, 2001; Aramburu, 2006; Carneiro, 2000; Darroch, 2005; Gloet and Terziovski, 2004; Huber, 1999; Jantunen, 2005; Johnson, 2000; Murray and Blackman, 2006). Research on information systems (IS) and information technology (IT) for example delves into issues of knowledge acquisition, creation and generation processes (e.g., Edwards et al., 2005; Gallivan et al, 2003; Griffith et al., 2003; Hendriks, 2000; Holsapple, 2005; Junnarkar and Brown, 1997; Kim and Lee, 2006; Kwan and Cheung, 2006; Lusignan, 2002).

Literature on team-work, collaboration, and cross-functional teams has also explored many of the facets of knowledge transfer, sharing, and creation (Mohamed et al., 2004;

Pinto et al., 1993; Politis, 2003; Pyoria, 2005; Van der Vegt and Bunderson, 2005; Zakaria et al., 2004; Cai, 2006; Kim and King, 2004). Research on collaboration in R\&D partnerships and technology joint-ventures has been conducted to investigate knowledge transfer, sharing, and adoption processes between organizations (Kotlarsky and Oshri, 2005; Kandemir and Hult, 2005). Finally, literature on strategic management, project management, and decision-science can also be found that explores KM issues such as of the application or utilization of knowledge (McCann and Buckner, 2004; Lytras and Pouloudi, 2003; Snyman and Kruger, 2004; Zheng, 2005; Bou-Llusar and Segarra-Cipres, 2006; Gagnon, 1999; Heinrichs, 2001; Janz and Prasarnphanich, 2003). 
Most authors discuss knowledge management and organizational learning theory in the context of several defined knowledge processes. However, there are quite a range of constructs presented in the literature that propose to constitute the basic knowledge processes and how they are defined. Tannembaum and Alliger (2000) considered four processes related to knowledge: knowledge sharing, knowledge accessibility, knowledge assimilation and knowledge application. They are explained as follows:

- knowledge sharing is the extent to which people share their knowledge;

- knowledge accessibility is the extent to which people have access to the information they need to make decisions, solve problems, perform job tasks and service customers;

- knowledge assimilation is the extent to which people learn or acquire the knowledge they need to perform well; and finally; and

- knowledge application is the extent to which people apply or use knowledge to effectively make decisions, solve problems and service customers.

Rastogi (2000) referred to a set of key knowledge operations needed for organizations to achieve competitive knowledge generation:

- identification of knowledge required for an effective implementation of competitive strategy;

- mapping existing and available knowledge (including expertise and skills);

- capturing existing knowledge through its formalized representation; 
- acquiring knowledge and information that is needed (including core skills or competencies);

- storing the existing, acquired, and created knowledge repositories in a way that facilitates retrieval and reuse;

- sharing knowledge through distribution as needed;

- applying knowledge (through actions, decisions, training etc.); and

- creating generating or discovering new knowledge (i.e., through $R \& D$, experimentation, creative thinking and innovation).

Probst et al. (2002) proposed eight fundamental knowledge processes:

- identification is the process where external knowledge is identified;

- acquisition refers to what expertise should be acquired from outside the firm;

- development is focused on generating new skills, new products, ideas and efficient processes;

- distribution is sharing and spreading knowledge already within the firm;

- utilization is activities to apply knowledge effectively; and

- preservation is the selective retention of knowledge.

Hedlund (1994) described knowledge processes as: knowledge storage (and retrieval), transfer, application (use), protection, acquisition or generation. Dalkir (2005) and others argued that these various knowledge processes interact with each other in a cyclelike pattern that facilitates knowledge flow and learning. Several authors have presented the notion that knowledge circulates as a result of these interactions as part of 
a "knowledge lifecycle", and an organization's base of knowledge enlarges through each iteration of the process (Chang Lee et al., 2005; del-Rey-Chamorro et al., 2003). Stabb et al. (2001) looked at knowledge processes only from a codified knowledge perspective. They proposed the following knowledge processes:

- creation or import (information or knowledge needs to be created or converted so that they fit the conventions of the company);

- capture (knowledge items have to be captured in order to determine their importance and how they mesh with the company's vocabulary conventions);

- retrieval and access (this step satisfies the searches and queries for knowledge by the knowledge worker); and

- use (the knowledge worker will not only recall knowledge items, but will process them for further use).

Thus it is clear that although there are common themes and elements in much of the literature there is a distinct lack of consensus on the definitions of knowledge processes. The range of terms and the different perspectives offered is perhaps a reflection of the newness of the discipline and the diversity of its origins in a number of disciplines. For the purposes of this research, a simple and inclusive definition of the five most basic and widely accepted knowledge processes was thought to be most appropriate. These five widely used knowledge processes were chosen on the basis that they could easily be shown to encompass most of the other knowledge processes discussed in the literature. The five processes chosen were: knowledge acquisition and adoption (KA), knowledge 
sharing and transfer (KS), knowledge generation and validation (KG), knowledge retention and storage (KR), and knowledge utilization and application (KU). These knowledge processes are found frequently in the literature and the interpretation chosen in this research is summarized below:

- $\underline{\text { knowledge a }}$ aquisition and adoption (KA) (e.g. as used by Holsapple and Jones, 2004; Aramburu, 2006; Bergman, 2004) meaning the process of obtaining and introducing new external knowledge (whether tacit or explicit) into the organization. This is interpreted to include knowledge identification and selection when for the purposes of acquisition. It also includes knowledge adoption and assimilation from sources outside the organization (e.g. industry best practices, new technologies, etc. as described by Hwang (2005), or Sussman and Siegal (2003)).

- knowledge sharing and transfer (KS) (e.g. as used by Nonaka and Takeuchi (1995); Meckler, 2001; Syed and Fytton, 2004; Bou-Llusar and Segarra-Cipres, 2006). This construct will be interpreted as the processes for exchange of knowledge within the organization (directly or indirectly) and as also incorporating the concepts of knowledge sharing or distribution (as defined by Brauner et al., 2006; Cabrera et al., 2006; Hendriks, 1999; Hoof and Rider, 2004);

- knowledge generation and validation (KG) (e.g. as used by Heinrichs, 2001; Foss et al., 2006). This construct implies the formation or production of new 
knowledge and incorporates knowledge development or adaptation processes. It may also include knowledge identification and selection processes when associated with internal knowledge generation processes.

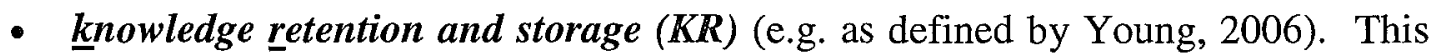
is the process of keeping knowledge (whether tacit or explicit) within the organization and maintaining its availability. It incorporates the related concepts of knowledge capture, preservation, storage, retrieval, accessibility, identification and protection in the context of long-term knowledge sustainability.

- knowledge utilization and application (KU) (e.g. as defined by Verkasalo et al.;1998; or Koskinen, 2003). This incorporates the concepts of knowledge application or use.

The following sub-sections will elaborate on each of these concepts further.

\subsubsection{Knowledge Acquisition and Adoption (KA) Processes}

Many different definitions of knowledge acquisition can be found, some focusing strictly on tacit knowledge, others on explicit, still others on both. Several authors take the view that knowledge acquisition is a process of an organization or sub-unit acquiring an external knowledge base (e.g., Diakoulakis et al., 2004), with the resulting benefits of expanding the firm's knowledge pool, increased organizational learning, and the further empowerment of a work environment. Holsapple and Jones (2004) concur with 
this view and define knowledge acquisition as acquiring knowledge from external sources and making it suitable for subsequent use. They describe knowledge acquisition activity as beginning with identifying knowledge in the external environment, and concluding with transforming it into a representation that can be employed by the organization (i.e. "adoption").

Other authors see knowledge acquisition more on an individual level. Pham and Swierczek (2006) defined knowledge acquisition as the development or creation of skills, insights, or relationships. Joshi (1998) defined knowledge capture in a similar manner to what most other authors see as knowledge acquisition: "the process of extracting, collecting, and/or gathering knowledge from external sources that is deemed to be of sufficient reliability, relevance, and importance, and organizing it by distilling, refining, orienting, interpreting, packaging, assembling, and/or transforming it into usable representations". Mykytyn et al. (1994) defined knowledge acquisition as "acquiring information directly from domain experts". Politis (2005) states new knowledge is acquired by reading, listening to someone, observing, experiencing events or thinking. For conceptual clarity, and to distinguish knowledge acquisition from the concepts of knowledge transfer and sharing and knowledge generation or creation, this study defines knowledge acquisition and adoption (KA) along the lines of Holsapple and Jones (2004) and considers it the process of obtaining existing external knowledge, whether tacit or explicit, that was not previously held by the organization and transforming it into a form suitable for use. This distinguishes it clearly from the 
process of obtaining knowledge from others within the organization through knowledge sharing and transfer processes.

\subsubsection{Knowledge Sharing and Transfer (KS) Processes}

Argote \& Ingram (1999) define knowledge transfer as the process through which one unit (e.g., group, department, or division) is affected by the experience of another. Zander and Kogut (1995) explored the effect on knowledge transfer due to the nature of knowledge being transferred based on its codifiability, teachability, complexity, system dependence, and product observability. Knowledge transfer is also defined as the application of prior knowledge to new learning situations (KcKeough, 95). Minbaeva et al. (2003) view knowledge transfer as a dynamic process between sender and receiver encompassing several stages from transmission of viable knowledge to its final application by the receiver. Szulanski (2000) defined four main stages of knowledge transfer: initiation, implementation, ramp-up, and integration.

Many authors concur that knowledge transfer practices play a key role in organizational learning and knowledge utilization (Alavi and Leidner, 2001; Argote and Ingram, 2000;

Ford and Chan, 2003; Nonaka, 1994). Nonaka and Takeuchi (1995) provide useful insight into knowledge transfer processes and strategies. Figure 5 highlights how knowledge can be transformed and transferred between individuals (tacit to tacit), between individuals and codified form (tacit to explicit), and vice-versa, through 
processes they refer to as socialization, externalization, combination, and internalization.

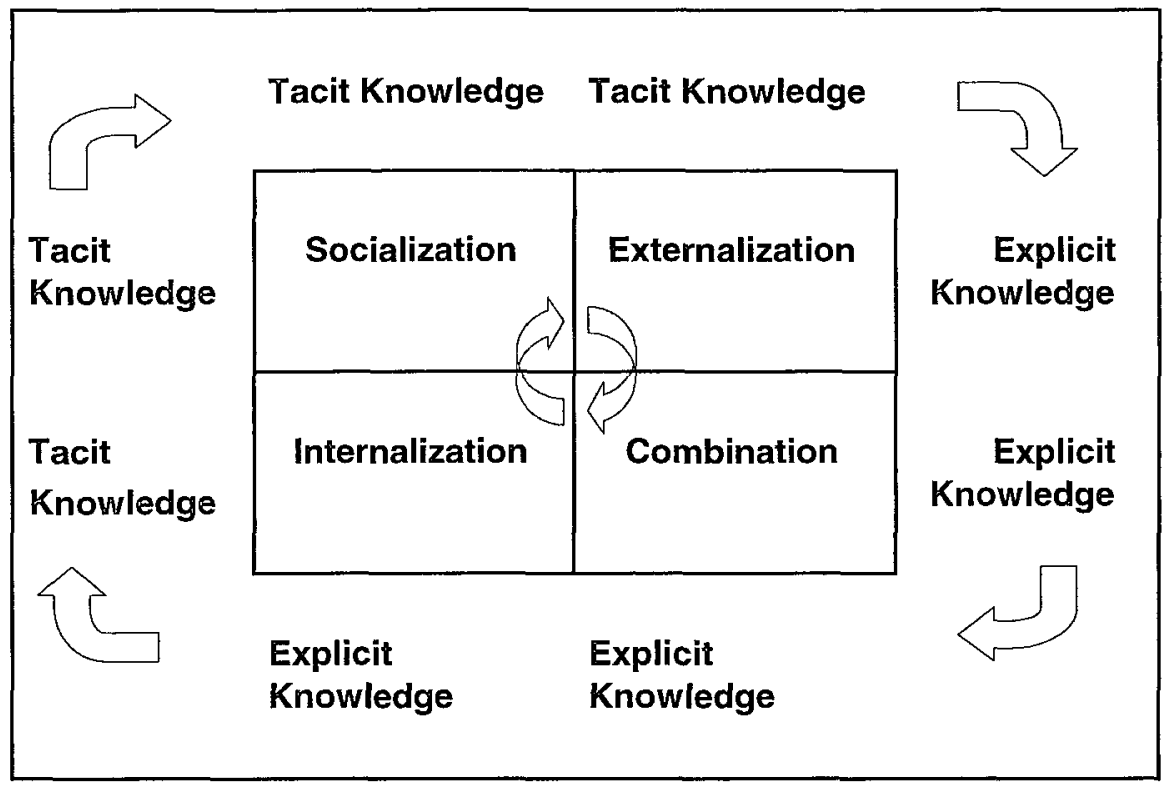

Figure 5. Knowledge Transfer Processes (Nonaka and Takeuchi, 1994)

In this context socialization is the process of sharing experience and creating tacit knowledge, for example common mental models or specialized skill-sets. Externalization is the process of articulating tacit knowledge into explicit concepts through for example, metaphors, analogies, concepts, hypotheses or models. Combination is the process of reconfiguring and reshaping information, ideas, and concepts through sorting, integrating, or combining into new knowledge frameworks or systems. Finally, internalization is the process of learning by doing, whereby explicit knowledge is embodied into an individual's tacit knowledge. Many other authors present similar concepts, and such models aid in developing an understanding and view of the firm and its work processes in terms of knowledge processes. Nonaka and 
Takeuchi (1995) emphasized that learning and knowledge creation occur when tacit knowledge is transferred between individuals (either directly or indirectly).

A review of the literature did not reveal knowledge-sharing to be as well-defined a construct (Sharrat and Usoro, 2003). Information sharing is a term applied to a wide range of exchanges and does not necessarily lead to the creation of new knowledge, however, knowledge-sharing intrinsically implies the transfer of new knowledge to the recipient (Van Beveren, 2002). As defined by Nonaka and Takeuchi (1995), and for the purposes of this research, knowledge transfer is defined to include knowledge sharing processes and the construct "knowledge sharing and transfer" (KS) processes will be used.

\subsubsection{Knowledge Generation and Validation Processes (KG)}

The processes of knowledge creation and knowledge generation are somewhat synonymous in the literature. Nonaka and Takeuchi (1995) defined knowledge creation as the capability of a firm to create new knowledge, disseminate it throughout the organization, and embody it in products, services, and systems. Further work by these same authors along with Umemoto (1996) expanded on this definition and argued knowledge creation was the process that organizationally amplifies the knowledge generated by individuals and crystallizes it as part of the knowledge system of the organization. Many organizational learning theorists share this view, however they tend to take a wider view of this as the organizational learning process and include 
knowledge acquisition in the process as well (e.g. Korneef, 2000). IAEA (2006b) defines knowledge creation as "a process that results in new knowledge, or organizes current knowledge in new ways making use of existing knowledge. Holsapple and Jones (2004) define knowledge generation as "producing knowledge by either discovery or derivation from existing knowledge".

An important aspect of knowledge generation or creation is validation. Knowledge validation, evaluates the quality of knowledge by comparing it to other independent sources of knowledge (Mengshoel and Delab, 1993). From an expert systems perspective, validation concerns both the quality of a system's decisions and advice and the correctness of the reasoning techniques used. It also applies to both a knowledge base and the inferences made using the knowledge base (Mengshoel and Delab, 1993). Gaines (1989) identifies several types of knowledge validation from a technology perspective in research on knowledge based systems (KBS), including: objective (compared to empirical approaches); pragmatic (used in applications); foundational (compared to another discipline's "deep knowledge"); referential (comprehensibility of explanations in a relevant reference community); subjective performance (compared to expert behavior in applications); and subjective knowledge (compared to expert rationale in communication with a reference community).

Other aspects of knowledge creation include knowledge identification, selection, and adaptation. Holsapple and Jones (2004) define knowledge selection as "selecting needed knowledge from internal sources and making it suitable for subsequent use". 
Thus knowledge creation may also include knowledge identification, selection and adaptation processes when associated with internal knowledge generation processes. In this research study, knowledge generation or creation will be defined to include processes of development/formation of new knowledge from within the organization and incorporates internal knowledge identification, selection, adaptation and validation processes. The construct name "knowledge generation and validation" or simply "knowledge generation" (KG) will be used.

\subsubsection{Knowledge Retention and Storage (KR) Processes}

Knowledge retention is discussed in different contexts. For example, it may be in the context of a mitigating action to protect against knowledge loss due to attrition, or in the context of long-term knowledge preservation for future use, or in the context of maintaining personnel competencies through training. For example, IAEA TECDOC 1510 (LAEA, 2006) defines knowledge retention as a process that identifies the critical knowledge and positions in an organization, and the methods to be used for addressing potential knowledge loss through attrition. In this context, $\mathrm{KR}$ is an ongoing process that requires continual updating to meet changing business needs. Alternatively, many formal training programs require confirmation of knowledge retention (e.g. Watkins, 2001). In this context, it refers to an individual employee's knowledge retention.

The concept of intellectual capital is based on the idea that knowledge is an asset or stock and exists both in the minds of an organization's employees as well as being 
embedded explicitly as structural capital in document artifacts, products, processes, or systems of the organization. Sanchez and Heene (1997) argue knowledge retention is a learning process and represents the information and knowledge flows that lead to a change in the stock of beliefs (i.e. read as "useful knowledge assets") within the organization. The concept of intellectual capital developed primarily out of the financial management literature, partly as a way of accounting for and rationalizing the valuation of many technology and innovation firms whose market capitalization far exceeded their tangible asset base. Stewart (1997) defines intellectual capital is an intangible intellectual asset that has been formalized, captured, and leveraged to create wealth (i.e. as a higher-valued asset). In general, there is agreement in the literature that intellectual capital includes human capital (competencies and capabilities in people), structural capital (i.e. organizational routines, methods, and process), and relational capital (relationships and networks) (Sveiby, 1997).

IAEA (2006b) defines knowledge preservation as "a process of maintaining an organizational system of knowledge and capabilities that preserves and stores perceptions, actions and experiences over time and secures the possibility of recall for the future". The document goes on to state "the preservation of knowledge is an important building block within the knowledge management field. Organizations that intentionally manage their experiences for them to be available for the future have to master three basic processes of knowledge management: select, from the large number of organizational events, persons or experts and processes, only those that are worth 
preserving; store their experience in a suitable form; and ensure the setting up and operation of the organizational memory".

The IAEA (2006b) also sees knowledge capture as an important part of the knowledge preservation process and define it as "a process of capturing the knowledge available within an individual or organization and making it available". It is represented as a process that requires "effective architectures, techniques, and standards for organizing and presenting content effectively; understanding what knowledge to capture, and how to document it in order to maximize its usefulness to others; and efficient systems and tools that centralize knowledge content and make it easy to store, access, and maintain". A knowledge repository is defined (IAEA, 2006b) as any identified location (physical or electronic) to store explicit knowledge and from where it can be retrieved. This research will take a broad definition of the construct of "knowledge retention and storage" $(\mathrm{KR})$ as a process that includes knowledge selection, preservation and storage (whether as human capital, structural capital, or relational capital). It also incorporates the related concepts of knowledge identification, capture, retrieval, accessibility and protection when considered in the context of knowledge retention and storage.

\subsubsection{Knowledge Utilization and Application (KU)}

Tannembaum and Alliger (2000) considered knowledge application as the extent to which people apply or use knowledge to effectively make decisions, solve problems and service customers. Rastogi (2000) saw knowledge utilization as applying knowledge 
through actions, decisions, training etc.. Probst et al. (2002) defined knowledge utilization as activities to apply knowledge effectively. Stabb et. Al. (2001) described knowledge utilization as not only recall of knowledge items, but re-processing of them for further use. Nevis et al. (1995) proposed knowledge utilization as the integration of learning so it is broadly available and can be generalized to new situations. Knowledge utilization as defined by Verkaskalo (1998) is the ability of organizations to transfer knowledge from those who have it (the provider) to those needing it (receiver) such that it can be applied in daily decision making. Koskinen (2003) argues that tacit knowledge utilization is a complex construct that cannot be easily measured. He posits several factors influence utilization, and these can be grouped into internal (under the control of the individual, such as memory, communication, and motivational systems) and external factors.

Although a range of definitions for knowledge utilization exist, in general, most authors discuss the construct in a general sense as "the use of or application of knowledge or a knowledge base to day-to-day challenges, problems and decisions faced by the firm". This definition is suitable for the purposes of this research and the construct of "knowledge utilization and application (KA) processes" is defined generally to apply to the use of individual, group, or company-wide knowledge, in tacit or explicit forms, and may refer to a very specific knowledge and application or collectively to a wide corporate knowledge base and its general usage. 


\subsection{Knowledge Management Practices}

The preceding discussion provided insight into the meaning of knowledge and a description of knowledge processes. This is a useful theoretical basis from which to commence a discussion on the practice of knowledge management (KM), and more specifically, KM practices. To clarify and understand what is meant by the term knowledge management practices, it is helpful to first review the definition of management. There is general agreement in the literature that management is a process that combines components of both leadership and administrative control and coordination, the latter of which includes planning and budgeting, organizing and staffing, controlled problem solving, and predicting results (Amidon, 1997). Amidon (1997) further defines leadership as the act of setting the vision for the future, aligning people, motivating and inspiring employees, and facilitating and creating needed change.

Holsapple and Singh (2003) conveniently define knowledge management in terms of the same three management elements of leadership, control, and coordination. Knowledge control is defined as ensuring that needed knowledge resources and processors are available in sufficient quantity and quality subject to required protection and constraints. Quality is controlled with respect to two dimensions: knowledge validity (accuracy and consistency) and knowledge utility (relevance and importance). Knowledge coordination is defined as guiding the conduct of knowledge management in an organization. It involves managing dependencies among knowledge resources, among 
knowledge manipulation activates, between knowledge resources and other resources (i.e. financial, human, and material), and between knowledge resources and KM activities. It also involves marshalling sufficient skills for executing various activities, arrangement of those activities in time, and integrating knowledge processing with an organization's operations. Finally, knowledge leadership is defined as establishing the enabling conditions for achieving fruitful knowledge management through other knowledge management activities. When effective, it should be a catalyst by inspiring, mentoring, setting examples, engendering trust and respect, instilling a cohesive and creative culture, establishing a vision, listening, learning, teaching, and knowledge sharing. It sets the tone (i.e. shapes the culture) for knowledge coordination, control, and measurement.

However, a review of knowledge management literature soon reveals that there are many differing perspectives on and definitions of KM. Table 2 provides a summary of many of the perspectives of knowledge management found in the literature.

Table 2. Definitions of Knowledge Management

\begin{tabular}{|c|l|}
\hline Author(s) & \multicolumn{1}{c|}{ Perspective on KM } \\
\hline O'Leary (1998) & $\begin{array}{l}\text { KM is 'managing the organization's knowledge by creating, structuring, } \\
\text { dissemination and applying it to enhance organizational performance'. }\end{array}$ \\
\hline $\begin{array}{c}\text { Alavi and Leidner } \\
\text { (1999) }\end{array}$ & $\begin{array}{l}\mathrm{KM} \text { is 'the process to acquire, organize, and communicate knowledge of } \\
\text { employees so others may be more effective in their work'. }\end{array}$ \\
\hline Andriessen (2004) & KM is 'organizing and optimizing K-processes'. \\
\hline Salisbury (2003) & $\begin{array}{l}\mathrm{KM} \text { is 'the deployment of a comprehensive system that enhances the growth of } \\
\text { an organization's knowledge'. }\end{array}$ \\
\hline Suguraman (2000) & $\begin{array}{l}\mathrm{KM} \text { is 'a process that allows business process automation and enhances } \\
\text { communication and collaboration between internal and external constituents'. }\end{array}$ \\
\hline Marshal (1997) & $\begin{array}{l}\mathrm{KM} \text { is seen as "intellectual capital management" or "knowledge asset } \\
\text { management", where the asset or capital being referred to encompass all forms } \\
\text { of tacit and explicit (including codified) knowledge in the organization. Thus, } \\
\mathrm{KM} \text { is the harnessing of intellectual capital. }\end{array}$ \\
\hline
\end{tabular}




\begin{tabular}{|c|l|}
\hline Author(s) & \multicolumn{1}{|c|}{ Perspective on KM } \\
\hline Malhotra (1998) & $\begin{array}{l}\text { KM is seen as: "catering to the critical issues or organizational adoption, } \\
\text { survival and competence in face of increasingly discontinuous environmental } \\
\text { change. Essentially, it embodies organizational processes that seek synergistic } \\
\text { combination of data and information processing capacity of information } \\
\text { technologies, and the creative and innovative capacity of human beings". }\end{array}$ \\
\hline $\begin{array}{c}\text { Skyrme and } \\
\text { Amidon (1997) }\end{array}$ & $\begin{array}{l}\mathrm{KM} \text { is 'the explicit and systematic management of vital knowledge and its } \\
\text { associated processes of creating, gathering, organizing, diffusion, use and } \\
\text { exploitation. It requires turning personal knowledge into corporate knowledge } \\
\text { that can be widely shared throughout an organization and appropriately applied'. }\end{array}$ \\
\hline $\begin{array}{l}\text { Van der Spek and } \\
\text { Spijkervet (1997) }\end{array}$ & $\begin{array}{l}\text { KM is 'the explicit control and management of core competencies in an } \\
\text { organization'. }\end{array}$ \\
\hline Wiig (1997) & $\begin{array}{l}\text { KM is a process of systematic and explicit application of knowledge to increase } \\
\text { returns to the organization and is achieved by processes to disseminate expert } \\
\text { knowledge across the organization. }\end{array}$ \\
\hline Hanley, 1999 & $\begin{array}{l}\text { KM is centred around people and the processes they use to share information } \\
\text { and build knowledge. }\end{array}$ \\
\hline APQC, 2000 & $\begin{array}{l}\text { KM is the conscious strategy of putting both tacit and explicit knowledge in to } \\
\text { action by creating context, infrastructure, and learning cycles that enable people } \\
\text { to find and use the collective knowledge of the enterprise. }\end{array}$ \\
\hline Knapp (1998) & $\begin{array}{l}\text { KM is the process of transferring intellectual capital to value. This involves } \\
\text { processes such as innovation and knowledge creation, acquisition, organization, } \\
\text { application, sharing, and replenishment. }\end{array}$ \\
\hline Newman (1992) & $\begin{array}{l}\text { KM is 'the processes that govern the creation, dissemination, and utilization of } \\
\text { knowledge'. }\end{array}$ \\
\hline O'Leary (1998) & $\begin{array}{l}\text { KM is 'managing the organization's knowledge by creating, structuring, } \\
\text { dissemination and applying it to enhance organizational performance'. }\end{array}$ \\
\hline
\end{tabular}

Although various definitions of "knowledge management" exist in the literature, most are consistent with the notion that $\mathrm{KM}$ is a coordinated approach taken to establish, build, and maintain the organization's core competencies, information resources, and knowledge processes to improve organizational performance.

Many authors state the objective of $\mathrm{KM}$ is to promote and enable the building and maintenance of knowledge and knowledge processes (both tacit and explicit) within the firm. A commonly stated goal of KM is to make knowledge workers more effective and efficient. $\mathrm{KM}$ is often described as a collective set of practices that aim to promote the creation or acquisition of new knowledge and to enable continuous improvement, 
thereby reducing the cost of being effective and increasing the pace of innovation. Thus the practice of $\mathrm{KM}$ often includes initiatives such as preserving existing knowledge, reducing the loss of intellectual capital (IC) from employees who leave, increasing collaboration to enhance knowledge sharing, improving the skill level of employees, and increasing the productivity of workers by making knowledge accessible. Holsapple and Singh (2003) state that the practice of KM is concerned with ensuring that "the right knowledge is available in the right form to the right processors (i.e. human or computer) at the right time for the right cost". $\mathrm{KM}$ focuses on enabling increased access to knowledge that in turn helps staff do the right things, and do them right.

Sanchez and Heene (1997) present knowledge management as a higher form of explicit information management. Information management is generally related to the organization, archival and retrieval of data in a structured manner. They distinguish and characterize $\mathrm{KM}$ as a larger set of management activities aimed at improving the reproduction, explanation, application, and integration of articulated knowledge (i.e. explicit information in a contextual manner). Thus information management is a subset of KM activity focused on the structured organization, storage, and retrieval of explicit information and data.

Meyers (1996) present a contrary view that "knowledge must be seen as tied to the personal or human element...it resides in people's heads, (and) ...individuals must identify, interpret and internalize knowledge. He argues that the representation of knowledge is mechanical, digital, visual (and so on) and for knowledge to provide a 
company with sustained competitive advantage, it should not be dependent on a given individual. Meyer's believed that KM offers only the ability to identify and then manage organizational knowledge to the extent it has been captured by an organization's systems, processes, products, rules, and cultures. This view of $\mathrm{KM}$ is contradictory to many other authors who focus on either aspects (i.e. practices) of KM related to individual tacit knowledge or explicit knowledge or both.

KM literature integrates elements of theory and principles from many management "schools of thought" including: information theory and information systems theory, business process re-engineering, quality management, organizational behaviour, complex systems theory (including social-technical systems theory), human resource management, intellectual capital (or intellectual asset) theory, innovation management, organizational learning, organizational culture, performance management, and cognitive theory. It has also be argued by various authors that KM practices are complementary to (or an element of) quality management systems, integrated management systems and corporate governance approaches.

As a subject of research, $\mathrm{KM}$ has a wide domain. There are many inter-related and sometimes overlapping perspectives and theories on $\mathrm{KM}$ described in the literature and the concepts are often contradictory or have multiple interpretations. The following discussion will explore the topic of knowledge management practices further.

$\mathrm{KM}$ includes a range of practices that help an organization identify, create, represent, distribute and enable the assimilation and use of its knowledge. A review of the 
literature reveals this range of practices to be wide and varied (see Table 3). Further, from Table 3 it can be seen these practices cover a range of aspects of $\mathrm{KM}$ including: activities focused on knowledge retention and loss prevention (such as knowledge and capability replacement through recruitment and initial training activities); activities focused on knowledge building (such as through employee development initiatives); activities focused on knowledge capture, storage, retrieval and re-use (such as use of information technology including Intranet portals, resource locators/navigators, information repositories); or activities focused on knowledge transfer (such as coaching and mentoring by subject matter experts).

Coombs and Hull (1998) outlined the importance of research focused on the study of knowledge management practices. They describe various attributes of a KM practice as including: the characteristics of the practice (i.e. generation, transfer, or utilization of knowledge); the domain of the practice (i.e. technical, market, or procedures); the performance intent of the practice; and the degree of formality of the practice. Other factors mentioned in the literature include the pervasiveness of the practice (i.e. how widespread it is in the organization), the maturity of the practice, the effectiveness of the practice, the acceptance of the practice. The early stage in the development of KM as a discipline is evidenced by the lack of clarity and consistency in the literature on what best practices are and how they should be defined and applied. Table 3 provides a summary of (and useful insight into) the range of activities encompassed by KM practice. 
Table 3. Summary of KM Methods and Tools Found in the Literature

\begin{tabular}{|l|l|l|}
\hline \multicolumn{1}{|c|}{$\begin{array}{c}\text { KM Method or } \\
\text { Practice }\end{array}$} & \multicolumn{1}{|c|}{ Author(s) } & \multicolumn{1}{c|}{ Description of Approach } \\
\hline $\begin{array}{l}\text { Implementation of KM } \\
\text { programs/systems in } \\
\text { general }\end{array}$ & $\begin{array}{l}\text { IAEA (2006b); Arora (2002); Young } \\
\text { (2006); Chang and Ahn (2005); } \\
\text { Corso et al. (2003); Treasury Board } \\
\text { of Canada Secretariat (2002); Chen } \\
\text { Liu (2003); Edwards (2005); Feng et } \\
\text { al. (2004); Gallivan (2003); }\end{array}$ & $\begin{array}{l}\text { Holistic approaches to implementing KM in an organization that } \\
\text { combine methods and tools to address both tacit and explicit } \\
\text { knowledge. This includes establishing KM strategy and goals and } \\
\text { implementation of a company-wide KM System. }\end{array}$ \\
\hline Wikis & $\begin{array}{l}\text { Schaffert (2008); Parker and Chao } \\
\text { (2007) }\end{array}$ & $\begin{array}{l}\text { The collaborative creation of knowledge content through an online } \\
\text { media. }\end{array}$ \\
\hline $\begin{array}{l}\text { Communities of } \\
\text { Practice }\end{array}$ & $\begin{array}{l}\text { Wenger (1998, 2000, 2002); } \\
\text { Wheatley (2002) }\end{array}$ & $\begin{array}{l}\text { A voluntary group of peer practitioners who share lessons learned, } \\
\text { methods and best practices in a given discipline or work } \\
\text { specialization. }\end{array}$ \\
\hline $\begin{array}{l}\text { Computer based training } \\
\text { (CBT) }\end{array}$ & $\begin{array}{l}\text { Uebel et al. (2001); Pedroni, M. } \\
\text { (2007). }\end{array}$ & $\begin{array}{l}\text { Computer systems and software and training content that supports } \\
\text { e-learning, usually at the user's own pace. }\end{array}$ \\
\hline Training Simulators & $\begin{array}{l}\text { Leo et al. (2000); Chavez-Mercado } \\
\text { et al. (1997); Lowthert (1995) }\end{array}$ & $\begin{array}{l}\text { Hands-on computer simulation models that permit users to } \\
\text { experience, through direct interaction with in a virtual (modeled) } \\
\text { environment, a representation of a real world system and its } \\
\text { behavior, possibly in an interactive life-like manner. }\end{array}$ \\
\hline $\begin{array}{l}\text { Knowledge Elicitation } \\
\text { Interviews (e.g. } \\
\text { employee exit } \\
\text { interview) }\end{array}$ & $\begin{array}{l}\text { Coffey and Hoffman (2003); } \\
\text { Hoffman et al. (1995); Klein (1992); } \\
\text { Gross et al. (2002); Hanes and Gross } \\
\text { (2002) }\end{array}$ & $\begin{array}{l}\text { Most often in the form of exit interviews, they are a form of tacit } \\
\text { knowledge capture from subject matter experts. }\end{array}$ \\
\hline $\begin{array}{l}\text { Knowledge Codification } \\
\text { and Snis (2001). }\end{array}$ & $\begin{array}{l}\text { (1999); Hall (2006); Sorensen } \\
\text { the explicit documentation of knowledge on methods or processes } \\
\text { thacit know-how (e.g. design documentation, guidelines, } \\
\text { procedures) }\end{array}$ \\
\hline
\end{tabular}




\begin{tabular}{|c|c|c|}
\hline $\begin{array}{l}\text { KM Method or } \\
\text { Practice }\end{array}$ & Author(s) & Description of Approach \\
\hline $\begin{array}{l}\text { Knowledge self-capture } \\
\text { (e.g. daylog, decision } \\
\text { summary) }\end{array}$ & Piktialis and Greenes, 2007. & $\begin{array}{l}\text { Efforts by an individual to document knowledge acquired. This } \\
\text { may be either to capture experience and insights in a very general } \\
\text { sense or may be related to a specific task or work process. }\end{array}$ \\
\hline $\begin{array}{l}\text { Mentoring and } \\
\text { Coaching }\end{array}$ & $\begin{array}{l}\text { Burrel and Safi (2008); Piktialis and } \\
\text { Greenes, } 2007 .\end{array}$ & $\begin{array}{l}\text { The interactive transfer of knowledge from more experienced to } \\
\text { less experienced staff. A dynamic and reciprocal relationship that } \\
\text { may take the form of managerial, group or team, or one-on-one } \\
\text { coaching and mentoring. }\end{array}$ \\
\hline $\begin{array}{l}\text { Information Processing } \\
\text { and Management }\end{array}$ & King (2006) & $\begin{array}{l}\text { Methods and systems to support information management } \\
\text { processes including records and data management practices. }\end{array}$ \\
\hline Document Management & Charles (2002). & $\begin{array}{l}\text { Systems and technology to support the archival and retrieval of } \\
\text { documents, electronic artifacts, drawings etc. }\end{array}$ \\
\hline Peer Assist & Eldridge (1994); IAEA (2008) & $\begin{array}{l}\text { Peers from different teams or organizations share their } \\
\text { experiences, insights, and knowledge with a team that has } \\
\text { requested assistance. }\end{array}$ \\
\hline $\begin{array}{l}\text { Action Reviews (i.e. } \\
\text { experience reviews) }\end{array}$ & Piktialis and Greenes, 2007 & $\begin{array}{l}\text { Action reviews are quick and simple team learning process held } \\
\text { while work is being performed, usually during a break in the task } \\
\text { or activity. A post-job briefing is a retrospective review of } \\
\text { recently completed work assignment, task, or activity to clarify or } \\
\text { establish and reinforce the lessons learned. A pre-job briefing is a } \\
\text { preparatory review in advance of a work assignment, task or } \\
\text { activity to familiarize, clarify or re-establish knowledge needed } \\
\text { and reinforce the past lessons learned. Intent is to reinforce on- } \\
\text { the-job learning during the experience. }\end{array}$ \\
\hline Story Telling & Mitchell (2000). & $\begin{array}{l}\text { To transfer experience, values, and lessons learned through the } \\
\text { recollection of previous incidents, or past circumstances or events. }\end{array}$ \\
\hline $\begin{array}{l}\text { Data Mining and Full- } \\
\text { Text Searching }\end{array}$ & Li et al. (2006) & $\begin{array}{l}\text { Computer based tools to enable the searching and extracting of } \\
\text { data or information from existing repositories to extract } \\
\text { information and support knowledge re-use or generation. }\end{array}$ \\
\hline
\end{tabular}




\begin{tabular}{|c|c|c|}
\hline $\begin{array}{l}\text { KM Method or } \\
\text { Practice }\end{array}$ & Author(s) & Description of Approach \\
\hline $\begin{array}{l}\text { Business Environment } \\
\text { Intelligence Gathering }\end{array}$ & Heinrichs (2001) & $\begin{array}{l}\text { Process and tools used to survey and acquire relevant information } \\
\text { on a firms business environment. }\end{array}$ \\
\hline $\begin{array}{l}\text { Cross-Functional } \\
\text { Teams, Team Learning }\end{array}$ & $\begin{array}{l}\text { Griffith et al. (2003); Huber (1999); } \\
\text { Lewis (2004); Mohamed et al. } \\
\text { (2004); Pinto et al. (1993); Politis } \\
\text { (2003) }\end{array}$ & $\begin{array}{l}\text { The formation of project or work teams comprised of members } \\
\text { from different disciplines and/or departments and/or production } \\
\text { phases to facilitate knowledge sharing. }\end{array}$ \\
\hline $\begin{array}{l}\text { Knowledge Loss Risk } \\
\text { Assessments }\end{array}$ & IAEA (2005) & $\begin{array}{l}\text { Method and process of assessing critical knowledge and resources } \\
\text { in a firm and its risk of being lost due to attrition. }\end{array}$ \\
\hline Intranet Portals & Marshall (1987) & $\begin{array}{l}\text { Internal organizational website to provide common } \\
\text { communication port and ease of access to various company } \\
\text { resources including links to various repositories, databases, } \\
\text { document archives etc. }\end{array}$ \\
\hline $\begin{array}{l}\text { Enterprise Application } \\
\text { Software (e.g. } \\
\text { Enterprise Resource } \\
\text { Planning Systems) }\end{array}$ & $\begin{array}{l}\text { Jones et al. (2003, 2006); Haugh } \\
(2004) ; \text { Ke and Wei (2006); EPRI } \\
(2006)\end{array}$ & $\begin{array}{l}\text { Advanced enterprise business software that integrates various } \\
\text { information sub-systems such as procurement, work-management, } \\
\text { resource planning, inventory management, production planning, } \\
\text { and finance. Such systems typically embed knowledge of business } \\
\text { processes and facilitate information and work flow. }\end{array}$ \\
\hline KM Assessment Tools & $\begin{array}{l}\text { Kankanhalli et al. (2004); Carpenter } \\
\text { et al. (2003); CMA (1999); Marti } \\
\text { (2004); Marti (2001); McDougall } \\
\text { and Hinks (2000); CMBG (2004); } \\
\text { Chang Lee et al. (2005); Ahmed et } \\
\text { al. (1994); Ahn et al. (2004); } \\
\text { Birchall and Tovstiga (2002); } \\
\text { Bornemann and Sammer (2003); } \\
\text { Cordero (1989); De Jager (1999); } \\
\text { del-Rey-Chamorro et al. (2003); } \\
\text { Grossman (2006) }\end{array}$ & $\begin{array}{l}\text { Various quantitative or qualitative methods to assess or measure } \\
\text { the extent, effectiveness, or quality of knowledge management } \\
\text { practices or processes. Most are based on benchmarking methods. }\end{array}$ \\
\hline
\end{tabular}




\begin{tabular}{|c|c|c|}
\hline $\begin{array}{l}\text { KM Method or } \\
\text { Practice }\end{array}$ & Author(s) & Description of Approach \\
\hline $\begin{array}{l}\text { Pay for Knowledge } \\
\text { Schemes }\end{array}$ & $\begin{array}{l}\text { Gupta (1987); Celani and Weber } \\
(1997) .\end{array}$ & $\begin{array}{l}\text { Remuneration system that includes financial rewards for } \\
\text { employees who achieve and maintain skill competencies or } \\
\text { knowledge in specified areas. }\end{array}$ \\
\hline IS/IT Infrastructure & $\begin{array}{l}\text { Marti (2004); Baldwin (2001); } \\
\text { Ahituv et al., (1994); Bach (2004); } \\
\text { Hendriks (1999, 2000); Holsapple } \\
\text { (2005); Junnarkar and Brown } \\
\text { (1997); Kim and Lee (2006); Pyoria } \\
\text { (2005); Tippins and Sohi (2003); } \\
\text { EPRI (2006) }\end{array}$ & $\begin{array}{l}\text { Design and implementation of IS and IT tools and infrastructure in } \\
\text { an organization to facilitate knowledge management processes } \\
\text { (capture, transfer, storage etc.) }\end{array}$ \\
\hline $\begin{array}{l}\text { Concept maps, } \\
\text { knowledge maps, } \\
\text { ontological models }\end{array}$ & $\begin{array}{l}\text { Eppler, M.J. (2001). } \\
\text { Novak (1995); Coffey et al. (2004); } \\
\text { Burnett et al. (2004) }\end{array}$ & $\begin{array}{l}\text { Graphical (diagrammatic) techniques to show associations, } \\
\text { linkages, structure, and inter-relationships in concepts or } \\
\text { knowledge domains }\end{array}$ \\
\hline $\begin{array}{l}\text { Intellectual Asset } \\
\text { Management } \\
\text { Approaches }\end{array}$ & $\begin{array}{l}\text { Guthrie (2001); Petty and Guthrie } \\
\text { (2000); Marti (2001); Andriessen } \\
\text { and Tissen (2000); Bontis (2001); } \\
\text { Brooking (1996a,b; 1998); } \\
\text { Contractor (2000); Edvinsson and } \\
\text { Malone (1997). }\end{array}$ & $\begin{array}{l}\text { Management strategies that recognize the value of intellectual } \\
\text { assets (or intellectual capital) and focus on enabling their creation } \\
\text { and utilization. }\end{array}$ \\
\hline $\begin{array}{l}\text { Promoting a Knowledge } \\
\text { Sharing Culture or } \\
\text { Knowledge } \\
\text { Management Culture }\end{array}$ & $\begin{array}{l}\text { Moon and Park (2002) } \\
\text { Jones et al. (2003) } \\
\text { Know and Gao (2004); Small } \\
\text { (2005); Caulkins (2004); Aramburu } \\
\text { et al. (2006); Balthazard and Cooke } \\
\text { (2004); Bergman et al. (2004); } \\
\text { Hanley (1999); Janz and } \\
\text { Prasarnphanich (2003); Kandemir } \\
\text { and Hult (2005) }\end{array}$ & $\begin{array}{l}\text { Management practices aimed at establishing and maintaining an } \\
\text { organizational culture that facilitates and rewards knowledge } \\
\text { sharing. }\end{array}$ \\
\hline
\end{tabular}




\begin{tabular}{|c|c|c|}
\hline $\begin{array}{l}\text { KM Method or } \\
\text { Practice }\end{array}$ & Author(s) & Description of Approach \\
\hline $\begin{array}{l}\text { Online Resource } \\
\text { Locators }\end{array}$ & Wills et al. (2002) & $\begin{array}{l}\text { Online databases or lists that help to find human or other } \\
\text { knowledge resources (e.g. subject matter expert locator). }\end{array}$ \\
\hline $\begin{array}{l}\text { Benchmarking and Self- } \\
\text { Assessment }\end{array}$ & $\begin{array}{l}\text { Marti (2001); Bin Syed-Ikhsan and } \\
\text { Rowland (2004); McDougall and } \\
\text { Hinks (2000); Kaplan and Norton } \\
(1992,1996) ; \text { Leseure and Brookes } \\
(2004)\end{array}$ & $\begin{array}{l}\text { Methods and tools for assessing the relative effectiveness of } \mathrm{KM} \\
\text { practices or processes at a point in time or over time. }\end{array}$ \\
\hline $\begin{array}{l}\text { Workforce Planning and } \\
\text { Succession Planning }\end{array}$ & & Methods and tools to assess the requirements for specific skills \\
\hline $\begin{array}{l}\text { Leadership } \\
\text { Development }\end{array}$ & & $\begin{array}{l}\text { Special training and assignments to build knowledge and } \\
\text { experience in potential leadership candidates. }\end{array}$ \\
\hline $\begin{array}{l}\text { Use of Retired or } \\
\text { Retiring Specialists }\end{array}$ & & $\begin{array}{l}\text { Initiatives that tap into the expertise of recently retired employees. } \\
\text { For example, as trainers or mentors, or to codify specialized } \\
\text { knowledge in the form of guidelines. }\end{array}$ \\
\hline $\begin{array}{l}\text { Directed Recruitment } \\
\text { and New Staff Training } \\
\text { Strategies }\end{array}$ & $\begin{array}{l}\text { Simo and Nyobe (no pub. date); } \\
\text { OECD (2000) }\end{array}$ & $\begin{array}{l}\text { Approaches focused on new hiring and developing specialized } \\
\text { skill sets needed to meet organizational needs, including new } \\
\text { graduate pipeline development programs such as efforts to } \\
\text { promote, strengthen, and improve undergraduate university or } \\
\text { college training programs. This may be in the form of } \\
\text { scholarships, research chairs, curriculum development, joint } \\
\text { research collaboration, work-terms and internships, adjunct } \\
\text { professors, joint training programs etc. }\end{array}$ \\
\hline $\begin{array}{l}\text { Formal Training and } \\
\text { Human Resource } \\
\text { Development Programs }\end{array}$ & IAEA (2004) & $\begin{array}{l}\text { Formal training program development and delivery, typically with } \\
\text { specific quality and performance objectives. }\end{array}$ \\
\hline $\begin{array}{l}\text { Operational Feedback } \\
\text { and Experience } \\
\text { Learning Systems }\end{array}$ & IAEA (2006); IAEA/OEDC (2001) & $\begin{array}{l}\text { Formal mechanisms to report, capture, assess, and correct } \\
\text { organizational failures or short-comings. Typically the focus is } \\
\text { put on identifying root causes and implementing corrective actions } \\
\text { to ensure organizational learning and improvement. }\end{array}$ \\
\hline
\end{tabular}




\begin{tabular}{|c|c|c|}
\hline $\begin{array}{c}\text { KM Method or } \\
\text { Practice }\end{array}$ & Author(s) & Description of Approach \\
\hline $\begin{array}{l}\text { Process Oriented } \\
\text { Knowledge } \\
\text { Management }\end{array}$ & $\begin{array}{l}\text { Malhotra (2005); Maier, R., and } \\
\text { Remus (2003); NEI (2003); IAEA } \\
\text { (2006a) }\end{array}$ & $\begin{array}{l}\text { A philosophy and approach to KM whereby } \mathrm{KM} \text { thinking and } \\
\text { practices are embedded or incorporated directly into the } \\
\text { organizations decision, business, and work processes. }\end{array}$ \\
\hline $\begin{array}{l}\text { Decision Support } \\
\text { Systems }\end{array}$ & DeSanctis (1982); Belardo (1982) & $\begin{array}{l}\text { Specialized computer software to provide enhanced capability for } \\
\text { management, operations, or maintenance decisions. Such systems } \\
\text { often embed decision rules and optimization functions based on } \\
\text { past expert judgment. }\end{array}$ \\
\hline $\begin{array}{l}\text { Knowledge or } \\
\text { Information Audits }\end{array}$ & $\begin{array}{l}\text { Hull et al. (2000); Liebowitz et al. } \\
\text { (2000); Burnett et al. (2004) }\end{array}$ & $\begin{array}{l}\text { Systematic approach to review and assess the adequacy of } \\
\text { information and knowledge flows and stores in an organizational } \\
\text { (or unit). }\end{array}$ \\
\hline $\begin{array}{l}\text { Enterprise/ Nuclear } \\
\text { Asset Management }\end{array}$ & $\begin{array}{l}\text { Backlund and Hannu (2002); } \\
\text { Harazim and Ferguson (2004); } \\
\text { IAEA (2005b); INPO (2001); IAEA } \\
\text { (2002b); Peterson (2003) }\end{array}$ & $\begin{array}{l}\text { Methods and tools to support the life-cycle management of } \\
\text { facilities and equipment. Condition assessment, equipment } \\
\text { reliability, surveillance and health monitoring, and maintenance } \\
\text { strategy are aspects. }\end{array}$ \\
\hline $\begin{array}{l}\text { Design Basis } \\
\text { Information } \\
\text { Management }\end{array}$ & CMBG (2004); Harris (2004) & $\begin{array}{l}\text { Specifically for production facilities, systems and tools to enable } \\
\text { effective configuration control, modification and maintenance of } \\
\text { design basis information. This may involve the integration of } \\
\text { several engineering tools such as CADD, electrical wiring } \\
\text { databases, parts management, equipment reliability databases, etc. }\end{array}$ \\
\hline $\begin{array}{l}\text { Knowledge or Risk } \\
\text { Informed Decision } \\
\text { Processes }\end{array}$ & $\begin{array}{l}\text { IAEA (2001); Kaner et al. (2004); } \\
\text { Makis et al. (2000) }\end{array}$ & $\begin{array}{l}\text { Methods and tools to permit better informed decisions through the } \\
\text { incorporation of equipment condition and economic, production or } \\
\text { safety significance. }\end{array}$ \\
\hline $\begin{array}{l}\text { Human Performance } \\
\text { Improvement Programs }\end{array}$ & $\begin{array}{l}\text { Bahr and Bier (2000); } \\
\text { Bhattacharya (2005); IAEA (2001) }\end{array}$ & $\begin{array}{l}\text { Specific training programs and work methods that focus on } \\
\text { reducing human error. }\end{array}$ \\
\hline $\begin{array}{l}\text { External Knowledge } \\
\text { Adoption }\end{array}$ & $\begin{array}{l}\text { Hwang (2005); Sussman and Siegal } \\
(2003)\end{array}$ & $\begin{array}{l}\text { Various methods may include external specialized training, } \\
\text { technology licensing, technology adoption, directed external } \\
\text { R\&D, custom system development, technology transfer from other } \\
\text { organizations. }\end{array}$ \\
\hline
\end{tabular}


A useful view of knowledge management practices is provided by Holsapple and Singh (2003). They view KM as a combination of "knowledge manipulation activities" performed by "knowledge processors" within the firm. They defined the "Knowledge Chain Model", an ontology that identifies five primary or major "knowledge manipulation" activities and four secondary or minor activities, all of which result in organizational learning. The five primary activities (actually knowledge processes by most other authors) are: knowledge acquisition activities (acquiring knowledge from eternal sources and making it suitable for subsequent use); knowledge selection (selecting needed knowledge from internal sources and making it suitable for subsequent use); knowledge generation (producing knowledge by either discovery or derivation from existing knowledge); knowledge internalization (altering the state of an organization's knowledge resources by distributing and storing acquired, selected, or generated knowledge) and knowledge externalization (embedding knowledge into organizational outputs for release into the environment).

The four secondary activities described Holsapple and Singh (essentially KM practices) are: knowledge leadership (establishing conditions that enable and facilitate fruitful conduct of $\mathrm{KM}$ ); knowledge coordination (managing dependencies among $\mathrm{KM}$ activities to ensure that proper processes and resources are brought to bear adequately at appropriate times); knowledge control (ensuring that needed knowledge processors and resources are available in sufficient quality and quantity, subject to security requirements); and knowledge measurement (assessing values of knowledge resources, knowledge processors, and their deployment). The authors posit that the 
competitiveness of an organization is directly related to organizational learning processes, and the Knowledge Chain Model explains the determinants of this learning. The model helps to explain how, in the author's words: "an organization's participants need to be cultivated, harnessed, and organized in the performance of these activities". Although their reference terminology differs from the "knowledge process" view of many other authors, this is a useful perspective in that it emphasizes the nature of knowledge management as "a coordinated effort of knowledge manipulation activities" that directly influence and enhance organizational learning processes. Further, their work is significant in that the authors provide a distinction between knowledge processes and the knowledge management practices intended to manipulate them. Their findings support the theoretical model proposed in this research.

Many authors argue that in order for the practice of $\mathrm{KM}$ to be effective, it is not just the sum of $\mathrm{KM}$ activities undertaken in an organization that is important. $\mathrm{KM}$ must be implemented in a coordinated company-wide fashion, as a "system", and within the context of clear strategy and goals (IAEA, 2006b; Arora, 2002; Young, 2006; Chang and Ahn, 2005; Corso et al., 2003; Treasury Board of Canada Secretariat, 2002; Chen Liu, 2003; Edwards, 2005; Feng et al., 2004; Gallivan, 2003; Haugh et al., 2004; and Starns and Odom, 2006). In a most general sense, much of the literature agrees that when $\mathrm{KM}$ is viewed and managed strategically as a corporate-wide system, it is referred to as a Knowledge Management System (KMS). However, there are several different perspectives in the literature as to what exactly constitutes a knowledge management system. These include views of KMS: 
- as an integrated component of the quality management system (QMS), which incorporates quality of knowledge-based processes (e.g. Carlin et al., 2007, or the Malcolm Baldrige Award as described in Smith (2008));

- as an important component of an integrated management system (IMS). The IAEA (2006) defines an integrated management system as "a set of interrelated or interacting elements (system) for establishing policies and objectives and enabling the objectives to be achieved in an efficient and effective way. The management system integrates all elements of an organization into one coherent system to enable all of the organization's objectives to be achieved. These elements include the structure, resources and processes. Personnel, equipment and organizational culture as well as the documented policies and processes are parts of the management system. The organization's processes have to address the totality of the requirements on the organization".

- as a key aspect of a process-oriented management system (e.g., the processoriented knowledge management described by Maier and Remus, 2002)

- as an organizational memory system (OMS), which is essentially a set of repositories of information and knowledge that the organization has acquired and retains (Huber, 1999)

- as an integrated corporate-wide $\mathrm{KM}$ approach with performance measurement and continuous improvement incorporated (e.g. Arora (2002); Young (2006); Chang and Ahn (2005); Corso et al. (2003); Chen Liu (2003); Edwards (2005); Feng et al. (2004); Gallivan (2003); Haugh et al. (2004)) 
- as an enterprise knowledge management system (EKMS), which is "an integrating framework that unites what individuals and corporations do with what they know. It is more than just another information management system. Whereas enterprise resource planning (ERP) systems and content management systems (CMSs) deal strictly with data and information, the EKMS deals with human-centered, experience based understanding that is actionable. The EKMS focuses on capturing people's knowledge with an intent to make it available to others for reuse and growth. That means: capturing, storing, accessing, searching, mining, extracting and using that knowledge." (EPRI, 2006).

- as an information management system (IMS). For example, Alavi and Leidner (2001) describe KMS as information systems that are geared toward enabling users to assign meaning to information and to capture some of their knowledge in information and/or data. Similarly, Davenport et al. (1998) define KMS as a tool to effect the management of knowledge and is manifested in a variety of implementations including document repositories, expertise databases, discussion lists, and context-specific retrieval systems incorporating collaborative filtering technologies.

There is value in all of these perspectives. It can be argued that no one of them is entirely correct and that an effective KMS may incorporate aspects of each of them. However, it is clear many author's agree on the following: if across an organization the collective set of management initiatives and practices aimed at improving knowledge processes are planned and coordinated in a strategic manner to address that 
organization's business needs, then these $\mathrm{KM}$ practices will have maximum benefit. Thus approaching $\mathrm{KM}$ in a strategic manner is in itself a KM practice.

In the literature on $\mathrm{KM}$, many practitioners and academics alike emphasize the importance of measures that assess the effectiveness of KM practices and knowledge processes, and provide feedback to enable the organization to identify shortcomings and improve over time (e.g. IAEA (2006b); Arora (2002); Young (2006); Chang and Ahn (2005); Corso et al. (2003); Treasury Board of Canada Secretariat (2002); Chen Liu (2003); Edwards (2005); Feng et al. (2004); Gallivan (2003); Haugh et al. (2004); Starns and Odom (2006)). Holsapple and Singh (2003) argue that knowledge measurement should be defined as: "the valuation of knowledge resources and knowledge processors, including quantitative methods, qualitative assessment, performance review, and benchmarking. It is a basis for evaluation of control, coordination, and leadership; for identifying and recognizing value-adding processors and resources; for assessing and comparing the execution of KM activities; and for evaluating the impacts of an organization's conduct of KM on bottom-line performance". Other authors provide similar definitions and most agree that in order to improve $\mathrm{KM}$, it must first be measured. However, there is much disagreement in the literature as to what aspects of KM should be the focus of measurement.

\subsubsection{Knowledge Management Measurement}

In general, the $\mathrm{KM}$ measurement and assessment approaches can be categorized into three basic types: methods that focus on attributes of the knowledge inputs; methods 
that focus on attributes of the knowledge processes (or processors); and methods that focus on the knowledge outputs (e.g. direct measures such as complexity, quantity of information, correctness properties of the knowledge itself as a product or output, or measures that focus on the value or benefit of the knowledge produced, expressed in a variety of forms ranging from measures of economic contribution to intangible benefits).

Assessment measures also vary widely in scope or orientation. For example, they may be based on corporate wide, group or individual measures, they may take a product focus, or even a capability focus (e.g. measures of increased absorptive capacity). Knowledge or knowledge process assessment is not objective (Cohen, 1998). Glazer (1991) proposed that as knowledge and its application is contextual, its measurement should be based on the perceptions of those who use it. Finally, methods may be categorized based on other factors such as: quality of knowledge processes, maturity of KM practices, extent of use of KM practices, measures of competence, assessment of knowledge base, and a host of intellectual asset and intellectual capital valuation tools. he Most Admired Knowledge Enterprises (MAKE) award is an annual international award to recognize best KM practices in knowledge-driven organizations in Asia, Europe, North America, India and Japan. This award assesses companies against a composite set of measures on eight KM performance dimensions (Murray and Greene, 2006):

- Creating a corporate knowledge-driven culture

- Developing knowledge workers through senior management leadership 
- Delivering knowledge-based products/solutions

- Maximizing enterprise intellectual capital

- Creating an environment for collaborative knowledge sharing

- Creating a learning organization

- Delivering value based on customer knowledge

- Transforming enterprise knowledge into shareholder value.Table 4 summarizes some of the various KM assessment approaches and tools found in a review of the literature. This literature is useful in that it is rich in $\mathrm{KM}$ practices and provides an excellent summary of what is considered KM "best practice" as well as providing many proven $\mathrm{KM}$ measures.

Table 4. Measurement of Knowledge and Knowledge Management

\begin{tabular}{|l|l|l|}
\hline \multicolumn{1}{|c|}{ Author(s) } & \multicolumn{1}{|c|}{ Name of Tool } & \multicolumn{1}{c|}{ Description of Tool } \\
\hline $\begin{array}{l}\text { Perez and Hynes } \\
\text { III, 1999 }\end{array}$ & $\begin{array}{l}\text { Tool for Assessing KM } \\
\text { Initiatives }\end{array}$ & $\begin{array}{l}\text { 5-dimensional K-assessment tool. Allows } \\
\text { knowledge workers to assess technology, } \\
\text { process, context, people, and content of the } \\
\text { KM process and highlight trouble spots and } \\
\text { increase the probability of the success of KM } \\
\text { initiatives. }\end{array}$ \\
\hline Lev, 1997 & $\begin{array}{l}\text { Knowledge Capital } \\
\text { Scoreboard }\end{array}$ & $\begin{array}{l}\text { A practitioner tool for measuring the economic } \\
\text { consequences of investment in knowledge } \\
\text { assets. }\end{array}$ \\
\hline Guthrie, 2001 & $\mathrm{n} / \mathrm{a}$ & $\begin{array}{l}\text { Literature Review of Tools. Summarized IC } \\
\text { Measurement Tools available. }\end{array}$ \\
\hline Petty and Guthrie, & $\mathrm{n} / \mathrm{a}$ & $\begin{array}{l}\text { Literature Review of Tools. Summarized IC } \\
\text { Measurement Tools available. }\end{array}$ \\
\hline Grossman, 2006 & $\mathrm{n} / \mathrm{a}$ & $\begin{array}{l}\text { Literature Review. Overview of KM } \\
\text { Assessment Approaches }\end{array}$ \\
\hline O'Dell et al., 2003 & $\mathrm{n} / \mathrm{a}$ & $\begin{array}{l}\text { Tool to Determine the State of KM in Your } \\
\text { Organization. A high-level questionnaire } \\
\text { aimed at obtaining an initial high-level } \\
\text { assessment of the maturity of KM in a firm. } \\
\text { Three levels are identified and can best be } \\
\text { summarized as "embarking stage", "exploring } \\
\text { stage", and "practicing stage". }\end{array}$ \\
\hline
\end{tabular}




\begin{tabular}{|c|c|c|}
\hline Author(s) & Name of Tool & Description of Tool \\
\hline Sveiby, 1997 & $\begin{array}{l}\text { Intangible Asset } \\
\text { Monitor }\end{array}$ & Knowledge Assets (IC) Measurement Model \\
\hline Stewart, 1997 & Economic Value Added & Knowledge Assets (IC) Measurement Model \\
\hline O'Dell et al., 1999 & $\mathrm{n} / \mathrm{a}$ & $\begin{array}{l}\text { Survey Tool to Assess Impediments to } \\
\text { Creating a Knowledge Sharing Culture. } \\
\text { Questionnaire that looks at the leading } \\
\text { impediments to knowledge sharing in } \\
\text { organizations. }\end{array}$ \\
\hline $\begin{array}{l}\text { Bornemann and } \\
\text { Sammer, } 2003\end{array}$ & $\begin{array}{l}\text { KM Activity } \\
\text { Prioritization }\end{array}$ & $\begin{array}{l}\text { Assessment methodology to prioritize } \\
\text { knowledge management related activities to } \\
\text { support organizational excellence. }\end{array}$ \\
\hline IAEA, 2005 & $\begin{array}{l}\text { Knowledge Loss Risk } \\
\text { Assessment }\end{array}$ & $\begin{array}{l}\text { Assess Risk of Core Competency Loss Due to } \\
\text { Attrition }\end{array}$ \\
\hline $\begin{array}{l}\text { Edvinsson and } \\
\text { Malone, } 1997\end{array}$ & Skandia Navigator & Knowledge Assets (IC) Measurement Model \\
\hline Sullivan, 2000 & $\begin{array}{l}\text { Intellectual Asset } \\
\text { Valuation }\end{array}$ & Knowledge Assets (IC) Measurement Model \\
\hline $\begin{array}{l}\text { Andriessen and } \\
\text { Tiessen, } 2000\end{array}$ & The Value Explorer & Knowledge Assets (IC) Measurement Model \\
\hline Stewart, 1997 & $\begin{array}{l}\text { Calculated Intangible } \\
\text { Value }\end{array}$ & Knowledge Assets (IC) Measurement Model \\
\hline $\begin{array}{l}\text { Intellectual } \\
\text { Capital } \\
\text { Management } \\
\text { Group }\end{array}$ & IC Tools & Knowledge Assets (IC) Measurement Model \\
\hline $\begin{array}{l}\text { Ahmed et al., } \\
1999\end{array}$ & IC Tools & Knowledge Assets (IC) Measurement Model \\
\hline CMA, 1999 & $\begin{array}{l}\text { CMA Report on } \\
\text { Measuring Knowledge } \\
\text { Assets }\end{array}$ & Knowledge Assets (IC) Measurement Model \\
\hline de Gooijer, 2000 & KM Assessment & $\begin{array}{l}\text { A Knowledge Management Performance } \\
\text { Scorecard }\end{array}$ \\
\hline Arthur Andersen & KM Assessment & $\begin{array}{l}\text { Knowledge Management Assessment Tool } \\
\text { (KMAT) }\end{array}$ \\
\hline $\begin{array}{l}\text { Teleos and The } \\
\text { KNOW Network }\end{array}$ & $\begin{array}{l}\text { The International Most } \\
\text { Admired Knowledge } \\
\text { Enterprises (MAKE) } \\
\text { Award }\end{array}$ & KM Assessment Criteria \\
\hline $\begin{array}{l}\text { British Standards } \\
\text { Institute }\end{array}$ & $\begin{array}{l}\text { Knowledge } \\
\text { Management PAS 2001: } \\
\text { A Guide to Good } \\
\text { Practice }\end{array}$ & KM Assessment Approach \\
\hline
\end{tabular}




\begin{tabular}{|l|l|l|}
\hline \multicolumn{1}{|c|}{ Author(s) } & \multicolumn{1}{|c|}{ Name of Tool } & \multicolumn{1}{c|}{ Description of Tool } \\
\hline $\begin{array}{l}\text { Canadian Institute } \\
\text { of Knowledge } \\
\text { Management }\end{array}$ & Frid Framework & KM Assessment Approach \\
\hline $\begin{array}{l}\text { American } \\
\text { Productivity \& } \\
\text { Quality Centre }\end{array}$ & $\begin{array}{l}\text { KM Roadmap to } \\
\text { Success }\end{array}$ & KM Assessment Approach \\
\hline $\begin{array}{l}\text { KM Standards } \\
\text { Australia }\end{array}$ & $\begin{array}{l}\text { Interim KM Standard } \\
\text { AS5037(Int) }\end{array}$ & KM Assessment Approach \\
\hline Rapley (1997) & $\begin{array}{l}\text { Accounting for the } \\
\text { Value Generated from } \\
\text { Innovation }\end{array}$ & Knowledge Assets (IC) Measurement Model \\
\hline $\begin{array}{l}\text { European } \\
\text { Standardisation } \\
\text { Committee }\end{array}$ & $\begin{array}{l}\text { European Guide to } \\
\text { Knowledge } \\
\text { Management }\end{array}$ & $\begin{array}{l}\text { KM Assessment Approach addressing a wide } \\
\text { range of issues in several categories. }\end{array}$ \\
\hline $\begin{array}{l}\text { Paulzen et al. } \\
\text { (2002) }\end{array}$ & $\begin{array}{l}\text { Knowledge Process } \\
\text { Quality Model (KPQM) }\end{array}$ & $\begin{array}{l}\text { Quality model for assessing knowledge } \\
\text { processes includes four dimensions: maturity } \\
\text { stage, knowledge activity, management area } \\
\text { and assessment structure. }\end{array}$ \\
\hline
\end{tabular}

As seen from Table 4, there are many approaches to assess $\mathrm{KM}$ in an organization. However, for the purposes of the current research, although specific measures can be utilized from each approach, these approaches are in general found to be very general and make little or no distinction between KM practices and knowledge processes (a key aspect of the research model, to be subsequently elaborated upon). However, several authors have emphasized that various quality attributes of knowledge processes in organizations are a key factor in organizational learning and performance (Linderman et al., 2004; Elliot, 1998; Barber and Munive-Hernandez, 2006; Bose, 2004; Buyukozkan, 2004). Glazer (1991) also concluded that because the production and application of knowledge is contextual, its measurement should be based on the perceptions of those who use it. Therefore knowledge process measures should be developed in the specific 
context of organizations based on measures that best reflect their knowledge objectives. Jennex et al. (2008) found that effectiveness is a valid measure of knowledge process quality (Jennex et al., 2008).

Combining both ideas, measures of knowledge process quality may be expressed in terms of "effectiveness" measures in language and terminology that managers can understand and interpret meaningfully in the context of their organizational objectives. Similarly, it can be argued that KM practice measures should be developed based on the perceptions of those who use them. The level of support for KM practices (in an organization) are indicated by the extent of use of $\mathrm{KM}$ practices. Thus specific measures can be developed based on the extent of use of accepted KM best practices. The IAEA (2006b) has applied this approach successfully to the measurement of KM practices and a similar approach is adopted for this research. The preceding discussion has provided a basis to discuss knowledge management practice and its measurement. The next section will provide an outline of the salient and distinguishing aspects of KM in NPPs.

\subsubsection{Knowledge Management in Nuclear Power Plants}

To better understand $\mathrm{KM}$ within a nuclear plant organizational framework, it is important to recognize and understand some of the unique and perhaps peculiar characteristics of these organizations. O'Dell et al. (2003) describe widely adopted strategies for knowledge management that provide a useful framework to facilitate this 
discussion. Their KM strategies (italics) and the relevance to NPPs (indented subbullets) are:

a) KM as a business strategy: this is prevalent in organizations that believe KM and knowledge sharing are key to their ability to compete and grow. These organizations often see knowledge as their product and pursue KM because they firmly believe it will have a significant positive impact on the profitability of the enterprise.

- In NPPs, this perspective is of secondary importance, as growth and competition are not primary drivers within nuclear plant organizations (the focus of study). However, the belief that KM will have an impact on profitability is seen as a strategic driver.

b) Transfer of Knowledge and Best Practices: the most widespread strategy, it focuses on systematic approaches to the reuse of knowledge and the transfer of best practices, with the goal of improving operations, products, or services. Sharing tacit and explicit knowledge enables the organization to operate more effectively and efficiently.

- In NPPs, this KM strategy is of primary importance. There is a very strong industry wide "culture of sharing" in the nuclear power industry. This culture is born out of a common and shared risk that a single nuclear accident could have serious repercussions globally on the societal and political support for the continued operation of existing plants. This creates a strong motivation by governments, regulators, owners, and 
operators to participate in the sharing of best practices via communities of practice and other mechanisms.

c) Personal responsibility for knowledge - organizations operating under this strategy believe that people are the engine of knowledge and should be supported in, and responsible for, identifying, maintaining, and expanding their own knowledge. They are also expected to understand, increase, and share their knowledge assets. These organizations realize their employees are their most valuable asset and need to be able to use their knowledge - both personal and that of the collective enterprise - to the benefit of the company. There is also a trend toward making teams and communities of practice responsible for critical bodies of organizational knowledge.

- In NPPs, this strategy is of primary importance. There is a strong safety culture at NPPs built on the premises that excellence in training, employee competency, responsibility for safety, a questioning attitude, and individual human performance excellence are all of high importance.

d) Intellectual Asset Management - emphasizes organizational level management of intellectual assets such as patents, technologies, operational and management practices, customer relations, organizational arrangements, and other structural knowledge assets. Effective exploitation of these valuable assets can help the organization increase is competitive advantage. There is an increasing movement to measure the value of the organizational knowledge assets. 
- In NPPs, this strategy is of primary importance, but not from the intellectual asset exploitation or technology development/innovation perspective. Rather, the emphasis is on leveraging intellectual assets such as operational and maintenance practice excellence, plant life-cycle asset management, organizational structure and safety culture, organizational performance management practices and structural assets such as configuration management of the plant design basis.

e) Innovation and Knowledge Creation - this strategy emphasizes innovation and the creation of new knowledge through basic and applied research and development. The development of unique knowledge and expertise increases the organization's competitive value.

- In NPPs, this perspective is of secondary importance and an approach to $R \& D$ external to firm is usually taken, although process innovation and creation of new knowledge (at least from an operational expertise perspective) is still important.

O'Dell et al. further point out the importance of understanding why KM is important in a given organization and to clearly link this understanding to the value proposition and business rationale for embarking on any KM initiative. They posit three fundamental value propositions: customer intimacy, product-to-market excellence, and operational excellence. Though the first two are important in most industries, the later is clearly the primary value proposition and business driver for NPP organizations. Although it can be argued that power production is "product-to-market" activity, it is not consistent with 
the spirit of the construct (which was intended to focus on product and technology innovation (i.e. R\&D) for delivery to external markets), and further, the process and activities associated with power production are primarily and fundamentally associated with operational excellence. Thus from the NPP perspective, the prominent value proposition for KM lies clearly in improving operational excellence. This establishes the context for organizational performance in this study.

Introducing and maintaining an effective knowledge management program in a nuclear plant is a multi-faceted problem that is difficult and challenging. Many utilities are making progress towards the implementation of knowledge management practices and support systems, but in general, progress lags other industries and higher than expected implementation effort is being experienced (IAEA, 2006). Further, nuclear utilities are not always be able to determine the effectiveness of $\mathrm{KM}$ initiatives, and in many cases are not be able to identify key areas in need of improvement. As a result, knowledge management initiatives in NPPs have not always provided all of the expected benefits (EPRI, 2006). This underscores the need for effective KM strategy, implementation, measurement, and ongoing improvement.

It can be argued that KM in NPP organizations involves additional challenges not faced by most industries. Some of the issues faced by nuclear plant operators include: a complex technology base and infrastructure (i.e. both from a design basis perspective and from and operations and management perspective); lengthy technology and plant life-cycles (i.e. design through to decommissioning may range from 60-100 years); highly capital-intensive plants (i.e. 6 to 8 billion dollars in capital cost per plant); a 
heavy reliance on multi-disciplinary technologies and expertise (e.g., physics, chemistry, material science, civil, process and mechanical engineering, etc.); competing operational objectives (i.e., safety, economics, and production); potentially high hazards that must be systematically managed to demonstrably low tolerable risks; and finally, the ongoing need for coordination of complex physical and human systems. In addition, stringent requirements for safety, security, environmental protection, equipment qualification, nuclear quality assurance, and equipment/design configuration management must be met, and all in the context of a regulated industry environment.

Despite all these challenges, NPP's have been unknowingly implementing a wide range of KM activities for a long time without explicitly recognizing it as such. There are many examples of long established KM practices in NPPs: formal equipment reliability programs, the systematic approach to training, processes and information systems for the configuration management of design basis information, rigorously documented operational procedures, plant work management systems, outage planning systems, prejob briefing practices, document management systems, and risk-informed asset management programs. However, many NPPs have not yet adopted a systematic or strategic approach to $\mathrm{KM}$, and $\mathrm{KM}$ practices are not seen as an integral part of the management system. A corporate wide strategy and implementation plan are often lacking.

The nuclear industry in most countries is a maturing industry. Figure 6 provides data on the number of plants by age (initial commercial operating date) across the entire population of NPPs globally. The typical design life of NPPs built prior to 2000 is 
about 30 years. It is evident that a large percentage of plants have reached or are approaching their design life. A 30 year design life unfortunately also coincides with the typical duration of an NPP career and recent high retirement rates in NPPs in many countries have highlighted their vulnerability to the loss of tacit knowledge. An aging global fleet of nuclear plants is in need either refurbishment or decommissioning and at the same time many new build projects are being planned and launched, creating high demand for specialized nuclear skills. Pro-active measures aimed at knowledge retention have been needed.

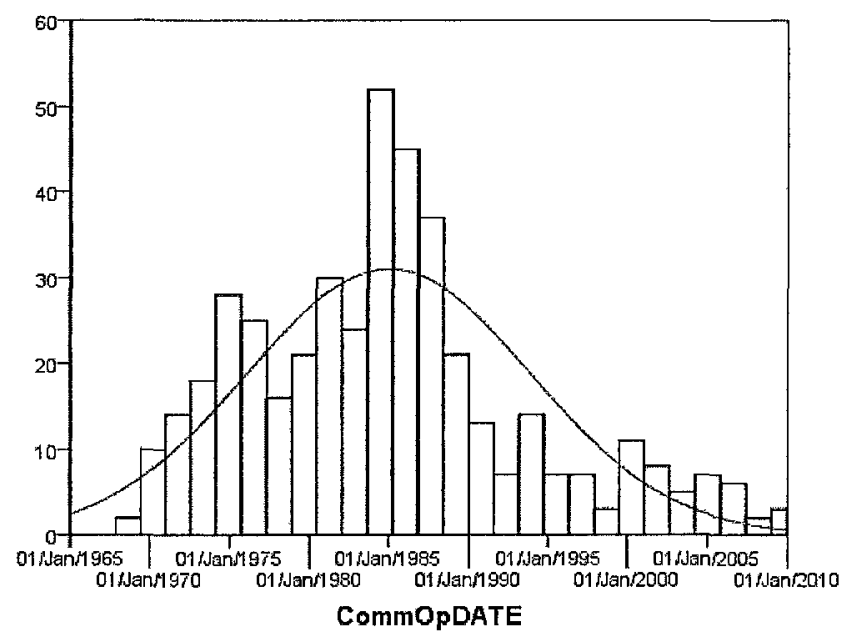

Figure 6. Summary of all NPPs by start-up date (WANO, 2010)

There is also concern in the industry over the lack of a "pipeline" of new nuclear program graduates with adequate technical skills to replace retiring NPP specialists and nuclear knowledge-workers. This is due to a long-term global decline in the number and size of university programs targeting nuclear specific knowledge and skills, a trend which started in the late 1980's (IAEA, 2007). There are only a handful of undergraduate nuclear engineering and science programs operating today, and an even 
smaller sub-set of these offer a post-graduate level program. There is also a recognition that it takes many years of on-the-job training to build the competencies and expertise needed for many NPP positions (e.g. a qualified nuclear operator or site fueling physicist). In light of all this, it is no surprise that knowledge retention has become a real priority at most NPPs in recent years (IAEA, 2007).

NPPs are very knowledge-driven organizations. Wiig (1999) provides a useful model to help appreciate this (Figure 7). Intelligent-acting "knowledge workers" go about their day-to-day work routines in all areas of the organization (operations, maintenance, technical support, training, etc.). Learning and continuous improvement occurs through experience gained and lessons learned.

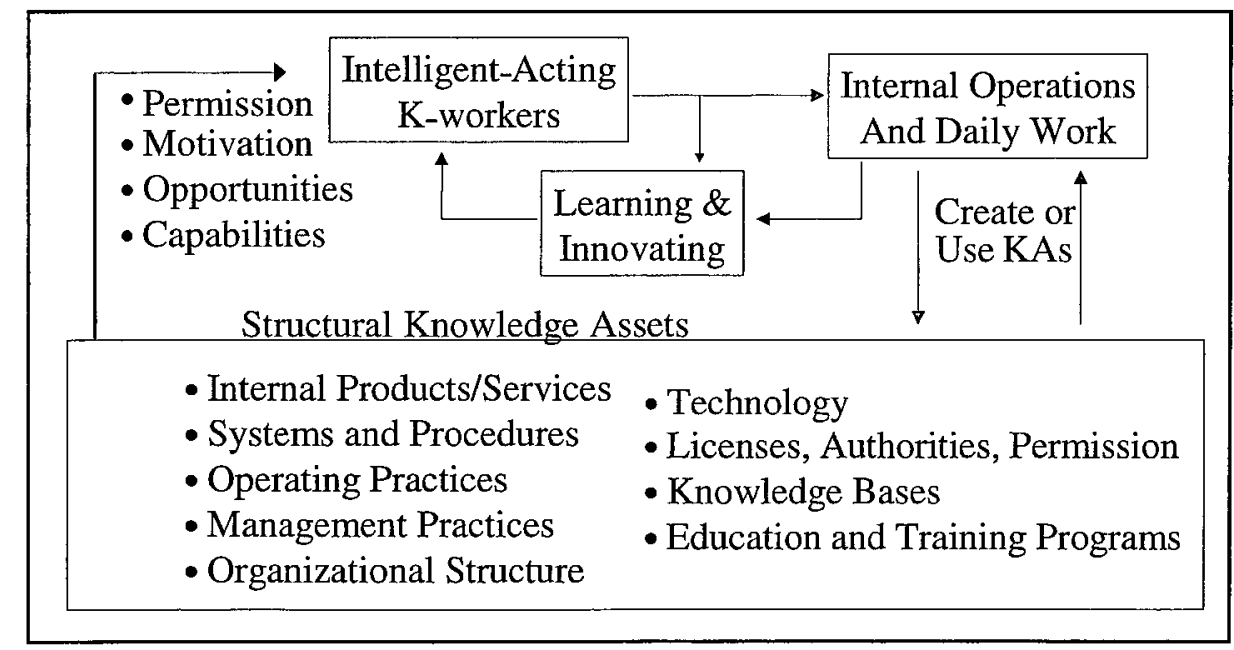

Figure 7. NPP as Knowledge-Driven Enterprise (adapted from Wiig, 1999)

Though-out the course of carrying out work activity, "structural knowledge assets" are created, and these may take many forms (e.g. internal products and services, information systems, operating and management practices, organizational roles and responsibilities and structure are defined, in-house technology may be developed and applied, explicit 
knowledge bases are created such as documentation or archives, and education and training programs are developed and captured).

Wiig's model highlights that learning and innovation occur on a continuous basis, and this enables effective utilization and improvement of the structural knowledge assets. All of this occurs within an organizational culture and factors such as empowerment and authority structures that enable or permit action, incentives and rewards that provide motivation, opportunities to acquire or utilize knowledge, and in general, the capabilities and capacity for effective problem solving all contribute to a pro-active knowledge driven organization. In the NPP context, the various organizational units of the NPP (e.g., operations, maintenance, technical support, training, human resources, safety and licensing, etc.) all contribute to and make use of the collective knowledge base of the organization. This can be viewed as an "integrated and shared knowledge base" (Figure 8). It represents the sum total of tacit knowledge (i.e. skills, experience base, expertise that contribute to the competencies of the organizations work-force), and the explicit knowledge that is captured and represented in information assets, artifacts and archives (such as databases, information systems, documents, procedures, work processes, design manuals, operational records, etc.). 


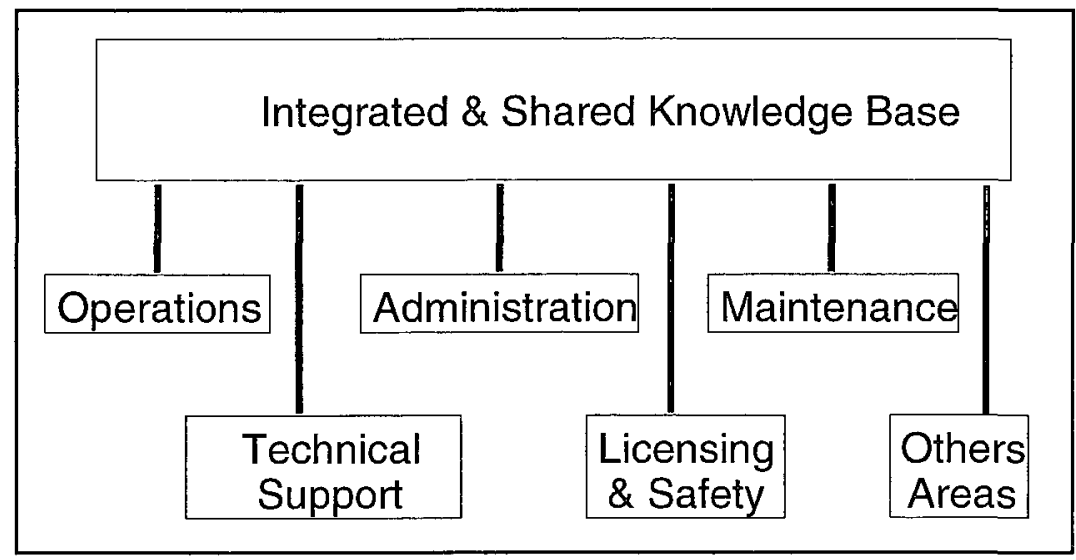

Figure 8. The NPP Integrated and Shared Knowledge Base

The information systems and information technology infrastructure in NPPs play an important role to support the integration and sharing functions of the knowledge base by enabling data, information, and knowledge to be stored, accessed, and flow. It facilitates knowledge capture, retention, transformation, and re-use. In an operating NPP, the complexity and inter-dependencies of these information management (IM) systems are significant. There are, in a typical plant, a large number of complex and heterogeneous technology (and sometime obsolete legacy) systems. Collectively, the number, complexity, and inter-dependencies of these systems (both in terms of automatic or manual information flows, and/or mutual support for organizational knowledge/work processes) present a formidable integration challenge in most NPPs. These systems include: computer aided design (CAD) model and electronic drawings repository; electronic operating procedures; operations and maintenance history data management systems; document and records management systems; real-time plant state information systems; outage planning and work control systems; plant configuration management (of design basis); quality control/surveillance records database; system 
health monitoring and surveillance information system; maintenance program information systems; models and codes; equipment reliability system, etc.

The knowledge base required to operate an NPP efficiently spans many disciplines including chemistry and corrosion specialists; electrical engineers, human factors experts, control engineers, civil engineers, software engineers, process and mechanical engineers; applied physicists; thermohydraulics experts; information technology specialists, material science experts, radiation protection and health physicists, highly trained operations staff, equipment reliability experts, nuclear life-cycle asset management specialists, performance management professionals, nuclear operations training instructors, simulation technology specialists, licensing and nuclear safety engineers, nuclear work planning and control specialists, and the traditional professional roles in finance, human resources, legal and procurement, etc.. Deep knowledge of plant operations, performance, safety, reliability, and regulatory compliance issues is required on the part of most staff relative to their roles and responsibilities. This is all in the context of a comprehensive and necessary framework of governing policies and procedures. In short, the investment in, and maintenance of, the organizational knowledge base to operate NPPs is significant.

From a work-flow and work process perspective, KM practices can be applied to everyday work in an NPP organizational environment. KM gives organizations a new viewpoint from which to examine aspects of the organization's business processes. For example, any given work activity can be reviewed in terms of the expertise (i.e. competencies and experience) needed to perform the task; the roles and interactions of 
the various participants involved in the task; the information needs, flows, products, and inter-dependencies on the participants; and the work procedures, methodologies, and decision processes involved (see Figure 9).

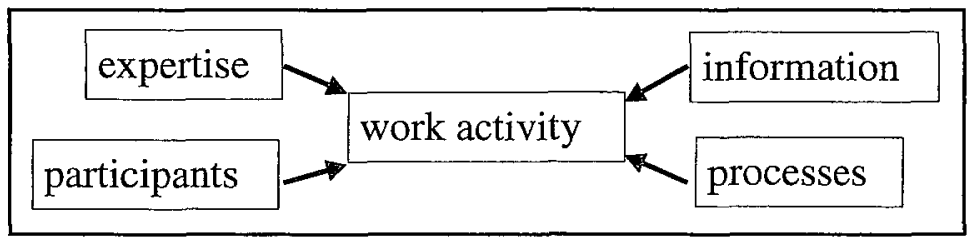

Figure 9. Viewing Work Processes from a KM Perspective

A KM perspective gives rise to many questions that help to understand the knowledge and knowledge process dependencies associated with a work activity. What are the critical knowledge processes involved? What are the characteristics of the relevant knowledge needed? Who has or should have this knowledge? What mechanisms are needed for the generation, sharing and utilization of this knowledge? What organizational conditions, processes, and changes are needed to make the work more efficient and effective (e.g. organizational structure, incentives, culture etc.)? KM offers a constructive perspective on a problem in terms of identifying knowledge process needs, gaps, and potential solutions. Some examples of possible knowledge or knowledge process gaps that might be revealed by a KM self-assessment review in an NPP would include:

- The Human Resources department has not understood the expertise needed in hiring staff for the technical support unit or engineering department.

- IT is not aware of the strategic business value of plant process historical data records and fails to adequately back-up the system hard drives. 
- The responsibility for updating work procedures is not clear and as a result key work procedures remain ambiguous despite corrective actions requesting they be improved.

- Maintenance experts are too busy to mentor junior staff and are effectively discouraged from doing so because their workload and deadlines will not permit it.

- Information records/logs are produced by operations for maintenance personnel without understanding why (i.e. who will use it and how) and as a result data is not logged correctly or completely.

- Data is captured in the work management system but not in a form it can be used.

- Data and information cannot be shared between the work management system, the parts inventory database, and the parts procurement database with the result that it takes too long to retrieve data, information is out of date, and errors are made.

KM provides a fresh viewpoint from which to examine and optimize work activity in terms of knowledge processes. In the context of NPP organizations this is significant because work activities and work processes (such as day-to-day operations and maintenance decision processes) are necessary, numerous, and complex. They are needed to ensure standardized methods, work control, and safe practices and are in the form of policy, process, and procedures. In NPPs, an emphasis on documented work processes and procedures must be taken out of necessity in order to deal with the 
complexity of the technology, the work environment, and work processes. There are many simultaneous requirements imposed on the NPP organizational work processes because of the need for compliance to safety, security, environmental protection, production reliability, regulatory oversight, equipment qualification, and quality assurance (QA) requirements. At the enterprise level, $\mathrm{KM}$ objectives aim to build, maintain, and capitalize on the alignment and synergies of the knowledge process requirements of organizational business/work processes and facilitate the building and maintenance of organizational knowledge in a strategic manner to enable its better utilization and ultimately achieve superior organizational performance.

\subsubsection{Importance of Communities of Practice}

The importance of nuclear communities of practice (COPs) as a vehicle for knowledge transfer, sharing and retention, and their overall importance in achieving and maintaining a high level of plant performance has been stated in several sources (Jones, 2003; Eldridge et al., 1994; Haber et al. 1991; Hill, 2001; IAEA, 1999; IAEA 2006; IAEA 2001; IAEA 2001b; Fox and Maidment, 1996; Karns, 2005; Lowthert, 1996; Schinzel, 2002). In the US, most if not all operating NPPs participate actively in nuclear COP programs and regularly perform and report on self-assessments based on the benchmarking programs established in those COPs (NEI, 2003). This fact alone has been cited as an important contributor to improved overall US NPP fleet performance over the past two decades (NEI, 2003). 
Wenger and Snyder (2000) define communities of practice as "groups of people informally bound together by shared expertise and passion for a joint enterprise". They argue the primary output of a COP is knowledge and the benefits typically include improved problem solving, exchange of best practices, and professional skills development and retention. Wenger (1998) defines a community of practice as "a group of people that share a common concern, set of problems, or a passion about a topic, and who deepen their knowledge and expertise in this area by interacting on an ongoing basis". Wenger et al. (2002) further propose that a COP can be described along three dimensions: what it is about (i.e. its joint enterprise as understood by its members); how it functions (i.e. the mutual agreement that binds members into a social entity); and what capability it has produced (i.e. the shared repertoire of communal resources, such as routines, sensibilities, artifacts, vocabulary, styles etc. that members develop over time). Wheatley (2002) describes COPs as "a rich environment where people with common experiences and shared problems or needs find each other, exchange ideas, develop trusting relationships, and form a community that promotes knowledge and experience sharing". In the US, the Nuclear Energy Institute (NEI) has taken the lead in establishing a number of COPs. These include (NEI, 2003):

\section{- INPO Work Management Working Group}

- INPO Work Management Working Group

- NEI RP Task Force

- Nuclear Information Technology Strategic Leaders

- Electric Utility Cost Group-Nuclear Committee (Data) 
- Nuclear Information Management Strategic Leaders (NIRMA)

- Nuclear Human Resources Group

- Licensing Actions Task Force (LATF)

- NEI EP Managers Task Force

- Fire Protection CoP

- Nuclear Supply Chain Strategic Leadership

- Nuclear Asset Management Task Force

- Configuration Management Benchmarking Group

- Industry Equipment Reliability Working Group

Typically these have a clear charter, organizing committee, and are strongly supported by the utilities, the US Nuclear Regulatory Commission (NRC), and NEI. These formal working groups facilitate the ongoing dissemination of nuclear operations and maintenance best practices based on a strong culture of experience sharing of lessons learned between NPP specialist peers. The US NPP experience with COPs is summarized in the following (NEI, 2003):

COPs have provided a network of process experts for solving problems... In this way COPs facilitate knowledge management across the industry, preserving and improving industry-wide competencies.

In summary, Communities of Practice have provided several key benefits to the US nuclear industry. These include (NEI, 2003):

- enable the building and future direction of process knowledge; 
- provide for cost insights;

- assist in the development of meaningful performance indicators;

- encourage practical strategies for standardization; and

- optimize efficiency.

NEI has played a lead role in establishing these COPs. It can be argued that the high participation rate of US NPPs in COPs and the associated benchmarking and dissemination of best practices has been a significant contributor to the steady performance improvements of US NPPs over the last 2 decades. Figures 10, 11 and 12 respectively provide key average performance statistics for the US NPP fleet that highlight the outstanding improvements that have been made in 3 key areas: average reactor "scram" rates (i.e. automatic safety system shut-downs per 7000 hours of operation); average refueling outage days; and capacity factor. Average reactor "scrams" is considered a good overall indicator as many types of events ranging from operator errors to equipment failures can result in the initiating event that triggers an unplanned automatic shut-down. Thus it is a good overall measure of safety performance. Figure 10 shows recent improvements in US NPPs. 


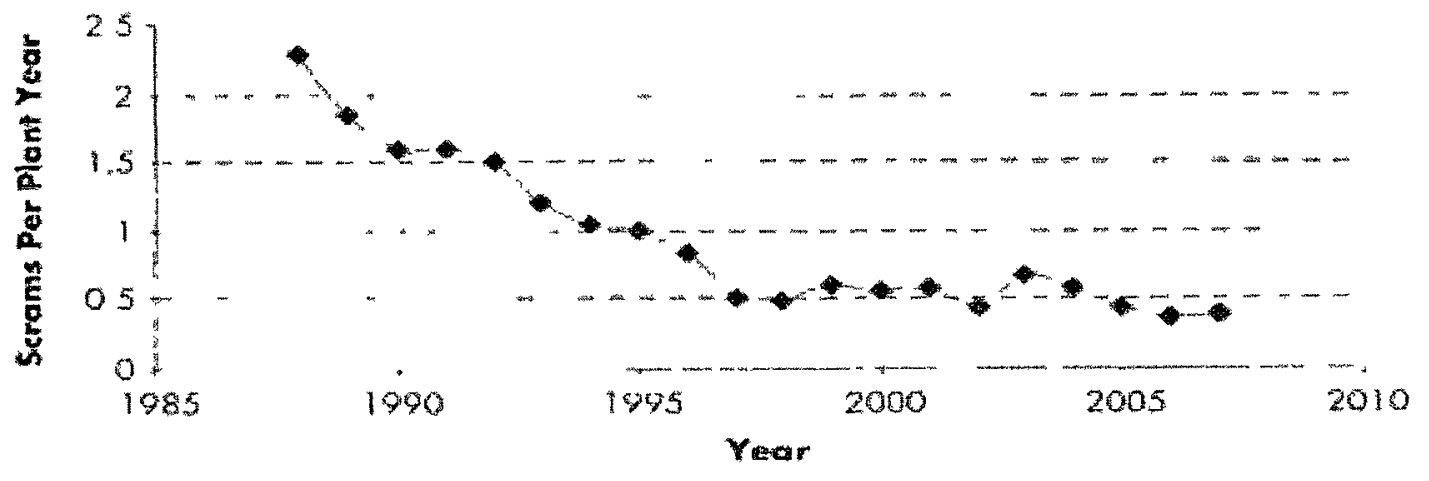

Figure 10. US NPP “Scram” Rates (Nuclear News, June 2009)

Refueling outage days is an indicator of organizational effectiveness. Outage duration is the result of many factors including logistics and work planning, work management, parts management, operations-to-field maintenance coordination, etc. See Figure 11.

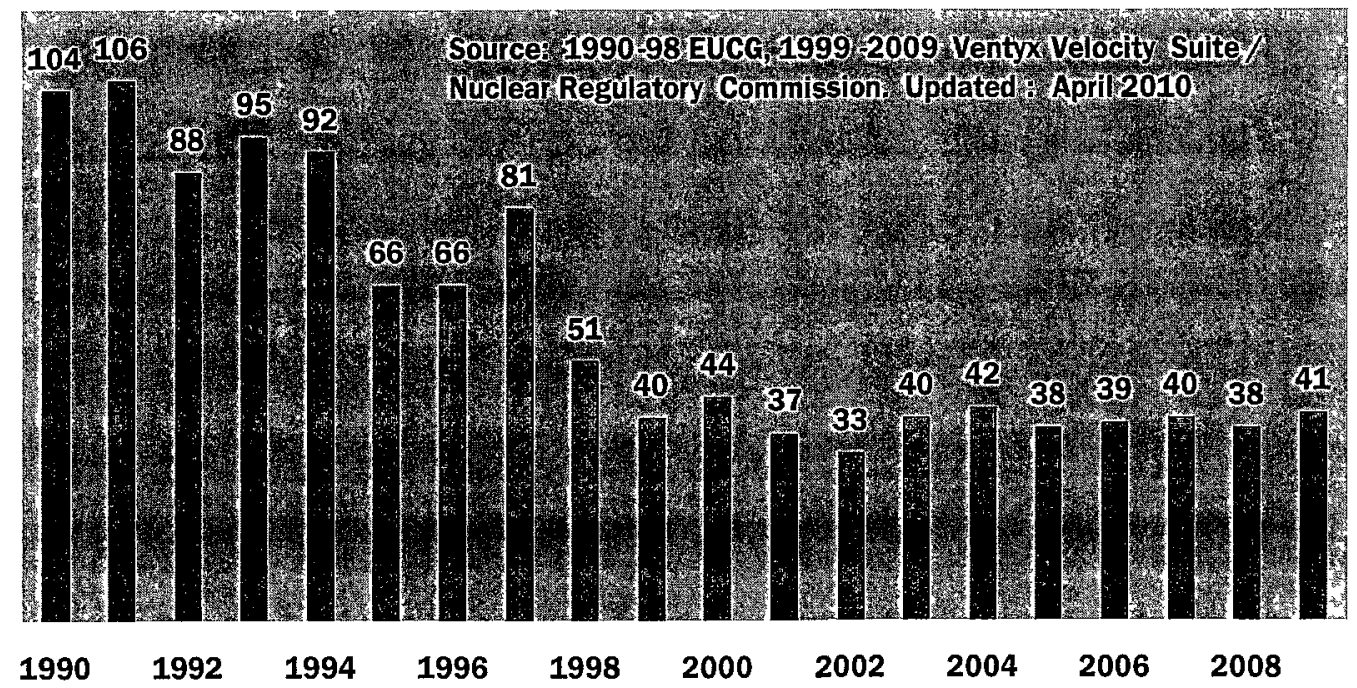

Figure 11. US Fleet Refueling Outage Days (NEI Website, 01/11)

Finally, capacity factor (CF, which is similar to capability factor) is also considered a good overall measure of plant performance. Once again, many factors collectively contribute to CF. It is a good overall indicator of organizational performance. Figure 12 shows steady US NPP improvements. 


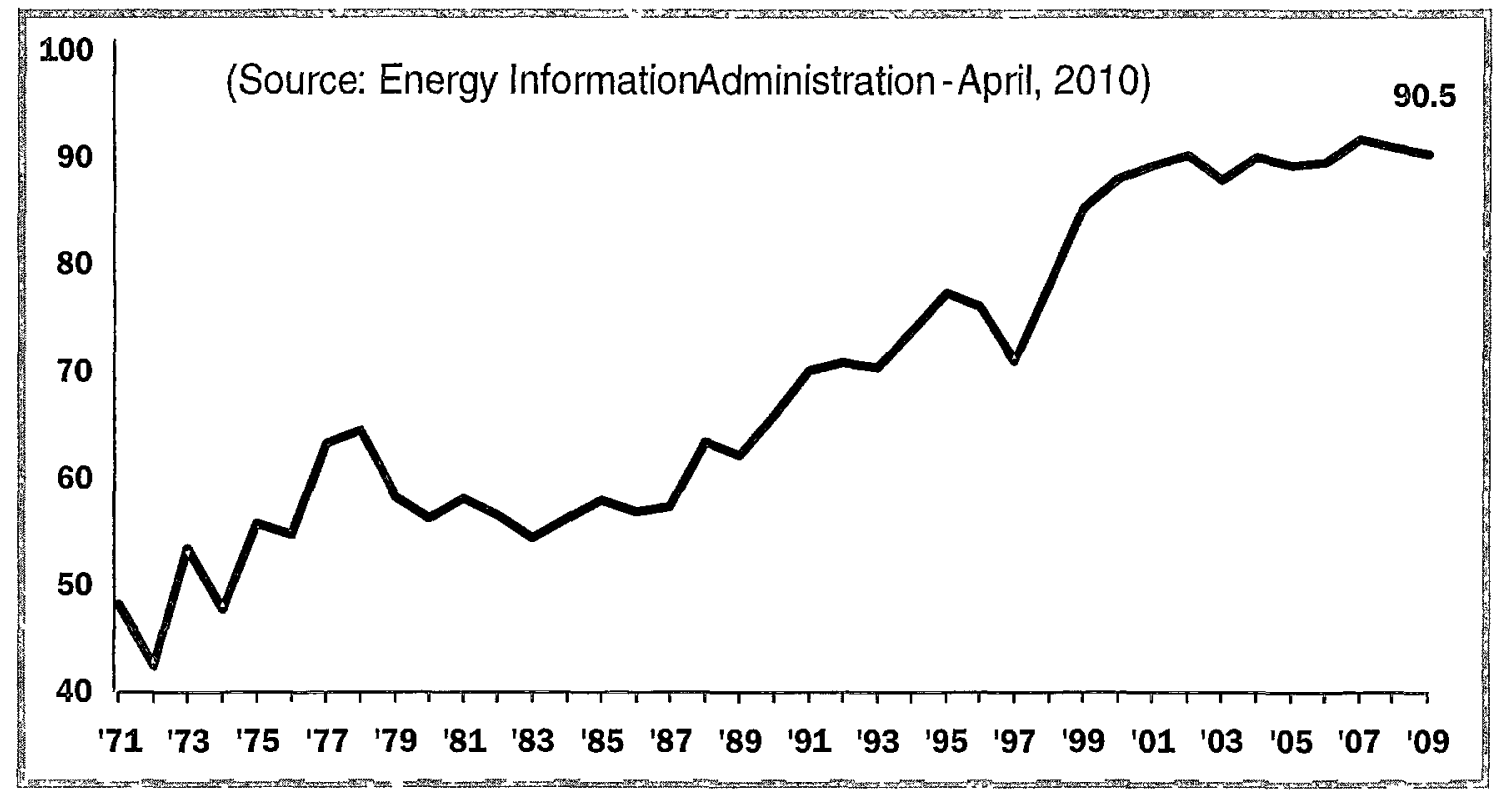

Figure 12. US Fleet Capacity Factor (NEI Website, Jan/11)

The long-term steady improvement in the US NPP fleet performance is dramatic. Although it is attributed to several factors, the impact of COPs must be recognized as key driver (NEI, 2006). Similar industry peer COP organizations exist in other countries and regions such as across EDF's fleet of nuclear plants in France (Ballay and Vannieuwenhuyse, 2004). Thus COP involvement and utility self-assessment benchmarking is thought to be an important factor in NPP knowledge acquisition and adoption processes regarding operational best practices through-out the industry.

\subsection{Organizational Performance}

In order to further explore and better understand the link between KM and NPP plant performance, this section will build on the discussion thus far with: i.) a review key findings from the literature on organizational performance; ii.) a summary and 
discussion of the literature specifically addressing NPP performance; iii.) a discussion of the literature linking KM to organizational performance; iv.) and finally, a discussion and summary of the literature on the role of $\mathrm{KM}$ in achieving sustained organizational performance in the nuclear plant context.

There has been considerable research published in the area of organizational performance, but the literature is still characterized by lack of consistency in the definition of terms (O'Donnel and Duffy, 2002). Neely et al. (1995) pointed out that although many studies have been published on performance, only a few of them explicitly define performance itself. Therefore, there is "massive disagreement as to what performance is" (Meyer and Gupta, 1994) and "few people agree on what performance really means: it can mean anything from efficiency, to robustness, or resistance, or return on investment, or plenty of other definitions never fully specified" (Lebas, 1995). As a result, numerous different terms are used to describe performance. They include among others: effectiveness, efficiency, productivity, quality, and capability. The differences between these constructs can be appreciated by reviewing some of the definitions of performance found in the literature.

A number of authors offer explicit definitions of performance (Cordero, 1989; Dwight, 1995; Neely et al., 1995; Rolstadas, 1998), while others discuss specific dimensions of performance (Moseng and Bredrup, 1993). Definitions of performance differ significantly in their scope. Dwight (1995) understood performance as "the level to which a goal is attained". However, other authors, for example Cordero (1989), Gleason and Barnum (1986), and Neely et al. (1995) viewed performance as a more 
encompassing construct and pointed out the need to address not only the achievement of goals, but also how this achievement is obtained. Therefore, their definition of performance stated that it is both "the efficiency and effectiveness of an action". They understood effectiveness as "the extent to which an objective has been achieved" ("doing the right thing", measuring outputs to determine if they help accomplish objectives), while efficiency was defined as "the degree to which resources have been used economically" ("doing things right", measuring resources to determine whether minimum amount are used in production of outputs).

Other authors extended this definition even further and identified more dimensions of performance, not only effectiveness and efficiency (Sink et al., 1984; Sink, 1985; Sink and Tuttle, 1989). Sink and colleagues' model claims that the performance of an organizational system is a complex interrelationship between the following seven performance dimensions: effectiveness (which involves doing the right things, at the right time, with the right quality, etc.; defined as actual output/expected output); efficiency (defined as resources expected to be consumed/resources actually consumed); productivity (the traditional ratio of output/input); innovation (seen as a key element in sustaining and improving performance); profitability/'budgetability' (which represents the ultimate goal for any organization); quality and quality of work life. Some authors pointed out the need to consider also several other dimensions of performance. For example, Feltham and Xie (1994) identified congruence (defined as the consistency and alignment of management priorities and actions with strategy) as another dimension of performance. Neely et al. (1995) and others (Leong et al, 1990, Fitzgerald et al, 1991) 
added flexibility to the list and argued that the four main dimensions of performance are: quality, time, cost (includes productivity), and flexibility. Slightly different dimensions of performance were identified by Moseng and Bredrup (1993). They looked at performance as the integration of three dimensions: efficiency, effectiveness and adaptability. The first two dimensions are similar to those used by Sink and Tuttle (1989), while the third expresses to which extent the company is prepared for future changes.

The notion of "future" with regard to performance is also described in a study by Lebas (1995) who pointed to the fact that performance is not so much about past achievements, as generally accepted, but about the future, i.e. the capability of the unit being evaluated. His definition of performance stated that performance "is about deploying and managing well the components of the causal model that lead to the timely attainment of stated objectives within constraints specific to the firm and to the situation." He argued that a well-performing organization is one that will achieve its objectives, not necessarily one that has already achieved them. Therefore, his understanding of performance is focused around capability and future potential of the organization. Lebas (1995) also pointed out that the definition and operationalization of performance is very subjective: it can be defined by different parameters in each firm, defining it to match its strategy and vision, subject to the external constraints of the market.

The literature on performance distinguishes between the terms productivity and performance. Some authors use these terms interchangeably. However, other authors see 
productivity explicitly as only one of many dimensions of performance (i.e. a measure of efficiency) (Sink et al., 1984; Clark and Fujimoto, 1991). Viewed as a dimension of performance, productivity is generally understood as a ratio of total outputs of the organization relative to the inputs used to generate them. Stainer and Nixon (1997) distinguish between partial productivity (a ratio relating single output to a single input, such as labor, materials, or capital); total factor productivity (a ratio of total output in financial terms to a single input or a ratio of a single output to total input); and total productivity (a ratio of total output to total input).

In this study, performance is measured as a dependent variable and on an organizational level. The focus will be on two performance dimensions widely discussed in the literature: organizational effectiveness, measured in terms of how well organizational goals are met; and operational performance, measured in terms of industry-wide operational efficiency ratios. This will be discussed further in the following sections.

\subsubsection{Organizational Performance of NPPs}

Organizational performance in production environments has been determined to be the direct result of a range of factors in many industries (Alikhan, 1991; Bhattacharya, 2005; Bititci et al., 1997; Coes, 1990; Condu, 2005; Djerdjouri, 2005; Eldridge et al., 1995; Haber et al., 1991; IAEA, 2006; IAEA, 2001; Ittner and Larcher, 1998; ). Many organizational performance factors have in turn been shown to be directly associated with knowledge management and organizational learning (Ahn et al., 2004; Bach, 2004; Baldwin, 2001; Brockman and Morgan, 2003; Thomas and Keithley, 2002; Nelson and 
Cooprider, 1996; Jie, 2001; Hall, 2002; De Gooijer, 2000; Bou-Llusar and SegarraCipres, 2006; Panayides, 2004; Malhotra, 2005; Calantone et al. 2002; Marques, 2006; Schack, 2004).

Organizational performance in NPPs involves several considerations. This section will elaborate on these issues. Porter (1985) points out that competitive advantage can be achieved by either low product cost or product differentiation. For nuclear power plants, the only differentiation that can be achieved in the product (electricity) is the reliability of production, and competitive advantage can only be gained through lower production costs. This creates a delicate balance between competing objectives for NPPs. Production costs are primarily fuel costs, operations (primarily labour) costs, and maintenance costs. As fuel costs are fairly consistent across the industry for a given reactor type, operations and maintenance costs are the remaining variables management has control over. However, a critical balance has to be achieved between these cost components and overall plant operational safety and reliability. Too much of a reduction in operations and maintenance spending can have severe financial consequences (e.g. unplanned outages or in the worst case, a nuclear accident). Too much spending on operations and maintenance drives costs up and makes a plant a poor performer economically, and thus not competitive. It is clear then that the key differentiator for NPP competitiveness is the achievement of efficiency and effectiveness in operations and maintenance while maintaining high performance in terms of production capacity, reliability, and safety. 
IAEA Technical Series Report 437 on Economic Performance Indicators (IAEA, 2006a) for NPPs states:

Electricity generating facilities such as nuclear power plants focused primary management attention on strategic and tactical matters having to do with plant operating performance, safety and reliability... Plant safety and reliability were most often the sole determinants of management decision-making and consequently the focus of key performance measures of success. With deregulation and the open market pricing of electricity, the business and financial success of operating nuclear plants must be considered to a much greater extent, along with the successful achievement of safety and reliability objectives.

A comprehensive study by the IAEA titled: "Evaluating and Improving Nuclear Power Plant Operating Performance" (IAEA, 1999) looked at plant performance statistics and examined in detailed case studies the best management practices at six NPPs (in different countries and off different reactor types, three pressurized water reactors (PWRs), one pressurized heavy water reactor (PHWR), one Russian (WWER) reactor, and one boiling water reactor (BWR) with leading performance records). Figure 13 shows the steady improvement in fleet performance in the period 1980 to 1996 in terms of "energy availability factor" (EAF). This trend has continued. 


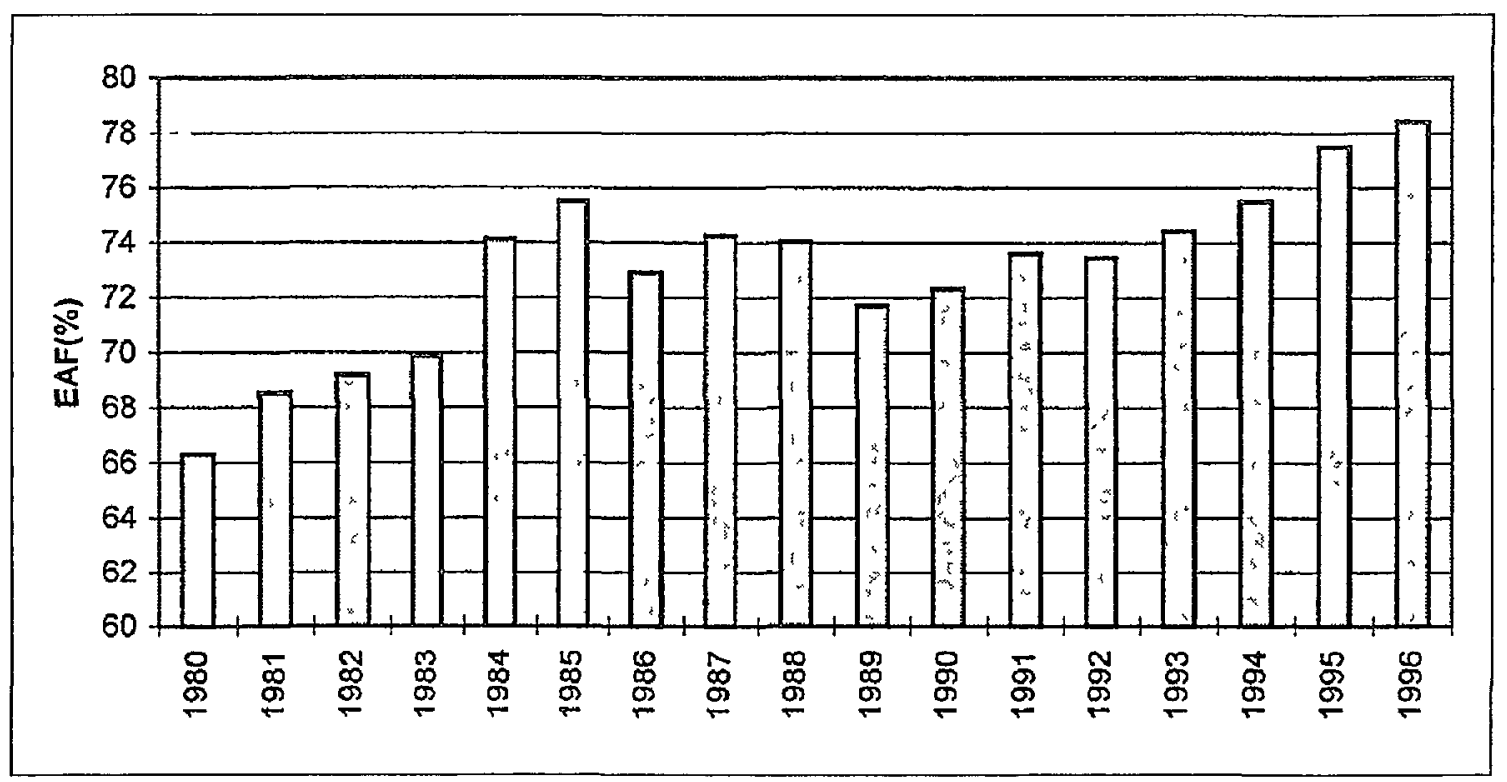

Figure 13. World Avg. Energy Availability Factors (IAEA, ‘99)

The report states these improvements can be attributed to a process of learning from experience, and that a number of initiatives taken by the Member States and the IAEA have had a significant role in the achievement of sustained improvement in the overall performance of the plants. However, another of the findings was that the evaluation of NPP technical performance requires the consideration of many factors over and above the traditional performance indicators (e.g. WANO indicators), and these include for example: environmental impacts, industrial safety, radiological protection, safety indicators, scram (automatic safety system shut-down) rates, and fuel reliability are among some of the more important indicators. This study builds on these findings by developing specific effectiveness measures for each. The IAEA report also states:

It is generally considered that good technically-performing plants have consistently good results in all areas. Even if the correlation between all 
these factors, as ingredients for good performance, is not obvious, there seems to be a link between them... Identifying quantitatively the contribution of one good practice to the improvement of plant energy availability factor is extremely difficult, because all else must be held constant, which is not possible.

These findings have influenced the approach taken is this research, and the approach taken will be to use a collective set of effectiveness measures that will overcome this limitation and show a link to operational performance efficiency measures.

Significant performance improvements have been made across the global fleet of NPPs in shifting to a more proactive and preventive maintenance and improved equipment reliability. Figure 14 shows the trend towards higher ratios of planned to unplanned energy unavailability factor (EUF) despite the effects of ageing, and this has been attributed (IAEA, 1999) to organizational learning from operational experience. However, given the range of management practices that may influence performance, it is unclear what combination of factors can be attributed most to these gains. This thesis specifically examines this question further in terms of the role KM practices play and their influence on NPP organizational performance. 


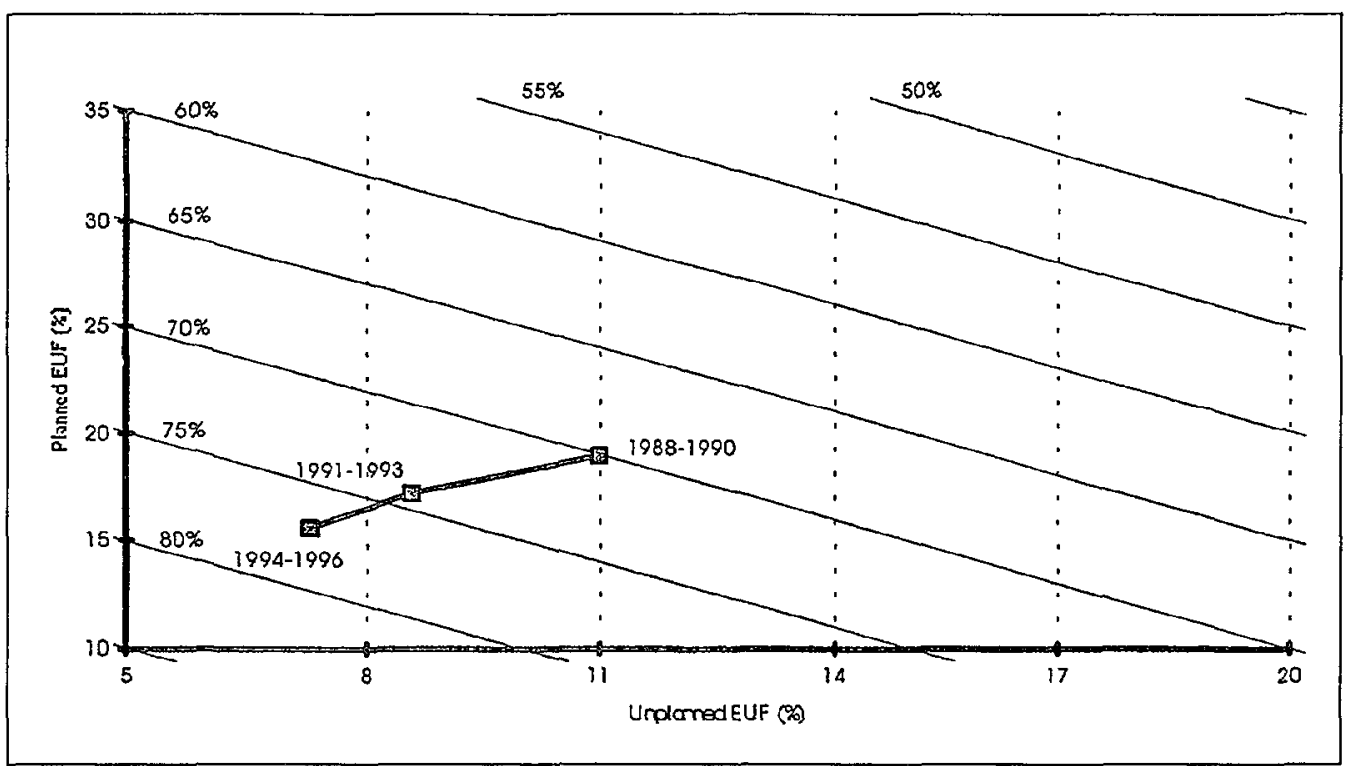

Figure 14. Avg. Planned \& Unplanned Energy Unavailability Factors (IAEA, ‘99)

Another of the findings was the result of a survey of planned and unplanned energy unavailability factors (EUF) for all NPPs. Figure 15 below shows the average values by reactor type and the marked difference between reactor designs can been seen. This finding also highlights the need to compare NPP operational performance within their peer group by reactor type to properly account for performance limitations inherent in the plant design (i.e. a moderating factor beyond management's control). Therefore, in this study, controlling for plant type (as a moderating variable) will be factored into the analysis. 


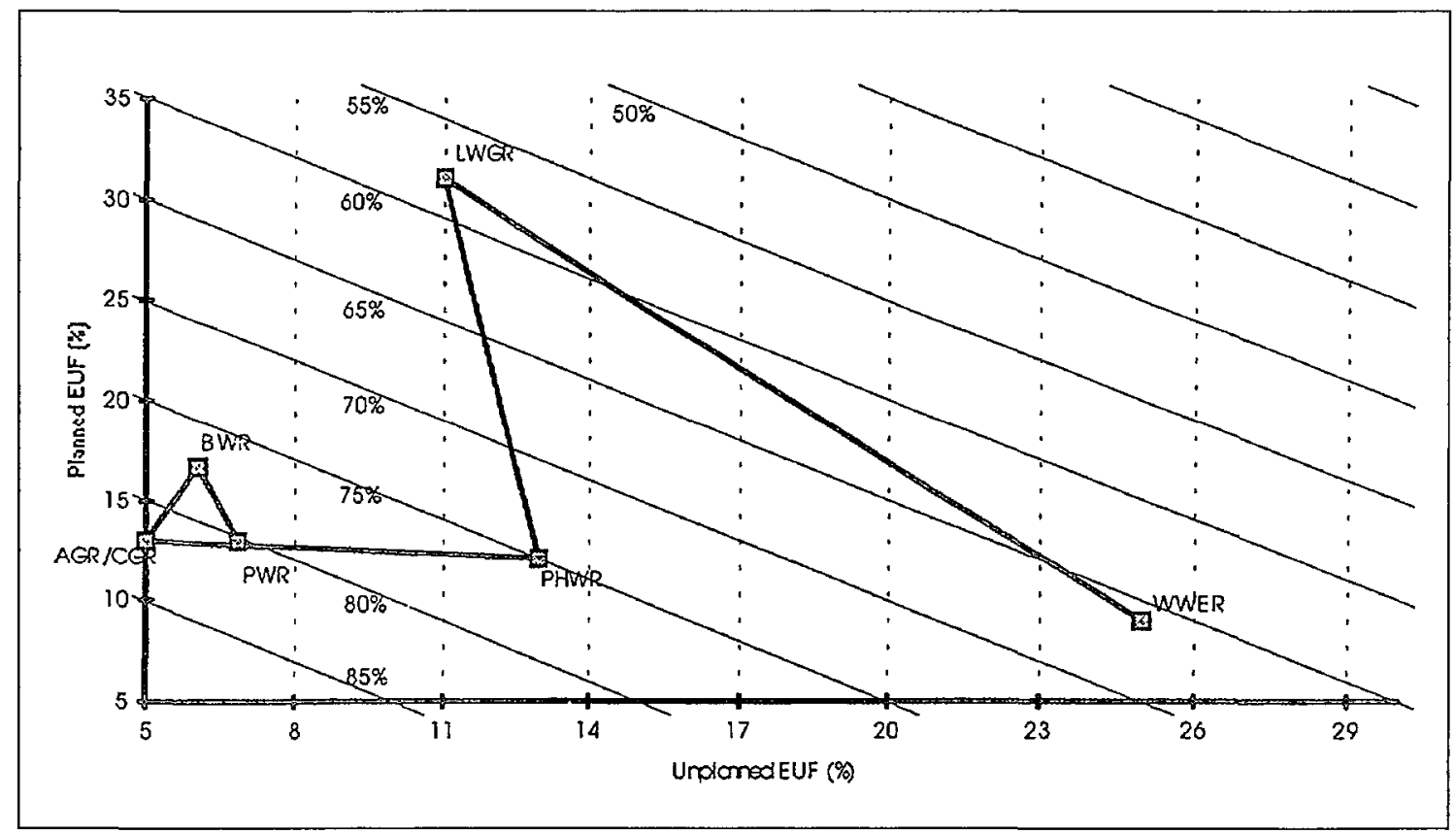

Figure 15. Planned \& Unplanned EUF by Reactor Type (IAEA, ‘99)

The main findings and conclusions of the study were that there was a wide agreement between countries, utilities, and plants regarding what strategies are important to achieve improved plant operational performance. The strategies included: strong focus on company philosophy, mission, vision, and values; clear goals and objectives; promoting self-assessment and ongoing performance improvement; focus on riskinformed reliability centered maintenance; engaged and open management culture; implementing best practices (work management, quality assurance, use of technological tools); and a strong focus on safety.

\subsubsection{Linking Knowledge Processes and KM to Organizational Performance}

Numerous authors have argued that knowledge directly impacts firm performance. Some example findings from the literature include: 
- knowledge, combined with effective management is a driver of organizational performance (Brown \& Duguid, 2000; Davenport \& Prusak, 1998).

- organizational learning theorists (Ross, 1986; Hohen and Levinthal 1989; Argote, Beckman and Epple, 1990; Alder, 90; Darr, Argote and Epple, '95) argue that as companies develop greater experience with a particular technology, process, or methodology, they improve operational efficiencies resulting in decreased costs or increased outputs.

- Stein and Zwass (1995) view organizational memory as the means by which knowledge from the past is brought to bear on present activities, thus influencing organizational effectiveness.

- organizational learning is an important element of organizational performance (Hoerr, 1999; Rheem, 1995; Brown, 1998).

Tacit and explicit knowledge together comprise the organization's intellectual capital and this is found in people (human capital) and organizational structures (structural capital) (Stewart, 1997). Organizations are able to utilize human capital to achieve their objectives, and as human capital increases due to learning and experience, it becomes more useful to the organization. Likewise structural capital (in the form of processes, documents, information systems, and procedures) represents codified or explicit knowledge. It facilitates organizational memory and allows explicit knowledge to be reused, tacit knowledge to be applied, and creates value for the organization. However, Stewart (1997) points out that intellectual capital can only be utilized effectively in the 
context of organizational strategy, which offers a rationale for meaningful alignment of goals and provides direction and priority to organizational activities.

Several authors take a resource-based view of competitive advantage from knowledge and argue that profits are derived from a firm's core competence. Management aims at exploring, coordinating and applying knowledge resources effectively in the firm (Dierickx and Cool, 1989; Amit and Schoemaker, 1993; Grant, 1996). Much of this management literature shares a focus on development and maintenance of core competence. Development of organizational resources and their effective deployment became an early central point in strategic management thinking and was seen to be a primary driver of firm performance (Porter, 1980). However, much of the early literature explaining performance differentiation was premised simply on the heterogeneity or uniqueness of the resource mix between firms (Barney, 1991; Peteraf, 1993) and did not emphasize organizational learning and knowledge management as key factors. Nonaka and Takeuchi (1995) also viewed knowledge as a competitive resource. Figure 16 shows the connection between knowledge creation, continuous innovation, and competitive advantage.

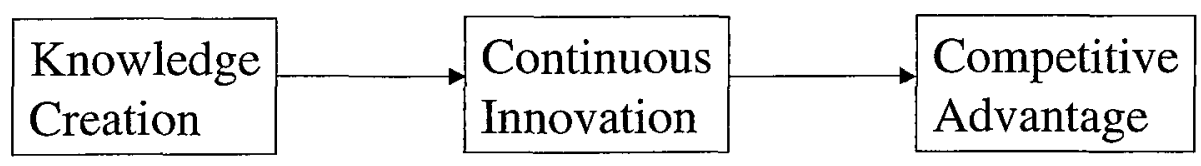

Figure 16. KM for Competitive Advantage (Nonaka and Takeuchi, '95)

Strong support can be found in the literature establishing the link between specific knowledge processes (e.g., knowledge acquisition, selection, generation, and internalization) and organizational performance defined in terms of competitiveness: 
- the link between knowledge acquisition practices and performance factors such as superior firm reputation (Wright, 1999; Leonard-Barton, 1995), firm innovativeness (Wright, 1999; Leonard-Barton, 1995; Stewart, 1997), and firm productivity (Leonard-Burton, 1995; Stewart (1997) has been documented;

- the mechanisms by which knowledge selection practices support performance factors such as innovation (Applegate et al., 1996); agility (Laudon and Laudon, 1997; Applegate et al., 1996); and productivity (Wright, 1998; Laudon and Laudon, 1998) has been documented.

Chen and Mohamed (2007) found that knowledge acquisition and utilization play important roles in the building an organizational knowledge base. Their research showed empirically that the higher the intensity of these two activities, the larger the organizational knowledge pool which, in turn, demands greater knowledge dissemination capacity. This dissemination capacity enables more active and intense responses to organizational changes and needs, and facilitates and stimulates acquisition and utilization of new tacit knowledge, thus improving business performance.

Senge (1999) saw the process of acquiring knowledge (i.e. building the corporate knowledge base) as a learning process integrating technical expertise, methodological knowledge, social competence and "knowing where" (i.e. to acquire it and apply it). All of this is viewed in the organizational context and helps establish the link both to organizational learning and individual and firm performance. He viewed knowledge as 
creating the "capacity for effective action", which depends on "an individual's or firm's efficiency and effectiveness to search, acquire, and deploy knowledge" (see Figure 3).

Pham and Swierczek (2006) conducted empirical research on link between knowledge processes and firm performance and found a statistically significant impact for factors related to the organizational learning on firm performance. They concluded that:

- knowledge acquisition has significant impact on both performance improvement and organizational climate,

- knowledge sharing has significant impact only on organizational climate,

- knowledge utilization has significant impact on performance improvement and organizational climate.

Several KM frameworks can be found in the literature. For instance, Carluccil and Schiuma (2006) define a KM framework for the identification of the key organization knowledge assets as the basis of company's business performance improvement. Their KM implementation process helps management to visualize how knowledge assets contribute to organizational performance and to define and evaluate what KM initiatives need to be implemented in order to develop those knowledge assets, which in turn supports the company's value creation. Their reference model is based on four key knowledge processes: knowledge organization; knowledge dissemination and analysis; knowledge use, and knowledge creation. These processes operate at the individual and group level, at the organization level, at the customer interface level, and at the enterprise level (see Figure 17). 


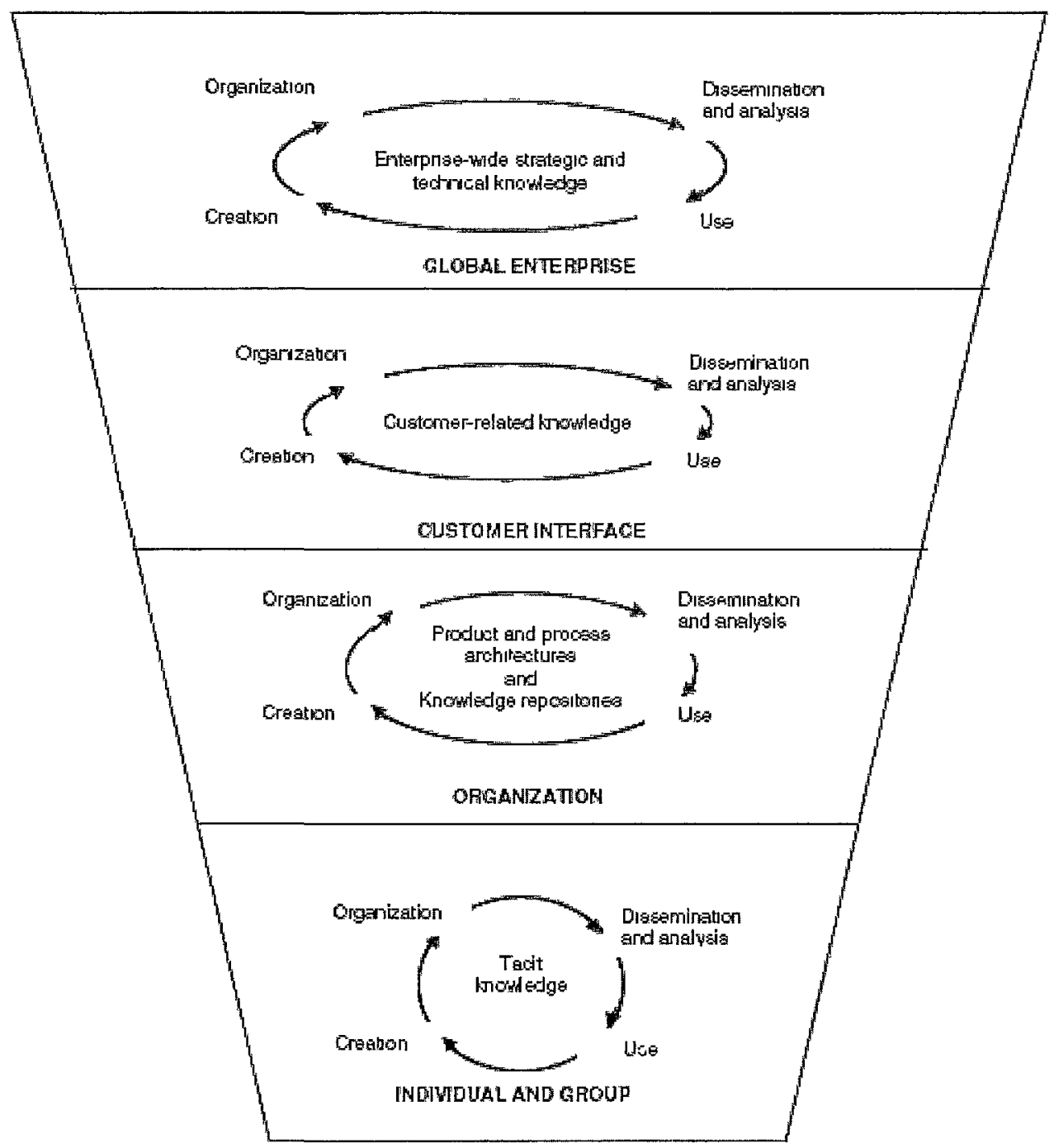

Figure 17. Knowledge Asset Value Spiral (Carlucci1 \& Schiuma, ‘06)

Finally, numerous authors have argued that $\mathrm{KM}$ practices directly impact firm performance. Some example findings from the literature include:

- effective KM will create an environment conducive of continuous learning, that will help the organization recognize its knowledge assets, and that will help secure knowledge that is critical to its overall success (Wiig, 1997). 
- effective KM will expand the resources available to the organization and will enable more intelligent decisions and problem solving (Lank, 1997).

- effective KM will help organizations become more competitive by increasing productivity and reducing costs (Grayson and O'Dell, 1998).

- successful organizations are those that continuously review their processes looking for ways to improve (Hoerr, 1999).

- when complex knowledge was involved, weak ties between team members had a negative impact on knowledge sharing and team performance (Hansen, 1999).

- research has been done to show how knowledge measurement promotes productivity (Wah, 1999; Perez and Hynes III, 1999);

- a strong association between knowledge control and productivity has been shown to exist (Mullen, 1996); and

- the direct relationship between knowledge coordination and firm productivity and agility has been found (McCune, 1999).

- Holsapple and Singh (2003) viewed KM as supporting competitiveness across several dimensions: productivity (e.g. lower cost, greater speed), quality, dependability, organizational agility (e.g. flexibility, responsiveness, change proficiency), and innovation.

- leadership commitment has a significant positive impact on both performance improvement and organizational climate (Pham and Swierczek, 2006). 
- incentives have a significant positive impact only on performance improvement (Pham and Swierczek, 2006).

- staff interaction has a significant positive impact only on organizational climate (Pham and Swierczek, 2006).

The view of KM activities in a value-consequence chain was described by Wiig (1997) as the "KM Benefits Chain" (Figure 18). He showed how the effects of knowledge benefits cascade to intermediate benefits and ultimately organizational benefits. For example, faster access to knowledge (knowledge management benefit) leads to faster problem solving (intermediate benefit), which in turn leads to better and faster innovation (the organizational benefit).

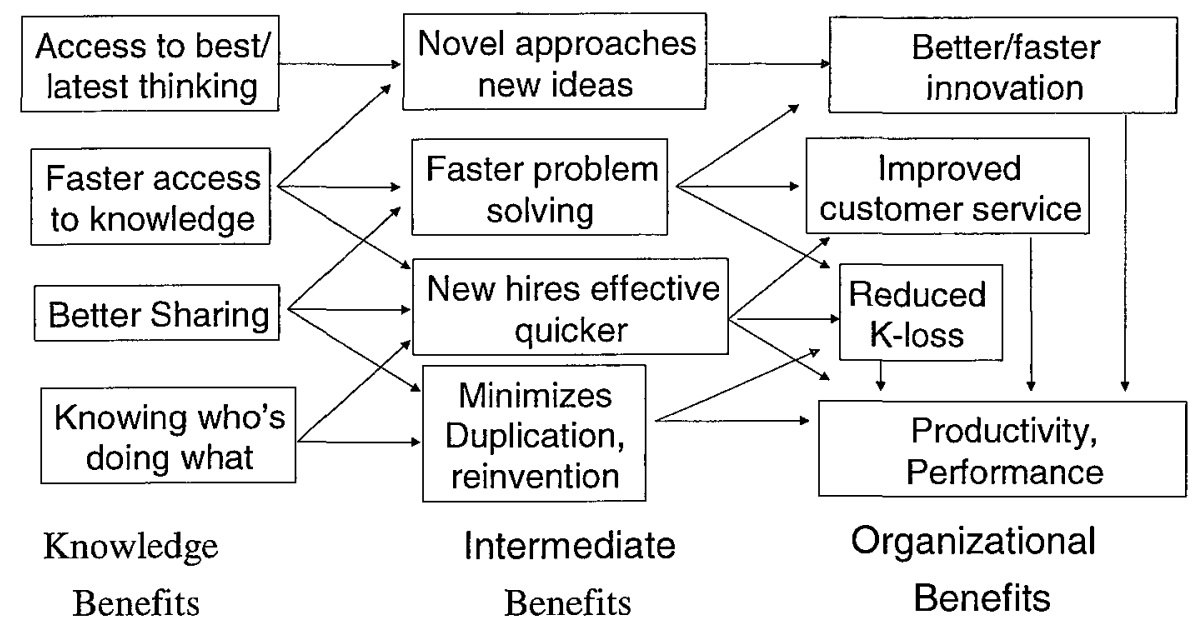

Figure 18. KM Benefits Chain (adapted from Wiig, 1997)

Firestone and McElroy (2004) argue that the literature on KM, organizational learning (OL) and complex adaptive systems (CAS) as three schools of thought are quite intimately related and can in fact be combined. In their view, decisions and actions are 
performed in firms by agents who perform planning, acting, monitoring, and evaluating behaviours in decision execution cycles. They view organizations as having three tiers of business processes: operational business processes, knowledge processes, and processes for managing knowledge processes. Operational processes are the routine decisions and actions that use knowledge. Knowledge processes involve the production and integration of knowledge and produce outcomes. They view the outcomes, i.e. knowledge, as an encoded, tested, evaluated, and still surviving structure of information that helps the adaptive system (agent) that developed it to adapt. The knowledge outcomes include:

- tested, evaluated, and surviving beliefs or belief predispositions (in minds), and

- tested, evaluated, and surviving, sharable (objective), linguistic formulations (knowledge claims)

Firestone and McElroy (2004) essentially view the collective set of processes for managing knowledge processes as "knowledge management". This perspective helps to understand the link between KM practices, knowledge processes and firm performance. They define $\mathrm{KM}$ formally as "the set of processes that seeks to change the organization's present pattern of knowledge processing to enhance both it and its knowledge outcomes". They also point out that management can and should directly influence and enable effective knowledge processing and thus organizational learning. In effect, they summarize what much of the literature agrees upon: management practices and actions can build, enhance, and maintain the organizational knowledge base by directly influencing knowledge processes. Also of interest with respect to the 
present research is their clear conceptual link from $\mathrm{KM}$ practices, to knowledge processes, to operational processes and outcomes. Firestone and McElroy (2004) state: “KM doesn't directly manage knowledge outcomes, but only impacts (knowledge) processes, which in turn impact outcomes". This supports the key underlying suppositions of this study and much of the literature: effective KM practices directly impact knowledge processes and this in turn promotes organizational learning. Organizational learning helps build an integrated and shared knowledge base, which in turn creates an improved capacity for effective action. As a result, better decisions and actions are taken, operational effectiveness increases, and firm performance results. The present research builds on these basic theoretical underpinnings. They are found through-out the literature and form the high-level conceptual basis for the research model.

In summary, there is strong support in the literature to establish a positive relationship of knowledge, knowledge processes, and $\mathrm{KM}$ practices to organizational performance. In simple terms, KM practices influence the quality of knowledge processes, which in turn facilitate organizational learning and building of knowledge. This improves a firm's capacity for effective action and will directly impact performance. The next section will explore these relationships further by discussing them specifically in the context of NPPs organizations. 


\subsubsection{KM in the Context of NPP Performance}

From the preceding discussion, one can conclude that KM has the potential to greatly influence NPP organizational performance by influencing knowledge processes to enhance organizational learning. However, to better understand this relationship it is important to consider the distinct nature of NPP organizations relative to other industry organizations. To gain further insights on this, this section will discuss the NPP organizational context.

An IAEA study (1999) found a number of issues were very important to achieve the successful implementation of NPP performance strategies. These have been summarized in Table 5, and categorized in terms of what type of KM practice (as applicable) they are considered to be associated with. These findings reinforce the argument that KM practices enhance organizational performance.

Table 5. Issues Important to NPP Performance Improvement (IAEA, '99)

\begin{tabular}{|l|l|}
\hline \multicolumn{1}{|c|}{$\begin{array}{c}\text { Important Issues Identified as Related to } \\
\text { Achievement of Improved NPP Technical } \\
\text { Performance (IAEA, 1999) }\end{array}$} & \multicolumn{1}{|c|}{ Related KM Practices } \\
\hline $\begin{array}{l}\text { Organizational process documents clearly reflecting } \\
\text { responsibilities and delegation of assignments between } \\
\text { headquarters, site management, and department work } \\
\text { teams. }\end{array}$ & $\begin{array}{l}\text { Effectiveness of business (i.e. } \\
\text { knowledge-driven decision) } \\
\text { processes. }\end{array}$ \\
\hline $\begin{array}{l}\text { Goals, expectations, and business plan evaluated and } \\
\text { updated periodically to provide a systematic approach to } \\
\text { performance enhancement. }\end{array}$ & $\begin{array}{l}\text { Focus on quality and continuous } \\
\text { improvement, organizational } \\
\text { learning }\end{array}$ \\
\hline $\begin{array}{l}\text { Atmosphere of openness, in which mistakes are brought } \\
\text { to management's attention to be used as opportunities for } \\
\text { learning. }\end{array}$ & $\begin{array}{l}\text { Culture of openness and trust, } \\
\text { knowledge sharing and support } \\
\text { for learning. }\end{array}$ \\
\hline Management committed to quality. & $\begin{array}{l}\text { Focus on documentation of } \\
\text { process, feedback measures, } \\
\text { continuous improvement } \\
\text { (learning). }\end{array}$ \\
\hline
\end{tabular}




\begin{tabular}{|l|l|}
\hline \multicolumn{1}{|c|}{$\begin{array}{l}\text { Important Issues Identified as Related to } \\
\text { Achievement of Improved NPP Technical } \\
\text { Performance (IAEA, 1999) }\end{array}$} & \multicolumn{1}{|c|}{ Related KM Practices } \\
\hline $\begin{array}{l}\text { Involvement of all employees in quality assurance by } \\
\text { identifying weak points in the quality of plant operations, } \\
\text { finding their causes, and devising corrective measures. }\end{array}$ & $\begin{array}{l}\text { Focus on operational } \\
\text { experience, corrective actions, } \\
\text { continuous improvement, and } \\
\text { organizational learning }\end{array}$ \\
\hline $\begin{array}{l}\text { Management responsible for developing job skills, } \\
\text { managerial and leadership potential through training, } \\
\text { emphasizing the pursuit of higher education, and job } \\
\text { rotation to increase common knowledge for the entire } \\
\text { staff. }\end{array}$ & $\begin{array}{l}\text { Support for knowledge } \\
\text { retention, organizational } \\
\text { learning, knowledge transfer } \\
\text { processes. }\end{array}$ \\
\hline $\begin{array}{l}\text { Improving the quality of operating procedures and } \\
\text { providing high quality operating aids to minimize human } \\
\text { related errors and maximize timely, effective response to } \\
\text { abnormal conditions. One important operating aid is a } \\
\text { computerized information system to provide real time } \\
\text { plant status. }\end{array}$ & $\begin{array}{l}\text { Knowledge codification, } \\
\text { knowledge retention, technology } \\
\text { support (advanced operational } \\
\text { support systems). }\end{array}$ \\
\hline $\begin{array}{l}\text { Improved plant reliability through effective maintenance } \\
\text { policies, planning and performance. Use of condition } \\
\text { based decision analysis with detailed databases and } \\
\text { online monitoring of plant equipment could reduce } \\
\text { preventive maintenance. }\end{array}$ & $\begin{array}{l}\text { Knowledge codification, focus } \\
\text { on information flow, continuous } \\
\text { improvement, and advanced } \\
\text { operational support systems. }\end{array}$ \\
\hline $\begin{array}{l}\text { Use of operating experience information for plant } \\
\text { upgrades, procedural enhancements, and process } \\
\text { improvements to improve technical support. }\end{array}$ & $\begin{array}{l}\text { Focus on feedback from lessons } \\
\text { learned, knowledge sharing, } \\
\text { continuous improvement } \\
\text { through organizational learning }\end{array}$ \\
\hline $\begin{array}{l}\text { Interaction and communication between and among } \\
\text { groups improved by using "customer-supplier" } \\
\text { relationship between departments and establishment of } \\
\text { interdisciplinary teams to address urgent or specific } \\
\text { problems. }\end{array}$ & $\begin{array}{l}\text { Open and trusting } \\
\text { communications, knowledge } \\
\text { sharing and transfer. }\end{array}$ \\
\hline
\end{tabular}

In the NPP context, KM is important for several reasons. To begin with, the long lifecycle of nuclear facilities (i.e. typically 60-100 years) coupled with the complex technology base create significant knowledge retention and re-use challenges. Figure 19 highlights the need for knowledge management over each phase of the plant life-cycle. Information and knowledge created and captured during one phase may be quite important during later phases such as refurbishment or even decommissioning. For 
example, the challenges of equipment failures, technology obsolescence, or design improvements over time create the need for ongoing design changes throughout the facility's life. Over such long time periods and due to the need for many plant changes, configuration management of the plant design basis and the keeping of detailed records (e.g. history and rationale of plant changes) become essential to maintain a safe and economic plant. The methods or tools (whether technology-oriented or manual) by which the plant design knowledge base is maintained over the life of the plant become important. For example, design information captured during design or manufacturing phases may prove essential to achieving a safe and cost effective decommissioning.

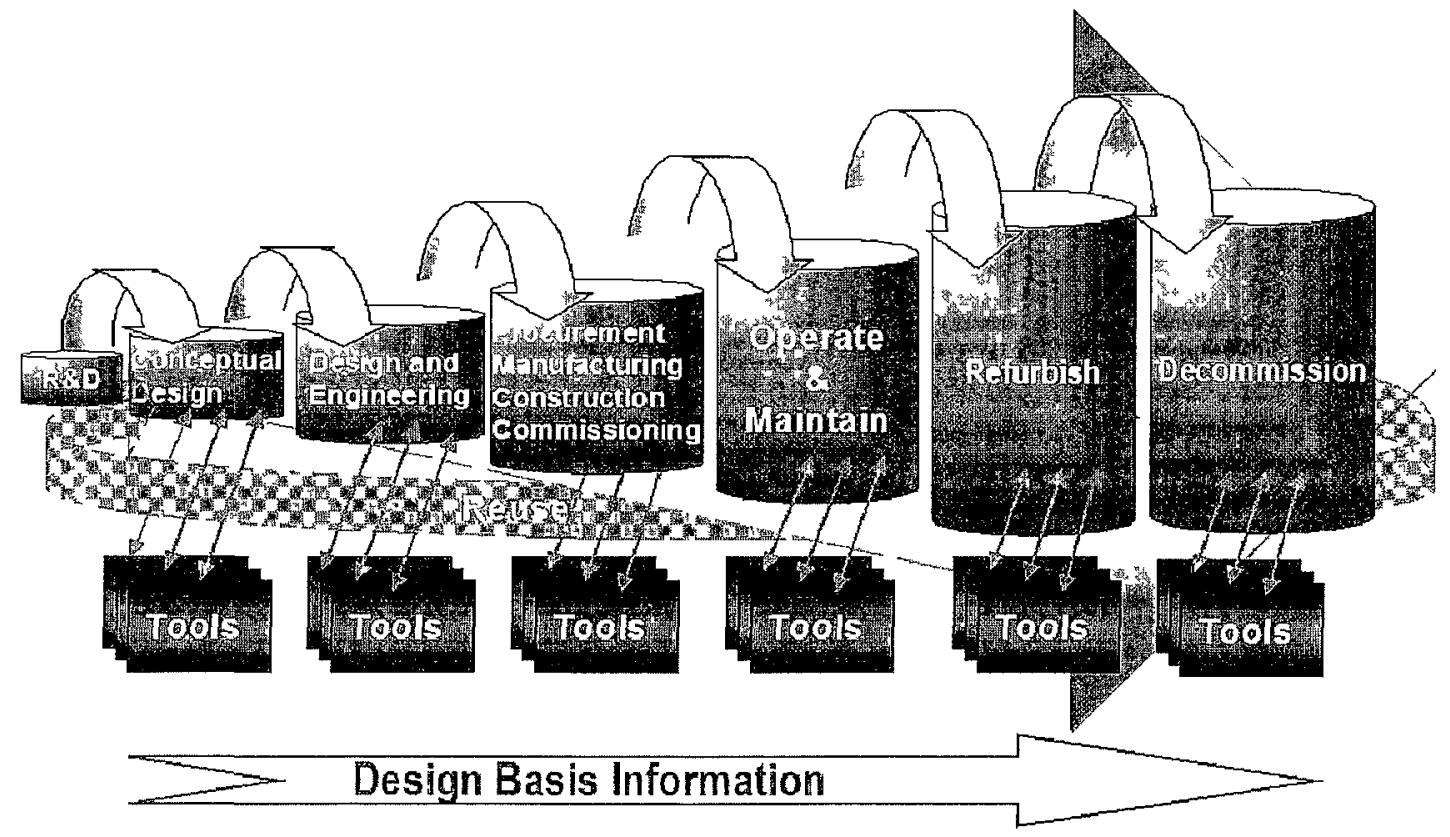

Figure 19. Nuclear Plant Life-cycle (Olmstead and Didsbury, 2002).

There is agreement in the literature that to implement KM effectively at an enterprise level in an organization a strategic approach is required (Hedlund, 1994; Grant, 1996; Hitt and Ireland, 1995). A key success factor is seen to be the 'fit' or 'alignment' of 
people (and their competencies), business and work processes (i.e. procedures, management controls, work practices, methods etc.), IS infrastructure (i.e. information systems/technology), and plant and equipment (i.e. the physical plant technology base) (Bou-Llusa and Segarra-Cipres, 2006; IAEA, 2006b). See Figure 20.

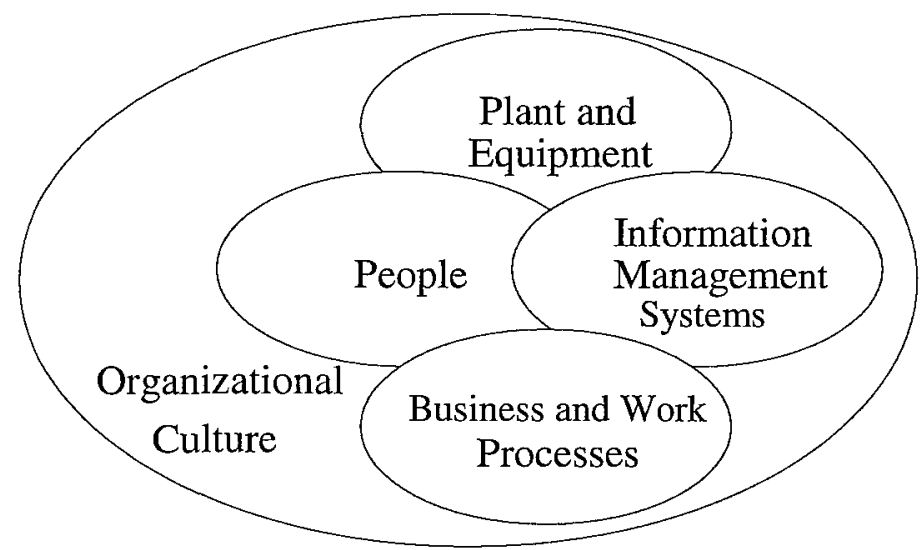

Figure 20. Alignment of Knowledge Processes (adapted from IAEA, ‘06b)

In NPPs this alignment is important due to the complex technical work environment, interdisciplinary knowledge requirements, and the need to work to well-defined processes and procedures. It also must be achieved in the context of the organizational culture. In order to better comprehend KM requirements in NPPs and to appreciate what is meant by this alignment, it is useful to understand the context of NPP management systems in general. The IAEA (2006c) defines the management system at nuclear power plants as: a set of interrelated or interacting elements (system) for establishing policies and objectives and enabling the objectives to be achieved in an effective and efficient way. The management system integrates all elements of an organization into one coherent system to enable all of the organization's objectives to be achieved. These elements include the organizational structure, resources and processes. 
Personnel, equipment and organizational culture, as well as the documented policies and processes, are part of the management system. The organization's processes have to address the totality of the requirements on the organization (e.g. as established in IAEA safety standards and other international codes and standards).

A well-established example of a NPP management system is the Nuclear Energy Institute's (NEI) "Standard Nuclear Performance Model" (SNPM). The model identifies and standardizes the description and scope of all the major processes needed in an operating NPP based on accepted best practices. The SNPM model (see Figure 21) is represented by:

- core processes ("those processes most directly related to nuclear safety and that accomplish a key business function, the core processes are Operate Plant, Work Management, Manage Configuration, Equipment Reliability and Materials and Services"),

- enabling processes ("a process that is applied in support of one or more core processes, the enabling processes are Management Processes and Support Services, Training, Nuclear Fuel and Loss Prevention"), and

- sub-processes ("a description for a subset of process steps within the bounds of a single core or enabling process). 


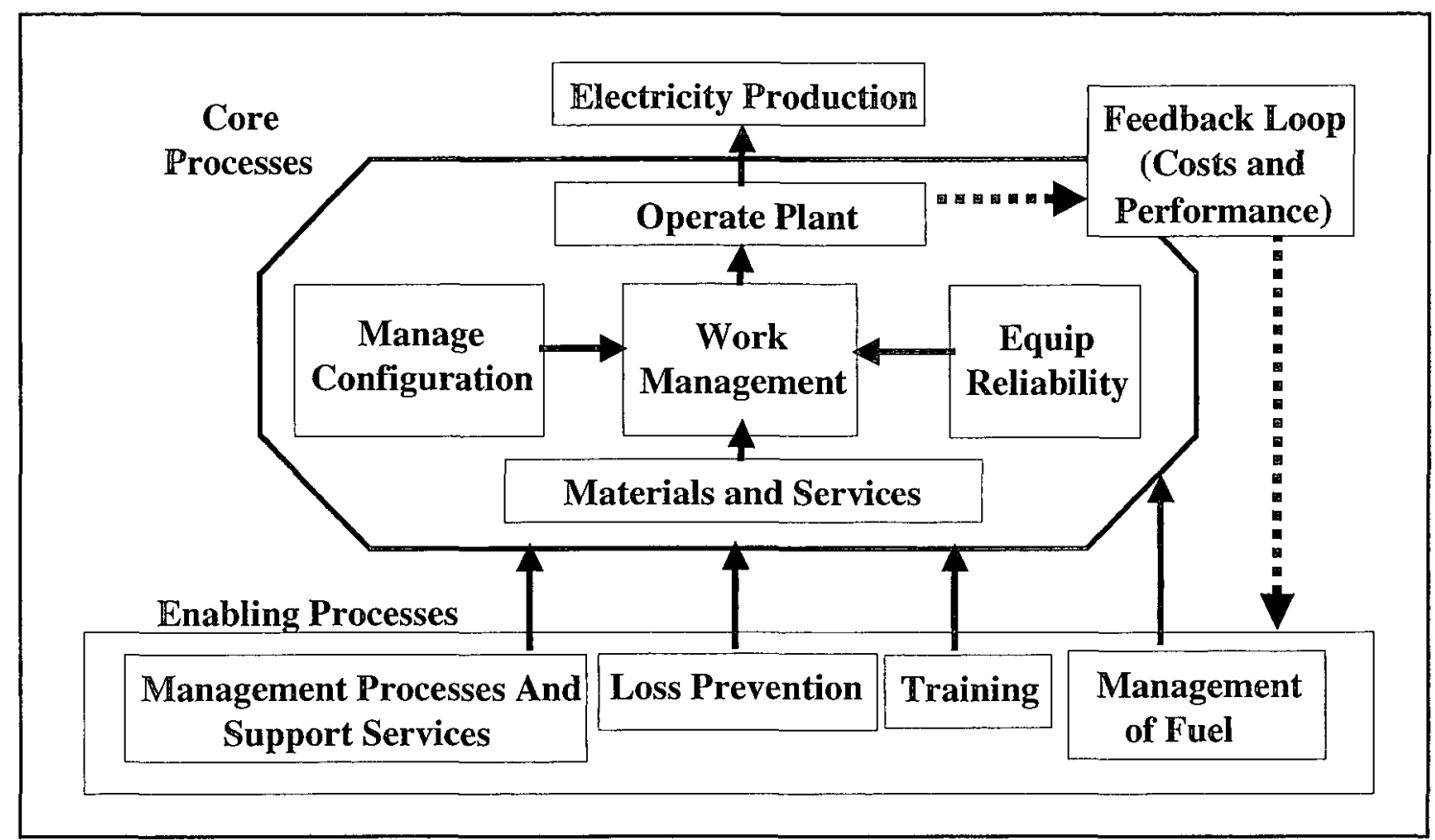

Figure 21. The Standard Nuclear Performance Model (SNPM) (NEI, 2003)

The overriding goal of any nuclear plant and of course the SNPM is electricity production. The SNPM is a useful framework in terms of identifying and discussing the key NPP business processes, all of which are knowledge-driven. For example, any given process such as work management can be looked at in terms of various knowledge process characteristics, such as:

- The requirements for data or information inputs, flows and outputs, and the need for capture, processing, integration, storage or retention, distribution, retrieval, and visualization of this data or information.

- The participants in the process, the knowledge required as a prerequisite or acquired or generated through the process. This may include temporal issues 
(speed or sequencing or parallel flow or dependencies or delays of information or knowledge flows)

- The explicit knowledge base embedded in the process as defined and as codified in policy, procedures, guidelines, and accepted practice.

- The information technology that supports or automate the process, including work-flow and decision rules that may embed methodological knowledge

- The importance for data/information integrity/correctness, clarity, security, quality, objective/subjective nature, completeness, standardization/consistency, and the knowledge verification or validation processes.

- The complexity, sensitivity, criticality, information density, interpretability, and possible social aspects (e.g. empowerment, responsibility etc.) of the information or knowledge flows in the process.

- The alignment of tacit and explicit knowledge processes with respect to the business needs of the processes in terms of work-flow and/or decision processes, considering the process requirements.

- The alignment and integration of the knowledge processes with related or interfacing knowledge processes of other core or enabling processes in the model.

- Measurement and feedback to enable continuous improvement of the quality of the knowledge processes. 
In summary, the SNPM provides a process-oriented business framework that enables the identification and analysis of the knowledge inputs, knowledge processes and processors, knowledge flows and stores, and knowledge outputs. The key assumption being that in a knowledge driven organization such as a NPP, productive and effective decision and work processes will result in increased organizational performance on every dimension. A key hypothesis of this research is that this will occur when the organization has high quality knowledge processes, defined as "knowledge processes that have been have been optimized to meet or exceed the requirements of the business processes". This builds on the concepts advanced by McElroy (see Mierzejewska, 2004) who describes the link between $\mathrm{KM}$ and performance as follows:

organizational performance is determined by the quality of actions taken by employees in the performance of work, which is determined by the quality of decisions made related to these actions, which is determined by the quality of decision-making, which is related to the quality of knowledge utilized in the context of each decision, which is a related to the quality of knowledge processes in the firm, which is influenced by the quality of KM.

The challenge of achieving sustained high level of operational performance in today's NPPs can be described as a problem of optimizing human behaviour and action in a complex socio-technical work environment, within a large framework of operating constraints, to achieve the simultaneous optimization of numerous goals, and according to a large array of business and work processes. Nuclear plant organizations are heavily knowledge-dependent and their operational needs demand a high level of knowledge- 
based infrastructure in the form of human capital, underlying base technology (of the plant), and work methodology. One could argue the terms "knowledge-worker" and "knowledge organization" are all the more appropriate in the multi-disciplinary work environs of nuclear plant organizations. For these reasons, NPP managers are interested in understanding and influencing the factors that affect the building and retention of the corporate knowledge base and its utilization.

Thus McElroy's ideas help explain why effectiveness and productivity in NPPs stem from the quality of decisions and actions taken in every-day work and at all levels in the organization. McElroy's theory on the quality of knowledge and knowledge processes is complementary to Senge's (1999) view of organizational knowledge as supporting (employees') capacity for effective action and Wiig's (1997) KM benefits chain. Thus it is incumbent upon NPP management to ensure, both as a strategic and tactical objective, the establishment, protection, and improvement of knowledge and knowledge processes (i.e. this "capacity for effective action") in order to achieve high levels of performance. Thus KM can be viewed as a necessary planned and coordinated effort, using whatever practical and effective means are available and within the control management, to influence the quality of knowledge processes and increase knowledge.

Affecting these relationships are additional important factors that influence human behavior such as motivation, commitment, trust, leadership, empowerment, accountability and a common vision. These factors are seen in the literature to be associated with the corporate culture. The next section will explore how organizational culture may play a role in these relationships. 


\subsection{Organizational Culture}

There are many differing definitions of organizational culture in the literature. O’Dell et al. (2003) saw organizational culture as "the unique combination of expectations, written and unwritten rules, and social mores that dictates the everyday actions and behaviors of each employee". Sashkin and Rosenbach (1996) see organizational culture centered around four key issues: adaptation (i.e., how people deal with external forces and the need to change); goal achievement (i.e., the nature of organizational goals, how they are defined, and their importance); coordination (i.e., how people work together to get the job done); and shared values and beliefs (i.e., the degree to which people in the organization generally agree that these values and beliefs are important and should guide their actions. Schein (1990) defined organizational culture as: "a pattern of basic assumptions, invented, discovered, or developed by a given group as it learns to cope with its problems of external adaptation and internal integration, that has worked well enough to be considered valid and, therefore is to be taught to new members as the correct way to perceive, think, and feel in relation to those problems". Thus clearly, organizational culture influences communication and knowledge processes.

Cummings and Worley (1997) argue that the beliefs, attitudes and assumptions underlie an organization's culture and are a key factor. These basic assumptions influence how employees perceive and interpret the firm's problems and how they should be dealt with. They include assumptions about the firm's relation to its environment, about human nature and relationships. Thus culture influences employee behavior and motivation, and ultimately attitudes towards learning, communication, knowledge 
acquisition, and knowledge sharing. Several authors have argued that organizational culture affects knowledge sharing and transfer efficiency (Hansen (1999); Ribiere (2001), Yeung et al (1999). O'Dell et al. (2003) found that organizational culture is often "the most important factor in the success of knowledge management initiatives, and it is definitely the most complex to change". They found that few organizations start-off with cultures having strong support for knowledge sharing and collaboration. In most firms, employees are motivated and rewarded for personal knowledge and performance, and this falls far short of delivering the benefits that can be achieved when knowledge management and sharing is practiced. In an earlier study (O'Dell et al., 2000), the authors investigated impediments to the implementation of knowledge sharing approach in organizations and found, from empirical data, the following seven leading factors:

- Employees do not have time to share knowledge

- Employees have a strong not-invented-here culture

- Employees are scattered geographically or there are organizational barriers making it difficult to share knowledge

- Employees are afraid that sharing knowledge will make them less valuable to the organization

- Employees are unwilling to share their knowledge

- Organizations are unaware of the importance of knowledge sharing

- Organizational leadership does not care about sharing knowledge 
It is clear that that cultural barriers may impede $\mathrm{KM}$ and knowledge processes. Other roadblocks to success identified by the O'Dell et al. in subsequent work (2003) include: lack of common perspectives; lack of time; non-aligned rewards systems; lack of motivation; lack of absorptive capacity; lack of formal communications; lack of emphasis placed on implicit (tacit) knowledge; and the lack of technology or the expertise or use it.

O'Dell et al. (2003) point out that KM initiatives should not be expected or intended as drivers of cultural change in the organization and have little chance of success in this respect. Rather, the author's posit that the core cultural values are needed to be in place to enable $\mathrm{KM}$ processes to be most effective. In organizations with cultures that are not conducive to KS, leadership by example is the most powerful method to facilitate change, and the higher up an individual is in the organization, the more significant the likely impact. Another important aspect of culture is rewards and recognition. In many organizations, recognition and rewards are needed to encourage employees to share their expertise, and whether tangible or intangible in nature, acknowledge the importance and value of the knowledge contributions employees make (O'Dell et al., 2003).

Other indicators of culture found in the literature include trust between members of the organization (Zucker, 1986; and Rempel et al., 1985; and Nelson and Cooprider, 1996) and openness between members of the organization (Stata, 1989; Hamel, 1991; and Badaracco, 1991; and Kanter, 1983). Anderson and Narus (1990) found that repeated group exchange builds trust, and this in turn leads to increased communication and increased knowledge sharing. Research by Badaracco (1991) found a strong link 
between knowledge sharing and openness in the organization. They found that communication and knowledge sharing occurred to higher levels when relationships are not hampered by constraints that give rise to lack of openness.

Davenport et al. (1998) found that to achieve effective knowledge management in organizations, strong support from senior management was important. Their work highlighted several examples of senior management support including: communicating knowledge management, learning and knowledge sharing are important to the firm's success; providing adequate funding and resources for infrastructure (e.g. information technology); and clarifying what types of knowledge are important to the organization.

There is arguably a strong link between organizational culture (including safety culture) and knowledge sharing culture. In NPPs, safety culture is a dominant component of organizational culture (IAEA, 2005). The IAEA Safety Guide (2006c) defines safety culture as the assembly of characteristics and attitudes in organizations and individuals which establishes that, as an overriding priority, protection and safety issues receive the attention warranted by their significance. One of the stated objectives of safety culture is "to improve safety performance of the organization through the planning, control and supervision of safety-related activities in normal, transient, and emergency situations", which assumes a knowledge-driven (i.e. training and experience based) approach to safety-related decision making.

The WANO nuclear industry guideline "Principles for a Strong Nuclear Safety Culture" (WANO, 2006) states "...culture is a key ingredient in the overall success of the plant. 
Organizational culture is the shared basic assumptions that are developed in an organization as it learns and copes with problems. The basic assumptions that have worked well enough to be considered valid are taught to new members of the organization as the correct way to perceive, think, act, and feel. Culture is the sum total of a group's learning. Culture is for the group what character and personality are for the individual." The same guideline defines safety culture as "an organization's values and behaviours-modeled by its leaders and internalized by its members-that serve to make nuclear safety the overriding priority".

Implied in the WANO definition is the notion that nuclear power plants are designed, built, and operated (and intended) to produce power in a safe, reliable, efficient manner; that the concept of safety culture applies to every employee in the nuclear organization, from the board of directors to the individual contributor; that the focus is on nuclear safety, although the same principles apply to radiological safety, industrial safety, and environmental safety; and that nuclear safety is the first priority at a nuclear station. The strength of a facility's safety culture could lie anywhere along a broad continuum, depending on the degree to which the attributes of safety culture are embraced.

The following principles are described in the WANO Guideline:

- Everyone is personally responsible for nuclear safety.

- Leaders demonstrate commitment to safety.

- Trust permeates the organization.

- Decision-making reflects safety first. 
- Nuclear technology is recognized as special and unique.

- A questioning attitude is cultivated.

- Organizational learning is embraced.

- Nuclear safety undergoes constant examination.

It is clear that the constructs of organizational culture, safety culture, and knowledge sharing culture are based on similar shared factors of trust, leadership, rewards, shared vision, personal responsibility, support for learning, questioning attitude, and communication. Even though safety culture is a somewhat intangible concept, it is possible to determine, based on observable attributes, whether a station tends toward one end of the continuum or the other. Another important point is the link between safety and organizational performance. The same WANO report (2006) on nuclear safety culture concludes: "commercial nuclear electric generating plants are designed, built, and operated to produce electricity. Safety, production, and cost control are necessary goals for the operation of such a plant. These outcomes are quite complementary, and most plants today achieve high levels of safety, impressive production records, and competitive costs, reinforced by decisions and actions made with a long-term view. This perspective keeps safety as the overriding priority for each plant and for each individual associated with it."

Thus the aspects of a healthy and positive organizational culture are completely consistent with those identified in the literature for a strong organizational safety culture, these in turn are also consistent with the dimensions of a strong knowledge 
sharing culture, and all have been identified as important enablers of NPP organizational performance. The following section explores the impact of organizational culture on performance.

\subsubsection{Influence of Organizational Culture on Performance}

Several researchers have investigated the link between organizational culture and performance (Haber et al. (1991); Barney (1986); Lee and Yu (2004); Chatman and Jehn (1994), Gorden (1985) and Kotter and Heskett (1992)). Barney (1986) argued that culture may be a source of competitive advantage if it adds value, is rare (i.e. has attributes dissimilar to other firms) and not easily imitated. Lee and Yu (2004) defined culture as a set of values and beliefs held by employees. Thus strong organizational cultures imply homogeneity and pervasiveness of these values. In a very large empirical study, they found that different industries had different cultural profiles and cultural variation is greater across industries than within them. Their research also suggests that organizational cultures do evolve to "fit" industry dynamics and demands. Their findings are consistent with research by Chatman and Jehn (1994), Gordon (1985) and Kotter and Heskett (1992) which earlier found strong evidence that "cultural fit" directly accounts for some portion of organizational performance. Findings by Quick (1992) and Kotter and Heskett (1992) suggest that culture may only be an intermediary factor or moderator of the effect of other necessary factors on organizational performance, such as effective leadership. Ahanotu (1998) found that empowerment was a key factor of culture influencing productivity and performance in production environments. 
Another important aspect of organizational culture is the concept of shared vision (Levine (2006). There is a strong link between shared vision and organizational performance (Hoe, 2007; Levine, 2006). Calantone et al. (2002) found that shared vision has a positive effect on an organization's innovativeness, and this in turn has a positive effect on organizational performance. There is general agreement in the literature on what shared vision means:

- Thoms and Greenberger (1998) define vision as an image created in the mind of the leaders that followers react to positively when it reflects their values, shows an ideal future, and contains enough information to provide direction for future behaviour, or more precisely "the ability to create a positive image of an organization in the future".

- Bass and Stogdill (1990) see vision the creation of an image of a desired future state of the organization that serves as a guide for interim strategies, decisions, and behaviors.

- Nanus (1992) defined vision as a realistic credible attractive future for the organization, and an articulation of a destination toward which the organization should aim, a future that in important ways is better, more successful, or more desirable than the present.

- Kirkpatrick and Locke (1996) describe vision as a general view of the end goals that should challenge the status quo but is within the realm of possibility and focuses attention on desired outcomes. 
Nanus argues that having the right vision solicits commitment and energizes the organization, creates meaning and focus, establishes standards of excellence, and helps bridge the present to the future. Based on this view, it can be argued that vision imparts direction and generates motive towards organization goals, and can be viewed as an important enabler of performance.

From these findings it can be argued that organizational culture plays an important role influencing the impact of $\mathrm{KM}$ practices. In the context of the current research, it is seen

as playing an important role in moderating the effect of $\mathrm{KM}$ practices on the quality of knowledge processes in an organization. It is also seen to moderate the subsequent effect of quality of knowledge processes on organizational performance. Finally, it is also believed organizational culture moderates the effect information systems and technology has on quality of knowledge processes. The next section will investigate the role of information systems and information technology in enabling knowledge processes and ultimately affecting organizational performance.

\subsection{Information Systems and Information Technology Support}

The information management (IM) infrastructure (i.e. as defined by the collective set of IS/IT and DSS systems) is seen as a key to enabling and support of effective KM, and as a necessary element in establishing and optimizing a corporate-wide KM System in NPPs (EPRI, 2006). Most NPP design organizations see the IM infrastructure in NPPs as a way to future cost reduction and competitiveness. IM systems can be in the form of any IS, IT, or decision support systems (DSS). The importance of IM systems and 
tools is due to several factors (Alavi and Leidner, 2001): they permit a means of data/information management or conversion (e.g. data capture, transfer, organization, storage, and archival or retrieval) (see also Huber, 1998); they provide interpreted or interpretable information; they aid in knowledge generation (i.e. capture and learning, innovation); they can provide a means to identify plant system patterns and behaviours; they can provide tools to test and verify assumptions, technical premises about the plant state etc.; they can (in the case of DSSs) capture tacit knowledge in the form of "decision logic or criteria"; they can be useful support tools for capturing, presenting, or tracking processes, procedures, and work task execution; and finally, they can help maintain and make available the various data and information on the large volume of rules, constraints, sequences, inter-dependencies, guidelines, limits and conditions needed for safe and reliable plant operation. It is clear that information technology plays an important role in facilitating KM in NPPs.

The impact of information technology and information systems on organizations has been researched from many different perspectives. Authors have measured IT/IS usage in several ways including: frequency of use (Culnan, 1993); motivation to use (De Sanctis, 1982); utilization of the outputs of the system (Jennex and Olfman, 2002); and diversity of software packages used for work (Thompson et al., 1991). There is also a body of literature that focuses on the quality of IT and information systems from the user's perspective. Some of the attributes described in the literature include: functionality and flexibility (Dewitz, 1996); ability to bring past information to bear on the present (Jennex and Olfman, 2002); response time, accessibility, ease of use, 
reliability, ease of learning (Belardo, Karwan, and Wallace, 1982); convenience of access, flexibility, response time (Bailey and Pearson, 1983); accuracy, level of detail, relevance (Ahituv, Neumann, and Riley, 1994); and precision, completeness, timeliness, and source (Alter, 1999)

A report by EPRI (1998) found that many IS and DSS systems at US NPPs were developed over time and out of need and within the constraints of the technology and resources of the day. The study found that many plants have obsolete systems that have evolved into complex systems and infrastructures (some have migrated to newer platforms multiple times). It was found that due to reasons such as cost of updating training programs or work procedures and the lack of design documentation, these systems have been difficult to change or replace (i.e. they have become entrenched in these organizations). In such cases, the work-process (knowledge-processes) are often constrained by the level of support provided by these IT systems and the cost and complexity of changing or improving them may be prohibitive.

DeLone and McLean (1992) provide a useful "IT and IS System Success Model" to understand how information management systems and infrastructure impact both individual and organizational performance (Figure 22). Essentially their research has established that two key factors, the quality of the system (i.e. its design and overall capabilities, features, and effectiveness) and the quality of the information itself contained within the system (completeness, correctness, etc.) will have a significant impact on system use and user satisfaction, which in turn will determine the utility and impact (benefit) obtained by individual users and collectively the organizational impact. 
In the KM context, these straight-forward principles still apply, however the KM perspective views the individual impact and organizational benefit specifically in terms of information and data flow, knowledge capture, storage and retrieval, knowledge sharing, and organizational learning.

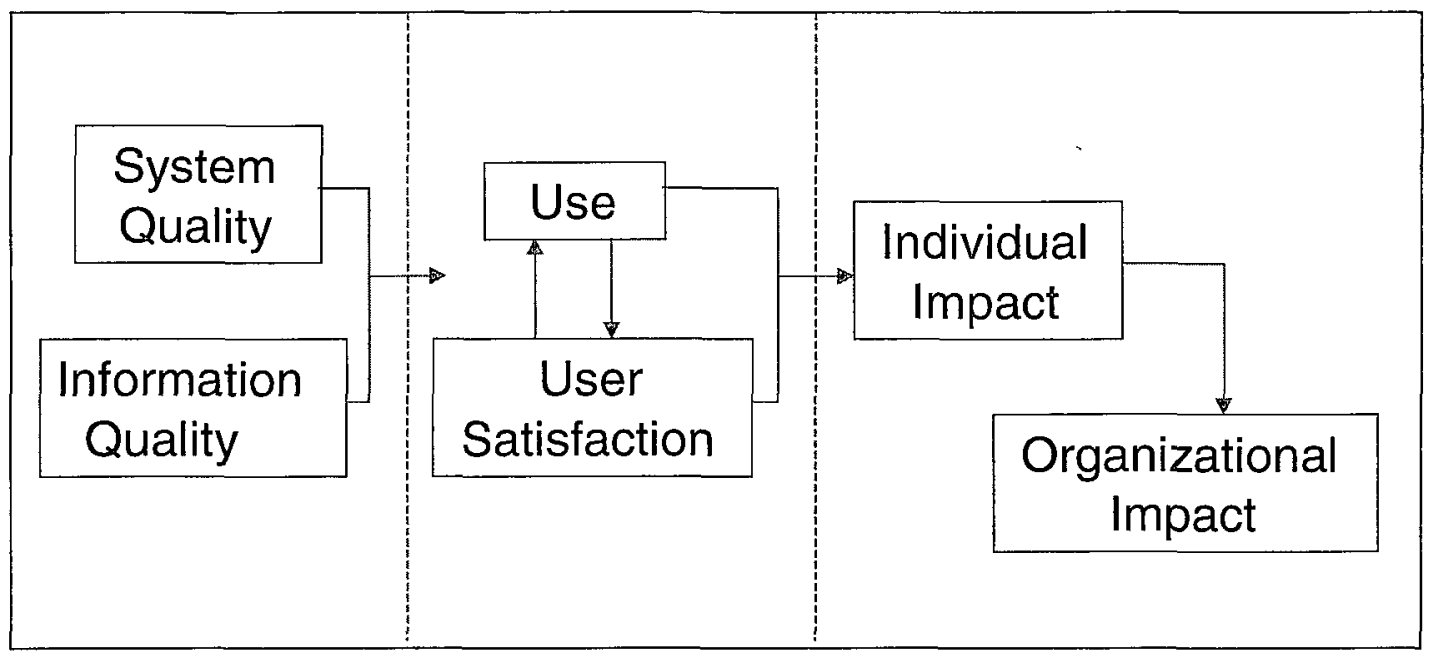

Figure 22. IS/IT System Success Model (adapted, DeLone \& McLean, ‘92)

Another way of looking as this is that IS/IT systems offer the ability to integrate data and information from several sources and complement tacit knowledge. In effect, one can view these systems (whether stand-alone or integrated systems) as providing an extension of aspects of our human capability for acquiring data and information by making it more accessible and providing for the integration of data into information. These systems also improve or enhance human capability for communication (exchanging data and information), memory (retaining data and information), organizing data into information (filtering, storing), and interpreting (visualization and analysis). It can then be argued these properties provide enhanced "contextual and actionable (explicit) knowledge" which in turn enables more effective utilization and 
accumulation of tacit knowledge. A synergy and feedback effect occurs by improving the availability, accessibility, and richness of data and information, and providing a fuller knowledge-oriented decision/action context, which in turn generates information and data that can be captured by the IS/IT system. This process adds relevance and meaning by providing and aiding in information building and sense-making, and facilitates overall comprehension and opportunity to utilize knowledge. Figure 23 illustrates this synergy effect.

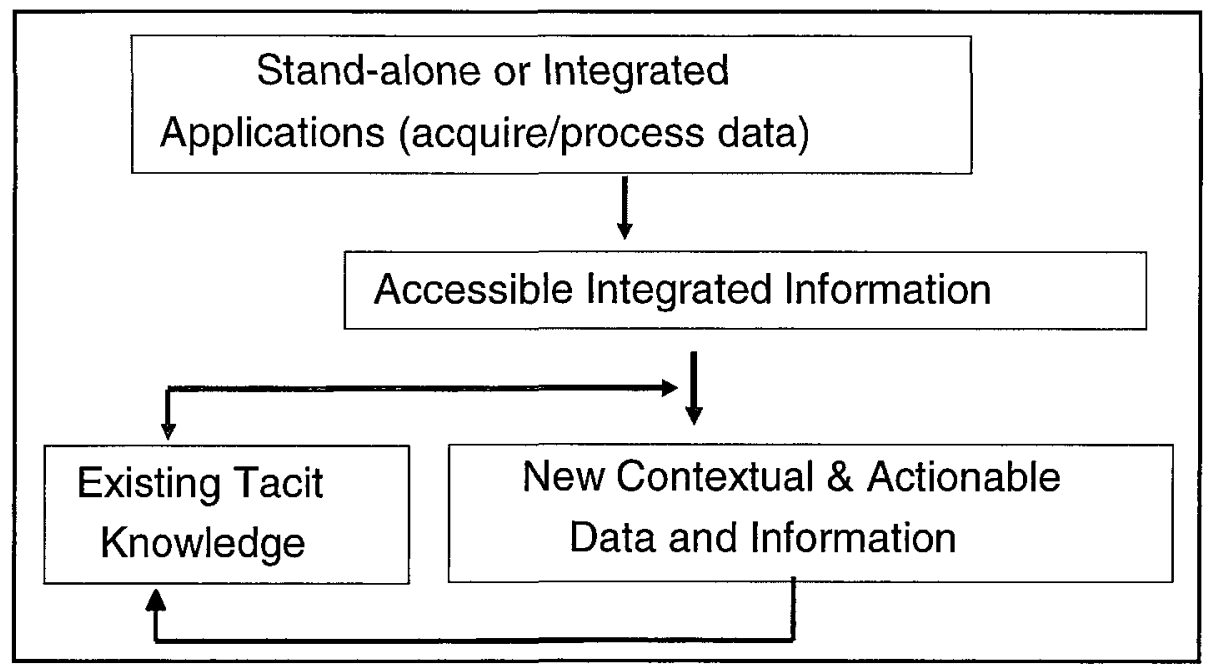

Figure 23. Synergy and Feedback Effect of IT/IS Support

In summary, information technology is seen as a key enabler of the quality of knowledge processes and thus it plays an important role in organizational effectiveness. The extent of technology support is thought to have a significant positive influence the efficiency of knowledge flows and stores in the organization (Bach, 2004). IT/IS is seen as playing a vital role in strengthening the information management infrastructure, the shared knowledge base, and improving knowledge processes (Bach, 2004). As such, 
"technology support" will be retained as an important independent variable construct in the theoretical model developed as part of this research.

\subsection{Conclusions}

The role of KM is particularly important in technology-intensive industries such as the nuclear industry (Beraha et al., 2005). Further, literature supports the argument that knowledge management practices focused on building and maintaining human competencies in nuclear power plants, enable operational and safety performance improvements, including reductions in operational and personnel safety risk (Bhattacharya, 2005, Gharib, 2005). Nuclear power plants (NPPs) around the globe are beginning to recognize the strategic importance of $\mathrm{KM}$ in achieving sustained overall operational performance (IAEA, 2006).

Although a large number of qualitative studies have examined $\mathrm{KM}$ and its impact on performance, few empirical studies have been reported that directly explore the relationship (Keskin, 2005; Lee et al., 2005). Further, there is considerable disagreement in the literature and a lack of consensus on basic theory and relationships (Lewis, 2004). In particular, there is little or no qualitative research on extent of use of KM practices in the context of NPPs (IAEA, 2006; EPRI, 1999) and again little or no empirical research linking KM to NPP operational performance (Lowthert, 1996).

In summary, the literature review provides a strong theoretical basis that supports the notion of a strong link between KM practices and knowledge processes. However, 
empirical research is needed to understand the mechanisms by which these relationships occur in the NPP facilities. Further, the role of organizational culture, in particular its ability to enhance the effect of $\mathrm{KM}$ on learning processes and in turn their impact on organizational performance has not been adequately explored. The next section proposes and describes a theoretical framework to explain these relationships, states a corresponding set of research hypotheses, and defines the constructs and measures needed as a research framework to confirm them. 


\section{Theoretical Frammework}

This chapter describes the research model proposed by this research. The research questions are posed, and the model is presented as derived from the literature review. The various constructs in the model are summarized, specific hypotheses developed, the measures described.

\subsection{Research Question}

The preceding literature review provides some useful insights into the role and influence knowledge management practices may have in organizations, and specifically with respect to knowledge processes and organizational learning. Technology support has also been established as an important precursor. Further, the literature provides useful insights into their collective effects on organizational effectiveness and operational performance. However, little or no research has been done to date to specifically examine and explain these relationships. The fundamental question pursued by this research is:

To what extent does the level of support for knowledge management practices in NPP organizations impact organizational performance, and by what mechanisms does this occur?

In order to answer this fundamental question, research is required to gain a better understanding of the contributing factors and their interactions in NPP organizations. The literature review provided a broad overview of the various $\mathrm{KM}$ practices in use 
today. However, no research has been done to identify what practices are in use in NPPs and to what extent. Further, it is clear from the literature that knowledge processes are believed to play an important role in organizational learning and ultimately organizational performance. Again however, no empirical research has been done to investigate and substantiate this in the nuclear context. Various authors have researched the role of organizational culture and although there is general agreement on its importance, the theoretical model to explain and predict the effect of culture on knowledge processes is lacking. Again little work has been done to apply and confirm the theoretical basis in the context nuclear power production facilities. It is clear from the literature that organizational culture may moderate the effect of support for knowledge practices on the quality of knowledge processes, and subsequently the effect of the quality of knowledge processes on organizational performance. Thus the research question can be broken down into several more specific components:

- To what extent are knowledge management practices currently supported and in use by managers in operating NPPs?

- To what extent does support for knowledge management practices impact the quality of knowledge processes?

- To what extent does the level of technology support (i.e. in terms of the effectiveness of information systems and information technologies) impact the quality of knowledge processes?

- To what extent does the quality of knowledge processes impact organizational performance? 
- To what extent does a supportive organizational culture moderate the impact of support for knowledge management practices on quality of knowledge processes?

- To what extent does a supportive organizational culture moderate the impact of technology support on the quality of knowledge processes?

- To what extent does a supportive organizational culture moderate the impact of the quality of knowledge processes on organizational performance?

The answers to these questions are of great interest to NPP owners and operators. Little if any qualitative or quantitative management research has been done on nuclear plant organizations, likely due to their inaccessible nature. In order to probe into these research questions, a theoretical model of the impact of KM practices on NPP performance was developed based on the application of existing theory discussed in the previous chapter, and adapted to the NPP organizational environment. The following section describes the proposed research model, explains its grounding in current theory and the hypotheses that are derived from it.

\subsection{Research Model}

Based on the findings of the literature review, a simple conceptual model is derived. Figure 24 illustrates the basic elements (or constructs) of the proposed model to be used in this study, and this will be referred to as the "Knowledge Management Performance Model" (KMPM). The elements include: support for knowledge management practices (i.e. degree to which management is supporting those practices that are known 
to influence employee behaviour and action to positively affect knowledge processes); level of support for technology (i.e. IT/IS technology); the quality of knowledge processes (i.e. the extent to which knowledge processes meet the requirements of the organization's business processes); organizational performance (i.e. the degree to which the organizational goals have been achieved). Although conceptually simple and based on fundamentals in the literature, the KMPM provides an integrated perspective and a clear conceptual understanding the role of $\mathrm{KM}$ in organizational performance that is consistent with organizational learning theory.

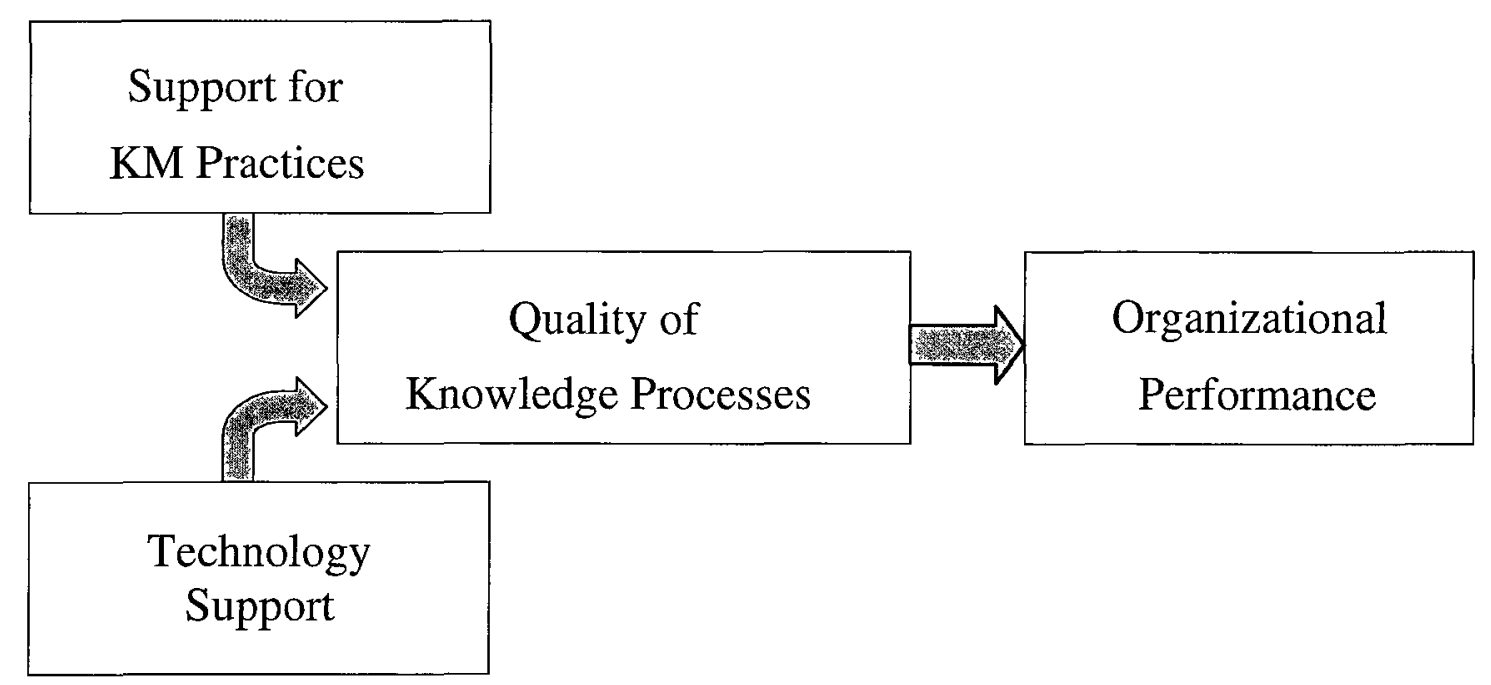

Figure 24. The "Knowledge Management Performance Model" (KMPM)

\subsubsection{The Full Research Model}

As can be seen from Figure 24, the proposed high-level model consists of four constructs: support for KM practices, (organizational) technology support, quality of knowledge processes, and organizational performance. This section will explain the 
assumptions behind the development of each construct in the proposed model that lead to the development of the full detailed research model. Descriptions and derivations of the specific sub-constructs and measures needed to operationalize the model as a basis for the research study are provided.

To begin with, the first construct "support for KM practices" represents a latent construct that measures the extent of organizational support for $\mathrm{KM}$, where $\mathrm{KM}$ is assumed to be the collective set of actions/practices implemented by management to influence the quality of knowledge processes and represents the upper part of the lefthand side (i.e. one of the independent or exogenous factors) of the proposed research model. The construct is operationalized by measuring the extent of use of specific management practices (explained in detail in the next section) known to affect/change the quality of knowledge processes. The IAEA Guidelines $(2006 \mathrm{~b}, 2008)$ provide a useful categorization of $\mathrm{KM}$ practices that can be used to form this construct as a composite of several sub-constructs. These categories are the basis for the IAEA's approach to the assessment of KM at NPPs and have been developed and fine-tuned over several years with direct input from NPP managers as part of the IAEA station KM assist visits. The sub-constructs (each supported by several measures that are explained later in more detail) are:

- KM strategy and planning (KMS) - the extent to which corporate wide KM policy and strategy has been established and the planning to implement it have been put in place. 
- Support for organizational learning (SOL) - the extent to which management provides sufficient resources and enables various mechanisms for individual, group, or institutional level learning.

- Process Management (related) Practices (PMP) - the extent to which management establishes and maintains effective knowledge-oriented business processes (e.g. process-oriented KM practices).

- Information Management Practices (IMP) - the extent to which effective information management practices have been implemented (i.e. that support knowledge processes)

- Organizational Performance Management (related) Practices (OPM) - the extent to which knowledge oriented or knowledge process oriented organizational performance management practices have been put in place.

- Training Related Practices (TRP) - the extent to which industry best practices for training (especially with respect to organizational core competencies) have been put in place.

- Human Resource (related) Practices (HRP) - the extent to which HR related practices aimed at competency development and knowledge retention have been put in place.

Note that although a direct effect could be hypothesized from $\mathrm{KM}$ practices to organizational performance and this can be supported in the literature, the model presumes the effect is primarily through the intermediate constructs of quality of 
knowledge processes and supportive organizational culture. Tests for direct effects will be included in the analysis however.

The second construct, organizational technology support (OTS) measures the level of organizational support for the use of computers, information systems and network technology using measures of the "effective use of" various widely used technologies (i.e. as an effective proxy for organizational support, and based on DeLone and McLean, 1992). It measures this on two dimensions: conventional information systems and technology (IST), and advanced operational (decision) support systems (OSS). IST measures how effectively conventional application of IT and IS technology is used and OSS measures how effectively NPP-specific advanced operational (decision) support systems are utilized. The basis for both constructs will be explained in further detail in the next section. Although a direct effect could be hypothesized from organizational technology support (OTS) to organizational performance and can be supported in the literature, the model presumes the entire effect is through the intermediate construct of quality of knowledge processes. Though not a main hypothesis, it is possible that OTS also impacts organizational performance through supportive organizational culture as an intermediate construct. Tests to explore for this and possible direct effects to organizational performance will be included in the analysis.

The third construct, quality of knowledge processes (QKP), is based on the five widely accepted key knowledge processes discussed in the literature review:

- knowledge acquisition and adoption (KA), 
- knowledge generation and validation $(\mathrm{KG})$,

- knowledge sharing and transfer (KS),

- knowledge retention and storage (KR), and

- knowledge utilization and application (KU).

The right hand side of the model, the dependent variable is "organizational performance". Organizational performance (or more specifically NPP performance) is made up of two constructs: organizational effectiveness $(O E)$ and operational performance $(O P)$, the former based directly on established measures of performance in the literature (explained below) and the later being published industry measures of production reliability and safety (i.e. actual plant data). Note that several authors have established empirically that operational performance is an outcome of organizational effectiveness. Thus "organizational performance" is seen to be comprised of both organizational effectiveness and operational performance. Both components are well established in the literature as constructs, and their measures are easily tailored to NPP operational objectives (explained below).

As discussed in Chapter 2, the academic and practitioner literature supports the notion that knowledge processes are influenced by organizational culture and it plays an significant role in organizational effectiveness (Haber et al., 1991). In particular, a number of researchers have identified that culture influences organizational learning and ultimately affects performance (Barney (1986); Lee and Yu (2004); Chatman and Jehn 
(1994)). Gordon (1985) found that "cultural fit" affects organizational performance. Quick (1992) and Kotter and Heskett (1992) suggest that culture strongly influences organizational performance. Ahanotu (1998) found that empowerment influenced productivity and performance in production environments. In addition, the concept of shared vision was found to impact organizational performance (Hoe, 2007; Levine, 2006). For these reasons, and given the findings from the literature review outlining the importance of cultural alignment with organizational objectives (e.g. safety, production, and knowledge sharing etc.), the construct "supportive organizational culture" (SOC) was included in the model as an intermediate variable and it is thought also to moderate the effect that "support for KM practices" has on the "quality of knowledge processes" and in turn, the effect "quality of knowledge processes" has on organizational performance.

Finally, physical plant factors such as plant age, plant design type, plant size (MWe output), and number of operating units at the site have been identified as factors in NPP performance (IAEA, 1999 and 2006a). However, there is no clear findings or agreement in the literature on the nature of these relationships. Plant type is hypothesized to moderate the relationship between organizational effectiveness and operational performance. It may also moderate the relationships between plant age, size, and number of units with organizational effectiveness or operational performance. Figure 25 illustrates the full detailed research model. 


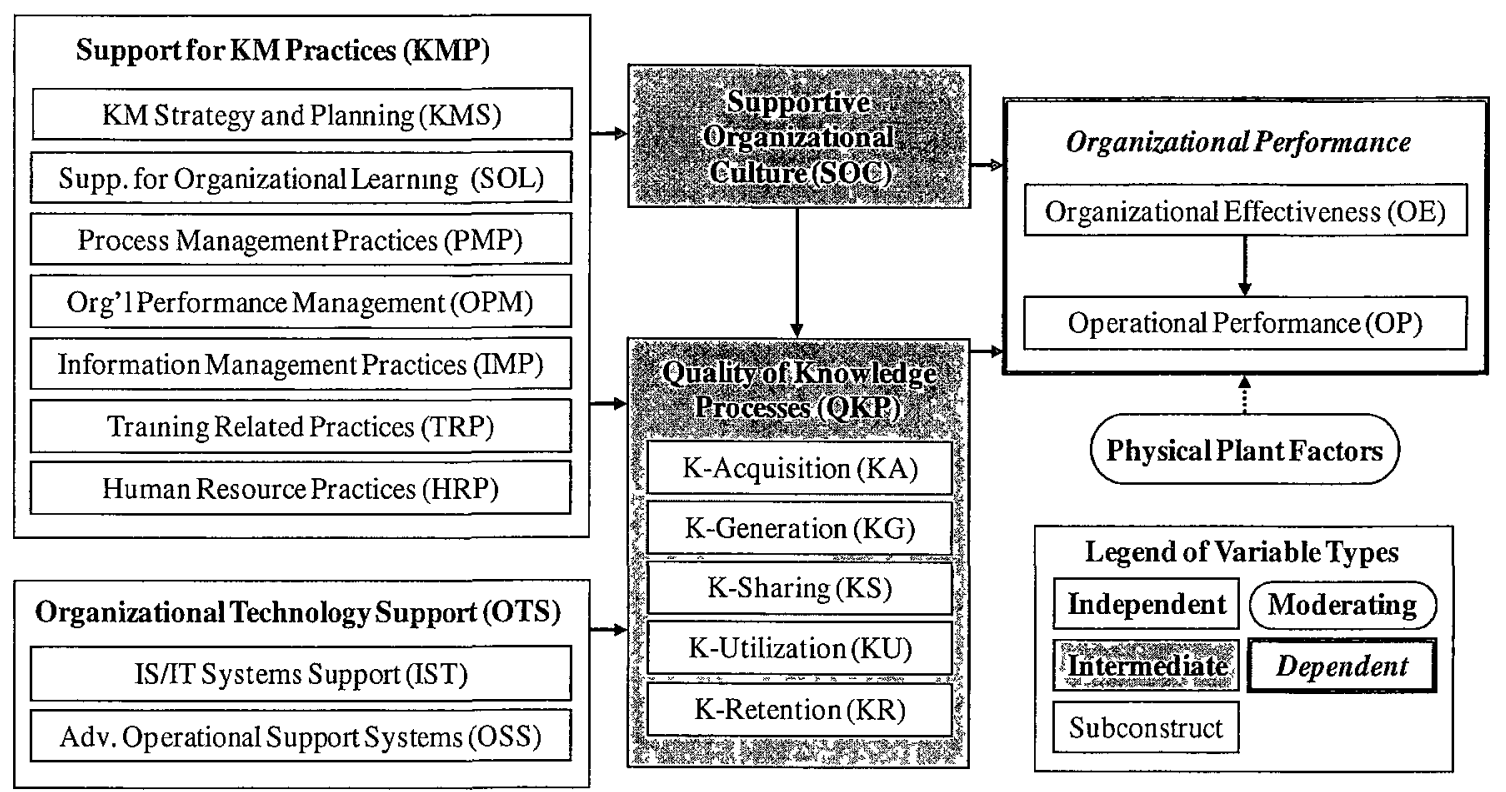

Figure 25. The NPP Knowledge Management Performance Model

The model in Figure 25 recognizes organizational performance may be conditioned by physical plant characteristics and shows a dotted line from the construct "physical plant factors" to organizational performance. This is to indicate the exploratory nature of this relationship (i.e. these effects hypothesized to exist but their direction is unknown at this time).

Several key assumptions based on the preliminary research (including the literature review, the NPP assist visits and the IAEA peer review) are made and underlie the research model. These can be summarized as:

- Quality knowledge processes in an organization, if aligned to company objectives, enables continuous organizational learning that results in an increased organizational knowledge base and its effective utilization. This 
results in more efficient and effective decision making, problem solving, and work processes and ultimately higher levels of sustained organizational performance.

- KM practices are the necessary planned and coordinated effort, using whatever practical and effective means are available and within the control management, to influence the quality of these knowledge processes.

- Organizational technology support (also a variable under control of management) enables and enhances the quality of knowledge processes and their impact on organizational performance.

- A reasonable degree of uniformity exists between all NPPs in terms of having the same or similar basic operating objectives, fundamental business processes and operating practices. These would include all key aspects of an integrated management system and include adequate organizational structure and financial stability.

- These basic operating objectives, fundamental business processes and operating practices are a necessary condition for any level of acceptable organizational performance but are not in themselves a sufficient condition to achieve sustained high performance.

- It is assumed that management support for effective $\mathrm{KM}$ practices, if implemented in a cohesive and integrated manner aligned with organizational 
objectives in NPPs are also a necessary condition to enable sustained high organizational performance.

- However, it is also recognized that even if these two necessary conditions are met (i.e. an organization has basic operating objectives, fundamental business processes and operating practices in combination with support for effective KM practices), other factors may also influence an NPP organization's ability to achieve sustained high performance and may also be necessary conditions.

- Finally, it is recognized that support for KM practices are not in themselves a sufficient condition to achieve sustained high organizational performance. A key assumption of this performance model is that the other necessary conditions to achieve sustained high organizational performance have been met.

\subsubsection{The Main Research Hypotheses}

Several interesting hypotheses can be developed and supported by the model in Figure 25. These are summarized below:

Hypothesis 1. Higher levels of overall support for KM practices (KMP) will positively influence the supportive organizational culture (SOC).

Hypothesis 2. Higher levels of supportive organizational culture (SOC) will positively influence the overall quality of knowledge processes $(Q K P)$.

Hypothesis 3. Higher levels of supportive organizational culture (SOC) will positively influence organizational effectiveness (OE). 
Figure 26 illustrates these relationships for $\mathrm{H} 1, \mathrm{H} 2$, and $\mathrm{H} 3$.

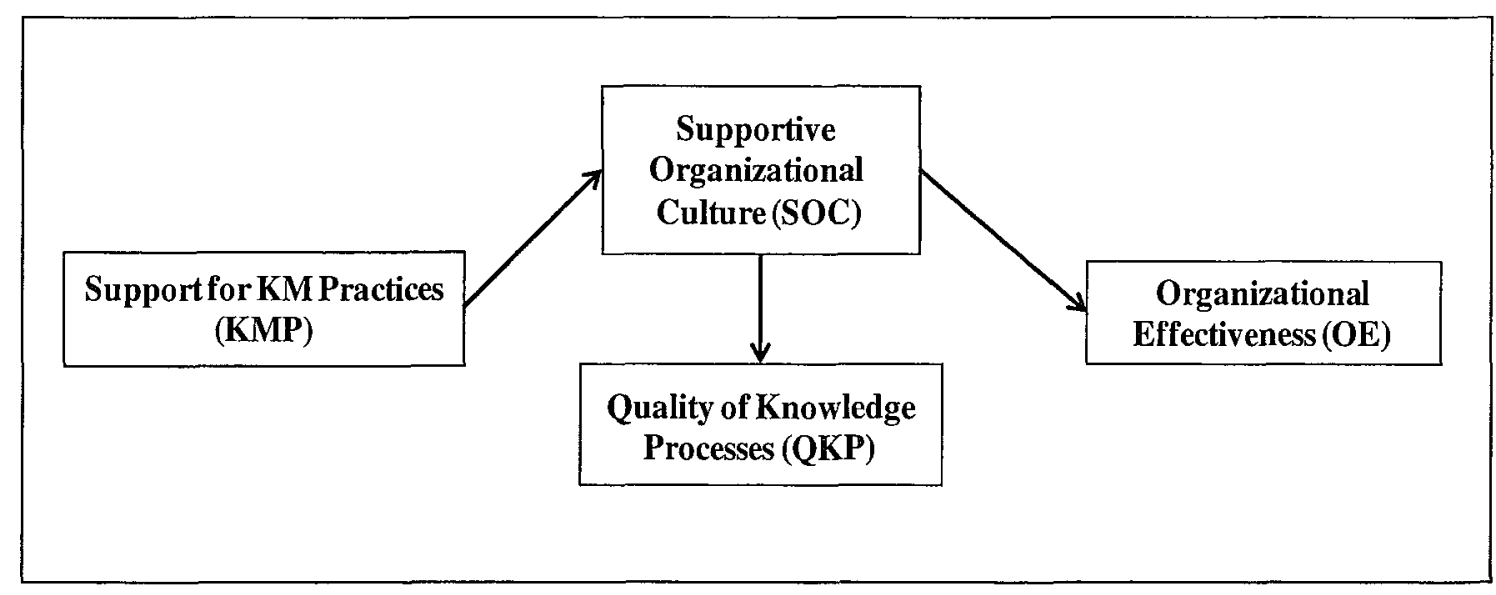

Figure 26. Links to Supportive Organizational Culture

Hypothesis 4. Higher levels of overall support for KM practices (KMP) will positively influence the overall quality of knowledge processes $(Q K P)$ directly, and indirectly through the intermediate effect of supportive organizational culture (SOC).

Hypothesis 5. Higher levels of overall support for KM practices (KMP) will positively influence organizational effectiveness (OE) through the intermediate effects of both overall quality of knowledge processes (QKP) and supportive organizational culture (SOC).

Hypothesis 6. Higher levels of organizational technology support (OTS), or individually information systems and technology support (IST) or advanced operational support systems (OSS), will positively influence overall quality of knowledge processes (QKP). 
Hypothesis 7. Higher levels of organizational technology support (OTS), or individually information systems and technology (IST) support or advanced operational support systems (OSS) support, will positively influence organizational effectiveness $(O E)$ through the intermediate effects of overall quality of knowledge processes (QKP).

Hypothesis 8. Higher overall quality of knowledge processes (QKP) will positively influence organizational effectiveness (OE).

Hypothesis 9. Higher organizational effectiveness $(O E)$ will positively influence operational performance $(O P)$.

Hypothesis 10. As an exploratory hypothesis, it is expected that plant factors (i.e. physical characteristics) including: a.) number of plant operating units at the site ("units"); b.) plant size ("size"); and c.) plant age in years since start-up ("age") will influence organizational effectiveness (OE) and operational performance $(O P)$ and the relationship between them; and d.) all of these relationships will all be moderated by plant design type ("type"). The direction of influence is not known.

A number of secondary (or sub-hypotheses) are also developed, but as the theoretical basis found in the literature for these relationships is not consistent or well established, they are considered exploratory. They are described in the following sub-sections. 


\subsubsection{Possible Links Amongst the QKPs and with $O E$}

At the core of the proposed "NPP Knowledge Management Performance Model" (KMPM) are the five quality of knowledge processes (QKPs). There is no shortage of literature characterizing the interactions between these key processes. Literature can be found supporting a link between any and all of them. Many authors put the emphasis on a given knowledge process (e.g. knowledge creation or knowledge sharing) and define the other knowledge processes in terms of their interaction with them. For example, Shankar and Gupta (2005) describe a model of organizational learning centered on knowledge utilization and its interaction with the other knowledge processes (see Figure 27).

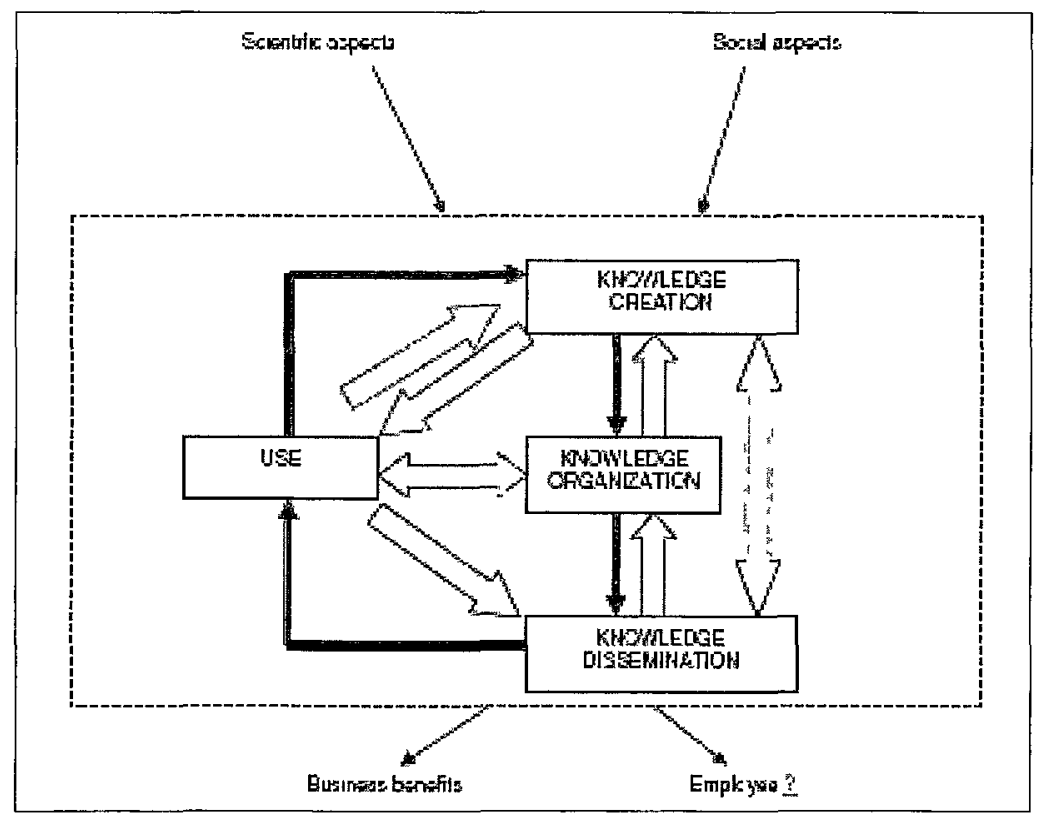

Figure 27. Framework for KM (Shankar and Gupta, 2005)

In the literature in general however, there is a lack of consistent theory and empirical research to explain the dominant interactions among the knowledge processes and their 
effects on organizational effectiveness (OE). There is no clear mechanism identified or agreed upon by which knowledge processes impact performance.

Lee and OuYang (2010) summarized the literature on the links between the knowledge processes in organizations and proposed the "KM/KMS Value Chain". This model emphasizes the dominant links (interdependencies and flows) found in the literature between the knowledge processes and purports to explain how knowledge management impacts on business performance. Based on Alavi (1997) and Alavi and Leidner (2001) knowledge management process categories: knowledge creation/acquisition, knowledge organization/storage (conversion), knowledge distribution (sharing and transfer), and knowledge application; they summarized previous empirical studies establishing the links between the knowledge processes, and argue this is the dominant "value chain" that influences organizational performance.

The findings of Lee and OuYang (2010) from the literature are summarized in Figure 28. Note that the construct "knowledge conversion" is defined as the combination of knowledge functions including storage, retrieval, repository, organize, assemble, integrate, document, and codification. Thus it is quite similar to the construct "knowledge retention and storage" used in this study. They found that the literature generally supports the following "value chain cycle" mechanism for organizational learning that builds and maintains the corporate knowledge base: that knowledge creation and acquisition processes promote and support knowledge retention and storage processes; these in turn promote and support knowledge sharing processes, these in turn 
promote and support knowledge utilization processes, and these in turn promote knowledge creation and acquisition processes (see Figure 28).

\begin{tabular}{|c|c|c|c|c|}
\hline KM/KMS Value Cham & $\rightarrow C$ Cieation/Acquisitio & $\longrightarrow \longrightarrow$ Conversion & $\longrightarrow$ Sharin & \multirow{2}{*}{$\underset{\text { Application }}{\text { Application }}$} \\
\hline Pentland (1995) & Construction & Organization & Distribution & \\
\hline Spender(1996) & Create & & Transfer & Use \\
\hline Demarest (1997) & Discennng & choosing a contaner & Dissemination & $\mid$ Use \\
\hline Daal et al (1998) & Creation & Dran-Up & Dissemination & Apply Er aluate \\
\hline Dat eaport et al (1998a) & Creation & & Transference & Asset Management \\
\hline 5 kgrme \& Amrdon (1998) & Create & & Iransfer & Use \\
\hline Teece (1998) & Create- & Axsemble Integrate & \begin{tabular}{|l|l|} 
Iransfer \\
\end{tabular} & Explot \\
\hline Verhasalo \& Lappalamen (1998) & Perception Arquistition & Documentation Retrieval & Iran15mission & Decasion mahing \\
\hline $\begin{array}{l}\text { Bulasubramanat et al (1999) } \\
\end{array}$ & Discover Caphise & Transform Cla suffy Manntang & Disseminate & \\
\hline Liebow itz (1999) & identafy Capture & Store & Share & Apply Sell \\
\hline Srrvary (1999) & Learning Generate & & Disseminate & \\
\hline Zack (1999) & -tcquistion Refinement & Storage/Retneva & Distnbution & Presentation \\
\hline Lee \& Yang (2000) & Acquistion Innoration & Frotectson Infegration & Dissemmation & \\
\hline A. Law \& Leidnea (2001) & Creation (Construction), & Storage/retrevelal & Iransfer & Application \\
\hline Gold et al (2001) & Acquistition & \begin{tabular}{|c|c|c|} 
Conversion & Protection \\
\end{tabular} & & Application \\
\hline Grover \& Day enport (2001) & Generation & Codification & Iranlsfer & Realization \\
\hline Shan et al (2001) & Acquistion Generation & Organzzation Integration & Distribution & $\begin{array}{l}\text { Decision sugpoit applicautons } \\
\text { Refinement zud Refreshnent } \\
\end{array}$ \\
\hline Shlin es al (2001) & Creation & Storage & Distribution & Application \\
\hline Litras et al $(2002)$ & Acquire Relate $/$ 'alue & Organze & Iransfer & Use Enable reuse \\
\hline Dartoch $(200 \hat{)})$ & Acquistron & & Dissemination & Responsur eness: \\
\hline Liebowitz \& Megbolugbe (2003) & $\begin{array}{l}\text { denthfic ation and capture } \\
\text { Creation }\end{array}$ & & Sharmg & application \\
\hline Remus \& Schub (2003) & Assess Refine & & Share Distrbbute & \\
\hline Smart at al $(2003)$ & Creation/adoptron Adaption & Enbodument & & Eraluation \\
\hline Shankar ef al $(2003)$ & Capture & Document Organizz & Shanng & Agply Reuse Evolic Feedback \\
\hline Liu et al (2004) & Obtaintug & Stontig & Shanng & \\
\hline Cu1 et al (2005) & Acquistion & Contersion & & Appicatron \\
\hline Tancrverds (2005) & Creatton & Integrate & Transfer & Leserage \\
\hline
\end{tabular}

Figure 28. The KM/KMS Value Chain (Lee \& OuYang, ‘10)

As there is clearly no consensus in the literature on the causal nature of the interactions between the knowledge processes, this study takes an exploratory approach. It assumes for now, based on the findings of Lee and OuYang, that the following 5 sub-hypotheses, if true, can be expected to be confirmed:

Hypothesis 11. Higher quality knowledge acquisition and adoption (KA) processes and higher quality knowledge generation and validation $(K G)$ processes will both have a positive influence on quality knowledge retention and storage (KR) processes. 
Hypothesis 12. Higher quality knowledge retention and storage $(K R)$ processes will have a positive influence on the quality of knowledge sharing and transfer $(K S)$ processes.

Hypothesis 13. Higher quality of knowledge sharing and transfer $(K S)$ processes will have a positive influence on quality of knowledge utilization and application $(K U)$ processes.

Hypothesis 14. Higher quality knowledge utilization and application (KU) processes will have a positive influence on both knowledge acquisition and adoption (KA) processes and knowledge generation and validation (KG) processes.

Feedback from the IAEA peer review and assist visits indicated that knowledge retention and storage (KR) and knowledge utilization and application (KU) processes was thought to directly influence organizational effectiveness. Literature from Shankar and Gupta (2005), IAEA (2006), Young, T. (2006), and Jennex et al. (2008)) also supported the contention. Therefore the following relationships (sub-hypotheses) were included as exploratory in nature and expected may be confirmed by this study:

Hypothesis 15. Higher quality knowledge utilization and application (KU) processes will have a positive influence on organizational effectiveness (OE).

Hypothesis 16. Higher quality knowledge retention and storage (KR) processes will have a positive influence on organizational effectiveness (OE). 
Figure 29 below illustrates all of these exploratory sub-hypotheses related to the quality of knowledge process inter-relationships and possible direct links to organizational effectiveness, all of which the literature predicts will be positive effects. The relationships are all shown as links with dotted lines to indicate the exploratory nature of these secondary hypotheses.

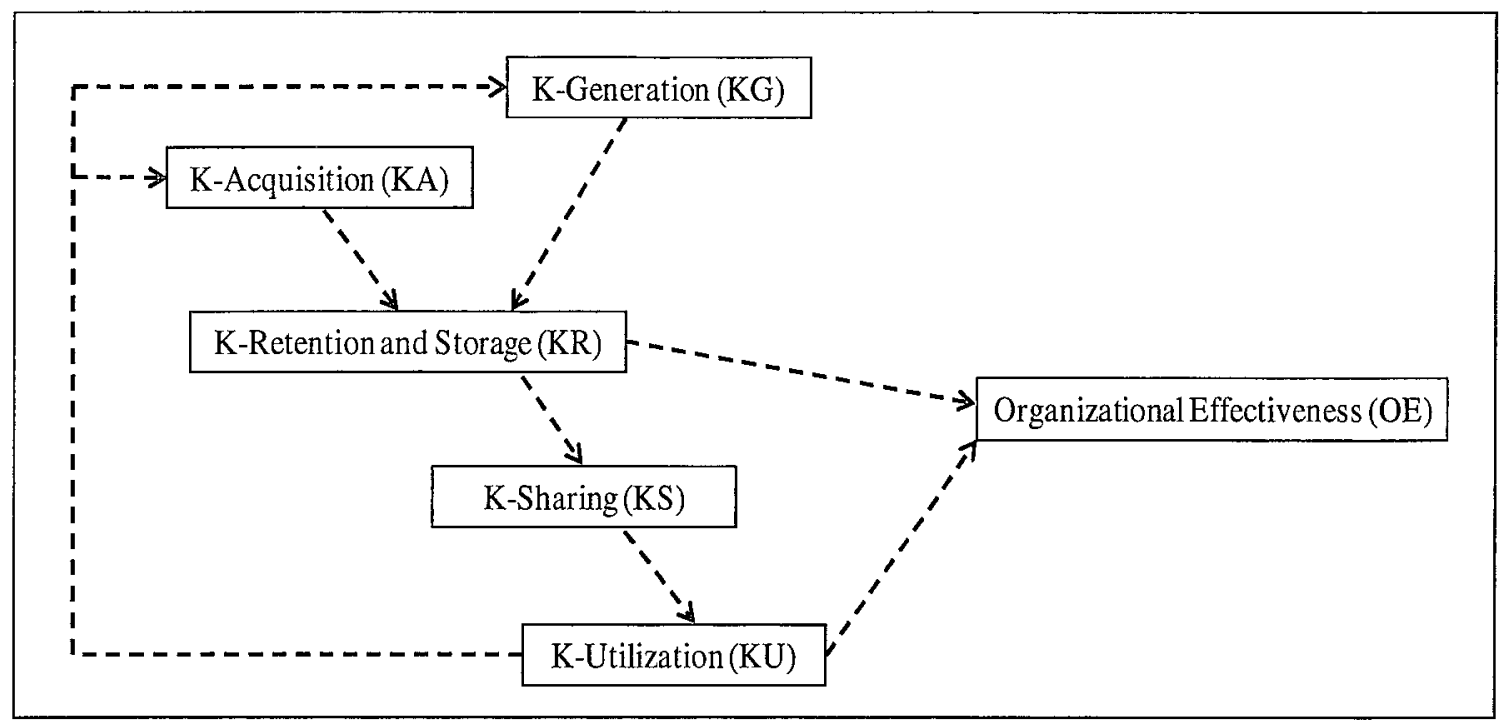

Figure 29. Expected Value-Chain Links Amongst the QKPs and with OE

\subsubsection{Possible Links from KMPs or OTSs to QKPs}

In addition to the above secondary hypotheses, the literature review findings support notion that the level of support for each specific KM practice (KMP) may have a direct influence on one or more specific knowledge processes. Similarly with OTS. Thus direct one-to-one links between each of the KM practices and OTS dimensions with each of the quality of knowledge processes was considered. Each of these possible links was explored in light of the literature review findings, peer review feedback, and the 
IAEA station assist visits to consider whether a direct relationship could be expected in the context of NPP organizations. As a result, several additional exploratory subhypotheses are proposed in this study and will be tested to see if they can be confirmed. They are summarized below and a graphical representation of each is provided.

Support for KM strategy and planning (KMS) is thought to influence all of the knowledge management processes.

Hypothesis 17. Higher support for KM strategy and planning (KMS) will have a positive influence on quality of knowledge acquisition (KA) processes, quality of knowledge sharing and transfer (KS) processes, quality of knowledge utilization (KU) processes, quality of knowledge generation (KG) processes, and quality of knowledge retention and storage (KR) processes. (see Figure 30).

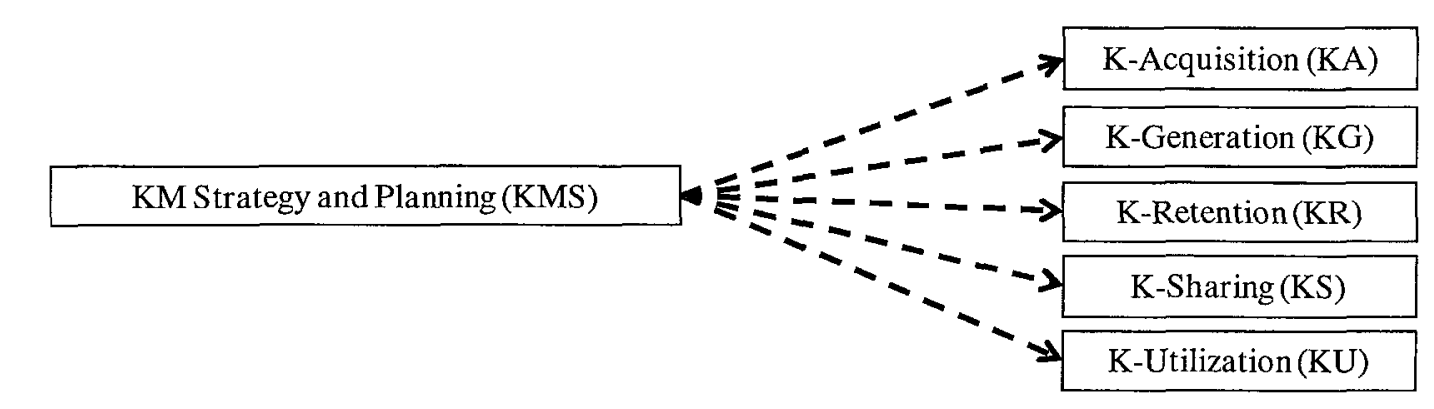

Figure 30. Hypothesized Links Between KMS and QKPs

Hypothesis 18. Higher support for organizational learning (SOL) and will have a positive influence on quality of knowledge acquisition (KA) processes, quality of knowledge generation and validation $(K G)$ processes, quality 
of knowledge retention and storage (KR), and quality of knowledge sharing and transfer $(K S)$ processes (see Figure 31).

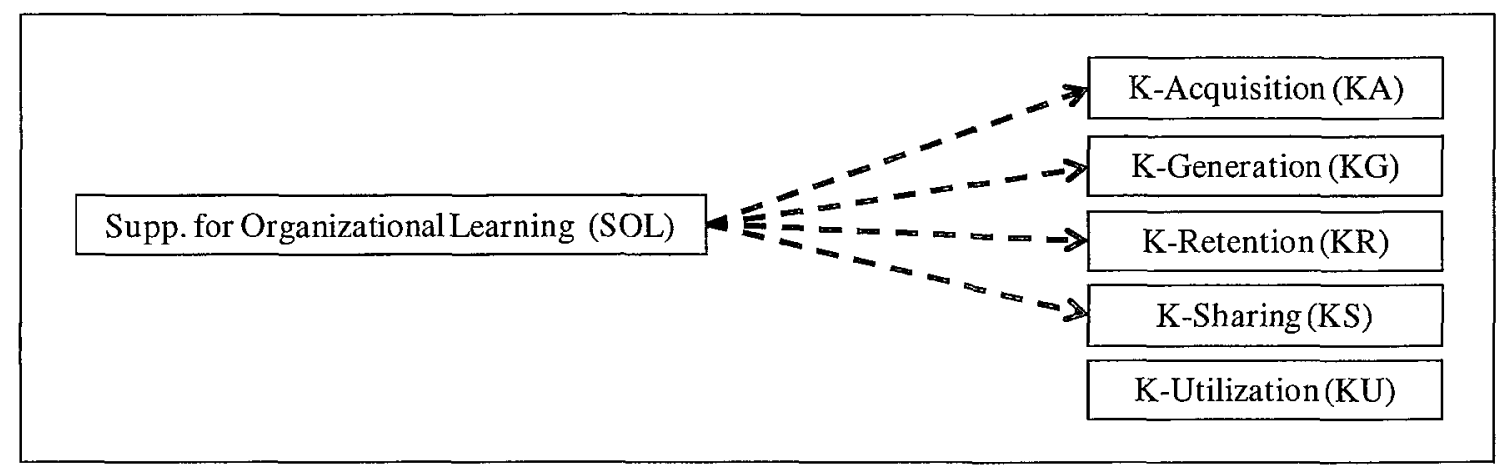

Figure 31. Hypothesized Links Between SOL and QKPs

Hypothesis 19. Higher support for (KM-supportive) human resource related practices (HRP) will have a positive influence on quality of knowledge acquisition (KA) processes, quality of knowledge sharing and transfer $(K S)$ processes, quality of knowledge utilization $(K U)$ processes, and quality of knowledge retention and storage $(K R)$ processes (see Figure 32).

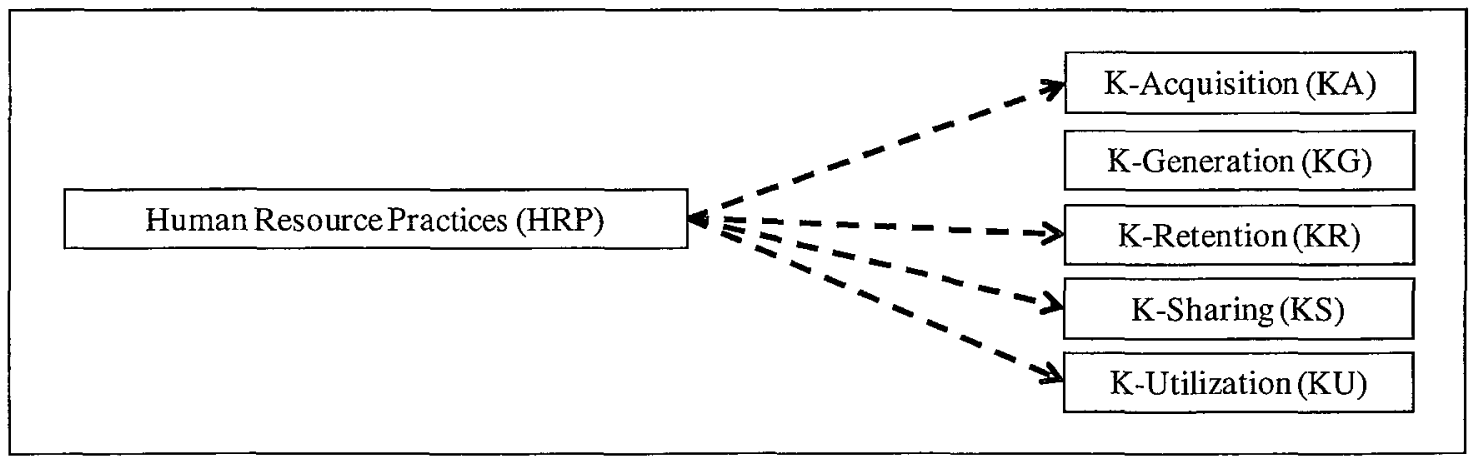

Figure 32. Hypothesized Links Between HRP and QKPs 
Hypothesis 20. Higher support for training related practices (TRP) will have a positive influence on quality of knowledge retention and storage (KR) processes, quality of knowledge utilization and application (KU) processes, and quality of knowledge sharing and transfer (KS) processes (see Figure 33).

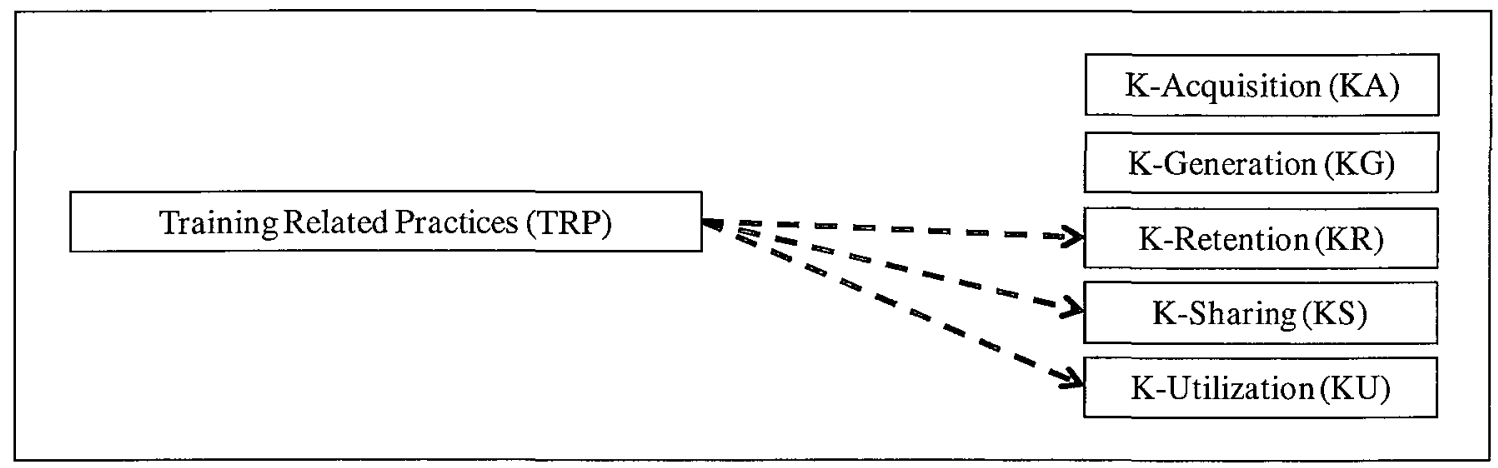

Figure 33. Hypothesized Links Between TRP and QKPs

Hypothesis 21. Higher support for information management practices (IMP) will have a positive influence on quality of knowledge sharing and transfer (KS) processes, quality of knowledge retention and storage $(K R)$ processes, quality of knowledge generation and validation $(K G)$ and quality of knowledge utilization and application (KU) processes (see Figure 34). 


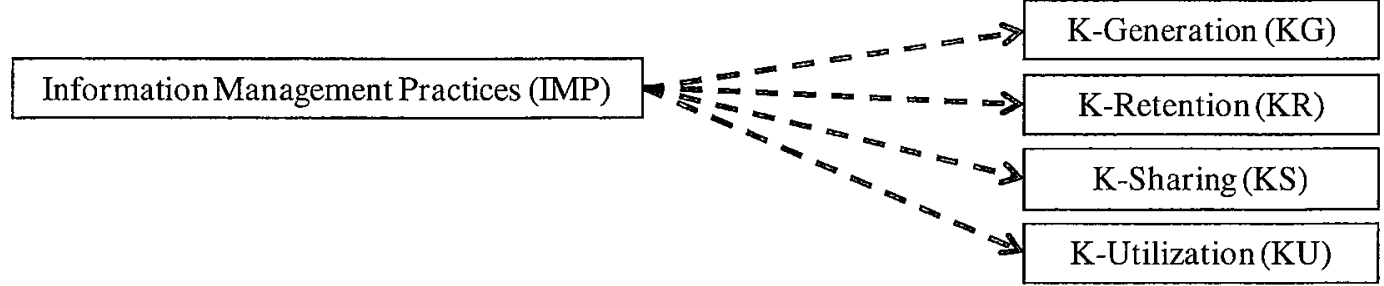

Figure 34. Hypothesized Links Between IMP and QKPs

Hypothesis 22. Higher levels of effectiveness of information systems and technology support (IST) will have a positive influence on quality of knowledge generation $(K G)$ processes, quality of knowledge utilization and application $(K U)$ processes, quality of knowledge retention and storage $(K R)$, and quality of knowledge sharing and transfer $(K S)$ processes (see Figure 35).

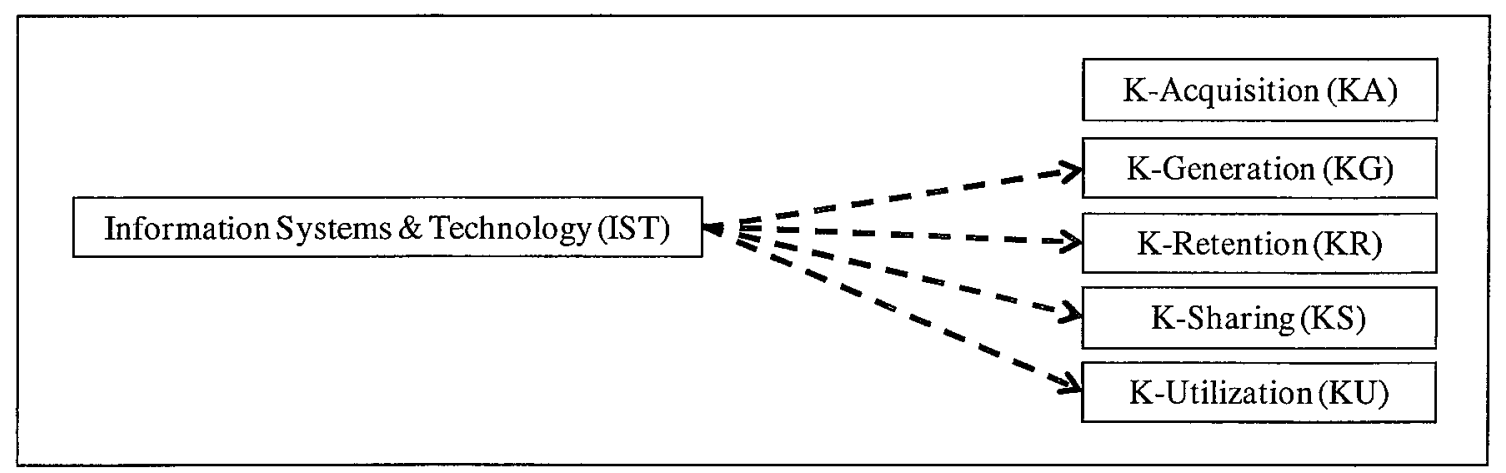

Figure 35. Hypothesized Links Between IST and QKPs

Hypothesis 23. Higher levels of effectiveness of advanced operational support systems (OSS) will have a positive influence on quality of knowledge utilization 
and application $(K U)$ processes and quality of knowledge generation and validation $(K G)$ processes (see Figure 36 ).

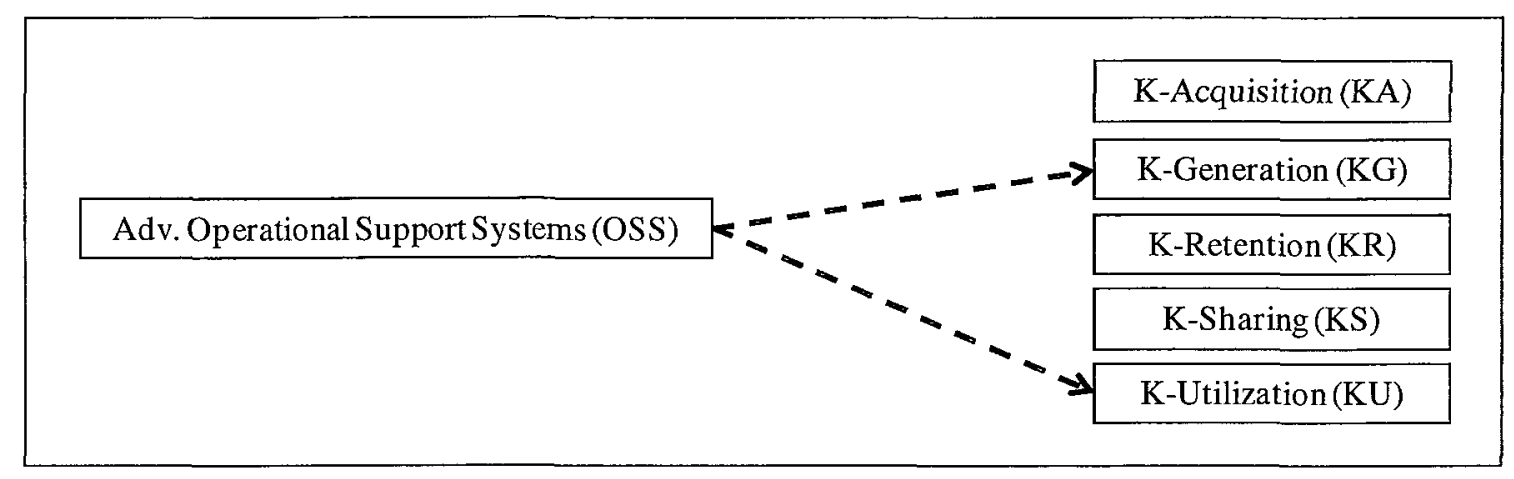

Figure 36. Hypothesized Links Between OSS and QKPs

Hypothesis 24. Higher support for process management practices (PMP) have a positive influence on quality of knowledge sharing and transfer (KS) processes, quality of knowledge utilization (KU) processes, and quality of knowledge retention and storage (KR) processes (see Figure 37).

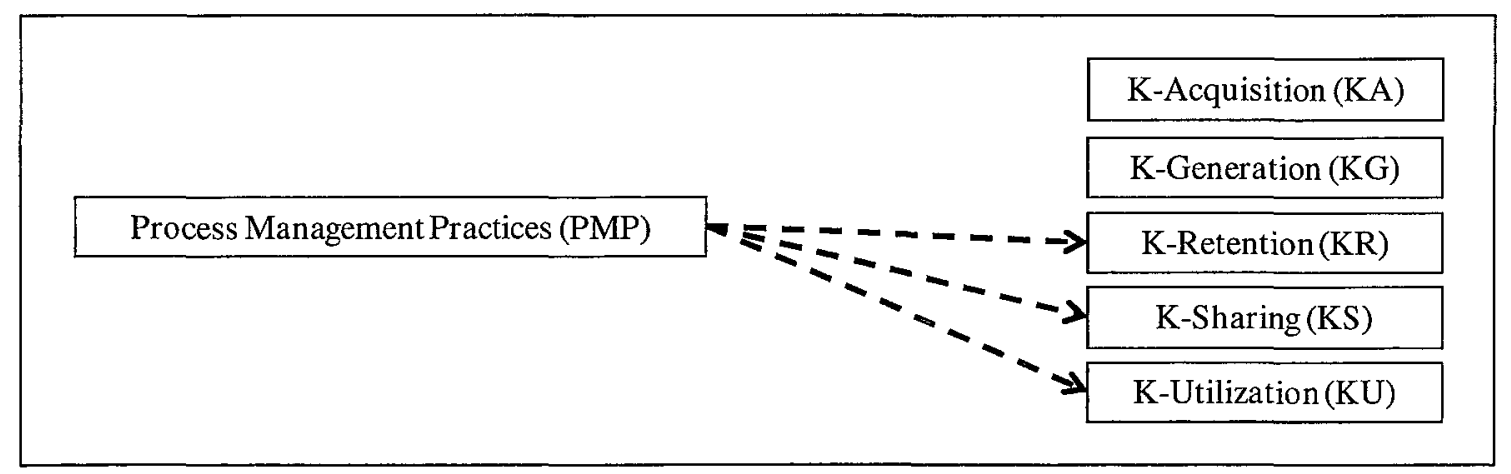

Figure 37. Hypothesized Links Between PMP and QKPs

Hypothesis 25. Higher support for organizational performance management (OPM) practices will have a positive influence on quality of knowledge 
retention and storage (KR) processes and quality of knowledge utilization and application (KU) processes (see Figure 38).

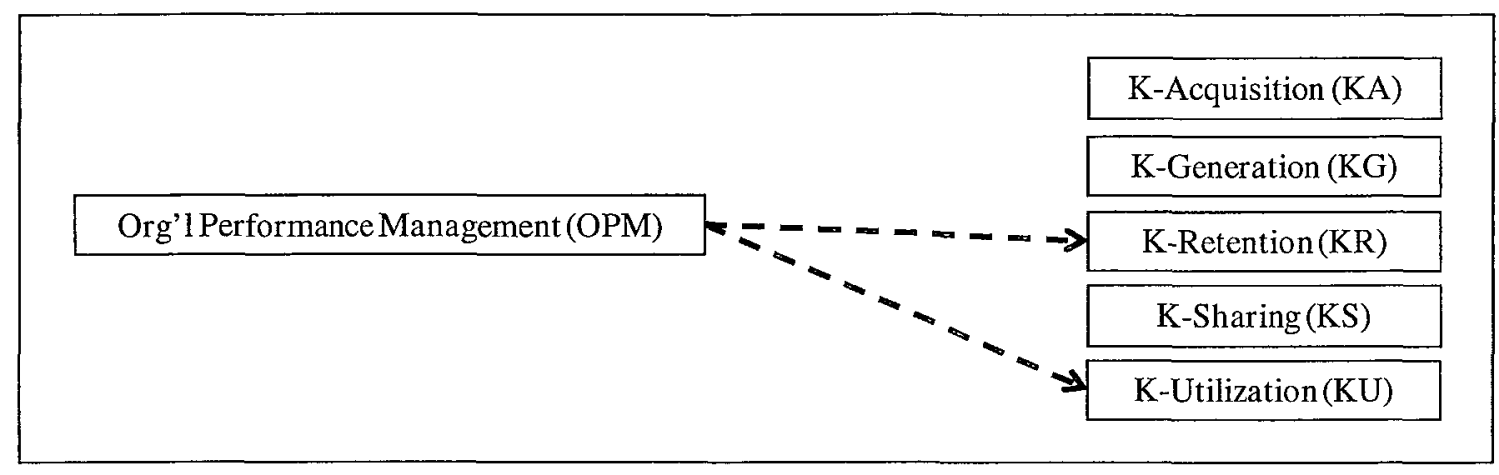

Figure 38. Hypothesized Links Between OPM and QKPs

\subsubsection{Possible Direct Links from PMP or $O P M$ to $O E$ and $O P$}

In addition to the above sub-hypotheses, the literature review findings, peer review feedback, and the IAEA station assist visits supported the possibility that the level of support for process management related practices (PMP) and organizational performance management related practices (OPM) may each have a direct influence on organizational effectiveness and/or operational performance. As a result, direct one-toone links between PMP and OPM with OE and OP were considered likely and the following additional exploratory sub-hypotheses are proposed in this study and will be tested to see if they can be confirmed. They are summarized below and a graphical representation is provided in Figure 39 with links shown as dotted lines to indicate an expected but exploratory hypothesis. 
Hypothesis 26. Higher support for process management practices (PMP) will have a positive influence on organizational effectiveness (OE) and operational performance $(O P)$ (see Figure 39).

Hypothesis 27. Higher support for organizational performance management (OPM) practices will have a positive influence on organizational effectiveness $(O E)$ and operational performance (OP) (see Figure 39).

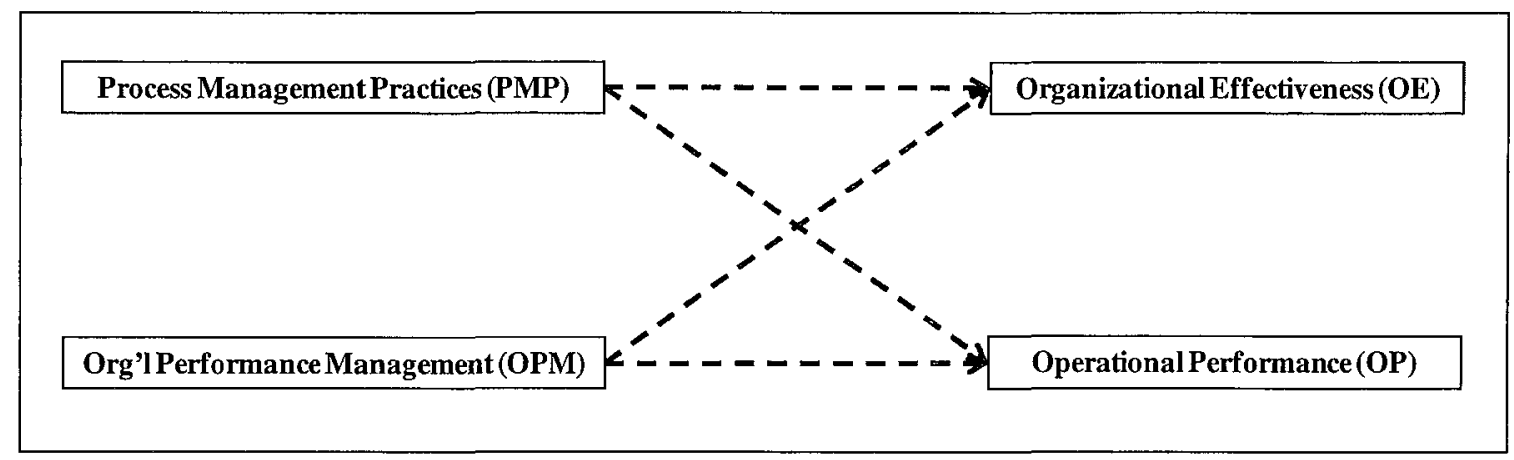

Figure 39. Possible PMP and OPM Links to OE and OP

\subsubsection{Possible Links to and from SOC}

Finally, it is expected from the literature review, prior empirical research, peer review feedback, and the IAEA NPP station visits that a supportive organizational culture (SOC) plays an important moderation role in many of the preceding relationships in NPP organizations. Several of the KMPs and the OTSs we considered and are expected to positively impact SOC and likewise SOC to positively impact all of the QKPs. The following relationships are exploratory but most are expected to be confirmed: 
Hypothesis 28. Higher support for KM strategy and planning (KMS), support for organizational learning (SOL), information management practices (IMP), training related practices (TRP), and human resource related practices (HRP) will each have a positive influence on supportive organizational culture (SOC).

Hypothesis 29. Higher level of supportive organizational culture (SOC) will have a positive direct influence on each of the quality of knowledge processes: quality of knowledge acquisition (KA), quality of knowledge generation and validation $(K G)$, quality of knowledge retention and storage $(K G)$, quality of knowledge sharing and transfer (KS), and quality of knowledge utilization and application $(K U)$.

Figure 40 below illustrates these relationships as dotted line links to indicate they were supported by the preliminary research but are exploratory in nature.

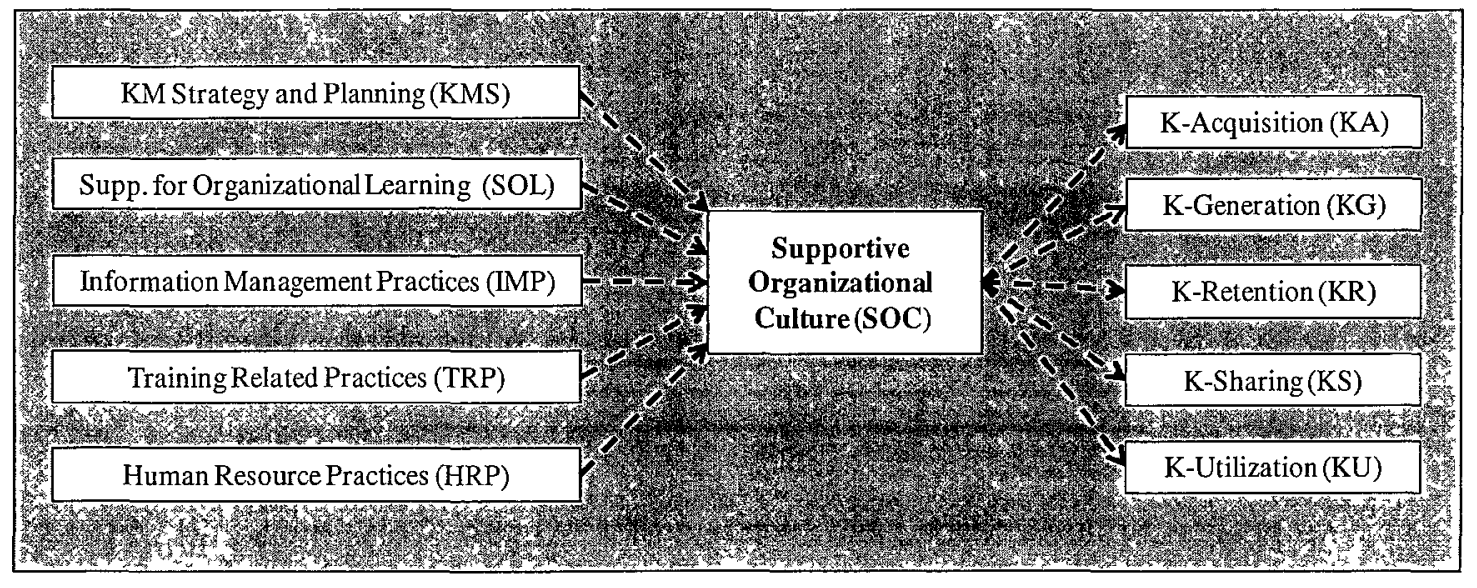

Figure 40. Hypothesized Links for KMPs and QKPs with SOC 


\subsection{Operationalizing the Model Constructs}

In any complex empirical research, it is important to carefully develop construct measures based on a range of considerations. The following sub-sections discuss each of the sub-constructs of the full model, and describes the theoretical and practical aspects behind the development of appropriate measures to 'operationalize' them. In each case, it will be explained how the indicator measures were developed and grounded in the literature. Note that in some cases judgment was used to tailor or adapt the specific wording of the construct measures (i.e. survey instrument questions) to improve their readability for the target respondents and suitability in the context of research objectives. This was based on four considerations: i.) preliminary research findings (i.e. from IAEA meetings and NPP station visits and the literature review) ii.) the author's personal nuclear industry experience, iii.) peer review from NPP managers, and iv.) input from nuclear industry KM experts at the IAEA.

\subsubsection{NPP Organizational Performance}

IAEA Technical Series Report 437 states:

Generally, nuclear plant performance indicators, whether operational or economic, are not mutually exclusive. Nuclear plant managements must simultaneously take into account a number of performance measures to ensure their safe, reliable and economic operation. In viewing nuclear power plant performance, not only is there a strong dependence between individual 
performance variables but, in addition, good performance along one particular measure is often correlated with good performance in most other key measures of success.

Recognizing this limitation, NPP organizational performance has been operationalized as two distinct constructs: organizational effectiveness and operational performance. The following sections explain the organizational effectiveness and operational performance constructs in further detail. They are both measures of primary performance objectives of NPPs organizations.

\subsubsection{Organizational Effectiveness}

Considering the findings from IAEA (1999), organizational effectiveness can be defined against the following top level management objectives for NPPs: managing external interfaces (public, regulator, suppliers); managing the production of documentation; managing organizational change; managing projects; managing operations; managing maintenance; managing safety; managing regulatory compliance, managing environmental objectives, and managing financial objectives. Specific "organizational effectiveness" measures were developed for each of these management objectives, resulting in the following:

a. The organization has difficulty making operational changes smoothly and in a timely manner ${ }^{1}$.

${ }^{1}$ Note: the measure has been intentionally worded as a negative statement to minimize the effect of positive response bias (Kuethe, 1959) and will be reverse coded. 
b. Projects involving multiple departments are typically behind schedule, over-budget, and not well coordinated ${ }^{1}$.

c. Safety objectives are consistently met or exceeded.

d. Quality of documentation (i.e. design, work-process and procedural documentation) needs to improve ${ }^{1}$.

e. Weekly operations objectives are regularly not met ${ }^{1}$.

f. The organization is effective at managing its external interfaces (i.e. the regulator, public, suppliers, contractors)

g. Environmental objectives are sometimes not met $^{1}$.

h. Maintenance objectives (e.g. level of corrective and preventive maintenance backlog) based on industry best practice are consistently met or exceeded.

i. Financial objectives are often not met $^{1}$.

j. Regulatory objectives are consistently met or exceeded.

A research study released by the Institute of Nuclear Power Operators (INPO, 2006) investigated the fundamentals for NPP operations, engineering, maintenance, radiological protection, chemistry, and training. The term "fundamentals" was defined in the document as the essential knowledge, skills, behaviors, and practices personnel need to apply to conduct their work effectively and properly and they are seen to be the foundation of successful NPP performance. In the INPO study a total of 33 NPPs were reviewed to identify strengths and weaknesses, in fundamentals. Considering this, and

${ }^{1}$ Note: the measure has been intentionally worded as a negative statement to minimize the effect of positive response bias (Kuethe, 1959) and will be reverse coded. 
the findings from a review of the Standard Nuclear Performance Model (SNPM) (NEI, 2003), the following high-level performance measures have been defined as additional organizational effectiveness measures that focus specifically on NPP operational effectiveness:

k. Maintenance technicians consistently conduct high-quality corrective and preventive maintenance.

1. The ratio of corrective to preventive maintenance is high relative to best performing NPPs of similar design ${ }^{1}$.

m. The plant chemistry program ensures the plant consistently operates within the chemistry specifications.

n. System and/or performance analysis engineers are not effective at resolving problems that affect plant safety or performance ${ }^{1}$.

o. Radiological conditions are effectively controlled (i.e. field levels are as low as reasonably achievable and dose control is effective).

p. Operators effectively act on changing plant conditions to ensure ongoing safe and reliable plant operation.

q. Recurrence of known and avoidable operational problems is not always prevented1.

r. Work planning and management is effective (e.g. planned work-scope is stable, little time is wasted waiting on approvals or parts)

${ }^{1}$ Note: the measure has been intentionally worded as a negative statement to minimize the effect of positive response bias (Kuethe, 1959) and will be reverse coded. 
s. The average number of critical component failures per year is low relative to other similar plants.

t. System health improvement initiatives are effective.

u. Corrective and preventive maintenance and outage work is completed on schedule and in a timely manner.

v. Financial resources (budgets) are adequate and allocated wisely.

Items are measured using a 5-point Likert scale where 1 indicates strongly disagree and 5 indicates strongly agree. An additional check-box is provided to for respondents to indicate "unable to rate". Item sequence has be re-ordered on the actual survey.

\subsubsection{Operational Performance}

WANO started tracking NPP performance in 1991 based on a set of simple but key quantitative overall plant performance indicators, each a measure of operational "efficiency". It is now recognized that the WANO performance indicators provide an excellent measure of how well a NPP is managed overall (WANO, 2005). WANO claims that as of 2005 reporting year, 97\% of operating NPPs reported on their indicators. These indicators are widely accepted and reported within the WANO membership, but are considered confidential and are not published other than in an aggregated manner. They are available primarily for the use of WANO members. WANO's indicators are well defined, fairly easy to derive, and accepted almost universally around the globe. They have been chosen for use in this study, however, only aggregated data can be reported. 
NPP stations that wish to respond anonymously were asked to provide the WANO performance indicators for their operations over the latest year reported (2009). For stations providing the name of their station, data from WANO was used directly. The WANO indicators (see Appendix E) used are:

a. Unit Capability Factor (WANO definition: the annual percentage of maximum energy generation that a plant is capable of supplying to the electrical grid, limited only by factors within control of plant management.)

b. Unplanned Capability Loss Factor (WANO definition: the percentage of maximum energy generation that a plant is not capable of supplying to the electrical grid because of unplanned energy losses, such as unplanned shutdowns or outage extensions. A low value indicates important plant equipment is well maintained and reliably operated and there are few outage extensions.)

c. Forced Loss Rate (WANO definition: is the percentage of energy generation during non-outage periods that a plant is not capable of supplying to the electrical grid because of unplanned energy losses, such as unplanned shutdown or load reductions. A low value indicates important plant equipment is well maintained and reliably operated.)

d. Unplanned Automatic SCRAMs ${ }^{1}$ per 7,000 Hours Critical (WANO definition: the mean scram (i.e. automatic shutdown) rate for approximately one year (i.e. 7000

${ }^{1}$ The term "SCRAM" refers to an unplanned actuation of the automatic safety systems to rapidly shutdown the reactor. To help remember its meaning the phase "safe core reactivity arrest made" is used. SCRAMs are often triggered spuriously resulting in loss of production. 
hours) of operation. Unplanned automatic scrams result in thermal and hydraulic transients that affect plant systems.)

The remaining WANO indicators are not standardized and are calculated differently for each reactor design and were deemed to be inappropriate for any valid statistical performance comparisons to be made across all NPPs and thus were not used: collective radiation exposure, industrial safety accident rate, safety system performance, fuel reliability, and chemistry performance.

The four WANO performance indicators used in this research focus on operational performance. In the "WANO Performance Indicator Programme Reference Manual" (2010) it states the indicators have been established on one or more of the following WANO criteria:

- The indicator provides a quantitative indication of nuclear safety, plant reliability, and plant efficiency or personnel safety.

- The indicators monitor fundamental results rather than the performance of intermediate processes or individual programme elements.

- The indicator has wide applicability.

- The indicator provides a meaningful perspective without a detailed knowledge of plant programmes and practices.

- The indicator is objective and fair.

- The indicator is amenable to goal setting.

- Data is available and reliable. 
- Indicators that primarily monitor plant reliability are chosen to reflect performance only in areas that can be controlled or influenced by plant management.

The four WANO indicators used are quantitative scale measures derived from actual plant performance. In addition, as a single event may significantly influence performance indicators in a given year, respondents are asked the following question:

a. Please indicate if a one-time extraordinary event affected these performance ratings. If so, please comment to clarify.

\subsubsection{Quality of Knowledge Processes}

The knowledge processes have been addressed widely in the literature and can be operationalized in many ways. For the purposes of the current research, an inclusive definition that captures the full breadth of the key knowledge process definitions (i.e. supported by the literature) was sought. The European Guide to Good Practice in Knowledge Management (CEN CWA 14924-1 2004) summarizes empirical research, practical experiences and the analysis of more than $150 \mathrm{KM}$ frameworks worldwide and states that this research has shown that there is strong agreement on the following five core knowledge processes. These are: knowledge identification; knowledge creation; knowledge sharing; knowledge use, and knowledge storage. Considering the findings from the review of the literature and the various terminology widely used to describe the main knowledge processes, the constructs used to describe these five core processes 
have been further clarified (broadened) in the following manner to better suit the objectives of this research:

- Knowledge identification is replaced with a wider scope construct of knowledge acquisition and adoption on the grounds that they are closely interrelated and the later two incorporate the former and are widely accepted and utilized in the literature (Grobelnik et al, 2005; Hornik and Ruf, 1997) and to fully capture the importance of bringing existing external knowledge into the organization on an ongoing basis.

- Similarly, knowledge creation is considered to be including both subcomponents: knowledge generation and validation processes (based on Jantunen, 2005; Holsapple et al., 2005). This recognizes the importance of validation processes in knowledge creation, which is particularly relevant in the nuclear plant operations context.

- Knowledge sharing is incorporated as part of the knowledge sharing and transfer construct and represents a wider and more general construct (based on Holsapple et al., 2005; Hyttinen and Rintala, 2005).

- Finally, the process of knowledge storage is replaced with the broader construct of knowledge retention and storage to reflect a greater emphasis on active memory and tacit knowledge (based in INPO, 2006) as opposed to storage of codification and explicit information only.

In summary, the sub-constructs used to comprise the primary knowledge processes (see Figure 41) are: knowledge acquisition and adoption (KA); knowledge generation and 
creation (KG); knowledge sharing and transfer (KS); knowledge utilization and application (KU); and knowledge retention and storage (KR). Thus the primary knowledge processes are represented in the research model as five interacting and intermediate sub-constructs, all well-established in the literature.

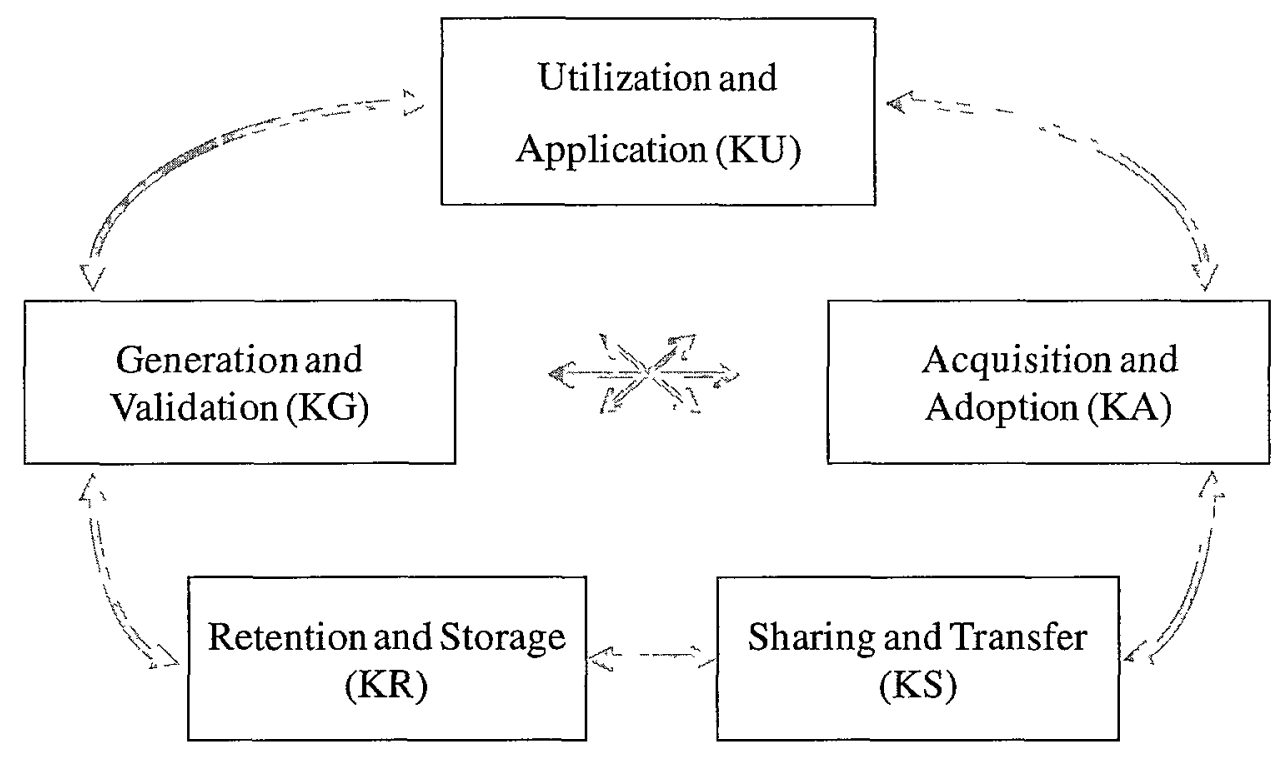

Figure 41. Primary Knowledge Process Sub-Constructs

Several authors have emphasized the link between knowledge processes and organizational learning and have argued that various quality attributes of knowledge processes in organizations are a key factor in organizational performance (Linderman et al., 2004; Elliot, 1998; Barber and Munive-Hernandez, 2006; Bose, 2004; Buyukozkan, 2004). Thus, the hypothesis that organizational performance in NPPs is directly influenced by the quality of knowledge processes (all measurable constructs).

The following sub-sections describe the measures used to operationalize each of the sub-constructs of "quality of knowledge processes", namely: knowledge acquisition and 
adoption (KA); knowledge generation and validation $(\mathrm{KG})$; knowledge sharing and transfer (KS); knowledge utilization and application (KU); and knowledge retention and storage (KR). The approach taken is justified on the bases of previous findings of Glazer (1991) and Jennex et al. (2008). First, Glazer (1991) concluded that the production and application of knowledge is contextual, its measurement should be based on the perceptions of those who use it. Therefore knowledge process measures are developed in the specific context of NPP organizations based on prior NPP research (IAEA 2006b, EPRI 2006) and on measures that best reflect the knowledge objectives

of NPPs. Secondly, that effectiveness is a valid measure of knowledge process quality (Jennex et al., 2008). The effectiveness measures are expressed in language and terminology that NPP managers can understand and interpret meaningfully in the context of the perceived organizational knowledge process requirements. The following sub-sections explain further the operationalization of the sub-constructs for each knowledge process quality dimension and the development of the specific measures used.

\subsubsection{Knowledge Acquisition and Adoption (KA)}

In order to operationalize the construct "quality of knowledge acquisition", the measures developed by Akgun et al. (2002) and Brockman and Morgan (2003) have been adapted. Respondents are asked indicate their level of agreement with each of the following statements about their organization: 
a. The organization has difficulty finding and hiring appropriately qualified graduates. 1

b. The organization excels at identifying and acquiring external technical information needed to operate and maintain the plant.

c. External information acquired is often not organized or stored in a maintainable and accessible way to facilitate use and re-use. ${ }^{1}$

Feedback from the trial survey and IAEA expert peer review also identified the following additional measures which were thought to be important indicators of knowledge acquisition process quality:

d. The organization is effective at acquiring knowledge from external (e.g. peer-plant) operating experiences.

e. The organization is highly effective at adopting external best practices.

f. The organization is good at capturing technical know-how and relevant design information related to services or products received from outside organizations.

Items are measured using a 5-point Likert scale where 1 indicates strongly disagree and 5 indicates strongly agree. An additional check-box is provided to for respondents to indicate "unable to rate".

${ }^{1}$ Note: the measure has been intentionally worded as a negative statement to minimize the effect of positive response bias (Kuethe, 1959) and will be reverse coded. 


\subsubsection{2. $\quad$ Knowledge Generation and Validation $(K G)$}

Holsapple and Singh (2003) describe knowledge generation (or creation) as an activity that produces knowledge by either discovering it or deriving it from existing knowledge, where the latter has resulted from acquisition, selection, and/or prior generation. Activities involved in creation of knowledge may include monitoring and evaluating selected or acquired knowledge in terms of its usability, producing knowledge from a base of existing knowledge by creating, synthesizing, analyzing, and constructing, and transferring the produced knowledge. Holsapple and Singh (2003) also describe knowledge derivation as the use of process knowledge (e.g. procedures and rules) and descriptive knowledge (e.g. data and information) to generate new process and/or descriptive knowledge by employing $\mathrm{KM}$ skills that are of an analytical, logical, and constructive nature. Although the result is "new" to the processor that derives it, it may have previously existed but not have been externalized or may already exist elsewhere in the organization. Finally, Holsapple and Singh (2003) describe knowledge discovery as a form of knowledge generation that is less structured than knowledge derivation and involves skills of creativity, imagination, experimentation, and/or synthesis. The construct "quality of knowledge generation and validation processes $(K G)$ " is intended to incorporate these elements and is operationalized with the following measures (adapted from IAEA 2006b, EPRI 2006):

a. NPP staff learn from operating experience and new and better ways of running the plant are seldom overlooked. 
b. Independent review processes are effective at validating proposed operational or design changes that may impact safety or production.

c. Employees lack the questioning attitude needed to challenge assumptions and investigate anomalies or uncertainties. ${ }^{1}$

d. Employees regularly create new innovative solutions by combining or adapting existing and/or (newly) acquired knowledge.

Feedback from the trial survey and IAEA expert peer review also identified the following additional measures which were thought to be important indicators of the quality of knowledge generation and validation processes:

e. The organization excels at generating, transforming, and presenting plant data as meaningful information.

f. Engineers have to spend too much time gathering and compiling data from many sources. ${ }^{1}$

Items are measured using a 5-point Likert scale where 1 indicates strongly disagree and 5 indicates strongly agree. An additional check-box is provided to for respondents to indicate "unable to rate".

\subsubsection{Knowledge Sharing and Transfer (KS)}

For the purposes of this research, the construct of knowledge transfer is considered closely inter-related with knowledge sharing and the later has been combined in a single

${ }^{1}$ Note: the measure has been intentionally worded as a negative statement to minimize the effect of positive response bias (Kuethe, 1959) and will be reverse coded. 
construct to represent the conveyance of tacit or explicit knowledge within the organization from one party to another, whether direct or indirect. The construct "quality of knowledge sharing and transfer" is based on measures adapted from IAEA (2006b) and operationalized with the following measures:

a. Findings, information, data, reports, or files generated in one area of the company are readily accessible to other areas.

b. Employees often do not know where in the organization to find specialized knowledge and information. ${ }^{1}$

c. The problem of hoarding (keeping) knowledge does not exist and employees willingly share their knowledge with co-workers.

d. Expertise and skills are not effectively transferred to junior staff from more experienced employees. ${ }^{1}$

e. Employees routinely and voluntarily share relevant information with other parts of the organization where it may be needed.

Items are measured using a 5-point Likert scale where 1 indicates strongly disagree and 5 indicates strongly agree. An additional check-box is provided to for respondents to indicate "unable to rate".

${ }^{1}$ Note: the measure has been intentionally worded as a negative statement to minimize the effect of positive response bias (Kuethe, 1959) and will be reverse coded. 


\subsubsection{Knowledge Utilization and Application (KU)}

The construct of "quality of knowledge utilization and application" is based on measures adapted from IAEA (2006b) and operationalized with the following measures:

a. Lessons learned from operating experience are incorporated in work practices, manuals, procedures and decision-making.

b. The organization is often not able to apply its knowledge effectively to solve difficult technical problems. ${ }^{1}$

c. Employees are consistently able to make important technical decisions correctly.

d. Employees are not always aware of and do not make effective use of each other's skills and expertise. ${ }^{1}$

e. Equipment replacement and design change decisions are based on a risk-informed decision process.

Items are measured using a 5-point Likert scale where 1 indicates strongly disagree and

5 indicates strongly agree. An additional check-box is provided to for respondents to indicate "unable to rate".

\subsubsection{Knowledge Retention and Storage (KR)}

The construct "quality of knowledge retention and storage" is based on measures adapted from IAEA (2006b) and is operationalized with the following measures:

\footnotetext{
${ }^{1}$ Note: the measure has been intentionally worded as a negative statement to minimize the effect of positive response bias (Kuethe, 1959) and will be reverse coded.
} 
a. Employees often lack an appropriate knowledge of the reactor and power plant fundamentals. $^{1}$

b. Employees have adequate knowledge/understanding of work processes (e.g. industrial and radiation safety work practices).

c. There is often a shortage of critical skills and experience due to unexpected departures and retirements. ${ }^{1}$

d. Plant design basis documents are easily located and are up-to-date and accurate.

e. Maintenance, operations, or technical support specialists lack adequate knowledge of specific systems and technologies to enable them to work effectively and safely.

Items are measured using a 5-point Likert scale where 1 indicates strongly disagree and 5 indicates strongly agree. An additional check-box is provided to for respondents to indicate "unable to rate".

\subsubsection{Support for Knowledge Management Practices}

This is a composite construct comprised of several sub-constructs as defined in the IAEA Guidelines (2006b, 2008), which provide a useful categorization of KM practices. Based on a review of the literature, and feedback from industry peers, these categories have been adapted to form a set of formative sub-constructs, each to measure a unique aspect of the composite factor, the "level of organizational support for KM practices". Each represents a set of measures of the level of support for the implementation (and

\footnotetext{
${ }^{1}$ Note: the measure has been intentionally worded as a negative statement to minimize the effect of positive response bias (Kuethe, 1959) and will be reverse coded.
} 
ongoing execution) of a set of practices associated with a thematic topic. These specific actions that are known to influence the quality of knowledge processes in their organizations. The following sub-sections describe the measures adapted for each of these constructs.

\subsubsection{Support for KM Strategy and Planning}

The sub-construct "support for KM strategy and planning" is operationalized in the following manner. Respondents are asked to indicate their level of agreement with each of the following statements about their organization:

a. The organization has a clear, documented high level knowledge management plan and goals.

b. Implementation of the knowledge management strategy is openly and actively supported by management.

c. Knowledge management roles and responsibilities are clearly defined and understood by managers and employees.

d. Other management strategies (e.g. human resources, information systems, operations, communications and maintenance plans) are closely aligned with the knowledge management strategy and plan.

e. The needs and gaps in the organizational knowledge base are periodically reviewed and the knowledge management strategy and plan is revised to address them 
Items are measured using a 5-point Likert scale where 1 indicates strongly disagree and 5 indicates strongly agree. An additional check-box is provided to for respondents to indicate "unable to rate".

\subsubsection{Support for Organizational Learning}

The sub-construct "support for $O L$ " is operationalized in the following manner. Respondents are asked to indicate their level of agreement with each of the following statements about their organization:

a. Knowledge creation and application (e.g., finding better methods, technology innovation) is encouraged, recognized and rewarded.

b. Sharing of knowledge is promoted and rewarded (e.g., experts are encouraged and rewarded to coach or mentor other employees).

c. Open communication and a no-blame approach to reporting problems and sharing lessons learned are promoted (e.g., regular communication is encouraged between maintenance and operations personnel).

d. Learning opportunities are encouraged (e.g., joining specialist groups or attending training seminars).

Items are measured using a 5-point Likert scale where 1 indicates strongly disagree and

5 indicates strongly agree. An additional check-box is provided to for respondents to indicate "unable to rate". 
Feedback from the trial survey, participation in industry workshops and the IAEA expert peer review also strongly identified the importance of nuclear industry "communities of practice" (COPs) as a key tool to improve organizational learning. Participation in COPs by NPPs is believed to be widely practiced in almost all countries having nuclear power production and in all the major key result areas important to nuclear plant safety and production performance. According to NEI (2003), the following 18 COPs are considered the important key result areas consistent with the main processes and sub-processes of SNPM model (NEI, 2003):
a. Equipment Reliability
b. Materials and Services (supply chain)
c. Information Technology
d. Business Services / Nuclear Asset Management
e. Information Management
f. Licensing / Regulatory Issues
g. Human Resources
h. Radiation Protection
i. Nuclear Fuel
j. Performance Monitoring / Improvement
k. Plant Operations
1. Chemistry Management
m. Work Management
n. Simulators 
o. Training

p. Cost Estimation and Management

q. Configuration Management

r. Fire Protection

Thus two indicators to measure organizational support for knowledge acquisition were included: the extent of NPP participation in COPs (i.e. measured as the total number of COP groups the NPPs is participating in); and the extent of internal benchmarking based on COP indicators practiced at the NPP (i.e. measured as the total number of COP groups the NPP is actively benchmarking against). The following question was asked for each of COPs listed above:

a. Please indicate which communities of practice (COP) your station organization participates in and whether regular self-assessment is done against the performance indicators or benchmarks from that COP group.

An additional question, "other COPs" was also asked in case any significant other COP groups exist. Finally, to gain additional insight into the nature and extent of COP group participation, respondents were asked:

b. Please also indicate whether COP participations include: local (e.g. national), regional (e.g. European), international (e.g. IAEA, EPRI, INPO or NEI), or Owner's Group based COPs. 


\subsubsection{Support for Process Management Practices}

The sub-construct "support for process management practices" is operationalized in the following manner. Respondents are asked to indicate their level of agreement with each of the following statements about their organization:

c. For all processes and procedures, priority is placed on ensuring the requirements, methods, inputs, outputs, interfaces, responsibilities, and workflow are documented correctly and maintained up to date.

d. Consideration of hazards and risk is built into all work and decision processes to ensure safety is not adversely impacted.

e. Procedures are aligned to knowledge and information requirements of both work tasks and decision processes

f. A process to measure and improve the quality and control of all business, work, and decision processes is defined and followed.

g. Comprehensive knowledge management procedures (e.g. for knowledge loss risk assessment) are documented and in use.

h. Knowledge management processes and procedures are extended to suppliers and technical support organizations

Items are measured using a 5-point Likert scale where 1 indicates strongly disagree and

5 indicates strongly agree. An additional check-box is provided to for respondents to indicate "unable to rate". 


\subsubsection{Support for Information Management Practices}

The sub-construct "support for IM practices" is focused on the non technologydependent aspects of information management. It is operationalized in the following manner. Respondents are asked to indicate their level of agreement with each of the following statements about their organization:

a. Licensing documents, design basis documents, procedures, specifications, drawings, and training materials are updated promptly to address plant changes and are maintained under configuration management.

b. Records, data, and logs are required to be complete, meaningful, accurate and accessible (e.g., logs, minutes, test results).

c. Data standards, metadata, document codes, subject indexes and filing systems are widely used to enable efficient information correlation, storage and retrieval.

d. Procedures ensure the needs for data and information safety, security, maintainability, accessibility, quality and preservation.

Items are measured using a 5-point Likert scale where 1 indicates strongly disagree and 5 indicates strongly agree. An additional check-box is provided to for respondents to indicate "unable to rate". 


\subsubsection{Support for Organizational Performance Management Practices}

The sub-construct "support for organizational performance management practices" is operationalized in the following manner. Respondents are asked to indicate their level of agreement with each of the following statements about their organization:

a. Independent external peer review assessments are conducted regularly (e.g. WANO, INPO, or IAEA-OSART reviews).

b. Self-assessments are widely used to stimulate learning and improve performance (e.g. benchmarking against best practices).

c. Performance objectives are established and monitored for all levels and areas of the organization (including for knowledge processes).

d. Performance objectives for operations, maintenance, and safety are based on objectives established by industry best practice.

e. The effectiveness of the management system (including knowledge management aspects) is regularly reviewed.

f. Ongoing processes for operational experience capture, review, analysis and corrective action are defined and followed.

Items are measured using a 5-point Likert scale where 1 indicates strongly disagree and

5 indicates strongly agree. An additional check-box is provided to for respondents to indicate "unable to rate". 


\subsubsection{Support for Training Related Practices}

The sub-construct "support for training related practices" is operationalized in the following manner. Respondents are asked to indicate their level of agreement with each of the following statements about their organization:

a. The organization incorporates principles of the "systematic approach to training" (SAT) in training programs.

b. Sufficient training is provided to achieve and maintain the required level of competence for all job positions.

c. Training material is reviewed to ensure it reflects lessons learning from operating experience and agrees with plant documentation.

d. Collaboration with universities and colleges ensures an appropriate supply of new graduates.

e. Other techniques are used for training (e.g. story-telling, concept mapping, pre-job briefings, informal seminars, mentoring programs etc.). Please specify

Items are measured using a 5-point Likert scale where 1 indicates strongly disagree and 5 indicates strongly agree. An additional check-box is provided to for respondents to indicate "unable to rate". 


\subsubsection{Support for Human Resource Related Practices}

The sub-construct "support for HR related practices" is operationalized in the following manner. Respondents are asked to indicate their level of agreement with each of the following statements about their organization:

a. Expected retirements and unexpected departures are regularly tracked and the resulting need for and availability of critical knowledge and job skills is acted upon.

b. New hiring is done long before experts depart to facilitate knowledge transfer and ensure the competency of replacements is developed in time.

c. Interviews with departing employees are routinely carried out well in advance to identify critical knowledge and experience and to facilitate knowledge capture and transfer.

d. Competency, training and knowledge sharing or transfer goals are identified, evaluated and rewarded in employee performance assessment.

e. Work assignments promote learning (e.g., job-rotations, team selections and staff assignments consider learning opportunities).

Items are measured using a 5-point Likert scale where 1 indicates strongly disagree and 5 indicates strongly agree. An additional check-box is provided for respondents to indicate "unable to rate". 


\subsubsection{Technology Support}

The construct "technology support" measures the extent to which IS/IT solutions and the advanced support systems (i.e. software solutions that support complex operational decision processes) effectively support the organization's needs (relative to NPP best practices). "Extent of use" measures, when applied to information systems technology are not as relevant as "effectiveness" measures when considering the impact on knowledge processes and organizational performance (DeLone and McLean, 1992). To indicate the level of organizational support for technology in NPPs, the two separate and identifiable sub-constructs discussed earlier are measured. These are:

- information systems and technology support (or "IT/IS support"), and

- support for advanced operational support systems (or "Advanced OSS support").

Both constructs are operationalized based on effectiveness measures of technology or system implementations considered most important or relevant to NPPs. These systems have been identified from previous comprehensive nuclear industry research (see IAEA 2006b, EPRI, 2006) and based on the findings that they are considered most important to supporting the informational needs of the organization. The sub-construct "information systems and technology support" is based on eight measures and is operationalized in the following manner. Respondents are asked to indicate how effectively each of the following technologies is used in their organization (i.e. with respect to organizational needs and relative to NPP norms):

a. Three dimensional (3D) virtual reality environments for training. 
b. Computer and/or web-based training.

c. Desktop (e.g. plant) training simulators.

d. Full scope main control room training simulators.

e. Electronic archives and databases (e.g. for document management, event reporting, maintenance records, etc.)

f. Enterprise application software (e.g. for financials, procurement, inventory management, work and outage management, etc.)

g. Intranet web portal with search/retrieval access to frequently used resources (e.g. documents, bulletins, contact lists, etc.)

h. Three-dimensional (3D) computer aided design (CAD) plant models and editable electronic drawings.

Similarly, the sub sub-construct "advanced operational support systems" has been based on the same IAEA and EPRI nuclear industry research findings. The construct is based on seven effectiveness measures and is operationalized in the following manner. Respondents are asked to indicate how effectively each of the following technologies is used in their organization (i.e. relative to NPP best practices):

a. Operational decision support systems (e.g. refuelling software)

b. Regularly updated (i.e. "living") probabilistic risk models of equipment reliability for maintenance and outage planning.

c. Real-time probabilistic risk models for operator evaluation and awareness of plant safety (i.e. "a safety monitor"). 
d. System health monitors (e.g. predictive maintenance tools such as vibration, acoustic, thermal, or other monitors).

e. Advanced model-based monitoring and diagnostics (e.g. physics, chemistry, boiler, feed water and thermal hydraulics models).

f. Advanced information exchange (e.g. hand-held computers, plant-wide equipment status monitoring, wireless communications).

g. Electronic (i.e. graphical) road-maps of business and decision processes or workflows (e.g. operational flow-sheets) with links to supporting procedures, related resources or documents.

h. Automated field data collection (i.e., smart instruments, field-bus, radio frequency identification (RFID) tagging, data logging, equipment monitors).

Items are measured for both sub-constructs using a 5-point Likert scale with the following definitions: "very effectively"; "effectively"; "somewhat effectively"; "not effectively"; and "not used at all". An additional check-box is provided for respondents to indicate "unable to rate".

\subsubsection{Supportive Organizational Culture}

Findings from prior research (IAEA, 2006b; IAEA 1999) indicate that organizational culture is a key factor influencing knowledge processes and ultimately the performance of NPPs. In the context of the research model, the level to which the organization has a "supportive organizational culture" is hypothesized to be a moderating variable, influencing both the effect that support for $K M$ practices has on the quality of 
knowledge processes, and the relationship between knowledge process quality and organizational performance. Recall that Sashkin and Rosenbach (1996) argued organizational culture centered around four key issues:

1. adaptation (i.e., how people deal with external forces and the need to change);

2. goal achievement (i.e., the nature of organizational goals, how they are defined, and their importance);

3. coordination (i.e., how people work together to get the job done); and

4. shared values and beliefs (i.e., the degree to which people in the organization agree that these values and beliefs are important and should guide their actions.)

For the purposes of this study, adaptation and shared values and beliefs are all seen to be more important, however, some specific additional dimensions introduced by O'Reilly et al. (1991) are thought to be missing. They described eight factors that define the key characteristics of an organizational culture:

1. Innovation and risk taking

2. Attention to detail

3. Orientation towards outcomes or results (outcome orientation)

4. Aggressiveness and competitiveness

5. Supportiveness

6. Emphasis on Growth and rewards

7. Collaborative and team orientation

8. Decisiveness 
Their research indicated these eight orthogonal factors are unambiguously defined, and in general, they approximate many of the dimensions to which the qualitative literature on culture has referred. Of these, innovation and risk taking and emphasis on growth and rewards (both similar to and considered part of adaptation), outcome orientation, collaborative and team orientation are seen to be distinct and in agreement with the literature review findings, and can be used as a basis for the development of appropriate measures. Attention to detail is part of outcome orientation. Finally, safety orientation (i.e. safety culture) is seen as an additional missing dimension, and although closely tied to and overlapping organizational culture, has its own distinct aspects and was included on the grounds that it cannot be ignored given its importance in the context of the present study. The WANO Guideline "Principles for a Strong Nuclear Safety Culture" describes the following principles as key to a strong nuclear safety culture:

1. Everyone is personally responsible for nuclear safety.

2. Leaders demonstrate commitment to safety.

3. Trust permeates the organization.

4. Decision-making reflects safety first.

5. Nuclear technology is recognized as special and unique.

6. A questioning attitude is cultivated.

7. Organizational learning is embraced.

8. Nuclear safety undergoes constant examination. 
The dimensions of organizational culture were also researched in research by Detert et al (2000). They reviewed the literature and confirmed empirically there is strong support for the following 8 dimensions of organizational culture (summary from Jones et al., 2006):

1. Orientation to change (stability vs. change). Extent to which organizations have a propensity to maintain a stable level of performance that is good enough' or a propensity to seek to always do better through innovation and change.

2. Control, coordination, and responsibility (concentrated vs. autonomous decision making). Extent to which organizations have decision making structures centered around a few vs. decision making structures centered around dissemination of decision making responsibilities throughout the organization.

3. Orientation to collaboration (isolation vs. collaboration). Extent to which organizations encourage collaboration among individuals and across tasks or encourage individual efforts over team-based efforts.

4. Basis of truth and rationality (hard data vs. personal experience). Extent to which organizations seek truth through systemic, scientific study using hard data or through personal experience and intuition.

5. Motivation (external vs. internal). Extent to which organizations deem that individuals are motivated by an internal desire to perform well or by external rewards and encouragement. 
6. Orientation to work (process vs. results). Extent to which individuals in organizations focus on work as an end (results) or to which they focus on the process by which work is done as a means to achieve other ends.

7. Orientation and focus (internal vs. external). Extent to which organizational improvements are driven by a focus on internal process improvements or by external stakeholder desires.

8. Nature of time horizon (short term vs. long term). Extent to which organizations focus on the long-term or the short-term.

Of these, and based on the literature review in the NPP context, the following are considered to be more important to NPP operational objectives and were included: orientation to change (similar to adaptation), control coordination and responsibility, orientation to collaboration, basis of truth and rationality, orientation to work, orientation and focus, and nature of time horizon. Motivation (as defined) is seen to be a product of the other factors and thus not seen to be a significant differentiator between NPPs and was not included as a measure. Considering these perspectives, the construct to measure supportive culture was based on the WANO Guide (2006), with specific wording of the measures adapted as appropriate. The following are the measures were adapted to address each of the above identified cultural dimensions (except goal achievement and aggressiveness, as these were not included). Note that some are negatively worded to reduce self-report bias and are reverse-coded.

Adaptation / Orientation to Change: 
a. Managers and employees often do not see learning, innovation, and improvement as a part of their jobs ${ }^{1}$.

b. Employees who innovate feel recognized and rewarded.

Attention to Detail / Outcome-orientation / Orientation to work (process vs. results).

c. There is a prevailing attitude and commitment to follow defined processes and fully comply with procedures.

Control, coordination, and responsibility

d. Employees often do not feel empowered to make decisions appropriate to their job duties ${ }^{1}$.

Shared Values and Beliefs:

e. There is shared vision, purpose, and expectations among employees and they see all their problems as mutual.

Supportiveness:

f. People are seen as the organisation's most valued asset.

g. Employees and managers are open-minded and respect each other's opinions and contributions.

Collaborative and Team Orientation / Orientation to collaboration:

h. There is a team-oriented approach throughout the station (e.g., employees trust, cooperate, and help each other).

Orientation and focus (internal vs. external)

${ }^{1}$ Note: the measure has been intentionally worded as a negative statement to minimize the effect of positive response bias (Kuethe, 1959) and will be reverse coded. 
i. Employees often do not feel responsible for plant performance and fail to demonstrate their commitment to it ${ }^{1}$.

j. Improvements are mostly driven by externally imposed requirements (e.g. regulatory rulings, owner influences) ${ }^{1}$.

Safety Orientation / Demonstrated Commitment to Safety:

k. Consideration of safety is clearly evident in employee and management actions and decisions.

Basis of truth and rationality / Attention to detail.

1. A questioning attitude is cultivated (i.e. information, approaches and decisions are carefully scrutinized).

Nature of time horizon (short term vs. long term):

$\mathrm{m}$. The organization is focused primarily on short-term goals ${ }^{1}$.

\subsubsection{Other Measurement Considerations}

Feedback from the IAEA expert peer review and observations during the IAEA workshops and station assist visits indicated that staffing levels, extent of use of contractors (both at power and during outages), and the educational demographics of the workforce may also be possible indicators of organizational effectiveness and correlate with plant performance. There were conflicting opinions on this topic and little prior research appears to have been done in the context of NPPs. There are known to be

${ }^{1}$ Note: the measure has been intentionally worded as a negative statement to minimize the effect of positive response bias (Kuethe, 1959) and will be reverse coded. 
widely varying practices associated with outage management and the use of contractors. There are also known to be widely varying staffing levels in different NPP sites. Finally, the influence of higher or lower proportions of engineering and/or applied science program trained university graduates on an NPP station's staff versus technical college diplomas or trades persons is another debated topic. As an exploratory research, the following demographic measures where included:

a. Please indicate the number of employees (excluding contractors) at your station.

b. Please indicate the typical number of full-time equivalent contractors during outages.

c. Please indicate the typical number of full-time equivalent contractors while at power.

d. Please indicate the percentage of employees with university degrees at your station.

Some basic demographic questions which were thought to be useful were also included for those respondents that wished to reply anonymously (i.e. choosing not to identify their NPP station). Respondents were asked to:

a. indicate the country your station is located in

b. indicate the number of operational units (i.e. power reactors) at your station

c. indicate the type of reactor (e.g. PWR, BWR, PHWR, LWCGR, or GCR etc.):

d. indicate the plant model (i.e. product) name (e.g. EPR, AP1000, VVER 440, etc.):

e. indicate the approximate age of each unit at their station (measured in years from completion of construction). 
Finally, stations willing to do so were asked to identify the name of their plant and to provide a contact number (in case any follow-up clarifications were needed) and to indicate the number of operations managers who helped complete this survey response. They were also invited to make any additional comments on or clarifications of their responses in a few blank lines for free text.

\subsection{Summary}

Chapter 3 has provided a summary of the research model and it's independent, intermediate, and dependent variables. The survey measurement instrument used is provided in English, Chinese, Russian, and French versions in Appendix A through D respectively. 


\section{Research Methodology}

This section describes the research methodology followed in this study. The research was based on a formal empirical study. The development of the empirical measures and the data collection procedure of the research are discussed in detail. The sampling frame and the data analysis methods will also be described.

\subsection{Research Design}

This research involves a two-step approach. First, preliminary research has been conducted both as a participant in several IAEA NPP workshops and site visit meetings on $\mathrm{KM}$ and a formal literature review has been completed and summarized in Chapter 2. Based on the findings of this preliminary research, a formal survey instrument (using mostly qualitative perception measures) was developed and administered to the global community of NPP organizations (through the cooperation of the IAEA).

\subsubsection{Background Research via Industry Participation}

The IAEA has a well-developed program to promote $\mathrm{KM}$ best practices in the nuclear industry. As part of the IAEA's series of KM meetings, conferences and workshops, the author was able to personally participate in the following (all of which allowed direct

interaction with NPP managers on in-depth presentations, forums, and discussions of KM issues related to NPPs): 
- IAEA Utility Workshop on Nuclear Knowledge Management (Trieste, Aug 2005)

- IAEA Meeting for the Development of Guidance on the Implementation of KM in NPPs (Vienna, Oct, 2005)

- IAEA Meetings on the Methods and Tools for Nuclear Knowledge Preservation (Vienna, Oct. 2006, Oct. 2007, and Oct. 2008).

- IAEA School of Nuclear Knowledge Management (Trieste, Sept 2006).

- IAEA Workshop with Ignalina NPP managers on implementing KM, Ignalina Lithuania (Mar, 2007)

- IAEA Workshop with Bruce Power NPP managers on implementing KM, Canada (April, 2007)

- IAEA Workshop with Ontario Power Generation Darlington NPP mangers on implementing KM, Canada (April, 2007)

- IAEA International Conference on Knowledge Management in Nuclear Utilities (Vienna, June 2007).

- Regional Workshop on Nuclear KM for Nuclear Utilities (Obninsk, June, 2007).

- Meeting of Senior Nuclear Industry Officials on Knowledge Management (Vienna, 2008).

- IAEA Meeting with the Canadian Nuclear Safety Commission (Ottawa, May 2008)

- IAEA Workshop with Zaparosze NPP mangers on implementing KM, Energator Ukraine (June, 2008). 
- IAEA-NEI-EPRI-DOE Joint Workshop on Process-Oriented KM (Rockville, Jul. 2008)

- ANS Utility Working Conference on KM (Amelia Island, Aug. 2008).

- IAEA Workshop on the establishment of a Nuclear KM Community of Practice (Oct, 2008)

- Meeting to discuss KM System implementation at Leibstadt NPP in Switzerland (Oct., 2008)

- IAEA, AECL, and COG Joint Utility Workshop on KM Methods and Tools (Toronto, Sep. 2009)

- IAEA Assist Visit to Chalk River Nuclear Labs (Chalk River, September, 2009).

- ANS Utility Working Conference - Track on KM (Amelia Island, Aug. 2010).

- $2^{\text {nd }}$ IAEA Meeting with the Canadian Nuclear Safety Commission (Toronto, Sept. 2010)

Involvement in the IAEA meetings afforded a unique opportunity to hear directly from NPP managers on the perceptions of KM issues in their organizations. It provided exposure to NPP managers from a wide cross-section of the industry, and a chance to gather their opinions, insights and experiences. This was helpful in the development of the quantitative research instrument. The next section will describe how the survey instrument was developed. 


\subsubsection{Empirical Survey}

Quantitative research approaches are in general based on statistical methods and are used to understand and characterize specific aspects or phenomena of the object of study. The development of a research instrument is a key part of the research process and may include a variety of measurement tools and techniques (Patton, 1990). Survey methodology was chosen in this study as the preferred data collection approach due to its versatility and general acceptance as a practical and proven technique for measuring perceptions of organizational learning (McGee and Prusak, 1993). The survey employed Likert-type scales on the grounds that the technique is well established in social sciences research and has been used in many empirical management studies including studies specific to the measurement of management perceptions of knowledge processes (e.g. Hitt and Ireland, 1997, Mowka and Vardarajan, 1990, Malhotra, 1997). Albaum (1997) conducted research on the measurement validity of various forms of Likert scales. In his study, he highlights the following:

- although a number of different types of scales or scale formats, including the semantic differential, Stapel scale, Likert scale, Thurstone differential scale, and direct rating scales exits, only one of them is most widely used, the Likert scale.

- when a Likert scale is used to measure attitude, the standard format consists of a series of statements to which a respondent is to indicate a degree of agreement or disagreement using the following options (or equivalent): strongly agree, agree, neither agree nor disagree, disagree, strongly disagree. 
- the scale is intended to measure direction and intensity of attitude.

- the scale is often used by researchers as a summated scale based on a small number of scale items (as few as two or three).

- as little as two or three categories can be used. Such scales are sufficient to meet criteria of test-retest reliability, concurrent validity, and predictive validity.

- a neutral response position can (and should) be provided.

Albaum (1997) points out that "Likert scales are used typically in social sciences research and are not ratio or even interval scales. They are ordinal only. For example, we cannot state the interval between "strongly agree" and "somewhat agree" is equivalent to the interval between "somewhat agree" and "neither agree nor disagree". Nor can we establish with any certainty that there is a zero point on such as scale. However, Likert scales are none-the-less used widely based on the assumption that we can treat these ordinal measures as though they were interval data. Statistical procedures are then applied (i.e. based on the assumption of normality of the data) and reasonable conclusions can be drawn from the inferences made".

Special care was taken in designing the measures such that the assumption of consistent scale intervals is preserved in the survey scale (i.e. wording) design. Note that several authors recommend a 7-point (minimum) or higher Likert scale be used to improve reliability and validity of the measure, however, when formative constructs are operationalized by combining multiple (i.e. 3 or more) indicators into a composite 
index, five-point scales are usually considered more than adequate (Neuman and Robson, 2008).

The factors (sub-constructs) proposed in the research model are based on summated scales by combining individual indicators into a single composite measure and taking the average score. Summated scales are a widely used technique and provide both a means of overcoming (i.e. reducing to some extent) the measurement error inherent in all measured variables and (when properly constructed) the ability to represent multiple aspects of a construct in a single measure (Hair et al., 2006). Any measures having negative correlations have been reverse-scored to avoid canceling out effects in the summated scale.

\subsubsection{Sampling Frame}

The empirical survey is specifically focused on NPPs and therefore the unit of analysis is the NPP station organization. This requires some clarification in that the definition of "NPP station organization" could be construed differently in cases where a parent organization or utility owns several nuclear plants. The focus of the survey sample was

directed specifically at each organization located at the nuclear power plant installation (i.e. the station "site organization"). Some NPP sites operate multiple nuclear plants, in which case the focus of interest is the aggregate site organization operating all the NPP units. In a few special cases (likely no more than 3 or 4), there is more than one station at a site (e.g. Bruce NPP has an A and B station). This may have occurred because the 
stations were built at different times and in some cases may even be different reactor types. These special cases were considered based on the whether or not a single common operational management organizational structure operates both stations, or whether they operate as independent organizational entities.

Due to the range of issues covered by the survey measures, a high level of organizational understanding is required to complete the survey meaningfully. Also, it was expected that many of the stations responding would have multiple individuals at the station assisting in completing the survey due to the range of questions involved. For this reason, the completion of the survey assumes a depth of knowledge about the organization that would only be found in very experienced senior operational managers who are well embedded in the organization and understand it. This level of understanding is, out of necessity, the norm for Plant Operating Manager (and similar senior operations manager) positions.

Therefore, the survey instrument was intended to be completed by senior operations managers at each station (e.g. Chief Nuclear Officer, Plant manager, Vice-President of Operations, Station Technical Manager, Director of Operations and Maintenance, or people in similar positions) who have a solid understanding of organizational practices and operational performance management processes, and who can solicit input from various departments or subordinates as needed to complete particular sections. It is recognized at the outset that soliciting survey responses from senior operations management positions would be a challenge and it would be difficult to get a high return 
rate. Access to this population is extremely difficult. For security reasons, the names and contact information for senior plant managers is often not published. These individuals are also extremely busy managers and it was expected that getting them interested and motivated to respond to a detailed survey would be a challenge. Thus the role of the IAEA as administer of the survey was quite important to the research success. It was recognized that NPP organizations would lend more credibility and recognition to the request if it was administered through the IAEA and this would significantly improve response rates. According to officials at the IAEA, an empirical survey of NPPs of this nature has not been previously attempted and there is strong interest in the research findings. Most NPP site organizations operate typically 1 to 4 reactor units, with a small percentage being in the range of 5 to 8 units, and the average being approximately 2.2 reactors per site. As there are 437 operating units at this time, the total population of "site organizations" is 204 .

\subsubsection{Survey Instrument Development}

There are many excellent guidelines published on the techniques and approaches to good survey design (e.g., Converse and Presser, 1986; DeMaio, 1983; Fowler, 1995; Gower, 1994; Platek et al., 1985; Schuman and Presser, 1996). The survey instrument has been developed along the principles set forth in these guides, and to the extent practical, however, the survey development has been based primarily on the methodology proposed by Moore and Benbasat (1991) which involves the following steps: operationalization of the constructs based on theory; developing and pre-testing 
an initial version of the instrument on knowledgeable managers and academics; revision of the questionnaire to address any feedback; and preparation of the survey kit (including cover letter to administer the survey).

Unit of analysis is each operating NPP station organization. There are anywhere from one to 8 operating reactors at an individual NPP station. However, there is typically one organization or "plant site operation", whether a single or multi-unit station, and organizational governance, accountabilities, policies, processes, and procedures are common across the organization. The intended sampling frame is therefore the entire global population of NPP station (site) organizations. Thus, despite the reactor performance data being available on a reactor basis, the survey was designed to solicit a response from each site organization. An invitation was sent via the IAEA to participate in the survey to all of the world's operating NPP stations. This population is well defined and finite (204 site organizations). The sampling element was an individual, the Station or Plant Manager (or his/her delegates), the target respondent of the survey.

As per survey design guidance of Bailey (1982), the following considerations were taken into account; anticipation of the sources of error, relevance of the study, relevance of individual questions to the study, relevance of questions to the respondents, questions with multiple answers or interpretations, ambiguous questions, level of wording, abstract vs. concrete questions, biased vs. neutral questions, sequencing of questions, and finally sensitive or threatening questions. Bailey also recommends at least one 
open-ended question be included in all surveys to allow respondents to comment on anything they feel was omitted. This was also done.

\subsubsection{Trial Survey}

The trial version of the survey was distributed by the IAEA to 15 NPPs specifically selected as "cooperative" stations with known contacts who had been recently involved in one of the IAEA's KM initiatives. A total of 8 responses were received (53.3\%), all of them usable. Response data was compiled and histograms were plotted for each of the measures and reasonably good "dispersion" of the responses was observed. This provided some confidence that each measure would provide adequate discrimination across a larger sample.

Direct feedback (via e-mail) on the survey was also solicited from these same 8 respondents and this also indicated the survey was well understood (i.e. the questions were clear) and the measures were deemed relevant. Several wording suggestions were received and some additional measures recommended. These changes were discussed and incorporated as appropriate during the IAEA expert peer review meeting which was held to review the trial survey and findings.

Once the full survey was conducted, it was possible to compare responses from the trial survey with responses from the full survey for the following stations: Darlington (Canada), Paks (Hungary), Daya Bay/Lingao (China), Ignalina (Lithuania), Kalinin (Russia), Entergy (USA), Susquahanna (USA), Temelin (Czech Republic), and 
Zaparosze (Ukraine). Table 15 (see Section 6.5, Reliability Screening of Measures) summarizes the results of the trial and final survey for these stations. Note that a total of 5 (multiple) responses were received from Daya Bay/Lingao NPP (a 4-unit station), and this included responses from the Maintenance Manager, Operations Manager, Plant Manager, Training Manager, and General Manager. This afforded a unique additional opportunity to confirm the reliability of measures (see Section 6.5, Reliability Screening of Measures).

\subsubsection{Peer Review of Survey Instrument}

Assessment of content validity was achieved by a panel of 8 nuclear industry experts assembled at an IAEA week-long workshop in June 2009. This meeting was expressly for the purpose of reviewing and validating the trial survey and findings prior to continuing with the full survey implementation. Participants included several ex-NPP managers and several IAEA and industry nuclear KM specialists. The survey questions (i.e. the indicator item measures for each construct) were carefully reviewed for:

- Meaningful measure and conceptual consistency with the construct

- Adequately captures the intent of the construct concept (scope, depth, completeness, etc)

- The possibility of misinterpretation of the question wording

- Willingness of respondents to reply to the question (i.e. sensitivity, confidentiality)

- Consistency and appropriateness of interpretation in various regions 
- Correct and consistent use of terminology, definitions

- Simplicity and clarity of wording

- Avoidance of repetition or overlapping in measures

- Likely range of response and calibration of wording (strength)

- Appropriate wording to minimize response bias

- Completeness of the construct (missing measures)

- Consistency with previous/existing measures

- Conflicts between practical (i.e. industry) versus theoretical (i.e. academic) literature meanings

- Adequate correspondence with and contribution to measuring the construct meaning

- Avoidance of overlap with other constructs being measured, elimination of repetition.

There was considerable discussion around the differences in organizational culture regionally. For example, it was generally expected that North American and European NPPs would be more likely to respond. It was also thought that in Russia, France and China, a local language version would increase response rates. Finally, it was recommended that any questions about external peer review ratings were too sensitive and confidential and should be removed from the survey. The following changes were recommended and made to the final version of the survey:

- The survey was translated into French, Russian, and Chinese. 
- The questions related to external peer review ratings were removed.

- A number of editorial wording improvements were made to the questions in the final version of the survey. This included some deliberate use of stronger or weaker adjectives on specific questions so the wording would "calibrate" the question appropriately to ensure a measurable range of responses was received and not just all 1's or 5's on the scale.

- Several new measures were added for construct completeness and strength.

- Two measures were reworded and relocated to other constructs for clarity and consistency.

\subsubsection{Translation Process}

The survey was translated from English into French, Russian, and Chinese so that it would be available in 4 major languages to increase the likelihood of response. An initial translation was made in each case, followed by a two step verification process: first an independent verification by a professional translator at the IAEA, then a less formal review by a native-language speaking nuclear industry manager (in each case) with knowledge of NPPs and knowledge management. This second step was quite useful to find many NPP/nuclear and KM -specific terms that the translators had not fully understood or miss-translated. Note that it was decided to complete the Chinese translation in a slightly different format that included both the English and the Chinese text provided for each question. This was done deliberately as the subject matter did not translate easily into Chinese and most station managers at Chinese NPPs speak English 
for technical and business reasons, so this was thought to be a way to improve comprehension and ensure correct interpretation.

\subsubsection{Validity and Reliability}

Schutt (1999) describes the three specific criteria needed to establish validity of quantitative research: measurement validity, generalizability, causal validity. He defines them as follows:

- measurement validity occurs when a measure actually measures what it was intended by the researcher.

- generalizability exists when a conclusion holds true for the group given a set of specified conditions.

- causal validity takes place when a conclusion about a relationship between variables is accurate.

Bailey $(1982, p 68)$ states that measurement validity has two parts: a.) face validity of the measuring instrument is the degree to which it is actually measuring the concept in question and not some other concept and b.) the measurement of the concept must be sufficiently accurate. He also points out that in addition to validity, a measure must have reliability, which implies consistency of measurement. Here validity implies reliability, however, reliability does not imply validity. And finally, whether assessing validity or reliability, the adequacy of a measure is dependent upon the accuracy or precision that is required. He points out that criterion validity (that the measure is 
consistent with other accepted measures of the same concept) and construct validity (that the measure can be substituted for another accepted measure of the same concept and the entire theory retested to obtain the same results) are also important. Bailey (1982) proposes that reliability for new measures can be determined by either a.) use of alternate forms of the same measure administered simultaneously (i.e. a parallel method of reliability assessment), or b.) by repeated measures (i.e. a test re-test method). To implement the first approach, separate reliability-check question pairs are often inserted into the survey, usually with oppositely biased wording (i.e. one positive and the other negative) in different parts of the survey (i.e. not adjacent questions). To implement the second approach, surveys are re-administered to the same respondents after a period of time. In the current study, the use of new constructs and measures are avoided as much as possible to minimize reliability concern. Reliability is achieved by using wellestablished constructs from the literature and the use (or slight adaptation) of proven measures. However, the second approach was also used by comparing repeated measures from the trail survey with the final, and in a few cases where multiple responses were received from the same organization and could be compared. The first approach was not employed due to minimized the length of the survey.

\subsubsection{Common Method Variance and Other Sources of Error}

Donaldson and Grant-Valone (2002) researched the effects of common method bias and self-report bias in business research. They state: 
In general, research participants want to respond in a way that makes them look as good as possible. Thus, they tend to under-report behaviors deemed inappropriate by researchers or other observers, and they tend to over-report behaviors viewed as appropriate. Self-report bias is particularly likely in organizational behavior research because employees often believe there is at least a remote possibility that their employer could gain access to their responses.

Borman (1991) reported that on average, peer and supervisor performance ratings were more accurate than self-ratings because of differential leniency effects in self-reports. ... ... The problem of self-report bias is compounded by the fact that when all variables in an organizational behavior study are based on one method of measurement, substantive findings are likely to be contaminated by shared method variance.

The authors proposed that motivational self-report bias is a function of four general factors:

1. the true state of affairs (i.e. the true score on the construct versus random or systematic error)

2. the nature of the construct of interest (e.g. sensitivity on the information)

3. dispositional characteristics of the respondent

4. situational characteristics of the measurement situation or environment. 
The preferred approach to estimate and reduce the effect of self-report bias in this study would be to obtain multiple responses from each site. This was not considered to be practical and was not attempted. However, in cases where multiple responses were received, these were examined and provided some confidence in the reliability of the measures (see Section 6.5). As an alternative, question 11 in Part $\mathrm{G}$ asked respondents to "please indicate the number of managers who helped complete this survey". This question was included to better understand how stations were completing the survey and the extent of possible individual response bias. If more participating managers helped prepare the response, it would tend to indicate less likelihood of individual response bias.

Bailey (1982) argues that errors in social research are in general simply a failure to collect data accurately. These errors can be either random or systematic. Random errors tend to cancel each other out and can be tolerated. Systematic errors do not cancel each other out and can result in erroneous findings, however, they can sometimes be detected and removed or minimized (by a correction). He describes how error can arise from a number of sources in each stage of research:

- During concept or hypothesis construction, including choice of operational definitions, errors can give rise to a lack of face validity;

- During construction of the instrument, a lack of reliability may arise due to ambiguity;

- During sampling, a lack of external validity due to sampling error may occur; 
- During data gathering, errors due to failure to control the environment, personal characteristics of respondents, relationship between the researcher and the respondent, defects of the research instrument, or misinterpretation can occur;

- During coding, incorrect recording of data is often encountered; and

- During data analysis, misuse or faulty interpretation of statistics can be a problem.

A concerted effort was made to minimize all of these sources of error. The first three items above were considered as part of construct and measurement design process and discussed earlier. Further consideration of these items was given during the trail survey and the expert peer review that followed (see Sections 4.1.4.1 and 4.1.4.2). Additional consideration of reliability issues including construct reliability screening and tests for discriminant validity are discussed in Section 6.5 "Reliability Analysis". Careful consideration for and control of the data gathering process was a priority and this is further described in Section 4.2 "Survey Distribution List and Distribution Method" below. The response data was carefully assessed for normality, skewness, missing data, and outliers and this is summarized in Sections 5.5 "Descriptive Data for Indicator Measures". A systematic data entry, coding, and screening process was followed and is described in Section 6 "Data Entry and Preparation". This included the application of standard practices for identification, assessment, and handling of outliers and missing data. Careful application of conservative statistical assumptions (e.g. cut-off frequencies, goodness of fit tests) were applied and documented throughout the statistical analysis. 


\subsection{Survey Distribution List and Distribution Method}

Appendix A contains the survey cover page provided to the IAEA with the survey. It provides a basic outline, states the objectives, and instructions for completion. The 4 different language versions of survey instrument can be found in Appendix A, B C and D. The survey and cover letter were approved by the Carleton Research Ethics Board prior to distribution.

The survey was not conducted as an anonymous survey; however, respondents had the option not to identify themselves or their station. The distribution of the survey with the assistance of the IAEA was seen as an important aspect of the survey implementation approach that ensured an adequate response rate. Further, respondents were reassured that data would be kept confidential and results would not be reported on individual stations (i.e. only aggregate statistics would be published). Finally, the appeal of the survey instrument as a useful "self-assessment" tool for participating NPPs for benchmarking purposes was emphasized and respondents were given the option to request a copy of a summary of the research findings if they participated. Respondents were also instructed that when completing the questionnaire, if they were unsure of the answer to a question, and it is not possible to get input from others within their organization who may be able to answer the question, it is preferred that they check the "unable to rate" box rather than guess at an answer.

Many regional and national nuclear power industry organizations hosts a number of important industry forums and workshops for senior NPP executives in any given year. 
Summary reports and workshop minutes are often available to participating organizations and participant contact lists are typically included that include the name, title, and e-mail address of the executives. A search of these contact lists from several source organizations was used to create an up-to-date e-mail distribution list that included plant/station managers, chief nuclear officers, and station vice-president of operations and site interface officers. Site interface officers are senior officials responsible for coordinating all formal communications between the site NPP management and external organizations such as regulators, government officials, and agencies like the IAEA.

Using the IAEA's current list of 437 operating NPPs as a reference list, a contact list was developed that contained at least 2 (and in most cases 3 or 4) senior management contact names at each station. This included the site interface/contract officer, and senior plant executive manager(s). The survey was initially distributed with the assistance of the IAEA to each NPP with a single direct and personalized e-mail to the manager(s) to be contacted for that station, with a copy to the station interface officer, and in the appropriate language(s) for the country where that NPP was located. The cover letter was attached (see Appendix A). Two follow-up electronic mailings were conducted. Figure 42 is an example of the typical e-mail text that was provided. 
Mr./Ms. <name of senior plant manger>

$<$ title e.g., Plant Manager or similar position of executive > (above repeated for each individual)

Copy to: Mr./Ms. <name of station interface officer $>$

Station Interface Officer

Dear $<$ Sir or Madam $>$ :

I would like to draw to your attention to the attached invitation to $<$ name of NPP $>$ to participate in an important global survey to study the link between Knowledge Management (KM) and NPP organizational and operational performance. The survey investigates the effect of $K M$ best practices on the quality of knowledge processes (i.e. knowledge transfer, retention, utilization etc.) and their resulting effects on organizational effectiveness and operational performance. It also explores whether information technology support and organizational culture have an influence on this relationship.

Many IAEA member states are participating in research project and we feel strongly that there will be significant benefits from the survey findings, including useful industry benchmark data to aid plants in KM self-assessments. Participating NPPs will be sent the final study findings, once available. The survey is available in 4 languages: English, French, Russian, and Chinese. It is in two formats: MSWord and PDF. The MS-Word version may be completed electronically and submitted by e-mail. The surveys and invitation letter asking NPPs to participate has been attached. The survey takes about 1-2 hours to complete and should be submitted directly to the IAEA. I have attached the <specify language $>$ versions for your convenience. Please feel free to contact me, $\mathrm{Mr}$. <name of officer $>$ at the IAEA if you have any questions. We hope your station will participate. Also your assistance in distributing the survey to other senior NPP station management contacts and promoting participation would be very much appreciated.

Thank you and best regards,

$M r .<$ name of officer $>$, IAEA

Figure 42. Personalized IAEA E-mail to Station Managers

The MS-Word version file that was attached was protected and had forms enabled for on-line electronic completion. Surveys could be returned to the IAEA in any of the following ways:

- E-mail back the IAEA a MS-Word version (i.e. completed electronically)

- E-mail back the IAEA a scanned version of a printed and hand-completed version

- Ground mail to IAEA a printed and hand-completed version

- Fax to IAEA of a printed and hand-completed version 
The second e-mail sent to each station was via bulk e-mail as a reminder by "blindcopy" to the remainder of the distribution who had not responded after a two week period. The third and final e-mail was again a bulk e-mail reminder by "blind-copy" to the (updated) remaining distribution who had not responded after a month with a final notice and a two week extended deadline.

\subsection{Questionnaire Response Rate}

A total of 118 individual survey responses were received. Two of these were essentially blank and could not be used, therefore 116 completed responses were considered. There was also a response from Korea that did not indicate the station nor provide operating performance data, so could not be used, reducing the total count of usable responses to 115. The respondents identified in many cases that the survey represented multiple units and in some cases multiple stations. In a few cases the response was a "fleet" response and was reporting on multiple stations, all of which were claimed to have similar "standardized" management practices. Thus a total of 124 station "site organizations" (i.e. slightly higher than the total number of survey responses) were reporting out of a total of 204 station organizations in total (60.8\%).

NPP stations range from single unit to 8 unit stations. On average there are 2 units per station. In a few cases, there were multiple stations at a single site. When considering the total number of units at each participating site, the responses represented a total of 253 reactor units or $57.9 \%$ of all 437 operating reactors. A total of 50 different operating organizations were included in the response. In 4 cases a fleet-wide response 
was provided and it was clearly stated in these cases that practices and management approaches were standardized across the fleet. Fleet responses were received from Entergy (USA), Exelon (USA), E.ON (Germany and Sweden), and NextEra (USA). Table 174 in Appendix F summarizes all of the NPP units from which data was obtained.

\subsection{Statistical Analysis Approach}

In order to investigate the impact of KM practices on NPP organizational performance, this study used a combination of standard statistical methods for testing the hypotheses based on the following techniques: construct reliability analysis, standard multivariate regression, and path analysis. Independent samples $\mathrm{T}$-tests and multi-group path analysis model techniques were also used to complement specific aspects of the analysis. Structural equation model (SEM) methods were attempted but due to the limited sample size and the large number of variables an acceptable SEM model fit could not be obtained at the measurement model level.

As a general guide to the multivariate analyses and interpretation, the guidelines provided by Hair et al. (2006) were followed and provided a framework for the key considerations to ensure a meaningful analysis. They included the following (paraphrased) basic rules:

- establishing the practical significance as well as statistical significance of results 
- recognizing the sample size affects on the results by considering statistical power and potential for over-fitting the model.

- knowing the data by looking at the influence of outliers, violations of assumptions, missing data and careful attention to diagnostic techniques.

- striving for model parsimony by avoiding specification errors of omitting critical predictor variables or including irrelevant variables.

- looking at the errors (residuals, misclassification of observations etc.)

- validating the results.

These considerations (in respective order) were incorporated in the analysis in the following ways:

- careful consideration was given to confirm the validity of each relationship found to be significant. This included consideration of content validity and consistency with theory. Relationships expected but found not to be (or close to being) significant were also carefully considered in terms of possible measurement limitations, or explanation in terms of direct versus indirect effects.

- Careful consideration of model goodness of fit statistics and the consideration of sample size on their meaningful interpretation. For example, consideration of the relationship between the number of parameters and required sample size.

- Careful screening of the data for skewness, kurtosis, missing data and outliers as well as confirmation of the reliability of the constructs. 
- Careful examination of and conservative application of overall goodness of fit statistics when accepting or comparing models, and favoring simple models over complex models. This includes considering all possible relationships (whether hypothesized or not) and applying a systematic approach to step-wise removal on non-significant relationships.

- When using path analysis, valuating model goodness of fit indicators relative to the saturated model. When applying regression analysis, considering bivariate residual plots.

- Overall, confirming results are consistent including comparing results from different methods, comparing incremental results based on a step-wise model development approach, and confirming findings are consistent with theory or carefully investigating and if necessary explaining any unexpected results.

The basic approach to statistical analysis described by Hair et al. (2006) provides a 6step structured framework for multivariate modeling and was followed:

i. The research problem and analysis objectives were defined and multivariate techniques to be used were selected.

ii. An analysis plan and data collection approach was developed.

iii. The data was collected and examined to evaluate the assumptions underlying the multivariate technique (this included graphical analysis, missing data analysis). In particular, in this stage, it was important to do the following:

a) identify, interpret, and decide on how to deal with outliers in the data. 
b) to understand and interpret the correlations and confirm (i.e. test) the underlying assumptions of the multivariate analysis techniques.

c) to assess the nature of missing data (random or systematic) and choose an appropriate method of correction.

iv. The multivariate model was estimated and the overall model fit assessed.

v. The variate(s) were interpreted.

vi. The multivariate model was validated.

Steps i. and ii. have already been discussed. The following sections describe steps iii. to vi. in the process. The multivariate statistical analysis that was performed on the data is described, and results interpreted and summarized to show how the model was validated. The methods and results obtained at each step are described. Due to the complexity of the data set, the number of variables and possible relationships (and associated parameters to be estimated), and limitations in the data set itself (i.e. sample size), no single multi-variable technique was sufficient in itself to fully analyze and interpret the data. The analysis was comprised of the following methods and stages:

- Uni-variate Data Analysis

- Bivariate Data Analysis

- Outlier and Missing Data Analysis

- Construct Reliability Analysis
- Linear Regressions

- Path Analysis (including some Multi-Group Model Analysis)

- Independent Sample T-tests

An explanation of each approach and the associated analysis for each is provided in the appropriate sections that follow. 


\section{Descriptive Statistics}

\subsection{Demographic Profile of Population (all stations)}

This section summarizes basic descriptive data to characterize the total population of NPPs. To begin with, Figure 43 below is a histogram showing the number of plants by type for the entire global population of NPPs. Plant types are:

- AGR-advanced gas reactor

- PWR - pressurized water reactor

- BWR - boiling water reactor

- FBR - fast breeder reactor

- VVER - Russian designed pressurized water reactor (actually a type of PWR)

- PHWR - pressurized heavy water reactor (e.g. CANDU)

- LWCGR - light water cooled gas reactor

- GCR - gas cooled reactor

There are significant differences in the design of these reactors and obviously this influences operations and maintenance and overall plant performance. The knowledge base and operating experience of the more popular reactor designs is also considerably greater. Tests for the moderating effect of plant type are summarized in Section 8.7. 


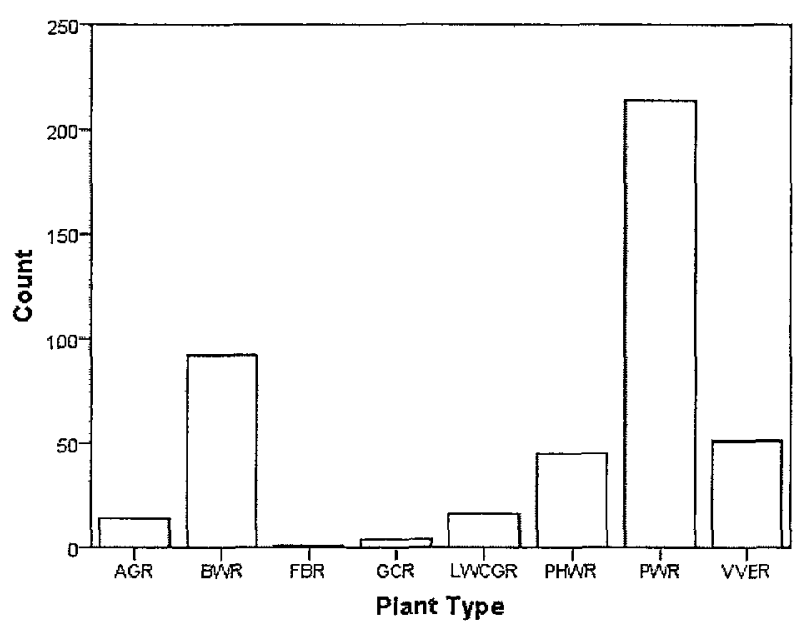

Figure 43. Summary of all NPPs by reactor type

Figure 44 below shows the distribution of plants by output rating (i.e. Megawatts electric production capacity). The largest plants built to date have a rated output of approximately 1600 MWe and are of Russian VVER design.

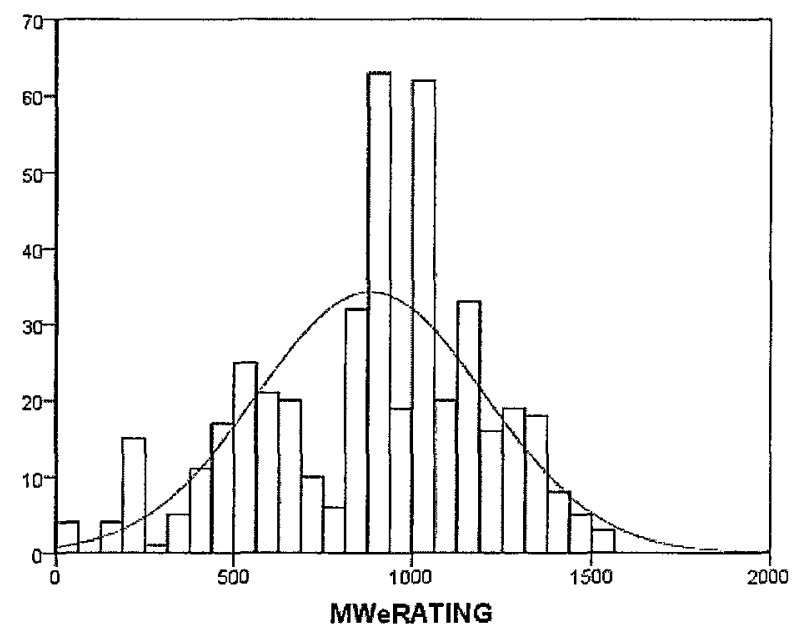

Figure 44. Summary of all NPPs by output rating (MWe)

Figure 45 below provides a profile of the age of the global fleet of NPPs based on the commercial operating date when the units first went into service. This date is in general 
a good indicator of plant age (and wear-out condition) but it is important to note that many exceptions exist. For example, there are units that only recently went into service but that had their construction started and halted for a decade or more before being commissioned and put into service (these are older plants, despite being "new"), and there are units that have been refurbished and despite being "old" are effectively new plants. In general, because plant construction slowed significantly since the 1990's the bulk of the existing NPPs are in the final half of their "planned" production life, however, many are being (or have plans to be) refurbished for significant plant life extensions (sometimes for an 30 additional years depending on the scope of refurbishment). While some plants have suffered de-ratings and increased outages due to plant aging and equipment degradation, other units have been upgraded, improved, and even up-rated for higher production, so plant age is not assumed to be correlated with operating performance.

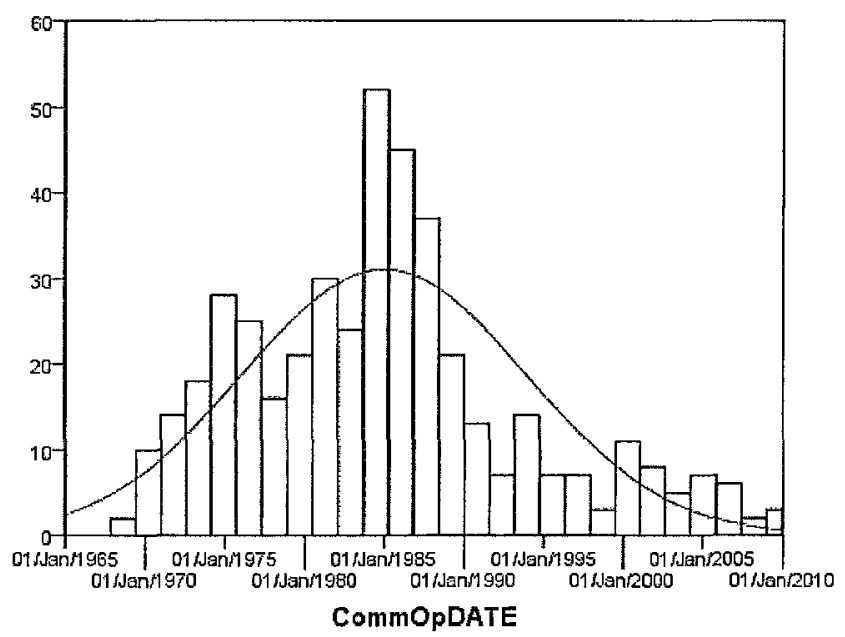

Figure 45. Summary of all NPPs by start-up date 
Table 6 below summarized the reactor types by country.

Table 6. Summary of all NPPs by Country and Reactor Type

\begin{tabular}{|c|c|c|c|c|c|c|c|c|}
\hline \multirow{2}{*}{ Country } & \multicolumn{9}{|c|}{ REACTOR TYPE } \\
\cline { 2 - 10 } & AGR & BWR & FBR & GCR & LWCGR & PHWR & PWR & Total \\
\hline Armenia & 0 & 0 & 0 & 0 & 0 & 0 & 1 & 1 \\
\hline Belgium & 0 & 0 & 0 & 0 & 0 & 0 & 7 & 7 \\
\hline Brazil & 0 & 0 & 0 & 0 & 0 & 0 & 2 & 2 \\
\hline Bulgaria & 0 & 0 & 0 & 0 & 0 & 0 & 2 & 2 \\
\hline Canada & 0 & 0 & 0 & 0 & 0 & 18 & 0 & 18 \\
\hline China & 0 & 0 & 0 & 0 & 0 & 2 & 9 & 11 \\
\hline Czech Republic & 0 & 2 & 0 & 0 & 0 & 0 & 6 & 8 \\
\hline Finland & 0 & 2 & 0 & 0 & 0 & 0 & 2 & 4 \\
\hline France & 0 & 0 & 0 & 0 & 0 & 0 & 58 & 58 \\
\hline Germany & 0 & 6 & 0 & 0 & 0 & 0 & 11 & 17 \\
\hline Hungary & 0 & 0 & 0 & 0 & 0 & 0 & 4 & 4 \\
\hline India & 0 & 2 & 0 & 0 & 0 & 16 & 0 & 18 \\
\hline Japan & 0 & 30 & 0 & 0 & 0 & 0 & 24 & 54 \\
\hline Korea & 0 & 0 & 0 & 0 & 0 & 4 & 16 & 20 \\
\hline Lithuania & 0 & 0 & 0 & 0 & 1 & 0 & 0 & 1 \\
\hline Netherlands & 0 & 0 & 0 & 0 & 0 & 0 & 1 & 1 \\
\hline Romania & 0 & 0 & 0 & 0 & 0 & 3 & 1 & 4 \\
\hline Russia & 0 & 0 & 1 & 0 & 15 & 0 & 15 & 31 \\
\hline S. Africa & 0 & 0 & 0 & 0 & 0 & 0 & 2 & 2 \\
\hline Slovakia & 0 & 0 & 0 & 0 & 0 & 0 & 4 & 4 \\
\hline Slovenia & 0 & 0 & 0 & 0 & 0 & 0 & 1 & 1 \\
\hline Spain & 0 & 2 & 0 & 0 & 0 & 0 & 6 & 8 \\
\hline Sweden & 0 & 7 & 0 & 0 & 0 & 2 & 3 & 12 \\
\hline Switzerland & 0 & 2 & 0 & 0 & 0 & 0 & 3 & 5 \\
\hline Taiwan & 0 & 4 & 0 & 0 & 0 & 0 & 2 & 6 \\
\hline UK & 14 & 0 & 0 & 4 & 0 & 0 & 1 & 19 \\
\hline Ukraine & 0 & 0 & 0 & 0 & 0 & 0 & 15 & 15 \\
\hline USA & 0 & 35 & 0 & 0 & 0 & 0 & 69 & 104 \\
\hline Total & 14 & 92 & 1 & 4 & 16 & 45 & 265 & 437 \\
\hline
\end{tabular}

\subsection{Demographic Profile of Responding Stations}

A summary of the basic demographics of survey response data are provided in this section. In most cases the data is presented in terms of the total number of responding NPPs in the survey response. This gives a perspective of the sample response data in terms of the global fleet of NPPs. Figure 46 below shows the total number NPPs per country in the survey response. The USA clearly had the highest number of reactor units represented. 


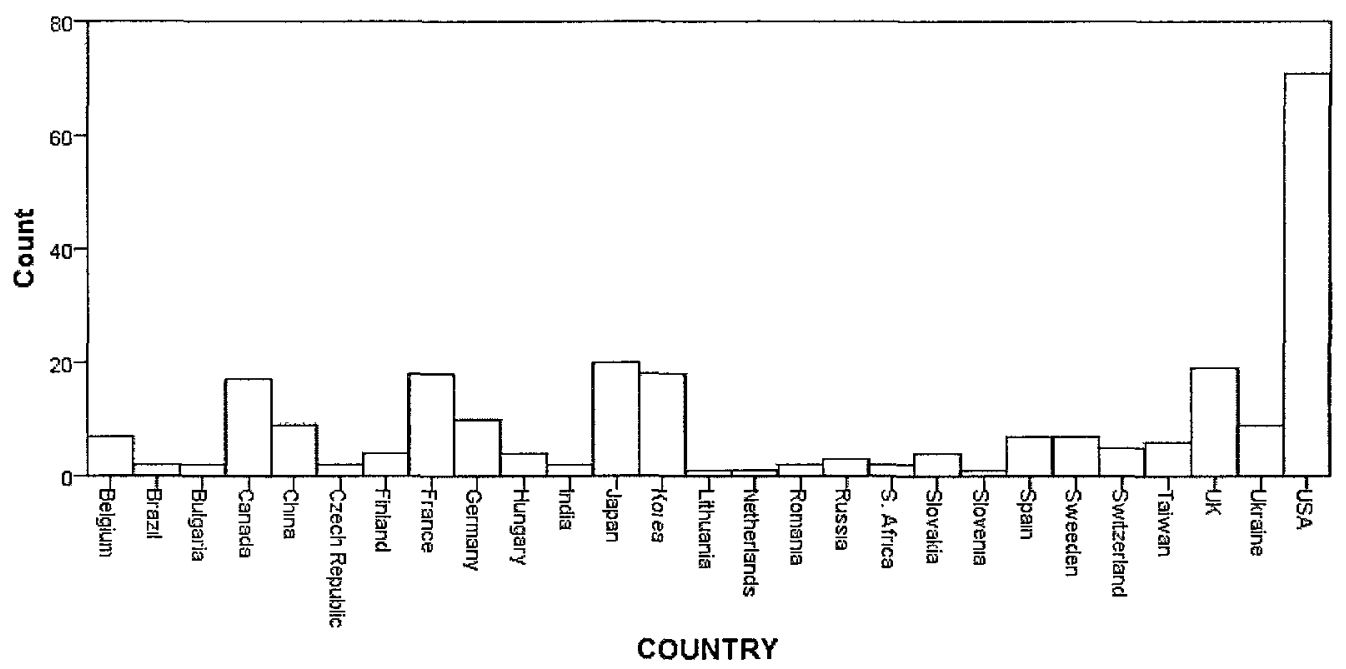

Figure 46. No. of Responding NPPs by country

Figure 47 shows the distribution of reactor types by country in the survey response data. Many countries have two and some have even three reactor types.

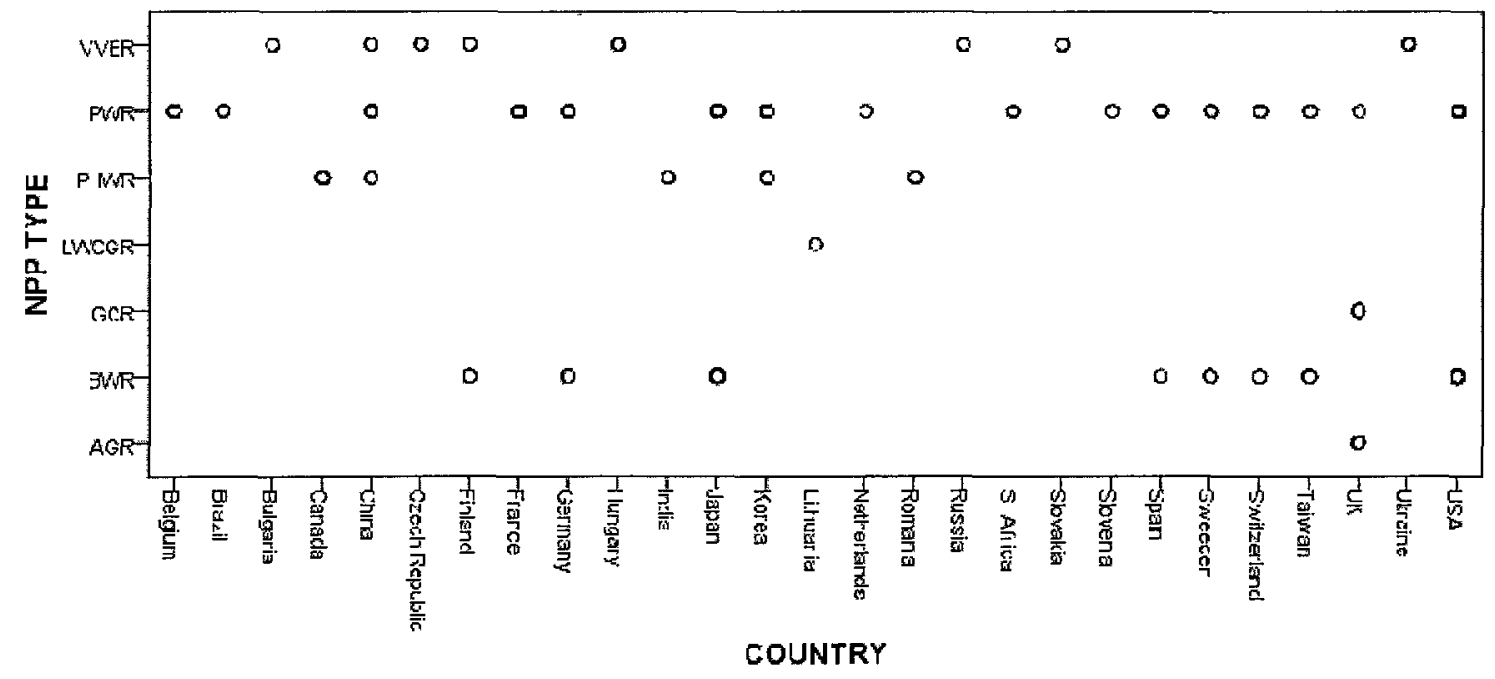

Figure 47. Responding NPPs by Reactor Type and Country

Table 7 below summarizes the response data by country with respect to the total NPP population and the NPPs included in the set of responding stations. 
Table 7. Responding NPPs by $\%$ of population and country

\begin{tabular}{|c|c|c|c|c|c|c|}
\hline \multirow[b]{2}{*}{ Country } & \multicolumn{2}{|c|}{ Total NPP Population } & \multicolumn{4}{|c|}{ NPPs in Sample Response } \\
\hline & Frequency & Percent & Frequency & $\begin{array}{l}\text { Percent of } \\
\text { Total NPPs } \\
\text { in Survey } \\
\text { Response }\end{array}$ & $\begin{array}{c}\text { Percent } \\
\text { Country } \\
\text { NPP } \\
\text { Population }\end{array}$ & $\begin{array}{c}\text { Percent } \\
\text { of Global } \\
\text { NPP } \\
\text { Population }\end{array}$ \\
\hline Armenia & 1 & .2 & 0 & .0 & .0 & .0 \\
\hline Belgium & 7 & 1.6 & 7 & 2.8 & 100.0 & 1.6 \\
\hline Brazil & 2 & .5 & 2 & .8 & 100.0 & .5 \\
\hline Bulgaria & 2 & .5 & 2 & .8 & 100.0 & .5 \\
\hline Canada & 18 & 4.1 & 17 & 6.7 & 94.4 & 3.9 \\
\hline China & 11 & 2.5 & 9 & 3.6 & 81.8 & 2.1 \\
\hline Czech Republic & 8 & 1.8 & 2 & .8 & 25.0 & .5 \\
\hline Finland & 4 & .9 & 4 & 1.6 & 100.0 & .9 \\
\hline France & 58 & 13.3 & 18 & 7.1 & 31.0 & 4.1 \\
\hline Germany & 17 & 3.9 & 10 & 4.0 & 58.8 & 2.3 \\
\hline Hungary & 4 & .9 & 4 & 1.6 & 100.0 & .9 \\
\hline India & 18 & 4.1 & 2 & .8 & 11.1 & .5 \\
\hline Japan & 54 & 12.4 & 20 & 7.9 & 37.0 & $\overline{4.6}$ \\
\hline Korea & 20 & 4.6 & 18 & 7.1 & 90.0 & 4.1 \\
\hline Lithuania & 1 & .2 & 1 & .4 & 100.0 & .2 \\
\hline Netherlands & 1 & .2 & 1 & .4 & 100.0 & .2 \\
\hline Romania & 4 & .9 & 2 & .8 & 50.0 & .5 \\
\hline Russia & 31 & 7.1 & 3 & 1.2 & 9.7 & .7 \\
\hline S. Africa & 2 & .5 & 2 & .8 & 100.0 & .5 \\
\hline Slovakia & 4 & .9 & 4 & 1.6 & 100.0 & .9 \\
\hline Slovenia & 1 & .2 & 1 & .4 & 100.0 & .2 \\
\hline Spain & 8 & 1.8 & 7 & 2.8 & 87.5 & 1.6 \\
\hline Sweden & 12 & 2.7 & 7 & 2.8 & 58.3 & 1.6 \\
\hline Switzerland & 5 & 1.1 & 5 & 2.0 & 100.0 & 1.1 \\
\hline Taiwan & 6 & 1.4 & 6 & 2.4 & 100.0 & 1.4 \\
\hline UK & 19 & 4.3 & 19 & 7.5 & 100.0 & 4.3 \\
\hline Ukraine & 15 & 3.4 & 9 & 3.6 & 60.0 & 2.1 \\
\hline USA & 104 & 23.8 & 71 & 28.1 & 68.3 & 16.2 \\
\hline Total & 437 & 100.0 & 253 & 100.0 & $\mathrm{n} / \mathrm{a}$ & 57.9 \\
\hline
\end{tabular}

Figure 48 shows the distribution of NPP units by size (output in MWe) for the sample. 


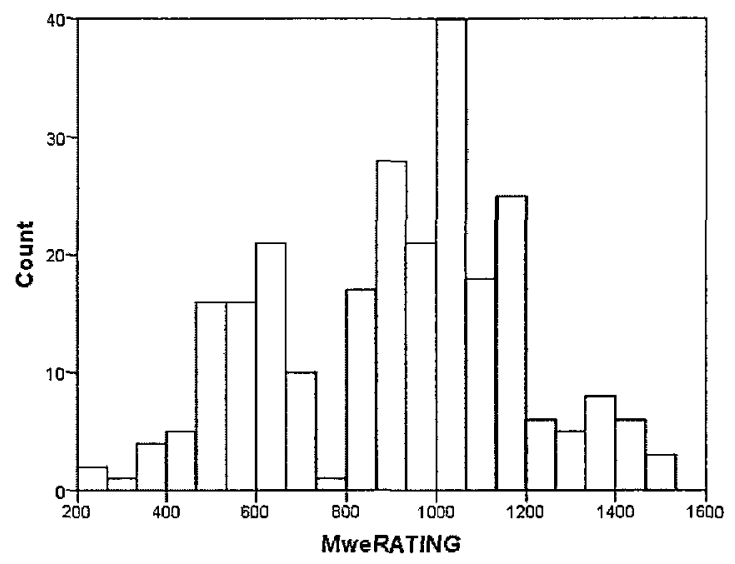

Figure 48. Breakdown of responding NPPs by plant output (MWe)

Figure 49 below shows the frequency occurrence of stations with $1,2,3,4,5,6$, and 7 units. It is interesting to note that a significant number of stations (almost 125) are 2unit stations.

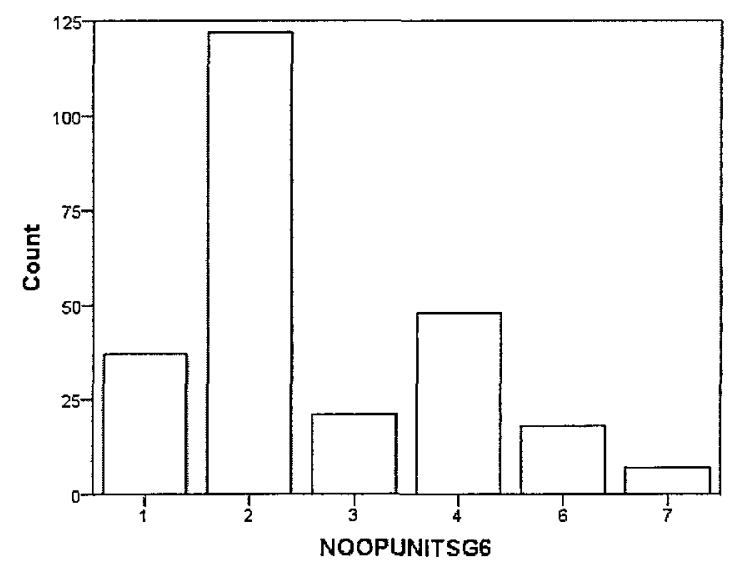

Figure 49. Breakdown of responding NPPs by no of units at site

Figures 50, 51, and 52 below show the relative distribution (counts) of NPPs responding to the survey in the following formats: operator vs. age; operator vs. plant output (MW 
electrical); and operator vs. plant type. It is clear to see that there exists a wide crosssection of the total population and that operators typically have a range of plants by age and size and even multiple types. Table 8 below summarizes the responses by country and reactor type for stations responding.

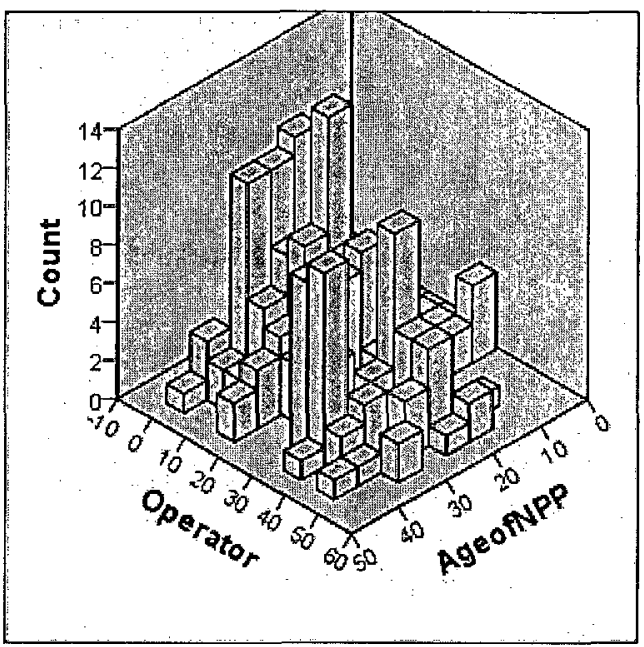

Figure 50. No. of NPPs by Operator by Age (yrs from start-up)

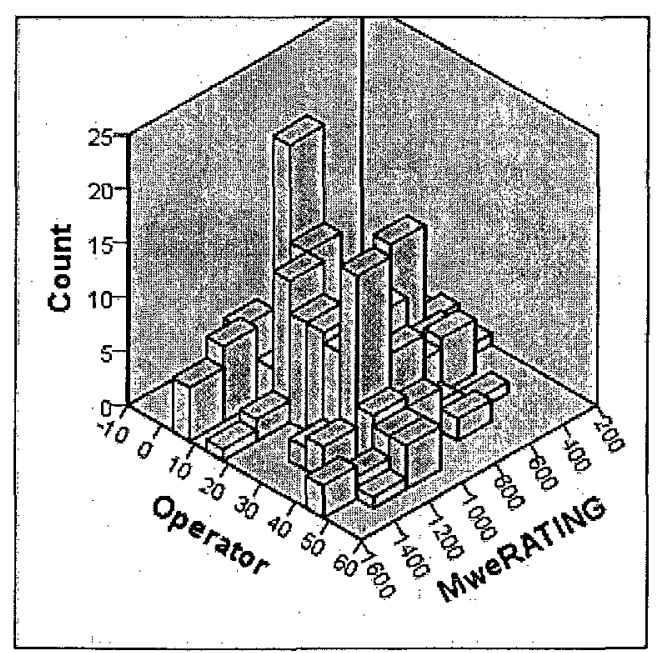

Figure 51. No. of Responding NPPs by Operator and Output (MWe) 


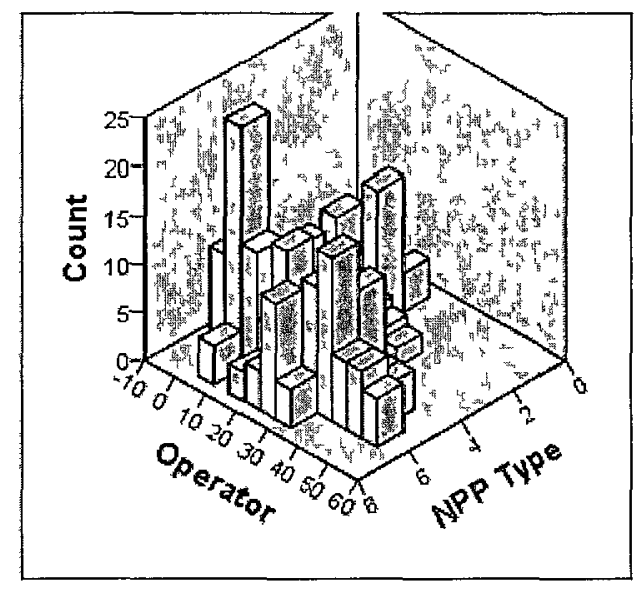

F1gure 52. No. of Responding NPPs by Operator and Plant Type

Table 8. Responding NPPs by Country and Reactor Type

\begin{tabular}{|c|c|c|c|c|c|c|c|}
\hline \multirow{2}{*}{ Country } & \multicolumn{7}{|c|}{ Count by REACTORTYPE } \\
\hline & AGR & BWR & GCR & LWCGR & PHWR & PWR & Total \\
\hline Belgıum & 0 & 0 & 0 & 0 & 0 & 7 & 7 \\
\hline Brazıl & 0 & 0 & 0 & 0 & 0 & 2 & 2 \\
\hline Bulgaria & 0 & 0 & 0 & 0 & 0 & 2 & 2 \\
\hline Canada & 0 & 0 & 0 & 0 & 17 & 0 & 17 \\
\hline Chına & 0 & 0 & 0 & 0 & 2 & 7 & 9 \\
\hline Czech Republic & 0 & 0 & 0 & 0 & 0 & 2 & 2 \\
\hline Fınland & 0 & 2 & 0 & 0 & 0 & 2 & 4 \\
\hline France & 0 & 0 & 0 & 0 & 0 & 18 & 18 \\
\hline Germany & 0 & 4 & 0 & 0 & 0 & 6 & 10 \\
\hline Hungary & 0 & 0 & 0 & 0 & 0 & 4 & 4 \\
\hline Indıa & 0 & 0 & 0 & 0 & 2 & 0 & 2 \\
\hline Japan & 0 & 13 & 0 & 0 & 0 & 7 & 20 \\
\hline Korea & 0 & 0 & 0 & 0 & 4 & 14 & 18 \\
\hline Lithuania & 0 & 0 & 0 & 1 & 0 & 0 & 1 \\
\hline Netherlands & 0 & 0 & 0 & 0 & 0 & 1 & 1 \\
\hline Romania & 0 & 0 & 0 & 0 & 2 & 0 & 2 \\
\hline Russia & 0 & 0 & 0 & 0 & 0 & 3 & 3 \\
\hline S. Africa & 0 & 0 & 0 & 0 & 0 & 2 & 2 \\
\hline Slovakia & 0 & 0 & 0 & 0 & 0 & 4 & 4 \\
\hline Slovenia & 0 & 0 & 0 & 0 & 0 & 1 & 1 \\
\hline Spain & 0 & 1 & 0 & 0 & 0 & 6 & 7 \\
\hline Sweden & 0 & 4 & 0 & 0 & 0 & 3 & 7 \\
\hline Switzerland & 0 & 2 & 0 & 0 & 0 & 3 & 5 \\
\hline Taiwan & 0 & 4 & 0 & 0 & 0 & 2 & 6 \\
\hline UK & 14 & 0 & 4 & 0 & 0 & 1 & 19 \\
\hline Ukraıne & 0 & 0 & 0 & 0 & 0 & 9 & 9 \\
\hline USA & 0 & 26 & 0 & 0 & 0 & 45 & 71 \\
\hline Total & 14 & 56 & 4 & 1 & 27 & 151 & 253 \\
\hline
\end{tabular}


Figure 53 below shows the number of units by each operator within the sample.

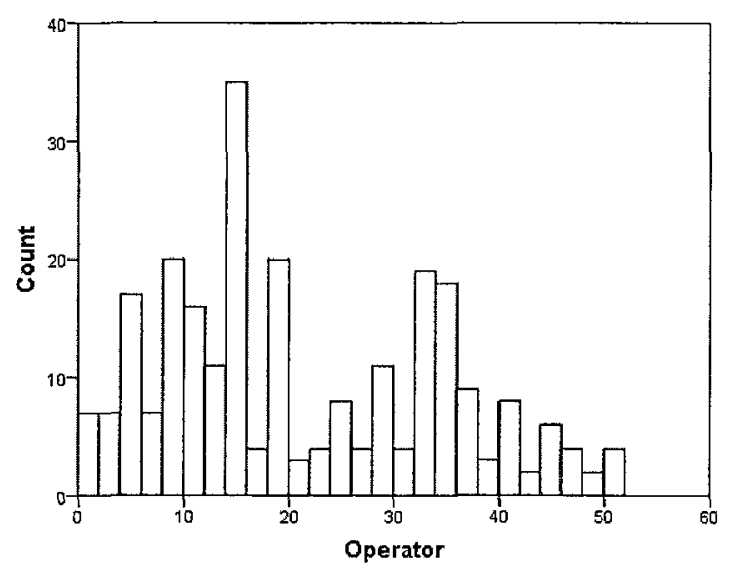

Figure 53. No. of responding NPPs by operator

Survey question G1 asked respondents to "please indicate the number of employees (excluding contractors) at your station". This question was included to obtain demographic data and was not used in the analysis, however, the data does give an indication of the range in staffing levels at the responding stations. Figure 54 below shows the distribution of responses to question G1:

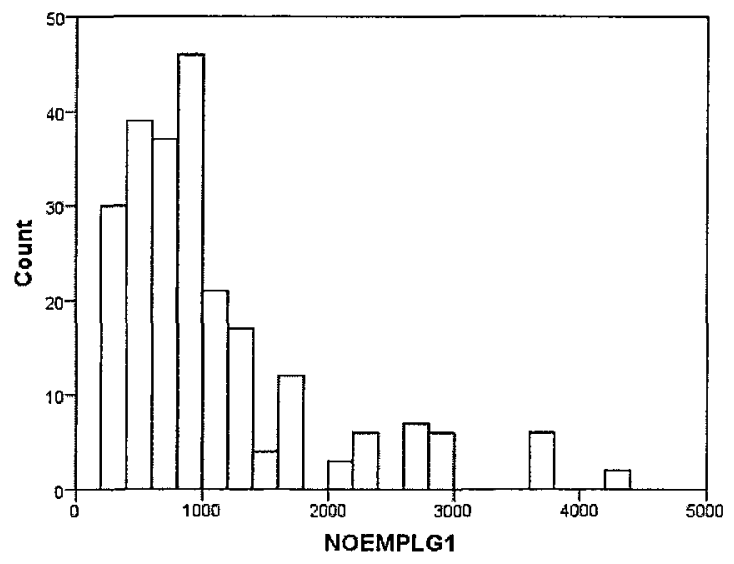

Figure 54. No. stations by no. of employees 
Figures 55 and 56 show the responses to survey questions G2 and G3 which were: "please indicate the typical number of full-time equivalent contractors during outages" (G2) and "please indicate the typical number of full-time equivalent contractors while at power" (G3). These questions were included to obtain demographic data and were not used in the analysis, however, the data does give an indication of the range in contractor staffing levels at the responding stations.

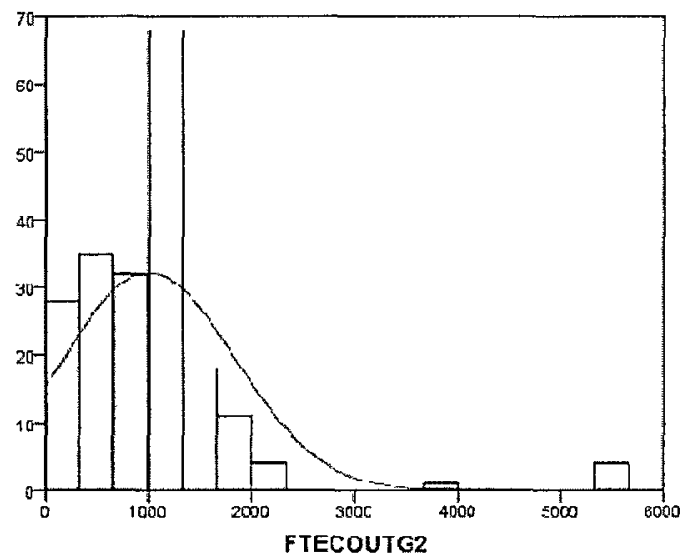

Figure 55. No. of stations by FTE contractors in outage

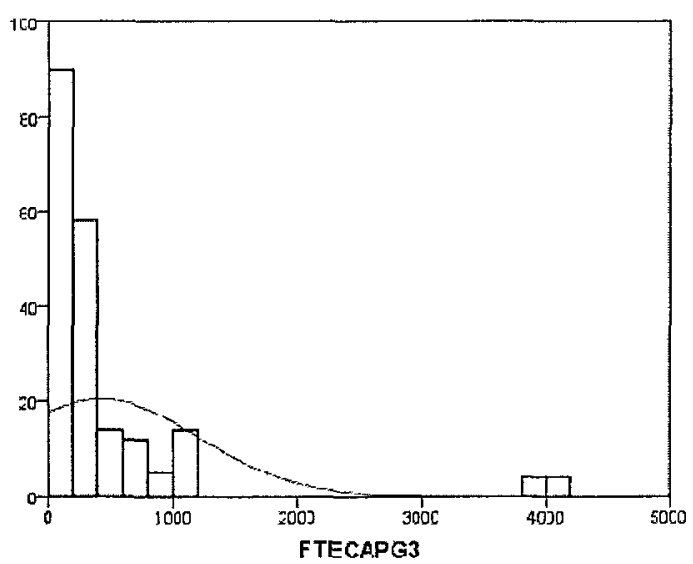

Figure 56. No. of stations by FTE contractors at power 
Figure 57 below summarizes responses to question G4: "please indicate the percentage of employees with university degrees at your station". This question was included to obtain demographic data and was not used in the analysis, however, the data does give an indication of the range in percentage of staff having university degrees across the responding stations.

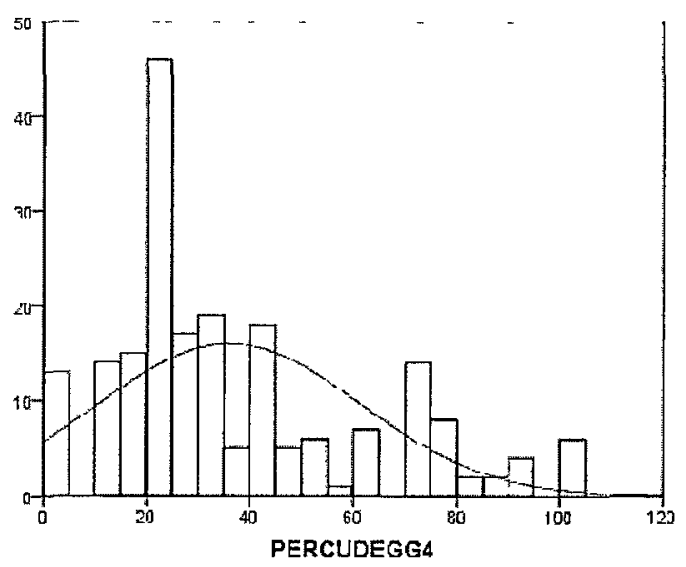

Figure 57 . No. Station by $\%$ of staff university grads

Question 10 was added based on feedback from the expert review at the IAEA in Vienna. It was tabulated for each community of practice (COP) group response as the total count of both measures (i.e. both "regular participant" and "doing benchmarking" for each COP group). Figure 58 below shows the distribution of responses binned up to $36(\max )$. Note that a large number of responses were either a zero (indicating either a true zero or were missing data and got coded as zero), or were at the top end of the binned scale at 36 (i.e. many respondents checked all answers). This skewed the data and made it questionable to do any statistical analysis (i.e. non-normal data). It is recommended this measure be retained in future if the survey is repeated but the question wording be revised to improve the quality of the response data. This measure 
was not used in the analysis. Had it been a reliable measure, it could have been included as one of the formative measures in the support for organizational learning (SOL) construct in terms of participation and the organizational performance management (OPM) construct in terms of benchmarking.

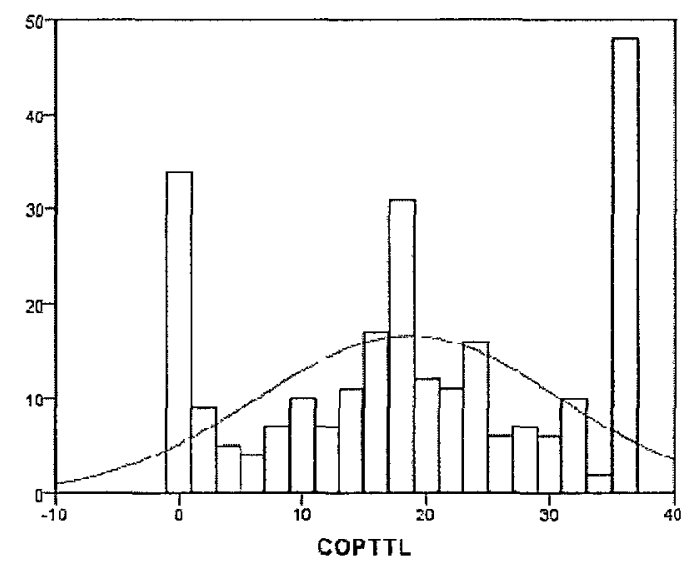

Figure 58. No. of NPPs by COP total-score

Table 59 below shows the age distribution of the NPPs in the sample. It is clear that a large percentage of stations are 20 years of age or older.

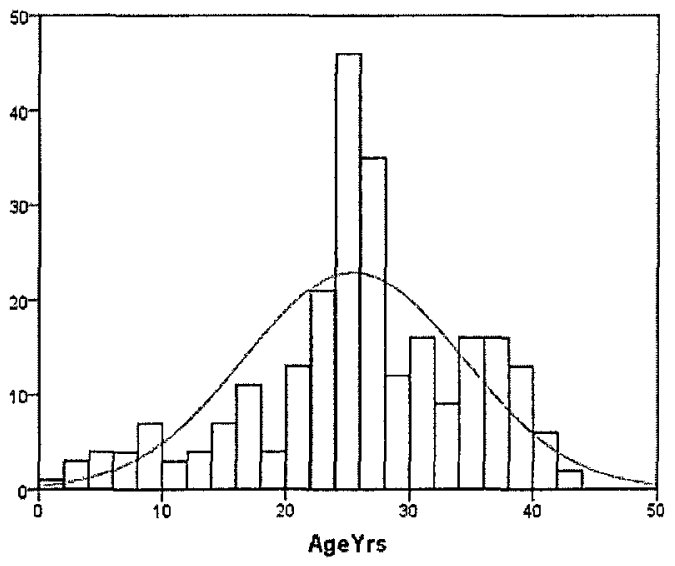

Figure 59. No. of NPPs by Age (in years since start-up) 
Figure 60 shows a cross-plot of reactor size by type from the sample. It is interesting to note the wide range of reactor sizes for BWR and PWR type designs which is fairly representative of the total population.

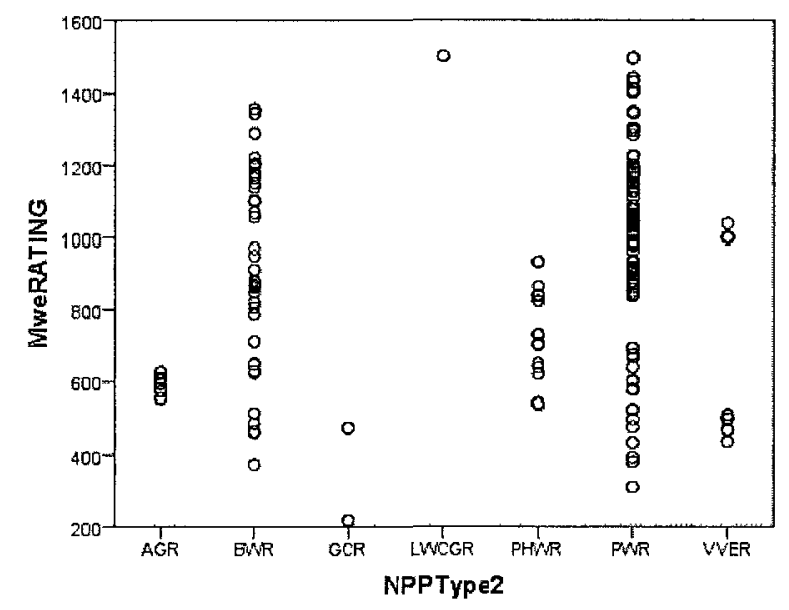

Figure 60. NPP Output (MWe) by Reactor Type

Figure 61 below is a histogram for Question 11 in Part G "please indicate the number of managers who helped complete this survey". This question was included to better understand the extent of possible personal bias (i.e. more participating managers would tend to minimize individual response bias). It was not used in the statistical analysis. 


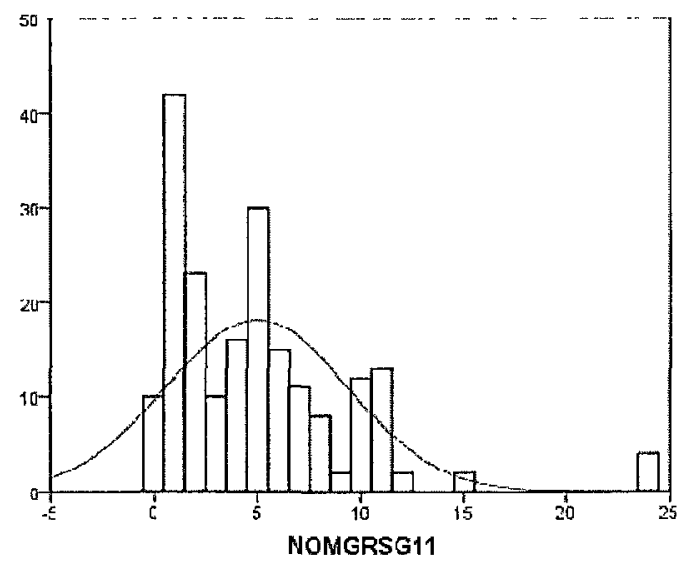

Figure 61. No. of NPPs by no. of responding managers

\subsection{Operational Performance Measures}

This study used four WANO indicators for operating performance: Unit Capability Factor (UCF), Unit Capability Loss (UCL) Rate Factor, Forced Loss Rate (FLR), and Unplanned Automatic SCRAMs per 7000 Hours Critical (UA7) (see Section 3.3.1.2 and Appendix E for a full definition of each). The term "SCRAM" refers to a "safe corereactivity arrest made" (i.e. an actuation of the automatic safety systems to rapidly shutdown the reactor).

For the purposes of this study, the raw WANO indicator data were re-scaled. A transformation was applied to each indicator such that the scale ranges from a maximum of 100 (best performance) to a minimum of zero (worst performance). WANO uses 4 different scaling schemes that limit the reporting range of the raw production data to "normal expected operating bands" but help to spread out the ratings for easier 
interpretation (i.e. reduce the skew and kurtosis somewhat as most stations are reporting in the operating band close to full production most of the time). This approach tends to result in indicator values considered outside of the "normal" operating band being assigned to one of the scale limits. Based on expert feedback received during the IAEA peer review meeting, it was decided that for the purposes of this study a wider scale transformation scheme should be used based on the raw plant performance data, however the scale limits should be adjusted to include a wider definition of performance for better discrimination of top and bottom performers. Table 9 below summarizes the wider indices used in this study.

Table 9. Derivation of Operating Performance Indicators

\begin{tabular}{|c|c|c|}
\hline Performance & Raw Indicator Value & Assigned Indicator Point INDEX Value (\%) \\
\hline $\begin{array}{l}\text { Unit Capability Factor } \\
\text { (UCF) }\end{array}$ & $\begin{aligned} \mathrm{UCF}_{\mathrm{RAW}} & >98 \\
40<=\mathrm{UCF}_{\mathrm{RAW}} & <=98 \\
\mathrm{UCF}_{\mathrm{RAW}} & <40\end{aligned}$ & $\begin{array}{l}\mathrm{UCF}_{\mathrm{IND}}=100 \\
\mathrm{UCF}_{\mathrm{IND}}=\left(\mathrm{UCF}_{\mathrm{RAW}}-40\right) * 1.724138 \\
\mathrm{UCF}_{\mathrm{IND}}=0\end{array}$ \\
\hline $\begin{array}{l}\text { Unit Capability Loss } \\
\text { (UCL) Rate Factor }\end{array}$ & $\begin{aligned} \mathrm{UCL}_{\mathrm{RAW}} & >40 \\
0<\mathrm{UCL}_{\mathrm{RAW}} & <=40 \\
\mathrm{UCL}_{\mathrm{RAW}} & <=0\end{aligned}$ & $\begin{array}{l}\mathrm{UCL}_{\mathrm{IND}}=0 \\
\mathrm{UCL}_{\mathbb{N D}}=\left(40-\mathrm{UCL}_{\mathrm{RAW}}\right) * 2.5 \\
\mathrm{UCL}_{\mathbb{I N D}}=100\end{array}$ \\
\hline $\begin{array}{l}\text { Forced Loss Rate } \\
\text { (FLR) }\end{array}$ & $\begin{aligned} \text { FLR RAW } & >25 \\
0<\mathrm{FLR}_{\text {RAW }} & <=25 \\
\mathrm{FLLR}_{\mathrm{RAW}} & <=0\end{aligned}$ & $\begin{array}{l}\mathrm{FLR}_{\mathrm{IND}}=0 \\
\mathrm{FLRR}_{\mathrm{IND}}=\left(25-\mathrm{FLR} \mathrm{RAW}_{\mathrm{R}}\right)^{* 4} \\
\mathrm{FLR}_{\mathrm{IND}}=100\end{array}$ \\
\hline $\begin{array}{l}\text { Unplanned Automatic } \\
\text { SCRAMS per } 7000 \\
\text { Hours Critical (UA7) }\end{array}$ & 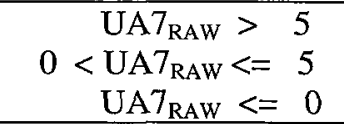 & $\begin{array}{l}\mathrm{UA} 7_{\mathrm{IND}}=0 \\
\mathrm{UA} 7_{\mathrm{IND}}=\left(5-\mathrm{UA} 7_{\mathrm{RAW}}\right) * 20 \\
\mathrm{UA} 7_{\mathrm{IND}}=100\end{array}$ \\
\hline $\begin{array}{l}\text { Combined Performance } \\
\text { Index (CPerfINDEX) }\end{array}$ & $\mathrm{n} / \mathrm{a}$ & CPerfINDEX $=[\mathrm{UCF}+(\mathrm{UCL}+\mathrm{FLR}) / 2+\mathrm{UA} 7] / 3$ \\
\hline
\end{tabular}

Note also that based on expert peer review feedback, UCL and FLR were deemed to be measuring much the same effect and were combined (averaged) to avoid over-weighting the aggregate scale too heavily towards these two measures. Thus the combined performance index used was an average of 3 effects: UCF, the average of UCL and FLR, and UA7. 


\subsection{Operational Performance Data}

Industry experts all agree that only 3-year indicators should be used as they remove the effects of refueling outages which are typically every 18-24 months for most NPPs (WANO, 2005). The WANO Reference Guide (2010) states: “indicators have received extensive international review and constitute a uniform set of indicators suitable for international use. The period values based on 3-years intervals provide the most suitable indication of long-term operational performance". Therefore only the 3-year operational performance indicator data for UCF, UCL, FLR, and UA7 was used in the analysis.

Figure 62 below shows histograms of each of the operational performance indicators for the NPP units responding to this survey. The raw indicator data histogram (raw count frequencies) for UCF, UCL, FLR, and UA7 is plotted (for each respectively), followed by the calculated (transformed) index indicator data histogram (for each respectively). Note that UCL, FLR, and UA7 are all "negative" indices. For this reason they all have reverse coded transformation indexes to allow them to be used in a combined scale, labeled "CPerfINDEX" (see Table 9 above for exact transformation). This explains why the histograms for UCL, FLR, and UA7 raw data are skewed towards zero while the histograms for UCL, FLR and UA7 indices are skewed towards $100 \%$. 


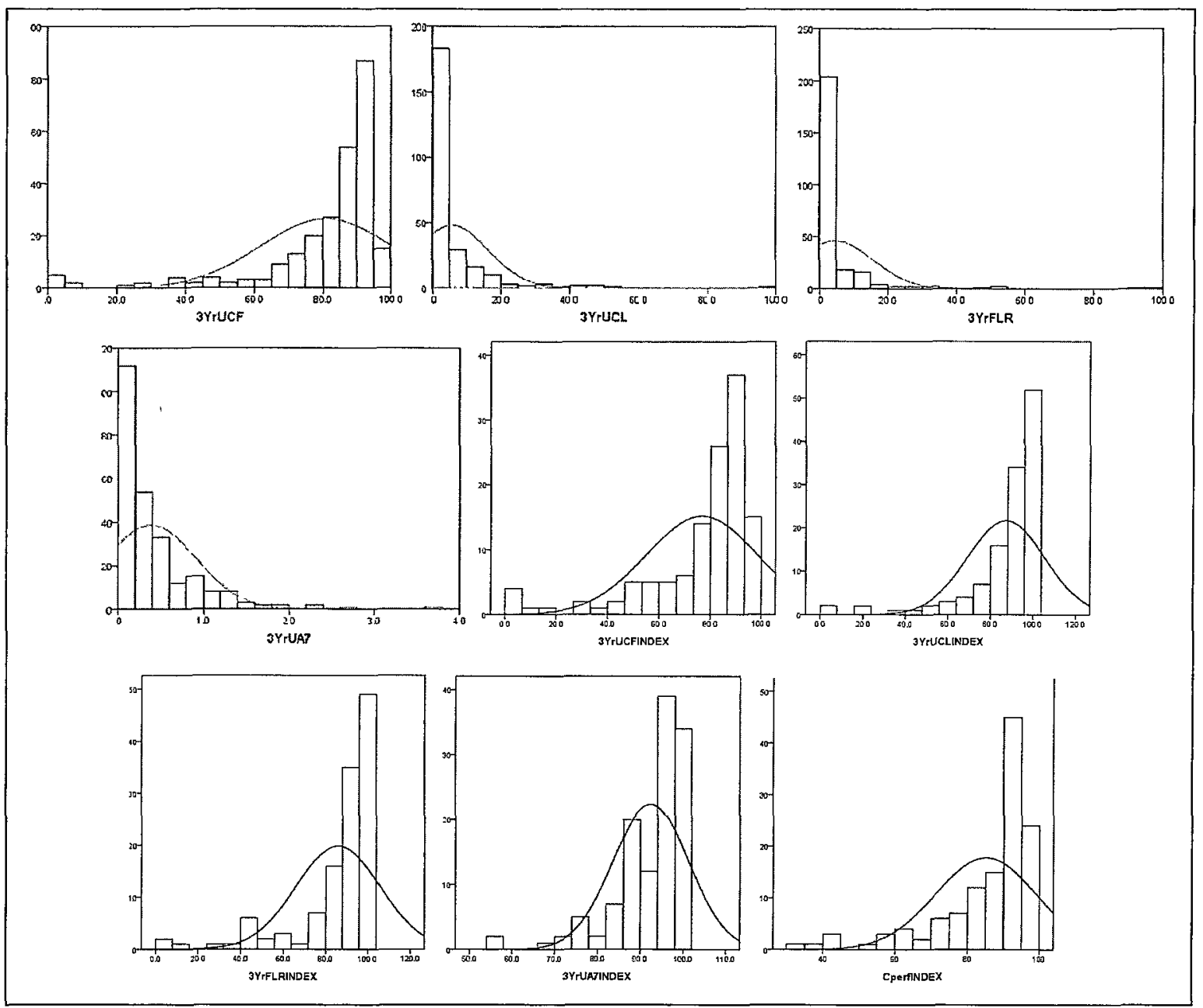

Figure 62. Responding NPPs - Raw \& Indexed Op'1 Performance Histograms

It is clear that these indices are all skewed and non-normal. To resolve this problem and produce a valid indicator based on the data that can support statistical analysis, the 4 indices were combined and averaged, then binned to normalize the data. Table 10 summarizes the transformation formula and binning to create the "CPerfNormBins" variable. Note that UCL and FLR were deemed to be measuring much the same effect and to avoid giving them too much weight were first averaged before combining with 
the other 2 remaining indices (i.e. rather than averaging all 4 indices, and this explains the denominator of 3 ).

Table 10. Normalization bins for combined performance index

\begin{tabular}{|c|c|}
\hline $\begin{array}{c}\text { Combined Performance Index CPerfINDEX Range } \\
\text { (Note: CPerfINDEX=(UCF+(UCL+FLR)/2+UA7)/3) }\end{array}$ & $\begin{array}{c}\text { Normalized Bin Number } \\
\text { Assignment (CPERFNormBins) }\end{array}$ \\
\hline $0<$ CPerfINDEX $<=25$ & 1 \\
\hline $25<$ CPerfINDEX $<=35$ & 2 \\
\hline $35<$ CPerfINDEX $<=60$ & 3 \\
\hline $60<$ CPerfINDEX $<=82$ & 4 \\
\hline $82<$ CPerfINDEX $<=92.7$ & 5 \\
\hline $92.7<$ CPerfINDEX $<=96.5$ & 6 \\
\hline $96.5<$ CPerfINDEX $<=97.8$ & 7 \\
\hline $97.8<$ CPerfINDEX $<=100$ & 8 \\
\hline
\end{tabular}

Figure 63 shows the histogram of the new measure when binned according to Table 10.

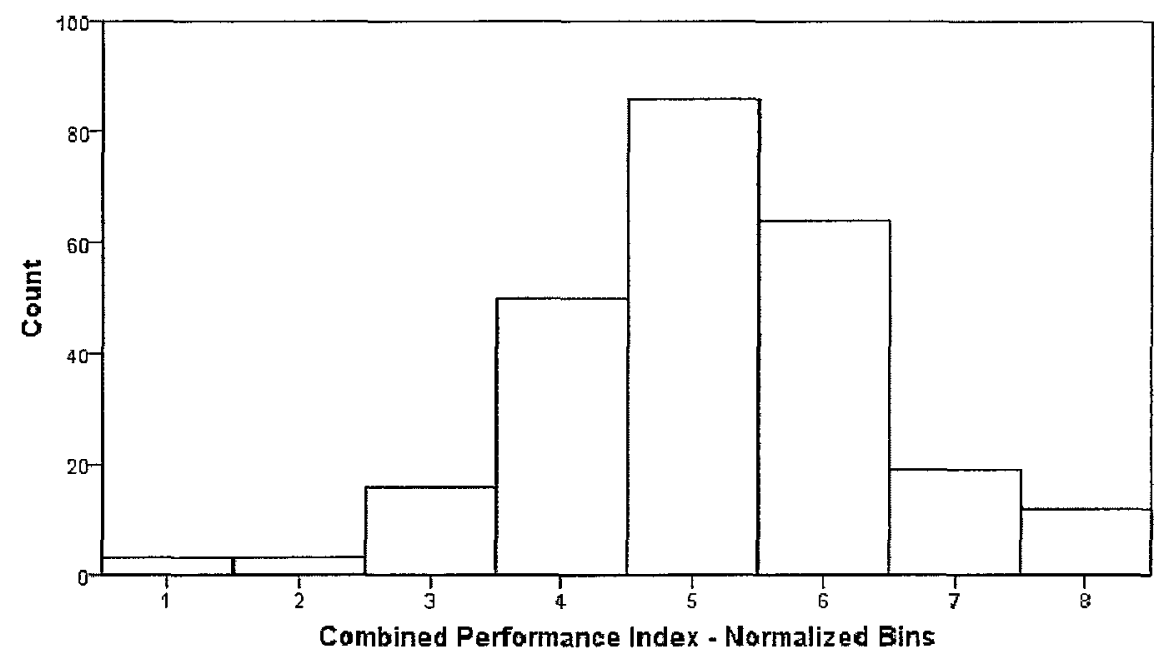

Figure 63. Responding NPPs by performance (normalized index)

This is the variable "CPERFNormBins" that will be referenced throughout. It is derived from CPerfINDEX and exhibits good normality. Table 11 below summarizes descriptive statistics for plant operating performance using CPerfINDEX by plant type 
and broken down by no. of units per site. Means, standard deviation and total counts are summarized.

Table 11. Responding NPP performance by plant type and units per site

\begin{tabular}{|c|c|c|c|c|}
\hline NPP Type & No. of Operating Units at Site & Mean & Std. Deviation & $\mathbf{N}$ \\
\hline \multirow[t]{2}{*}{ GCR } & 2 & 52.5 & 5.3 & 4 \\
\hline & Total & 52.5 & 5.3 & 4 \\
\hline \multirow[t]{3}{*}{ AGR } & 2 & 56.4 & 8.0 & 10 \\
\hline & 4 & 52.0 & 10.7 & 4 \\
\hline & Total & 55.7 & 8.5 & 14 \\
\hline \multirow[t]{5}{*}{ PHWR } & 1 & 37.6 & . & 1 \\
\hline & 2 & 80.8 & 12.7 & 12 \\
\hline & 4 & 81.7 & 6.4 & 8 \\
\hline & 6 & 86.0 & 2.8 & 6 \\
\hline & Total & 77.2 & 11.2 & 27 \\
\hline \multirow[t]{2}{*}{ LWCGR } & 1 & 87.0 & . & 1 \\
\hline & Total & 87.0 & . & 1 \\
\hline \multirow[t]{6}{*}{ BWR } & 1 & 86.7 & 12.7 & 15 \\
\hline & 2 & 92.0 & 3.8 & 26 \\
\hline & 3 & 56.8 & 8.0 & 3 \\
\hline & 4 & 84.5 & 4.9 & 5 \\
\hline & 7 & 59.9 & 3.7 & 7 \\
\hline & Total & 87.0 & 8.7 & 56 \\
\hline \multirow[t]{6}{*}{ PWR } & 1 & 89.2 & 8.0 & 20 \\
\hline & 2 & 89.9 & 6.1 & 56 \\
\hline & 3 & 87.7 & 1.9 & 11 \\
\hline & 4 & 81.9 & 4.5 & 27 \\
\hline & 6 & 80.0 & 3.5 & 6 \\
\hline & Total & 88.5 & 5.9 & 120 \\
\hline \multirow[t]{5}{*}{ VVER } & 2 & 88.6 & 4.1 & 12 \\
\hline & 3 & 83.9 & 2.8 & 6 \\
\hline & 4 & 92.3 & 0.8 & 4 \\
\hline & 6 & 90.4 & 0.6 & 6 \\
\hline & Total & 88.2 & 3.1 & 28 \\
\hline \multirow[t]{7}{*}{ Total } & 1 & 86.7 & 11.9 & 37 \\
\hline & 2 & 85.3 & 9.2 & 120 \\
\hline & 3 & 81.9 & 6.1 & 20 \\
\hline & 4 & 80.5 & 6.4 & 48 \\
\hline & 6 & 85.4 & 2.9 & 18 \\
\hline & 7 & 59.9 & 3.7 & 7 \\
\hline & Total & 84.9 & 8.7 & 250 \\
\hline
\end{tabular}




\subsection{Descriptive Data for Indicator Measures}

As part of the test for normality, the skewness and kurtosis of the measured variables must be shown to be acceptable. Skewness is a measure of asymmetry of a distribution. Curran et al. (1996) state that excessive skewness occurs when values exceed $+/-2$. Kurtosis is a measure of how "peaked" a distribution is relative to the normal distribution. Positive kurtosis indicates more clustered distribution and negative kurtosis values indicate more dispersion (flatness). If the kurtosis exceeds 2 standard errors the data is likely non-normal (Curran et al, 1996). Table 12 below summarizes descriptive statistics for all of the survey measures used and includes minimum, maximum, mean, standard deviation, skewness and kurtosis. Note that in all cases the summated scales do not exceed the limits for skewness or kurtosis and are considered acceptably normal in their distributions to be used in the statistical analysis. The list below explains the variable names (i.e. a legend) for Table 12 (and used throughout the remaining analysis):

- KMS - KM Strategy and Planning (summated scale)

- SOL - Support for Organizational Learning (summated scale)

- PMP - Process Management Related Practices (summated scale)

- OPM - Organizational Performance Management Related Practices (summated scale)

- IMP - Information Management Related Practices (summated scale)

- TRP - Training Related Practices (summated scale) 
- HRP - Human Resource Related Practices (summated scale)

- IST - Information Systems and Technology Support (summated scale)

- OSS - Advanced Operational Support Systems (summated scale)

- KA - Quality of Knowledge Acquisition Processes (summated scale)

- KG - Quality of Knowledge Generation and Validation Processes (summated scale)

- KS - Quality of Knowledge Sharing and Transfer Processes (summated scale)

- KU - Quality of Knowledge Utilization and Application Processes (summated scale)

- KR - Quality of Knowledge Retention and Storage Processes (summated scale)

- SOC - Supportive Organizational Culture (summated scale)

- $\mathrm{OE}$ - Organizational Effectiveness (summated scale)

Table 12. Descriptive Statistics for Constructs

\begin{tabular}{|c|c|c|c|c|c|c|c|c|c|}
\hline \multirow{2}{*}{ Item } & \multirow{2}{*}{\begin{tabular}{|c|}
$\mathbf{N}$ \\
Statistic \\
\end{tabular}} & \multirow{2}{*}{$\begin{array}{c}\text { Min } \\
\text { Statistic }\end{array}$} & \multirow{2}{*}{$\begin{array}{c}\text { Max } \\
\text { Statistic }\end{array}$} & \multirow{2}{*}{$\begin{array}{c}\text { Mean } \\
\text { Statistic }\end{array}$} & \multirow{2}{*}{$\begin{array}{l}\text { Std. Dev. } \\
\text { Statistic }\end{array}$} & \multicolumn{2}{|c|}{ Skewness } & \multicolumn{2}{|c|}{ Kurtosis } \\
\hline & & & & & & Statistic & S.E. & Statistic & S.E. \\
\hline KMS & 123 & 1.4 & 5.0 & 3.458 & .8434 & -.010 & .218 & -.666 & .433 \\
\hline SOL & 124 & 2.5 & 5.0 & 4.125 & .5946 & -.334 & .217 & -.599 & .431 \\
\hline PMP & 124 & 2.5 & 5.0 & 3.724 & .6493 & .084 & .217 & -.647 & .431 \\
\hline OPM & 123 & 2.9 & 5.0 & 4.192 & .4970 & -.067 & .218 & -.738 & .433 \\
\hline $\mathrm{IMP}$ & 124 & 2.5 & 5.0 & 4.353 & .4765 & -.558 & .217 & .727 & .431 \\
\hline TRP & 124 & 2.5 & 5.0 & 4.234 & .4930 & -.296 & .217 & .585 & .431 \\
\hline $\mathrm{HRP}$ & 124 & 1.8 & 5.0 & 3.374 & .7933 & .045 & .217 & -.923 & .431 \\
\hline IST & 122 & 1.0 & 5.0 & 3.710 & .7727 & -1.094 & .219 & 2.117 & .435 \\
\hline OSS & 120 & 1.0 & 5.0 & 3.266 & .8575 & -.102 & .221 & -.239 & .438 \\
\hline $\mathrm{KA}$ & 124 & 1.5 & 5.0 & 3.538 & .6796 & -.159 & .217 & .520 & .431 \\
\hline $\mathrm{KG}$ & 123 & 2.0 & 5.0 & 3.580 & .5475 & .148 & .218 & .600 & .433 \\
\hline $\mathrm{KS}$ & 123 & 2.0 & 5.0 & 3.645 & .6632 & .293 & .218 & -.534 & .433 \\
\hline $\mathrm{KU}$ & 124 & 2.6 & 5.0 & 3.886 & .5422 & .139 & .217 & -.249 & .431 \\
\hline $\mathrm{KR}$ & 123 & 2.4 & 5.0 & 4.096 & .5522 & -.588 & .218 & .049 & .433 \\
\hline SOC & 123 & 2.4 & 4.9 & 3.867 & .5998 & -.396 & .218 & -.573 & .433 \\
\hline $\mathrm{OE}$ & 123 & 2.5 & 5.0 & 3.894 & .5192 & -.116 & .218 & .114 & .433 \\
\hline
\end{tabular}




\section{Data Entry and Preparation}

Data was compiled from the surveys and coded manually into MS Excel. All completed surveys were verified once entered to confirm correct coding. As the changes made to the final survey affected on a small number of questions, the trial survey data could be used as part of the final data set. This resulted in a small amount of missing data for the new questions that were added.

A systematic correction of reverse coded questions (these are identified Section 3.2) was made. This was done using software search/replace function in a rigorous manner to eliminate human error. As an ordinal 5-point Likert scale was used in all cases, the following translations were applied to those specific responses: all 1's were converted to 5's and vice-versa; and all 2's were converted to 4's and vice-versa. Summated scales were calculated using the "average (item list)" function which ignores missing values.

\subsection{Coding of Multiple Unit Responses}

There are anywhere from 1 to 8 operating reactors at an individual NPP station. However, there is typically only one organization or "plant site operation", whether a single or multi-unit station. There are also cases where a single owner operates multiple NPP stations. The intended sampling frame is the entire global population of 204 NPP station (site) organizations. Thus, despite the reactor performance data being available 
on a reactor basis, it was only practical for the survey to be designed to solicit a response from each site organization.

Figure 64 shows a possible fleet configuration that helps to visualize some of the various cases that show how NPP operators may be organized. Each light grey box represents an organizational (business) unit, usually each at a different site. The darker grey box highlights a special case where at a single site, two NPP station organizations exist. This may happen when NPPs were built at the same site many years apart and with difference reactor types.

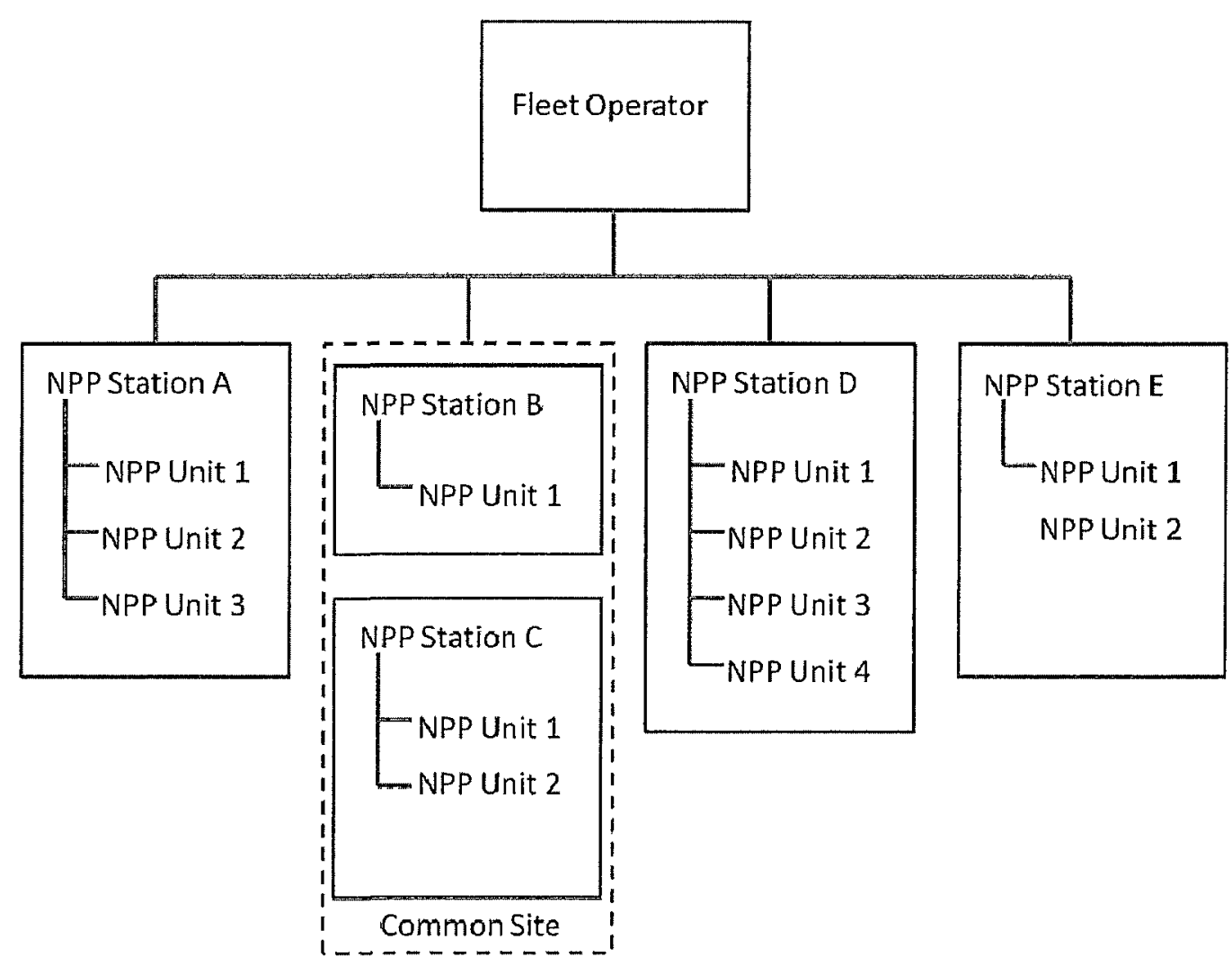

Figure 64. Example of possible NPP fleet configuration 
As a result of the variations in station organizations, there were differences in the survey responses that affect coding of the data. Some specific cases need to be considered and an explanation of response coding used in each case is provided. They are:

1. Cases where the station has multiple units and a single response was intended to represent all units at the station.

2. Cases where an owner operates multiple stations (i.e. a fleet) and it was indicated that the single response was to be used for all the stations represented because operating policy and practices are standardized across the organization.

3. Cases where multiple responses were received for a single unit (reactor) station.

4. Cases where a single response was received for one unit of a multiple unit station.

5. Cases where a response was received for one or more individual unit(s) of a multiple unit station in addition to a station wide or fleet wide response

The data was coded for each of these respective cases using consistent rules in the following manner:

1. For cases where the station has multiple units and a single response was intended to represent all units at the station, the average of the operating performance data for all of the reactor units at the station was used.

2. For cases where an owner operates multiple stations (i.e. a fleet) and it was indicated that the single response was to be used for all the stations represented because operating policy and practices are standardized across the organization, 
the organizational data was applied to the operating performance data for each station, and in the case of multiple unit stations, rule 1 also applies.

3. For cases where multiple responses were received for a single unit (reactor) station, an average of the organizational data responses was used (this results in scalar data instead of ranked data) so the station data remained as a single response case in the data set.

4. For cases where a single response was received for one unit of a multiple unit station, the organizational data was assumed to apply to the entire station on the basis that it is the same organization and as per rule 1 . above, the production data was averaged for all the units at the station.

5. For cases where a response was received for one or more individual unit(s) of a multiple unit station in addition to a station wide or fleet wide response, an average of the responses was used as the organizational data for the station (this results in scalar data instead of ranked data). Equal weighting was given to the unit level response as to the station-wide or fleet response to create a single response case in the dataset for the station.

\subsection{Data Quality and Screening}

This section describes the various steps take to ensure data quality including data screening. For this research, the "rules of thumb" outlined by Hair et al. (2006) for methods of detection and interpretation of univariate, bivariate, and multivariate outliers was applied. Outliers were carefully described, profiled and categorized prior to making 
decisions on retention or deletion. Univariate outliers were identified for each variable as candidates for removal however were only deleted if bivariate or multivariate considerations indicated a problem. For bivariate outliers, scatter-plots (see Appendix G) were used and again only more serious cases removed unless high-level multi-variate analysis indicated a problem. For multivariate outliers, Mahalanobis $\mathrm{D}^{2}$ measure was used as recommended by Hair et al. (2006).

To get a cursory understanding of the nature of correlations, bivariate profiling of relationships using a scatter-plot matrix of selected indicators was used (see Appendix G). To confirm the statistical assumptions, checks were performed to confirm the four important statistical assumptions (Hair et. al., 2006): normality, homoscedasticity, linearity, and absence of correlated errors. A brief discussion of each follows:

- Univariate normality was examined for each factor, and for those determined to be non-normal, the acceptability (given the sample size, which when increased decreases the detrimental effect of non-normality) was determined based on an examination of two measures of shape: kurtosis (i.e. the peakedness or flatness of the data) and the skewness (i.e. how balanced or symmetric the data is). Refer back to Section 5.5, Table 12 for kurtosis and skew values for each factor.

- Homoscedasticity refers to the assumption that dependent variables(s) exhibit equal levels of variance across the range of predictor variable(s) and it is desirable because the variance of the dependent variable being explained in the relationship should not be concentrated in only a limited range of the 
independent values (Hair et al., 2006). Tests of homoscedasticity included both graphical (i.e. standardized residuals) and statistical tests (i.e. multi-group path model analysis at low vs high range of the predictor). In the case of operating performance data, transformations were applied to correct for homoscedasticity (i.e. scaling and normalization as described in Sections 5.3 and 5.4).

- Departures from linearity were investigated using scatter-plots and by the examination of residuals. If non-linearity was detected, explicit models for the relationship were tested and a transformation was applied where possible to linearize the variable.

- Absence of correlated errors refers to the basic assumption that errors are uncorrelated and there is a need to confirm this. Possible causes of correlated errors were identified and an analysis of variables was conducted for each causal factor. The use of scatter-plots and the examination of residuals was a key tool for this purpose. The use of actual performance data obtained independently of the survey responses also minimizes likelihood of correlated errors.

\subsection{Missing Data}

The approach described by Hair et al. (2006) to identifying and selecting missing data was followed, and this involved a 4-step (paraphrased) process:

i. the type of missing data was determined, including what is ignorable or nonignorable missing data, and whether it is due to known or unknown missing data processes etc. 
ii. the extent of missing data was determined, and whether it is low enough so as not to affect the results, even if it operates in a non-random manner, and choose (based on a number of considerations) which cases to delete or retain.

iii. the randomness of the missing data processes was diagnosed (i.e. missing at random versus missing completely at random).

iv. the imputation method was selected.

Table 13 below summarizes the missing data counts and percentages for each of the indicator variables. The total count, mean, and standard deviation are also provided as univariate statistics. Of the 114 measures the majority have less than $10 \%$ missing data and only 10 cases had missing data between $10-16 \%$. Only a single indicator, item 52 with variable "AOSSB2i" has an extremely high missing value count (241) or 93.1\%. This variable was for Question 2i on advanced operational support systems and asked about the extent of "other (i.e. AOSS) systems in use" and it is left out of the analysis. Only 16 case of 114 total had \%-missing levels between 5-10\%. This meant the vast majority of cases had less than $5 \%$ missing data ( 87 out of 114 or $76 \%$ ).

In addition to Table 13, SPSS "Missing Value Analysis" (MVA) function was used to look for missing data patterns. The measures associated with each construct were examined. Table 14 below shows the typical output generated. No significant issues were identified. It was concluded that the data can be assumed to be missing at random (MAR). 
Table 13. Univariate Statistics

\begin{tabular}{|c|c|c|c|c|c|}
\hline Measure & $\mathbf{N}$ & Mean & S.D. & Missing & $\%$ \\
\hline KMSa & 122 & 3.677 & .9521 & 7 & 5.4 \\
\hline KMSb & 122 & 3.725 & .9268 & 7 & 5.4 \\
\hline KMSc & 122 & 3.203 & 1.0577 & 7 & 5.4 \\
\hline KMSd & 121 & 3.394 & 1.0159 & 8 & 6.2 \\
\hline KMSe & 110 & 3.274 & 1.1178 & 19 & 14.7 \\
\hline SOLa & $\overline{123}$ & 4.028 & .8229 & 6 & 4.7 \\
\hline SOLb & 123 & 3.836 & .9071 & 6 & 4.7 \\
\hline SOLC & 123 & 4.435 & .6506 & 6 & 4.7 \\
\hline SOLd & 124 & 4.228 & .7148 & 5 & 3.9 \\
\hline $\mathrm{PMPa}$ & 123 & 4.136 & .6606 & 6 & 4.7 \\
\hline PMPb & 123 & 4.493 & .5245 & 6 & 4.7 \\
\hline PMPC & 119 & 4.014 & .7405 & 10 & 7.8 \\
\hline$\overline{\mathrm{PMPd}}$ & 123 & 3.850 & .7657 & 6 & 4.7 \\
\hline PMPe & 110 & 3.097 & 1.1111 & 19 & 14.7 \\
\hline PMPf & $\overline{108}$ & 2.461 & 1.2486 & 21 & 16.3 \\
\hline $\mathrm{IMPa}$ & 122 & 4.414 & .5921 & 7 & 5.4 \\
\hline $\mathrm{IMPb}$ & 122 & 4.537 & .5095 & 7 & 5.4 \\
\hline $\mathrm{IMPC}$ & 119 & 4.130 & .7508 & 10 & 7.8 \\
\hline IMPd & 118 & 4.398 & .5904 & 11 & 8.5 \\
\hline OPMa & 123 & 4.932 & .2487 & 6 & 4.7 \\
\hline OPMb & 123 & 4.293 & .5983 & 6 & 4.7 \\
\hline OPMc & 123 & 4.140 & .6843 & 6 & 4.7 \\
\hline OPMd & 123 & 4.463 & .5531 & 6 & 4.7 \\
\hline OPMe & 123 & 3.812 & .8352 & 6 & 4.7 \\
\hline OPMf & 123 & 4.251 & .6758 & 6 & 4.7 \\
\hline TRPa & 122 & 4.503 & .7203 & 7 & 5.4 \\
\hline TRPb & 122 & 4.217 & .6132 & 7 & 5.4 \\
\hline TRPC & 123 & 4.254 & .5530 & 6 & 4.7 \\
\hline TRPd & 119 & 3.870 & 1.0498 & 10 & 7.8 \\
\hline $\mathrm{TRPe}$ & 118 & 3.965 & .7507 & 11 & 8.5 \\
\hline $\mathrm{HRPa}$ & 123 & 3.977 & .8037 & 6 & 4.7 \\
\hline $\mathrm{HAPb}$ & 123 & 3.144 & 1.0924 & 6 & 4.7 \\
\hline HRPc & 122 & 3.036 & 1.0527 & 7 & 5.4 \\
\hline HRPd & 123 & 3.171 & 1.1543 & 6 & 4.7 \\
\hline $\mathrm{HRPe}$ & 123 & 3.530 & .8915 & 6 & 4.7 \\
\hline ISTa & 117 & 2.063 & 1.1245 & 12 & 9.3 \\
\hline ISTb & 119 & 3.589 & 1.0379 & 10 & 7.8 \\
\hline ISTC & 119 & 3.103 & 1.4682 & 10 & 7.8 \\
\hline ISTd & 121 & 4.587 & 1.0009 & 8 & 6.2 \\
\hline ISTe & 122 & 3.963 & 1.0572 & 7 & 5.4 \\
\hline ISTf & 120 & 4.101 & 1.0000 & 9 & 7.0 \\
\hline ISTg & 119 & 3.996 & 1.0830 & 10 & 7.8 \\
\hline ISTh & 110 & 2.564 & 1.1769 & 19 & 14.7 \\
\hline OSSa & 113 & 3.488 & 1.2509 & 16 & 12.4 \\
\hline OSSb & 116 & 3.237 & 1.4119 & 13 & 10.1 \\
\hline OSSc & 115 & 3.176 & 1.4591 & 14 & 10.9 \\
\hline OSSd & 119 & 3.859 & .8814 & 10 & 7.8 \\
\hline OSSe & 114 & 3.177 & 1.0446 & 15 & 11.6 \\
\hline OSSf & 119 & 3.468 & 1.0299 & 10 & 7.8 \\
\hline OSSg & 113 & 2.907 & 1.1428 & 16 & 12.4 \\
\hline OSSh & 111 & 2.920 & 1.1163 & 18 & 14.0 \\
\hline OSSi & 10 & 4.3000 & 1.33749 & 119 & 92.2 \\
\hline $\mathrm{KAa}$ & 123 & 3.542 & 1.0949 & 6 & 4.7 \\
\hline $\mathrm{KAb}$ & 114 & 3.663 & .8053 & 15 & 11.6 \\
\hline KAC & 119 & 3.156 & 1.0774 & 10 & 7.8 \\
\hline $\mathrm{KAd}$ & 124 & 3.982 & .7903 & 5 & 3.9 \\
\hline $\mathrm{KAe}$ & 111 & 3.515 & .9800 & 18 & 14.0 \\
\hline
\end{tabular}

\begin{tabular}{|c|c|c|c|c|c|}
\hline Measure & $\mathbb{N}$ & Mean & S.D. & Mlssing & $\%$ \\
\hline KAf & 106 & 3.544 & .7080 & 23 & 17.8 \\
\hline $\mathrm{KGa}$ & 123 & 3.621 & .9053 & 6 & 4.7 \\
\hline $\mathrm{KGb}$ & 122 & 4.021 & .7095 & 7 & 5.4 \\
\hline $\mathrm{KGc}$ & 116 & 4.003 & .6989 & 13 & 10.1 \\
\hline KGd & 123 & 3.620 & .7395 & 6 & 4.7 \\
\hline $\mathrm{KGe}$ & 123 & 3.526 & .7511 & 6 & 4.7 \\
\hline KGf & 122 & 2.751 & 1.0190 & 7 & 5.4 \\
\hline $\mathrm{KSa}$ & 123 & 3.530 & 1.0603 & 6 & 4.7 \\
\hline $\mathrm{KSb}$ & 123 & 3.439 & .9144 & 6 & 4.7 \\
\hline $\mathrm{KSc}$ & 123 & 3.853 & .8456 & 6 & 4.7 \\
\hline KSd & 123 & 3.472 & .9257 & 6 & 4.7 \\
\hline $\mathrm{KSe}$ & 110 & 3.988 & .8016 & 19 & 14.7 \\
\hline $\mathrm{KUa}$ & 123 & 4.196 & .6504 & 6 & 4.7 \\
\hline KUb & 123 & 3.951 & .8951 & 6 & 4.7 \\
\hline KUC & 121 & 4.026 & .6444 & 8 & 6.2 \\
\hline KUd & 122 & 3.478 & .9056 & 7 & 5.4 \\
\hline KUe & 119 & 3.789 & 1.0689 & 10 & 7.8 \\
\hline $\mathrm{KRa}$ & 121 & 4.263 & .8358 & 8 & 6.2 \\
\hline $\mathrm{KRb}$ & 122 & 4.220 & .5590 & 7 & 5.4 \\
\hline$\overline{K R c}$ & 122 & 3.644 & .9421 & 7 & 5.4 \\
\hline KRd & 120 & 4.026 & .7519 & 9 & 7.0 \\
\hline $\mathrm{KRe}$ & 122 & 4.324 & .7317 & 7 & 5.4 \\
\hline SOCa & 123 & 3.868 & .9771 & 6 & 4.7 \\
\hline $\mathrm{SOCb}$ & 123 & 3.697 & .7383 & 6 & 4.7 \\
\hline SOCC & 123 & 4.292 & .7611 & 6 & 4.7 \\
\hline SOCd & 122 & 3.443 & .9349 & 7 & 5.4 \\
\hline SOCe & 123 & 3.704 & .7970 & 6 & 4.7 \\
\hline SOCf & 123 & 3.867 & .9542 & 6 & 4.7 \\
\hline $\mathrm{SOCg}$ & 122 & 4.043 & .7005 & 7 & 5.4 \\
\hline SOCh & 123 & 3.955 & .7467 & 6 & 4.7 \\
\hline SOCi & 123 & 4.066 & .7797 & 6 & 4.7 \\
\hline $\mathrm{SOCj}$ & 123 & 4.656 & .5086 & 6 & 4.7 \\
\hline SOCk & 122 & 3.260 & 1.1991 & 7 & 5.4 \\
\hline $\mathrm{SOCl}$ & 123 & 4.239 & .5739 & 6 & 4.7 \\
\hline SOCm & 121 & 3.612 & 1.1557 & 8 & 6.2 \\
\hline OEa & 123 & 3.549 & 1.0459 & 6 & 4.7 \\
\hline $\mathrm{OEb}$ & 123 & 3.999 & .7921 & 6 & 4.7 \\
\hline$\overline{O E C}$ & 120 & 3.469 & 1.1607 & 9 & 7.0 \\
\hline OEd & 123 & 4.521 & .6056 & 6 & 4.7 \\
\hline $\mathrm{OEe}$ & 122 & 3.352 & 1.1457 & 7 & 5.4 \\
\hline OEf & 122 & 4.314 & .7749 & 7 & 5.4 \\
\hline $\mathrm{OEg}$ & 122 & 4.047 & .8325 & 7 & 5.4 \\
\hline OEh & 124 & 4.478 & .6882 & 5 & 3.9 \\
\hline $\mathrm{OEI}$ & 124 & 3.173 & .8825 & 5 & 3.9 \\
\hline $\mathrm{OEj}$ & 124 & 4.581 & .6482 & 5 & 3.9 \\
\hline OEk & 122 & 4.481 & .7497 & 7 & 5.4 \\
\hline $\mathrm{OEI}$ & 124 & 3.499 & .9140 & 5 & 3.9 \\
\hline OEm & 117 & 3.724 & .9696 & 12 & 9.3 \\
\hline OEn & 122 & 3.544 & .8830 & 7 & 5.4 \\
\hline OEo & 124 & 3.930 & .7153 & 5 & 3.9 \\
\hline OEp & 123 & 4.417 & .7455 & 6 & 4.7 \\
\hline $\mathrm{OEq}$ & 122 & 3.561 & .9931 & 7 & 5.4 \\
\hline $\mathrm{OEr}$ & 121 & 3.626 & 1.0472 & 8 & 6.2 \\
\hline OEs & 122 & 4.105 & .8902 & 7 & 5.4 \\
\hline OEt & 119 & 3.711 & .9063 & 10 & 7.8 \\
\hline OEu & 120 & 3.531 & 1.0816 & 9 & 7.0 \\
\hline $\mathrm{OEv}$ & 118 & 3.552 & 1.1343 & 11 & 8.5 \\
\hline
\end{tabular}


Table 14. Missing Value Analysis

\begin{tabular}{|c|c|c|c|c|c|}
\hline \multirow[b]{2}{*}{ Number of Cases } & \multicolumn{5}{|c|}{ Missing Patterns } \\
\hline & KMSa & KMSb & KMSc & KMSd & KMSe \\
\hline 229 & & & & & \\
\hline 15 & & & & & $\mathrm{X}$ \\
\hline 4 & & & & $\bar{X}$ & $\bar{x}$ \\
\hline 11 & $\bar{X}$ & $\mathrm{X}$ & $\bar{x}$ & $\bar{x}$ & $\bar{x}$ \\
\hline
\end{tabular}

The approach to handling missing data can influence the results of the analysis. Omitting cases with missing values can greatly reduce the effective sample size while the use of inappropriate methods for estimating the missing data can introduce bias in the parameter estimates (Hair et al., 2006). There are several approaches to handling missing data. In this study, a simple imputation scheme was applied to missing data items. If the missing data item was part of a response case for which there was a second (multiple) response, the other response case data item was used, otherwise the average value for all the item measures of the construct for that case response was used. In a few response cases all or most of the item measures of a given construct were missing and these cases were omitted from the specific analysis. This resulted in a slightly reduced " $n$ " in only a few of the analyses (i.e. usually there were no case omissions and $n=124$, however there were several instances where one case was omitted and $n=123$, and there were two or three instances where 2 cases were omitted and $n=122$ ).

\subsection{Outliers}

In order to systematically identify outliers, categorical regressions were completed for each construct. The construct measurement items were each individually regressed against the summated scale and cross-plotted for bivariate outlier analysis. A number of 
"candidate for deletion" data points were identified in each case but these were only deleted if clear evidence existed. The categorical regressions were performed for every measure against its summated scale as the dependent variable.
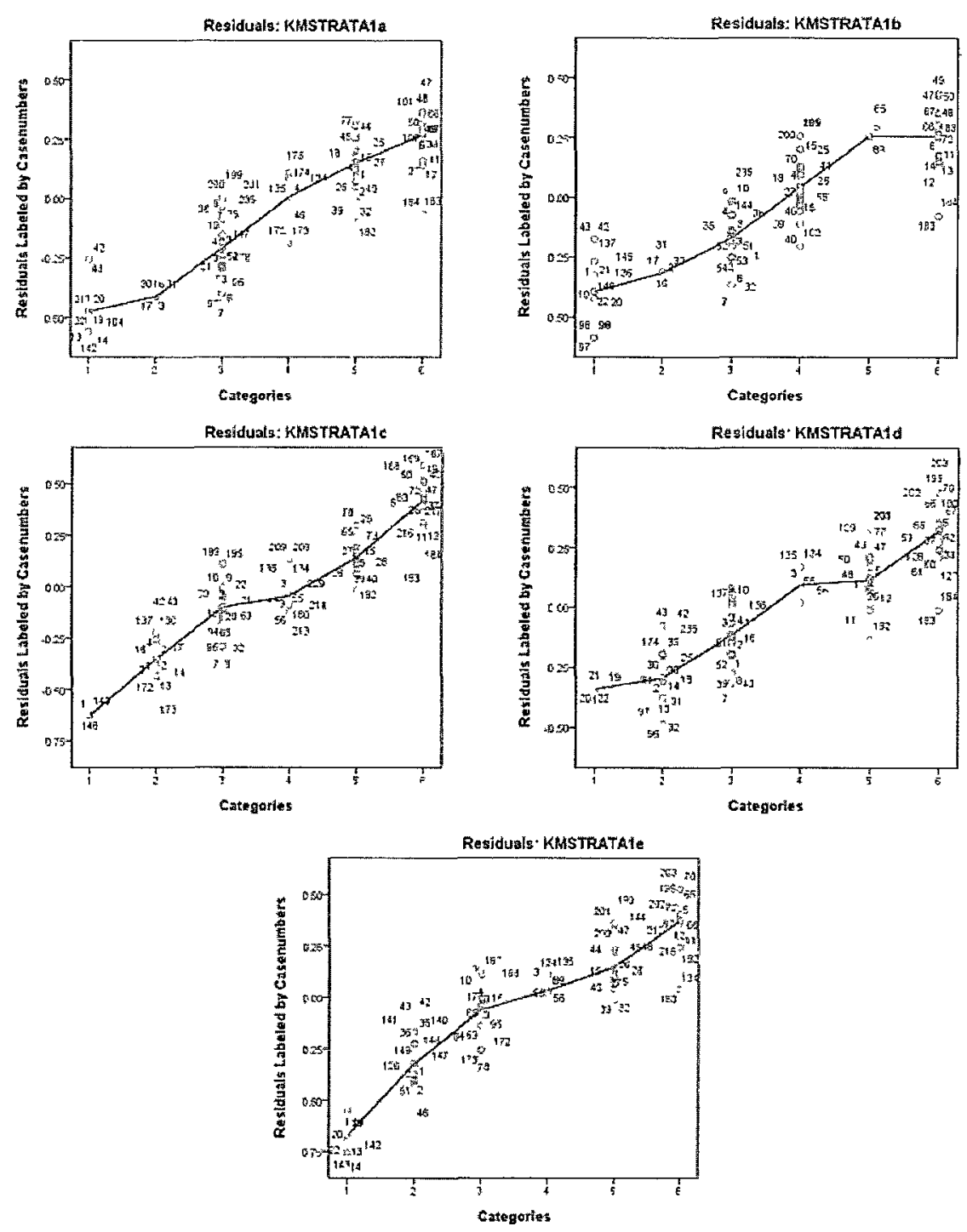

Figure 65. Example of residuals plotted by response category

The scatter plots in Figure 65 are an example with dependent variable KMS (i.e. the summated scale factor for "Support for KM Strategy and Planning"). Note that in all 
the regressions in Figure 65, individual item measures were significant at .001 or lower indicating high correlations. In this example, candidate outliers for deletion are cases 42(a,b), 43(a,b), 182(d,e), 183(a,b,d,e) and $184(a, b, d)$ but none appear to be serious outliers and they were not removed. In summary, the number of outliers removed for constructs affected were:

- PMP - 4 deletions

- IMP -13 deletions

- OPM -6 deletions

- TR -6 deletions

- IST - 28 deletions
- OSS -24 deletions

- $\mathrm{KG}-10$ deletions

- $\mathrm{KU}-5$ deletions

- $\mathrm{KR}-16$ deletions

- $\mathrm{SOC}-15$ deletions

\subsection{Reliability Screening of Measures}

A useful indication of measurement reliability is consistency of results on repeated measures. In this study, it was possible to consider a number of cases where the same individual responded at two different times (on the trial survey and on the final survey) or where two different individuals responded from the same station (i.e. a multiple case response). Thus data from the trial surveys, plus the data from stations with multiple responses provided an opportunity to confirm reliability. Table 15 below shows the percent change in scale response for these cases where data was available. Any changes greater than $20 \%$ are bolded. Note although two responses show consistent large percentage change, this was not seen across the rows of the table indicating in general, there was no pattern of inconsistent response. Further the $\%$ change seems to be a constant bias in most cases indicating a consistent response bias across most of the survey questions for the individual respondent. This is not perceived to be a problem. 
Table 15. Repeated Measure \%-Change Response

\begin{tabular}{|c|c|c|c|c|c|c|c|c|c|c|c|c|c|c|c|c|c|c|c|c|c|}
\hline & & ial v & $s \mathrm{Fil}$ & & & & & & & & ultip & le $\mathrm{C}$ & ase & Resp & jons & & & & & & \\
\hline NPP Name & 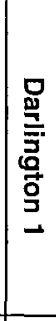 & 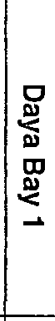 & 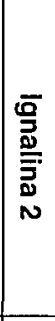 & $\begin{array}{l}-1 \\
\mathbf{0} \\
\mathbf{3} \\
\mathbf{0} \\
\mathbf{3} \\
-\end{array}$ & 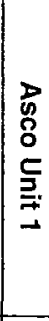 & $\begin{array}{l}8 \\
8 \\
\stackrel{8}{0} \\
\stackrel{0}{1} \\
-\end{array}$ & 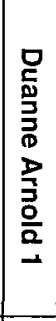 & 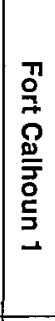 & 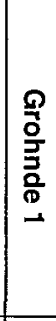 & $\begin{array}{l}\text { 을 } \\
\overline{\overline{\bar{E}}} \\
\text { 옴 } \\
\stackrel{0}{-}\end{array}$ & 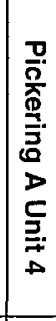 & 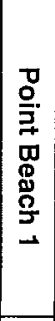 & 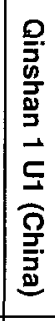 & 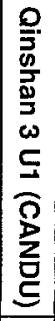 & 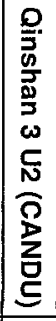 & 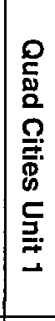 & 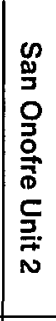 & 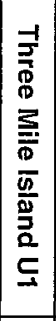 & 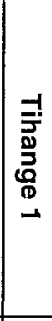 & 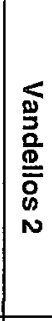 & 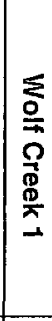 \\
\hline KM Strategy (KMS) & ثें & $\stackrel{!}{ \pm}$ & $\underset{\Phi}{2}$ & $\dot{\omega}$ & 8 & 乌ે & $\frac{1}{6}$ & $\stackrel{\infty}{\omega}$ & ț & : & $\stackrel{t}{\vec{v}}$ & 立 & ب́ & : & O & O & $\begin{array}{l}\vec{\omega} \\
\dot{\omega}\end{array}$ & $\underset{\infty}{\dot{\infty}}$ & $\overrightarrow{\dot{\Xi}}$ & $\stackrel{\omega}{\omega}$ & $\stackrel{\dot{c}}{c}$ \\
\hline $\begin{array}{l}\text { Supp for Learning } \\
\text { (SOL) }\end{array}$ & 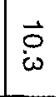 & ì & $\dot{9}$ & $\begin{array}{l}\dot{1} \\
0 \\
0\end{array}$ & $\vec{N}$ & $\bigcup_{\infty}^{\infty}$ & $\overrightarrow{0}$ & $\because$ & 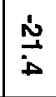 & $\because$ & $\frac{\dot{\vec{\omega}}}{\omega}$ & 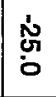 & $\overrightarrow{0}$ & $\stackrel{\dot{\Delta}}{\dot{\Xi}}$ & $\stackrel{\dot{\Xi}}{\doteq}$ & 岕 & $\underset{\omega}{\omega}$ & 今ે & $\begin{array}{l}\text { 心் } \\
\stackrel{\alpha}{\circ}\end{array}$ & 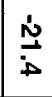 & $\begin{array}{l}\underset{\omega}{\dot{\omega}} \\
\stackrel{\omega}{2}\end{array}$ \\
\hline $\begin{array}{l}\text { Process Mgmt } \\
\text { Pract (PMP) }\end{array}$ & $\stackrel{\infty}{\infty}$ & 家 & 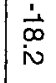 & $\begin{array}{l}\dot{1} \\
\dot{\infty} \\
\dot{\text { iv }}\end{array}$ & ज़ & $\begin{array}{l}\dot{\omega} \\
\dot{\omega} \\
\dot{\omega}\end{array}$ & 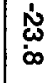 & $\vec{\infty}$ & 恿 & $\because$ & $\stackrel{\infty}{\omega}$ & $\underset{\infty}{N}$ & ب̂̉ & $\stackrel{\rho}{v}$ & $\stackrel{g}{v}$ & $\dot{\bar{\sigma}}$ & $\begin{array}{l}\tilde{\omega} \\
\dot{\omega}\end{array}$ & స્心 & $\stackrel{\infty}{v}$ & 蕞 & $\stackrel{+}{v}$ \\
\hline $\begin{array}{l}\text { Info Mgmt Pract } \\
\text { (IMP) }\end{array}$ & $\vec{\Xi}$ & $\stackrel{\dot{\vec{\prime}}}{ }$ & $\dot{\vec{\sigma}}$ & $\dot{\dot{a}}$ & $\stackrel{\phi}{\omega}$ & 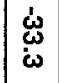 & సે. & $\begin{array}{l}\vec{v} \\
\infty\end{array}$ & : & : & ì & نั & بر & $\because$ & : & $\underset{\omega}{\omega}$ & $\vec{\omega}$ & ְִ & $\frac{\dot{\vec{t}}}{\omega}$ & ì & $\stackrel{\sim}{\dot{\omega}}$ \\
\hline $\begin{array}{l}\text { Org Perf Mgmt } \\
\text { (OPM) }\end{array}$ & $\vec{\omega}$ & 品 & O̊ & $\omega_{\infty}^{\prime}$ & $\stackrel{\varphi}{\longrightarrow}$ & $\stackrel{\dot{H}}{\dot{v}}$ & $\stackrel{\stackrel{v}{v}}{v}$ & $\vec{\forall}$ & $\stackrel{\stackrel{N}{v}}{v}$ & 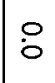 & $\underset{\infty}{\dot{\infty}}$ & : & $\because$ & $\because$ & $\because$ & 今̈ & $\stackrel{\sim}{0}_{\infty}^{1}$ & $\dot{\dot{\omega}}$ & 응 & $\frac{\dot{\vec{N}}}{\dot{N}}$ & $\stackrel{\dot{\omega}}{\dot{\omega}}$ \\
\hline $\begin{array}{l}\text { Training Rel } \\
\text { Practices } \\
\text { (TRP) }\end{array}$ & $\overrightarrow{\dot{\omega}}$ & i & $\vec{\omega}$ & $\overrightarrow{i v}$ & $\stackrel{\omega}{\omega}$ & $\mid \begin{array}{l}\overrightarrow{0} \\
\text { or }\end{array}$ & $\dot{\overrightarrow{\vec{\omega}}}$ & 응 & $\frac{\dot{\vec{D}}}{\infty}$ & $\therefore$ & $\begin{array}{l}\vec{\omega} \\
\sigma\end{array}$ & ir & $\stackrel{\circ}{\circ}$ & ب. & $\stackrel{\dot{\phi}}{\dot{ }}$ & $\underset{⿱ 亠 凶}{\omega}$ & $\vec{\circ}$ & $\dot{\vec{u}}$ & 8 & $\underset{\infty}{\dot{v}}$ & $\stackrel{\dot{\phi}}{\stackrel{g}{\nu}}$ \\
\hline $\begin{array}{l}\text { HR Rel'd Practices } \\
\text { (HRP) }\end{array}$ & $\stackrel{\circ}{\circ}$ & $\dot{\vdots}$ & $\vec{\omega}$ & 응 & $\circ$ & 岕 & ثั & $\stackrel{N}{\sim}$ & $\stackrel{\dot{\phi}}{\vec{\phi}}$ & or & ơ & $\vec{\sigma}$ & $\stackrel{\dot{b}}{\dot{\sigma}}$ & $\stackrel{\dot{\vec{t}}}{\dot{\omega}}$ & $\frac{\dot{\vec{b}}}{\dot{\omega}}$ & 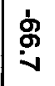 & 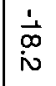 & 'b & 웅 & ì & $\underset{\omega}{\tilde{\omega}}$ \\
\hline IS\&T Support (IST) & 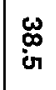 & $\stackrel{\omega}{-}$ & $\dot{\omega}_{i}^{\dot{\omega}}$ & $\stackrel{1}{0}$ & $\stackrel{\leftrightarrow}{\omega}$ & 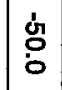 & $\stackrel{g}{y}$ & ட் & $\stackrel{f}{\circ}$ & 응 & $\stackrel{\vec{N}}{-}$ & 岂 & ڤ્ & $\dot{\vec{v}}$ & $\begin{array}{l}\dot{\vec{V}} \\
0\end{array}$ & $\stackrel{0}{v}$ & $\stackrel{p}{\omega}$ & $\overrightarrow{c \pi}$ & 点 & $\stackrel{9}{v}$ & $\stackrel{b}{\dot{D}}$ \\
\hline K Acq (KA) & $\vec{i}$ & & $\stackrel{P}{\circ}$ & $\because$ & $\dot{\vec{\phi}}$ & $\begin{array}{l}\vec{\omega} \\
i \\
\omega\end{array}$ & $\stackrel{b}{\dot{c}}$ & $\underset{\infty}{\stackrel{\infty}{\infty}}$ & $\stackrel{+}{D}$ & : & í & $\underset{N}{N}$ & $\stackrel{\tilde{\sim}}{\stackrel{\Delta}{g}}$ & $\stackrel{+}{0}$ & $\dot{1}$ & 岁 & $\stackrel{\dot{\Xi}}{\doteq}$ & $\begin{array}{l}\dot{1} \\
\dot{\omega}\end{array}$ & $\stackrel{t}{N}$ & $\stackrel{\text { N }}{\Xi}$ & $\vec{\omega}$ \\
\hline $\begin{array}{l}\text { K Gen } \\
\text { (KG) }\end{array}$ & $\stackrel{i v}{\omega}^{\omega}$ & $\overrightarrow{\dot{\omega}}$ & $\stackrel{1}{0}$ & 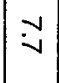 & $\begin{array}{l}\dot{\vec{\infty}} \\
\dot{\infty}\end{array}$ & $\stackrel{\sim}{\omega}$ & ! & $\vec{c}$ & 点 & $\therefore$ & $\begin{array}{l}\dot{b} \\
\dot{\sigma}\end{array}$ & $\stackrel{\vec{\omega}}{\dot{\omega}}$ & 它 & $\therefore$ & $\therefore$ & 空 & 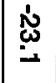 & $\dot{\infty}$ & ơ & $\frac{\dot{\omega}}{\sigma}$ & $\dot{\omega}$ \\
\hline K Sharing (KS) & $\stackrel{\dot{\omega}}{\omega}$ & $\stackrel{ }{\forall}$ & $\overrightarrow{\dot{\omega}}$ & $\stackrel{\phi}{\omega}$ & \% & $\dot{\vec{\sigma}}$ & $\dot{\vec{a}}$ & $\stackrel{\widetilde{\omega}}{\tilde{\omega}}$ & 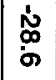 & $\dot{d}$ & ờ & $\frac{\dot{\vec{N}}}{\dot{N}}$ & $\stackrel{t}{\dot{c}}$ & í & $\begin{array}{l}\text { í } \\
\text { ir }\end{array}$ & $\stackrel{\dot{N}}{\stackrel{\Delta}{\Delta}}$ & $\stackrel{\sim}{.}$ & $\stackrel{\dot{0}}{-1}$ & 웅 & $\dot{\vec{v}}$ & $\stackrel{\frac{1}{v}}{\stackrel{c}{a}}$ \\
\hline $\begin{array}{l}\text { K Utilization } \\
\text { (KU) }\end{array}$ & or & $\underset{\sim}{\omega}$ & $\overrightarrow{\vec{\infty}}$ & $\because$ & N & $\begin{array}{l}\dot{\vec{\omega}} \\
\dot{\omega}\end{array}$ & $\underset{\omega}{\dot{\omega}}$ & $\overrightarrow{i r}$ & 悹 & 응 & $\stackrel{\dot{\vec{\Delta}}}{\dot{\infty}}$ & $\ddot{\omega}$ & $\frac{\dot{t}}{\mathrm{~N}}$ & 웅 & $\circ$ & 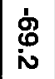 & 웅 & $\begin{array}{l}0 \\
0 \\
0\end{array}$ & $\begin{array}{l}\dot{1} \\
0 \\
0\end{array}$ & 웅 & $\vec{\omega}$ \\
\hline K Retention (KR) & $0 \pi$ & $\stackrel{\sim}{\sim}$ & $\begin{array}{l}\vec{\omega} \\
\omega\end{array}$ & $\because$ & هั & $\stackrel{\dot{\omega}}{\stackrel{\sigma}{v}}$ & $\dot{\vec{\omega}}$ & $\mid \begin{array}{l}0 \\
0 \\
\infty\end{array}$ & \pm & $\because$ & $\stackrel{\dot{\sigma}}{\stackrel{9}{v}}$ & N & $\stackrel{+}{\dot{c}}$ & $\stackrel{\dot{\vec{p}}}{\dot{\omega}}$ & $\frac{\dot{\vec{p}}}{\dot{\omega}}$ & $\frac{9}{i n}$ & 웅 & $\dot{\vec{\Xi}}$ & 今ે & $\dot{d}$ & $\overrightarrow{0}$ \\
\hline $\begin{array}{l}\text { Supp Org Culture } \\
\text { (SOC) }\end{array}$ & $\ddot{c}$ & $\overrightarrow{\mathrm{o}}$ & $\frac{1}{\omega}$ & 웅 & 衤 & $\dot{\vec{v}}$ & ì & $\vec{\partial}$ & $\underset{\substack{\dot{\infty} \\
\dot{\infty}}}{\mid}$ & 웅 & $\begin{array}{l}\dot{\vec{\omega}} \\
0\end{array}$ & $\dot{\vec{\sigma}}$ & $\dot{c}_{\dot{\sigma}}$ & $\stackrel{9}{y}$ & $\stackrel{\dot{\phi}}{v}$ & $\begin{array}{l}1 \\
5 \\
0 \\
0\end{array}$ & $\stackrel{m}{\omega}$ & $\dot{\overrightarrow{\vec{A}}}$ & 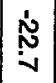 & \begin{tabular}{|l}
$\dot{\vec{\omega}}$ \\
$\dot{\omega}$
\end{tabular} & بُ \\
\hline Org Effect (OE) & $\stackrel{\circ}{=}$ & $\vec{\sigma}$ & क் & $\stackrel{N}{\infty}$ & $\stackrel{1}{\prime \prime}$ & $\frac{\dot{\omega}}{\vec{G}}$ & $\stackrel{\dot{v}}{\dot{\Xi}}$ & $\vec{T}$ & ثิ & $\stackrel{\circ}{\circ}$ & $\stackrel{\circ}{\circ}$ & in & ஸे & $\dot{\dot{\omega}}$ & $\dot{\phi}$ & 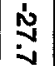 & $\underset{\infty}{+}$ & $\begin{array}{l}\infty \\
\infty \\
\infty\end{array}$ & $\begin{array}{l}\dot{\vec{\omega}} \\
\dot{\omega}\end{array}$ & i⿱宀 & $\stackrel{\vec{G}}{\leftrightarrow}$ \\
\hline
\end{tabular}

Note: changes greater than $20 \%$ have been bolded. 


\subsection{Construct Reliability}

This study was based on the use of several summated scale constructs (all measured). Construct reliability analysis was performed to ensure the integrity of each construct. Hair et al. (2006) states that reliability analysis for summated scales is commonly determined by using three measures of internal consistency amongst the scale measures: Cronbach's alpha should exceed 0.7, inter-item correlations should exceed 0.3 , and item-to-total correlations should exceed 0.5 . Using these criteria, the following section summarizes the reliability analysis on a construct-by-construct basis. SPSS "reliability analysis" function was used to perform the analysis.

\subsubsection{Support for KM Strategy and Planning (KMS)}

Table 16 summarizes the inter-item and item-to-total correlations for the summated scale KMS. All values are acceptable and the alpha is .899 for this scale. No changes were required.

Table 16. Inter-Item \& Item-to-Total Correlations for KMS

\begin{tabular}{|c|c|c|c|c|c|c|}
\hline & Cronbach's Alpha (5 items) $=.899$ & \\
\hline & KMSa & KMSb & KMSc & KMSd & KMSe & $\begin{array}{c}\text { KMS (summated } \\
\text { scale) }\end{array}$ \\
\hline KMSa & 1.000 & & & & & \\
\hline KMSb & .689 & 1.000 & & & & \\
\hline KMSc & .598 & .627 & 1.000 & & & \\
\hline KMSd & .591 & .548 & .657 & 1.000 & & \\
\hline KMSe & .675 & .591 & .654 & .782 & 1.000 & \\
\hline KMS (summated scale) & .836 & .809 & .842 & .851 & .883 & 1.000 \\
\hline
\end{tabular}




\subsubsection{Support for Organizational Learning (SOL) Practices}

Table 17 summarizes the inter-item and item-to-total correlations for the summated scale SOL. Although one inter-item correlation for is below 0.3 , the item-to-total correlations are acceptable. The alpha is .749 and is also acceptable. For this reason, no changes were made to this scale.

Table 17. Inter-Item \& Item-to-Total Correlations for SOL

\begin{tabular}{|c|c|c|c|c|c|}
\hline \multicolumn{6}{|c|}{ Cronbach's Alpha (4 items) =.749 } \\
\hline & SOLa & SOLb & SOLc & SOLd & SOL (summated scale) \\
\hline SOLa & 1.000 & & & & \\
\hline SOLb & .591 & 1.000 & & & \\
\hline SOLc & .334 & .155 & 1.000 & & \\
\hline SOLd & .512 & .539 & .430 & 1.000 & \\
\hline SOL (summated scale) & .824 & .797 & .583 & .807 & 1.000 \\
\hline
\end{tabular}

\subsubsection{Support for Process Management KM Practices (PMP)}

Table 18 summarizes the inter-item and item-to-total correlations for the summated scale PMP. All values are acceptable and the alpha is .837 for this scale. No changes were required.

Table 18. Inter-Item \& Item-to-Total Correlations for PMP

\begin{tabular}{|c|c|c|c|c|c|c|c|}
\hline \multicolumn{8}{|c|}{ Cronbach's Alpha (6 items) =.837 } \\
\hline & PMPa & PMPb & PMPc & PMPd & PMPe & PMPf & $\begin{array}{c}\text { PMP (summated } \\
\text { scale) }\end{array}$ \\
\hline PMPa & 1.000 & & & & & & \\
\hline PMPb & .452 & 1.000 & & & & & \\
\hline PMPc & .440 & .577 & 1.000 & & & & \\
\hline PMPd & .518 & .416 & .561 & 1.000 & & & \\
\hline PMPe & .343 & .347 & .476 & .417 & 1.000 & & \\
\hline PMPf & .538 & .389 & .566 & .536 & .753 & 1.000 & \\
\hline PMP (summated scale) & .685 & .625 & .770 & .737 & .798 & .887 & 1.000 \\
\hline
\end{tabular}




\subsubsection{Support for Info Management Related KM Practices (IMP)}

Table 19 summarizes the inter-item and item-to-total correlations for the summated scale IMP. The alpha is .711 for this scale. Although 2 inter-item correlations for IMPa are below 0.3, the item-to-total is acceptable. Item $\mathrm{IMPa}$ is related to the subject of design basis documentation configuration management and is deemed by NPP managers to be an important aspect of information management in NPPs (high content validity). For this reason, no changes were made to this scale.

Table 19. Inter-Item \& Item-to-Total Correlations for IMP

\begin{tabular}{|c|c|c|c|c|c|}
\hline \multicolumn{6}{|c|}{ Cronbach's Alpha (4 items) =.711 } \\
\hline & IMPa & IMPb & IMPc & IMPd & IMP (summated scale) \\
\hline IMPa & 1.000 & & & & \\
\hline IMPb & .345 & 1.000 & & & \\
\hline IMPc & .159 & .569 & 1.000 & & \\
\hline IMPd & .256 & .538 & .495 & 1.000 & \\
\hline IMP (summated scale) & .576 & .809 & .786 & .776 & 1.000 \\
\hline
\end{tabular}

\subsubsection{Support for OPM Related KM Practices}

Table 20 summarizes the inter-item and item-to-total correlations for the summated scale OPM. The alpha is .772 for this scale. Although the alpha is acceptable, three inter-item and the item-to-total correlations are lower than desirable for OPMa. However, this question asked if "independent external peer review assessments are conducted regularly" and industry expert opinion consistently rated this issue as very important to organizational performance management. For this reason this question is considered to have high content validity and was not removed from the scale. 
Table 20. Inter-Item \& Item-to-Total Correlations for OPM

\begin{tabular}{|c|c|c|c|c|c|c|c|}
\hline & \multicolumn{7}{|c|}{ Cronbach's Alpha (6 items) $=.772$} \\
\hline & OPMa & OPMb & OPMc & OPMI & OPMe & OPMf & $\begin{array}{c}\text { OPM (summated } \\
\text { scale) }\end{array}$ \\
\hline OPMa & 1.000 & & & & & & \\
\hline OPMb & .327 & 1.000 & & & & & \\
\hline OPMc & .056 & .534 & 1.000 & & & & \\
\hline OPMd & .356 & .470 & .217 & 1.000 & & & \\
\hline OPMe & .120 & .494 & .494 & .394 & 1.000 & & \\
\hline OPMf & .243 & .546 & .460 & .252 & .442 & 1.000 & \\
\hline OPM (summated scale) & .368 & .813 & .726 & .612 & .785 & .735 & 1.000 \\
\hline
\end{tabular}

6.6.6. Support for Training Related KM Practices (TRP)

Table 21 summarizes the inter-item and item-to-total correlations for the summated scale TRP. Inter-item and item-to-total correlations are lower than acceptable for 2 inter-item correlations and low for several others. The alpha is .652 for this scale and is marginal, and 2 item-to-total correlations were acceptable but low.

Table 21. Inter-Item \& Item-to-Total Correlations for TRP

\begin{tabular}{|c|c|c|c|c|c|c|}
\hline \multicolumn{7}{|c|}{ Cronbach's Alpha (5 items) =.652 } \\
\hline & TRPa & TRPb & TRPc & TRPd & TRPe & $\begin{array}{c}\text { TRP (summated } \\
\text { scale) }\end{array}$ \\
\hline TRPa & 1.000 & & & & & \\
\hline TRPb & .324 & 1.000 & & & & \\
\hline TRPc & .412 & .700 & 1.000 & & & \\
\hline TRPd & .071 & .307 & .193 & 1.000 & & \\
\hline TRPe & .262 & .572 & .559 & .048 & 1.000 & \\
\hline TRP (summated scale) & .581 & .803 & .774 & .584 & .672 & 1.000 \\
\hline
\end{tabular}

Based on a review of the questions it was decided that item TRPd was week in terms of content validity with respect to the other items and was removed. Table 22 below shows the result of this change. One inter-item correlation is lower than desired but the item-to-total correlations are more than acceptable and the alpha is .748 for this scale. No further changes were required to this scale. 
Table 22. Inter-Item \& Item-to-Total Correlations for Reduced TRP

\begin{tabular}{|c|c|c|c|c|c|}
\hline \multicolumn{6}{|c|}{ Cronbach's Alpha (4 items) $=.748$} \\
\hline & TRPa & TRPb & TRPc & TRPe & TRP (summated scale) \\
\hline TRPa & 1.000 & & & & \\
\hline TRPb & .296 & 1.000 & & & \\
\hline TRPc & .378 & .681 & 1.000 & & \\
\hline TRPe & .234 & .550 & .572 & 1.000 & \\
\hline TRP (summated scale) & .644 & .805 & .834 & .783 & 1.000 \\
\hline
\end{tabular}

\subsubsection{Support for Human Resource Related KM Practices (HRP)}

Table 23 summarizes the inter-item and item-to-total correlations for the summated scale HRP. All values are acceptable and the alpha is .849 for this scale. No changes were required.

Table 23. Inter-Item \& Item-to-Total Correlations for HRP

\begin{tabular}{|c|c|c|c|c|c|c|}
\hline \multicolumn{7}{|c|}{ Cronbach's Alpha (5 items) = .849 } \\
\hline & HRPa & HRPb & HRPc & HRPd & HRPe & $\begin{array}{c}\text { HRP (summated } \\
\text { scale) }\end{array}$ \\
\hline HRPa & 1.000 & & & & & \\
\hline HRPb & .625 & 1.000 & & & & \\
\hline HRPc & .455 & .485 & 1.000 & & & \\
\hline HRPd & .547 & .661 & .590 & 1.000 & & \\
\hline HRPe & .420 & .421 & .533 & .598 & 1.000 & \\
\hline HRP (summated scale) & .748 & .816 & .780 & .872 & .738 & 1.000 \\
\hline
\end{tabular}

\subsubsection{Effectiveness of Info Systems \& Technology Support (IST)}

Table 24 summarizes the inter-item and item-to-total correlations for the summated scale IST. Inter-item correlations are lower than acceptable for items ISTa and ISTh. ISTa has a poor item-to-total scale correlation and ISTh is marginal. It was also clear from station visits that the subject matter of question ISTa, "3D virtual reality training environments" was too new and unfamiliar to most respondents. ISTa was removed 
from the analysis but ISTh retained due to strong NPP manager opinions giving this high content validity. With this change, although some inter-item correlations were still less than 0.3 , the item-to-total correlations are acceptable (all greater than 0.5 ) and the resulting overall alpha of .812 is good. No further changes were made on the basis that high inter-item correlations are not expected in all cases for these measures.

Table 24. Inter-Item \& Item-to-Total Correlations for IST

\begin{tabular}{|c|c|c|c|c|c|c|c|c|c|}
\hline \multicolumn{8}{|c|}{ Cronbach's Alpha (8 items) $=.741$} \\
\hline & ISTa & ISTb & ISTc & ISTd & ISTe & ISTf & ISTg & ISTh & IST (summated scale) \\
\hline ISTa & 1.000 & & & & & & & & \\
\hline ISTb & .102 & 1.000 & & & & & & & \\
\hline ISTc & .457 & .347 & 1.000 & & & & & & \\
\hline ISTd & .263 & .449 & .074 & 1.000 & & & & & \\
\hline ISTe &. .002 & .241 & .364 & .577 & 1.000 & & & & \\
\hline ISTf & -.232 & .401 & .107 & .737 & .612 & 1.000 & & & \\
\hline ISTg & -.180 & .269 & .263 & .575 & .741 & .661 & 1.000 & & \\
\hline ISTh & .368 & .013 & .589 & .004 & .346 & -.035 & .114 & 1.000 & \\
\hline IST (summated scale) & .326 & .567 & .735 & .580 & .772 & .601 & .668 & .556 & \\
\hline
\end{tabular}

\subsubsection{Effectiveness of Operational Support Systems (OSS)}

Table 25 summarizes the inter-item and item-to-total correlations for the summated scale OSS. Due to a high number of missing values, OSSi was not considered an acceptable measure and was removed from the analysis. Of the remaining items, although 4 inter-item correlations are lower than 0.3 , the item-to-total correlations are acceptable (all greater than 0.5) and the overall alpha is good. No further changes were made on the basis that high inter-item correlations are not expected in all cases for these measures. 
Table 25. Inter-Item \& Item-to-total Correlations for OSS

\begin{tabular}{|c|c|c|c|c|c|c|c|c|c|}
\hline \multicolumn{8}{|c|}{ Cronbach's Alpha (9 items) $=.860$} \\
\hline & OSSa & OSSb & OSSc & OSSd & OSSe & OSSf & OSSg & OSSh & OSS (summaied scale) \\
\hline OSSa & 1.000 & & & & & & & & \\
\hline OSSb & .680 & 1.000 & & & & & & & \\
\hline OSSc & .457 & .800 & 1.000 & & & & & & \\
\hline OSSd & .608 & .542 & .496 & 1.000 & & & & & \\
\hline OSSe & .600 & .477 & .458 & .570 & 1.000 & & & & \\
\hline OSSf & .457 & .400 & .434 & .553 & .365 & 1.000 & & & \\
\hline OSSg & .318 & .242 & .168 & .373 & .294 & .346 & 1.000 & & \\
\hline OSSh & .394 & .247 & .316 & .381 & .419 & .603 & .519 & 1.000 & \\
\hline OSS (summated scale) & .783 & .792 & .750 & .767 & .719 & .708 & .559 & .661 & \\
\hline
\end{tabular}

\subsubsection{Quality of Knowledge Acquisition (KA) Processes}

Table 26 summarizes the inter-item and item-to-total correlations for the summated scale KA. Inter-item correlations are lower than 0.3 for KAa's correlations. The itemto-total correlations are acceptable (i.e. $>0.5$ ) except for KAa. Although the overall alpha is acceptable at .748 , item KAa is removed from the analysis and the results are summarized in Table 27. In hindsight the wording of this question "the organization has difficulty finding and hiring appropriately qualified graduates" is not "close" in terms of content validity with the other KA measures. With this change, alpha improves to .809 and although 2 inter-item correlations are low, the item-to-total values are well above .5 . No further changes were required.

Table 26. Inter-Item \& Item-to-Total Correlations for KA

\begin{tabular}{|c|c|c|c|c|c|c|c|}
\hline \multicolumn{8}{|c|}{ Cronbach's Alpha (6 items) $=.748$} \\
\hline KAa & KAa & KAb & KAc & KAd & KAe & KAf & KA (summated scale) \\
\hline KAb & 1.000 & & & & & & \\
\hline KAc & .096 & 1.000 & & & & & \\
\hline KAd & .108 & .179 & 1.000 & & & & \\
\hline KAe & .066 & .617 & .141 & 1.000 & & & \\
\hline KAf & .041 & .560 & .448 & .736 & 1.000 & & \\
\hline KA(summated scale) & .221 & .514 & .429 & .590 & .698 & 1.000 & \\
\hline
\end{tabular}


Table 27. Inter-Item \& Item-to-Total Correlations for Reduced KA

\begin{tabular}{|c|c|c|c|c|c|c|}
\hline \multicolumn{7}{|c|}{ Cronbach's Alpha (5 items) $=.809$} \\
\hline & KAb & KAC & KAd & $\mathrm{KAe}$ & KAf & $\begin{array}{c}\text { KA (summated } \\
\text { scale) }\end{array}$ \\
\hline $\mathrm{KAb}$ & 1.000 & & & & & \\
\hline KAC & .179 & 1.000 & & & & \\
\hline KAd & .617 & .141 & 1.000 & & & \\
\hline $\mathrm{KAe}$ & .560 & .448 & .736 & 1.000 & & \\
\hline KAf & .514 & .429 & .590 & .698 & 1.000 & \\
\hline KA (summated scale) & .725 & .626 & .773 & .894 & .823 & 1.000 \\
\hline
\end{tabular}

\subsubsection{Quality of Knowledge Generation (KG) Processes}

Table 28 summarizes the inter-item and item-to-total correlations for the summated scale KG. Although inter-item correlations are lower than 0.3 for $\mathrm{KGf} \leftarrow \rightarrow \mathrm{KGc}, \mathrm{KGf}$ $\leftrightarrow \mathrm{KGd}, \mathrm{KGf} \leftarrow \rightarrow \mathrm{KGe}, \mathrm{KGe} \leftarrow \rightarrow \mathrm{KGc}$ and $\mathrm{KGc} \leftarrow \rightarrow \mathrm{KGa}$, the item-to-total correlations are acceptable (all $>.5$ ) and the overall alpha is acceptable at .758 .

Table 28. Inter-Item \& Item-to-Total Correlations for KG

\begin{tabular}{|c|c|c|c|c|c|c|c|}
\hline \multicolumn{8}{|c|}{ Cronbach's Alpha (6 items) $=.758$} \\
\hline & KGa & KGb & KGc & KGd & KGe & KGf & $\begin{array}{c}\text { KG (summated } \\
\text { scale) }\end{array}$ \\
\hline KGa & 1.000 & & & & & & \\
\hline KGb & .415 & 1.000 & & & & & \\
\hline KGC & .220 & .364 & 1.000 & & & & \\
\hline KGd & .352 & .508 & .300 & 1.000 & & & \\
\hline KGe & .539 & .398 & .238 & .443 & 1.000 & & \\
\hline KGf & .421 & .368 & .197 & .238 & .275 & 1.000 & \\
\hline KG (summated scale) & .748 & .733 & .536 & .675 & .703 & .662 & 1.000 \\
\hline
\end{tabular}

\subsubsection{Quality of Knowledge Sharing \& Transfer (KS) Processes}

Table 29 summarizes the inter-item and item-to-total correlations for the summated scale KS. The inter-item and item-to-total correlations are acceptable and the alpha is .795 for this scale. No changes were required. 
Table 29. Inter-Item \& Item-to-Total Correlations for KS

\begin{tabular}{|c|c|c|c|c|c|c|}
\hline & $\mathrm{KSa}$ & $\mathrm{KSb}$ & $\mathrm{KSc}$ & $\mathrm{KSd}$ & $\mathrm{KSe}$ & $\begin{array}{c}\text { KS (summated } \\
\text { scale) }\end{array}$ \\
\hline $\mathrm{KSa}$ & 1.000 & & & & & \\
\hline $\mathrm{KSb}$ & .572 & 1.000 & & & & \\
\hline $\mathrm{KSc}$ & .308 & .360 & 1.000 & & & \\
\hline $\mathrm{KSd}$ & .527 & .554 & .441 & 1.000 & & \\
\hline KSe & .366 & .311 & .530 & .428 & 1.000 & \\
\hline KS (summated scale) & .776 & .764 & .688 & .800 & .681 & 1.000 \\
\hline
\end{tabular}

\subsubsection{Quality of Knowledge Utilization (KU) Processes}

Table 30 summarizes the inter-item and item-to-total correlations for the summated scale KU. Although inter-item correlations are low for $\mathrm{KUe} \leftrightarrow \rightarrow \mathrm{KUd}$, KUe $\leftrightarrow \rightarrow$ KUc, KUe $\leftrightarrow$ KUa, KUc $\leftrightarrow$ KUb and KUa $\leftrightarrow$ KUb, the item-to-total correlations are acceptable (all $>.5$ ) and the overall alpha is low but acceptable (i.e. greater than 0.6 but less than 0.7 ). No changes were made to this scale as all the item questions were deemed to have high content validity.

Table $30 . \quad$ Inter-Item \& Item-to-Total Correlations for KU

\begin{tabular}{|c|c|c|c|c|c|c|}
\hline \multicolumn{7}{|c|}{ Cronbach's Alpha (5 items) $=.651$} \\
\hline & KUa & KUb & KUc & KUd & KUe & $\begin{array}{c}\mathrm{KU} \text { (summated } \\
\text { scale) }\end{array}$ \\
\hline KUa & 1.000 & & & & & \\
\hline KUb & .195 & 1.000 & & & & \\
\hline KUc & .336 & .214 & 1.000 & & & \\
\hline KUd & .306 & .316 & .516 & 1.000 & & \\
\hline $\mathrm{KUe}$ & .232 & .470 & .199 & .097 & 1.000 & \\
\hline KU (summated scale) & .570 & .712 & .634 & .667 & .675 & 1.000 \\
\hline
\end{tabular}

\subsubsection{Quality of Knowledge Retention \& Storage (KR) Processes}

Table 31 summarizes the inter-item and item-to-total correlations for the summated scale KR. Although 3 inter-item correlations are low, the item-to-total correlations are 
acceptable (all $>.5$ ) and the overall alpha is .773 and is acceptable (i.e. greater than 0.7 ). No changes were made.

Table 31. Inter-Item \& Item-to-Total Correlations for KR

\begin{tabular}{|c|c|c|c|c|c|c|}
\hline \multicolumn{7}{|c|}{ Cronbach's Alpha (5 items) $=.773$} \\
\hline & KRa & KRb & KRc & KRd & $\mathrm{KRe}$ & $\begin{array}{c}\text { KR (summated } \\
\text { scale) }\end{array}$ \\
\hline KRa & 1.000 & & & & & \\
\hline KRb & .438 & 1.000 & & & & \\
\hline KRc & .370 & .518 & 1.000 & & & \\
\hline KRd & .201 & .415 & .284 & 1.000 & & \\
\hline KRe & .754 & .481 & .497 & .277 & 1.000 & \\
\hline KR (summated scale) & .761 & .743 & .756 & .579 & .823 & 1.000 \\
\hline
\end{tabular}

\subsubsection{Supportive Organizational Culture (SOC)}

Table 32 summarizes the inter-item and item-to-total correlations for the summated scale SOC. Only item SOCc has an item-to-total correlation below 0.5 and for this reason is removed. Once SOCc is omitted, there are 4 other inter-item correlations below 0.3 but this is considered acceptable given the item-to-total correlations are above 0.5 and the alpha is high. No further changes were made.

Table 32. Inter-Item \& Item-to-Total Correlations for SOC

\begin{tabular}{|c|c|c|c|c|c|c|c|c|c|c|c|c|c|c|}
\hline \multicolumn{15}{|c|}{ Cronbach's Alpha (5 items) = .902 } \\
\hline & SOCa & SOCb & SOCc & socd & soce & socf & $\mathrm{SOCg}$ & SOCh & SOCi & $S O C \mathbf{j}$ & SOCk & SOCl & $\mathrm{sOCm}$ & SOC* \\
\hline SOCa & 1.000 & & & & & & & & & & & & & \\
\hline SOCb & .592 & 1.000 & & & & & & & & & & & & \\
\hline SOCc & .161 & .372 & 1.000 & & & & & & & & & & & \\
\hline SOCd & .537 & .486 & .114 & 1.000 & & & & & & & & & & \\
\hline $\mathrm{SOCe}$ & .348 & .476 & .380 & .628 & 1.000 & & & & & & & & & \\
\hline SOCf & .555 & .645 & .201 & .575 & .515 & 1.000 & & & & & & & & \\
\hline SOCg & .528 & .568 & .270 & .584 & .450 & .792 & 1.000 & & & & & & & \\
\hline SOCh & 490 & .558 & .351 & .537 & .577 & .712 & .731 & 1.000 & & & & & & \\
\hline SOCi & .426 & .375 & .476 & .407 & .373 & .363 & .502 & .469 & 1.000 & & & & & \\
\hline SOCj & .383 & .588 & .417 & .368 & .334 & .507 & .552 & .529 & .473 & 1.000 & & & & \\
\hline SOCk & .442 & .302 & -.168 & .521 & .289 & .491 & .406 & .395 & .224 & .211 & 1.000 & & & \\
\hline SOCl & .427 & .438 & .259 & .513 & .427 & .377 & .438 & .556 & .344 & .438 & .351 & 1.000 & & \\
\hline $\mathrm{SOCm}$ & .344 & .348 & -.082 & .622 & .566 & .440 & .358 & .341 & .155 & .147 & .609 & .446 & 1.000 & \\
\hline SOC $^{*}$ & .712 & .738 & .361 & .796 & .716 & .812 & .792 & .794 & .596 & .612 & .632 & .654 & .652 & 1.000 \\
\hline
\end{tabular}




\subsubsection{Organizational Effectiveness (OE)}

This scale has a large number of items (22 in total) and cannot easily be shown on a page. High inter-item correlations were not expected as there are various dimensions of "effectiveness" including safety, production, and equipment reliability that may not correlate closely but capture the scope of this formative construct (i.e. it is not unidimensional). This explains why a large number of inter-item correlations are below 0.3. However, only 3 items have very low item-to-total correlations (ie. below .4). Of these, items OEd and OEj were close to .4 and were retained. The third, question OEc was reviewed and deemed to be easily miss-interpreted and was removed from the analysis. With this item removed alpha is .904 and no further changes were made. Table 33 summarizes inter-item correlations of this scale.

Table 33. Summary Statistics for OE Summated Scale

\begin{tabular}{|c|c|c|c|c|c|c|c|}
\hline \multicolumn{8}{|c|}{ Cronbach's Alpha (21 items) $=.904$} \\
\hline & Mean & Minimum & Maximum & Range & $\begin{array}{l}\text { Maximum / } \\
\text { Minimum }\end{array}$ & Variance & $\mathrm{N}$ of Items \\
\hline $\begin{array}{c}\text { Inter-Item } \\
\text { Correlations }\end{array}$ & .330 & -.116 & .771 & .887 & -6.639 & .031 & 22 \\
\hline
\end{tabular}

\subsubsection{Discriminant Validity}

Discriminant validity is the extent to which a construct discriminates from other constructs. It ensures a construct is able to account for more of the variance of the observed variables associated with it than the variance caused by either measurement 
error (included unmeasured influences) or other constructs within the conceptual framework (Farrel, 2009). The approach used in this study to confirm discriminant validity is a widely used approach developed by Fornell and Larcker (1981) and adapted by Farrel (2009) based on the correlation matrix of all the constructs in the model. Essentially the approach is to confirm the sum of squared factor loadings for each construct exceeds the square of each of the corresponding correlations. Table 34 presents this data and confirms the criteria is met.

Table 34. Squared Correlation Matrix \& Discriminant Validity

\begin{tabular}{||c|c|c|c|c|c|c|c|c|c|c|c|c|c|c|c|c|c|}
\hline & KMS & SOL & PMP & IMP & OPM & TRP & HRP & IST & OSS & KA & KG & KS & KU & KR & SOC & OE & OP \\
\hline KMS & 0.676 & & & & & & & & & & & & & & & & \\
\hline SOL & 0.248 & 0.604 & & & & & & & & & & & & & & & \\
\hline PMP & 0.346 & 0.305 & 0.704 & & & & & & & & & & & & & & \\
\hline IMP & 0.065 & 0.047 & 0.180 & $\mathbf{0 . 7 3 4}$ & & & & & & & & & & & & & \\
\hline OPM & 0.004 & 0.072 & 0.473 & 0.256 & 0.777 & & & & & & & & & & & & \\
\hline TRP & 0.151 & 0.215 & 0.524 & 0.404 & 0.244 & 0.724 & & & & & & & & & & & \\
\hline HRP & 0.291 & 0.349 & 0.676 & 0.062 & 0.142 & 0.162 & $\mathbf{0 . 7 2 4}$ & & & & & & & & & & \\
\hline IST & 0.001 & 0.015 & 0.165 & 0.040 & 0.127 & 0.005 & 0.047 & 0.767 & & & & & & & & & \\
\hline OSS & 0.002 & 0.108 & 0.293 & 0.038 & 0.130 & 0.053 & 0.065 & 0.410 & 0.748 & & & & & & & & \\
\hline KA & 0.095 & 0.245 & 0.539 & 0.153 & 0.311 & 0.219 & 0.270 & 0.099 & 0.204 & 0.789 & & & & & & & \\
\hline KG & 0.159 & 0.197 & 0.543 & 0.195 & 0.255 & 0.249 & 0.228 & 0.099 & 0.155 & 0.615 & 0.684 & & & & & & \\
\hline KS & 0.262 & 0.415 & 0.612 & 0.205 & 0.239 & 0.320 & 0.416 & 0.076 & 0.118 & 0.442 & 0.480 & 0.742 & & & & & \\
\hline KU & 0.132 & 0.171 & 0.462 & 0.345 & 0.157 & 0.226 & 0.239 & 0.179 & 0.168 & 0.310 & 0.441 & 0.364 & 0.668 & & & & \\
\hline KR & 0.298 & 0.338 & 0.554 & 0.116 & 0.049 & 0.300 & 0.417 & 0.018 & 0.070 & 0.262 & 0.323 & 0.483 & 0.386 & 0.733 & & & \\
\hline SOC & 0.367 & 0.506 & 0.586 & 0.120 & 0.072 & 0.230 & 0.440 & 0.056 & 0.073 & 0.301 & 0.417 & 0.465 & 0.352 & 0.464 & 0.787 & & \\
\hline OE & 0.321 & 0.183 & 0.542 & 0.203 & 0.055 & 0.181 & 0.257 & 0.094 & 0.104 & 0.249 & 0.347 & 0.372 & 0.507 & 0.504 & 0.497 & 0.750 & \\
\hline OP & 0.000 & 0.005 & -.042 & 0.014 & 0.020 & 0.007 & 0.004 & 0.009 & 0.022 & 0.025 & 0.024 & 0.023 & 0.012 & 0.003 & 0.006 & 0.035 & 0.728 \\
\hline
\end{tabular}

Note 1: Diagonals should be greater than corresponding rows and columns (Fornell and Larcker, 1981 and adapted by Farrel, 2009) to confirm adequate discriminant validity. Note 2: Bolded diagonals are the proportion of each variable's variance that is explained (i.e. shared variance) and can be defined as the sum of squared factor loadings calculated using SPSS factor analysis. Values under the diagonals are the squared standardized correlations from the correlation matrix. 


\section{Linean $\mathbb{R e g r e s s i o n}$ Assessments}

To begin to investigate the nature of the possible links between specific KM practices and the quality of knowledge processes (both overall and against its individual dimensions), multiple linear regressions were performed. This helped to explore the nature and strength of the relationships between the various sub-constructs of "support for KM practices" and "quality of K-processes", both as a composite summated scale and for each of its constructs: knowledge acquisition, knowledge sharing and transfer, knowledge retention and storage, and knowledge utilization and application. These were examined to see if any significant associations exist. Similarly, multiple linear regressions were used to explore possible direct relationships between specific knowledge processes and organizational performance (both organizational effectiveness and operational performance dimensions).

In the regressions that follow, significance levels of 0.05 were used as a cut-off and upper and lower bounds of the $95 \%$ confidence interval were determined not to include zero for a relationship to be considered supported in the data. Significance results of interest are in bolded text and are discussed in the interpretations. In most cases a scatter-plot to show the residuals provides a visual indication of the data fit for the regression and confirms assumptions about homoscedasticity. Also, observed power (i.e. defined by Hair et al. as the probability of detecting as statistically significant a specified level of $R^{2}$ (the coefficient of determination) at a specific significance level for 
a specific sample size) is reported only when it was found to be below the recommended 0.8 level.

\subsection{Linking Support for KMPs and OTSs to Quality of KA Processes}

Table 35 summarizes the significant results from the regression analysis of all the KMPs and OTSs combined on KA after non-significant factors were eliminated one at a time.

Table 35. Regression OPM+HRP+OSS $\rightarrow \mathrm{KA}$

\begin{tabular}{|c|c|c|c|c|c|c|}
\hline Dependent Variable: KA & & & \multicolumn{2}{|c|}{$95 \%$ Confidence Interval } \\
\cline { 5 - 7 } Parameter & $\mathbf{B}$ & Std. Error & $\mathbf{t}$ & Sig. & Lower Bound & Upper Bound \\
\hline OPM & .415 & .106 & 3.906 & .000 & .205 & .626 \\
\hline HRP & .290 & .059 & 4.893 & .000 & .172 & .407 \\
\hline OSS & .207 & .051 & 4.093 & .000 & .107 & .307 \\
\hline
\end{tabular}

From Table 35, it is clear that that only OPM, HRP, and OSS were found to have significant relationship with KA at the 0.05 level. Figure 66 below shows a scatterplot of the residuals for $\mathrm{OPM}+\mathrm{HRP}+\mathrm{OSS} \rightarrow \mathrm{KA}$. This is the standardized residual scatterplot produced by SPSS and shows the predicted vs. observed values, predicted vs. standard residuals, and observed vs. standard residual plots (and vice-versa). 


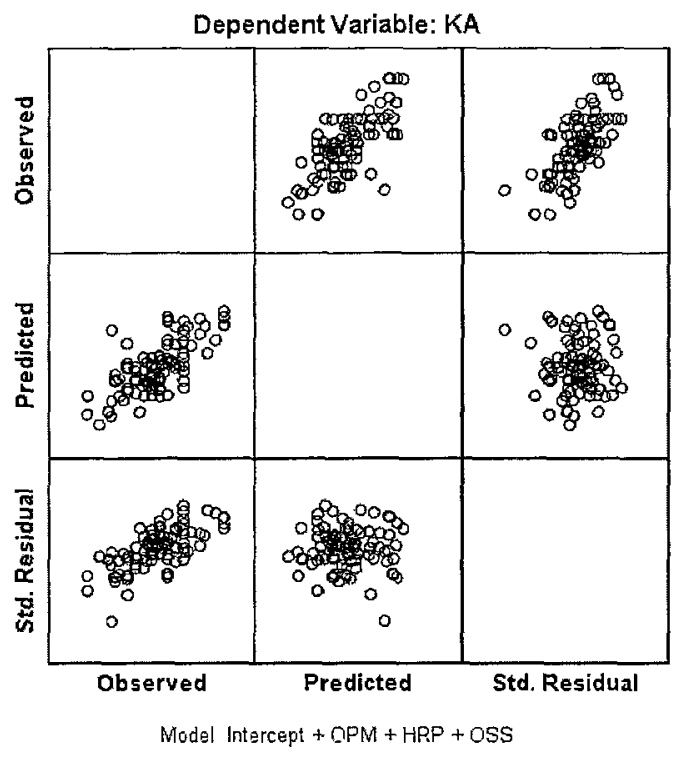

Figure 66. Scatterplot - residuals for OPM+HRP+OSS $\rightarrow \mathrm{KA}$

This standard format scatterplot provides much useful information for the following reasons. In general, the predicted vs. standard residual plot should indicate a random plot otherwise it would indicate some other systematic process is occurring. This is because linear regression assumes the independent and dependant variables are normal and tests whether a linear relationship exists between them. If these assumptions hold, then the residuals, which are the differences between the obtained and predicted dependant variable values, should also be normally distributed about each predicted dependant variable value (Hair, et. Al, 2006). Thus the residuals scatterplot should be an uncorrelated plot to confirm the residuals are independent, and in addition they ideally should behave somewhat normally (i.e. having a central tendency for each value of the predicted score). Ideally, a symmetrical trailing off pattern is expected, however, skewness and kurtosis of the data may also be revealed. 
In addition, the residuals scatterplot can be used to detect obvious signs of heteroscedasticity, which occurs when the variance of one or both variables is changes over the range of the other. This will be revealed in an asymmetric residuals scatterplot. Finally, the standardized residuals plot also provides a clear visual indication of the bivariate correlation between predicted vs. observed data values. The presence of outliers, non-linearity, and the strength of the correlation can be visually confirmed. This standard format residuals scatterplot will be used repeatedly throughout this section

Figure 67 shows diagrammatically the links suggested by the analysis consistent with the literature.

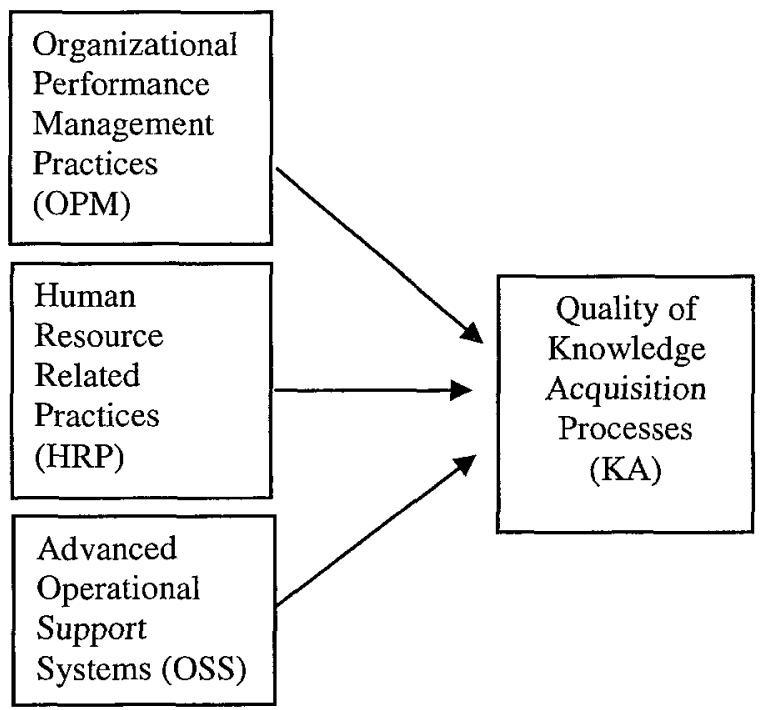

Figure 67. KMP and OTS Links to Quality of KA Processes 


\subsection{Linking Support for $\mathbb{K M P S}$ and OTSs to Quality of $\mathbb{K S}$ Processes}

Table 36 summarizes the significant results from the regression analysis of all the KMPs and OTSs combined on KS after non-significant factors were eliminated one at a time.

Table 36. Regression HRP+IMP+SOL $\rightarrow$ KS

\begin{tabular}{|c|c|c|c|c|c|c|}
\hline Dependent Variable:KS & & & \multicolumn{2}{|c|}{ 95\% Confidence Interval } \\
\cline { 5 - 7 } Parameter & $\mathbf{B}$ & Std. Error & $\mathbf{t}$ & Sig. & Lower Bound & Upper Bound \\
\hline HRP & .295 & .062 & 4.742 & .000 & .172 & .418 \\
\hline IMP & .418 & .091 & 4.587 & .000 & .237 & .598 \\
\hline SOL & .404 & .083 & 4.879 & .000 & .240 & .568 \\
\hline
\end{tabular}

From the regression analysis as summarized in Table 36 , it is clear that that only HRP, IMP, and SOL have significant relationship with KS at the 0.05 level. Figure 68 below shows a scatterplot of the residuals for HRP+IMP+SOL $\rightarrow \mathrm{KS}$ and Figure 69 shows diagrammatically the links suggested by the analysis consistent with the literature.

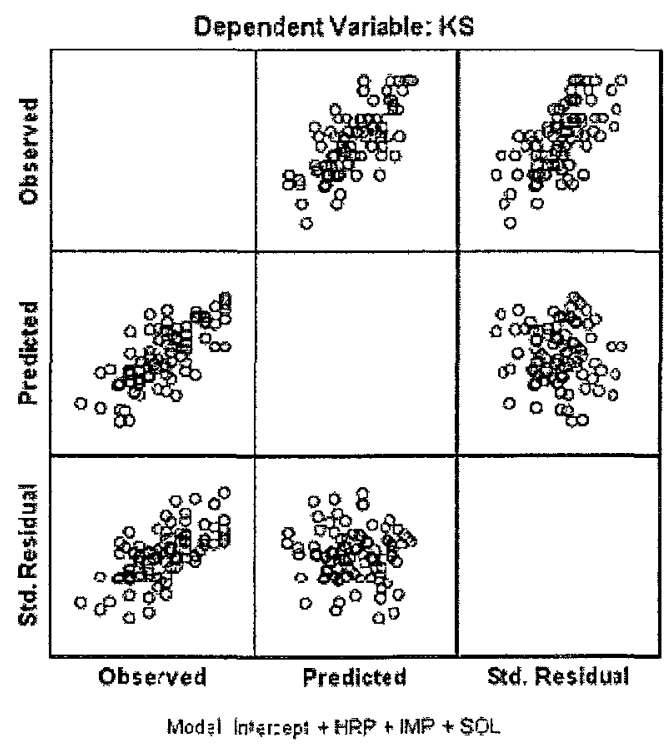

Figure 68. Residuals for $\mathrm{HRP}+\mathrm{IMP}+\mathrm{SOL} \rightarrow \mathrm{KS}$ 


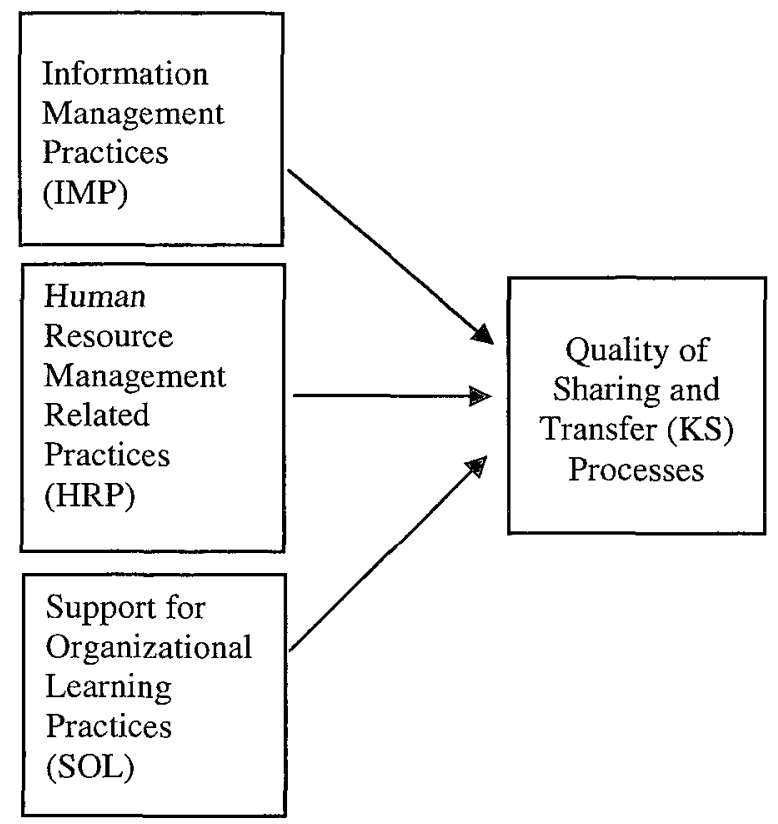

Figure 69. KMP Links to Quality of KS Processes

\subsection{Linking Support for KMPs and OTSs to Quality of KR Processes}

Table 37 summarizes the significant results from the regression analysis of all the KMPs and OTSs combined on KR after non-significant factors were eliminated one at a time.

Table 37. Regression HRP+TRP+OPM $\rightarrow \mathrm{KR}$

\begin{tabular}{|c|c|c|c|c|c|c|}
\hline Dependent Variable:KR & & & \multicolumn{2}{|c|}{ 95\% Confidence Interval } \\
\cline { 5 - 7 } Parameter & $\mathbf{B}$ & Std. Error & $\mathbf{t}$ & Sig. & Lower Bound & Upper Bound \\
\hline HRP & .355 & .054 & 6.533 & .000 & .247 & .463 \\
\hline TRP & .409 & .095 & 4.293 & .000 & .220 & .597 \\
\hline OPM & -.197 & .097 & -2.028 & .045 & -.390 & -.005 \\
\hline
\end{tabular}

From the regression analysis as summarized in Table 37, it is clear that that only HRP, TRP, and OPM have significant relationship with KR at the 0.05 level. Note that OPM correlates negatively with $\mathrm{KR}$, however, has an upper bound close to zero and is close to 
the .05 cutoff. This relationship is not as expected and is therefore suspect. It will be discussed later on in the context of the full Path Analysis model. Figure 70 below shows a scatterplot of the residuals for HRP+TRP $+O P M \rightarrow K R$ and Figure 71 shows diagrammatically the links suggested by the analysis consistent with the literature.

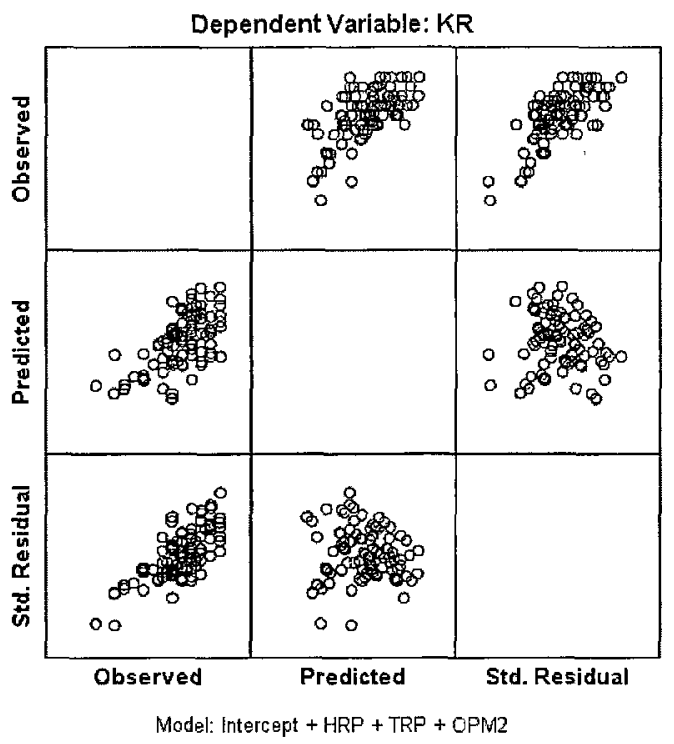

Figure 70. Residuals for OPM+TRP $+\mathrm{HRP} \rightarrow \mathrm{KR}$ 


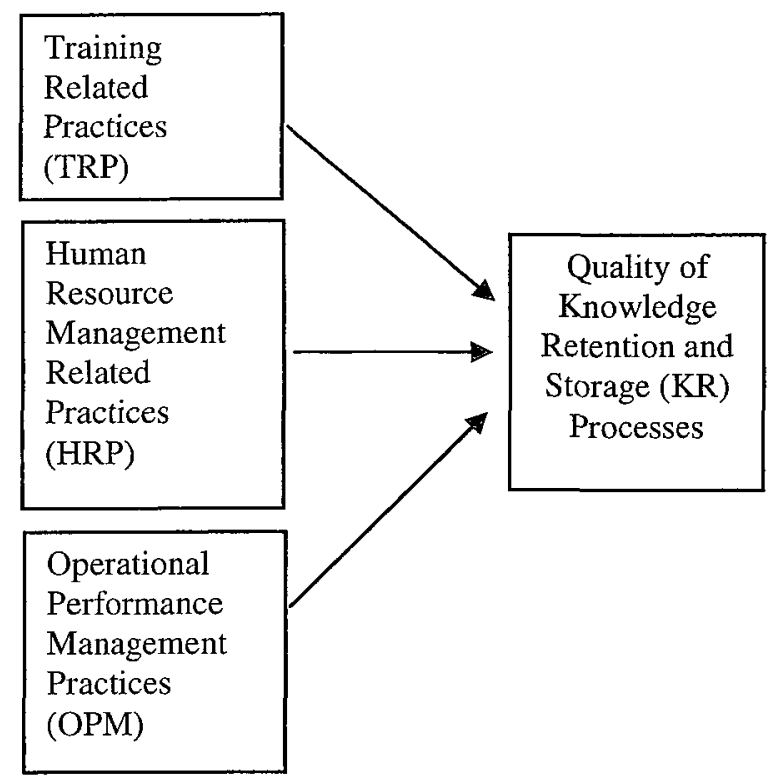

Figure 71. KMP Links to Quality of KR Processes

\subsection{Linking Support for KMPs and OTSs to Quality of KG Processes}

Table 38 summarizes the significant results from the regression analysis of all the KMPs and OTSs combined on KG after non-significant factors were eliminated one at a time.

Table 38. Regression OPM+KMS $\rightarrow \mathrm{KG}$

\begin{tabular}{|c|c|c|c|c|c|c|}
\hline \multicolumn{7}{|c|}{ Dependent Variable:KG } \\
\hline \multirow[b]{2}{*}{ Parameter } & \multirow[b]{2}{*}{ B } & \multirow[b]{2}{*}{ Std. Error } & \multirow[b]{2}{*}{$t$} & \multirow[b]{2}{*}{ Sig. } & \multicolumn{2}{|c|}{$\mathbf{9 5} \%$ Confidence Interval } \\
\hline & & & & & Lower Bound & Upper Bound \\
\hline OPM & .571 & .088 & 6.450 & .000 & .395 & .746 \\
\hline KMS & .255 & .047 & 5.455 & .000 & .162 & .347 \\
\hline
\end{tabular}

From the regression analysis as summarized in Table 38, it is clear that that only KMS and OPM have significant relationship with $\mathrm{KG}$ at the 0.05 level. Figure 72 below shows a scatterplot of the residuals for $\mathrm{OPM}+\mathrm{KMS} \rightarrow \mathrm{KG}$ and Figure 73 shows diagrammatically the links suggested by the analysis consistent with the literature. 


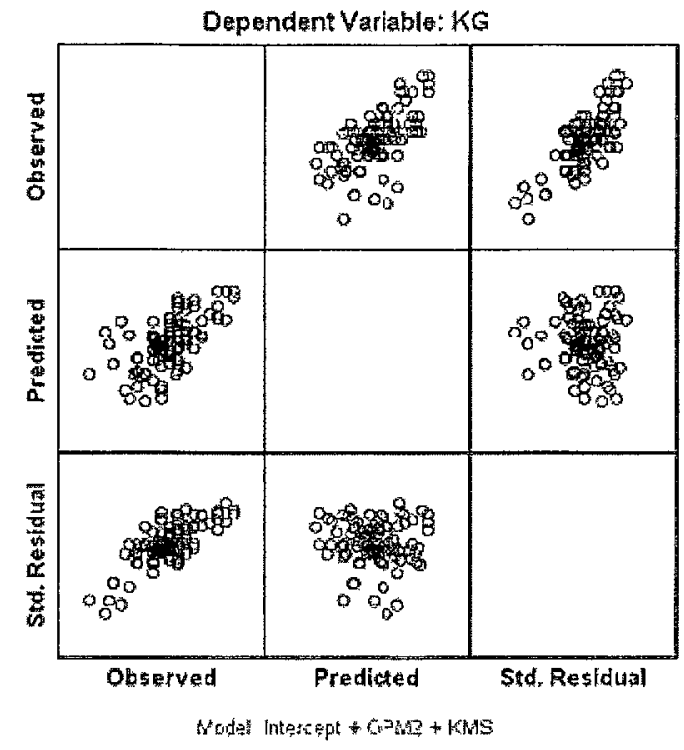

Figure 72. Residuals for $\mathrm{OPM}+\mathrm{KMS} \rightarrow \mathrm{KG}$

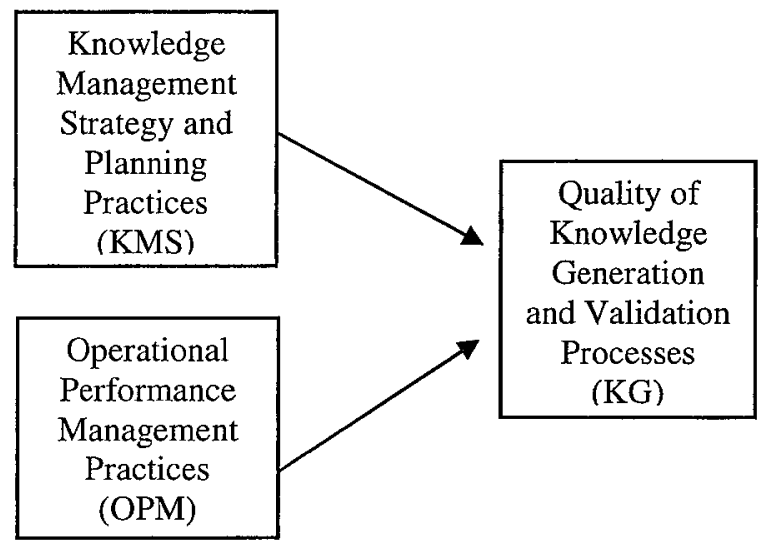

Figure 73. KMP Links to Quality of KG Processes

\subsection{Linking Support for KMPs and OTSs to Quality of KU Processes}

Table 39 summarizes the significant results from the regression analysis of all the KMPs and OTSs combined on $\mathrm{KU}$ after non-significant factors were eliminated one at a time. 
Table 39. Regression IMP+HRP+IST $\rightarrow \mathrm{KU}$

\begin{tabular}{|c|c|c|c|c|c|c|}
\hline \multicolumn{7}{|c|}{ Dependent Variable:KU } \\
\hline \multirow[b]{2}{*}{ Parameter } & \multirow[b]{2}{*}{ B } & \multirow[b]{2}{*}{ Std. Error } & \multirow[b]{2}{*}{$\mathbf{t}$} & \multirow[b]{2}{*}{ Sig. } & \multicolumn{2}{|c|}{$95 \%$ Confidence Interval } \\
\hline & & & & & Lower Bound & Upper Bound \\
\hline IMP & .419 & .077 & 5.452 & .000 & .267 & .571 \\
\hline HRP & .235 & .047 & 5.005 & .000 & .142 & .328 \\
\hline IST & .224 & .051 & 4.370 & .000 & .123 & .326 \\
\hline
\end{tabular}

Table 39 shows the result of regressions of KMPs on KU. Only IMP, HRP, and IST were significant at the 0.05 level. Figure 74 below shows a scatterplot of the residuals for IMP+HRP+IST $\rightarrow$ KU. Figure 75 illustrates the links suggested by these findings.

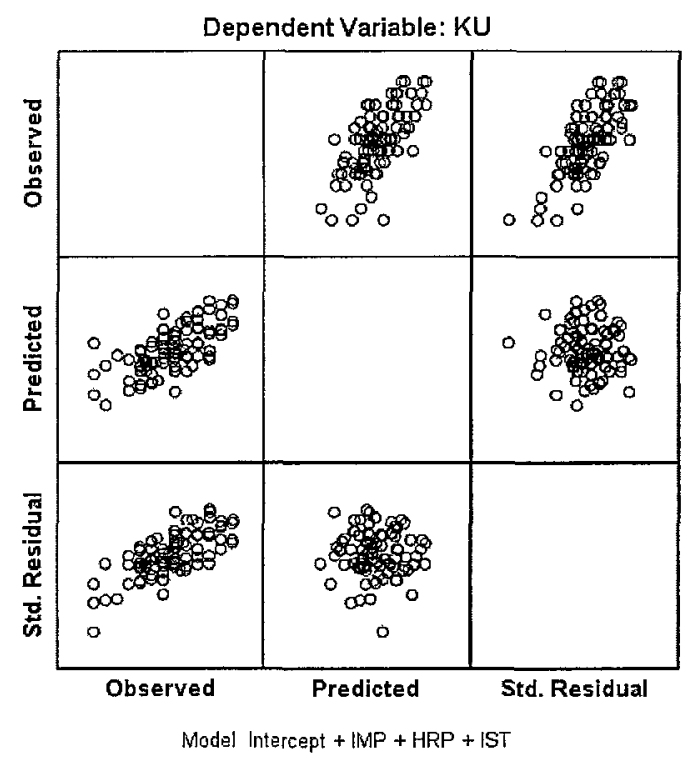

Figure 74. Residuals for IMP+HRP+IST $\rightarrow \mathrm{KU}$ 


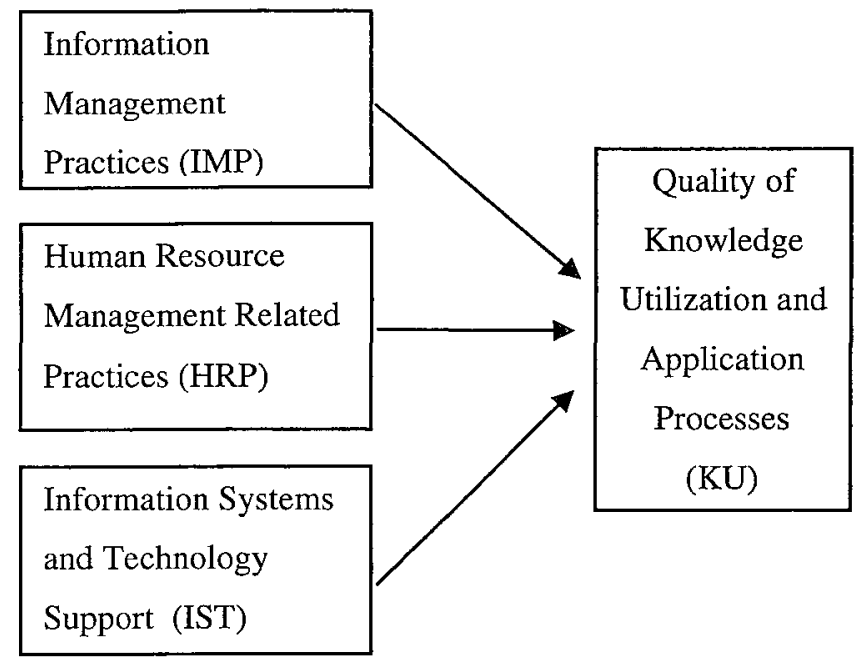

Figure 75. KMP and OTS Links to Quality of KU Processes

\subsection{Linking Support for KMPs and OTSs to SOC}

Table 40 summarizes the significant results from the regression analysis of all the KMPs and OTSs combined on SOC after non-significant factors were eliminated one at a time.

Table 40. Regression IMP+HRP+IST+SOL+KMS $\rightarrow$ SOC

\begin{tabular}{|c|c|c|c|c|c|c|}
\hline Dependent Variable:SOC & & & \multicolumn{2}{|c|}{$95 \%$ Confidence Interval } \\
\cline { 5 - 7 } Parameter & B & Std. Error & $t$ & Sig. & Lower Bound & Upper Bound \\
\hline IMP & .168 & .066 & 2.555 & .012 & .038 & .297 \\
\hline HRP & .156 & .049 & 3.173 & .002 & .059 & .253 \\
\hline IST & .097 & .042 & 2.283 & .024 & .013 & .180 \\
\hline SOL & .405 & .063 & 6.462 & .000 & .281 & .530 \\
\hline KMS & .169 & .044 & 3.856 & .000 & .082 & .256 \\
\hline
\end{tabular}

From the regression analysis as summarized in Table 40, it is clear that that only IMP, HRP, IST, SOL, and KMS have significant relationship with SOC at the 0.05 level. Figure 76 below shows a scatterplot of the residuals for the regression 
$\mathrm{IMP}+\mathrm{HRP}+\mathrm{IST}+\mathrm{SOL}+\mathrm{KIMS} \rightarrow \mathrm{SOC}$ and Figure 77 shows diagrammatically the links suggested by the analysis consistent with the literature.

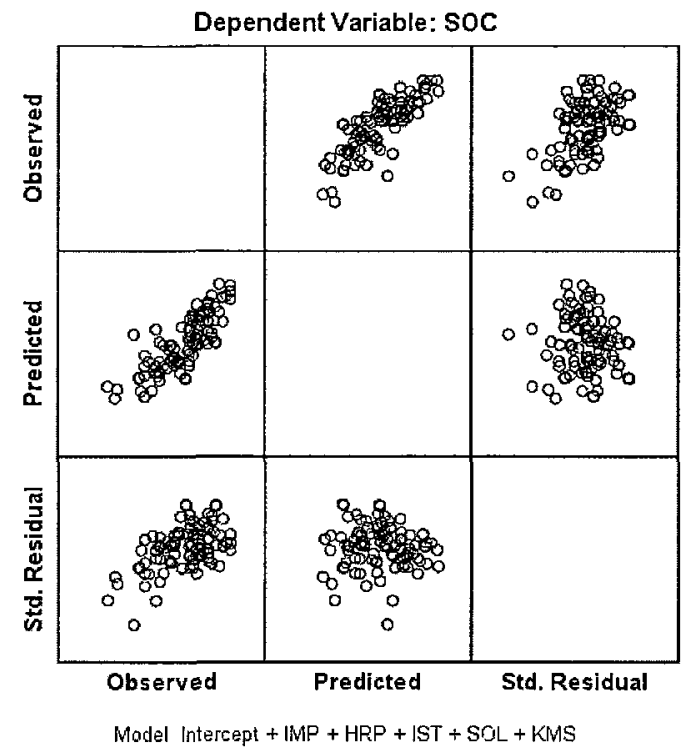

Figure 76. Residuals for IMP+HRP+IST+SOL+KMS $\rightarrow$ SOC

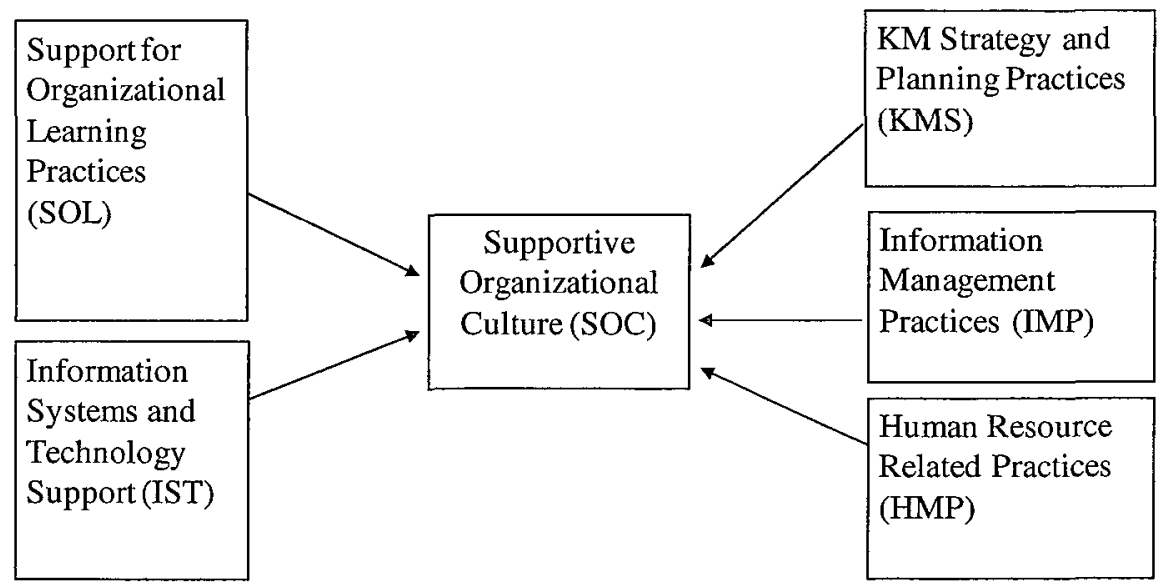

Figure 77. KMP and OTS Links to Supportive Organizational Culture (SOC) 


\subsection{Linking SOC to $Q \mathbb{K P}$ and $O \mathbb{E}$}

Similarly, to explore the possible impact supportive organizational culture (SOC) has on the quality of knowledge processes and organizational effectiveness, a series of linear regressions were run for SOC on the QKPs. Table 41 below summarize these findings. As per the theory summarized from the literature review the causal relationship for all of these is interpreted to be from SOC to each of the QKP constructs.

Table 41. Regression SOC $\rightarrow$ KA

\begin{tabular}{|c|c|c|c|c|c|c|}
\hline Dependent Variable:KA & & & \multicolumn{2}{|c|}{$95 \%$ Confidence Interval } \\
\cline { 5 - 7 } Parameter & $\mathrm{B}$ & Std. Error & $\mathrm{t}$ & Sig. & Lower Bound & Upper Bound \\
\hline SOC & .628 & .086 & 7.318 & .000 & .458 & .798 \\
\hline
\end{tabular}

From Table 41 it can be seen that SOC strongly correlates with KA and with high significance. Figure 78 below shows a scatterplot of residuals for SOC $\rightarrow$ KA.

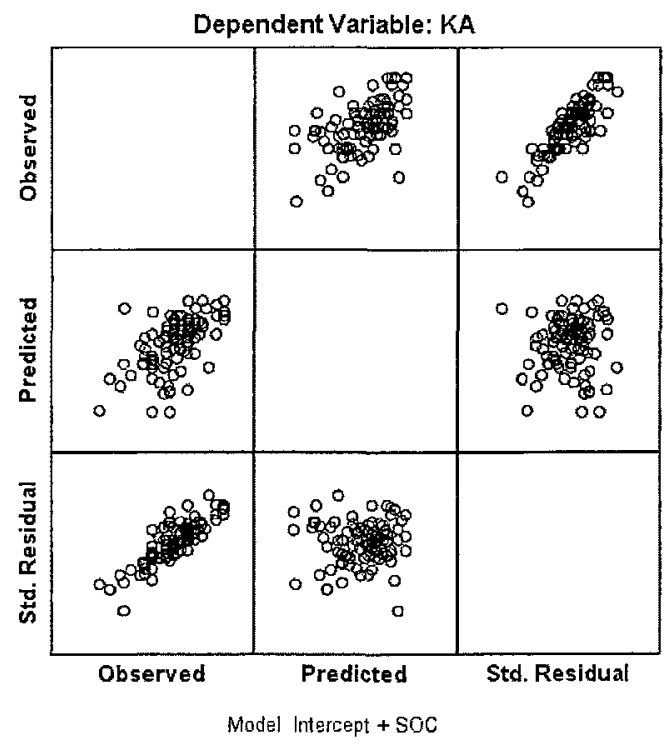

Figure 78. Residuals of SOC $\rightarrow \mathrm{KA}$ 
Table 42 below summarizes the regression of SOC $\rightarrow$ KG.

Table 42. Regression SOC $\rightarrow \mathrm{KG}$

\begin{tabular}{|c|c|c|c|c|c|c|}
\hline Dependent Variable:KG & & & \\
\hline & & & & & \multicolumn{2}{|c|}{$95 \%$ Confidence Interval } \\
\cline { 5 - 7 } Parameter & $\mathrm{B}$ & Std. Error & $\mathrm{t}$ & $\mathrm{Sig}$. & Lower Bound & Upper Bound \\
\hline SOC & .572 & .065 & 8.856 & .000 & .444 & .700 \\
\hline
\end{tabular}

From Table 42 we can see that SOC strongly correlates with KG and with high significance. Figure 79 below shows a scatterplot of residuals for SOC $\rightarrow$ KG.

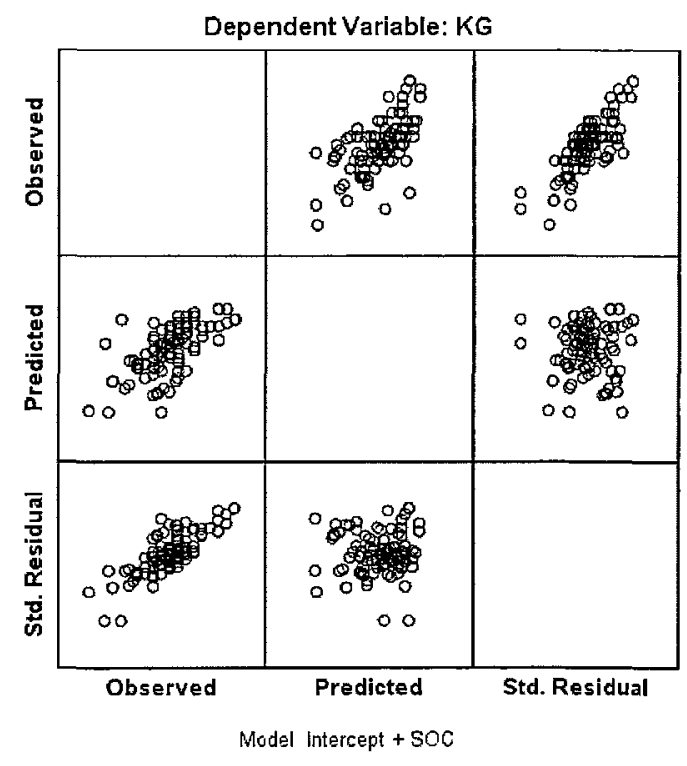

Figure 79. Residuals of $\mathrm{SOC} \rightarrow \mathrm{KG}$

Table 43 below summarizes the correlation of SOC $\rightarrow$ KS.

Table 43. Regression SOC $\rightarrow \mathrm{KS}$

\begin{tabular}{|c|c|c|c|c|c|c|}
\hline Dependent Variable:KS & & & \multicolumn{2}{|c|}{$95 \%$ Confidence Interval } \\
\cline { 5 - 7 } Parameter & B & Std. Error & $\mathbf{t}$ & Sig. & Lower Bound & Upper Bound \\
\hline SOC & .753 & .077 & 9.764 & .000 & .600 & .905 \\
\hline
\end{tabular}


From Table 43 we can see that SOC strongly correlates with KS and with high significance. Figure 80 below shows a scatterplot of residuals for SOC $\rightarrow \mathrm{KS}$.

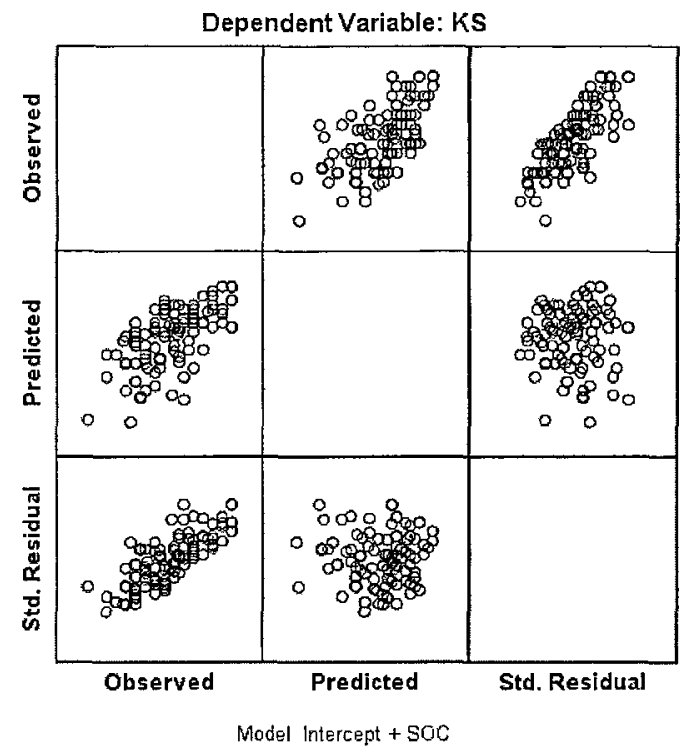

Figure 80. Residuals of SOC $\rightarrow \mathrm{KS}$

Table 44 summarizes the correlation of SOC $\rightarrow \mathrm{KU}$.

Table 44. Regression SOC $\rightarrow \mathrm{KU}$

\begin{tabular}{|c|c|c|c|c|c|c|}
\hline Dependent Variable:KU & \multicolumn{3}{|c|}{} \\
\hline & & & & & \multicolumn{2}{|c|}{$95 \%$ Confidence Interval } \\
\cline { 5 - 7 } Parameter & $\mathrm{B}$ & Std. Error & $\mathrm{t}$ & Sig. & Lower Bound & Upper Bound \\
\hline SOC & .538 & .066 & 8.183 & .000 & .408 & .668 \\
\hline
\end{tabular}

From Table 44 we can see that SOC strongly correlates with $\mathrm{KU}$ and with high significance. Figure 81 below shows a scatterplot of residuals for SOC $\rightarrow \mathrm{KU}$. 


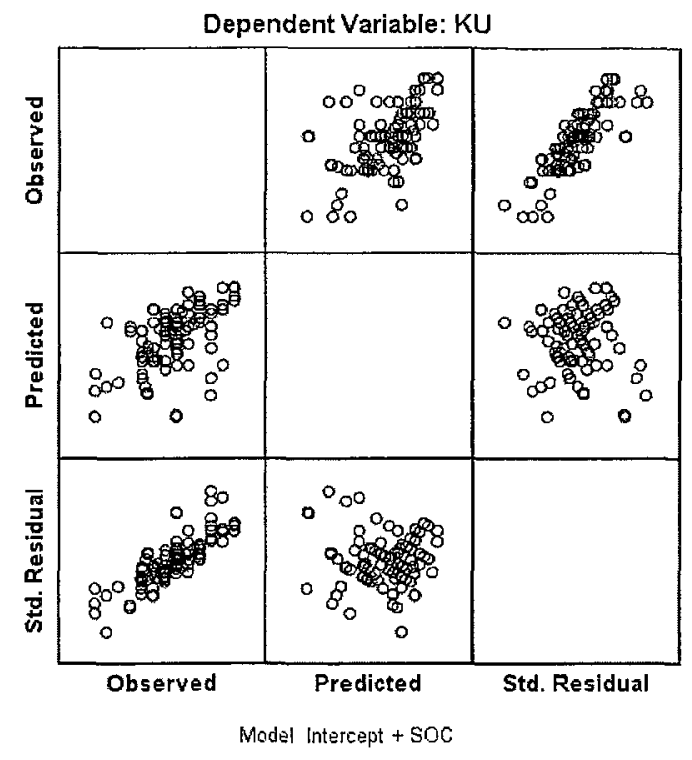

Figure 81. Residuals of $\mathrm{SOC} \rightarrow \mathrm{KU}$

Table 45 summarizes the correlation of $\mathrm{SOC} \rightarrow \mathrm{KR}$.

Table 45. Regression SOC $\rightarrow \mathrm{KR}$

\begin{tabular}{|c|c|c|c|c|c|c|}
\hline Dependent Variable:KR & & \multicolumn{2}{|c|}{$95 \%$ Confidence Interval } \\
\cline { 5 - 7 } Parameter & $\mathrm{B}$ & Std. Error & $\mathrm{t}$ & Sig. & Lower Bound & Upper Bound \\
\hline SOC & .616 & .062 & 9.859 & .000 & .492 & .739 \\
\hline
\end{tabular}

From Table 45 we can see that SOC strongly correlates with $\mathrm{KR}$ and with high significance. Figure 82 below shows a scatterplot of residuals for SOC $\rightarrow \mathrm{KR}$. 


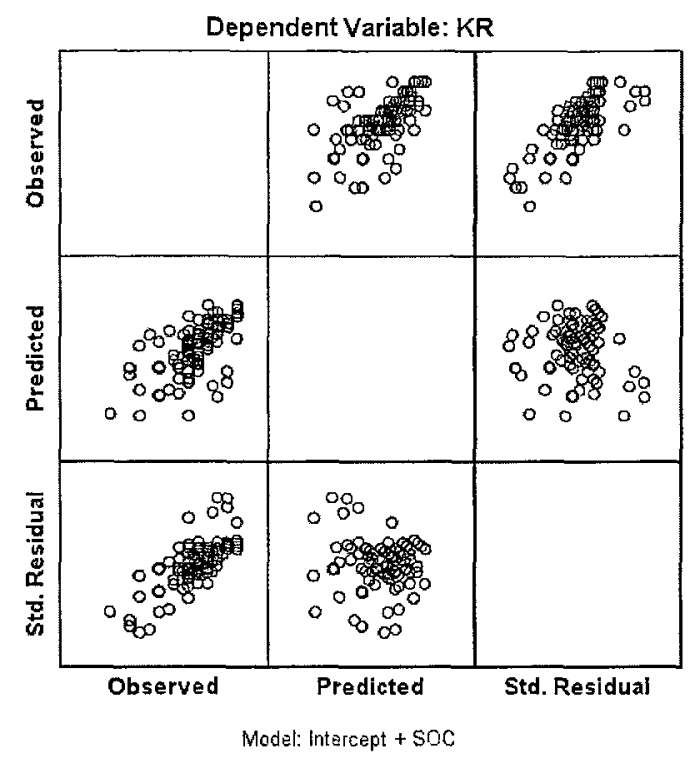

Figure 82. Residuals of SOC $\rightarrow \mathrm{KR}$

Table 46 summarizes the correlation of SOC $\rightarrow$ OE.

Table 46. Regression $\mathrm{SOC} \rightarrow \mathrm{OE}$

\begin{tabular}{|c|c|c|c|c|c|c|}
\hline \multicolumn{2}{|c|}{ Dependent Variable:OE } & & & \multicolumn{2}{|c|}{ 95\% Confidence Interval } \\
\cline { 5 - 7 } Parameter & $\mathrm{B}$ & Std. Error & $t$ & Sig. & Lower Bound & Upper Bound \\
\hline SOC & .600 & .060 & 10.079 & .000 & .482 & .718 \\
\hline
\end{tabular}

From Table 46 we can see that SOC strongly correlations with OE and with high significance. Figure 83 below shows a scatterplot of residuals for $\mathrm{SOC} \rightarrow \mathrm{OE}$. 


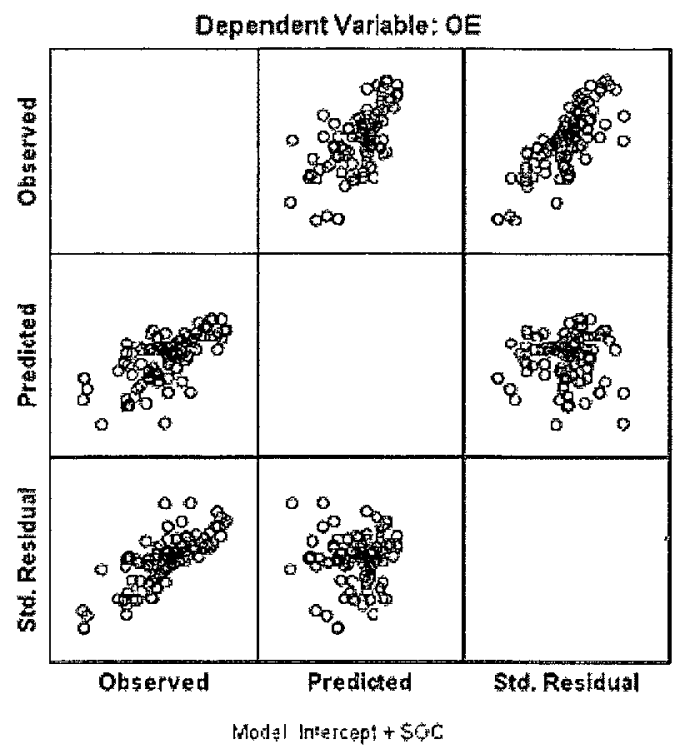

Figure 83. Residuals of SOC $\rightarrow$ OE

Figure 84 below summarizes all of these preliminary findings.

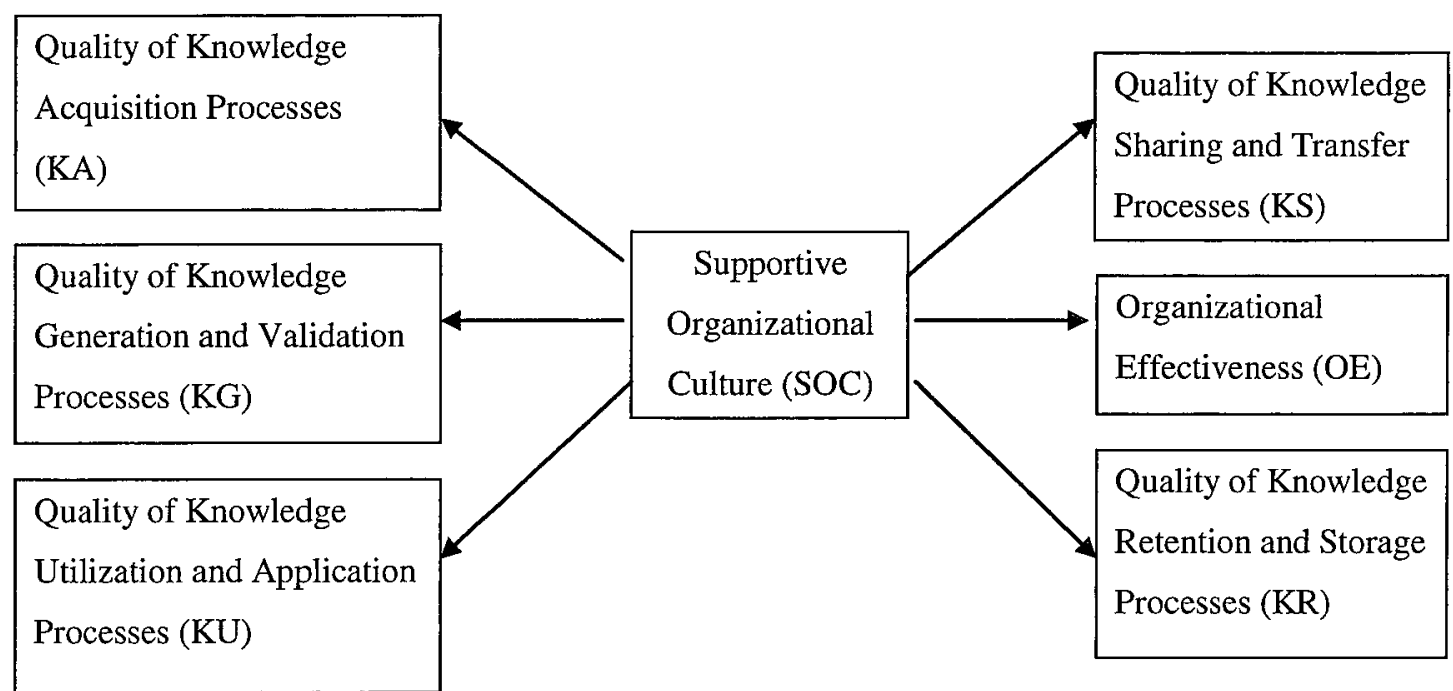

Figure 84. Supportive Organizational Culture Links to QKPs and OE 


\subsection{Role of SOC in Effects of Support for KMPS/OTSs on QKRS}

This section summarizes the results of linear regressions to explore the hypothesis that SOC significantly influences the relationships between both support for knowledge management practices (KMP) with the quality of knowledge processes (QKP) and organizational technology support (i.e. OTS, which includes factors OSS and IST) with the QKP. SOC is hypothesized to be an intermediate and moderating variable. Given the findings of Section 7.6 linking KMPs and IST to SOC and Section 7.7 linking SOC to $\mathrm{KA}, \mathrm{KG}, \mathrm{KS}, \mathrm{KU}$, and $\mathrm{KR}$ it can definitely be concluded that SOC plays an important role as an intermediate variable. Part of the effect that both the KMPs and OTSs have on the QKPs is via SOC.

In Sections 7.1 to 7.5 many direct links between specific KMPs to specific QKPs as well as links between OSS $\rightarrow \mathrm{KA}$ and IST $\rightarrow \mathrm{KU}$ were found to be significant. It is also hypothesized that SOC plays an important role (simultaneously) as a moderator of these relationships. This will be explored further in Section 8.15 using Path Analysis techniques.

\subsection{Inter-relationships Among $Q K P$ Constructs}

Regressions were run for all the possible "many-to-one" relationships amongst the quality of knowledge process constructs and their possible links to organizational effectiveness $(\mathrm{OE})$ to explore what relationships could be supported in the data. Tables 47 through 51 and Figure 91 summarize these findings. Note that these regressions 
show a linear dependency exists but do not imply a causal relationship (i.e. the direction must be interpreted based on theory). For this reason they are shown temporarily with a bi-directional "dotted line" link <---> to indicate the causal nature of the relationship is not yet determined and it could be causal in either direction. However, these relationships help to understand the significant linear relationships that exist and when combined with theory, will guide the selection of feasible causal path links using path analysis. As will be shown later in the analysis, this aid in finding a valid causal model incorporating all of the constructs and with a good model fit.

\subsubsection{Influence of other QKPs on Quality of KA Processes}

Table 47 summarizes the significant results from the regression analysis of all the other QKPs together on KA.

Table 47. Regression $\mathrm{KG}+\mathrm{KS}+\mathrm{KU}+\mathrm{KR}<--->\mathrm{KA}$

\begin{tabular}{|c|c|c|c|c|c|c|}
\hline Dependent Variable:KA & & & \multicolumn{2}{|c|}{$95 \%$ Confidence Interval } \\
\cline { 5 - 7 } Parameter & B & Std. Error & $t$ & Sig. & Lower Bound & Upper Bound \\
\hline KG & .672 & .098 & 6.841 & .000 & .478 & .867 \\
\hline KS & .239 & .086 & 2.784 & .006 & .069 & .409 \\
\hline
\end{tabular}

From Table 47 above, it is clear only KG and KS are significant at 0.05 or lower level. Figure 85 below shows a scatterplot of residuals for $\mathrm{KG}+\mathrm{KS}<--->\mathrm{KA}$. 


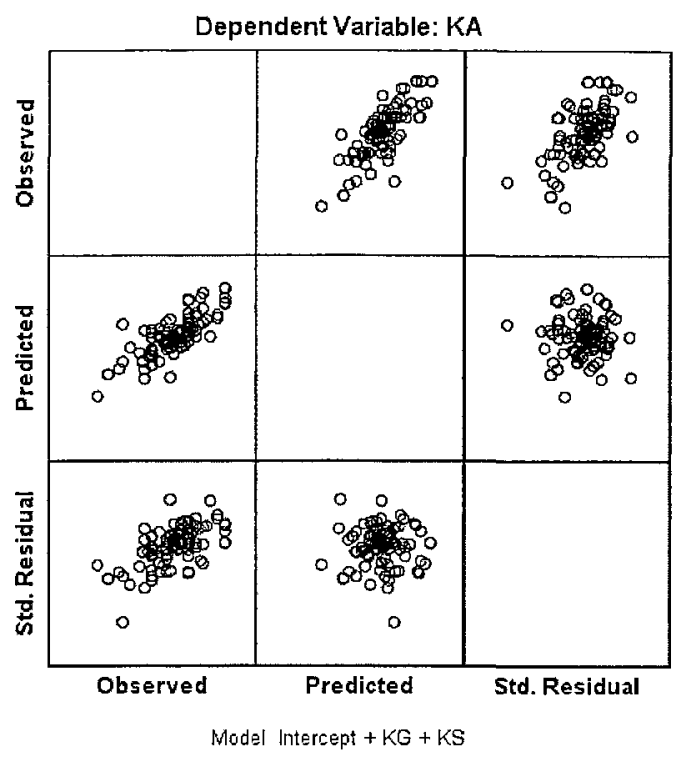

Figure 85. Residuals of $\mathrm{KG}+\mathrm{KS}<--->\mathrm{KA}$

From hypothesis 11 , we expect a link from $\mathrm{KA} \rightarrow \mathrm{KR}$, so the findings do not supports this. Also from hypothesis 14 , we expected a link from $\mathrm{KU} \rightarrow \mathrm{KA}$ which is also not supported by the data. Instead the data supports links between $\mathrm{KA}<--->\mathrm{KS}$ and $\mathrm{KA}<---$ $>\mathrm{KG}$, both with direction yet to be determined.

\subsubsection{Influence of other QKPs on Quality of KS Processes}

Table 48 summarizes the significant results from the regression analysis of all the other QKPs together on KS.

Table 48. Regression $\mathrm{KG}+\mathrm{KU}+\mathrm{KR}+\mathrm{KA}<--->\mathrm{KS}$

\begin{tabular}{|c|c|c|c|c|c|c|}
\hline \multicolumn{2}{|l|}{ Dependent Variable:KS } & & & & \multicolumn{2}{|c|}{$95 \%$ Confidence Interval } \\
\cline { 5 - 7 } Parameter & B & Std. Error & $t$ & Sig. & Lower Bound & Upper Bound \\
\hline KG & .312 & .118 & 2.634 & .010 & .077 & .546 \\
\hline KR & .502 & .086 & 5.839 & .000 & .332 & .672 \\
\hline KA & .262 & .094 & 2.784 & .006 & .076 & .448 \\
\hline
\end{tabular}


From Table 48 above, it is clear that only KG, KR, and KA are significant at the 0.05 level. Figure 86 below shows a residuals scatterplot of $K G+K R+K A<-->K S$.

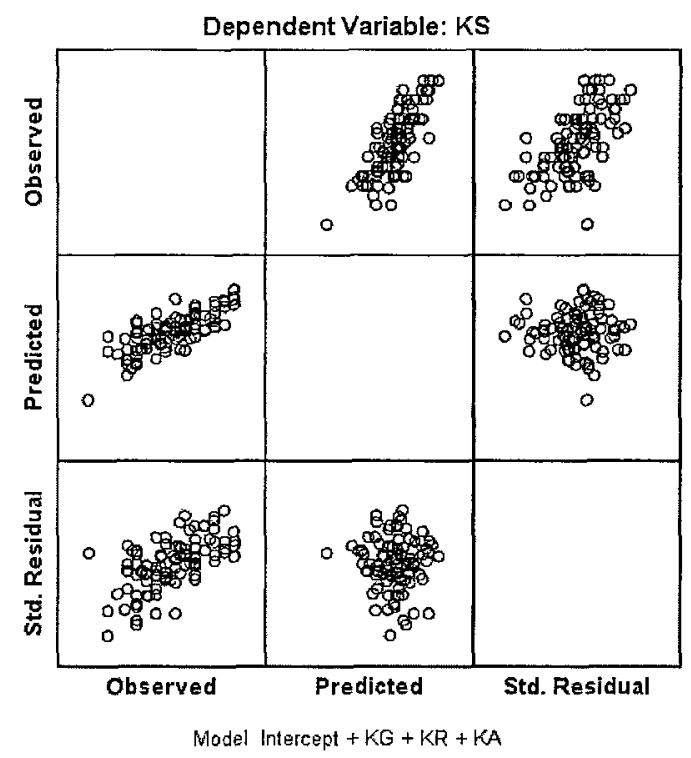

Figure 86. Residuals of $\mathrm{KG}+\mathrm{KR}+\mathrm{KA}<--->\mathrm{KS}$

From hypothesis 12 we expect a link $\mathrm{KR} \rightarrow \mathrm{KS}$ and from hypothesis 13 we expect a link $\mathrm{KS} \rightarrow \mathrm{KU}$. The findings support only the KR $\rightarrow \mathrm{KS}$ link. However, the findings also support links from $\mathrm{KG}<--->\mathrm{KS}$ and $\mathrm{KA}<--->\mathrm{KS}$ (both with direction to be determined). Recall the same $\mathrm{KA}<--->\mathrm{KS}$ relationship was also significant in the previous regression in Section 7.9.1 which looked at all possible links to KA. 


\subsubsection{Influence of other QKPs on Quality of KR Processes}

Table 49 summarizes the significant results from the regression analysis of all the other QKPs together on KR.

Table 49. Regression $\mathrm{KG}+\mathrm{KU}+\mathrm{KA}+\mathrm{KS}<--->\mathrm{KR}$

\begin{tabular}{|c|c|c|c|c|c|c|}
\hline Dependent Variable:KR & \multicolumn{3}{|c|}{} \\
\cline { 6 - 8 } & & & & & \multicolumn{2}{|c|}{$95 \%$ Confidence Interval } \\
\hline Parameter & $\mathrm{B}$ & Std. Error & $\mathrm{t}$ & Sig. & Lower Bound & Upper Bound \\
\hline KU & .316 & .086 & 3.661 & .000 & .145 & .487 \\
\hline KS & .452 & .077 & 5.839 & .000 & .299 & .606 \\
\hline
\end{tabular}

From Table 49 above, it is clear that only KU and KS are significant at the 0.05 level. Figure 87 below shows a residuals scatterplot of KS+KU<--->KR.

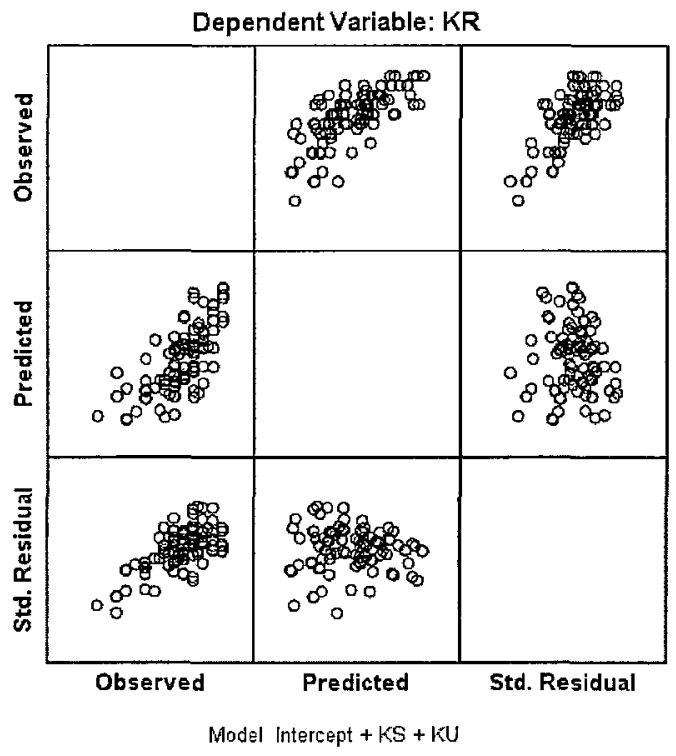

Figure 87. Residuals of $\mathrm{KS}+\mathrm{KU}<--->\mathrm{KR}$

From hypothesis 11 , links $\mathrm{KA} \rightarrow \mathrm{KR}$ and $\mathrm{KG} \rightarrow \mathrm{KR}$ were expected, and from hypothesis 12, a link $\mathrm{KR} \rightarrow \mathrm{KS}$ was expected. However the findings support only $\mathrm{KR} \rightarrow \mathrm{KS}$. 
However, a significant link exists between $\mathrm{KU}<--->\mathrm{KR}$ with direction yet to be determined.

\subsubsection{Influence of other QKPs on Quality of KG Processes}

Table 50 summarizes the significant results from the regression analysis of all the other QKPs together on KG.

Table 50. Regression $\mathrm{KU}+\mathrm{KA}+\mathrm{KS}+\mathrm{KR}<--->\mathrm{KG}$

\begin{tabular}{|c|c|c|c|c|c|c|}
\hline Dependent Variable:KG & & & \multicolumn{2}{|c|}{ 95\% Confidence Interval } \\
\cline { 5 - 7 } Parameter & B & Std. Error & $t$ & Sig. & Lower Bound & Upper Bound \\
\hline KU & .212 & .070 & 3.005 & .003 & .072 & .351 \\
\hline KA & .428 & .063 & 6.841 & .000 & .304 & .552 \\
\hline KS & .181 & .069 & 2.634 & .010 & .045 & .317 \\
\hline
\end{tabular}

From Table 50 above, it is clear that only KU, KA, and KS are significant at the 0.05 level. Figure 88 below shows a residuals scatterplot of $\mathrm{KA}+\mathrm{KS}+\mathrm{KU}<--->\mathrm{KG}$. 


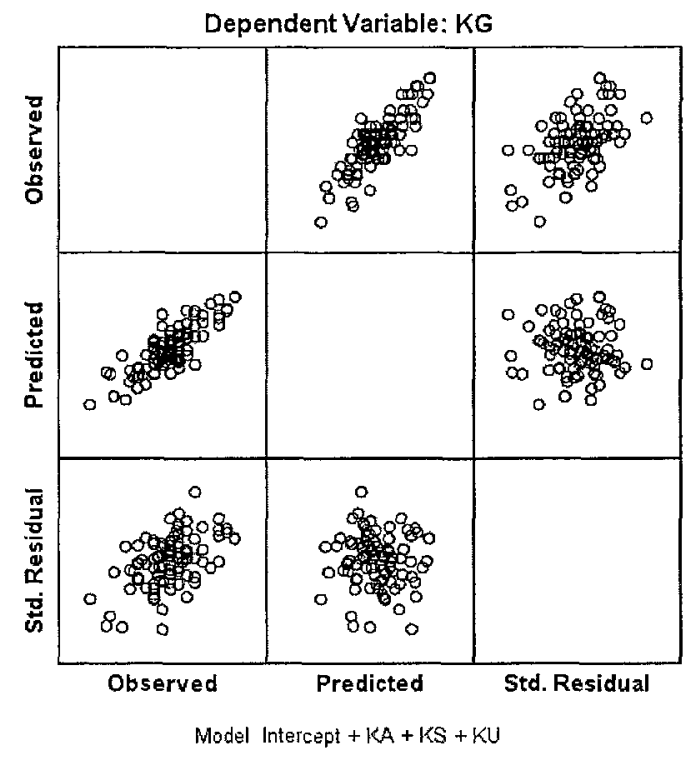

Figure 88. Residuals of $\mathrm{KA}+\mathrm{KS}+\mathrm{KU}<--->\mathrm{KG}$

From hypothesis 14 , it is clear that a link $\mathrm{KU} \rightarrow \mathrm{KG}$ was hypothesized and this is supported in the data. However, the findings clearly also support the following links: $\mathrm{KA}<--->\mathrm{KG}$ and $\mathrm{KS}<--->\mathrm{KG}$. Recall the link $\mathrm{KA}<--->\mathrm{KG}$ was earlier found to be significant in Section 7.9.1 and its direction is yet to be determined. Also in Section 7.9.2 the link $\mathrm{KS}<--->\mathrm{KG}$ was earlier found to be significant and its direction is yet to be determined.

7.9.5. Influence of other QKPs on Quality of KU Processes

Table 51 summarizes the significant results from the regression analysis of all the other QKPs together on KU. 
Table 51. Regression $\mathrm{KA}+\mathrm{KS}+\mathrm{KR}+\mathrm{KG}<--->\mathrm{KU}$

\begin{tabular}{|c|c|c|c|c|c|c|}
\hline Dependent Variable:KU & \multicolumn{3}{|c|}{} \\
\hline & & & & \multicolumn{2}{|c|}{$95 \%$ Confidence Interval } \\
\cline { 5 - 7 } Parameter & B & Std. Error & $\mathrm{t}$ & Sig. & Lower Bound & Upper Bound \\
\hline KR & .328 & .090 & 3.661 & .000 & .151 & .506 \\
\hline KG & .341 & .113 & 3.005 & .003 & .116 & .566 \\
\hline
\end{tabular}

From Table 51 above, it is clear that only $\mathrm{KR}$ and $\mathrm{KG}$ are significant at 0.05 level. Figure 89 below shows a residuals scatterplot of $\mathrm{KG}+\mathrm{KR}<--->\mathrm{KU}$.

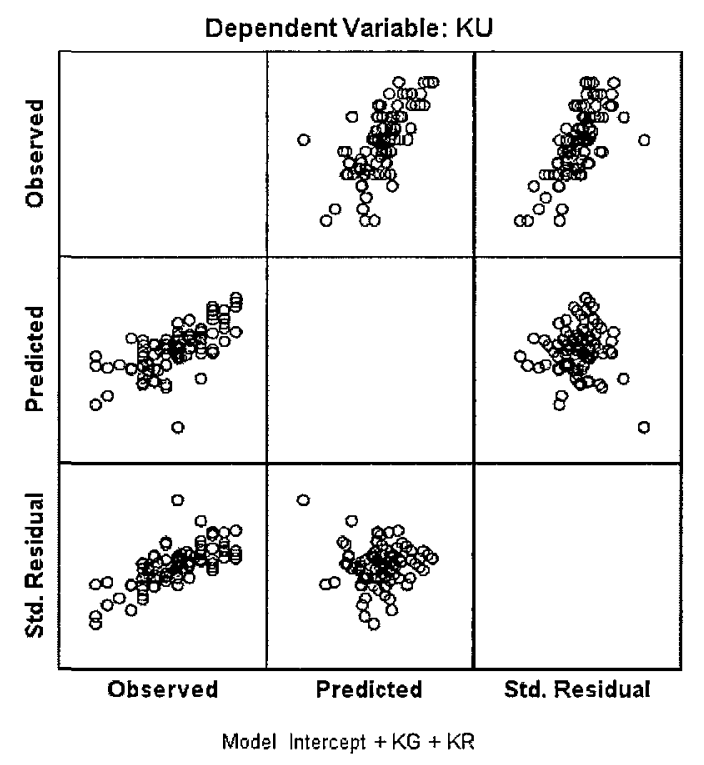

Figure 89. Residuals of $\mathrm{KG}+\mathrm{KR}<--->\mathrm{KU}$

From hypothesis 13 a link $\mathrm{KS} \rightarrow \mathrm{KU}$ was expected, however the findings do not support this. Also, from hypothesis 14 a link from $\mathrm{KU} \rightarrow \mathrm{KA}$ and from $\mathrm{KU} \rightarrow \mathrm{KG}$ was expected and the findings support on the link $\mathrm{KU} \rightarrow \mathrm{KG}$. Instead, the findings support a link from $\mathrm{KR}<--->\mathrm{KU}$ and the direction is not yet known. 


\subsubsection{Linking QKPs with Organizational Effectiveness (OE)}

Table 52 summarizes the significant results from the regression analysis of all the QKPs together on $\mathrm{OE}$.

Table 52. Regression $\mathrm{KA}+\mathrm{KS}+\mathrm{KR}+\mathrm{KG}+\mathrm{KU}<--->\mathrm{OE}$

\begin{tabular}{|c|c|c|c|c|c|c|}
\hline \multicolumn{2}{|l|}{ Dependent Variable: $\mathrm{OE}$} & & & \multicolumn{2}{|c|}{$95 \%$ Confidence Interval } \\
\cline { 6 - 7 } Parameter & $\mathrm{B}$ & Std. Error & $\mathrm{t}$ & Sig. & Lower Bound & Upper Bound \\
\hline $\mathrm{KR}$ & .361 & .078 & 4.639 & .000 & .207 & .516 \\
\hline $\mathrm{KU}$ & .385 & .076 & 5.037 & .000 & .234 & .536 \\
\hline
\end{tabular}

From Table 52 above, it is clear that only KR and KU are significant at 0.05 level.

Figure 90 below shows a residuals scatterplot of $\mathrm{KR}+\mathrm{KU}<--->\mathrm{OE}$.

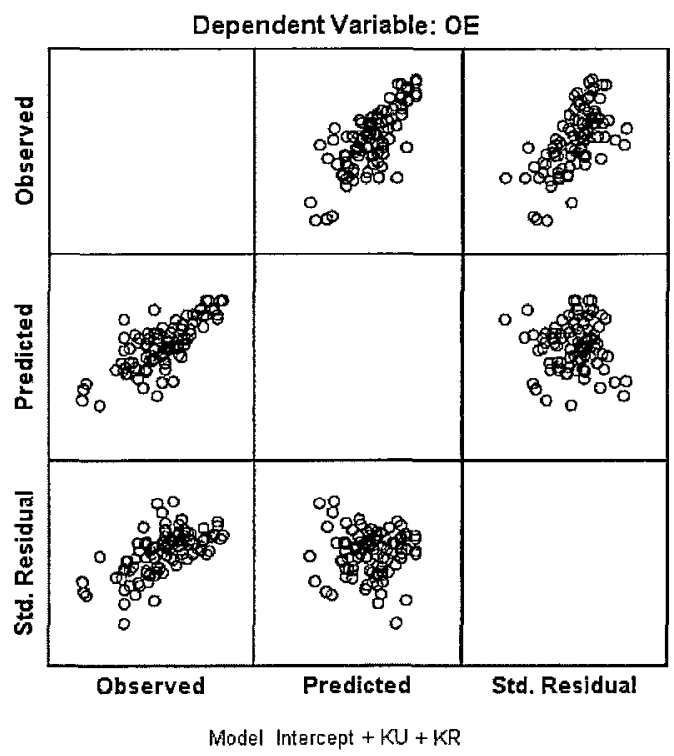

Figure 90. Residuals of $\mathrm{KR}+\mathrm{KU}<--->\mathrm{OE}$

From hypothesis 15 and 16 links from $\mathrm{KU} \rightarrow \mathrm{OE}$ and from $\mathrm{KR} \rightarrow \mathrm{OE}$ respectively were expected. The findings support both of these links. 


\subsubsection{Summary of Findings for Quality of Knowledge Processes}

Combining these results, and in light of the earlier findings from the literature about the direction of the expected (hypothesized) links that were found to be significant, the linear regression analysis supports the preliminary findings as shown in Figure 91. All links are shown as "dotted line arrows" until their validity and direction can be further confirmed using path analysis. Note that the expected links from $K A \rightarrow K R$, from $\mathrm{KS} \rightarrow \mathrm{KU}$, and from $\mathrm{KG} \rightarrow \mathrm{KR}$ (see Figure 29) could not be supported by the data and are omitted from Figure 91. All relationships shown are at the $\mathrm{p}=0.05$ or better significance level with the assumed direction shown if the link was hypothesized in Chapter 2 (i.e. as supported in the preliminary research). For those relationships shown in Figure 91 for which the causal relationship has not yet been determined, a two-way arrow is used to show a significant relationship exists (i.e. it is not implying a two-way relationship).

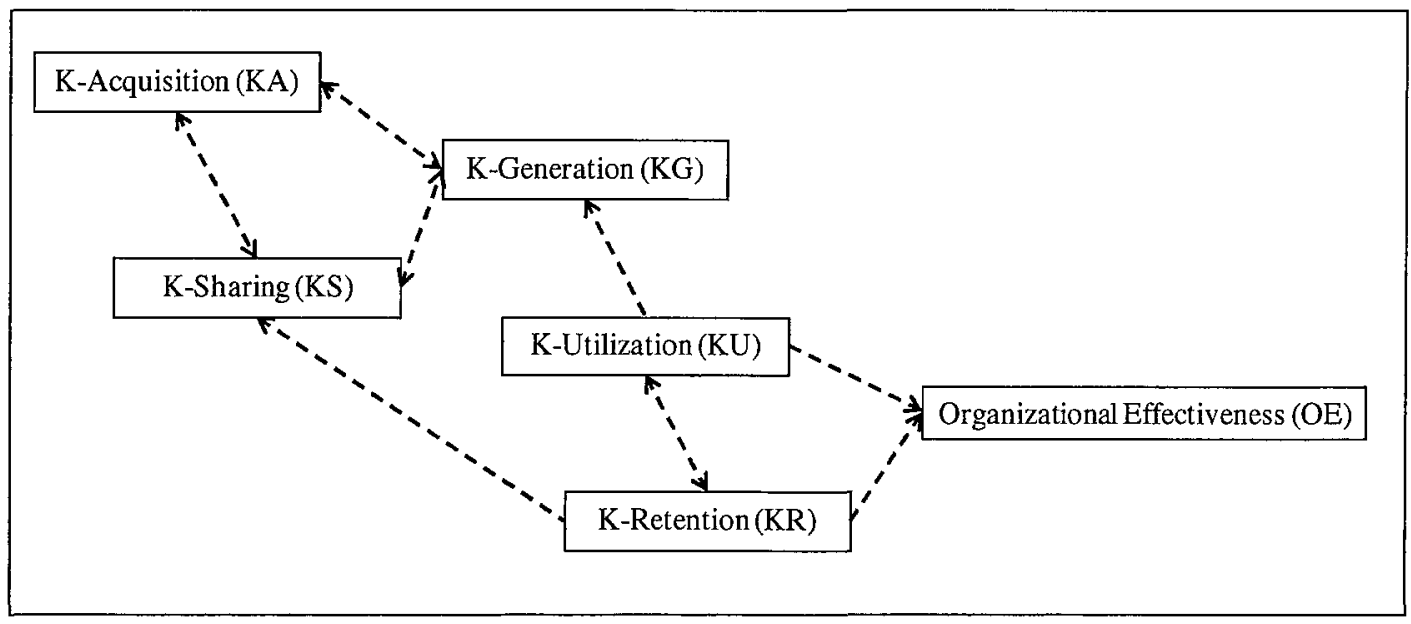

Figure 91. Prelim. Findings - Links Among QKPs and to OE 


\subsection{Linking KMPs and OTSs to Organizational Effectiveness (OE)}

Although not hypothesized in the full research model (see Figure 25), it is possible there are direct effects between KMPs and OE or OTSs and OE. To be thorough and systematic in the analysis, linear regressions were run to investigate the total indirect effects of each of the KMP constructs and OTS constructs with organizational effectiveness (OE). These will be compared with the final full model developed using path analysis. It is useful additional information to help guild the interpretation when either selecting between competing models, interpreting anomalous findings, or understanding the predictive limitations of either the full model or its underlying measures. The findings obtained from this step are not to be construed as necessarily valid as the important intermediate effects of SOC and the QKPs are being ignored. Table 53 summarizes the reduced factor set after non-significant variables were dropped from the model. Only KMS, PMP, IMP, and IST remained as significant at the .05 level. Note all these relationships to $\mathrm{OE}$ are positive, fairly strong effects, and with reasonable observed power $(>0.8)$.

Table 53. Regression KMS+PMP+IMP+IST $\rightarrow \mathrm{OE}$

\begin{tabular}{|c|c|c|c|c|c|c|}
\hline \multicolumn{2}{|l|}{ Dependent Variable:OE } & & & \multicolumn{2}{|c|}{$95 \%$ Confidence Interval } \\
\cline { 6 - 7 } Parameter & $\mathrm{B}$ & Std. Error & $\mathbf{t}$ & Sig. & Lower Bound & Upper Bound \\
\hline KMS & .223 & .053 & 4.223 & .000 & .118 & .327 \\
\hline PMP & .169 & .074 & 2.293 & .024 & .023 & .314 \\
\hline IMP & .228 & .089 & 2.570 & .011 & .052 & .404 \\
\hline IST & .146 & .046 & 3.147 & .002 & .054 & .237 \\
\hline
\end{tabular}

Figure 92 below shows a scatterplot of residuals for KMS+PMP+IMP+IST $\rightarrow \mathrm{OE}$. 


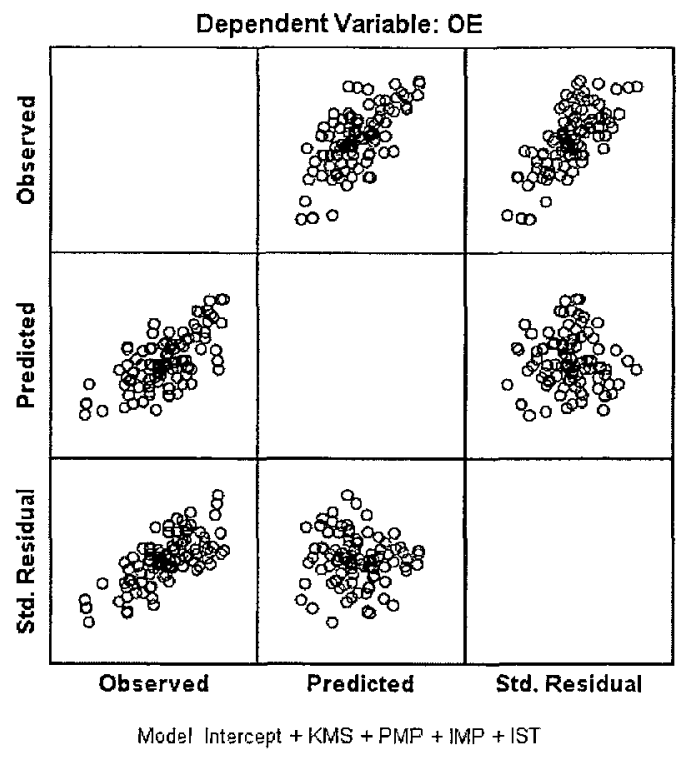

Figure 92. Residuals of KMS+PMP+IMP+IST $\rightarrow \mathrm{OE}$

To summarize these findings, Figure 93 illustrates all the significant direct links to OE. Although these relationships can be supported by theory from the literature, the purpose of this research is to go beyond the traditional simplistic interpretations and attempt and identify and understand the underlying mechanisms supporting each relationship. These results will be compared to the final full model using path analysis methods. 


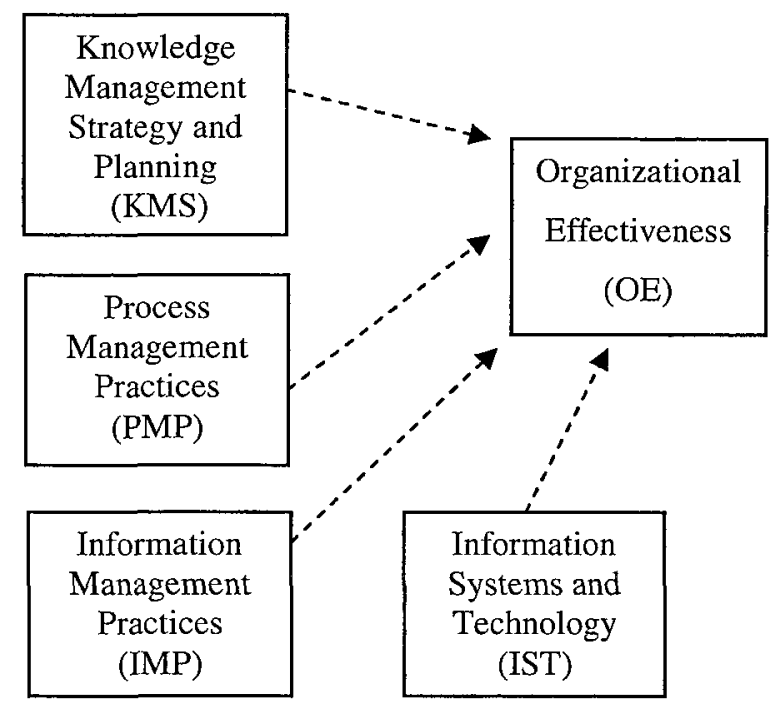

Figure 93. Possible KMP Links to Organizational Effectiveness

However, it should be kept in mind that, consistent with the proposed KMPM model, it is quite likely that the intermediary role of the QKPs and SOC will account for these correlations once included in a more complex integrated model. Thus it should not be concluded at this point that these are necessarily valid direct causal effects. For this reason they are shown as "dotted lines" (i.e. to be confirmed) in Figure 93.

\subsection{Linking Organizational Effectiveness to Operational Performance}

Linear regressions were run to investigate direct links from organizational effectiveness (OE) with organizational performance (OP) (i.e. the bin normalized variable "CPERFNormBins" referred to earlier) and the raw organizational performance indicator data (OP_raw) (i.e. earlier referred to as the combined performance index, or 
"CPerfINDEX"). Table 54 and 55 summarize these findings. Observed power is reported here to emphasize the strength of these important findings.

Table 54. Regression of $\mathrm{OE} \rightarrow \mathrm{OP}$

\begin{tabular}{|c|c|c|c|c|c|c|c|}
\hline \multicolumn{2}{|l|}{ Dependent Variable:OP } \\
\hline Parameter & $\mathrm{B}$ & $\begin{array}{c}\text { Std. } \\
\text { Error }\end{array}$ & $\mathrm{t}$ & Sig. & Lower Bound & Upper Bound & $\begin{array}{c}\text { Observed } \\
\text { Power }\end{array}$ \\
\hline OE & .535 & .189 & 2.831 & .005 & .161 & .909 & .802 \\
\hline
\end{tabular}

From Table 54 it can be seen that $\mathrm{OE}$ was found to be significant at 0.005 . Figure 94 below shows a scatterplot of residuals for $\mathrm{OE} \rightarrow \mathrm{OP}$.

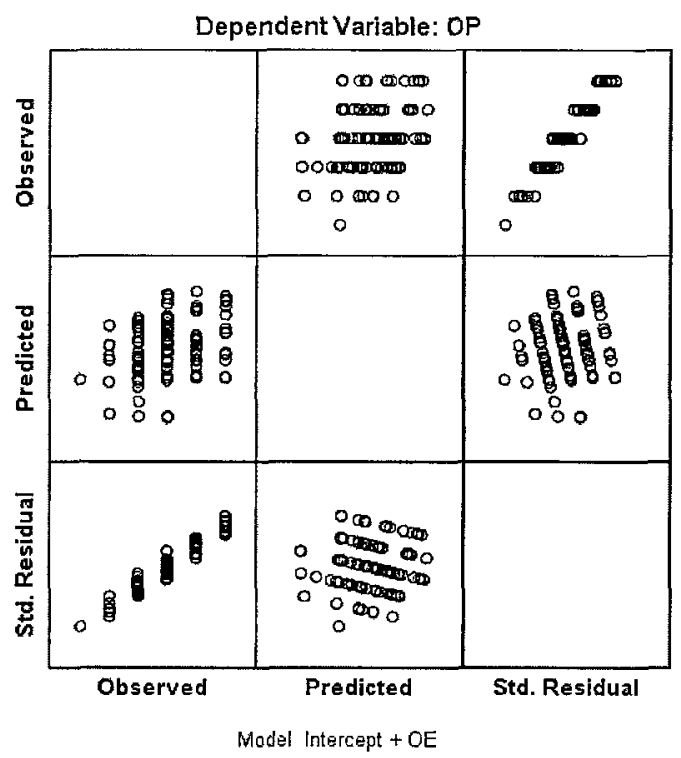

Figure 94. Residual Plot of $\mathrm{OE} \rightarrow \mathrm{OP}$

Table 55. Regression OE $\rightarrow$ OP_raw (CPerfINDEX)

\begin{tabular}{|c|c|c|c|c|c|c|c|}
\hline \multicolumn{8}{|c|}{ Dependent Variable:OP_raw } \\
\hline \multirow[b]{2}{*}{ Parameter } & \multirow[b]{2}{*}{ B } & \multirow{2}{*}{$\begin{array}{l}\text { Std. } \\
\text { Error }\end{array}$} & \multirow[b]{2}{*}{$t$} & \multirow[b]{2}{*}{ Sig. } & \multicolumn{2}{|c|}{$95 \%$ Confidence Interval } & \multirow{2}{*}{$\begin{array}{c}\text { Observed } \\
\text { Power }\end{array}$} \\
\hline & & & & & Lower Bound & Upper Bound & \\
\hline OE & 5.821 & 2.386 & 2.440 & .016 & 1.097 & 10.545 & .677 \\
\hline
\end{tabular}


From Table 55 it can be seen that $\mathrm{OE}$ was found to be significant at .016. Figure 95 below shows a scatterplot of residuals for $\mathrm{OE} \rightarrow$ OP_raw. Note that the observed power was expected to be lower due to the skew and kurtosis effects of the raw data discussed earlier (refer to Sections 5.4 and 5.5), however the result (.677) is better than expected.

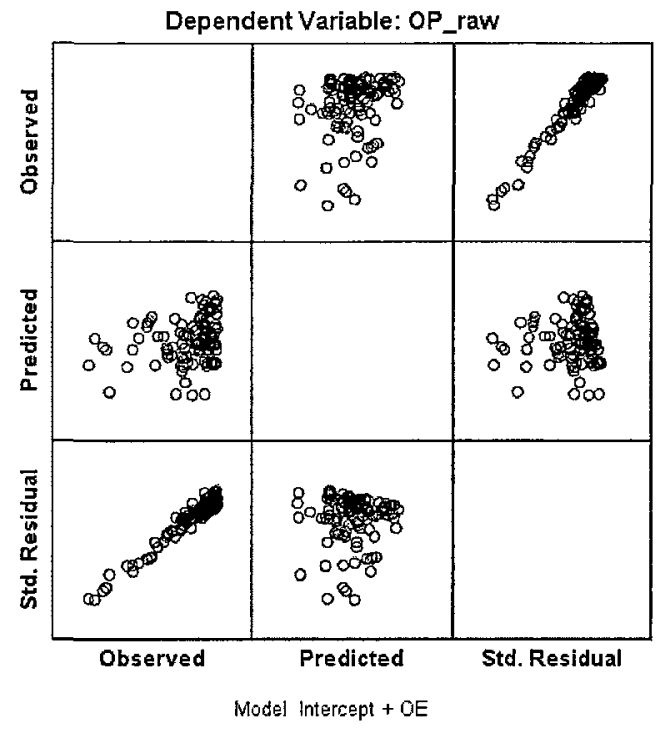

Figure 95. Residual Plot of $\mathrm{OE} \rightarrow \mathrm{OP} \_$raw

Because (as expected) the operational performance indicators are skewed towards optimal production values, it is difficult to visualize clearly the relationship from the scatterplot of Figure 95. To better illustrate and visualize the general trend line for these relationships, Figures $96-98$ show ribbon plots of:

- Mean of CperfINDEX vs. OE (i.e. "ORGEFFAVG") and Mode of CFERFNormBins (i.e. OP, the bin normalized operational performance index “CperfINDEX”) vs. OE (i.e. "ORGEFFAVG") .

- Mode of OP_raw (i.e. raw data with no indexed values) vs. OE, and 
- OP (i.e. bin normalized operational performance index "CFERFNormBins") vs. mean of OE (i.e. "ORGEFFAVG").

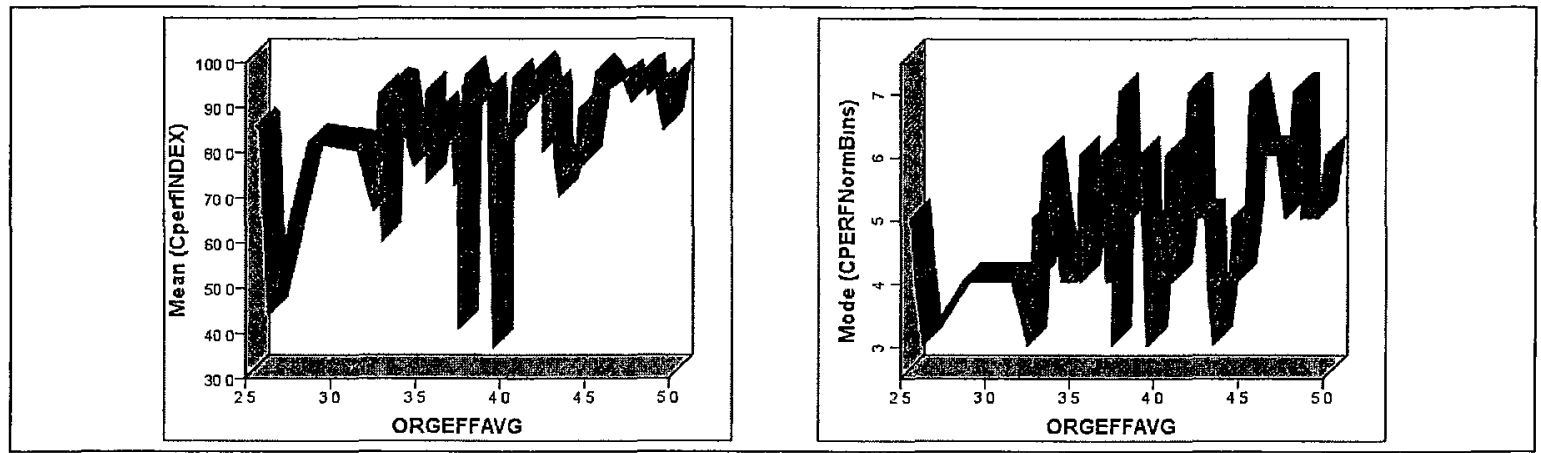

Figure 96. Mean OP_raw \& Mode OP vs. OE

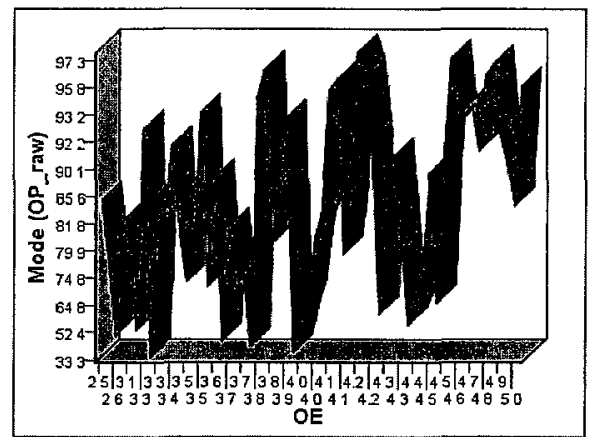

Figure 97. Mode OP_raw vs. OE

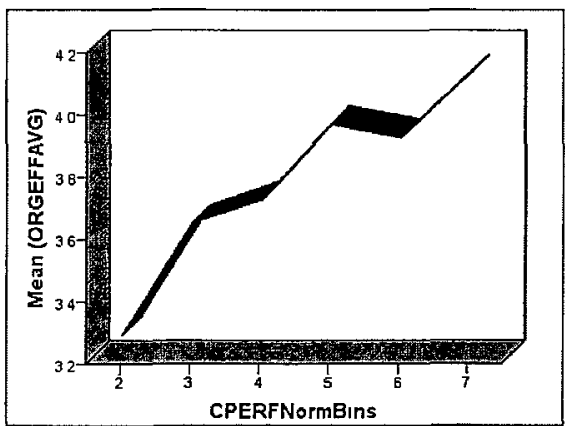

Figure 98. Mean OE vs. OP 
From Figures $96-98$ it is easy to see visually the positive relationship between these factors (i.e. $\mathrm{OE} \rightarrow \mathrm{OP}$ ).

\subsection{Effect of Plant Size, Units and Age on $O E$ and $O P$}

Linear regression was run for Size + Units + Age $\rightarrow$ OE. Table 56 summarizes the results. Plant size (MWe Rating), although significant with $\mathrm{p}=.026$ has a $\beta$ of .000 with an upper and lower bound almost zero, so can be ignored. Age is significant at $p=.000$ and $\beta$ of -.019 . Units is significant with $p=.043$ and $\beta$ of -.074 . It can be concluded that plant age and number of site units both have a statistically significant negative influence on organizational effectiveness.

Table 56. Regression Size + Units + Age $\rightarrow$ OE

\begin{tabular}{|c|c|c|c|c|c|c|}
\hline Dependent Variable: OE \\
\hline & & Std. & & & \multicolumn{2}{|c|}{$95 \%$ Confidence Interval } \\
\cline { 6 - 7 } Parameter & B & Error & t & Sig. & Lower Bound & Upper Bound \\
\hline Units & -.074 & .036 & -2.046 & .043 & -.145 & -.002 \\
\hline AgeYrs & -.019 & .005 & -3.621 & .000 & -.030 & -.009 \\
\hline
\end{tabular}

Linear regression was run for Size + Units + Age $\rightarrow$ OP. Table 57 summarizes the results. Plant size (MWe Rating), although significant with $\mathrm{p}=.038$, it has a $\beta$ of .001 with an upper and lower bound almost zero, so can be ignored. Age is not significant at $\mathrm{p}=.805$ and $\beta$ of -.003 and Units is significant with $\mathrm{p}=.006$ and $\beta$ of -.221 respectively. It can be concluded that the number of site units has a negative impact on operating performance. 
Table 57. Regression Units + Age $\rightarrow$ OP

\begin{tabular}{|c|c|c|c|c|c|c|c|}
\hline Dependent Variable: OP & \multicolumn{3}{|c|}{} \\
\hline & & $\begin{array}{c}\text { Std. } \\
\text { Parameter }\end{array}$ & $\mathrm{B}$ & Error & $\mathrm{t}$ & Sig. & \multicolumn{2}{|c|}{ 95\% Confidence Interval } & $\begin{array}{c}\text { Observed } \\
\text { Power }\end{array}$ \\
\hline Units & -.221 & .079 & -2.796 & .006 & -.377 & -.064 & .792 \\
\hline
\end{tabular}

\subsection{Finite Population Considerations}

A total of 122 usable responses were received. This might be considered a small sample size, however, it represents an actual return rate of about $60 \%$ of stations, and is a high percentage of the total population. When the population size is small and is known, the sampling error $(\varepsilon)$ can be reduced by multiplying it by the square root of the "finite population correction factor" $(f p c)$, where $f p c=(N-n) /(N-1)$ (Jobson, 1999). In this study, with the total finite population $N=204$ units, and the $60 \%$ return rate $(\mathrm{n}=124$ units), the reduction in sampling error expected using the equation above is calculated as 1- $\operatorname{sqrt}[(204-124) /(204-1)]$ or about $37 \%$.

Similarly, the variance $x$ should be multiplied by fpc to correct for the effect of a finite population, so $x_{\text {corrected }}=(f p c) *\left(\sigma^{2} / n\right)=[(N-n) /(N-1)] \sigma^{2} / n$, and the effect can be significant (Jobson, 1999). This correction can be an important factor to consider in a statistical analysis with a finite population. It can be applied to linear regressions using a case weighting variable in SPSS. It is not possible to apply fpc corrections to a path analysis using AMOS. As a result, in order to be able to directly compare the results from the previous linear regressions with the regression path weights obtained from the subsequent Path Analysis, fpc corrections were not applied to these regressions. However, it is safe to conclude that the significance obtained without $f p c$ will be a more 
conservative parameter estimate and that the actual statistical significance and observed power will be much better than actually reported.

\subsection{Summary of Linear Regressions}

In summary, the preceding linear regressions support the hypotheses in general and suggest many specific significant links between the constructs. Although the findings from the linear regressions are significant, they look at the relationships in relative isolation. Standard multiple linear regression techniques allow many-to-one relationships to be examined and provide valuable insights into the data, the findings must be interpreted with caution. In the context of a larger system of interacting constructs, standard linear regression methods do not provide an adequate means to examine the integrated effects simultaneously. To achieve a simultaneous analysis of these relationships a more advanced technique, referred to as path analysis, can be used. The findings obtained from the linear regressions provide a basic understanding from which to build and test a fuller integrated model based on the observed relationships. 


\section{Path Analysis Assessment}

Path Analysis is a useful methodology based on linear regression techniques to model causal relationships. It utilizes simple bivariate correlations to estimate the relationships in a system of structural equations (Hair et al., 2006). It enables a formal definition of dependent and independent variable relationships using a diagramming technique known as "path diagrams" that is a simplified version of the structure model representations in CFA or SEM. The hypothesized relationships are represented with uni-directional arrows to show causal dependency and bi-directional arches to show association without dependency (i.e. correlation). The absence of a path indicates that no relationship is thought to exist. A path coefficient is associated with each causal dependency relationship (uni-directional arrow) on the diagram. It is derived from the standardized regression coefficients that influence that particular path. They are interpreted much like regression coefficients, with the exception that being standardized coefficients they imply that with a unit change in the standard deviation of the independent variable a change in the dependence variable with result that is the product of the path coefficient and the dependent variable's standard deviation (Agresti and Finley, "86, p512). The approach assumes any residual for each dependent variable are uncorrelated with other dependent variable residuals. Paths are evaluated as both direct and indirect (i.e. through an intermediate variable). The method is based on decomposing the bivariate correlation between any two variables into the sum of the 
"compound paths" connecting them, where compound paths are determined by the researcher and evaluated following 3 basic rules (Hair et al., 2006, p763):

1. after going forward on an arrow, the path cannot go backward again; but the path can go backward as many times as necessary before going forward

2. the path cannot go through the same variable more than once, and

3. the path can include only one curved arrow (correlation variable pair).

Thus the correlation between any two constructs is the sum of the direct and indirect path coefficients. The coefficients then represent their causal relationships (or correlation estimates) between constructs.

In summary, models are represented in Path Analysis with visual diagrams, called "path diagrams". Paths from one construct to another are shown with arrows to show relationships as predicted by theory. Path Analysis provides a methodology for estimating the strengths of the relationships depicted by the arrows using the covariance matrix for input: it decomposes the bivariate correlation between any two variables into the sum of the compound paths connecting the two variables and thus solves the causal relationship based on correlations among all the constructs for the specified model (Hair, et al., 2006).

AMOS software was used to perform the path analysis. It is widely used for both structural equation modeling and path analysis and has been shown to provide results equivalent to Lisrel (Clayton and Pett, 2008). In AMOS path diagrams, the numbers on the arrows indicate the "loadings" or "weights" of one factor on another, and are similar 
to regression coefficients but are standardized. The number in the top right corner of all endogenous variables (i.e. variables dependent on other variables) is the "squared multiple correlation" and represents the extent to which the measured variable's variance is explained by the other factors acting on it. The Amos 18 User's Guide (Arbuckle, 2008) describes the squared multiple correlation as follows:

$R^{2}=1-\frac{\hat{\sigma}_{\text {residual }}^{2}}{\hat{\sigma}_{\text {Bndogenous }}^{2}}$, where $\hat{\sigma}_{\text {Bndoggnous }}^{2}$ is the estimated (implied) variance of the endogenous variable and $\hat{\sigma}_{\text {residzal }}^{2}$ is the estimated (implied) variance of the associated residual variable.

\subsection{Summary of Goodness of Fit (GOF) Statistics Used}

In order to ensure a model is statistically valid, a number of goodness of fit (GOF) statistics have been developed as guidelines. AMOS provides several standard goodness of fit statistics. The AMOS fit statistics used in this study are (from Arbuckle, 2008):

1. NPAR - number of distinct parameters to be estimated by the model.

2. CMIN $-\chi^{2}$ statistic (i.e. "chi"'-squared statistic).

3. DF - Degrees of freedom for the model (number of variables less number of free parameters)

4. $\quad \mathrm{P}$ - the $\mathrm{p}$-value based on $\chi^{2}$ statistic. In this case, a non-significant result (i.e. $>0.05$ ) indicates the estimated correlation matrix is not statistically different 
from the observed correlation matrix. Note however, that because $\chi^{2}$ statistic is sensitive to both the number of parameters and sample size, in models with more than 100 cases (and here $n=124$ ), it will often be significant. It is also affected by the size of the correlations in the model: the larger the correlations, the poorer the fit. For these reasons alternative measures of fit have been developed. Other fit statistics should be used and $\chi^{2}$ statistic should not be relied upon solely.

5. CMIN/DF - the ratio of $\chi^{2} / \mathrm{DF}$ in the range of 3 to 1 indicates acceptable fit between the hypothetical model and the sample data (Carmnines\&McIver,1981). Different researchers have recommended using ratios as low as 2 or as high as 5 to indicate a reasonable fit (Marsh and Hocevar, 1985).

6. The Bentler \& Bonett (1980) "normed fit index" (NFI), provides a ration of the fit of the saturated model (perfect fit) to the fit of the independence model (worse fit). In general, models with overall fit indices approaching 0.9 or higher are acceptable.

7. Similarly, Bollen's (1986) "relative fit index" (RFI) is obtained from the NFI. RFI values close to 1 indicate a very good fit.

8. Bollen's (1989) incremental fit index (IFI) is similar to NFI and RFI and values close to 1 indicate a very good fit.

9. The Tucker-Lewis Index (TFI) (Bollen, 1989) is also known as the BentlerBonett non-normed fit index (NNFI) typically ranges between zero and one. TLI values close to 1 indicate a very good fit. 
10. The comparative fit index (CFI; Bentler, 1990) is identical to the McDonald and Marsh (1990) relative noncentrality index ( RNI) except that the CFI is truncated to fall in the range from 0 to 1 . CFI values close to 1 indicate a very good fit.

11. RMSEA - Root mean square error of approximation (Browne and Cudeck 1993). It is a "badness of fit" index. RMSEA estimates the lack of fit of a model to the estimated population covariance matrix. If the fit is good, RMSEA should be small. RMSEA values of 0.05 or less indicate a close fit, a value of about .08 or less would indicate a reasonable fit (Browne and Cudeck, 1993). Several authors state that values between .08 and .95 RMSEA also indicate a reasonable fit. Most authors agree that a model with RMSEA greater than 0.95 would be considered poor."

12. HI-90 - is the upper (high-side) $90 \%$ confidence interval for RMSEA.

13. PLCOSE - tests the null hypothesis that RMSEA is no greater than .05. If PCLOSE is less than .05 , we reject the null hypothesis and conclude that the computed RMSEA is greater than .05, indicating lack of a close fit.

14. HN05 - Hoelter's "critical N" is provided in some cases and indicates the largest sample size for which one would accept the hypothesis that a model is correct. Critical $\mathrm{N}$ for significance levels of 0.05 are provided.

15. AIC - Akaike's Information Criterion is based on the standard goodness-of-fit $\chi^{2}$ statistic but includes a penalty for complexity. It is used to compare two models, including non-nested models. The model with the lower AIC is considered the 
best fit. AIC is included as GOF statistic when it is appropriate to compare competing models.

Table 58 below is an example that shows the standard reporting format to be used when reporting model GOFs for each Path Analysis Model developed. It includes all of the indicators explained above.

Table 58. Format for Reporting GOF (fictitious acceptable fit values)

\begin{tabular}{|c|c|c|c|c|c|c|c|c|c|c|c|c|c|c|}
\hline MPAR & CMIN & DF & P & CMIN/DF & NFI & RFI & IFI & TLI & CFI & RMSEA & HI 90 & PCLOSE & HN05 & AIC \\
\hline 16 & 5.176 & 4 & .27 & 1.294 & .985 & 944 & .997 & .987 & .987 & .048 & .149 & .422 & 122 & 287.33 \\
\hline
\end{tabular}

In addition to the GOF statistics, the regression weights, standardized regression weights, and squared multiple correlations will be reported. In cases where it is appropriate, the means, standardized total effects, standardized direct effects, and standardized indirect effects will also be reported.

\subsection{Hypothesis Testing via Stepwise Development of Path Models}

In order to develop appropriate models and a meaningful strategy for testing the hypotheses, a stepwise incremental model development strategy was employed, beginning with a simple top-level model based on summary scales, and developing several sub-models with increasing successive levels of detail to isolate and understand the construct iterations. Finally, a fully integrated model incorporating all of the constructs was developed and tested. The follow sub-sections describe the various models and their fit statistics and discuss the hypothesis findings they do or do not 
support. Reference is made as appropriate back to the preliminary findings from the linear regression analysis to aid in the interpretation of the results.

\subsection{Path Model 1: Top-level Model with KMPs}

Path Analysis Model 1 (see Figure 99) was developed to test the following hypotheses:

Hypothesis 1. Higher levels of overall support for KM practices (KMP) will positively influence the supportive organizational culture (SOC).

Hypothesis 2. Higher levels of supportive organizational culture (SOC) will positively influence the overall quality of knowledge processes $(Q K P)$.

Hypothesis 3. Higher levels of supportive organizational culture (SOC) will positively influence organizational effectiveness (OE).

Hypothesis 4. Higher levels of overall support for KM practices (KMP) will positively influence the overall quality of knowledge processes $(Q K P)$ directly, and indirectly through supportive organizational culture (SOC).

Hypothesis 5. Higher levels of overall support for KM practices (KMP) will positively influence organizational effectiveness $(O E)$ through the intermediate effects of both overall quality of knowledge processes (QKP) and supportive organizational culture (SOC).

Hypothesis $8 . \quad$ Higher overall quality of knowledge processes $(Q K P)$ will positively influence organizational effectiveness (OE).

Hypothesis 9. Higher organizational effectiveness (OE) will positively influence operational performance $(O P)$. 
A composite summated scale was created for "support for KM practices" ("KMP") from all its related summated-scale sub-constructs. Likewise a composite summated scale was created for "quality of knowledge processes" ("QKP") from the 5 sub-construct summate scales. The variable "SOC" is the summated scale for "supportive organizational culture" (all measures included), "OE" is the summated scale for all "organizational effectiveness" measures, and "OP" is the "operational performance" measure "CPERFNormBins" discussed earlier. Figure 99 shows the resulting model and analysis results. Note the composite construct names used here are used in all subsequent top-level models. An additional composite (not used in Figure 99 but later on) is organizational technology support (OTS), which includes an average of both IST and OSS summated scale constructs.

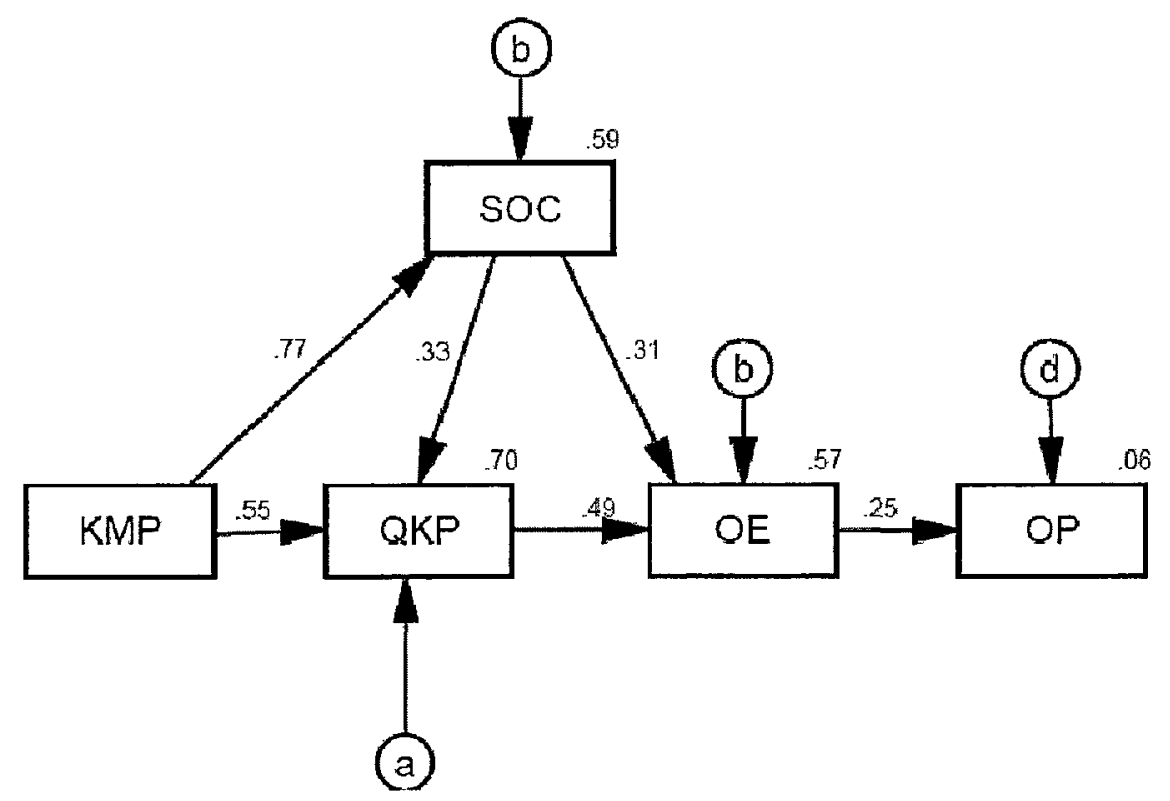

Figure 99. Path Diagram for Model 1 
Table 59. Model 1 - Goodness of Fit Statistics

\begin{tabular}{|c|c|c|c|c|c|c|c|c|c|c|c|c|c|}
\hline NPAR & CMIN & DF & P & CMIN/DF & NFI & RFI & IFI & TFI & CFI & RMSEA & HI 90 & PCLOSE & HN05 \\
\hline 16 & 4.240 & 4 & .375 & 1.060 & .989 & .957 & .999 & .997 & .999 & .022 & .137 & .531 & 287 \\
\hline
\end{tabular}

From Table 59, we can conclude the model is clearly a good fit according to all GOF statistics and statistically significant conclusions can be drawn from it. Table 60 below summarizes the regression weights and p-values for each link in the model. All relationships are significant at 0.005 level or better (note that in Table 60 and all subsequent tables $\mathrm{p}=* * *$ indicates $\mathrm{p}<.001)$. It is interesting to note the high path weight from KMP $\rightarrow$ SOC. Supportive organizational culture is strongly influenced by supportive KM practices. This is clearly an important relationship.

Table 60. Model 1 Regression Weights

\begin{tabular}{|c|c|c|c|c|c|}
\hline Link & Estimate & S.E. & C.R. & P & Standardized Estimates \\
\hline SOC <-- KMP & 1.010 & .077 & 13.195 & $* * *$ & .766 \\
\hline QKP <-- SOC & .280 & .065 & 4.319 & $* * *$ & .333 \\
\hline QKP <-- KMP & .611 & .085 & 7.169 & $* * *$ & .553 \\
\hline OE <-- QKP & .508 & .095 & 5.366 & $* * *$ & .489 \\
\hline OE <-- SOC & .274 & .079 & 3.444 & $* * *$ & .314 \\
\hline OP <--- OE & .526 & .186 & 2.830 & .005 & .248 \\
\hline
\end{tabular}

Table 61. Model 1 Squared Multiple Correlations

\begin{tabular}{|l|c|}
\hline Construct & Estimate \\
\hline SOC & .588 \\
\hline QKP & .699 \\
\hline OE & .570 \\
\hline OP & .061 \\
\hline
\end{tabular}

From the squared multiple correlations in Table 61 we can conclude that the explained variance of supportive organizational culture (SOC) is $\sim 59 \%$, the explained variance of quality of knowledge processes is $\sim 70 \%$ and the explained variance of organizational 
effectiveness is $57 \%$. As expected, the explained variance of operational performance is rather low at about $6 \%$.

The standardized regression weightings help to understand the path coefficients in terms of standard deviations of each construct. A standardized regression weighing value of +0.3 for example means that a change of one standard deviation in the value of the causal construct will cause a corresponding +'ve change of 0.3 standard deviations in the dependant construct. Tables 62,63 , and 64 summarize the standardized total, direct, and indirect effects.

Table 62. Model 1 Standardized Total Effects

\begin{tabular}{|c|c|c|c|c|}
\hline Construct & KMP & SOC & QKP & OE \\
\hline SOC & .766 & .000 & .000 & .000 \\
\hline QKP & .808 & .333 & .000 & .000 \\
\hline OE & .636 & .477 & .489 & .000 \\
\hline OP & .157 & .118 & .121 & .248 \\
\hline
\end{tabular}

Table 63. Model 1 Standardized Direct Effects

\begin{tabular}{|c|c|c|c|c|}
\hline Construct & KMP & SOC & QKP & OE \\
\hline SOC & .766 & .000 & .000 & .000 \\
\hline QKP & .553 & .333 & .000 & .000 \\
\hline OE & .000 & .314 & .489 & .000 \\
\hline OP & .000 & .000 & .000 & .248 \\
\hline
\end{tabular}

Table 64. Model 1 Standardized Indirect Effects

\begin{tabular}{|c|c|c|c|c|}
\hline Construct & KMP & SOC & QKP & OE \\
\hline SOC & .000 & .000 & .000 & .000 \\
\hline QKP & .256 & .000 & .000 & .000 \\
\hline OE & .636 & .163 & .000 & .000 \\
\hline OP & .157 & .118 & .121 & .000 \\
\hline
\end{tabular}

It is interesting to note the weighting coefficient for the indirect effect KMP $\rightarrow \mathrm{SOC} \rightarrow$ $\mathrm{QKP}$ is 0.256 . The total indirect effects of $\mathrm{KMP}$ on $\mathrm{OE}$ via path $\mathrm{KMP} \rightarrow \mathrm{SOC} \rightarrow \mathrm{OE}$ 
and path $\mathrm{KMP} \rightarrow \mathrm{QKP} \rightarrow \mathrm{OE}$ is 0.636 . Also, the indirect effect of $\mathrm{SOC} \rightarrow \mathrm{QKP} \rightarrow \mathrm{OE}$ has a standardized weight of 0.163 . These relationships are important and show the strength of the role that SOC plays as an intermediate variable "leveraging" the effect of the KMPs. Finally, the indirect effects on operational performance (OE) are noteworthy:

- $\mathrm{KMP} \rightarrow \mathrm{QKP} \rightarrow \mathrm{OE} \rightarrow \mathrm{OP}$ plus $\mathrm{KMP} \rightarrow \mathrm{SOC} \rightarrow \mathrm{QKP} \rightarrow \mathrm{OE} \rightarrow \mathrm{OP}$ plus $\mathrm{KMP}$ $\rightarrow \mathrm{SOC} \rightarrow \mathrm{OE} \rightarrow \mathrm{OP}$ combine to create a standardized indirect KMP loading of 0.157 on OP;

- $\mathrm{SOC} \rightarrow \mathrm{OE} \rightarrow \mathrm{OP}$ plus $\mathrm{SOC} \rightarrow \mathrm{QKP} \rightarrow \mathrm{OE} \rightarrow \mathrm{OP}$ combine to create a standardized indirect SOC loading of 0.118 on OP; and finally

- $\mathrm{QKP} \rightarrow \mathrm{OE} \rightarrow \mathrm{OP}$ has a standardized indirect loading of 0.121 on OP

All of these effects are strong, are statistically significant, and support the hypotheses. Table 65 provides an overall summary of the findings from Model 1 against the hypotheses.

Table 65. Summary of Model 1 Findings

\begin{tabular}{|c|c|c|}
\hline Hypothesis & Sign & Conclusion \\
\hline$H 1: K M P \rightarrow S O C$ & + & supported \\
\hline$H 2: S O C \rightarrow Q K P$ & + & supported \\
\hline$H 3: S O C \rightarrow O E$ & + & supported \\
\hline$H 4 a: K M P \rightarrow Q K P$ & + & supported \\
\hline$H 4 b: K M P \rightarrow S O C \rightarrow Q K P$ & + & supported \\
\hline$H 5 a: K M P \rightarrow Q K P \rightarrow O E$ & + & supported \\
\hline$H 5 b: K M P \rightarrow S O C \rightarrow O E$ & + & supported \\
\hline$H 8: Q K P \rightarrow O E$ & + & supported \\
\hline$H 9: O E \rightarrow O P$ & + & supported \\
\hline
\end{tabular}




\subsection{Path Model 2: Top-level Model with OTS}

Path Model 2 (see Figure 100) was developed to test hypotheses H6a and H7a which state:

Hypothesis 6a. Higher levels of organizational technology support (OTS) will positively influence overall quality of knowledge processes $(Q K P)$.

Hypothesis 7a. Higher levels of organizational technology support (OTS) will positively influence organizational effectiveness (OE) through the intermediate effects of overall quality of knowledge processes $(Q K P)$.

Figure 100 shows the Path Analysis Model 2 path diagram for the top level model with OTS on the left-hand-side instead of KMP. This model examines the effects of OTS alone on the other constructs without the influence of KMP.

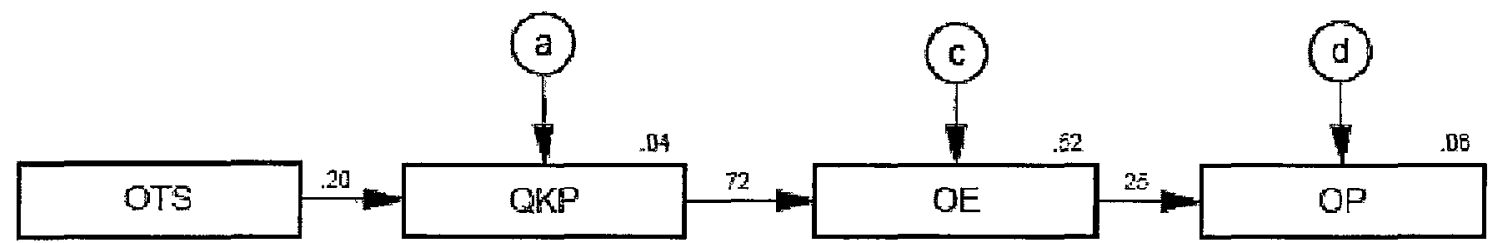

Figure 100. Path Diagram for Model 2

Table 66. Model Goodness of Fit Statistics for Path Model 2

\begin{tabular}{|c|c|c|c|c|c|c|c|c|c|c|c|c|c|}
\hline NPAR & CMIN & DF & P & CMINDF & NFI & RFI & IFI & TLI & CFI & RMSEA & HI 90 & PCLOSE & HN05 \\
\hline 11 & 3.206 & 3 & .361 & 1.069 & .970 & .899 & .998 & .993 & .998 & .023 & .153 & .496 & 313 \\
\hline
\end{tabular}

From Table 66, we can conclude the model is clearly a good fit according to all GOF statistics and statistical inference conclusions can be drawn from it. Table 67 below 
summarizes the regression weights and p-values for each link in the model. All relationships are significant at 0.05 level or better.

Table 67. Model 2 Regression Weights

\begin{tabular}{|c|c|c|c|c|c|}
\hline Link & Estimate & S.E. & C.R. & P & Standardized Estimate \\
\hline QKP <-- OTS & .110 & .050 & .196 & .027 & .196 \\
\hline OE <-- QKP & .750 & .065 & .724 & $* * *$ & .724 \\
\hline OP <-- OE & .529 & .186 & .248 & .005 & .248 \\
\hline
\end{tabular}

Table 68. Model 2 Squared Multiple Correlations

\begin{tabular}{|c|c|}
\hline Construct & Estimate \\
\hline QKP & .038 \\
\hline OE & .525 \\
\hline OP & .062 \\
\hline
\end{tabular}

Table 69. Model 2 Standardized Total Effects

\begin{tabular}{|c|c|c|c|}
\hline Construct & OTS & QKP & OE \\
\hline QKP & .196 & .000 & .000 \\
\hline OE & .142 & .724 & .000 \\
\hline OP & .035 & .180 & .248 \\
\hline
\end{tabular}

Table 70. Model 2 Standardized Direct Effects

\begin{tabular}{|c|c|c|c|}
\hline Construct & OTS & QKP & OE \\
\hline QKP & .196 & .000 & .000 \\
\hline OE & .000 & .724 & .000 \\
\hline OP & .000 & .000 & .248 \\
\hline
\end{tabular}

Table 71. Model 2 Standardized Indirect Effects

\begin{tabular}{|c|c|c|c|}
\hline Construct & OTS & QKP & OE \\
\hline QKP & .000 & .000 & .000 \\
\hline OE & .142 & .000 & .000 \\
\hline OP & .035 & .180 & .000 \\
\hline
\end{tabular}

From the analysis of Model 2, it is clear that organizational technology support has a positive influence on quality of knowledge processes, however, only $4 \%$ of the variance of QKP is explained by OTS so its role should not be overstated. The standardized 
regression weight for OTS $\rightarrow$ QKP is $0.196 \quad(\mathrm{p}=0.027)$. The total indirect effect of organizational technology support on organizational effectiveness is 0.142 standard weightings. The total indirect effect of organizational technology support (OTS) on operational performance is 0.035 standard weightings. Table 72 below summarizes the finding for hypothesis $\mathrm{H} 6$ and $\mathrm{H} 7$.

Table 72. Summary of Model 2 Findings

\begin{tabular}{|c|c|c|}
\hline Hypothesis & Sign & Conclusion \\
\hline H6a: $\quad$ OTS $\rightarrow Q K P$ & + & Supported \\
\hline H7a: $O T S \rightarrow O K P \rightarrow O E$ & + & Supported \\
\hline
\end{tabular}

\subsection{Path Model 3: Top-level Model with IST}

Path Model 3 (see Figure 101) was developed to test hypotheses $6 \mathrm{~b}$ and $7 \mathrm{~b}$ related to information and systems technology support (IST) and its impact on QKP and OE which stated:

Hypothesis 66 . Higher levels of information systems and technology support (IST) will positively influence overall quality of knowledge processes $(Q K P)$.

Hypothesis 7b. Higher levels of information systems and technology support (IST) will positively influence organizational effectiveness (OE) through the intermediate effects of overall quality of knowledge processes (QKP). 


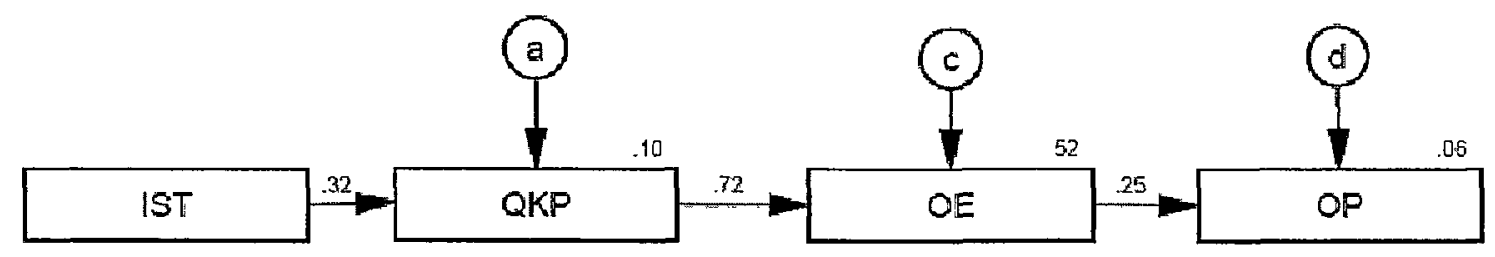

Figure 101. Path Diagram for Model 3

Table 73. Model Goodness of Fit Statistics for Path Model 3

\begin{tabular}{|c|c|c|c|c|c|c|c|c|c|c|c|c|c|c|}
\hline NPAR & CMIN & DF & $\mathbf{P}$ & CMIN/DF & NFI & RFI & IFI & TLI & $\mathrm{CFI}$ & RMSEA & HI 90 & PCLOSE & HN05 & AIC \\
\hline 11 & 1.720 & 3 & .632 & .573 & .985 & .949 & 1.012 & 1.042 & 1.000 & .000 & .120 & $\begin{array}{l}.737 \\
\end{array}$ & 582 & 23.720 \\
\hline
\end{tabular}

From Table 73, we can conclude the model is clearly a good fit according to all GOF statistics and statistical inference conclusions can be drawn from it. Table 74 below summarizes the regression weights and p-values for each link in the model. All relationships are significant at 0.005 level or better.

Table 74. Model 3 Regression Weights

\begin{tabular}{|c|c|c|c|c|c|}
\hline Link & Estimate & S.E. & C.R. & P & Standardized Estimate \\
\hline QKP <-- IST & .208 & .056 & 3.726 & $* * *$ & .320 \\
\hline OE <-- QKP & .750 & .065 & 11.605 & $* * *$ & .724 \\
\hline OP <--- OE & .529 & .186 & 2.838 & .005 & .248 \\
\hline
\end{tabular}

Table 75. Model 3 Standardized Regression Weights

\begin{tabular}{|ll|c|}
\hline \multicolumn{2}{|c|}{ Link } & Estimate \\
\hline QKP $<--$ & IST & .320 \\
\hline OE $<--$ & QKP & .724 \\
\hline OP $<---$ & OE & .248 \\
\hline
\end{tabular}

Table 76. Model 3 Squared Multiple Correlations

\begin{tabular}{|c|c|}
\hline Construct & Estimate \\
\hline QKP & .103 \\
\hline OE & .525 \\
\hline OP & .062 \\
\hline
\end{tabular}


Table 77. Model 3 Standardized Total Effects

\begin{tabular}{|c|c|c|c|}
\hline Construct & IST & QKP & OE \\
\hline QKP & .320 & .000 & .000 \\
\hline OE & .232 & .724 & .000 \\
\hline OP & .058 & .180 & .248 \\
\hline
\end{tabular}

Table 78. Model 3 Standardized Direct Effects

\begin{tabular}{|c|c|c|c|}
\hline Construct & IST & QKP & OE \\
\hline QKP & .320 & .000 & .000 \\
\hline OE & .000 & .724 & .000 \\
\hline OP & .000 & .000 & .248 \\
\hline
\end{tabular}

Table 79. Model 3 Standardized Indirect Effects

\begin{tabular}{|c|c|c|c|}
\hline Construct & IST & QKP & OE \\
\hline QKP & .000 & .000 & .000 \\
\hline OE & .232 & .000 & .000 \\
\hline OP & .058 & .180 & .000 \\
\hline
\end{tabular}

From the analysis of Model 3, it is clear that information systems and technology (IST) support has a positive influence on quality of knowledge processes and explains about $10 \%$ of the variance of QKP. The standardized regression weight for IST $\rightarrow$ QKP is $0.320(\mathrm{p}<0.001)$. The total indirect effect of information systems and technology (IST) support on organizational effectiveness via IST $\rightarrow$ QKP $\rightarrow$ OE is 0.232 standard weightings. The total indirect effect of information systems and technology (IST) support on operational performance (OP) via IST $\rightarrow$ QKP $\rightarrow$ OE $\rightarrow$ OP is 0.058 standard weightings. Table 80 below summarizes the finding from Model 3 for hypothesis $\mathrm{H} 6 \mathrm{~b}$ and $\mathrm{H} 7 \mathrm{~b}$. 
Table 80. Summary of Model 3 Findings

\begin{tabular}{|c|c|c|}
\hline Hypothesis & Sign & Conclusion \\
\hline$H 6 b: I S T \rightarrow Q K P$ & + & Supported \\
\hline$H 7 b: \quad I S T \rightarrow Q K P \rightarrow O E$ & + & Supported \\
\hline
\end{tabular}

\subsection{Path Model 4: Top-level Model with Advanced OSS Support}

Path Model 4 was developed to test hypotheses $6 \mathrm{c}$ and $7 \mathrm{c}$ related to support for advanced operational support systems (OSS) and its impact on QKP and OE which state:

Hypothesis 6c. Higher levels of advanced operational support systems technology (OSS) will positively influence overall quality of knowledge processes (QKP).

Hypothesis 7c. Higher levels of advanced operational support systems technology (OSS) will positively influence organizational effectiveness (OE) through the intermediate effects of overall quality of knowledge processes $(Q K P)$.

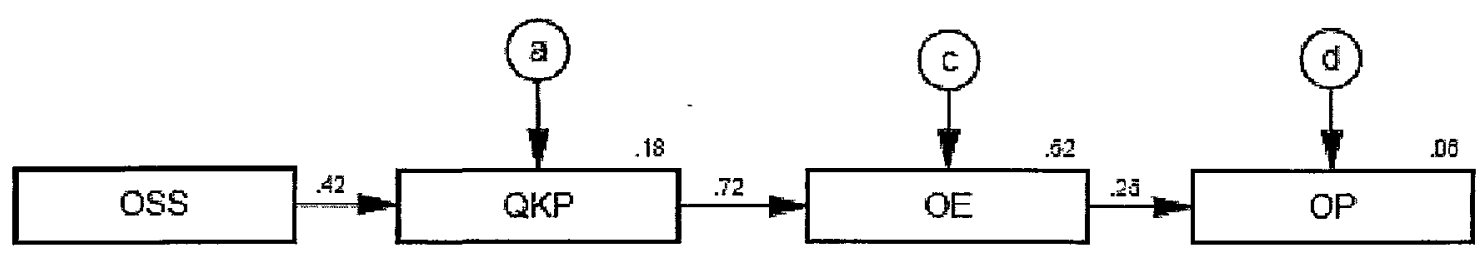

Figure 102. Path Diagram for Model 4

Table 81. Model Goodness of Fit Statistics for Path Model 4

\begin{tabular}{|c|c|c|c|c|c|c|c|c|c|c|c|c|c|}
\hline NPAR & CMIN & DF & P & CMIN/DF & NFI & RFI & IFI & TLI & CFI & RMSEA & HI 90 & PCLOSE & HN05 \\
\hline 11 & .820 & 3 & .845 & .273 & .993 & .977 & 1.018 & 1.065 & 1.000 & .000 & .083 & .896 & 1220 \\
\hline
\end{tabular}


From Table 81, we can conclude the model is clearly a good fit according to all GOF statistics and statistical inference conclusions can be drawn from it. Table 82 below summarizes the regression weights and p-values for each link in the model. All relationships are significant at 0.005 level or better.

Table 82. Model 4 Regression Weights

\begin{tabular}{|c|c|c|c|c|c|}
\hline Links & Estimate & S.E. & C.R. & P & Standardized Estimate \\
\hline QKP $<--$ OSS & .246 & .048 & 5.091 & $* * *$ & .421 \\
\hline OE <-- QKP & .750 & .065 & 11.605 & $* * *$ & .724 \\
\hline OP <-- OE & .529 & .186 & 2.838 & .005 & .248 \\
\hline
\end{tabular}

Table 83. Model 4 Standardized Regression Weights

\begin{tabular}{|lc|c|}
\hline \multicolumn{2}{|c|}{ Link } & Estimate \\
\hline QKP $<---$ & OSS & .421 \\
\hline OE $<---$ & QKP & .724 \\
\hline OP $<---$ & OE & .248 \\
\hline
\end{tabular}

Table 84. Model 4 Squared Multiple Correlations

\begin{tabular}{|c|c|}
\hline Factor & Estimate \\
\hline QKP & .177 \\
\hline OE & .525 \\
\hline OP & .062 \\
\hline
\end{tabular}

Table 85. Model 4 Standardized Total Effects

\begin{tabular}{|c|c|c|c|}
\hline Factor & OSS & QKP & OE \\
\hline QKP & .421 & .000 & .000 \\
\hline OE & .305 & .724 & .000 \\
\hline OP & .076 & .180 & .248 \\
\hline
\end{tabular}

Table 86. Model 4 Standardized Direct Effects

\begin{tabular}{|c|c|c|c|}
\hline Factor & OSS & QKP & OE \\
\hline QKP & .421 & .000 & .000 \\
\hline OE & .000 & .724 & .000 \\
\hline OP & .000 & .000 & .248 \\
\hline
\end{tabular}


Table 87. Model 4 Standardized Indirect Effects

\begin{tabular}{|c|c|c|c|}
\hline Factor & OSS & QKP & OE \\
\hline QKP & .000 & .000 & .000 \\
\hline OE & .305 & .000 & .000 \\
\hline OP & .076 & .180 & .000 \\
\hline
\end{tabular}

From the analysis of Model 4 (see Table 84), it is clear that advanced operational support systems (OSS) has a positive influence on quality of knowledge processes and explains about $18 \%$ of the variance of QKP in this model. The standardized regression weight for OST $\rightarrow$ QKP is $0.421 \quad(\mathrm{p}<0.001)$. The total indirect effect of advanced operational support systems (OSS) on organizational effectiveness (OE) is 0.305 standard weightings. The total indirect effect of advanced operational support systems $(\mathrm{OSS})$ on operational performance $(\mathrm{OP})$ via $\mathrm{OST} \rightarrow \mathrm{QKP} \rightarrow \mathrm{OE}$ is 0.076 standard weightings. Table 88 below summarizes the finding for hypothesis $\mathrm{H} 6 \mathrm{~b}$ and $\mathrm{H} 7 \mathrm{~b}$.

Table 88. Summary of Model 3 Findings

\begin{tabular}{|c|c|c|}
\hline Hypothesis & Sign & Conclusion \\
\hline H6c: OSS $\rightarrow$ QKP & + & Supported \\
\hline H7c: OSS $\rightarrow Q K P \rightarrow O E$ & + & Supported \\
\hline
\end{tabular}

\subsection{Path Model 5\&6: Top-level Model of Plant Age \& Units by Type}

Path Models 5 and 6a,b (see Figures 103 and 104) were developed to test the hypotheses H10a, H10b, H10c and H10d which stated:

Hypothesis 10. The plant factors (i.e. physical characteristics) including: a.) number of plant operating units at the site ("units"); b.) plant size ("size"); and c.) plant age in years since start-up ("ageyrs") will influence 
organizational effectiveness $(O E)$ and operational performance (OP) and the relationship between them; $d$.) all of these relationships will all be moderated by plant design type ("type").

Several nuclear industry studies (IAEA, 1999) refer to plant age, plant size and number of units at site as physical plant factors influencing organizational effectiveness and operational performance. Path Model 5 was developed to evaluate these effects. Initially, all three factors were included, plant age, plant size and number of units at site, each with links to both OE and OP. All possible combinations and permutations were examined for significance. Both size $\rightarrow \mathrm{OE}$ and size $\rightarrow \mathrm{OP}$ were found to be not significant. Also, units $\rightarrow \mathrm{OE}$ and ageyrs $\rightarrow$ OP were not significant. The reduced model (all responding stations included) is shown in Figure 103. These findings, although mostly consistent with the earlier preliminary linear regression findings of Section 7.12, differ in that the link Units $\rightarrow \mathrm{OE}$ (which just made the earlier regression cut-off at $\mathrm{p}=.043$ ) drops out of the integrated path analysis model as not significant. Looking again at Table 56 from the earlier regressions, it is clear that not only is the regression coefficient small $(B=-0.074)$ but the upper bound of the $95 \%$ confidence interval is almost zero (-.002) indicating a questionable result. In the path analysis model, AMOS solves the variables as a system of linear equations, and thus considers interaction effects. The result being that units $\rightarrow \mathrm{OE}$ proves to be not significant. 


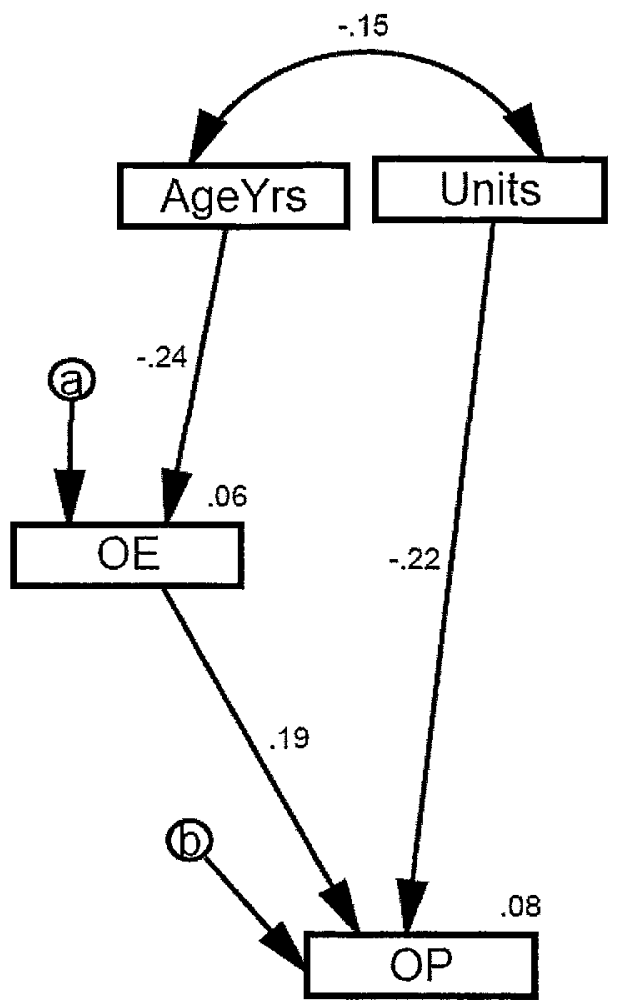

Figure 103. Model 5-Impact of Age and No. of Units on OE \& OP

Table 89. GOF for Model 5 - Impact of Age and No. of Units

\begin{tabular}{|c|c|c|c|c|c|c|c|c|c|c|c|c|c|c|c|}
\hline Model & NPAR & CMIN & $\begin{array}{c}\text { D } \\
\text { F }\end{array}$ & P & $\begin{array}{c}\text { CMIN/ } \\
\text { DF }\end{array}$ & NFI & RFI & IFI & TLI & CFI & RMSEA & HI 90 & PCLOSE & HN05 & AIC \\
\hline 5 & 8 & 3.727 & 2 & .155 & 1.864 & .857 & .570 & .928 & .741 & .914 & .084 & .217 & .241 & 196 & 19.727 \\
\hline
\end{tabular}

Table 89 shows the GOF test results for the model. From Table 89, although RFI and TLI are lower than desirable, it is concluded the model is an acceptable fit according to most GOF statistics and statistical inference conclusions can be drawn from it. Table 90 below summarizes the regression weights and p-values for each link in the model. All relationships are significant at 0.05 level or better. 
Table 90. Model 5 Regression Weights

\begin{tabular}{|c|c|c|c|c|c|}
\hline Link & Estimate & S.E. & C.R. & P & Standardized Estimate \\
\hline OE <-- AgeYrs & -.014 & .005 & -2.728 & .006 & -.241 \\
\hline OP <--- OE & .416 & .188 & 2.216 & .027 & .193 \\
\hline OP <-- Units & -.197 & .077 & -2.557 & .011 & -.223 \\
\hline
\end{tabular}

It is interesting to note that plant age has a negative effect only on organizational effectiveness and from Table 91 we can see this accounts for $6 \%$ of the explained variance. Similarly, the number of units at site also has a negative effect but only on OP. More than $8 \%$ of the explained variance of OP is explained by a combination of OE and No. of units at site. Tables 92-94 summarize the standardized total, direct, and indirect effects for the model. In this model (see Figure 92), Ageyrs has a total standardized weighting of -.241 on OE. No. of units has a total standardized weighting of -.223 on OP. Finally, OE has a total standardized weighting on OP of .193.

Table 91. Model 5 Squared Multiple Correlations

\begin{tabular}{|c|c|}
\hline Factor & Estimate \\
\hline OE & .058 \\
\hline OP & .084 \\
\hline
\end{tabular}

Table 92. Model 5 Standardized Total Effects

\begin{tabular}{|c|c|c|c|}
\hline \multirow{2}{*}{ Factor } & \multicolumn{3}{|c|}{ Estimate } \\
\cline { 2 - 4 } & AgeYrs & Units & OE \\
\hline OE & -.241 & .000 & .000 \\
\hline OP & -.046 & -.223 & .193 \\
\hline
\end{tabular}

Table 93. Model 5 Standardized Direct Effects

\begin{tabular}{|c|c|c|c|}
\hline \multirow{2}{*}{ Factor } & \multicolumn{3}{|c|}{ Estimate } \\
\cline { 2 - 4 } & AgeYrs & Units & OE \\
\hline OE & -.241 & .000 & .000 \\
\hline OP & .000 & -.223 & .193 \\
\hline
\end{tabular}


Table 94. Model 5 Standardized Indirect Effects

\begin{tabular}{|c|c|c|c|}
\hline \multirow{2}{*}{ Factor } & \multicolumn{3}{|c|}{ Estimate } \\
\cline { 2 - 4 } & AgeYrs & Units & OE \\
\hline OE & .000 & .000 & .000 \\
\hline OP & -.046 & .000 & .000 \\
\hline
\end{tabular}

The influence of these factors on organizational effectiveness (OE) and operational performance (OP) and the influence of $\mathrm{OE} \rightarrow \mathrm{OP}$ is also thought to be contingent on plant type. To test for the presence of these effects, AMOS "multi-group analysis" feature was used. However, when examined by plant type, the sample size for each plant type was too small and a model fit could not be found (i.e. the model in AMOS would not converge to a solution). As a compromise, the sample was analyzed for the two largest groups in the sample only: PWR and BWR type plants (with a total of 72 and 31 cases respectively). VVER plants are considered a sub-type of PWR and included in the PWR group. With this split, an adequate model fit was obtained on the simple top-level model and the two plant types were tested, one against the other for parameter differences. 

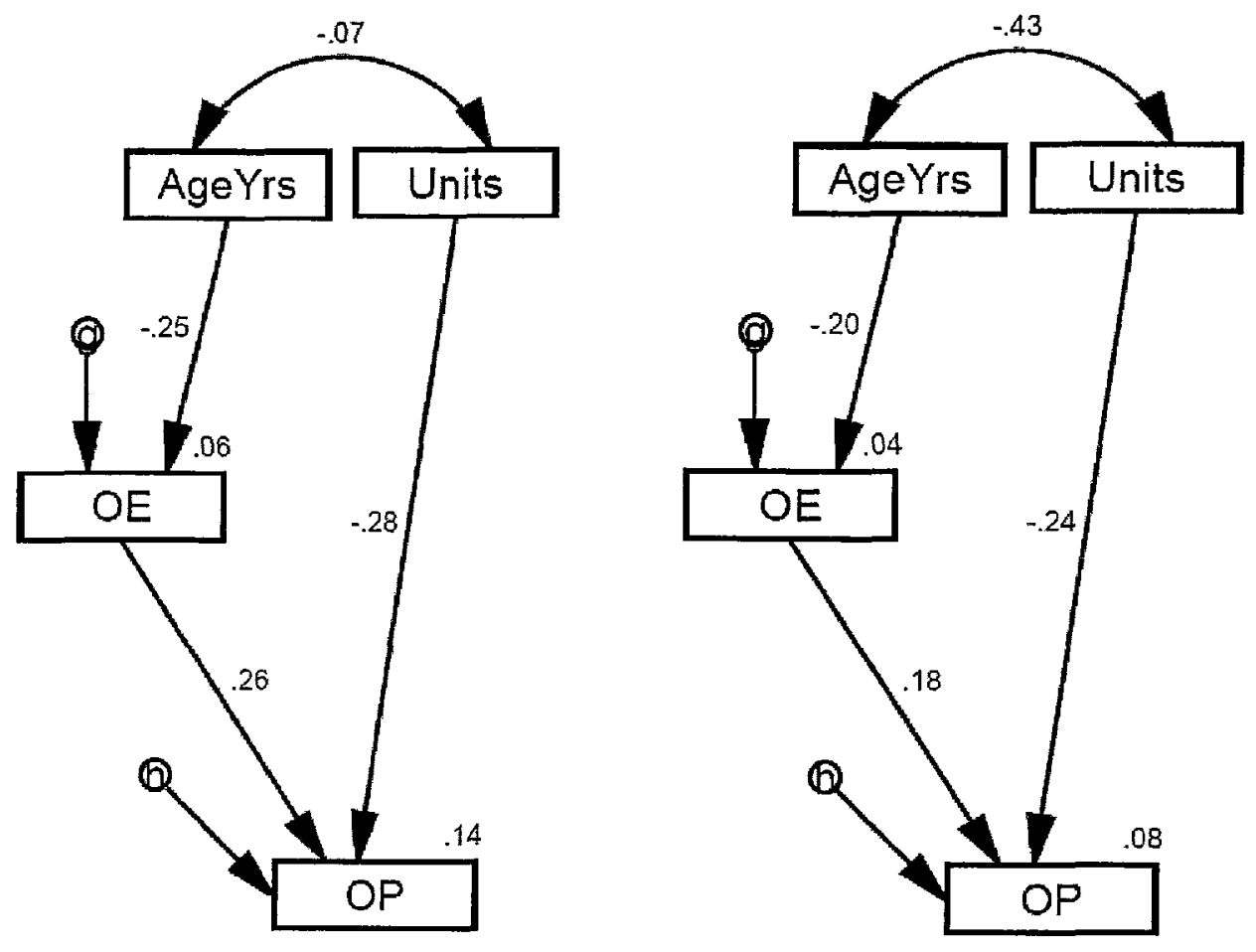

Figure 104. $\quad$ Model 6a,b - PWR (left) vs. BWR (right) Plants

Table 95. GOF for Model 6a \& 6b (PWR vs. BWR)

\begin{tabular}{|c|c|c|c|c|c|c|c|c|c|c|c|c|c|c|c|}
\hline Model & $\frac{\widetilde{z}}{\mathrm{z}}$ & 当 & 告 & A & 密 & $\bar{z}$ & 实 & $\underline{9}$ & 寻 & 胥 & 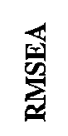 & 呑 & 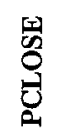 & 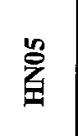 & $\stackrel{\varrho}{4}$ \\
\hline Unconstrained & 24 & .454 & 4 & .978 & .114 & .983 & .949 & 1.155 & 1.713 & 1.00 & .000 & .000 & .986 & 2112 & 48.45 \\
\hline $\begin{array}{c}\text { Structural } \\
\text { weights }\end{array}$ & 21 & 2.150 & 7 & .951 & .307 & .920 & .863 & 1.243 & 1.557 & 1.00 & .000 & .000 & .975 & 662 & 44.15 \\
\hline $\begin{array}{c}\text { Structural } \\
\text { intercepts }\end{array}$ & 19 & 2.333 & 9 & .985 & .259 & .913 & .884 & 1.372 & 1.596 & 1.00 & .000 & .000 & .994 & 734 & 40.33 \\
\hline $\begin{array}{c}\text { Structural } \\
\text { means }\end{array}$ & 17 & $\begin{array}{c}17.35 \\
9\end{array}$ & 11 & .098 & 1.578 & .355 & .297 & .601 & .535 & .574 & .076 & .140 & .239 & 116 & 51.35 \\
\hline
\end{tabular}

Table 95 shows the GOF test results of the 2-group analysis. From Table 95, it can be concluded the structural intercepts model is the best fit and can be assumed to be correct and statistical inference conclusions can be drawn from the models. The model 
comparison feature in AMOS indicates there is a statistical significance in the difference in structural means between the two groups with p-value of .001 if the structural intercepts model is assumed to be correct (see Table 96), which has already been established. Table 97 shows the estimate for the means of the two groups: PWRs have a mean age of 24.6 years and a mean number of units per station site of 2.2 NPPs. BWRs have a mean age of 30.8 years and a mean number of units per station site of 1.8 NPPs.

Table 96. Model 6a,b Comparison (assumes structural intercepts correct)

\begin{tabular}{|c|c|c|c|c|c|c|c|}
\hline Model & DF & CMIN & $\mathbf{P}$ & $\begin{array}{c}\text { NFI } \\
\text { Delta-1 }\end{array}$ & $\begin{array}{c}\text { IFI } \\
\text { Delta-2 }\end{array}$ & $\begin{array}{c}\text { RFI } \\
\text { rho-1 }\end{array}$ & $\begin{array}{c}\text { TLI } \\
\text { rho2 }\end{array}$ \\
\hline Structural means & 2 & 15.026 & .001 & .558 & .838 & .588 & 1.060 \\
\hline
\end{tabular}

Table 97. Model 6a,b Means (assumes structural intercepts equal)

\begin{tabular}{|c|c|c|c|c|c|c|c|c|}
\hline \multirow{2}{*}{ Factor } & \multicolumn{4}{|c|}{ PWR Group } & \multicolumn{4}{|c|}{ BWR Group } \\
\cline { 2 - 9 } & Estimate & S.E. & C.R. & P & Estimate & S.E. & C.R. & P \\
\hline AgeYrs & $\mathbf{2 4 . 6 3 8}$ & 1.028 & 23.973 & $* * *$ & $\mathbf{3 0 . 7 7 5}$ & 1.108 & 27.783 & $* * *$ \\
\hline Units & $\mathbf{2 . 1 9 4}$ & .137 & 16.029 & $* * * *$ & $\mathbf{1 . 7 7 4}$ & .215 & 8.265 & $* * *$ \\
\hline
\end{tabular}

Table 98 below summarizes the p-values and standardized regression weights for each inter-factor relationship for both plant type groups to highlight the differences. The loading of ageyrs $\rightarrow$ OE for PWRs is almost $-24.8 \%$ while for BWRs loading ageyrs $\rightarrow$ $\mathrm{OE}$ is about $4 \%$ less at only $-20.4 \%$. It is also evident that plant type moderates $\mathrm{OE} \rightarrow$ OP relationship: for PWRs OE $\rightarrow$ OP loads at $25.6 \%$ while for BWRs OE $\rightarrow$ OP loads at only $18.2 \%$, almost a $7 \%$ difference. Finally, it is clear that no. of units $\rightarrow$ OP loads at $27.8 \%$ for PWRs but only $23.7 \%$ for BWRs. Table 99 summarizes the squared multiple correlations assuming the structural intercepts equal model is correct. Model 6a shows that the PWR data explains $6 \%$ of the variance in OE and $14 \%$ of the variance 
in OP while Model $6 \mathrm{~b}$ shows that the BRW data explains $4 \%$ of the variance in $\mathrm{OE}$ and $8 \%$ of OP.

Table 98. Model 6a,b Regression Weights (structural intercepts equal)

\begin{tabular}{|c|c|c|c|c|c|c|}
\hline Link & Estimate & S.E. & C.R. & P & $\begin{array}{c}\text { Standardized Estimate - } \\
\text { PWR }\end{array}$ & $\begin{array}{c}\text { Standardized Estimate - } \\
\text { BWR }\end{array}$ \\
\hline OE <--- AgeYrs & -.014 & .005 & -2.606 & .009 & -.248 & -.204 \\
\hline OP <-- OE & .511 & .200 & 2.549 & .011 & .256 & .182 \\
\hline OP <-- Units & -.233 & .081 & -2.891 & .004 & -.278 & -.237 \\
\hline
\end{tabular}

Table 99. Model 6a,b Squared Mult. Correlations (structural intercepts equal)

\begin{tabular}{|c|c|c|}
\hline Factor & $\begin{array}{c}\text { Estimate - } \\
\text { PWR }\end{array}$ & $\begin{array}{c}\text { Estimate - } \\
\text { BWR }\end{array}$ \\
\hline OE & .061 & .042 \\
\hline OP & .140 & .082 \\
\hline
\end{tabular}

Tables 100-102 summarize the standardized total, direct, and indirect effects for Models $6 a$ and $6 b$.

Table 100. Model 6a,b Stand. Total Effects (structural intercepts equal)

\begin{tabular}{|c|c|c|c|c|c|c|}
\hline \multirow{2}{*}{ Factor } & \multicolumn{3}{|c|}{ Estimate- } & \multicolumn{3}{c|}{ Estimate - } \\
& BWR & \multicolumn{3}{c|}{} \\
\cline { 2 - 7 } & AgeYrs & Units & OE & AgeYrs & Units & OE \\
\hline OE & -.248 & .000 & .000 & -.204 & .000 & .000 \\
\hline OP & -.063 & -.278 & .256 & -.037 & -.237 & .182 \\
\hline
\end{tabular}

Table 101. Model 6a,b Stand. Direct Effects (structural intercepts equal)

\begin{tabular}{|c|c|c|c|c|c|c|}
\hline \multirow{3}{*}{ Factor } & \multicolumn{3}{|c|}{ Estimate- } & \multicolumn{3}{c|}{ Estimate - } \\
& PWR & \multicolumn{3}{c|}{} \\
\cline { 2 - 7 } & AgeYrs & Units & OE & AgeYrs & Units & OE \\
\hline OE & -.248 & .000 & .000 & -.204 & .000 & .000 \\
\hline OP & .000 & -.278 & .256 & .000 & -.237 & .182 \\
\hline
\end{tabular}

Table 102. Model 6a,b Stand. Indirect Effects (structural intercepts equal)

\begin{tabular}{|c|c|c|c|c|c|c|}
\hline \multirow{3}{*}{ Factor } & \multicolumn{3}{|c|}{ Estimate- } & \multicolumn{3}{c|}{ Estimate - } \\
\cline { 2 - 7 } & AgeYrs & Units & OE & AgeYrs & Units & OE \\
\hline OE & .000 & .000 & .000 & .000 & .000 & .000 \\
\hline OP & -.063 & .000 & .000 & -.037 & .000 & .000 \\
\hline
\end{tabular}


Table 103 summarizes the findings from Models 5 and $6 a, b$. In this table (and hereafter) the notation $\left.O P\right|_{\text {type }}$ means "OP is controlled for plant type".

Table 103. Summary of Model 5 and 6a,b Findings

\begin{tabular}{|l|c|l|}
\hline Hypothesis & $\begin{array}{c}\text { Direction } \\
(+/ \text { - sign) } \\
\text { Predicted, Actual }\end{array}$ & \multicolumn{1}{|c|}{ Conclusion } \\
\hline H1Oal: Units $\left.\rightarrow O P\right|_{\text {type }}$ & $t b d$, -'ve & $\begin{array}{l}\text { Supported } \\
\text { (for PWR } v s \text { BWR) }\end{array}$ \\
\hline H1Ob1: Size $\left.\rightarrow O P\right|_{\text {type }}$ & $t b d, n / a$ & Not Supported \\
\hline H1Oc1: Age $\left.\rightarrow O P\right|_{\text {type }}$ & $t b d, n / a$ & Not Supported \\
\hline H1Oa2: Units $\left.\rightarrow O E\right|_{\text {type }}$ & $t b d, n / a$ & Not Supported \\
\hline H10b2: Size $\left.\rightarrow O E\right|_{\text {type }}$ & $t b d, n / a$ & Not Supported \\
\hline H1Oc2: Age $\left.\rightarrow O E\right|_{\text {type }}$ & $t b d$, -'ve & $\begin{array}{l}\text { Supported } \\
\text { (for PWR } \text { vs } B W R \text { ) }\end{array}$ \\
\hline H1Od: OE $\left.\rightarrow O P\right|_{\text {type }}$ & + 've, +'ve & $\begin{array}{l}\text { Supported } \\
\text { (for PWR } \text { vs } B W R \text { ) }\end{array}$ \\
\hline
\end{tabular}

As with the earlier findings, Models 5 and 6 do not support the hypotheses that plant size effects either OE or OP. However, plant age and number of site units do have a negative effect on $\mathrm{OE}$ and $\mathrm{OP}$ respectively. In addition, Model 6 findings confirm that these effects, and the effect of $\mathrm{OE} \rightarrow \mathrm{OP}$ are all moderated by plant type. In specific, it can be concluded from Model 6 that the negative effect of the number of units on operating performance is about $4 \%$ stronger for PWR plants than for BWR plants. It can also be concluded that the negative effect of age of the plant on organizational effectiveness is about 5\% stronger for PWRs than BWRs. Finally, it can be concluded that the effect of $\mathrm{OE} \rightarrow \mathrm{OP}$ is about $8 \%$ stronger for PWRs than for BWRs and this effect explains about $6 \%$ more of the variance in OP for PWRs as opposed to BWRs. 


\subsection{Path Model 7\&8: Interaction Amongst QKP Constructs}

Path Models 7 and 8 were developed to test the following hypotheses related to interactions amongst the quality of knowledge process (QKP) constructs. Recall hypothesis 11-14 state:

Hypothesis 11a. Higher quality knowledge acquisition and adoption (KA) processes will have a positive influence on quality knowledge retention and storage (KR) processes.

Hypothesis $11 b$. Higher quality knowledge generation and validation $(K G)$ processes will have a positive influence on quality knowledge retention and storage $(K R)$ processes.

Hypothesis 12. Higher quality knowledge retention and storage (KR) processes will have a positive influence on the quality of knowledge sharing and transfer $(K S)$ processes.

Hypothesis 13. Higher quality of knowledge sharing and transfer (KS) processes will have a positive influence on quality of knowledge utilization and application $(K U)$ processes.

Hypothesis 14. Higher quality knowledge utilization and application (KU) processes will have a positive influence on knowledge acquisition and adoption (KA) processes.

Path Diagram for Model 7 was initially based on the findings from the linear regressions. Note that findings of the linear regression did not support the links KA $\rightarrow$ 
$\mathrm{KR}, \mathrm{KG} \rightarrow \mathrm{KR}$, and $\mathrm{KU} \rightarrow \mathrm{KA}$, however, these paths were initially included for completeness and reconfirmed this finding. Figure 105 below shows the resulting model with the non significant paths removed. Note that a link from KS $\rightarrow \mathrm{KU}$ was not significant for the linear regression model but in Path Model 7 it was. To ensure no valid links had been overlooked, all possible link combinations were tested in a systematic approach. The final path model strongly agrees with the linear regression findings.

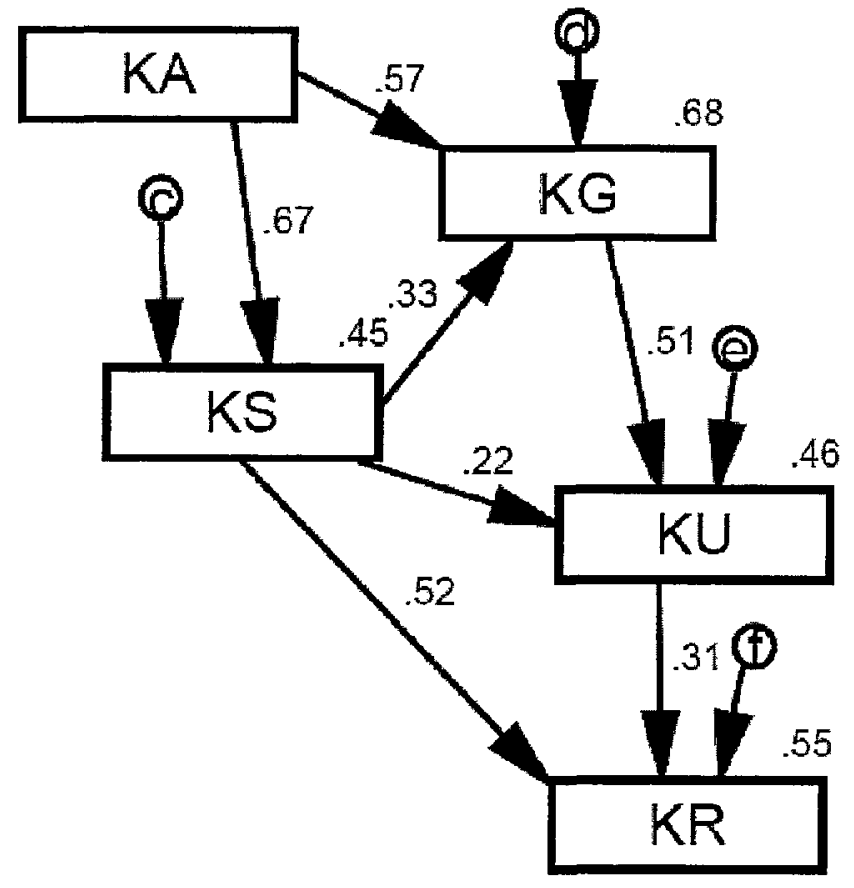

Figure 105. Path Diagram for Model 7

Table 104. Model GOF Statistics for Model 7

\begin{tabular}{|c|c|c|c|c|c|c|c|c|c|c|c|c|c|c|}
\hline NPAR & CMIN & DF & P & CMIN/DF & NFI & RFI & IFI & TLI & CFI & RMSEA & HI 90 & PCLOSE & HN05 & AIC \\
\hline 17 & .345 & 3 & .951 & .115 & .999 & .996 & 1.007 & 1.036 & 1.000 & .000 & .000 & .969 & 2903 & 34.345 \\
\hline
\end{tabular}


From Table 104, we can conclude the model is clearly an excellent fit according to all GOF statistics and statistical inference conclusions can be drawn from it. Table 105 below summarizes the regression weights and p-values for each. All the relationships are significant at 0.05 level.

Table 105. Model 7 Regression Weights

\begin{tabular}{|c|c|c|c|c|c|}
\hline Link & Estimate & S.E. & C.R. & $\mathbf{P}$ & Standardized Estimate \\
\hline $\mathrm{KS}<\cdots \mathrm{KA}$ & 654 & 065 & 10036 & $x=\infty$ & 672 \\
\hline KG $<-\cdots \mathrm{KA}$ & 464 & 056 & 8294 & $\infty \times$ & 571 \\
\hline KG $<\cdots-\mathbf{K S}$ & 273 & 058 & 4754 & $* \approx * \pi$ & 327 \\
\hline $\mathrm{KU}<---\mathrm{KG}$ & 495 & 092 & 5361 & 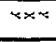 & 505 \\
\hline $\mathrm{KU}<-\cdots \mathrm{KS}$ & 182 & 077 & 2357 & 018 & 222 \\
\hline KR $<--\mathrm{KS}$ & 429 & 063 & 6855 & $* \times * *$ & 515 \\
\hline $\mathrm{KR}<--\mathrm{KU}$ & 314 & 076 & 4112 & $x \times *$ & 309 \\
\hline
\end{tabular}

Table 106 summarizes the squared multiple correlations. The model explains $45 \%$ of the variance of $\mathrm{KS}, 68 \%$ of the variance of $\mathrm{KG}, 46 \%$ of the variance of $\mathrm{KU}$, and $55 \%$ of the variance of $K R$.

Table 106. Model 7 Squared Multiple Correlations

\begin{tabular}{|c|c|}
\hline Factor & Estimate \\
\hline KS & 451 \\
\hline KG & 684 \\
\hline KU & 464 \\
\hline KR & 545 \\
\hline
\end{tabular}

Several authors (e.g. Young (2006), Koskinen (2003), Verkasalo and Lappalainen (1998)) have conducted research that proposed theory or advanced findings that knowledge retention (KR) leads to knowledge utilization (KU). A competing path model (Model 8, see Figure 106) was created to examine the goodness of fit with the link KR $\rightarrow \mathrm{KU}$ replacing KU $\rightarrow \mathrm{KR}$. Note that in this model the $\mathrm{KS} \rightarrow \mathrm{KU}$ link drops out as not significant. 


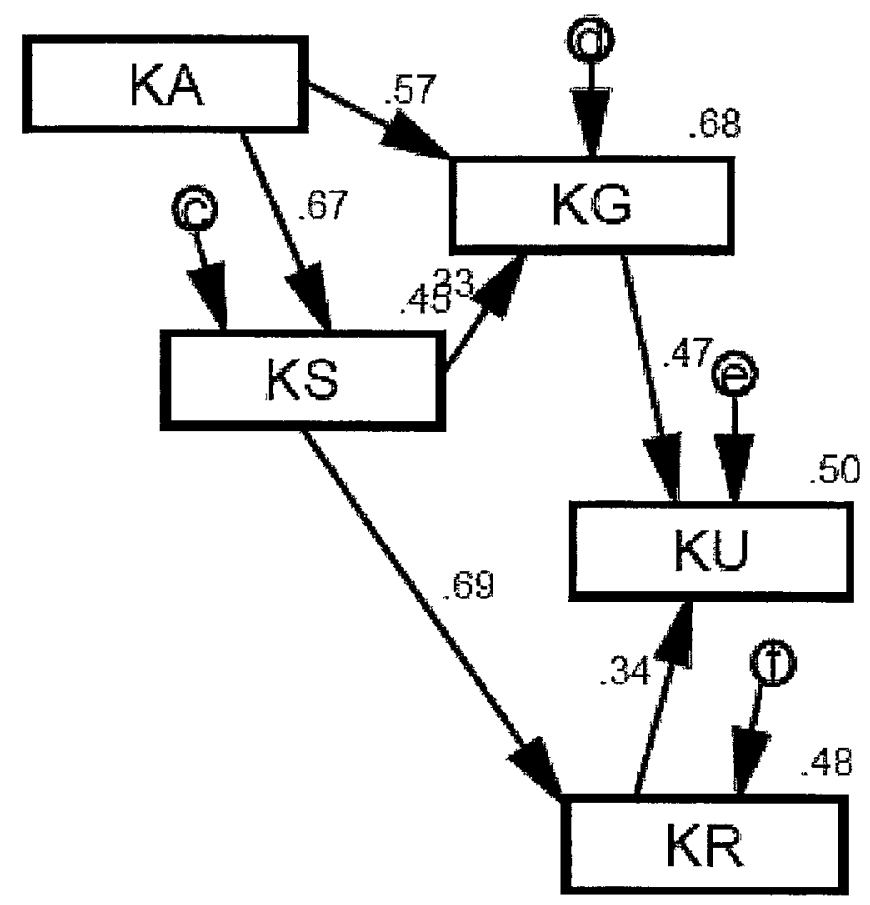

Figure 106. Path Diagram for Model 8

Table 107. Model GOF Statistics for Model 7

\begin{tabular}{|c|c|c|c|c|c|c|c|c|c|c|c|c|c|c|}
\hline NPAR & CMIN & DF & P & CMIN/DF & NFI & RFI & IFI & TLI & CFI & RMSEA & HI 90 & PCLOSE & HN05 & AIC \\
\hline 16 & 3.925 & 4 & .416 & .981 & .990 & .962 & 1.000 & 1.001 & 1000 & .000 & .132 & .571 & 310 & 35925 \\
\hline
\end{tabular}

From Table 107, we can conclude the model is also an excellent fit according to all GOF statistics and statistical inference conclusions can be drawn from it if we accept it as theoretically sound. Table 108 below summarizes the regression weights and pvalues for each relationship. All the relationships are significant at better than the .001 level. 
Table 108. $\quad$ Model 8 Regression Weights

\begin{tabular}{|c|c|c|c|c|c|}
\hline Links & Estimate & S.E. & C.R. & P & Standardized Estimates \\
\hline $\mathrm{KS}<--\mathrm{KA}$ & 654 & 065 & 10041 & $* * *$ & 672 \\
\hline $\mathrm{KG}<--\mathrm{KA}$ & 464 & 056 & 8301 & $* \times *$ & 570 \\
\hline $\mathrm{KR}<--\mathrm{KS}$ & 579 & 054 & 10646 & $\times \times *$ & 694 \\
\hline $\mathrm{KG}<--\mathrm{KS}$ & 274 & 058 & 4771 & $\times \times *$ & 328 \\
\hline $\mathrm{KU}<--\mathrm{KG}$ & 458 & 071 & 6465 & $\times \times *$ & 474 \\
\hline $\mathrm{KU}<--\mathrm{KR}$ & 332 & 071 & 4663 & $* * *$ & 342 \\
\hline
\end{tabular}

Table 109 below compares the GOF statistics for Model 7 and 8 . Although the fit for these two models is similar, Model 7 is slightly superior in that PCLOSE is .969 for Model 7 versus .571 for Model 8 and AIC is also slightly lower indicating a more parsimonious model. Given that there is a larger body of literature supporting the relationship that knowledge utilization processes give rise to knowledge retention processes (i.e. supporting hypothesis 14 that $\mathrm{KU} \rightarrow \mathrm{KR}$ is positive), Model 7 is assumed to be correct. However, it is possible there is a two-way effect. Unfortunately path analysis has limits as a methodology and cannot represent 2-way relationships. Table 110 below shows the difference between the two models in terms of ability to explain the construct variance. The most notable differences are that Model 8 explains $22 \%$ more variance for $\mathrm{KG}$ and Model 7 explains $20 \%$ more variance for $\mathrm{KR}$.

Table 109. GOF Comparison of Model 7 vs. 8

\begin{tabular}{|c|c|c|c|c|c|c|c|c|c|c|c|c|c|c|c|c|}
\hline Model & NPAR & CMIN & $\begin{array}{c}\text { D } \\
\text { F }\end{array}$ & P & $\begin{array}{c}\text { CMIN } \\
\text { DF }\end{array}$ & NFI & RFI & IFI & TLI & CFI & RMSEA & HI 90 & PCLOSE & HN05 & AIC \\
\hline 7 & 17 & 345 & 3 & 951 & 115 & 999 & 996 & 1007 & 1036 & 1000 & 000 & 000 & 969 & 2903 & 34345 \\
\hline 8 & 16 & 3925 & 4 & 416 & 981 & 990 & 962 & 1000 & 1001 & 1000 & 000 & 132 & 571 & 310 & 35925 \\
\hline
\end{tabular}


Table 110. Model 7 \& 8 Squared Multiple Correlations

\begin{tabular}{|l|c|c|}
\hline Factor & $\begin{array}{c}\text { Model 8 } \\
\text { Estimate }\end{array}$ & $\begin{array}{c}\text { Model 7 } \\
\text { Estimate }\end{array}$ \\
\hline $\mathrm{KS}$ & .452 & .451 \\
\hline $\mathrm{KR}$ & .482 & .684 \\
\hline $\mathrm{KG}$ & .685 & .464 \\
\hline $\mathrm{KU}$ & .502 & .545 \\
\hline
\end{tabular}

Both Model 7 and Model 8 are similar in overall structure and did not support many of the hypothesized links between the quality of knowledge processes. However, the findings are strong evidence that for NPPs, these relationships are the more dominant ones. Although the link between $\mathrm{KA} \rightarrow \mathrm{KR}$ and $\mathrm{KG} \rightarrow \mathrm{KR}$ were not supported directly, it is clear from the path diagram that evidence exists for a strong indirect link. These links will be discussed in more detail once the full model is developed. Table 111 summarizes the findings based on the preferred Model 7 analysis results.

Table 111. Summary of Model 7 Findings

\begin{tabular}{|c|c|c|}
\hline Hypothesis & Sign & Conclusion \\
\hline$H 11 a: K A \rightarrow K R$ & + & Not Supported $^{*}$ \\
\hline$H 11 b: K G \rightarrow K R$ & + & Not Supported $^{*}$ \\
\hline$H 12: K R \rightarrow K S$ & + & Not Supported \\
\hline$H 13: K S \rightarrow K U$ & + & Supported \\
\hline$H 14: K U \rightarrow K A$ & + & Not Supported \\
\hline Not Hypothesized (new) & & \\
\hline$K A \rightarrow K G$ & + & Supported \\
\hline$K A \rightarrow K S$ & + & Supported \\
\hline$K G \rightarrow K U$ & + & Supported \\
\hline$K S \rightarrow K R$ & + & Supported \\
\hline$K S \rightarrow K G$ & + & Supported \\
\hline$K U \rightarrow K R$ & + & Supported \\
\hline
\end{tabular}

*Note however an indirect link is supported. 


\subsection{Path Model 9: Interactions Between KMPs and QKPS}

Path Model 9 was developed to test the hypotheses 17-25 for specific predicted interactions amongst the constructs related to support for $\mathrm{KM}$ practices and the individual quality of knowledge process $(\mathrm{QKP})$ constructs. The relevant hypotheses are listed below:

Hypothesis 17. Higher support for KM strategy and planning (KMS) will have a positive influence on quality of knowledge acquisition (KA) processes, quality of knowledge sharing and transfer $(K S)$ processes, quality of knowledge utilization (KU) processes, quality of knowledge generation (KG) processes, and quality of knowledge retention and storage (KR) processes.

Hypothesis 18. Higher support for organizational learning (SOL) and will have a positive influence on quality of knowledge acquisition (KA) processes, quality of knowledge generation and validation $(K G)$ processes, quality of knowledge retention and storage $(K R)$ and quality of knowledge sharing and transfer $(K S)$ processes.

Hypothesis 19. Higher support for (KM-supportive) human resource related practices (HRP) will have a positive influence on quality of knowledge acquisition (KA) processes, quality of knowledge sharing and transfer $(K S)$ processes, quality of knowledge utilization $(K U)$ processes, and quality of knowledge retention and storage $(K R)$ processes. 
Hypothesis 20. Higher support for training related practices (TRP) will have a positive influence on quality of knowledge retention and storage (KR) processes, quality of knowledge utilization and application (KU) processes, and quality of knowledge sharing and transfer (KS) processes.

Hypothesis 21. Higher support for information management practices (IMP) will have a positive influence on quality of knowledge sharing and transfer (KS) processes, quality of knowledge retention and storage $(K R)$ processes, quality of knowledge generation and validation $(K G)$ and quality of knowledge utilization and application $(K U)$ processes.

Hypothesis 22. Higher levels of effectiveness of information systems and technology support (IST) will have a positive influence on quality of knowledge generation (KG) processes, quality of knowledge utilization and application $(K U)$ processes, quality of knowledge retention and storage (KR), and quality of knowledge sharing and transfer (KS) processes.

Hypothesis 23. Higher levels of effectiveness of advanced operational support systems (OSS) will have a positive influence on quality of knowledge utilization and application $(K U)$ processes and quality of knowledge generation and validation $(K G)$ processes.

Hypothesis 24. Higher support for process management practices (PMP) have a positive influence on quality of knowledge sharing and transfer (KS) 
processes, quality of knowledge utilization (KU) processes, and quality of knowledge retention and storage $(K R)$ processes.

Hypothesis 25. Higher support for organizational performance management (OPM) practices will have a positive influence on quality of knowledge retention and storage $(K R)$ processes and quality of knowledge utilization and application $(K U)$ processes.

Figure 107 shows the path diagram for Model 9a. The core of this model is based on the significant QKP links found in Model 7. Initially all links between KMP to all QKPs were included and links with either non-significant p-values or estimates that spanned or were close to zero were systematically removed in several iterations until only significant links remained (at 0.05 level or better). Many of the links dropped out. The remaining links were fairly consistent with the earlier linear regression findings with two exceptions. As part of the model fitting process, the link $\mathrm{KS} \rightarrow \mathrm{KU}$ dropped out as non-significant. Recall this link was earlier hypothesized and supported in Model 7. Table 112 summarizes the goodness of fit statistics for Model 9a. 


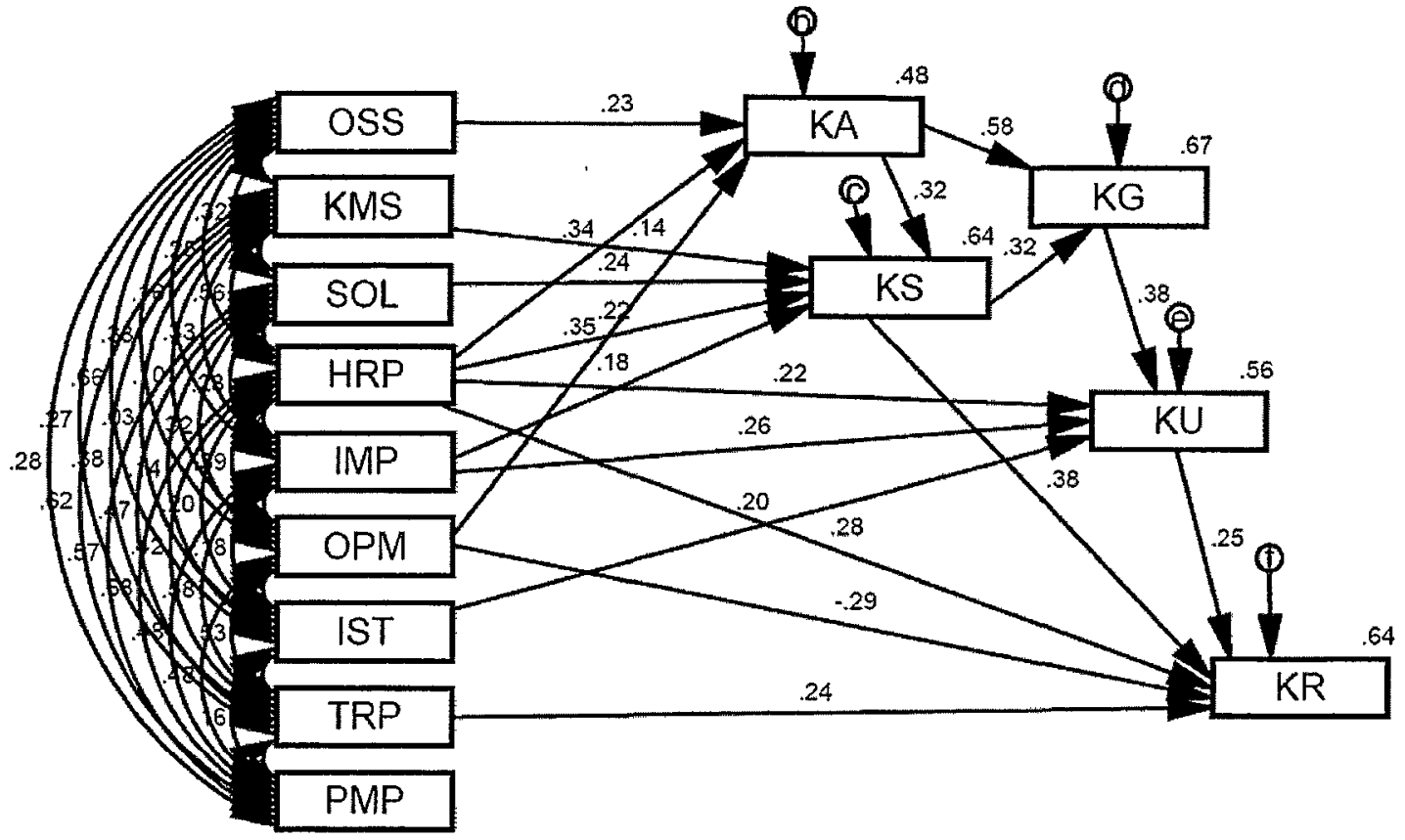

Figure 107. Path Diagram for Model 9a (with OPM $\rightarrow$ KR)

Table 112. Model Goodness of Fit Statistics for Path Model 9a

\begin{tabular}{|c|c|c|c|c|c|c|c|c|c|c|c|c|c|}
\hline NPAR & CMIN & DF & P & CMIN/DF & NFI & RFI & IFI & TLI & CFI & RMSEA & HI 90 & PCLOSE & AIC \\
\hline 83 & 37.916 & 36 & .382 & 1.053 & .966 & .902 & .998 & .995 & .998 & .020 & .067 & .809 & 203.916 \\
\hline
\end{tabular}

From Table 112, we can conclude the model is an excellent fit according to all GOF statistics and statistical inference conclusions can be drawn from it. As the models become more complex, the CMIN/DF, RMSEA and PCLOSE statistics, and AIC become more important and useful for model fit and comparisons.

An unexpected finding was the link OPM $\rightarrow \mathrm{KR}$ was significant and negative. Recall this relationship was hypothesized to be positive. After discussion with several industry experts, three possible explanations have been found for this result. First of all, a given 
NPP will tend to become much more critical in its assessment of the quality of KR processes as the organization becomes more mature in the application of systematic OPM methods and approaches. The OPM measures used in this study are all highly focused on KR objectives and included: conducting regular external peer review assessments; self-assessments such as benchmarking against best practices to stimulate learning; and ongoing processes for operational experience capture, review, analysis and corrective action. Thus NPPs with a high OPM program maturity may have a much more critical view of the KR processes (i.e. a systematic bias).

Secondly, it is possible that stations with high levels of maturity in OPM practices have confidence in their ability to acquire, build and retain critical knowledge within the organization and are not so concerned about tacit knowledge loss due to attrition, and therefore expend less effort on ensuring tacit knowledge retention. The link between OPM $\rightarrow$ KA supports this reasoning. Either of or both of these arguments may explain the negative correlation. Finally, it is also possible that the measures used for the KR and OPM constructs fail to measure some aspects of these constructs.

In order to assess the impact of removing the link OPM $\rightarrow \mathrm{KR}$, Model $9 \mathrm{~b}$ was created. Figure 108 depicts Model 9b, a variation of Model 9a with link OMP $\rightarrow$ KR removed. 


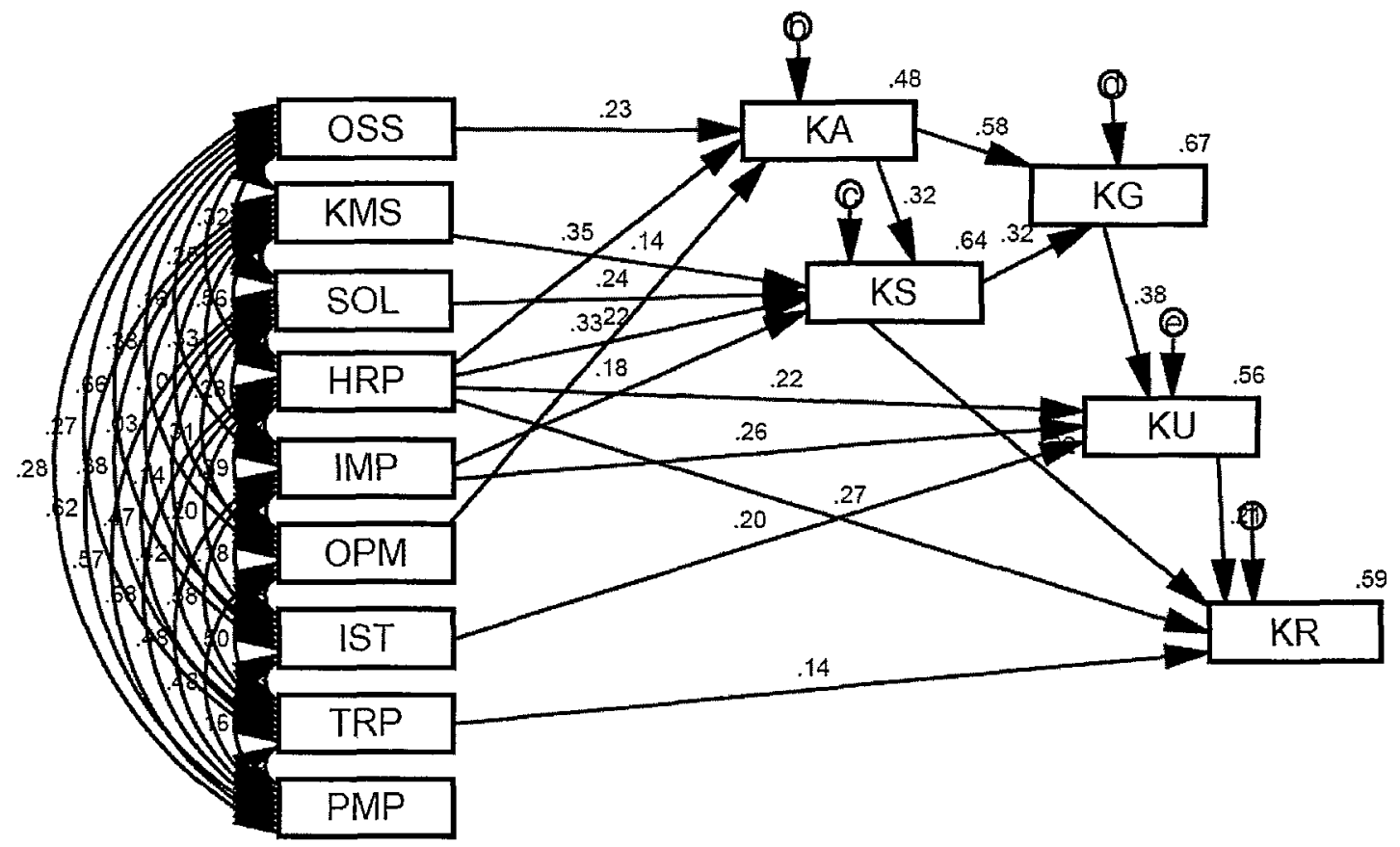

Figure 108. $\quad$ Path Diagram for Model 9b (without OPM $\rightarrow \mathrm{KR}$ )

Table 113 summarizes the fit statistics for both models 9a and 9b. From Table 113, we can conclude the model fit for Model $9 \mathrm{~b}$ is slightly reduced but is still very good according to all GOF statistics and statistical inference conclusions can be drawn from it.

Table 113. Model Goodness of Fit Statistics for Path Model 9a and 9b

\begin{tabular}{|c|c|c|c|c|c|c|c|c|c|c|c|c|c|c|}
\hline Model & NPAR & CMIN & DF & P & CMIN/DF & NFI & RFI & IFI & TLI & CFI & RMSEA & HI 90 & PCLOSE & AIC \\
\hline 9a & 83 & 37.916 & 36 & .382 & 1.053 & .966 & .902 & .998 & .995 & .998 & .020 & .067 & .809 & 203.916 \\
\hline 9b & 82 & 56.655 & 37 & .020 & 1.531 & .950 & .857 & .982 & .945 & .981 & .064 & .096 & .228 & 220.655 \\
\hline
\end{tabular}

Table 114 summarizes the regression weights and p-values for each relationship for both models $9 \mathrm{a}$ and $9 \mathrm{~b}$. The relevant links involving $\mathrm{KMP} \rightarrow \mathrm{QKP}$ construct interactions are 
highlighted in shaded rows. All the relationships are significant at or very close to 0.05 level or better.

Table 114. Model 9a,b Regression Weights

\begin{tabular}{|c|c|c|c|c|c|c|c|c|c|c|}
\hline & \multicolumn{5}{|c|}{ Model 9a (with OPM $\rightarrow$ KR) } & \multicolumn{5}{|c|}{ Model 9b (without OPM $\rightarrow$ KR) } \\
\hline Link & Estimate & S.E. & C.R. & $\mathbf{P}$ & $\begin{array}{c}\text { Standardized } \\
\text { Estimates }\end{array}$ & Estimate & S.E. & C.R. & $\mathbf{P}$ & $\begin{array}{c}\text { Standardized } \\
\text { Estimates }\end{array}$ \\
\hline $\mathrm{KA}<--\mathrm{HRP}$ & .293 & .061 & 4.798 & $* * *$ & .342 & .299 & .061 & 4.887 & $* * *$ & .349 \\
\hline KA <--- OPM & .540 & .116 & 4.670 & $* * *$ & .347 & .524 & .118 & 4.457 & $* * *$ & .332 \\
\hline $\mathrm{KA}<---$ OSS & .181 & .056 & 3.225 & .001 & .231 & .183 & .056 & 3.270 & .001 & .235 \\
\hline $\mathrm{KG}<-\cdots \mathrm{KA}$ & .467 & .056 & 8.295 & $* * *$ & .584 & .467 & .053 & 8.823 & $* * * *$ & .583 \\
\hline$K G<---\quad K S$ & .264 & .058 & 4.565 & $* * *$ & .317 & .265 & .055 & 4.798 & **** & .318 \\
\hline $\mathrm{KR}<-\cdots \mathrm{HRP}$ & .195 & .050 & 3.889 & $* * *$ & .284 & .183 & .055 & 3.320 & $* * *$ & .267 \\
\hline $\mathrm{KR}<---\quad \mathrm{KS}$ & .316 & .066 & 4.800 & $* * *$ & .379 & .268 & .071 & 3.775 & $* * *$ & .320 \\
\hline $\mathrm{KR}<--\mathrm{KU}$ & .251 & .071 & 3.526 & $* * *$ & .247 & .216 & .075 & 2.868 & .004 & .211 \\
\hline $\mathrm{KR}<---\mathrm{OPM}$ & -.365 & .084 & -4.360 & $x: * *$ & -.293 & & & & & \\
\hline KR <--- TRP & .268 & .078 & 3.435 & $* * *$ & .243 & .159 & .075 & 2.120 & .034 & .144 \\
\hline KS <--- HRP & .180 & .063 & 2.876 & .004 & .219 & .181 & .065 & 2.771 & .006 & .221 \\
\hline $\mathrm{KS}<---\mathrm{IMP}$ & .245 & .088 & 2.787 & .005 & .179 & .241 & .081 & 2.980 & .003 & .176 \\
\hline $\mathrm{KS}<-\ldots \mathrm{KA}$ & .306 & .068 & 4.513 & $* * *$ & .320 & .307 & .063 & 4.838 & $* * *$ & .321 \\
\hline $\mathrm{KS}<---\mathrm{KMS}$ & .105 & .053 & 1.982 & .047 & .136 & .105 & .054 & 1.943 & .052 & .136 \\
\hline KS <--- SOL & .268 & .080 & 3.349 & $* * *$ & .245 & .266 & .077 & 3.454 & $* * *$ & .243 \\
\hline $\mathrm{KU}<---\mathrm{HRP}$ & .149 & .047 & 3.196 & .001 & .222 & .149 & .048 & 3.141 & .002 & .222 \\
\hline KU <--- IMP & .295 & .074 & 3.956 & $* * *$ & .263 & .295 & .071 & 4.133 & $* * * *$ & .263 \\
\hline KU $<---\quad$ IST & .136 & .043 & 3.128 & .002 & .196 & .136 & .043 & 3.123 & .002 & .196 \\
\hline $\mathrm{KU}<--\mathrm{KG}$ & .374 & .074 & 5.082 & $* * *$ & .381 & .374 & .072 & 5.220 & $* * *$ & .381 \\
\hline
\end{tabular}

Table 115 summarizes the squared multiple correlations for the parameters of Model 9a and $9 \mathrm{~b}$. From table 115 we see the explained variances (squared multiple correlations) for the QKP constructs have improved somewhat over Model 7.

Table 115. Model 9 Squared Multiple Correlations

\begin{tabular}{|c|c|c|}
\hline Factor & Estimate - Model 9a & Estimate - Model 9b \\
\hline KA & .484 & .477 \\
\hline KS & .644 & .642 \\
\hline KG & .670 & .670 \\
\hline KU & .563 & .563 \\
\hline KR & .642 & .590 \\
\hline
\end{tabular}


It is evident that removing the link OPM $\rightarrow \mathrm{KR}$ has little effect on the overall fit. For this reason, and due to uncertainty about the validity of this relationship, Model $9 \mathrm{~b}$ (without OMP $\rightarrow \mathrm{KR}$ ) is the preferred model going forward. Thus assuming Model $9 \mathrm{~b}$ to be correct, the several conclusions about hypotheses $17-25$ can be made. Table 116 summarizes these results assuming Model $9 \mathrm{~b}$ is correct.

Table 116. Summary of Model 9 Findings

\begin{tabular}{|c|c|c|}
\hline Hypothesized & Sign & Conclusion \\
\hline H17a: $\quad K M S \rightarrow K A$ & + & Not Supported \\
\hline$H 17 b: \quad K M S \rightarrow K S$ & + & Supported \\
\hline$H 17 c: \quad K M S \rightarrow K U$ & + & Not Supported \\
\hline$H 17 c: \quad K M S \rightarrow K G$ & + & Not Supported \\
\hline H17e: $\quad K M S \rightarrow K R$ & + & Not Supported \\
\hline H18a: $\quad$ SOL $\rightarrow K A$ & $t$ & Not Supported \\
\hline$H 18 b: \quad S O L \rightarrow K G$ & + & Not Supported \\
\hline H18c: $\quad S O L \rightarrow K R$ & + & Not Supported \\
\hline H18d: $\quad S O L \rightarrow K S$ & + & Supported \\
\hline H19a: $\quad H R P \rightarrow K A$ & + & Supported \\
\hline$H 19 b: \quad H R P \rightarrow K S$ & + & Supported \\
\hline H19c: $\quad H R P \rightarrow K U$ & + & Supported \\
\hline H19d: $\quad H R P \rightarrow K R$ & + & Supported \\
\hline H2Oa: $\quad T R P \rightarrow K R$ & + & Supported \\
\hline$H 20 b: \quad T R P \rightarrow K U$ & + & Not Supported \\
\hline$H 20 c: \quad T R P \rightarrow K S$ & + & Not Supported \\
\hline H21a: $\quad I M P \rightarrow K S$ & + & Supported \\
\hline$H 2 I b: \quad I M P \rightarrow K R$ & + & Not Supported \\
\hline H21c: $\quad I M P \rightarrow K G$ & + & Not Supported \\
\hline H2Id: $I M P \rightarrow K U$ & + & Supported \\
\hline$H 22 a: \quad I S T \rightarrow K G$ & + & Not Supported \\
\hline H2ba: $\quad I S T \rightarrow K U$ & + & Supported \\
\hline$H 22 c: \quad I S T \rightarrow K R$ & + & Not Supported \\
\hline$H 22 d: \quad I S T \rightarrow K S$ & + & Not Supported \\
\hline H23a: $\quad$ OSS $\rightarrow K U$ & + & Not Supported \\
\hline$H 23 b: \quad O S S \rightarrow K G$ & + & Not Supported \\
\hline H24a: $\quad P M P \rightarrow K S$ & + & Not Supported \\
\hline$H 24 b: \quad P M P \rightarrow K U$ & + & Not Supported \\
\hline$P M P \rightarrow K R$ & + & Not Supported \\
\hline H25a: $O P M \rightarrow K R$ & + & Not Supported \\
\hline$H 25 b: \quad O P M \rightarrow K U$ & + & Not Supported \\
\hline \multicolumn{3}{|l|}{ Not Hypothesized (new) } \\
\hline$O S S \rightarrow K A$ & + & Supported \\
\hline$O P M \rightarrow K A$ & + & Supported \\
\hline
\end{tabular}


Another general observation was that the relationships found tended to be very clearly defined in the data as significant and with a strong associated path weighting. Finally, the links between OSS $\rightarrow \mathrm{KA}$ and $\mathrm{OPM} \rightarrow \mathrm{KA}$ were not hypothesized and were unexpected findings. Their implications will be discussed further on in the analysis. These findings are generally consistent with the earlier preliminary analysis finding for the inter-relationships amongst the QKPs derived using multiple linear regression (see Figure 91) and clarify the causal model relationships.

\subsection{Path Model 10: Interactions of SOC with KMPs and QKPs}

Path Model 10 was developed to test the hypotheses $28-29$ related to specific predicted interactions of the construct supportive organizational culture (SOC) with each of the constructs related to support for KM practices and each of the quality of knowledge process (QKP) constructs. The relevant hypotheses are:

Hypothesis 28. Higher support for KM strategy and planning (KMS), support for organizational learning (SOL), information management practices (IMP), training related practices (TRP), and human resource related practices (HRP) will each have a positive influence on supportive organizational culture (SOC).

Hypothesis 29. Higher level of supportive organizational culture (SOC) will have a positive direct influence on each of the quality of knowledge processes: quality of knowledge acquisition (KA), quality of knowledge generation 
and validation $(K G)$, quality of knowledge retention and storage $(K G)$, quality of knowledge sharing and transfer $(K S)$, and quality of knowledge utilization and application (KU).

Figure 109 shows the path diagram for Model 10.

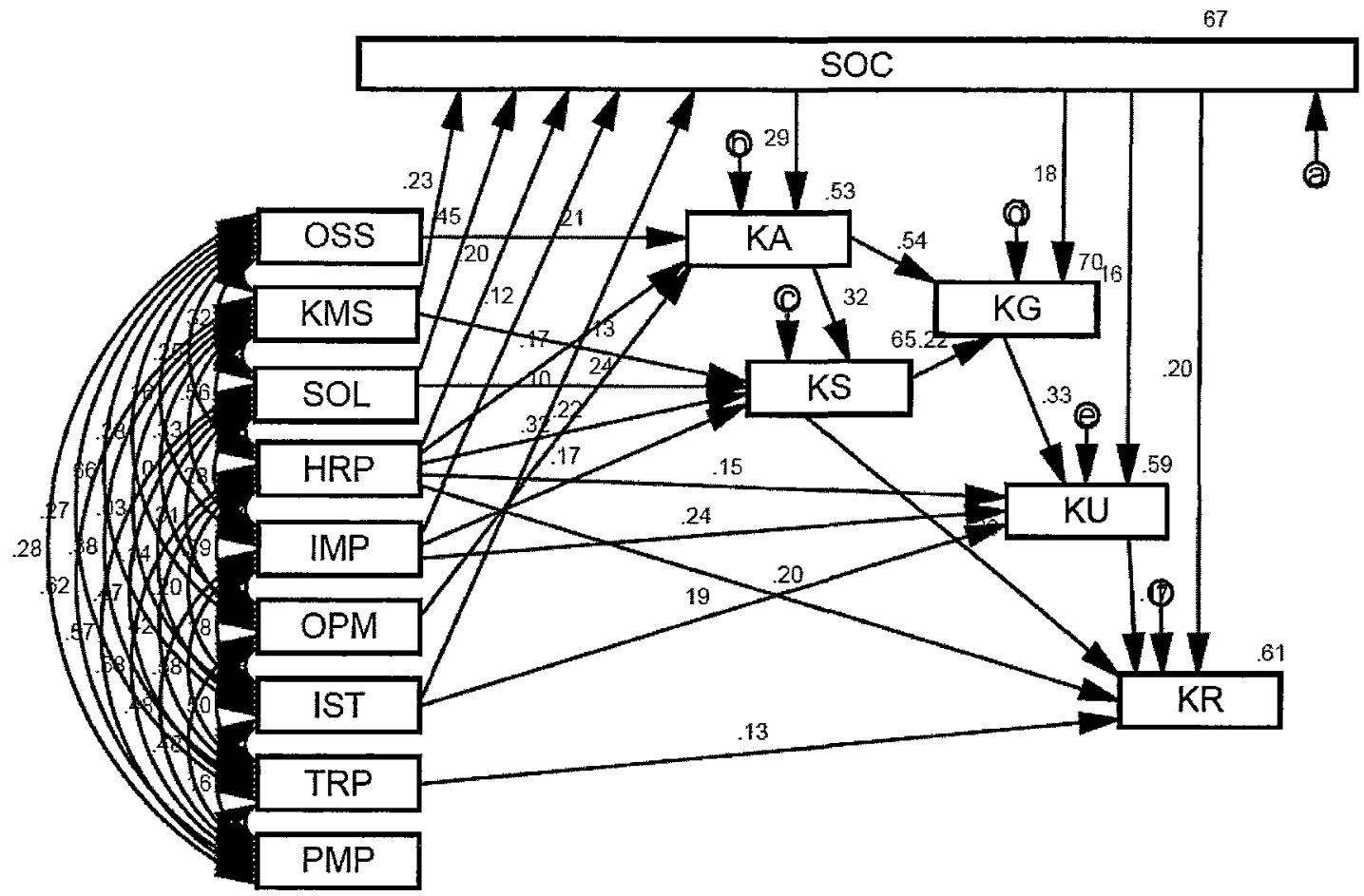

Figure 109. Path Diagram for Model 10

The construct supportive organizational culture has many interactions and it is quite central the overall thrust of the research. In order to present a readable path diagram that allows the QKP interactions and "logical flow" to be more visible, SOC is placed at the top of the diagram and interactions are shown mostly vertical in direction. Similarly, the KMPs are all to the left and are mostly horizontal lines to the QKPs. This improves 
readability somewhat on an otherwise busy and somewhat difficult to read path diagram. This format will be retained through-out the document for subsequent path models and it is hoped the consistent format will aid in reader familiarity and interpretation. Similarly, the same core QKPs are retained and presented in a similar layout.

Table 117 below summarizes the goodness of fit statistics for path Model 10 .

Table 117. Model GOF Statistics for Model 10

\begin{tabular}{|c|c|c|c|c|c|c|c|c|c|c|c|c|c|c|}
\hline NPAR & CMIN & DF & P & CMIN/DF & NFI & RFI & IFI & TLI & CFI & RMSEA & HI 90 & PCLOSE & AIC & HN05 \\
\hline 93 & 5441 & 42 & 095 & 1296 & 958 & 879 & 990 & 970 & 989 & 048 & 081 & 508 & 2404 & 137 \\
\hline
\end{tabular}

From Table 117, we can conclude path Model 10 is an excellent fit according to all GOF statistics and statistical inference conclusions can be drawn from it. Table 118 below summarizes the regression weights and $\mathrm{p}$-values for each relationship. The relevant links involving SOC are shown in shaded rows to highlight them. Note that 2 hypothesized relationships were slightly above the 0.05 significance level but were retained on the basis of strong theoretical support. Their inclusion improved the model fit. These links were: $\mathrm{SOC} \rightarrow \mathrm{KU}(\mathrm{p}=0.064)$ and $\mathrm{SOC} \rightarrow \mathrm{KR}(\mathrm{p}=0.018)$. The link IST $\rightarrow$ SOC was not a hypothesized relationship and although slightly above the 0.05 significance level $(\mathrm{p}=0.062)$ it was also retained on the basis of strong theoretical support in the literature (discussed below). The inclusion of this link also improved the model fit. All other relationships were significant at 0.05 or better indicating a good fit. Note also the path weights are fairly strong considering the number of parameters and this indicates meaningful relationships. 
Table 118. Model 10 Regression Weights

\begin{tabular}{|c|c|c|c|c|c|}
\hline Link & Estimate & S.E. & C.R. & P & Standardized Estimate \\
\hline KA <-- HRP & .146 & .071 & 2.042 & .041 & .169 \\
\hline KA <-- OPM & .505 & .112 & 4.499 & $* * *$ & .318 \\
\hline KA <--- OSS & .163 & .054 & 3.027 & .002 & .208 \\
\hline KA <-- SOC & .334 & .094 & 3.567 & $* * *$ & .294 \\
\hline KG <-- KA & .437 & .055 & 7.944 & $* * *$ & .542 \\
\hline KG <-- KS & .185 & .061 & 3.006 & .003 & .221 \\
\hline KG <-- SOC & .166 & .062 & 2.660 & .008 & .181 \\
\hline KR <-- HRP & .141 & .056 & 2.517 & .012 & .205 \\
\hline KR <--- KS & .217 & .071 & 3.070 & .002 & .261 \\
\hline KR <--- KU & .168 & .077 & 2.178 & .029 & .165 \\
\hline KR <--- SOC & .184 & .078 & 2.372 & .018 & .202 \\
\hline KR <--- TRP & .146 & .075 & 1.945 & .052 & .131 \\
\hline KS <--- HRP & .182 & .063 & 2.892 & .004 & .219 \\
\hline KS <-- IMP & .237 & .082 & 2.901 & .004 & .171 \\
\hline KS <-- KA & .309 & .065 & 4.757 & $* * *$ & .320 \\
\hline KS <-- KMS & .105 & .054 & 1.952 & .051 & .134 \\
\hline KS <--- SOL & .263 & .079 & 3.331 & $* * *$ & .237 \\
\hline KU <--- HRP & .103 & .053 & 1.931 & .053 & .151 \\
\hline KU <-- IMP & .273 & .073 & 3.746 & $* * *$ & .241 \\
\hline KU <--- IST & .133 & .043 & 3.110 & .002 & .191 \\
\hline KU <-- KG & .319 & .078 & 4.083 & $* * *$ & .325 \\
\hline KU <-- SOC & .145 & .078 & 1.855 & .064 & .161 \\
\hline SOC<-- HRP & .149 & .053 & 2.802 & .005 & .197 \\
\hline SOC<-- IMP & .156 & .071 & 2.193 & .028 & .124 \\
\hline SOC<-- IST & .079 & .042 & 1.870 & .062 & .102 \\
\hline SOC<-- KMS & .163 & .048 & 3.389 & $* * *$ & .228 \\
\hline SOC<-- SOL & .454 & .068 & 6.653 & $* * *$ & .450 \\
\hline
\end{tabular}

Table 119 summarizes the squared multiple correlations for Model 10 factors. From Table 119 it can be concluded that Model 10 explains a high level of the variance for all the QKPs and SOC.

Table 119. Model 10 - Squared Multiple Correlations

\begin{tabular}{|c|c|}
\hline Factor & Estimate \\
\hline SOC & .669 \\
\hline $\mathrm{KA}$ & .532 \\
\hline $\mathrm{KS}$ & .651 \\
\hline $\mathrm{KG}$ & .696 \\
\hline $\mathrm{KU}$ & .585 \\
\hline $\mathrm{KR}$ & .612 \\
\hline
\end{tabular}


From these findings we can draw several conclusions about hypotheses 25-28. Table 120 summarizes these results. Although the link IST $\rightarrow$ SOC was not originally hypothesized, further investigation reveals that this relationship is supported in the literature. Higher levels of support for IST enhance the supportive organizational culture through mechanisms like corporate Intranets, enterprise and collaboration software, and e-mail; all of which have been shown in the literature to enhance employee communications, improve organizational alignment/focus on strategic objectives and priorities, and promote collaborative teamwork (e.g. Huber and Daft, 1987; Pinto and Pinto, 1990; Johannessen et al., 1999). This relationship invites further research but is retained in the models going forward as a legitimate finding.

Table 120. Summary of Model 10 Findings

\begin{tabular}{|c|c|c|}
\hline Hypothesized & Sign & Conclusion \\
\hline H28a: $\quad K M S \rightarrow S O C$ & + & Supported \\
\hline H28b: $\quad S O L \rightarrow S O C$ & + & Supported \\
\hline H28c: $\quad I M P \rightarrow S O C$ & + & Supported \\
\hline H28d: $\quad T R P \rightarrow S O C$ & + & Not Supported \\
\hline$H 28 e: \quad H R P \rightarrow S O C$ & + & Supported \\
\hline$H 29 a: \quad S O C \rightarrow K A$ & + & Supported \\
\hline$H 29 b: \quad S O C \rightarrow K G$ & + & Supported \\
\hline$H 29 c: \quad S O C \rightarrow K R$ & + & Supported \\
\hline H29d: $\quad S O C \rightarrow K S$ & + & Not Supported \\
\hline$H 29 e: \quad S O C \rightarrow K U$ & + & Supported \\
\hline \multicolumn{3}{|l|}{ Not Hypothesized (new) } \\
\hline$I S T \rightarrow S O C$ & + & Supported \\
\hline
\end{tabular}

\subsection{Path Model 11: Full Model with OE Added}

Path Model 11 was developed to test hypothesis 15 and 16 related to the direct influence of $\mathrm{KU} \rightarrow \mathrm{OE}$ and $\mathrm{KR} \rightarrow \mathrm{OE}$; to test hypothesis 26a related to the influence of $\mathrm{PMP} \rightarrow \mathrm{OE}$; and to test hypothesis 27a related to the influence of $\mathrm{OPM} \rightarrow \mathrm{OE}$. 
These hypotheses are restated again for reference:

Hypothesis 15. Higher quality knowledge utilization and application $(K U)$ processes will have a positive influence on organizational effectiveness (OE).

Hypothesis 16. Higher quality knowledge retention and storage (KR) processes will have a positive influence on organizational effectiveness $(O E)$.

Hypothesis 26a. Higher support for process management practices (PMP) will have a positive influence on organizational effectiveness (OE).

Hypothesis 27a. Higher support for organizational performance management (OPM) practices will have a positive influence organizational effectiveness $(O E)$.

Path Model 11 was developed based on Model 10 but through a systematic process of adding and testing all possible links to the organizational effectiveness (OE) construct, and retaining only those that proved significant. The model is shown in Figure 110. The expected links from OPM $\rightarrow$ OE and PMP $\rightarrow$ OE were not significant and were removed. Table 121 summarizes the goodness of fit statistics for this model. 


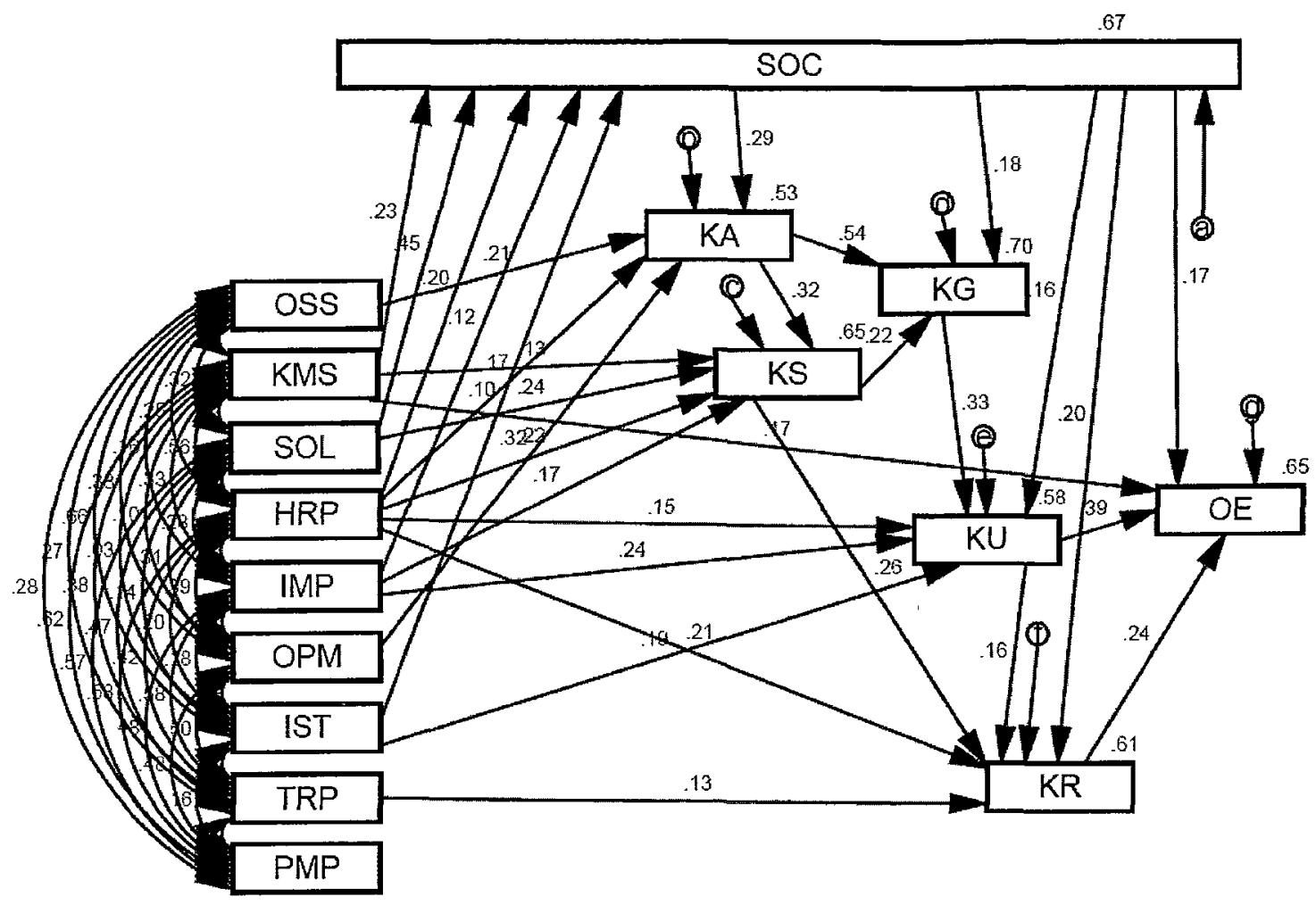

Figure 110. Path Model 11

Table 121. GOF Statistics for Model 11

\begin{tabular}{|c|c|c|c|c|c|c|c|c|c|c|c|c|c|c|}
\hline NPAR & CMIN & DF & P & CMIN/DF & NFI & RFI & IFI & TLI & CFI & RMSEA & HI 90 & PCLOSE & AIC & HN05 \\
\hline 99 & 68.454 & 53 & .075 & 1.292 & .952 & .877 & .989 & .969 & .988 & .048 & .078 & .522 & 266.454 & 133 \\
\hline
\end{tabular}

From Table 121, we can conclude the model is an excellent fit according to all GOF statistics and statistical inference conclusions can be drawn from it. Table 122 below summarizes the regression weights and p-values for each relationship. The relevant links involving $\mathrm{OE}$ are highlighted as shaded rows. 
Table 122. Model 11 Regression Weights

\begin{tabular}{|l|c|c|c|c|c|}
\hline Link & Estimate & S.E. & C.R. & P & Standardized Estimate \\
\hline SOC <--- IST & .079 & .042 & 1.860 & .063 & .101 \\
\hline SOC <-- HRP & .150 & .053 & 2.817 & .005 & .199 \\
\hline SOC <--- SOL & .455 & .068 & 6.669 & $* * *$ & .451 \\
\hline SOC <-- KMS & .161 & .048 & 3.342 & $* * *$ & .225 \\
\hline SOC <-- IMP & .155 & .071 & 2.182 & .029 & .123 \\
\hline KA <-- SOC & .334 & .094 & 3.566 & $* * *$ & .294 \\
\hline KA <-- HRP & .146 & .071 & 2.041 & .041 & .169 \\
\hline KA <-- OSS & .163 & .054 & 3.027 & .002 & .208 \\
\hline KA <-- OPM & .505 & .112 & 4.501 & $* * *$ & .318 \\
\hline KS <-- SOL & .263 & .079 & 3.338 & $* * *$ & .237 \\
\hline KS <-- KMS & .105 & .054 & 1.952 & .051 & .134 \\
\hline KS <--- KA & .308 & .065 & 4.753 & $* * *$ & .319 \\
\hline KS <--- IMP & .236 & .082 & 2.895 & .004 & .171 \\
\hline KS <-- HRP & .182 & .063 & 2.894 & .004 & .219 \\
\hline KG <--- KA & .437 & .055 & 7.947 & $* * *$ & .542 \\
\hline KG <-- SOC & .166 & .062 & 2.660 & .008 & .181 \\
\hline KG <--- KS & .185 & .061 & 3.007 & .003 & .221 \\
\hline KU <-- HRP & .103 & .053 & 1.931 & .054 & .151 \\
\hline KU <--- IMP & .273 & .073 & 3.748 & $* * *$ & .241 \\
\hline KU <--- KG & .319 & .078 & 4.083 & $* * *$ & .325 \\
\hline KU <-- SOC & .145 & .078 & 1.855 & .064 & .161 \\
\hline KU <-- IST & .133 & .043 & 3.110 & .002 & .191 \\
\hline KR <-- TRP & .145 & .075 & 1.932 & .053 & .130 \\
\hline KR <--- KU & .168 & .077 & 2.174 & .030 & .165 \\
\hline KR <--- KS & .218 & .071 & 3.087 & .002 & .262 \\
\hline KR <-- SOC & .183 & .078 & 2.350 & .019 & .200 \\
\hline KR <-- HRP & .143 & .056 & 2.549 & .011 & .207 \\
\hline OE <-- KU & .377 & .069 & 5.509 & $* * *$ & .394 \\
\hline OE <-- SOC & .149 & .072 & 2.064 & .039 & .173 \\
\hline OE <--- KR & .225 & .073 & 3.102 & .002 & .239 \\
\hline OE <--- KMS & .103 & .042 & 2.449 & .014 & .168 \\
\hline
\end{tabular}

The only significant QKP links to OE were found to be $\mathrm{KU} \rightarrow \mathrm{OE}$ with a standardized weighting of $0.370(\mathrm{p}<.001)$ and $\mathrm{KR} \rightarrow \mathrm{OE}$ with a standardized weighting of $0.249(\mathrm{p}$ $=.001)$. Both are large effects, and from Table 123 we can see the variance explained of $\mathrm{OE}$ is quite high at about $65 \%$. This supports hypothesis H15: KU $\rightarrow \mathrm{OE}$ (+'ve) and hypothesis H16: KR $\rightarrow$ OE (+'ve). This finding agrees with the earlier linear regression findings and supports hypothesis 15 and 16. 
The only KMP link to OE that was significant was $\mathrm{KMS} \rightarrow \mathrm{OE}$ with a standardized weighting of $0.168(p=.014)$. The hypothesized relationship PMP $\rightarrow$ OE (+'ve) was not significant the therefore Hypothesis H26a was not supported. The hypothesized relationship OPM $\rightarrow \mathrm{OE}$ (+'ve) was not significant the therefore Hypothesis $\mathrm{H} 27$ a was not supported. These finding were unexpected and invite further research. The direct link SOC $\rightarrow$ OE was also significant and although not hypothesized was retained on the grounds it can be supported in the literature. These findings will be discussed further in the context of Model 16 and the summary conclusions.

Table 123. Model 11 Squared Multiple Correlations

\begin{tabular}{|c|c|}
\hline Factor & Estimate \\
\hline SOC & .668 \\
\hline $\mathrm{KA}$ & .532 \\
\hline $\mathrm{KS}$ & .651 \\
\hline $\mathrm{KG}$ & .696 \\
\hline $\mathrm{KU}$ & .585 \\
\hline $\mathrm{KR}$ & .613 \\
\hline $\mathrm{OE}$ & .651 \\
\hline
\end{tabular}

Table 124 below summarizes the findings from the analysis with Model 11.

Table 124. Summary of Model 11 Findings

\begin{tabular}{|c|c|c|}
\hline Hypothesized & Sign & Conclusion \\
\hline$H 15: K U \rightarrow O E$ & + & Supported \\
\hline$H 16: K R \rightarrow O E$ & + & Supported \\
\hline$H 26 a: P M P \rightarrow O E$ & + & Not Supported \\
\hline$H 27 a: O P M \rightarrow O E$ & + & Not Supported \\
\hline Not Hypothesized (new) & & \\
\hline SOC $\rightarrow O E$ & + & Supported \\
\hline$K M S \rightarrow O E$ & + & Supported \\
\hline
\end{tabular}




\subsection{Path Model 12: Full Model with OP Added}

Path Model 12 was developed to re-test hypothesis H9 in the context of the full model. Hypothesis $\mathrm{H} 9$ was related to the link between $\mathrm{OE} \rightarrow \mathrm{OP}$ and is restated again for reference:

Hypothesis 9. Higher organizational effectiveness (OE) will positively influence operational performance $(O P)$.

Figure 111 shows the path diagram for Model 12. This model is similar to Model 11 but with one significant addition: the operational performance (OP) construct has been added to the bottom right of the path diagram. Table 125 summarizes the fit statistics. 


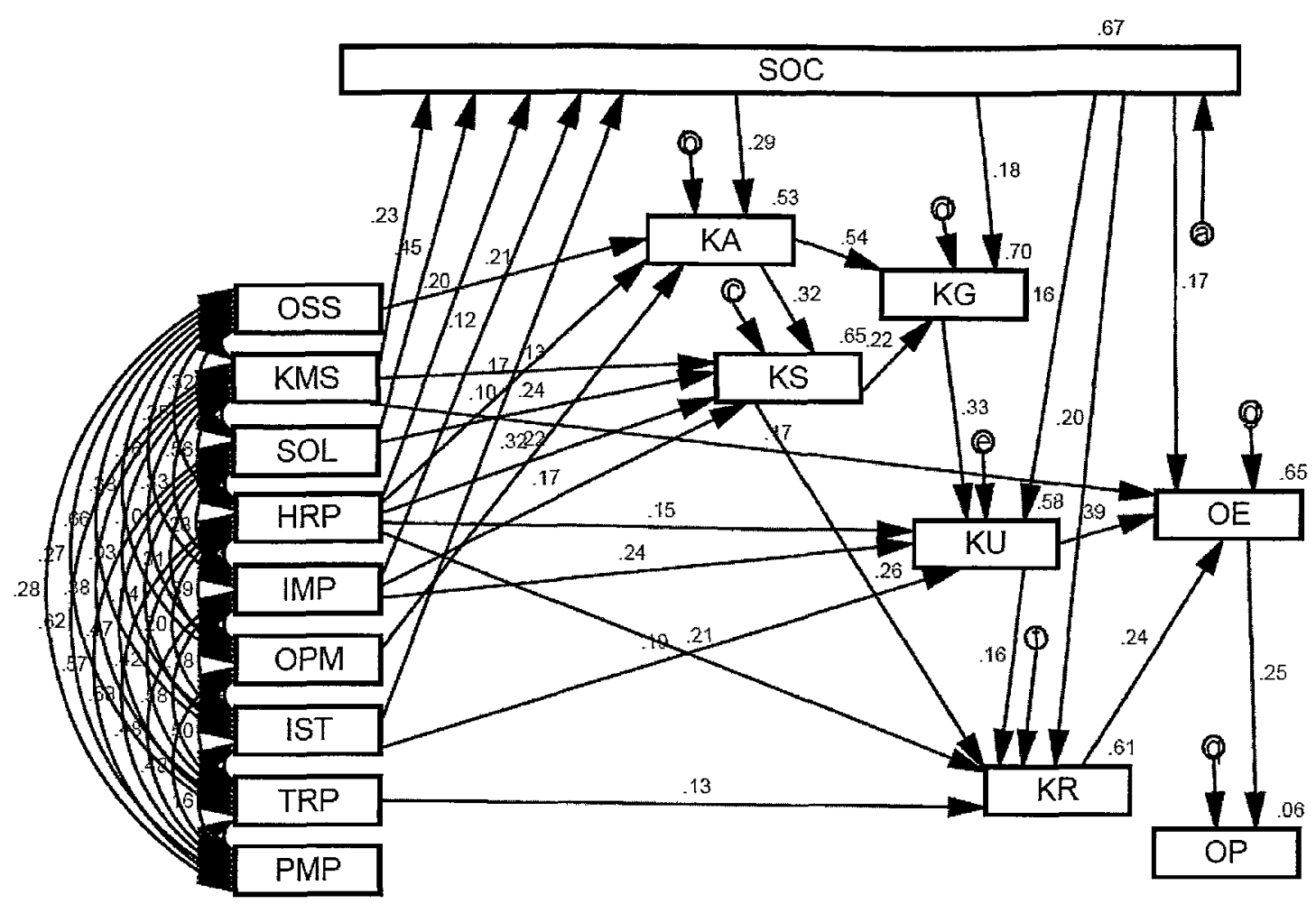

Figure 111. Path Diagram for Model 12

Table 125. Model GOF Statistics for Model 12

\begin{tabular}{|c|c|c|c|c|c|c|c|c|c|c|c|c|c|c|}
\hline NPAR & CMIN & DF & P & CMIN/DF & NFI & RFI & IFI & TLI & CFI & RMSEA & HI 90 & PCLOSE & AIC & HN05 \\
\hline 102 & 85.408 & 68 & .075 & 1.256 & .941 & .868 & .987 & .970 & .987 & .045 & .072 & .596 & 289.408 & 133 \\
\hline
\end{tabular}

From Table 125, we can conclude the model is an excellent fit according to all GOF statistics and statistical inference conclusions can be drawn from it. Table 126 below summarizes the regression weights and p-values for each relationship at the .05 level or better. The new link $\mathrm{OE} \rightarrow \mathrm{OP}$ is highlighted in grey. Note that although the links IST $\rightarrow \mathrm{SOC}, \mathrm{SOC} \rightarrow \mathrm{KU}, \mathrm{KMS} \rightarrow \mathrm{KS}, \mathrm{HRP} \rightarrow \mathrm{KU}$, and TRP $\rightarrow \mathrm{KR}$ were slightly above the .05 cutoff for significance they were retained in the model on the basis that they are supported theoretically and would likely be significant with a larger sample size. 
Table 126. Model 12 Regression Weights

\begin{tabular}{|c|c|c|c|c|c|}
\hline Link & Estimate & S.E. & C.R. & P & Standardized Estimate \\
\hline SOC <-- IST & 079 & 042 & 1860 & 063 & 101 \\
\hline SOC <-- HRP & 150 & 053 & 2817 & 005 & 199 \\
\hline SOC <-- SOL & 455 & 068 & 6669 & $* * * *$ & 451 \\
\hline SOC <-- KMS & 161 & 048 & 3342 & $* * *$ & 225 \\
\hline SOC <-- IMP & 155 & 071 & 2182 & 029 & 123 \\
\hline KA <-- SOC & 334 & 094 & 3566 & $* * *$ & 294 \\
\hline KA <-- HRP & 146 & 071 & 2041 & 041 & 169 \\
\hline KA <-- OSS & 163 & 054 & 3027 & 002 & 208 \\
\hline KA <-- OPM & 505 & 112 & 4501 & $* * *$ & 318 \\
\hline KS <-- SOL & 263 & 079 & 3338 & $* * *$ & 237 \\
\hline KS <-- KMS & 105 & 054 & 1952 & 051 & 134 \\
\hline KS <-- KA & 308 & 065 & 4753 & $* * *$ & 319 \\
\hline KS <-- IMP & 236 & 082 & 2895 & 004 & 171 \\
\hline KS <- HRP & 182 & 063 & 2894 & 004 & 219 \\
\hline KG <-- KA & 437 & 055 & 7947 & $* * *$ & 542 \\
\hline KG <- SOC & 166 & 062 & 2660 & 008 & 181 \\
\hline KG <-- KS & 185 & 061 & 3007 & 003 & 221 \\
\hline KU <-- HRP & 103 & 053 & 1931 & 054 & 151 \\
\hline KU <-- IMP & 273 & 073 & 3748 & $* * *$ & 241 \\
\hline KU <--- KG & 319 & 078 & 4083 & $* * * *$ & 325 \\
\hline KU <-- SOC & 145 & 078 & 1855 & 064 & 161 \\
\hline KU <--- IST & 133 & 043 & 3110 & 002 & 191 \\
\hline KR <-- TRP & 145 & 075 & 1932 & 053 & 130 \\
\hline KR <-- KU & 168 & 077 & 2174 & 030 & 165 \\
\hline KR <--- KS & 218 & 071 & 3087 & 002 & 262 \\
\hline KR <-- SOC & 183 & 078 & 2350 & 019 & 200 \\
\hline KR <-- HRP & 143 & 056 & 2549 & 011 & 207 \\
\hline OE <-- KU & 377 & 068 & 5512 & $* * *$ & 173 \\
\hline OE <-- SOC & 149 & 072 & 2062 & 039 & 239 \\
\hline OE <-- KR & 225 & 073 & 3104 & 002 & 168 \\
\hline OE <-- KMS & 103 & 042 & 2449 & 014 & 248 \\
\hline OP <--- OE & 533 & 188 & 2838 & 005 & \\
\hline
\end{tabular}

Table 127 summarizes the mean values for the KMPs in the model. 
Table 127. Model 12-KMP Means

\begin{tabular}{|c|c|c|c|c|}
\hline Factor & Estimate & S.E. & C.R. & P \\
\hline SOL & 4.125 & .053 & 77.262 & $* * *$ \\
\hline IMP & 4.353 & .043 & 101.735 & $* * *$ \\
\hline HRP & 3.374 & .071 & 47.367 & $* * *$ \\
\hline IST & 3.715 & .070 & 53.233 & $* * *$ \\
\hline KMS & 3.461 & .076 & 45.703 & $* * *$ \\
\hline OPM & 4.206 & .039 & 108.621 & $* * *$ \\
\hline OSS & 3.253 & .079 & 41.248 & $* * *$ \\
\hline TRP & 4.234 & .044 & 95.652 & $* * *$ \\
\hline PMP & 3.724 & .058 & 63.882 & $* * *$ \\
\hline
\end{tabular}

Table 128 summarizes the squared multiple correlations for Model 12.

Table 128. Model 12 Squared Multiple Correlations

\begin{tabular}{|c|c|}
\hline Factor & Estimate \\
\hline SOC & .668 \\
\hline $\mathrm{KA}$ & .532 \\
\hline $\mathrm{KS}$ & .651 \\
\hline $\mathrm{KG}$ & .696 \\
\hline $\mathrm{KU}$ & .585 \\
\hline $\mathrm{KR}$ & .613 \\
\hline $\mathrm{OE}$ & .651 \\
\hline $\mathrm{OP}$ & .062 \\
\hline
\end{tabular}

From Table 128, it is clear Model 12 explains a large percentage of the variance of the QKPS and SOC. However, the model predicts only about 6\% of the operational performance construct. Operational performance is known to be influenced by many other factors, some of which were already discussed such as random equipment failures, external (grid related) events, plant type, plant age, plant size, and the number of units at the site. For these reasons, it was not expected that a high level of variance would be explained and this result is not surprising. Table 129 summarizes the finding of this model. Table 130 provides the standardized total effects matrix for the model. 
Table 129. Summary of Model 12 Findings

\begin{tabular}{|c|c|c|}
\hline Hypothesized & Sign & Conclusion \\
\hline$H 9: \mathrm{OE} \rightarrow \mathrm{OP}$ & + & Supported \\
\hline
\end{tabular}

Table 130. Model 12 Standardized Effects

Part a.) Total Effects

\begin{tabular}{|c|c|c|c|c|c|c|c|c|c|c|c|c|c|c|c|}
\hline & IST & KMS & SOL & IMP & HRP & SOC & OSs & OPM & $\mathbf{K A}$ & KS & KG & TRP & KU & KR & $\mathrm{OE}$ \\
\hline SOC & .101 & .225 & .451 & .123 & .199 & .000 & .000 & .000 & .000 & .000 & .000 & .000 & .000 & .000 & .000 \\
\hline KA & .030 & .066 & .132 & .036 & .228 & .294 & .208 & .318 & .000 & .000 & .000 & .000 & .000 & .000 & .000 \\
\hline KS & .010 & .155 & .280 & .182 & .292 & .094 & .066 & .102 & .319 & .000 & .000 & .000 & .000 & .000 & .000 \\
\hline KG & .037 & .111 & .215 & .082 & .224 & .361 & .127 & .195 & .613 & .221 & .000 & .000 & .000 & .000 & .000 \\
\hline $\mathbf{K U}$ & .219 & .072 & .142 & .287 & .256 & 278 & .041 & .063 & .199 & .072 & .325 & .000 & .000 & .000 & .000 \\
\hline KR & .059 & .098 & .187 & .120 & .365 & .270 & .024 & .037 & .117 & .274 & .054 & .130 & .165 & .000 & .000 \\
\hline $\mathrm{OE}$ & .118 & .259 & .179 & .163 & .223 & .347 & .022 & .034 & .106 & .094 & .141 & .031 & .434 & .239 & .000 \\
\hline OP & .029 & .064 & .044 & .041 & .055 & .086 & .005 & .008 & .026 & .023 & .035 & .008 & .108 & .059 & .248 \\
\hline
\end{tabular}

Part b.) Direct Effects

\begin{tabular}{|c|c|c|c|c|c|c|c|c|c|c|c|c|c|c|c|}
\hline & IST & KMS & SOL & IMP & HRP & SOC & OSS & OPM & KA & KS & KG & TRP & KU & KR & OE \\
\hline SOC & .101 & .225 & .451 & .123 & .199 & .000 & .000 & .000 & .000 & .000 & .000 & .000 & .000 & .000 & .000 \\
\hline KA & .000 & .000 & .000 & .000 & .169 & .294 & .208 & .318 & .000 & .000 & .000 & .000 & .000 & .000 & .000 \\
\hline KS & .000 & .134 & .237 & .171 & .219 & .000 & .000 & .000 & .319 & .000 & .000 & .000 & .000 & .000 & .000 \\
\hline KG & .000 & .000 & .000 & .000 & .000 & .181 & .000 & .000 & .542 & .221 & .000 & .000 & .000 & .000 & .000 \\
\hline KU & .191 & .000 & .000 & .241 & .151 & .161 & .000 & .000 & .000 & .000 & .325 & .000 & .000 & .000 & .000 \\
\hline KR & .000 & .000 & .000 & .000 & .207 & .200 & .000 & .000 & .000 & .262 & .000 & .130 & .165 & .000 & .000 \\
\hline OE & .000 & .168 & .000 & .000 & .000 & .173 & .000 & .000 & .000 & .000 & .000 & .000 & .394 & .239 & .000 \\
\hline OP & .000 & .000 & .000 & .000 & .000 & .000 & .000 & .000 & .000 & .000 & .000 & .000 & .000 & .000 & .248 \\
\hline
\end{tabular}

Part c.) Indirect Effects

\begin{tabular}{|c|c|c|c|c|c|c|c|c|c|c|c|c|c|c|c|}
\hline & IST & KMS & SOL & IMP & HRP & SOC & Oss & OPM & KA & KS & $\mathbf{K G}$ & TRP & KU & $\mathbf{K R}$ & $\mathrm{OE}$ \\
\hline SoC & .000 & .000 & .000 & .000 & .000 & .000 & .000 & .000 & .000 & .000 & .000 & .000 & .000 & .000 & .000 \\
\hline $\mathrm{KA}$ & .030 & .066 & .132 & .036 & .058 & .000 & .000 & .000 & .000 & .000 & .000 & .000 & .000 & .000 & .000 \\
\hline KS & .010 & .021 & .042 & .012 & .073 & .094 & .066 & .102 & .000 & .000 & .000 & .000 & .000 & .000 & .000 \\
\hline KG & .037 & .111 & .215 & .082 & .224 & .180 & .127 & .195 & .071 & .000 & .000 & .000 & .000 & .000 & .000 \\
\hline $\mathrm{KU}$ & .028 & .072 & .142 & .047 & .105 & .117 & .041 & .063 & .199 & .072 & .000 & .000 & .000 & .000 & .000 \\
\hline KR & .059 & .098 & .187 & .120 & .158 & .070 & .024 & .037 & .117 & .012 & .054 & .000 & .000 & .000 & .000 \\
\hline OP & .029 & .064 & .044 & .041 & .055 & .086 & .005 & .008 & .026 & .023 & .035 & .008 & .108 & .059 & .000 \\
\hline
\end{tabular}

\subsection{Path Model 13: Competing Full Model with KR as Causal to KU}

Recall earlier in the analysis of the QKP construct interactions, Model 8 showed that an alternative "competing" model was possible with an interaction $\mathrm{KR} \rightarrow \mathrm{KU}$ and was also a good fit and could be supported in the literature. Path Model 13 (see Figure 112) was developed to revisit this issue in the context of the full model and consider its merit as a 
possible competing solution and also any interpretations given the links it supports amongst the quality of knowledge processes and the theoretical soundness of interpretations. Thus Path Model 13 results as an alternative final model when the link between quality of knowledge utilization and application processes (KU) and quality of knowledge retention and storage processes (KR) is reversed. Note that as a consequence of reversing the direction of this link, the links SOC $\rightarrow \mathrm{KU}$ and HRP $\rightarrow$ $\mathrm{KU}$ are no longer significant and drop out of the model. However, the explained variance of $\mathrm{KU}$ increases to $60 \%$. Table 131 summarizes the goodness of fit statistics for both models.

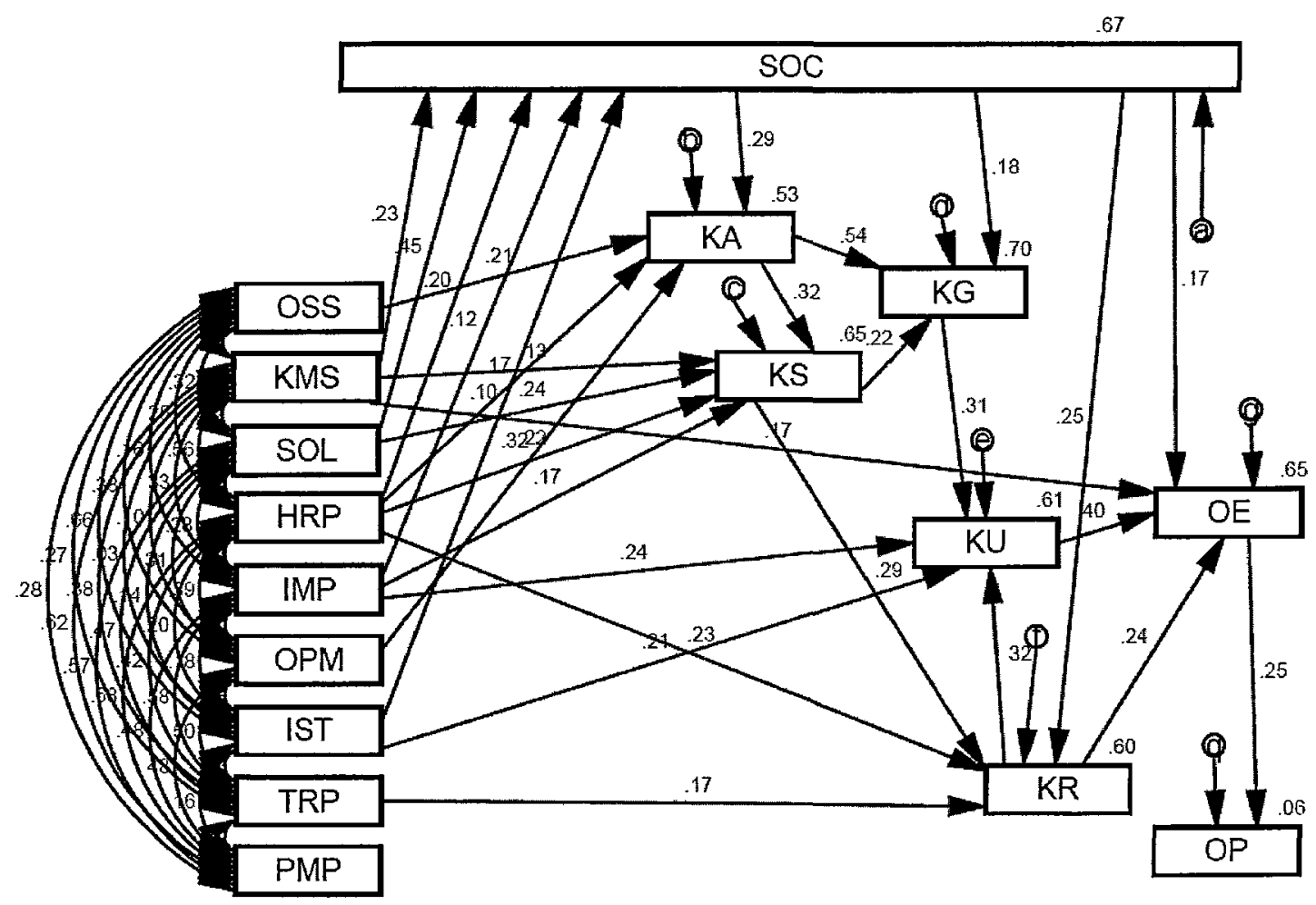

Figure 112. Path Diagram for Model 13 
Table 131. GOF Statistics for Models 12 and 13

\begin{tabular}{|c|c|c|c|c|c|c|c|c|c|c|c|c|c|c|c|}
\hline Model & NPAR & CMIN & DF & P & $\begin{array}{c}\text { CMIN } \\
\text { /DF }\end{array}$ & NFI & RFI & IFI & TLI & CFI & RMSEA & HI 90 & PCLOSE & AIC & HN05 \\
\hline 12 & 102 & 85.408 & 68 & .075 & 1.256 & .941 & .868 & .987 & .970 & .987 & .045 & .072 & .596 & 289.408 & 133 \\
\hline 13 & 100 & 83.635 & 70 & .127 & 1.195 & .942 & .874 & .990 & .977 & .990 & .039 & .067 & .707 & 283.635 & 139 \\
\hline
\end{tabular}

From Table 131, we can conclude Model 13 is an equally acceptable fit according to all

GOF statistics and statistical inference conclusions can be drawn from it. Table 132

below summarizes the regression weights and p-values for each relationship for both

Model 12 and 13 for comparative purposes.

Table 132. Model 12 \& 13 Regression Weights

\begin{tabular}{|c|c|c|c|c|c|c|c|c|c|c|}
\hline \multirow{2}{*}{$\frac{\text { Analog }}{\text { Link }}$} & \multicolumn{5}{|c|}{ Model 13} & \multicolumn{5}{|c|}{ Model 12} \\
\hline & Estimate & S.E. & C.R. & $\mathbf{P}$ & $\begin{array}{c}\text { Standardized } \\
\text { Estimate }\end{array}$ & Estimate & S.E. & C.R. & $\mathbf{P}$ & $\begin{array}{l}\text { Standardized } \\
\text { Estimate }\end{array}$ \\
\hline SOC $<-\cdots$ IST & .078 & .042 & 1.848 & .065 & .101 & .079 & .042 & 1.860 & .063 & .101 \\
\hline SOC <-- HRP & .150 & .053 & 2.816 & .005 & .199 & .150 & .053 & 2.817 & .005 & .199 \\
\hline SOC <-- SOL & .455 & .068 & 6.669 & $* * *$ & .451 & .455 & .068 & 6.669 & $* * *$ & .451 \\
\hline SOC <--- KMS & .161 & .048 & 3.341 & $* * *$ & .225 & .161 & .048 & 3.342 & $* * *$ & .225 \\
\hline SOC $<-1 \mathrm{MPP}$ & .155 & .071 & 2.181 & .029 & .123 & .155 & .071 & 2.182 & .029 & .123 \\
\hline $\mathrm{KA}<-\mathrm{SOC}$ & .335 & .094 & 3.569 & $* * *$ & .294 & .334 & .094 & 3.566 & $* * *$ & .294 \\
\hline KA <-- HRP & .146 & .071 & 2.039 & .041 & .169 & .146 & .071 & 2.041 & .041 & .169 \\
\hline KA <-- OSS & .163 & .054 & 3.023 & .002 & .207 & .163 & .054 & 3.027 & .002 & .208 \\
\hline KA <-- OPM & .505 & .112 & 4.501 & $* * *$ & .319 & .505 & .112 & 4.501 & $* * *$ & .318 \\
\hline $\mathrm{KS}<--\mathrm{SOL}$ & .262 & .079 & 3.319 & $* * *$ & .236 & .263 & .079 & 3.338 & $* * *$ & .237 \\
\hline $\mathrm{KS}<--\mathrm{KMS}$ & .105 & .054 & 1.948 & .051 & .134 & .105 & .054 & 1.952 & .051 & .134 \\
\hline $\mathrm{KS}<--\mathrm{KA}$ & .309 & .065 & 4.760 & $* * *$ & .320 & .308 & .065 & 4.753 & $* * *$ & .319 \\
\hline KS <--- IMP & .233 & .082 & 2.856 & .004 & .169 & .236 & .082 & 2.895 & .004 & .171 \\
\hline KS <--- HRP & .183 & .063 & 2.908 & .004 & .221 & .182 & .063 & 2.894 & .004 & .219 \\
\hline $\mathrm{KR}<--\mathrm{TRP}$ & .185 & .075 & 2.446 & .014 & .166 & .145 & .075 & 1.932 & .053 & .130 \\
\hline $\mathrm{KG}<\cdots \mathrm{KA}$ & .437 & .055 & 7.942 & $* * *$ & .542 & .437 & .055 & 7.947 & $* * *$ & .542 \\
\hline $\mathrm{KG}<--\mathrm{SOC}$ & .165 & .062 & 2.656 & .008 & .180 & .166 & .062 & 2.660 & .008 & .181 \\
\hline KR <--- KS & .244 & .070 & 3.466 & $* * *$ & .293 & .218 & .071 & 3.087 & .002 & .262 \\
\hline $\mathrm{KR}<--\mathrm{SOC}$ & .229 & .076 & 3.009 & .003 & .250 & .183 & .078 & 2.350 & .019 & .200 \\
\hline $\mathrm{KG}<---\mathrm{KS}$ & .186 & .061 & 3.028 & .002 & .223 & .185 & .061 & 3.007 & .003 & .221 \\
\hline $\mathrm{KR}<---\mathrm{HRP}$ & .156 & .057 & 2.740 & .006 & .225 & .143 & .056 & 2.549 & .011 & .207 \\
\hline $\mathrm{KU} \ll \mathrm{HRP}$ & & & & & & .103 & .053 & 1.931 & .054 & .151 \\
\hline $\mathrm{KU}<---\mathrm{IMP}$ & .278 & .071 & 3.901 & $* * *$ & .244 & .273 & .073 & 3.748 & $* * * *$ & .241 \\
\hline $\mathrm{KU}<---\mathrm{KG}$ & .301 & .070 & 4.317 & $* * *$ & .306 & .319 & .078 & 4.083 & $* * *$ & .325 \\
\hline $\mathrm{KU}<--\mathrm{SOC}$ & & & & & & .145 & .078 & 1.855 & .064 & .161 \\
\hline $\mathrm{KU}<---$ IST & .150 & .042 & 3.607 & $* * *$ & .215 & .133 & .043 & 3.110 & .002 & .191 \\
\hline $\mathrm{KU}<--\mathrm{KR}$ & .316 & .069 & 4.561 & $* * *$ & .320 & & & & & \\
\hline $\mathrm{KR}<---\mathrm{KU}$ & & & & & & .168 & .077 & 2.174 & .030 & .165 \\
\hline $\mathrm{OE}<--\mathrm{KU}$ & .378 & .067 & 5.598 & $* * *$ & .397 & .377 & .068 & 5.512 & $* * *$ & .394 \\
\hline
\end{tabular}




\begin{tabular}{|c|c|c|c|c|c|c|c|c|c|c|}
\hline Analog & \multicolumn{5}{|c|}{ Model 13 } & \multicolumn{4}{c|}{ Model 12 } \\
\hline Link & Estimate & S.E. & C.R. & P & $\begin{array}{c}\text { Standardized } \\
\text { Estimate }\end{array}$ & Estimate & S.E. & C.R. & P & $\begin{array}{c}\text { Standardized } \\
\text { Estimate }\end{array}$ \\
\hline $\mathrm{OE}<--\mathrm{SOC}$ & .149 & .070 & 2.124 & .034 & .174 & .149 & .072 & 2.062 & .039 & .173 \\
\hline $\mathrm{OE}<--\mathrm{KR}$ & .224 & .075 & 2.987 & .003 & .239 & .225 & .073 & 3.104 & .002 & .239 \\
\hline $\mathrm{OE}<--\mathrm{KMS}$ & .104 & .042 & 2.451 & .014 & .169 & .103 & .042 & 2.449 & .014 & .168 \\
\hline $\mathrm{OP}<--\mathrm{OE}$ & .533 & .188 & 2.831 & .005 & .248 & .533 & .188 & 2.838 & .005 & .248 \\
\hline
\end{tabular}

The weights and significance for the two models is quite similar on all parameters except for some expected variation for KU and KR. The non-significant links SOC $\rightarrow$ $\mathrm{KU}$ and $\mathrm{HRP} \rightarrow \mathrm{KU}$ that have been removed in Model 13 are shown as grayed-out in the Table 132. In Table 132 the links $\mathrm{KR} \rightarrow \mathrm{KU}$ and $\mathrm{KU} \rightarrow \mathrm{KR}$ are shown as grayedout for Model 12 and 13 respectively (i.e. one of these links is not present in each model).

Table 133 summarizes the squared multiple correlations. There is very little difference in the explained variance between the two models for all of the model parameters. Considering all, Path Model 12 is preferred and assumed to be the more correct, so is used going forward. As discussed with Model 8, further research is required to confirm and better understand the link $\mathrm{KR} \rightarrow \mathrm{KU}$.

Table 133. Model $12 \& 13$ Squared Multiple Correlations

\begin{tabular}{|c|c|c|}
\hline Factor & $\begin{array}{l}\text { Model 13 } \\
\text { Estimate }\end{array}$ & $\begin{array}{c}\text { Model 12 } \\
\text { Estimate }\end{array}$ \\
\hline KA & .668 & .668 \\
\hline KG & .532 & .532 \\
\hline KR & .651 & .651 \\
\hline KS & .697 & .696 \\
\hline KU & .598 & .585 \\
\hline OE & .608 & .613 \\
\hline OP & .648 & .651 \\
\hline SOC & .061 & .062 \\
\hline
\end{tabular}




\subsection{Path Model 14: Effect of Support for KMP High vs. KMP Low}

Path Model 14 was developed to better understand the effect of support for KM Practices (KMPs) on quality of knowledge processes (QKPs), supportive organizational culture (SOC), and organizational effectiveness (OE) (i.e. related to hypotheses 1-5, and 8). The dataset was split into two sub-groups according to stations with overall KMP higher than the mean KMP vs. stations with lower than the mean KMP value. Note that due to the smaller sample size after splitting the dataset, the construct operating performance (OP) had to be removed from the model in order to get an acceptable fit. AMOS multi-group analysis function was used to compare the two groups. Figure 113 shows Model 14a with KMP High and Figure 114 shows Model 14b with KMP low. The results of the 2-group analysis assume the structural weights and intercepts constrained to be equal between the two path models.

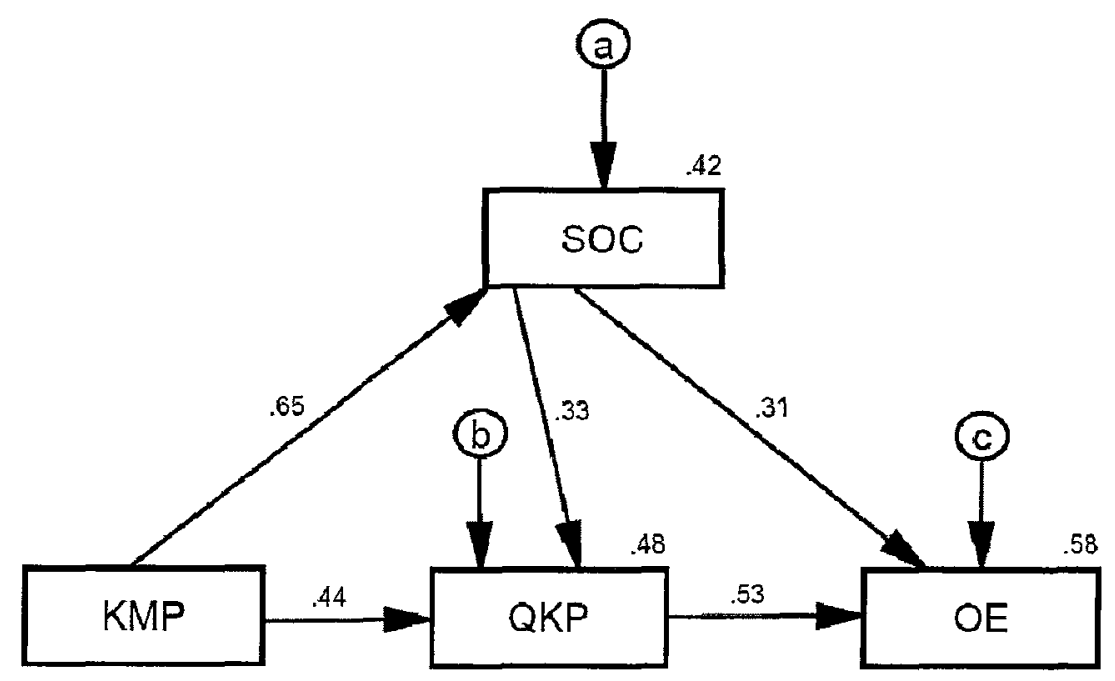

Figure 113. Model 14a: KMP High - Structural Weights \& Intercepts Equal 


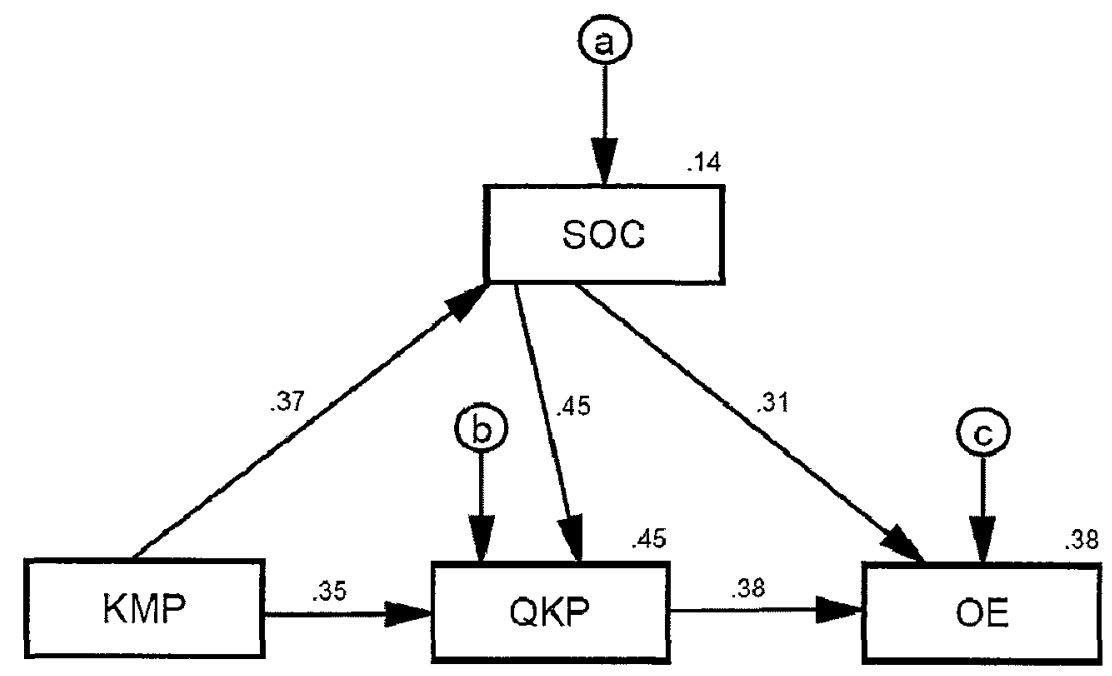

Figure 114. Model 14b: KMP Low - Structural Weights \& Intercepts Equal

Table 134 shows the goodness of fit test results of the 2-group analysis.

Table 134. Multi-Group Analysis of Models 14a,b (KMP High vs. Low)

\begin{tabular}{|c|c|c|c|c|c|c|c|c|c|c|c|c|c|c|c|}
\hline Model & $\begin{array}{c}\text { NPA } \\
\text { R }\end{array}$ & CMIN & DF & P & $\begin{array}{c}\text { CMI } \\
\text { N/DF }\end{array}$ & NFI & RFI & IFI & TLI & CFI & RMSEA & HI 90 & PCLOSE & AIC & HN05 \\
\hline $\begin{array}{c}\text { Unconstrained } \\
\text { Parameters }\end{array}$ & 26 & 3.946 & 2 & .139 & 1.973 & .978 & .871 & .989 & .932 & .989 & .090 & .222 & .221 & 55.946 & 184 \\
\hline $\begin{array}{c}\text { Structural } \\
\text { Weights }\end{array}$ & 21 & 15.236 & 7 & .033 & 2.177 & .917 & .858 & .953 & .918 & .952 & .099 & .167 & .106 & 57.236 & 112 \\
\hline $\begin{array}{c}\text { Structural } \\
\text { Intercepts }\end{array}$ & $\mathbf{1 8}$ & $\mathbf{1 8 . 1 0 6}$ & $\mathbf{1 0}$ & $\mathbf{. 0 5 3}$ & 1.811 & $\mathbf{. 9 0 1}$ & $\mathbf{. 8 8 2}$ & $\mathbf{. 9 5 3}$ & $\mathbf{. 9 4 3}$ & $\mathbf{. 9 5 3}$ & $\mathbf{. 0 8 2}$ & $\mathbf{. 1 4 2}$ & $\mathbf{. 1 7 3}$ & $\mathbf{5 4 . 1 0 6}$ & $\mathbf{1 2 3}$ \\
\hline $\begin{array}{c}\text { Structural } \\
\text { Means }\end{array}$ & 17 & 104.265 & 11 & .000 & 9.479 & .432 & .380 & .459 & .407 & .456 & .266 & .314 & .000 & 138.265 & 24 \\
\hline
\end{tabular}

From Table 134, it can be concluded the structural intercepts model is the best fit and can be assumed to be correct. The model comparison feature in AMOS indicates there is a statistical significance in the difference in structural means between the two groups with p-value of .000 if we assume the structural intercepts model is correct (see Table 135). Thus it is assumed that the structural weights and intercepts models are correct and GOF statistics indicate statistical inference conclusions can be drawn from the 
models. Table 136 summarizes the statistics for the mean value of KMP for the two groups.

Table 135. Model 14a,b Comparison (assume structural intercepts correct)

\begin{tabular}{|c|c|c|c|c|c|c|c|}
\hline Model & DF & CMIN & P & $\begin{array}{c}\text { NFI } \\
\text { Delta-1 }\end{array}$ & $\begin{array}{c}\text { IFI } \\
\text { Delta-2 }\end{array}$ & $\begin{array}{c}\text { RFI } \\
\text { rho-1 }\end{array}$ & $\begin{array}{c}\text { TLI } \\
\text { rho2 }\end{array}$ \\
\hline Structural means & 1 & 86.159 & .000 & .470 & .497 & .502 & .537 \\
\hline
\end{tabular}

Table 136. Model 14a,b Means (structural intercepts equal)

\begin{tabular}{|c|c|c|c|c|c|c|c|c|}
\hline \multirow{2}{*}{ Factor } & \multicolumn{4}{|c|}{ KMP High Group } & \multicolumn{4}{c|}{ KMP Low Group } \\
\cline { 2 - 9 } & Estimate & S.E. & C.R. & P & Estimate & S.E. & C.R. & P \\
\hline KMP & 4.211 & .044 & 94.956 & $* * *$ & 3.515 & .024 & 147.333 & $* * *$ \\
\hline
\end{tabular}

Table 137 below summarizes the regression weights and p-values for each inter-factor relationship for both groups to highlight the differences. It is quite evident that a large difference exists between the two groups. Table 138 shows the squared multiple correlations for the two groups. Going from KMP low to high sees an increase in explained variance of $27.9 \%$ for SOC, of $3.7 \%$ for QKP and of $20.4 \%$ for OE. For the latter of these, $58 \%$ of the variance of organizational effectiveness is explained by the KMP High group model whereas only $38 \%$ is explained by the data in the KMP Low group.

Table 137. Model 14a,b Regression Weights (structural intercepts equal)

\begin{tabular}{|c|c|c|c|c|c|c|}
\hline Link & Estimate & S.E. & C.R. & P & $\begin{array}{c}\text { Standardized Estimate - } \\
\text { KMP High }\end{array}$ & $\begin{array}{c}\text { Standardized Estimate - } \\
\text { KMP Low }\end{array}$ \\
\hline SOC <-- KMP & .933 & .084 & 11.111 & $* * *$ & .646 & .372 \\
\hline QKP <-- SOC & .316 & .059 & 5.356 & $* * *$ & .326 & .454 \\
\hline QKP <-- KMP & .615 & .083 & 7.382 & $* * *$ & .440 & .352 \\
\hline OE <-- QKP & .511 & .084 & 6.086 & $* * *$ & .531 & .380 \\
\hline OE <-- SOC & .290 & .075 & 3.881 & $* * *$ & .311 & .309 \\
\hline
\end{tabular}


Table 138. Model 14a,b Squared Mult. Correlations (structural intercepts equal)

\begin{tabular}{|c|c|c|}
\hline Factor & $\begin{array}{l}\text { Estimate - } \\
\text { KMP High }\end{array}$ & $\begin{array}{l}\text { Estimate - } \\
\text { KMP Low }\end{array}$ \\
\hline SOC & .417 & .138 \\
\hline QKP & .485 & .448 \\
\hline OE & .581 & .377 \\
\hline
\end{tabular}

Tables 139, 140, and 141 summarize the standardized total, direct, and indirect effects assuming the structural intercepts model is correct.

Table 139. Model 14a,b Stand. Total Effects (structural intercepts equal)

\begin{tabular}{|c|c|c|c|c|c|c|}
\hline \multirow{2}{*}{ Factor } & \multicolumn{3}{|c|}{ Estimate- } & \multicolumn{3}{c|}{ Estimate - } \\
\cline { 2 - 7 } & KMP High & SOC & QKP & KMP & SOC & QKP \\
\hline SOC & .646 & .000 & .000 & .372 & .000 & .000 \\
\hline QKP & .650 & .326 & .000 & .520 & .454 & .000 \\
\hline OE & .546 & .484 & .531 & .312 & .481 & .380 \\
\hline
\end{tabular}

Table 140. Model 14a,b Stand. Direct Effects (structural intercepts equal)

\begin{tabular}{|c|c|c|c|c|c|c|}
\hline \multirow{2}{*}{ Factor } & \multicolumn{3}{|c|}{$\begin{array}{c}\text { Estimate - } \\
\text { KMP High }\end{array}$} & \multicolumn{3}{c|}{ Estimate - } \\
\cline { 2 - 7 } & KMP LP & SOC & QKP & KMP & SOC & QKP \\
\hline SOC & .646 & .000 & .000 & .372 & .000 & .000 \\
\hline QKP & .440 & .326 & .000 & .352 & .454 & .000 \\
\hline OE & .000 & .311 & .531 & .000 & .309 & .380 \\
\hline
\end{tabular}

Table 141. Model 14a,b Stand. Indirect Effects (structural intercepts equal)

\begin{tabular}{|c|c|c|c|c|c|c|}
\hline \multirow{2}{*}{ Factor } & \multicolumn{3}{|c|}{$\begin{array}{c}\text { Estimate- } \\
\text { KMP High }\end{array}$} & \multicolumn{3}{c|}{ Estimate - } \\
& KMP Low \\
\cline { 2 - 7 } & KMP & SOC & QKP & KMP & SOC & QKP \\
\hline SOC & .000 & .000 & .000 & .000 & .000 & .000 \\
\hline QKP & .211 & .000 & .000 & .169 & .000 & .000 \\
\hline OE & .546 & .173 & .000 & .312 & .172 & .000 \\
\hline
\end{tabular}

From Tables 139, 140, and 141, it is clear that there are notable differences in these relationships when controlling for the level of support for KMP in NPPs. The loading of SOC $\rightarrow$ OE does not change between the two groups, however, the loadings of KMP $\rightarrow \mathrm{QKP}, \mathrm{KMP} \rightarrow \mathrm{SOC}$ and SOC $\rightarrow \mathrm{QKP}$ changes significantly. Thus the increase in 
loading of QKP $\rightarrow$ OE from .38 to .53 when going from group KMP Low to KMP High is due to a simultaneous increase in the loading of KMP $\rightarrow$ QKP from .35 to .44 and of $\mathrm{KMP} \rightarrow \mathrm{SOC}$ from .37 to .65 . However, when going from group KMP Low to KMP High the loading of SOC $\rightarrow$ QKPs drops $12 \%$ (i.e. from .45 to .33). The explained variance of SOC increases by $28 \%$ (i.e. from .138 to .417 ), the explained variance of QKP goes up by $4 \%$ (i.e. from .448 to .485 ) and the explained variance of $\mathrm{OE}$ increases by $21 \%$ (i.e. from .377 to .581 ). The overall effect of KMP on QKP increases by $13 \%$ (i.e. from .520 to .650 ). The overall effect of KMP on OE increases $23 \%$ (i.e. from .312 to .546 ). Thus it can be concluded that there are significant differences between NPPs with high levels of support for KMPs and those with low levels of support for KMPs. Higher levels of KMPs have a strong positive impact on organizational effectiveness.

\subsection{Path Model 15: Effect of SOC High vs. SOC Low}

Path Model 15 was developed to better understand the effect of a supportive organizational culture (SOC) on quality of knowledge processes (QKPs) and organizational effectiveness (OE) (i.e. related to hypotheses 2 and 3). In previous sections SOC has been established as an intermediate variable in the following: KMPs $\rightarrow$ SOC $\rightarrow$ QKPs, KMPs $\rightarrow$ SOC $\rightarrow$ OE, OTSs $\rightarrow$ SOC $\rightarrow$ QKPs, and OTSs $\rightarrow$ SOC $\rightarrow$ OE. Also in previous sections, many direct links between specific KMPs to specific QKPs as well as direct links between OSS $\rightarrow \mathrm{KA}$ and IST $\rightarrow \mathrm{KU}$ were found to be significant. Path Model 15 will also be used to determine whether SOC, in addition to 
its role as an intermediate construct may also (simultaneously) play a role as a moderator of these direct relationships.

Thus to establish whether moderation is occurring, and better understand the effects of SOC, multi-group analysis feature in AMOS path analysis can be used. The dataset was split into 2 sub-groups according to plants with overall SOC higher vs. lower than the mean SOC value of 3.9. Note that due to the smaller sample size after splitting the dataset, the factor operating performance (OP) had to be removed from the model in order to get an acceptable fit. Figures 115 and 116 show the results of the 2-group analysis (Models 15a,b), with the structural weights and intercepts constrained to be equal between the two path models.

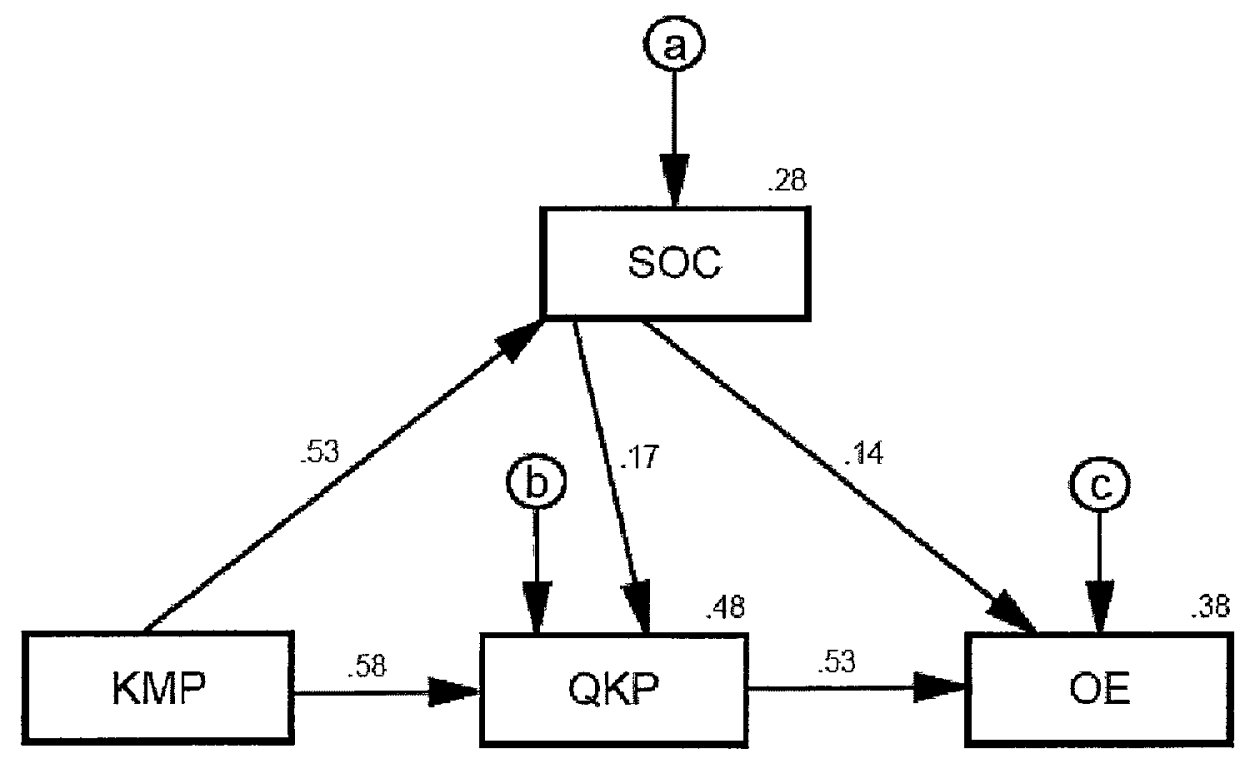

Figure 115. Model 15a: SOC High (structural weights assumed equal) 


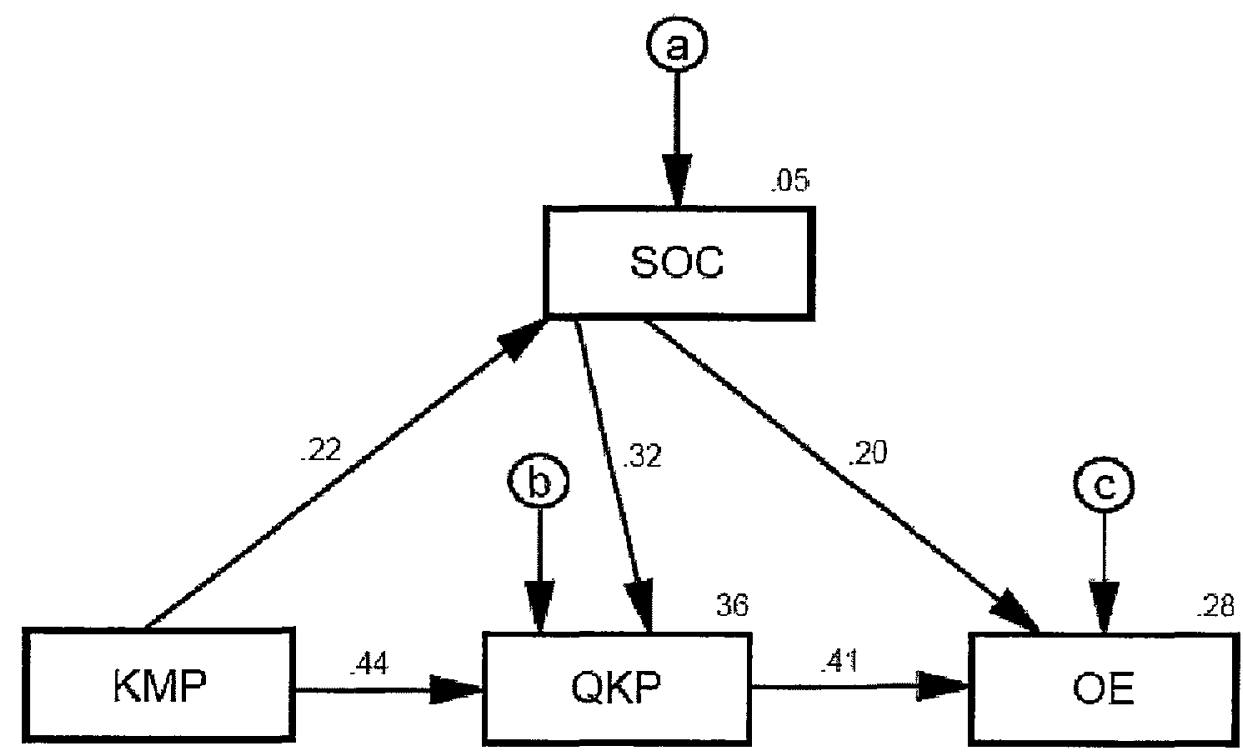

Figure 116. Model 15b: SOC Low (structural weights assumed equal).

Table 142 shows the GOF test results of the 2-group analysis.

Table 142. Multi-Group Analysis - Models 15a,b (SOC High vs. Low)

\begin{tabular}{|c|c|c|c|c|c|c|c|c|c|c|c|c|c|c|c|}
\hline Model & NPAR & CMIN & DF & P & $\begin{array}{c}\text { CMIN } \\
\text { IF }\end{array}$ & NFI & RFI & IFI & TLI & CFI & RMSEA & HI 90 & PCLOSE & AIC & HN05 \\
\hline $\begin{array}{c}\text { Unconstrained } \\
\text { Parameters }\end{array}$ & 26 & 2.83 & 2 & .243 & 1.416 & .982 & .889 & .995 & .965 & .994 & .000 & .200 & .341 & 54.831 & 255 \\
\hline $\begin{array}{c}\text { Structural } \\
\text { Weights }\end{array}$ & $\mathbf{2 1}$ & 15.64 & 7 & $\mathbf{. 0 2 9}$ & $\mathbf{2 . 2 3 5}$ & .898 & $\mathbf{. 8 2 5}$ & $\mathbf{. 9 4 1}$ & $\mathbf{. 8 9 5}$ & $\mathbf{. 9 3 9}$ & $\mathbf{. 0 3 1}$ & .170 & $\mathbf{. 0 9 5}$ & $\mathbf{5 7 . 6 4 4}$ & $\mathbf{1 0 9}$ \\
\hline $\begin{array}{c}\text { Structural } \\
\text { Intercepts }\end{array}$ & 18 & 107.49 & 10 & .000 & 10.749 & .300 & .160 & .321 & .174 & .311 & .238 & .335 & .000 & 143.493 & 22 \\
\hline $\begin{array}{c}\text { Structural } \\
\text { Means }\end{array}$ & 17 & 170.53 & 11 & .000 & 15.503 & -.110 & -.211 & -.119 & -.229 & .000 & .303 & .395 & .000 & 204.530 & 15 \\
\hline
\end{tabular}

From Table 142, it can be concluded the unconstrained parameters model is the best fit. However, the structural weights model is also a very close fit. The lower than desired pvalue can be attributed to the smaller split-sample size and is offset by an acceptable PCLOSE value of .095 . The CFI and AIC also indicate a good fit relative to the unconstrained parameters model, so for these reasons, and the fact that the structural weights model fits well to the full dataset, the structural weights model is assumed to be 
correct. The model comparison feature in AMOS indicates there is a statistical significance in the difference in structural means between the two groups with p-value of .000 if we assume the structural weights model to be correct (see Table 143). Table 144 summarizes the estimates of the means for KMP high and low groups.

Table 143. Model 15a,b Comparison (assuming structural weights correct)

\begin{tabular}{|c|c|c|c|c|c|c|c|}
\hline Model & DF & CMIN & P & $\begin{array}{c}\text { NFI } \\
\text { Delta-1 }\end{array}$ & $\begin{array}{c}\text { IFI } \\
\text { Delta-2 }\end{array}$ & $\begin{array}{c}\text { RFI } \\
\text { rho-1 }\end{array}$ & $\begin{array}{c}\text { TLI } \\
\text { rho2 }\end{array}$ \\
\hline Structural intercepts & 3 & 91.849 & .000 & .598 & .627 & .665 & .722 \\
\hline Structural means & 4 & 154.886 & .000 & 1.009 & 1.057 & 1.037 & 1.125 \\
\hline
\end{tabular}

Table 144. Model 15a,b Means for SOC High \& Low (structural weights equal)

\begin{tabular}{|c|c|c|c|c|c|c|c|c|}
\hline \multirow{2}{*}{ Factor } & \multicolumn{4}{|c|}{ SOC High Group } & \multicolumn{4}{c|}{ SOC Low Group } \\
\cline { 2 - 9 } & Estimate & S.E. & C.R. & P & Estimate & S.E. & C.R. & P \\
\hline KMP & $\mathbf{4 . 0 5 6}$ & .049 & 82.863 & $* * *$ & $\mathbf{3 . 5 0 9}$ & .031 & 114.508 & $* * *$ \\
\hline
\end{tabular}

Thus it is assumed that the structural weights model is correct and GOF statistics indicate statistical inference conclusions can be drawn from the models. Table 145 below summarizes the regression weights and p-values for each inter-factor relationship for both groups to highlight the differences. Table 146 summarizes the squared multiple correlations for the two sub-groups. Some interesting differences are that the $38 \%$ of variance of organizational effectiveness is explained by the SOC High group model whereas only $28 \%$ is explained by the data in the SOC Low group.

Table 145. Model 15a,b Regression Weights (structural weights equal)

\begin{tabular}{|c|c|c|c|c|c|c|}
\hline Link & Estimate & S.E. & C.R. & P & $\begin{array}{c}\text { Standardized Estimate - } \\
\text { SOC High }\end{array}$ & $\begin{array}{c}\text { Standardized Estimate - } \\
\text { SOC Low }\end{array}$ \\
\hline SOC <-- KMP & .348 & .067 & 5.226 & $* * *$ & .526 & .219 \\
\hline QKP <-- SOC & .280 & .085 & 3.301 & $* * *$ & .173 & .315 \\
\hline QKP <-- KMP & .624 & .087 & 7.206 & $* * *$ & .584 & .443 \\
\hline OE <-- QKP & .522 & .089 & 5.887 & $* * *$ & .534 & .413 \\
\hline OE <-- SOC & .223 & .109 & 2.047 & .041 & .141 & .199 \\
\hline
\end{tabular}


Table 146. Model 15a,b Squared Mult. Correlations (structural weights equal)

\begin{tabular}{|c|c|c|}
\hline Factor & $\begin{array}{l}\text { Estimate - } \\
\text { SOC High }\end{array}$ & $\begin{array}{c}\text { Estimate - } \\
\text { SOC Low }\end{array}$ \\
\hline SOC & .277 & .048 \\
\hline QKP & .477 & .357 \\
\hline OE & .377 & .279 \\
\hline
\end{tabular}

Tables 147, 148, and 149 summarize the standardized total, direct, and indirect effects assuming the structural weights equal model is correct.

Table 147. Model 15a,b Stand. Total Effects (structural weights equal for both)

\begin{tabular}{|c|c|c|c|c|c|c|}
\hline \multirow{2}{*}{ Factor } & \multicolumn{3}{|c|}{$\begin{array}{c}\text { Estimate - } \\
\text { SOC High }\end{array}$} & \multicolumn{3}{c|}{ Estimate - } \\
& SOC Low \\
\cline { 2 - 7 } & KMP & SOC & QKP & KMP & SOC & QKP \\
\hline SOC & .526 & .000 & .000 & .219 & .000 & .000 \\
\hline QKP & .675 & .173 & .000 & .512 & .315 & .000 \\
\hline OE & .435 & .234 & .534 & .255 & .330 & .413 \\
\hline
\end{tabular}

Table 148. Model 15a,b Stand. Direct Effects (structural weights equal)

\begin{tabular}{|c|c|c|c|c|c|c|}
\hline \multirow{2}{*}{ Factor } & \multicolumn{3}{|c|}{ Estimate - } & \multicolumn{3}{c|}{ Estimate - } \\
& SOC High & \multicolumn{3}{c|}{ SOC Low } \\
\cline { 2 - 7 } & KMP & SOC & QKP & KMP & SOC & QKP \\
\hline SOC & .526 & .000 & .000 & .219 & .000 & .000 \\
\hline QKP & .584 & .173 & .000 & .443 & .315 & .000 \\
\hline OE & .000 & .141 & .534 & .000 & .199 & .413 \\
\hline
\end{tabular}

Table 149. Model 15a,b Stand. Indirect Effects (structural intercepts equal)

\begin{tabular}{|c|c|c|c|c|c|c|}
\hline \multirow{2}{*}{ Factor } & \multicolumn{3}{|c|}{$\begin{array}{c}\text { Estimate - } \\
\text { SOC High }\end{array}$} & \multicolumn{3}{c|}{ Estimate - } \\
& SOC Low \\
\cline { 2 - 7 } & KMP & SOC & QKP & KMP & SOC & QKP \\
\hline SOC & .000 & .000 & .000 & .000 & .000 & .000 \\
\hline QKP & .091 & .000 & .000 & .069 & .000 & .000 \\
\hline OE & .435 & .092 & .000 & .255 & .130 & .000 \\
\hline
\end{tabular}

From Tables 147, 148, and 149 it is clear that there are notable differences in these relationships when controlling for the level of supportive organizational culture (SOC) in NPPs. The standardized loading of SOC $\rightarrow$ OE, KMP $\rightarrow$ QKP, KMP $\rightarrow$ SOC and SOC $\rightarrow$ QKP change significantly between the two groups. There is an increase in 
loading of QKP $\rightarrow \mathrm{OE}$ from .413 to .534 when going from group SOC Low to KMP High. There is also a simultaneous increase in the loading of KMP $\rightarrow$ QKP from .512 to .675 and in the loading of KMP $\rightarrow$ SOC from .219 to .526 . Although when going from group SOC Low to SOC High the total effect of SOC $\rightarrow$ QKPs decreases $14 \%$ (i.e. from .315 to .173), it is clear to see SOC moderates (increases) the effects of QKP $\rightarrow \mathrm{OE}$ and $\mathrm{KMP} \rightarrow \mathrm{QKP}$. The explained variance of QKP also increases by $12 \%$ (i.e. from .357 to .477 ) and the explained variance of $\mathrm{OE}$ increases by $10 \%$ (i.e. from .279 to .377 ). The direct effect SOC $\rightarrow$ OE decreases $6 \%$ (i.e. from .199 to .141 ) when going from group SOC Low to High. Thus it can be concluded that there are significant differences between NPPs with high supportive organizational culture (SOC) and NPPs with low supportive organizational culture (SOC), and that overall, these differences have a strong positive impact on organizational effectiveness. There is a statistically significant difference at better than the .001 level in the mean value of KMPs between the two sub-groups. Mean value of KMP is about 4.0 for SOC High group versus about 3.5 for the SOC Low group and this is thought to be the primary causal factor accounting for the difference in SOC between the two sub-groups and the subsequent effects on the other factors.

From these findings of we can conclude that SOC has a strong moderating variable effect on both the relationship between KMP $\rightarrow$ QKP, QKP $\rightarrow$ OE. As it has also been shown that support for KMPs strongly influence (increase) SOC, it can be concluded that higher levels of support for KM practices achieve a leveraged effect on QKP and $\mathrm{OE}$ though an increase in supporting organizational culture. 


\subsection{Path Model 16: Estimating Raw Operational Performance}

Path Model 16 was created to replace OP on the RHS of Model 12 with three of the four raw operational performance parameters: 3-year average unit capability factor (3YrUCF), 3-year average forced loss rate (3YrFLR), and 3-year average unplanned automatic scrams (3YrUA7) per 10,000 hours. The fourth raw operational performance parameter, 3-year average unplanned capability loss (3YrUCL) was not included for simplicity reasons, and because FLR and UCL measure much the same thing and are highly collinear. By using non-standardized values, this model can be used to predict the direct effects of each KMP or OTS construct on plant performance and thus estimate the magnitude of the economic benefit to be achieved with changes in each independent variable under management's control. For example, a step increase in the factor KMS can be translated into an estimated KWh production improvement (with all else assumed constant). The $\mathrm{KU} \rightarrow \mathrm{KR}$ causal relationship discussed previously was assumed to be correct and included. Figure 117 shows Path Model 16a with no direct effects links to the 3 operational performance factors. 


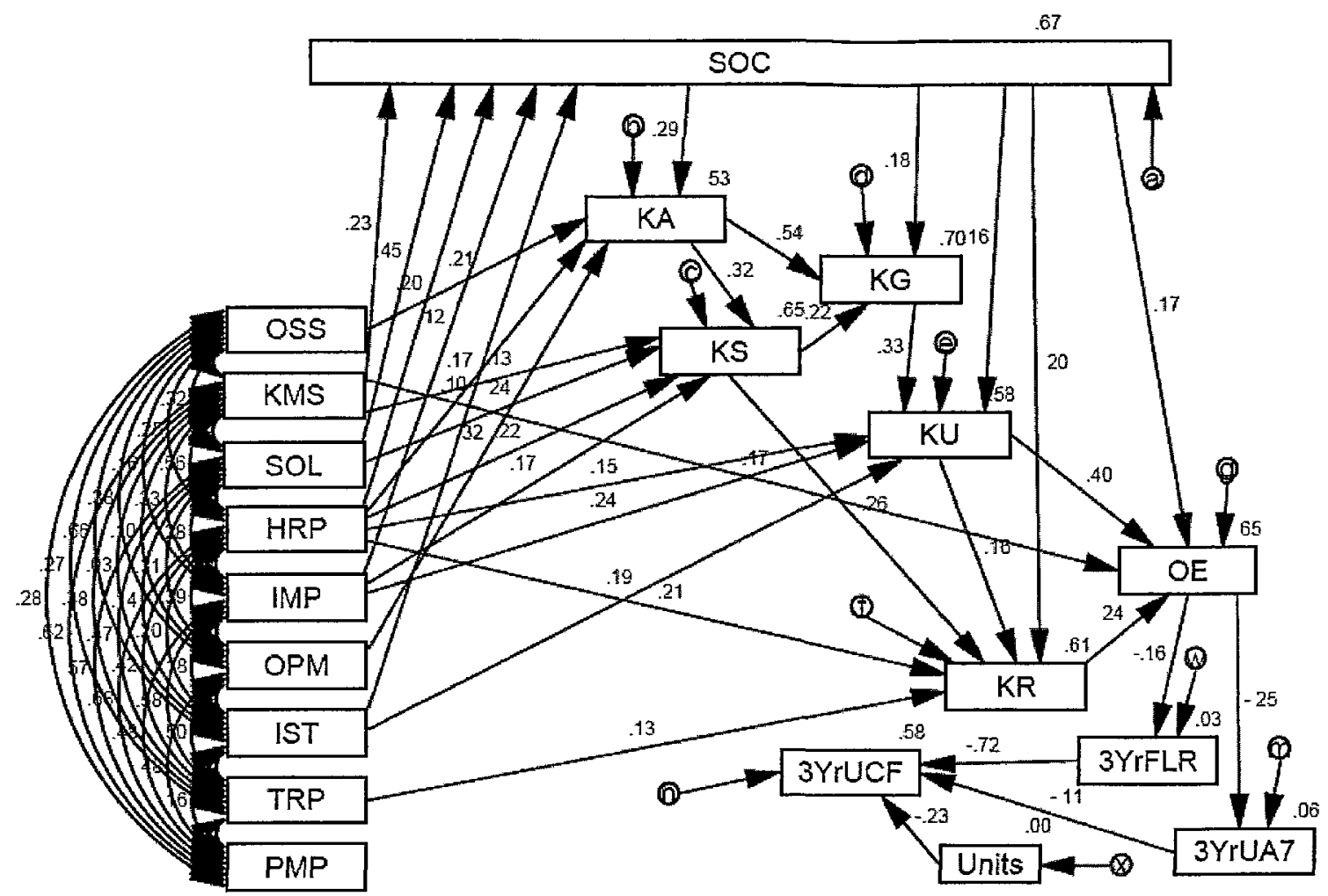

Figure 117. Path Model 16a: Full Model with Raw Performance Measures

Table 150 summarizes the goodness of fit statistics for Model 16a. Although PCLOSE is marginal, the reduction in GOF is expected because the raw operational performance factors are known to be skewed distributions. Therefore, the model fit is considered acceptable recognizing it is intended to predict approximate effect sizes and directions. Table 151 shows the direct effect regression weights for each link. Table 152 shows the mean values for the KMP and OTS factors in the model. Table 153 shows the model intercepts and Table 154 shows the squared multiple correlations for the endogenous factors. 
Table 150. Model 16a - GOF Statistics

\begin{tabular}{|c|c|c|c|c|c|c|c|c|c|c|c|c|c|c|}
\hline NPAR & CMIN & DF & P & $\begin{array}{c}\text { CMIN } \\
\text { IDF }\end{array}$ & NFI & RFI & IFI & TLI & CFI & RMSEA & HI 90 & PCLOSE & AIC & HN05 \\
\hline 112 & 185.156 & 118 & .000 & 1.569 & .889 & .802 & .957 & .918 & .954 & .067 & .085 & .073 & 409.156 & 100 \\
\hline
\end{tabular}

Table 151. Model 16a-Regression Weights

\begin{tabular}{|c|c|c|c|c|c|}
\hline Link & Estimate & S.E. & C.R. & $\mathbf{P}$ & Standardized Estimate \\
\hline SOC $<--$ KMS & .161 & .048 & 3.343 & $* * *$ & .225 \\
\hline SOC $<--$ HRP & .150 & .053 & 2.817 & .005 & .199 \\
\hline SOC $<---$ IMP & .155 & .071 & 2.182 & .029 & .123 \\
\hline SOC $<--$ SOL & .455 & .068 & 6.669 & $* * *$ & .451 \\
\hline SOC $<---$ IST & .079 & .042 & 1.860 & .063 & .101 \\
\hline $\mathrm{KA}<--$ SOC & .334 & .094 & 3.566 & $* * *$ & .294 \\
\hline $\mathrm{KA}<--$ OSS & .163 & .054 & 3.027 & .002 & .208 \\
\hline$<---\quad$ HRP & .146 & .071 & 2.041 & .041 & .169 \\
\hline KA $<--$ OPM & .505 & .112 & 4.501 & $* * *$ & .318 \\
\hline$<--\quad \mathrm{KA}$ & .308 & .065 & 4.753 & $* * *$ & .319 \\
\hline$<--\quad$ SOL & .263 & .079 & 3.338 & $* *:$ & .237 \\
\hline$<--\quad$ KMS & .105 & .054 & 1.952 & .051 & .134 \\
\hline$<--\quad$ IMP & .236 & .082 & 2.895 & .004 & .171 \\
\hline$<---\quad$ HRP & .182 & .063 & 2.894 & .004 & .219 \\
\hline$<\cdots \quad \mathrm{KA}$ & .437 & .055 & 7.947 & $* * *$ & .542 \\
\hline KG $<---$ SOC & .166 & .062 & 2.660 & .008 & .181 \\
\hline$<---\quad$ KS & .185 & .061 & 3.007 & .003 & .221 \\
\hline KU $<---$ KG & .319 & .078 & 4.083 & $* * *$ & .325 \\
\hline$<--\quad$ IST & .133 & .043 & 3.110 & .002 & .191 \\
\hline KU $<---$ SOC & .145 & .078 & 1.855 & .064 & .161 \\
\hline$<---\quad$ IMP & .273 & .073 & 3.748 & $* * *$ & .241 \\
\hline KU $<---$ HRP & .103 & .053 & 1.931 & .054 & .151 \\
\hline$<-\cdots \quad$ HRP & .143 & .056 & 2.549 & .011 & .207 \\
\hline$<--\quad \mathrm{KU}$ & .168 & .077 & 2.174 & .030 & .165 \\
\hline$<--$ SOC & .183 & .078 & 2.350 & .019 & .200 \\
\hline$<--\quad$ KS & .218 & .071 & 3.087 & .002 & .262 \\
\hline$<--\quad$ TRP & .145 & .075 & 1.931 & .053 & .130 \\
\hline$<-\quad$ SOC & .146 & .072 & 2.025 & .043 & .170 \\
\hline$<---\quad \mathrm{KU}$ & .379 & .068 & 5.530 & $* * *$ & .396 \\
\hline $\begin{array}{lll}\mathrm{OE} & <-- & \mathrm{KR} \\
\end{array}$ & .227 & .073 & 3.121 & .002 & .241 \\
\hline OE $<--$ KMS & .103 & .042 & 2.445 & .014 & .168 \\
\hline 3YrUA7<-- $\quad$ OE & -.209 & .073 & -2.856 & .004 & -.250 \\
\hline 3 3YFLR <--- OE & -3.256 & 1.789 & -1.819 & .069 & -.162 \\
\hline 3YrUCF $<--3$ YrFLR & -1.089 & .088 & -12.309 & $* * *$ & -.716 \\
\hline 3 YrUCF <--- 3YrUA7 & -4.007 & 2.124 & -1.886 & .059 & -.110 \\
\hline 3 YrUCF <--- Units & -2.925 & .746 & -3.923 & *** & -.228 \\
\hline
\end{tabular}


Table 152. Model 16a - Means

\begin{tabular}{|l|c|c|c|c|}
\hline Factor & Estimate & S.E. & C.R. & P \\
\hline SOL & 4.125 & .053 & 77.262 & $* * *$ \\
\hline IMP & 4.353 & .043 & 101.735 & $* * *$ \\
\hline HRP & 3.374 & .071 & 47.367 & $* * *$ \\
\hline IST & 3.715 & .070 & 53.233 & $* * *$ \\
\hline KMS & 3.461 & .076 & 45.703 & $* * *$ \\
\hline OPM & 4.206 & .039 & 108.621 & $* * *$ \\
\hline OSS & 3.253 & .079 & 41.248 & $* * *$ \\
\hline TRP & 4.234 & .044 & 95.652 & $* * *$ \\
\hline PMP & 3.724 & .058 & 63.882 & $* * *$ \\
\hline
\end{tabular}

Table 153. Model 16a - Intercepts

\begin{tabular}{|c|c|c|c|c|}
\hline Factor & \multicolumn{1}{|c|}{ Estimate } & \multicolumn{1}{c|}{ S.E. } & \multicolumn{1}{c|}{ C.R. } & P \\
\hline 3YrFLR & 17.184 & 7.024 & 2.446 & .014 \\
\hline 3YrUA7 & 1.202 & .287 & 4.187 & $* * *$ \\
\hline Units & 2.153 & .110 & 19.497 & $* * * *$ \\
\hline 3YrUCF & 96.284 & 2.056 & 46.830 & $* * *$ \\
\hline
\end{tabular}

Table 154. Model 16a - Squared Multiple Correlations

\begin{tabular}{|c|c|}
\hline Factor & Estimate \\
\hline SOC & .668 \\
\hline KA & .532 \\
\hline $\mathrm{KS}$ & .651 \\
\hline $\mathrm{KG}$ & .696 \\
\hline $\mathrm{KU}$ & .585 \\
\hline $\mathrm{KR}$ & .613 \\
\hline OE & .650 \\
\hline Units & .000 \\
\hline 3YrFLR & .026 \\
\hline 3YrUA7 & .062 \\
\hline 3YrUCF & .584 \\
\hline
\end{tabular}

Table 155, 156, and 157 summarize the total, direct, and indirect effects between all the factors in the model and the 3 raw operational performance factors. Having the raw performance index factors in the model and using the non-standardized parameters permits the estimation of the magnitude of direct and indirect effects on operational performance parameters. For example, from Table 155, a 1 unit increase in SOC results in a $1.3 \%$ increase in UCF. 
Table 155. Total Effects - Model 16a (non-standardized values)

\begin{tabular}{|c|c|c|c|c|c|c|c|c|c|c|c|c|c|c|c|c|c|c|c|}
\hline & SOL & KMS & IMP & IST & HRP & SOC & OSS & OPM & KA & KS & KG & TRP & KU & KR & OE & Units & 3YrFLR & 3YrUA7 & \begin{tabular}{|l|} 
Total \\
\end{tabular} \\
\hline 3YrFLR & -.504 & -.516 & -.577 & -.257 & -.473 & -.968 & -.043 & -.133 & -.263 & -.241 & -.432 & -.107 & -1.357 & -.738 & -3.256 & .000 & .000 & .000 & -9.865 \\
\hline 3YrUA7 & -.032 & .033 & -.037 & -.016 & -.030 & -.062 & -.003 & -.009 & -.017 & -.015 & -.028 & -.007 & -.087 & -.047 & -.209 & .000 & .000 & .000 & -0.632 \\
\hline 3YrUCF & .678 & .695 & .777 & .346 & .636 & 1.302 & .058 & .179 & .354 & .324 & .582 & .144 & 1.825 & .993 & 4.381 & -2.925 & -1.089 & -4.007 & 13.274 \\
\hline
\end{tabular}

Table 156. Direct Effects - Model 16a (non-standardized values)

\begin{tabular}{|c|c|c|c|c|c|c|c|c|c|c|c|c|c|c|c|c|c|c|}
\hline & SOL & KMS & IMP & IST & HRP & SOC & OSS & OPM & KA & KS & KG & TRP & $\mathrm{KU}$ & $\mathrm{KR}$ & $\mathrm{OE}$ & Units & 3YrFLR & 3 YrUA7 \\
\hline 3YrFLR & .000 & .000 & .000 & .000 & .000 & .000 & .000 & .000 & .000 & .000 & .000 & .000 & .000 & .000 & -3.256 & .000 & .000 & .000 \\
\hline 3YrUA7 & .000 & .000 & .000 & .000 & .000 & .000 & .000 & .000 & .000 & .000 & .000 & .000 & .000 & .000 & -.209 & .000 & .000 & .000 \\
\hline 3YrUCF & .000 & .000 & .000 & .000 & .000 & .000 & .000 & .000 & .000 & .000 & .000 & .000 & .000 & .000 & .000 & -2.925 & -1.089 & -4.007 \\
\hline
\end{tabular}

Table 157. Indirect Effects - Model 16a (non-standardized values)

\begin{tabular}{|c|c|c|c|c|c|c|c|c|c|c|c|c|c|c|c|c|c|c|c|c|c|c|c|}
\hline & SOL & KMS & IMP & IST & HRP & SOC & OSS & OPM & KA & KS & KG & TRP & KU & KR & OE & Units & 3YrFLR & 3YrUA7 \\
\hline 3YrFLR & -.504 & -.516 & -.577 & -.257 & -.473 & -.968 & -.043 & -.133 & -.263 & -.241 & -.432 & -.107 & -1.357 & -.738 & .000 & .000 & .000 & .000 \\
\hline 3YrUA7 & -.032 & -.033 & -.037 & -.016 & -.030 & -.062 & -.003 & -.009 & -.017 & -.015 & -.028 & -.007 & -.087 & -.047 & .000 & .000 & .000 & .000 \\
\hline 3YrUCF & .678 & .695 & .777 & .346 & .636 & 1.302 & .058 & .179 & .354 & .324 & .582 & .144 & 1.825 & .993 & 4.381 & .000 & .000 & .000 \\
\hline
\end{tabular}


To further explore how effectively Model $16 \mathrm{a}$ is predicting the raw operational performance factors, Model 16b was created to test if any direct links from the KMPs or OTSs improve the predictive power or fit of the model significantly. All possible additional link combinations between individual KMPs and OTSs with each of the 3 operational performance factors (i.e. 3YrUCF, 3YrFLR, and 3YrUA7) were tested and only the following relationships were significant and were added: OSS $\rightarrow$ 3YrUCF and PMP $\rightarrow$ 3YrUA7. Figure 118 illustrates the path model that results.

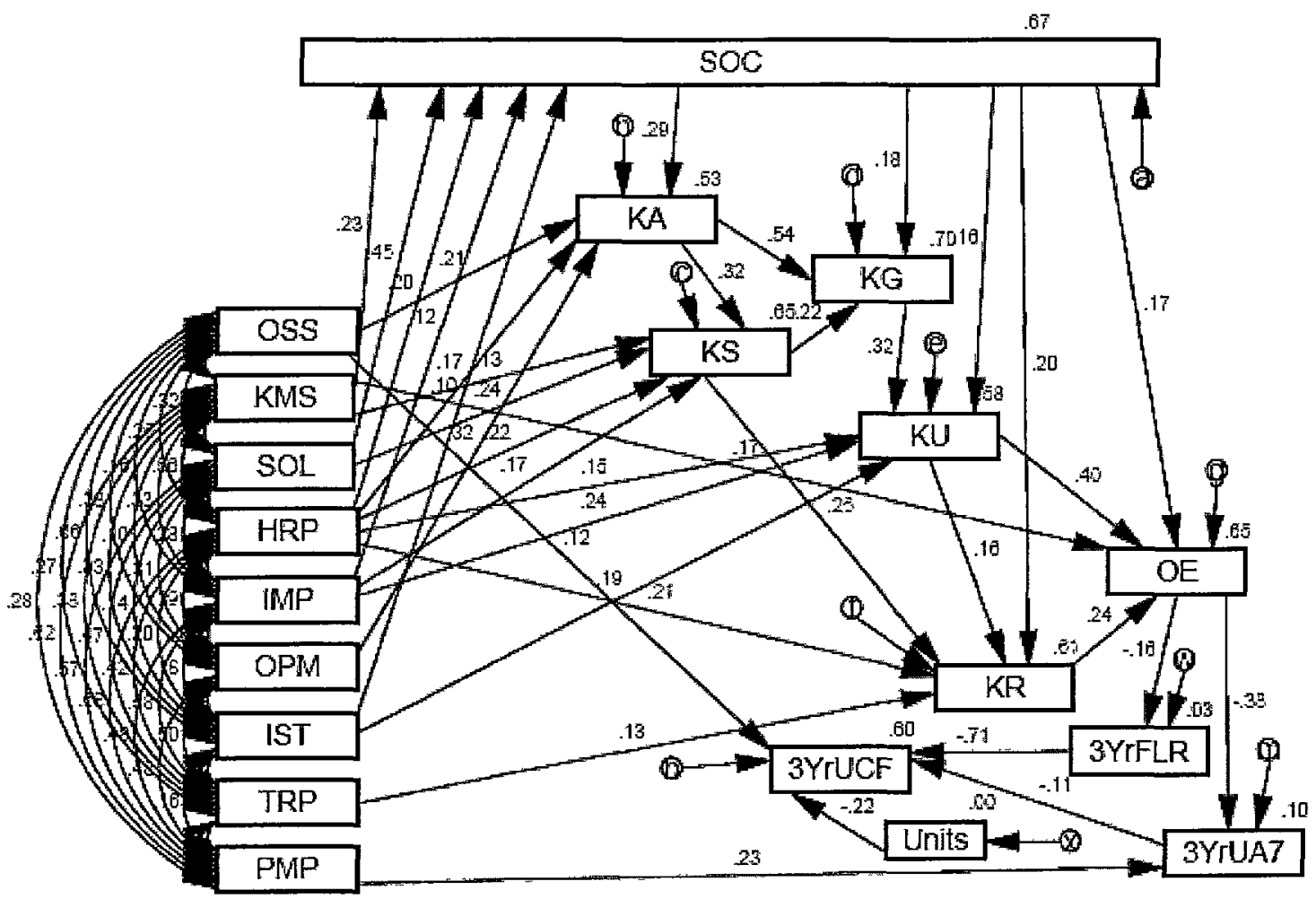

Figure 118. Path Model 16b: Direct KMP \& OTS Effects on Raw OP Measures 
Table 158 summarizes the goodness of fit statistics for Model 16b. From Table 158, we can conclude the model is an good fit according to all GOF statistics and statistical inference conclusions can be drawn from it.

Table 158. GOF Statistics for Model 16a,b

\begin{tabular}{|c|c|c|c|c|c|c|c|c|c|c|c|c|c|c|c|}
\hline Model & NPAR & CMIN & DF & $\mathbf{P}$ & $\begin{array}{c}\text { CMIN } \\
\text { /DF }\end{array}$ & NFI & RFI & IFI & TLI & CFI & RMSEA & HI 90 & PCLOSE & AIC & HN05 \\
\hline $16 a$ & 112 & 185.15 & 118 & .000 & 1.569 & .889 & .802 & .957 & .918 & .954 & .067 & .085 & .073 & 409.156 & 100 \\
\hline $16 \mathrm{~b}$ & 114 & 176.05 & 116 & .000 & 1.518 & .894 & .809 & .961 & .925 & .959 & .064 & .082 & .122 & 404.056 & 104 \\
\hline
\end{tabular}

Table 159 below summarizes the regression weights and $\mathrm{p}$-values for each relationship.

Table 159. Model 16b Regression Weights

\begin{tabular}{|c|c|c|c|c|c|c|}
\hline \multicolumn{2}{|r|}{ Link } & Estimate & S.E. & C.R. & $\mathbf{P}$ & Standardized Estimate \\
\hline SOC & $<---\quad$ KMS & .160 & .048 & 3.342 & $* * *$ & .225 \\
\hline SOC & $<---\quad$ HRP & .150 & .053 & 2.818 & .005 & .199 \\
\hline SOC & $<--\quad$ IMP & .155 & .071 & 2.183 & .029 & .123 \\
\hline SOC & $<---\quad$ SOL & .455 & .068 & 6.669 & $* * *$ & .451 \\
\hline SOC & $<---\quad$ IST & .079 & .042 & 1.868 & .062 & .102 \\
\hline $\mathrm{KA}$ & $<--$ SOC & .334 & .094 & 3.561 & $* * *$ & .293 \\
\hline $\mathrm{KA}$ & $<---\quad$ OSS & .165 & .054 & 3.059 & .002 & .210 \\
\hline $\mathrm{KA}$ & $<---$ HRP & .146 & .071 & 2.039 & .041 & .169 \\
\hline $\mathrm{KA}$ & $<---$ OPM & .504 & .112 & 4.501 & $* * *$ & .318 \\
\hline KS & $<---\quad \mathrm{KA}$ & .308 & .065 & 4.753 & ***** & .319 \\
\hline $\mathrm{KS}$ & $\begin{array}{ll}<-- & \text { SOL } \\
\end{array}$ & .263 & .079 & 3.338 & $* * *$ & .237 \\
\hline KS & $<--\quad$ KMS & .105 & .054 & 1.952 & .051 & .134 \\
\hline $\mathrm{KS}$ & $<---\quad$ IMP & .236 & .082 & 2.895 & .004 & .171 \\
\hline KS & $<-\quad$ HRP & .182 & .063 & 2.894 & .004 & .219 \\
\hline KG & $<\quad \mathrm{KA}$ & .437 & .055 & 7.946 & $* * *$ & .542 \\
\hline $\mathrm{KG}$ & $<--$ SOC & .166 & .062 & 2.660 & .008 & .181 \\
\hline KG & $<---\quad$ KS & .185 & .061 & 3.007 & .003 & .221 \\
\hline $\mathrm{KU}$ & $<---\quad \mathrm{KG}$ & .318 & .078 & 4.078 & $* * *$ & .325 \\
\hline $\mathrm{KU}$ & $<--\quad$ IST & .133 & .043 & 3.110 & .002 & .191 \\
\hline $\mathrm{KU}$ & $<-$ SOC & .145 & .078 & 1.853 & .064 & .161 \\
\hline $\mathrm{KU}$ & $<--\quad$ IMP & .273 & .073 & 3.752 & $* * *$ & .241 \\
\hline $\mathrm{KU}$ & $<---\quad$ HRP & .103 & .053 & 1.934 & .053 & .151 \\
\hline $\mathrm{KR}$ & $<--\quad$ HRP & .143 & .056 & 2.549 & .011 & .207 \\
\hline KR & $<--\quad \mathrm{KU}$ & .168 & .077 & 2.174 & .030 & .165 \\
\hline $\mathrm{KR}$ & $<-\quad$ SOC & .183 & .078 & 2.350 & .019 & .200 \\
\hline KR & $\begin{array}{ll}<-- & \text { TRP } \\
\end{array}$ & .145 & .075 & 1.932 & .053 & .130 \\
\hline KR & $<\cdots \quad$ KS & .218 & .071 & 3.088 & .002 & .262 \\
\hline $\mathrm{OE}$ & $<--$ SOC & .147 & .072 & 2.034 & .042 & .171 \\
\hline $\mathrm{OE}$ & $<--\quad \mathrm{KU}$ & .378 & .068 & 5.527 & $* * *$ & .395 \\
\hline $\mathrm{OE}$ & $<--\quad \mathrm{KR}$ & .226 & .073 & 3.119 & .002 & .241 \\
\hline $\mathrm{OE}$ & $<--\quad$ KMS & .103 & .042 & 2.447 & .014 & .168 \\
\hline 3YrUA7 & $<-\quad \mathrm{OE}$ & -.314 & .086 & -3.660 & $* * *$ & -.375 \\
\hline
\end{tabular}




\begin{tabular}{|l|c|c|c|c|c|}
\hline Link & Estimate & S.E. & C.R. & P & Standardized Estimate \\
\hline 3YrFLR $<--$ OE & -3.255 & 1.789 & -1.819 & .069 & -.162 \\
\hline 3YrUA7 <--- PMP & .151 & .068 & 2.219 & .026 & .227 \\
\hline 3YrUCF $<---$ OSS & 2.206 & 1.049 & 2.103 & .035 & .122 \\
\hline 3YrUCF $<--$ Units & -2.778 & .733 & -3.792 & $* * *$ & -.217 \\
\hline 3YrUCF $<---$ 3YrFLR & -1.077 & .087 & -12.385 & $* * *$ & -.712 \\
\hline 3YrUCF $<--$ 3YrUA7 & -3.970 & 2.088 & -1.902 & .057 & -.109 \\
\hline
\end{tabular}

Table 160 summarizes the squared multiple correlations and Table 161 summarizes the means for the model. Table 162 summarizes the intercepts for the 3 raw operational performance factors and the units factor.

Table 160. Model 16b Squared Multiple Correlations

\begin{tabular}{|c|c|}
\hline Factor & Estimate \\
\hline SOC & .668 \\
\hline $\mathrm{KA}$ & .533 \\
\hline $\mathrm{KS}$ & .651 \\
\hline $\mathrm{KG}$ & .696 \\
\hline $\mathrm{KU}$ & .585 \\
\hline $\mathrm{KR}$ & .613 \\
\hline $\mathrm{OE}$ & .650 \\
\hline 3YrFLR & .026 \\
\hline 3YrUA7 & .099 \\
\hline 3YrUCF & .596 \\
\hline
\end{tabular}

Table 161. Means for Path Model 16b

\begin{tabular}{|l|c|c|c|c|}
\hline Factor & Estimate & S.E. & C.R. & P \\
\hline SOL & 4.125 & .053 & 77.262 & $* * *$ \\
\hline IMP & 4.353 & .043 & 101.735 & $* * *$ \\
\hline HRP & 3.374 & .071 & 47.367 & $* * *$ \\
\hline IST & 3.716 & .070 & 53.236 & $* * *$ \\
\hline KMS & 3.461 & .076 & 45.703 & $* * *$ \\
\hline OPM & 4.206 & .039 & 108.621 & $* * *$ \\
\hline OSS & 3.255 & .079 & 41.368 & $* * *$ \\
\hline TRP & 4.234 & .044 & 95.652 & $* * *$ \\
\hline PMP & 3.724 & .058 & 63.882 & $* * *$ \\
\hline Units & 2.153 & .110 & 19.497 & $* * *$ \\
\hline
\end{tabular}


Table 162. Model 16b Intercepts for Operational Performance Factors

\begin{tabular}{|l|c|c|c|c|}
\hline Factor & Estimate & S.E. & C.R. & P \\
\hline 3YrFLR & 17.181 & 7.024 & 2.446 & .014 \\
\hline 3YrUCF & 87.161 & 3.956 & 22.031 & $* * *$ \\
\hline 3YrUA7 & 88.720 & 4.010 & 22.125 & $* * *$ \\
\hline Units & 2.153 & .110 & 19.497 & $* * *$ \\
\hline
\end{tabular}

Table 160 shows that almost $60 \%$ of the variance of $3 \mathrm{YrUCF}$ is explained by the model, about $10 \%$ of 3 YrUA7, and about 3\% of 3YrFLR. Table 163 to 165 show the nonstandardized total, direct and indirect effects on the raw operational performance factors. 
Table 163. Total Effects - Model 16b (non-standardized values)

\begin{tabular}{|c|c|c|c|c|c|c|c|c|c|c|c|c|c|c|c|c|c|c|c|c|c|c|c|c|}
\hline & SOL & KMS & IMP & IST & HRP & SOC & OSS & OPM & KA & KS & KG & TRP & KU & KR & OE & PMP & Units & 3YrFLR & 3YrUA7 \\
\hline 3YrFLR & -504 & -516 & -577 & -257 & -473 & -968 & -043 & -132 & -263 & -240 & -431 & -107 & -1355 & -737 & -3255 & 000 & 000 & 000 & 000 \\
\hline 3YrUA7 & -049 & -050 & -056 & -025 & -046 & -093 & -004 & -013 & -025 & -023 & -042 & -010 & 131 & 071 & -314 & 151 & 000 & 000 & 000 & 000 \\
\hline 3YrUCF & 736 & 754 & 843 & 376 & 690 & 1414 & 2270 & 193 & 384 & 351 & 630 & 156 & 1979 & 1076 & 4753 & -601 & -2778 & 1077 & -3970 \\
\hline
\end{tabular}

Table 164. Direct Effects - Model 16b (non-standardized values)

\begin{tabular}{|c|c|c|c|c|c|c|c|c|c|c|c|c|c|c|c|c|c|c|c|}
\hline & SOL & $\overline{K M S}$ & IMP & IST & HRP & $\overline{\mathrm{SOC}}$ & oss & OPM & $\mathrm{KA}$ & KS & KG & TRP & $\mathrm{KU}$ & KR & $\mathrm{OE}$ & $\overline{P M P}$ & Units & 3YrFLR & 3YrUA7 \\
\hline 3YrFLR & 000 & 000 & 000 & 000 & 000 & 000 & 000 & 000 & 000 & 000 & 000 & 000 & 000 & 000 & -3255 & 000 & 000 & 000 & 000 \\
\hline 3YrUA7 & 000 & 000 & 000 & 000 & 000 & 000 & 000 & 000 & 000 & 000 & 000 & 000 & 000 & 000 & -314 & 151 & 000 & 000 & 000 \\
\hline 3YrUCF & 000 & 000 & 000 & 000 & 000 & 000 & 2206 & 000 & 000 & 000 & 000 & 000 & 000 & 000 & 000 & 000 & -2778 & $\begin{array}{l}-1077 \\
\end{array}$ & -3970 \\
\hline
\end{tabular}

Table 165. Indirect Effects - Model 16b (non-standardized values)

\begin{tabular}{|c|c|c|c|c|c|c|c|c|c|c|c|c|c|c|c|c|c|c|c|c|c|c|c|c|c|c|c|}
\hline & SOL & KMS & IMP & IST & HRP & SOC & OSS & OPM & KA & KS & KG & TRP & KU & KR & OE & PMP & Units & 3YrFLR & 3YrUA7 \\
\hline 3YrFLR & -504 & -516 & -577 & -257 & -473 & -968 & -043 & -132 & -263 & -240 & -431 & -107 & -1355 & -737 & 000 & 000 & 000 & 000 & 000 \\
\hline 3YrUA7 & -049 & -050 & -056 & -025 & -046 & -093 & -004 & -013 & -025 & -023 & -042 & -010 & -131 & -071 & 000 & 000 & 000 & 000 & 000 \\
\hline 3YrUCF & 736 & 754 & 843 & 376 & 690 & 1414 & 063 & 193 & 384 & 351 & 630 & 156 & 1979 & 1076 & 4753 & -601 & 000 & 000 & 000 \\
\hline
\end{tabular}




\section{Discussion and Research Implications}

This chapter summarizes the major research findings and discusses the implications of this study. It also highlights the original contributions made to both theory and practice.

\subsection{Introduction}

The objective of this study was to investigate the role of KM in NPPs. This research explored several basic questions of interest to NPP managers such as:

- To what extent are NPPs site organizations implementing KM practices today?

- By what methods or practices can management influence knowledge processes and organizational learning to improve organizational effectiveness and operational performance?

- What effect does management support for KM practices have on NPP organizational effectiveness?

- What effect does management support for these KM practices have on plant operational performance?

Based on a review of the literature, a theoretical model was developed. A number of hypotheses were proposed relating management support for KM practices to the quality of knowledge processes and subsequently to organizational performance in NPPs. The moderating effect of a supportive organizational culture was also hypothesized. Based 
on the academic and practitioner literature, measures for all of the variables were developed and their reliability and validity was established through empirical study. Statistical analysis of the data was performed and the findings presented. A summary and interpretation of the findings follows.

\subsection{Summary of Findings}

The sampling frame was the entire global population of 204 nuclear power station organizations. A sample size of 124 respondents (60\%) was achieved and was an excellent cross-section of the total population. Prior to the analysis, data preparation and screening included coding verification, missing data analysis, removal of outliers, and checks for non-linearity. A combination of standard statistical methods were used in the data analysis including construct reliability analysis, standard multivariate regression and path analysis. Independent samples $\mathrm{T}$-tests and multi-group path analysis model techniques were also used to complement specific aspects of the analysis.

In general, the findings from the path analysis assessment are consistent with the initial simple regression analysis with the following noted differences: the additional link KMS $\rightarrow$ KS was found to be significant in the path model, and the links OPM $\rightarrow$ KG, $\mathrm{KMS} \rightarrow \mathrm{KG}, \mathrm{SOC} \rightarrow \mathrm{KS}, \mathrm{PMP} \rightarrow \mathrm{OE}, \mathrm{IMP} \rightarrow \mathrm{OE}$, and IST $\rightarrow \mathrm{OE}$ were not found to be significant in the full model. This was expected in that the model has shown that the quality of knowledge processes is the intermediary mechanism by which the KMPs impact organizational effectiveness. 
Table 166 provides a summary of the findings against each of the hypotheses.

Table 166. Summary of Findings by Hypothesis

\begin{tabular}{|c|c|c|c|c|c|}
\hline \multirow{2}{*}{ Hypothesis } & \multirow{2}{*}{$\begin{array}{c}\text { Hypothesized } \\
\text { Direction } \\
(+/-)\end{array}$} & \multirow{2}{*}{ Conclusion } & \multicolumn{3}{|c|}{$\begin{array}{c}\text { Standardized Estimate } \\
\text { (Effects) }\end{array}$} \\
\hline & & & Direct & Indirect & Total \\
\hline \multicolumn{6}{|l|}{ Model 1: } \\
\hline$K M P \rightarrow S O C$ & + & Supported & .715 & .000 & .715 \\
\hline$S O C \rightarrow Q K P$ & + & Supported & .410 & .000 & .410 \\
\hline$S O C \rightarrow O E$ & + & Supported & .314 & .200 & .514 \\
\hline$H 4 a: \quad K M P \rightarrow Q K P$ & + & Supported & .486 & .293 & .779 \\
\hline$H 4 b: \quad K M P \rightarrow S O C \rightarrow Q K P$ & + & Supported & $n / a$ & .293 & $n / a$ \\
\hline H5a: $\quad K M P \rightarrow Q K P \rightarrow O E$ & + & Supported & $n / a$ & .240 & $n / a$ \\
\hline$H 5 b: \quad K M P \rightarrow S O C \rightarrow O E$ & + & Supported & $n / a$ & .223 & $n / a$ \\
\hline \multicolumn{6}{|l|}{ Model 2: } \\
\hline$H 6 a: \quad O T S \rightarrow Q K P$ & + & Supported & .196 & .000 & .196 \\
\hline \multicolumn{6}{|l|}{ Model 3: } \\
\hline$H 6 b: \quad I S T \rightarrow Q K P$ & + & Supported & .320 & .000 & .320 \\
\hline \multicolumn{6}{|l|}{ Model 4: } \\
\hline$H \sigma c: \quad O S S \rightarrow Q K P$ & + & Supported & .421 & .000 & .421 \\
\hline \multicolumn{6}{|l|}{ Model 2: } \\
\hline$H 7 a: \quad O T S \rightarrow Q K P \rightarrow O E$ & + & Supported & $n / a$ & .142 & $n / a$ \\
\hline \multicolumn{6}{|l|}{ Model 3: } \\
\hline$H 7 b: \quad I S T \rightarrow Q K P \rightarrow O E$ & + & Supported & $n / a$ & .232 & $n / a$ \\
\hline \multicolumn{6}{|l|}{ Model 4: } \\
\hline$H 7 c: \quad O S S \rightarrow Q K P \rightarrow O E$ & + & Supported & $n / a$ & .305 & $n / a$ \\
\hline \multicolumn{6}{|l|}{ Model 1: } \\
\hline$H 8: \quad Q K P \rightarrow O E$ & + & Supported & .489 & .000 & .489 \\
\hline$H 9: \quad O E \rightarrow O P$ & + & Supported & .248 & .000 & .248 \\
\hline \multicolumn{6}{|l|}{ Model 5\&6: } \\
\hline H10al: Units $\left.\rightarrow O P\right|_{\text {type }}$ & $t b d$ & $\begin{array}{r}\text { Supported } \\
P W R \\
B W R \\
\end{array}$ & $\begin{array}{l}-.278 \\
-.237\end{array}$ & $\begin{array}{l}.000 \\
.000\end{array}$ & $\begin{array}{l}-.278 \\
-.237\end{array}$ \\
\hline H10b1: $\quad$ Size $\left.\rightarrow O P\right|_{\text {tvpe }}$ & $t b d$ & Not Supported & .000 & .000 & .000 \\
\hline HlOcl: Age $\left.\rightarrow O P\right|_{\text {type }}$ & $t b d$ & $\begin{array}{r}\text { Indirect Only } \\
P W R \\
B W R \\
\end{array}$ & $\begin{array}{l}.000 \\
.000\end{array}$ & $\begin{array}{l}-.065 \\
-.036\end{array}$ & $\begin{array}{l}-.065 \\
-.036\end{array}$ \\
\hline HlOa2: Units $\left.\rightarrow O E\right|_{\text {type }}$ & $t b d$ & Not Supported & .000 & .000 & .000 \\
\hline H10b2: $\quad$ Size $\left.\rightarrow O E\right|_{\text {type }}$ & $t b d$ & Not Supported & .000 & .000 & .000 \\
\hline$H 10 c 2:$ Age $\left.\rightarrow O E\right|_{\text {type }}$ & $t b d$ & $\begin{array}{r}\text { Supported } \\
P W R \\
B W R \\
\end{array}$ & $\begin{array}{l}-.248 \\
-.204\end{array}$ & $\begin{array}{l}.000 \\
.000\end{array}$ & $\begin{array}{l}-.248 \\
-.204\end{array}$ \\
\hline HIOd: $\left.O E \rightarrow O P\right|_{\text {type }}$ & $t b d$ & $\begin{array}{r}\text { Supported } \\
P W R \\
B W R \\
\end{array}$ & $\begin{array}{l}.256 \\
.182\end{array}$ & $\begin{array}{l}.000 \\
.000\end{array}$ & $\begin{array}{l}.256 \\
.182\end{array}$ \\
\hline \multicolumn{6}{|l|}{ Model 12: } \\
\hline H11a: $\quad K A \rightarrow K R$ & + & Indirect Only & .000 & .117 & .117 \\
\hline H11b: $\quad K G \rightarrow K R$ & + & Indirect Only & .000 & .054 & .054 \\
\hline H12: $\quad K R \rightarrow K S$ & + & Not Supported & .000 & .000 & .000 \\
\hline
\end{tabular}




\begin{tabular}{|c|c|c|c|c|c|}
\hline \multirow[t]{2}{*}{ Hypothesis } & \multirow{2}{*}{$\begin{array}{c}\text { Hypothesized } \\
\text { Direction } \\
(+/-)\end{array}$} & \multirow{2}{*}{ Conclusion } & \multicolumn{3}{|c|}{$\begin{array}{c}\text { Standardized Estimate } \\
\text { (Effects) }\end{array}$} \\
\hline & & & Direct & Indirect & Total \\
\hline$K S \rightarrow K U$ & + & Indirect Only & .000 & .072 & .072 \\
\hline$K U \rightarrow K A$ & + & Not Supported & .000 & .000 & .000 \\
\hline$K U \rightarrow O E$ & + & Supported & .394 & .039 & .434 \\
\hline H16: $\quad K R \rightarrow O E$ & + & Supported & .239 & .000 & .239 \\
\hline H17a: $\quad K M S \rightarrow K S$ & + & Supported $* *$ & .134 & .021 & .156 \\
\hline$H 17 b: \quad K M S \rightarrow K A$ & + & Indirect Only & .000 & .066 & .066 \\
\hline H18a: $\quad S O L \rightarrow K A$ & + & Indirect Only & .000 & .132 & .132 \\
\hline$H 18 b: \quad S O L \rightarrow K G$ & + & Indirect Only & .000 & 215 & .215 \\
\hline H18c: $\quad S O L \rightarrow K R$ & + & Indirect Only & .000 & .187 & .187 \\
\hline H18d: $\quad S O L \rightarrow K S$ & + & Supported & .237 & .042 & .280 \\
\hline H19a: $\quad H R P \rightarrow K A$ & + & Supported & .169 & .058 & .228 \\
\hline H19b: $\quad H R P \rightarrow K S$ & + & Supported & .219 & .073 & .292 \\
\hline$H 19 c: \quad H R P \rightarrow K U$ & + & Supported $* *$ & .151 & .105 & .256 \\
\hline H19d: $\quad H R P \rightarrow K R$ & + & Supported & .207 & .158 & .365 \\
\hline H2Oa: $\quad T R P \rightarrow K R$ & + & Supported ${ }^{* *}$ & .130 & .000 & .130 \\
\hline$H 2 O b: \quad T R P \rightarrow K S$ & + & Not Supported & .000 & .000 & .000 \\
\hline$H 20 c: \quad T R P \rightarrow K U$ & + & Not Supported & .000 & .000 & .000 \\
\hline H2la: $\quad I M P \rightarrow K S$ & + & Supported & .171 & .012 & .182 \\
\hline$H 21 b: \quad I M P \rightarrow K R$ & + & Indirect Only & .000 & .120 & .120 \\
\hline$H 21 c: \quad I M P \rightarrow K G$ & + & Not Supported & .000 & .082 & .082 \\
\hline H2ld: $\quad I M P \rightarrow K U$ & + & Supported & .241 & .047 & .287 \\
\hline H22a: $\quad I S T \rightarrow K U$ & + & Supported & .191 & .028 & .219 \\
\hline$H 22 b: \quad I S T \rightarrow K R$ & + & Indirect Only & .000 & .059 & .059 \\
\hline$H 22 c: \quad I S T \rightarrow K S$ & + & Indirect Only & .000 & .010 & .010 \\
\hline H23a: $\quad O S S \rightarrow K U$ & + & Indirect Only & .000 & .041 & .041 \\
\hline$H 23 b: \quad O S S \rightarrow K G$ & + & Indirect Only & .000 & .127 & .127 \\
\hline H24a: $\quad P M P \rightarrow K R$ & + & Not Supported & .000 & .000 & .000 \\
\hline$H 24 b: \quad P M P \rightarrow K U$ & + & Not Supported & .000 & .000 & .000 \\
\hline H25a: $\quad O P M \rightarrow K R$ & + & Indirect Only & .000 & .037 & .037 \\
\hline$H 25 b: \quad O P M \rightarrow K U$ & + & Indirect Only & .000 & .063 & .063 \\
\hline H26a: $\quad P M P \rightarrow O E$ & + & Not Supported & .000 & .000 & .000 \\
\hline$H 26 b: \quad P M P \rightarrow O P$ & + & Not Supported & .000 & .000 & .000 \\
\hline H27a: $O P M \rightarrow O E$ & + & Indirect Only & .000 & .034 & .034 \\
\hline$O P M \rightarrow O P$ & + & Indirect Only & .000 & .008 & .008 \\
\hline H28a: $\quad K M S \rightarrow S O C$ & + & Supported & .225 & .000 & .225 \\
\hline$H 28 b: \quad S O L \rightarrow S O C$ & + & Supported & .451 & .000 & .451 \\
\hline$H 28 c: \quad I M P \rightarrow S O C$ & + & Supported & .123 & .000 & .123 \\
\hline H28d: $\quad T R P \rightarrow S O C$ & + & Not Supported & .000 & .000 & .000 \\
\hline$H 28 e: \quad H R P \rightarrow S O C$ & + & Supported & .199 & .000 & .199 \\
\hline H29a: $\quad S O C \rightarrow K A$ & + & Supported & .294 & .000 & .294 \\
\hline$H 29 b: \quad S O C \rightarrow K G$ & + & Supported & .181 & .180 & .361 \\
\hline$H 29 c: \quad S O C \rightarrow K R$ & + & Supported & .200 & .070 & .270 \\
\hline H29d: $\quad S O C \rightarrow K S$ & + & Indirect Only & .000 & .094 & .094 \\
\hline$H 29 e: \quad S O C \rightarrow K U$ & + & Supported $* *$ & .161 & .117 & .278 \\
\hline
\end{tabular}

Note: All estimates are significant at $\mathrm{p}<=0.05$ unless noted by with double asterisk $(* *)$.

** relationship was slightly higher than 0.05 cut-off but link was not removed as it is expected and supported in theory. 
The following sections provide an interpretation of these results in the broader context of the research and draw conclusions and recommendations for management.

\subsection{Contribution of the Research}

The research represents the first study of its kind by providing an integrated $\mathrm{KM}$ and performance model for NPP site organizations. It also represents the first of its kind in terms of proposing and validating a causal model framework that explains the interaction between knowledge management practices and the quality of knowledge processes in the context of NPP organizations. Although further research is required, it is reasonable to speculate the findings may possibly be applicable to other similar knowledge-driven and technology-dependent production-oriented organizations in regulated industries where high consequence hazards must be managed to low tolerances for risk, such as the commercial airline industry or perhaps pharmaceutical manufacturing.

No prior empirical studies could be found in the literature that directly explore these relationships collectively as a framework. In the context of the nuclear power production industry, Lowthert (1996) reported that almost no empirical research on the topic of KM could be found, and little progress appears to have been made in the interim. The issue of KM practices and their links to organizational performance has not been previously researched and had not been well understood. No prior empirical research appears to have been done linking $\mathrm{KM}$ practices to organizational effectiveness 
as a framework for organizational learning (and performance), let alone in the specific context of NPPs. Further, very limited empirical research has been published on the determinants of NPP performance. In summary, after an extensive literature search, no similar theoretical framework nor formal empirical research appears to have been conducted specifically on the links between KM practices and quality of knowledge processes, nor on the moderating role of a supportive organizational culture, nor on the collective influence of these factors on organizational effectiveness and firm performance.

NPPs are difficult organizations for researchers to obtain access to. Despite the obvious interest from industry and need for NPP managers to understand and be able to influence and improve knowledge processes and organizational learning in their organizations, a consistent theoretical basis and meaningful guidance for practitioners on the subject had been lacking. This research provides significant insights for both. This research advances understanding of the application and benefit of KM in NPPs in several ways:

- It provides a better understanding of $\mathrm{KM}$ methods and practices currently being applied in NPPs. There had been little or no industry wide empirical research on this topic to date.

- It provides a better understanding of knowledge processes in NPPs. Specifically the links between knowledge acquisition, sharing, generation, utilization, and retention, and their links (direct and indirect) to organizational effectiveness. 
- It provides a better understanding of the impact of information systems, information technology, and advanced operational support systems technology on the quality of knowledge processes and organizational effectiveness in NPPs.

- It provides a better understanding of the importance and influence of a supportive organizational culture on the quality of knowledge processes, organizational effectiveness and ultimately organizational performance.

- It provides a better understanding of the impact that $\mathrm{KM}$ practices have on supportive organizational culture.

- It provides a better understanding of the impact of key physical plant characteristics on NPP organizational effectiveness and operational performance: namely reactor age, number of units at site, reactor type, and reactor size.

- The measurement instruments developed for this research constitute a reliable set of construct measures that provide a basis for future research and will support the ongoing assessment of KM practices, knowledge processes, and NPP performance by the industry. The IAEA plans to repeat the survey every 3-5 years and longitudinal data will provide an excellent basis for industry benchmarking.

- The study data also provides a useful basis for future benchmarking at the NPP organizational level for self-assessment and improvement. Currently no other equivalent industry tools or data exist. 
- The study provides an integrated theory and framework to explain the relationships between knowledge management practices, quality of knowledge processes, and organizational performance. The model is validated with empirical data and provides a quantitative tool to understand and predict the cause and effect relationships.

- Finally, the research has helped to identify several key issues and questions in need of further research.

In summary, this research has provided previously unavailable insights into the various KM practices in NPPs today and the relative benefits obtained by their implementation. The findings can help management understand how to benefit from implementing KM practices and justify strategic investment in KM. The insights obtained from this research will help managers to better understand how and why knowledge management practices are effective at improving organizational effectiveness, and by what mechanisms this occurs. In summary, the research provides much-needed insights and guidance to help NPP managers maximize the benefit from KM initiatives in future. A more detailed discussion and interpretation of specific findings follows.

\subsection{Relationship Between KMPs and QKPS}

A major finding of the study is that overall support for knowledge management practices positively impacts the quality of knowledge processes. Also higher levels of organizational technology support (OTS), or individually information systems and technology support (IST) or advanced operational support systems (OSS), positively 
influence overall quality of knowledge processes (QKP). The detailed findings of the study attribute these effects to the following sets of relationships between individual KMPs and the QKPs:

- Advanced operational support systems, human resource related practices, and operational performance management practices have a positive direct influence on the quality of knowledge acquisition processes.

- Knowledge management strategy and planning, support for organizational learning, human resource related practices, and information management practices all have a positive and direct impact on the quality of knowledge sharing and transfer processes.

- Human resource related practices, information management practices, and information systems and technology all have a positive and direct impact on the quality of knowledge utilization and application processes.

- Human resource related practices, organizational performance management practices, and training related practices have a positive and direct impact on the quality of knowledge retention and storage processes.

However, despite being predicted by theory and supported in the literature, the following expected relationships that were hypothesized were not supported by the research findings:

- Knowledge management strategy and planning was not found to have a direct impact on knowledge acquisition and adoption, knowledge generation and 
validation, knowledge utilization and application, or knowledge retention and storage.

- Support for organizational learning practices was not found to have a direct impact on knowledge acquisition and adoption, knowledge generation and validation, or knowledge retention and storage.

- Support for training related practices was not found to have a direct impact on quality of knowledge utilization and application, or quality of knowledge sharing and transfer processes.

- Support for information management practices was not found to have a direct impact on knowledge generation and validation, or knowledge retention and storage.

- Extent of use of information systems and technology was not found to have a direct impact on knowledge generation and validation, knowledge retention and storage, or knowledge sharing and transfer.

- Extent of use of advanced operational support systems was not found to have a direct impact on knowledge generation and validation, or knowledge utilization and application.

- Support for process management practices was not found to have a direct impact on knowledge retention and storage, or knowledge sharing and transfer, or knowledge utilization and application. 
- Support for organizational performance management practices was not found to have a direct impact on knowledge retention and storage or knowledge utilization and application.

These relationships could be investigated further in future research.

\subsection{Relationship Between KMPs and SOC}

An important finding of the study supporting hypothesis 1 is that higher levels of overall support for KM practices (KMP) positively influence the supportive organizational culture (SOC). The detailed findings of the study attribute this effect to the following KM practices: support for knowledge strategy and planning, support for organizational learning, human resource related practices, information process management practices, and extent of use of information systems and technology. Although training related practices was hypothesized to play a role it was not supported by the data. Instead, it was found that the extent of use of information systems and technology plays a direct role.

\subsection{Inter-relationships Amongst the QKPs}

Another finding of the study is that higher quality knowledge acquisition and adoption (KA) processes will have a positive influence on both the quality of knowledge generation and validation (KG) processes and the quality of knowledge sharing and transfer (KS) processes. However, the findings did not support a link between quality of 
knowledge acquisition processes and quality of knowledge retention and storage (KR) processes.

A conclusion of the study is that higher quality knowledge generation and validation (KG) processes have a positive influence on quality of knowledge utilization and application (KU) processes. However, the expected relationship between quality of knowledge generation and validation processes $(\mathrm{KG})$ and quality of knowledge retention and storage (KR) processes was not supported by the data. Only an indirect link KG $\rightarrow$ $\mathrm{KU} \rightarrow \mathrm{KR}$ was supported.

A conclusion of the study is that higher quality of knowledge sharing and transfer (KS) processes has a positive influence on both quality of knowledge retention and storage (KR) processes and on quality of knowledge generation and storage (KG) processes. However, the expected relationship between quality of knowledge sharing and transfer (KS) on quality of knowledge utilization and application (KU) processes was not supported in the data. Only an indirect link $\mathrm{KS} \rightarrow \mathrm{KG} \rightarrow \mathrm{KU}$ was supported.

A conclusion of the study is that higher quality of knowledge utilization and application (KU) processes has have a positive influence on quality of knowledge retention and storage (KR) processes. However, the data also supports a competing theory that the causal relationship between these constructs is in the reverse direction. It is likely that this is two-way and complementary relationship. Further research is needed to clarify this. 
Finally, a conclusion of the study is that knowledge retention and storage (KR) did not directly influence knowledge sharing and transfer processes (KS), nor any other quality knowledge process.

\subsection{Influence of QKPs on Organizational Effectiveness}

The study findings support hypothesis 8 that higher overall quality of knowledge processes $(\mathrm{QKP})$ positively influences organizational effectiveness (OE). Furthermore, the findings support hypothesis 15 and 16 , that higher quality knowledge utilization and application (KU) processes and higher quality knowledge retention and storage (KR) processes both have a direct and positive influence on organizational effectiveness (OE).

\subsection{Effect of KMPs on Organizational Performance}

The study findings clearly support hypothesis 5 , that higher levels of overall support for KM practices (KMP), through the intermediate effects of both overall quality of knowledge processes (QKP) and supportive organizational culture (SOC) positively influence organizational effectiveness (OE). An unexpected finding was the direct link between knowledge management strategy and planning (KMS) and organizational effectiveness (OE). The study findings did not support hypothesis 26 or 27, that higher support for process management practices (PMP) or organizational performance management (OPM) practices will have a positive influence on organizational effectiveness (OE) and operational performance (OP). However, from Path Model 16b, which investigated direct links from KMPs to the raw operational performance factors, 
two findings were observed: that support for operational support systems (OSS) has a positive and direct influence on unit capability factor (UCF); and that support for process management practices has a positive and direct influence on the unplanned automatic scram rate (UA7). This is likely explained by NPPs with higher support for process management practices likely operate more conservatively with respect to safety and follow "work-to-rule" practices more strictly. Thus it would be expected that operating margins would be set more conservatively and more strictly enforced, resulting in a higher unplanned automatic scram rates on average.

\subsection{Supportive Organizational Culture as Moderator}

The data analysis supports hypothesis 2 , that higher levels of supportive organizational culture (SOC) positively influence the overall quality of knowledge processes (QKP). More specifically, the study findings support Hypothesis 29 , and show that higher levels of supportive organizational culture (SOC) have a positive direct influence on quality of knowledge acquisition (KA), quality of knowledge generation and validation (KG), quality of knowledge retention and storage (KG), and quality of knowledge utilization and application (KU). The study findings did not support the hypothesis that supportive organizational culture directly positively influences quality of knowledge sharing and transfer (KS), however, they do show that there is an indirect and positive effect on KS through the quality of knowledge acquisition (i.e. SOC $\rightarrow \mathrm{KA} \rightarrow \mathrm{KS}$ ).

In addition, the study findings support hypothesis 3, that higher levels of supportive organizational culture (SOC) will positively influence organizational effectiveness 
(OE), and hypothesis $4 \mathrm{~b}$, that higher levels of overall support for KM practices (KMP) will positively influence the overall quality of knowledge processes (QKP) indirectly through the effect of supportive organizational culture (SOC) (i.e., KMP $\rightarrow \mathrm{SOC} \rightarrow$ QKP). The later indirect effect of SOC is as an intermediate and moderating variable.

Finally, Path Models $15 \mathrm{a}$ and $15 \mathrm{~b}$ helped to establish that supportive organizational culture (SOC) will positively influence (moderate) the strength of the relationship between the KMPs $\rightarrow$ QKP and in turn, again will positively influence (moderate) the strength of the relationship between QKPs and OE. This too is a pronounced effect and it can be concluded that SOC moderates (by increasing) the effect of QKP $\rightarrow$ KMP and the effect of KMP $\rightarrow \mathrm{OE}$ (at the same time).

\subsection{Findings related to Organizational Performance}

The study strongly confirmed the hypotheses 15 and 16 that stated quality of knowledge utilization and application (KU) processes and quality of knowledge retention and storage (KR) processes both directly affect organizational effectiveness. The study findings clearly supported hypothesis 9 , that higher organizational effectiveness (OE) positively influences operational performance (OP). The study findings also support hypotheses 10a1 and 10c2: that plant age influences organizational effectiveness (OE) and that the number of operating units at a site influences operational performance (OP) respectively. In both cases the effect was found to be negative. The study findings did not support hypothesis $10 \mathrm{a} 2$ that plant age influences operational performance (OP), or hypothesis $10 \mathrm{c} 1$ that the number of operating units at a site influences organizational 
effectiveness (OE). Finally, the study findings did not support hypothesis $10 \mathrm{~b} 1$ or $10 \mathrm{~b} 2$ that plant size (i.e. MWe output) influences operational performance (OP). It can be concluded it has no effect.

The finding that plant age negatively influences organizational effectiveness may be explained by a number of possible causes. For example, equipment and/or technology obsolescence may have a significant impact on the organizations ability to maintain the plant equipment efficiently and effectively. For example, skills availability in older analog or digital electronics is a challenge. As well supplier technical support and availability of qualified parts may be a problem in older plants. Further research is recommended. The finding that plant age does not influence operational performance is not surprising as the effects of plant aging across the global fleet would be expected to be offset by increased operational experience and resulting improvements in maintenance and operating practices, as well as ongoing equipment upgrades and design improvements. Further research is recommended to better understand these effects.

The finding that the number of units negatively influences operational performance needs further investigation. Most multi-unit plants have a large number of shared auxiliary and support systems supporting multiple units. It is possible that in many of these designs there is an increased vulnerability to equipment failures and maintenance outage due to single points of failure. Equipment failure frequencies of common equipment may also be a factor. The finding that the number of units has no significant effect on organizational effectiveness is thought to be the combination of two opposing 
effects: there is a larger pool of expertise at stations with more units which results in an expanded organizational knowledge base, however, there is also a tendency to achieve economies of scale by reduced staffing per unit and sharing resources across all units. Further research is recommended to better understand these effects.

\subsection{Unexpected Findings}

There were a number of unexpected findings in this study. Table 167 summarizes the significant relationships found in the data but not expected (i.e. not hypothesized).

Table 167. Relationships Found But Not Hypothesized

\begin{tabular}{|c|c|c|c|c|c|}
\hline $\begin{array}{c}\text { Significant Relationships Found } \\
\text { But Not Hypothesized } \\
(\text { Model 12) }\end{array}$ & \multirow{2}{*}{$\begin{array}{c}\text { Direction } \\
(+l-)\end{array}$} & Conclusion & \multicolumn{3}{|c|}{$\begin{array}{c}\text { Standardized Estimate } \\
\text { (Effects) }\end{array}$} \\
\cline { 4 - 6 } & & & Direct & Indirect & Total \\
\hline$K A \rightarrow K G$ & + & Supported & .542 & .071 & .613 \\
\hline$K A \rightarrow K S$ & + & Supported & .319 & .000 & .319 \\
\hline$K G \rightarrow K U$ & + & Supported & .325 & .000 & .325 \\
\hline$K S \rightarrow K R$ & + & Supported & .262 & .012 & .274 \\
\hline$K S \rightarrow K G$ & + & Supported & .221 & .000 & .221 \\
\hline$K U \rightarrow K R$ & + & Supported & .165 & .000 & .309 \\
\hline$I S T \rightarrow S O C$ & + & Supported & .101 & .000 & .101 \\
\hline$O S S \rightarrow K A$ & + & Supported & .208 & .000 & .208 \\
\hline$O P M \rightarrow K A$ & + & Supported & .318 & .000 & .318 \\
\hline$K M S \rightarrow O E$ & + & Supported & .168 & .091 & .259 \\
\hline
\end{tabular}

Note: All estimates are significant at $\mathrm{p}<=0.05$ except as noted by the asterisk $\left(^{*}\right)$. The $\mathrm{p}$-value of .061 for this relationship was slightly higher than 0.05 cut-off but the path link was not removed from the model as it was supported in theory.

The first 6 links in Table 167 define the core interactions amongst the quality of knowledge processes in the fitted model (see Path Model 12, Figure 110). These are a clearly dominant set of inter-relationships in the data and represent a significant finding of the study. In effect, this is the mechanism by which KMPs, together with SOC, influence organizational effectiveness. The pattern of flow between the QKP links is somewhat intuitive: quality of knowledge acquisition and adoption (KA) processes 
positively influence both quality of knowledge sharing and transfer (KS) processes and quality of knowledge generation and validation (KG) processes. The quality of knowledge generation and validation $(\mathrm{KG})$ processes in turn positively influence the quality of knowledge utilization and application (KU) processes, which in turn positively impacts on quality of knowledge retention and storage (KR) processes. It is also very likely that quality of knowledge retention and storage (KR) processes positively impacts on quality of knowledge utilization and application (KU) processes (i.e. in a 2-way relationship). In parallel, the quality of knowledge sharing and transfer (KS) processes positively impact quality of knowledge retention and storage (KR) processes. Finally, both the quality of knowledge utilization and application (KU) processes and the quality of knowledge retention and storage (KR) processes load positively on organizational effectiveness (OE). Figure 119 illustrates the "core NPP knowledge process model" that results. All relationships are significant positive effects.

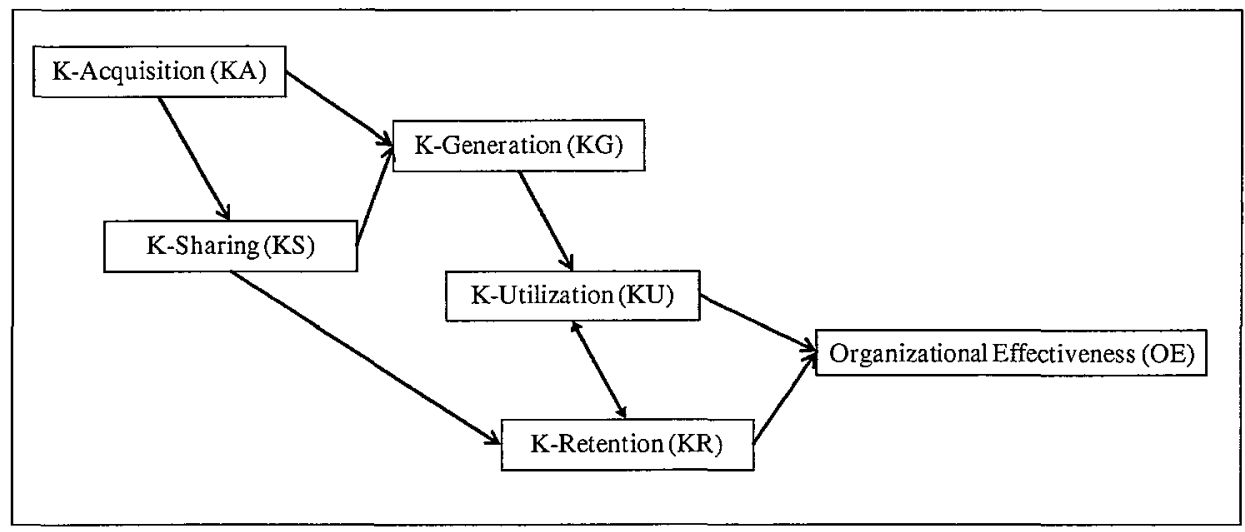

Figure 119. Core NPP Knowledge Process Model

Although the specific link IST $\rightarrow$ SOC was not originally hypothesized, it was found to be close to significant ( $\mathrm{p}=.063$ ) in the data (see Path Model 12) and was retained. 
Several authors report that increased effective usage of IST strengthens organizational culture by enhancing employee communications and improving collaborative teamwork through mechanisms like corporate Intranets, enterprise and collaboration software, and e-mail (Huber and Daft, 1987; Pinto and Pinto, 1990; Johannessen et al., 1999).

Another unexpected finding was that process management practices (PMP) were positively loading on the raw operational performance factor for the 3-year unplanned actuations (i.e. 3YrUA7, see Model 16b). This could be explained by the expectation that NPPs with a high level of support for process management practices would have a stronger organizational awareness and managerial focus (i.e. a heavier emphasis) on compliance to procedures. It is possible this may promote more conservative decision making with respect to safety. Thus at NPPs with higher support for PMP it would be expected that safety margins be established more conservatively and more strictly enforced. This may account for higher "scram" rates in that safety trip thresholds would be set correspondingly lower and more frequent (and conservative) scram events occurring. Further research is required to verify this.

The relationship OSS $\rightarrow$ KA was unexpected as the usage of OSS systems is primarily for internal information processing, and the primary benefits were expected to be in terms of knowledge generation, retention, and utilization. A review of the measures associated with OSS and KA constructs and feedback from industry experts resulted in the following proposed explanation. Advanced OSS technology is in general a relatively new phenomena in NPPs over the past decade and the industry is clearly in 
the process of technology adoption with some stations being early adopters and many others lagging. In general the process of acquiring and implementing OSS technology solutions is considered a major knowledge acquisition and adoption process itself, and therefore it is possible that NPPs with a high level of effective OSS usage would also be likely to report higher levels of quality of knowledge acquisition and adoption processes. Further research is required to test these propositions.

A significant and unexpected link was found between OPM $\rightarrow$ KA. In hindsight this should likely have been anticipated and hypothesized. The implementation and focus of OPM programs is fairly consistent across the industry with an emphasis on the adoption of industry best practices and lessons learned from other NPPs. The measures for OPM focus on external peer reviews, self assessment and benchmarking against external best practices, and operational experience reviews (which would include events from other stations). In hindsight, these aspects of OPM can be considered a form knowledge acquisition and adoption activity and stations with higher levels of support for OPM practices would be expected to report higher quality of knowledge acquisition and adoption processes.

Another unexpected finding was that the direction of the link from quality of knowledge retention and storage (KR) to quality of knowledge utilization and application (KU) was equally supported in the data (see Path Models 12 with KU as causal versus Path Model 13 with $\mathrm{KR}$ as causal). As discussed earlier, it is likely that this link is a two-way relationship. Theory supporting a relationship in either direction can be found in the 
literature. It is expected that further research will likely confirm this as a two-way interaction.

The hypotheses that were not supported in the findings (see Table 155) may be explained by one of the following possibilities:

- The relationship truly does not exist in the sample population and thus cannot be supported.

- The relationship exists but due to the small sample size and/or the weakness of the effect, the relationship cannot be established as significant.

- Due to a weakness in the measurement model, the dimensions of the constructs being measured were not able to capture the presence of the relationship adequately.

For example, the link between supportive organizational culture (SOC) and quality of knowledge sharing and transfer was expected but not found. It is possible that one or two of the possible explanations above may apply. 


\section{Conclusions}

It is widely claimed in the literature that $\mathrm{KM}$ practices are an important factor in achieving overall organizational effectiveness (Bou-Llusar and Segarra-Cipres, 2006) and that $\mathrm{KM}$ is an important driver of performance and essential to maintain competitive advantage (Panayides, 2004; Malhotra, 2005; Marques, 2006; Schack, 2004). This study investigated these relationships through empirical research specifically in NPPs. The preceding discussion has explained the theoretical basis, developed a research model and hypotheses, explained the research design methods, the sample frame, data collection approach, and data analysis methods to be employed. This study was conducted based on an empirical survey of the global population of NPPs. The data was analyzed according to the methodologies proposed and the results have been summarized and interpreted. The following chapter summarizes the key findings and implications of this study, discusses its limitations and presents recommendations for future research.

\subsection{Summary and Implications}

NPP managers are keenly aware of the importance of maintaining a high level of core competencies needed to successfully and safety operate a nuclear power plant to high levels of sustained operational performance. Serious nuclear accidents such as Chernobyl, Three-Mile Island, and Fukushima have high-lighted the critical importance of correct decision making in a high-consequence technically complex knowledge-based 
operational environment. Post-accident reviews have clearly indicated these events could (and should) have been avoided if knowledge and knowledge process gaps had been prevented. The difficulties associated with simultaneous high attrition of experienced nuclear industry specialists combined with shortages of knowledgeable replacement workers at a time when industry demand for their expertise is increasing has only further highlighted the vulnerability of NPPs to knowledge loss and the importance of effective knowledge management.

A review of the literature has revealed a lack of cohesive theory and research explaining the mechanisms by with managers, by applying knowledge management practices can effectively influence and strengthen organizational learning processes and build and sustain the integrated knowledge base to achieve higher levels of organizational performance. In particular, research and industry guidance is lacking in the context of the nuclear power industry.

This study has incorporated and extended the previous work presented in the literature and developed (for the first time) a more complete and integrated theory and framework that establishes the means by which $\mathrm{KM}$ practices, through the intermediary roles of knowledge processes and supportive organizational culture, are a key determinant of organizational effectiveness and ultimately operational performance in the context of NPPs. A formal research methodology was applied using well established methods and statistically significant findings have been presented. The reliability of measures, 
consistency of the dataset, and strength of the findings support many of the hypotheses, with some exceptions as noted and some additional unexpected findings.

There are significant business and economic implications of these findings for NPP managers. It is clear that a strong business case exists for organizational strategy, planning and resources to be applied to implement an effective KM system comprised of best $\mathrm{KM}$ practices. Benefits can be tangibly measured and include achievable sustained improvements in organizational effectiveness and operational performance. To illustrate the magnitude of the potential economic benefit of improved knowledge management practices as predicted by Model $16 \mathrm{~b}$ for a typical NPP, the annual production benefit from a representative 1 unit (on the ordinal scale) step increase for each of the KMPs can be estimated (e.g. going from a rating of 2.5 to 3.5 on each specific KMP or OTS scale). As an example, Table 168 shows the typical production (wholesale) electricity price and its dollar value per day and per year for a representative 1300 MWe size plant, assuming 100\% UCF. The raw (non standardized) regression "total effects" from Table 163 for each of the KMPs and OTSs are summarized in Table 169. However, to simplify the analysis, the unpredicted effect of PMP on UA7 is ignored for the moment and excluded. Table 170 summarizes the MW-h/day benefit achieved assuming a 1 unit (on the ordinal scale) step increase of each KMP, and the total effect from Table 169, and using the typical electricity price values derived in Table 168. Finally, Table 171 shows the model predicted annual benefit in millions of dollars per year $(\mathrm{M} \$ \mathrm{yr})$ for each factor. The economic incentive for operating performance improvement from improving KMPs is significant. Note that the minus 
sign is important... FLR and UA7 values represent a loss in production, so a negative "effect loading" value in Table 169 is a beneficial effect economically when considering either of these two indicators. This example is meant to be indicative only to point out the "approximate magnitude" of the overall potential benefits to be gained with improved KM practices.

Table 168. Typical NPP Production Price and Value

\begin{tabular}{|c|c|c|}
\hline Parameter & Value & Units \\
\hline Typical NPP output per day (1300 MWe rated unit) & $\$ 31,200$ & MWe \\
\hline Typical production price of electricity per KWh & 0.037 & $\$ / K W h$ \\
\hline Typical production price of electricity per MWh & 37 & $\$ / M W h$ \\
\hline Typical production value of electricity per day & 1.15 & $\mathrm{M} \$ / \mathrm{d}$ \\
\hline Typical production value of electricity per year & $\$ \quad 421.4$ & $\mathrm{M} \$ / \mathrm{yr}$ \\
\hline
\end{tabular}

Table 169. Total Effect of KMPs/OTSs on Raw Performance Factors

\begin{tabular}{|c|c|c|c|c|c|c|c|c|c|}
\hline $\begin{array}{c}\text { Non- } \\
\text { standardized } \\
\text { Loading }\end{array}$ & SOL & KMS & MMP & IST & HRP & SOC & OSS & OPM & TRP \\
\hline 3YrFLR & -0.50 & -0.52 & -0.58 & -0.26 & -0.47 & -0.97 & -0.04 & -0.13 & -0.11 \\
\hline 3YrUA7 & -0.05 & -0.05 & -0.06 & -0.03 & -0.05 & -0.09 & 0.00 & -0.01 & -0.01 \\
\hline 3YrUCF & 0.74 & 0.75 & 0.84 & 0.38 & 0.69 & 1.41 & 2.27 & 0.19 & 0.16 \\
\hline
\end{tabular}

Table 170. Predicted Effect of Unit Scale Increase of KMPs/OTSs (MWh/d)

\begin{tabular}{|c|c|c|c|c|c|c|c|c|c|}
\hline MWh/d & SOL & KMS & IMP & IST & HRP & SOC & OSS & OPM & TRP \\
\hline 3YrFLR & 149.39 & 152.94 & 171.02 & 76.17 & 140.20 & 286.92 & 12.75 & 39.12 & 31.71 \\
\hline 3YrUA7 & 14.52 & 14.82 & 16.60 & 7.41 & 13.63 & 27.57 & 1.19 & 3.85 & 2.96 \\
\hline 3YrUCF & 218.15 & 223.49 & 249.87 & 111.45 & 204.52 & 419.11 & 672.83 & 57.21 & 46.24 \\
\hline
\end{tabular}

Table 171. Predicted Effect of Unit Scale Increase of KMPs/OTSs in M\$/year

\begin{tabular}{|c|c|c|c|c|c|c|c|c|c|}
\hline M $\$ \mathbf{Y r} \mathbf{r}$ & SOL & KMS & IMP & IST & HRP & SOC & OSS & OPM & TRP \\
\hline 3YrFLR & $\$ 2.02$ & $\$ 2.07$ & $\$ 2.31$ & $\$ 1.03$ & $\$ 1.89$ & $\$ 3.88$ & $\$ 0.17$ & $\$ 0.53$ & $\$ 0.43$ \\
\hline 3YrUA7 & $\$ 0.20$ & $\$ 0.20$ & $\$ 0.22$ & $\$ 0.10$ & $\$ 0.18$ & $\$ 0.37$ & $\$ 0.02$ & $\$ 0.05$ & $\$ 0.04$ \\
\hline 3YrUCF & $\$ 2.95$ & $\$ 3.02$ & $\$ 3.38$ & $\$ 1.51$ & $\$ 2.76$ & $\$ 5.66$ & $\$ 9.09$ & $\$ 0.77$ & $\$ 0.62$ \\
\hline
\end{tabular}


To determine the total magnitude of the benefit in terms of improvements in UCF, UA7 and FLR assuming Model 16b, we can estimate this by adding the effects. Table 172 summarizes the calculation of this estimated effect and a total benefit.

Table 172. Predicted Production Benefit (ignoring PMP effect)

\begin{tabular}{|c|c|c|c|c|c|c|}
\hline & FLR & UA7 & UCF & $\begin{array}{c}\text { Total \% } \\
\text { Gain in } \\
\text { Generation }\end{array}$ & $\begin{array}{c}\text { MW- } \\
\text { h/d }\end{array}$ & $\mathbf{M S / Y r}$ \\
\hline Total & -3.58 & -0.35 & 7.43 & 11.36 & 3543 & $\begin{array}{l}\$ 47.9\end{array}$ \\
\hline
\end{tabular}

From the example of Table 172, we can see that a total production benefit on the order of $\sim \$ 48 \mathrm{M} /$ year is predicted by the model. This helps to highlight for managers the strong economic incentive for implementing KM programs in NPPs.

Some of the more important implications of the study findings for NPPs managers include:

- NPP organizations that have higher support for KM practices are realizing significant benefits in terms of organizational performance. This results in significant economic gains. Though not quantified in this study, it also likely results in real improvements in operational safety which, over the life of a station, also provides additional significant economic gains. To realize these benefits, manager's need to integrate KM strategy and policy into the NPPs 
integrated management system in a coordinated corporate-wide manner that ensures alignment of the KM system with business goals at all levels.

- KM practices, which are fully under management's control, have been shown to have a significant direct impact on the quality of knowledge processes in NPP organizations. This is both a direct effect and an indirect effect, the latter being achieved through the impact of a supportive organizational culture. Managers need to better understand $\mathrm{KM}$ philosophy and practices in order to adopt best practices appropriately and implement them successfully. Manager's need to recognize the importance of their personal corporate leadership and example to project and enable the changes needed for effective and successful adoption of KM practices and facilitate a supportive organizational culture.

- Investment in and achievement of effective organizational technology support (OTS) is a necessary condition to achieve high levels of sustained organizational performance. Advanced operational support systems (OSS) in particular have been shown to have a direct and positive impact on unit capability factor (UCF). Support for effective IS and IT technology (IST) has a direct and positive effect of reducing both forced loss rate (FLR) and unplanned actuations (UA7). IST has also been shown to positively affect supportive organizational culture. NPP managers need to recognize the importance of organizational technology support in enabling quality knowledge processes and improving operational performance. 
- Another important implication for NPP managers is that operational performance management (OPM) related $\mathrm{KM}$ practices have been found to directly improve the quality of knowledge acquisition and adoption processes. NPP managers need to recognize the importance of OPM practices in promoting and enhancing KA processes. This helps to find and bring new technology, methods, and expertise into the organization and promotes adopting them along with proven industry best practices.

- Finally, it has been shown that KM plays vital role in the achievement of operational goals, including high levels of nuclear safety. Serious accidents like Chernobyl, Three Mile Island, and Fukushima have clearly been shown to be the consequence of knowledge gaps and poor decision making, and could have and should have been prevented. It is incumbent upon NPP managers to recognize the importance of the link between support for KM practices and quality of knowledge processes and continuous organizational learning. This in turn ensures both the organizational knowledge base and core nuclear competencies are adequately maintained.

\subsection{Limitations of the Study}

There are some specific limitations and potential limitations of this research that should be recognized and understood to ensure the appropriate interpretation of the findings and to guide future research. To begin with, the generalizability of the findings to other industries must be considered carefully. Although the findings are statistically 
significant, the models developed and patterns of construct interaction may be quite specific to NPP organizations. As discussed in Section 2, NPPs have many unique organizational attributes. It is expected the findings may be applicable to other similar knowledge-driven and product technology-dependent production-oriented organizations in regulated industries where high consequence hazards must be managed to achieve low tolerances for risk such as the commercial airline industry. However, it is not known whether the same detailed models and inter-construct relationships would apply. In particular, the relationships between individual KMPs and QKPs, and the interrelationships amongst the QKPs may be applicable to organizations in such industries or may be the specific signature of NPP organizations only. Further research is required. However, it is likely that the main hypotheses apply to other industries and the importance of the sub-construct inter-relationships (i.e. the sub-hypotheses) will vary from industry to industry. For example, in a company designing and manufacturing advanced cell phones, the importance of and links to/from the quality of knowledge acquisition and adoption may be different.

The construct measures developed and used in this study are grounded in prior research. However, it is recognized that many have been adapted and tailored for the purposes and context of this study and this represents the first time some of them have been used. Although construct reliability was confirmed and overall fit of the models was good, it may still be possible to improve on the measures for future research. Further refinement, use and validation is desirable for any measures. In particular the measures for COP participation and measures for staffing levels had a high percentage of 
questionable or missing responses and could be improved. If these constructs were to be applied to study the same relationships in other industries, the measures would need to be tailored to that industry to ensure applicability and comprehension by respondents.

Another limitation of the study was due to the lack of maturity in the understanding and application of knowledge management in many NPPs. It is clear from the data, from station visits and from participation in several IAEA meetings on KM with NPP managers that interpretation of terminology and meanings is not always consistent or correct. This will definitely affect some percentage of the survey responses. It is likely that as more stations become versed in KM practices and terminology and expectations become more standardized across the industry that this issue and the corresponding response bias would diminish.

Other recognized limitations of the study are self-report bias and common method bias. As discussed earlier, these are common problems in social sciences research using surveys. Several measures were reverse coded to hopefully minimize the effect, however, it is recognized that individual station responses in particular may be vulnerable to this type of error. Question 11 in Part G, "please indicate the number of managers who helped complete this survey" was included and does provide some measure of the extent of possible individual response bias (i.e. the average was about 4 , and more participating managers would tend to indicate less likelihood of response bias). From the station assist visits it was observed that having the management team respond collectively to questions initiated much discussion and resulted in clarifications 
and appropriate "mental calibration" of mutually determined response rankings. Although this is subjective observation, it is believed that having multiple managers provide input improves response quality and reduces bias, and the practice should be encouraged and continued in future. One particular non-response weakness of the study was the lack of responses from Russian plants. Only a single Russian NPP participated. It is believed that without head office approval (i.e. the state nuclear utility responsible for all Russian NPPs), NPPs managers were not permitted or were reluctant to participate. It is hoped that if the survey is repeated in future this limitation could be overcome.

Common method bias was discussed earlier in the methodology section. It is recognized that correlations between variables measured with the same method are subject to common method bias and this may be a source of error in this study. However, the use of WANO production indicators as measure of operational performance helps to minimize this type of error.

As a rough measure of non-response bias, an independent samples t-test comparison of NPP operational performance data (3-year unit Capacity Factor) was performed for respondents versus non-respondents to see if there is any statistical difference. Table 173 and 174 summarize the results. There is a significant (i.e. to the .005 level) difference in means between the two groups with responding units having a 3.79 higher mean 3-year Unit Capacity Factor (CF). Although it is not believed to have adversely affected the study findings, it is possible there is a difference in respondent versus non- 
respondent groups that may affect the relationships among the variables being studied (Sackett and Larson, 1992). Although not large in magnitude, this difference does indicate a bias in the response rate towards higher performing plants.

Table 173. Indep. Samples T-test - Responding vs. Non-Responding NPPs

\begin{tabular}{|c|c|c|c|c|c|c|c|c|c|c|}
\hline & \multicolumn{2}{|c|}{$\begin{array}{l}\text { Levene's } \\
\text { Test }^{\star}\end{array}$} & \multicolumn{7}{|c|}{ t-test for Equality of Means } \\
\hline & & \multirow[b]{2}{*}{$\mathbf{F}$} & \multirow[b]{2}{*}{ Sig. } & \multirow[b]{2}{*}{$\mathbf{t}$} & \multirow[b]{2}{*}{ df } & \multirow{2}{*}{$\begin{array}{l}\text { Sig. (2- } \\
\text { tailed) }\end{array}$} & \multirow{2}{*}{$\begin{array}{c}\text { Mean } \\
\text { Differenc } \\
e\end{array}$} & \multirow{2}{*}{$\begin{array}{c}\text { Std. Error } \\
\text { Differenc } \\
\mathrm{e}\end{array}$} & \multicolumn{2}{|c|}{$\begin{array}{l}95 \% \text { Confidence } \\
\text { Interval of the } \\
\text { Difference }\end{array}$} \\
\hline & & & & & & & & & Lower & Upper \\
\hline \multirow{2}{*}{$\begin{array}{c}\text { 3-Yr Unit } \\
\text { CF }\end{array}$} & Equal variances assumed & .077 & .781 & 2.8 & 425 & .005 & 3.79 & 1.35 & 1.13 & 6.44 \\
\hline & $\begin{array}{c}\text { Equal variances not } \\
\text { assumed }\end{array}$ & & & 2.8 & 389 & .005 & 3.79 & 1.35 & 1.13 & 6.44 \\
\hline
\end{tabular}

*test for equal variance.

Table 174. Responding vs. Non-Responding Group Statistics

\begin{tabular}{|c|c|c|c|c|c|}
\hline & Responding & $\mathrm{N}$ & Mean & Std. Deviation & Std. Error Mean \\
\hline \multirow{2}{*}{ 3-Yr Unit CF } & $\mathrm{Y}$ & 246 & 83.4 & 13.8 & .88 \\
\cline { 2 - 6 } & $\mathrm{N}$ & 181 & 79.6 & 13.7 & 1.02 \\
\hline
\end{tabular}

A further limitation of the study is small sample size. Although a high percentage of the total population responded, it was not possible to obtain an adequate model fit using Structural Equation Model techniques with a sample size of only 124 stations. This may also be due in part to a higher sensitivity of SEM to non-normalities (i.e. presence of skew, kurtosis, and heteroscedasticity). The IAEA has expressed interest in repeating the NPP survey in future to obtain longitudinal data and benchmark data for the industry. If a higher response rate is obtained in future, SEM analysis could be used and additional analytical benefits achieved. Increasing the Likert scale to a 7 or 9 -point scale could also improve the normality of the data in future. The use of Lisrel for SEM 
analysis could also be explored (it is possible that it is better suited to analyze complex SEM models with a large number of parameters such as in this study).

As expected, the full model predicts only a limited percentage of overall variance in operational performance. There are a wide range of other possible influences on plant organizational performance parameters such as weather and power grid effects, owneroperator influences, no. of units as site, power output etc.. In future studies, it may be possible to greatly improve the parameter estimates and explained variance. One factor identified and investigated in this research is reactor type, and future research should consider the impact of this factor.

Organizational effectiveness (OE) was measured as a single complex summated scale variable. It did not get broken down into various sub-constructs. In particular it could be useful to decompose this measure into sub-constructs for safety, operational reliability, maintenance dimensions. Operational performance (i.e., OP dimensions) measures focused exclusively on 4 indicators: unit capability factor, unit capability loss, forced (outage) loss rate, and safety system unavailability. Direct links from the subconstruct dimensions of $\mathrm{OE}$ to the individual dimensions of $\mathrm{OP}$ could be included in future analyses, and provided the sample size is adequate the findings may prove interesting and important.

Basic linear regression and path analysis methods were used in this study. Path analysis permits a level of causal variable modeling to be addressed, however, is not able to model 2-way correlations such as the possible link $\mathrm{KU} \leftrightarrow \rightarrow \mathrm{KR}$ discussed in 
Path Model 13. SEM methodology, would be useful to support additional analysis. If higher response rates can be achieved on future repetitions of the NPP survey it should be possible to apply this technique.

Finally, it is possible the entire effect of KMPs and OTSs on OE and OP is only indirect and via QKPs and SOC. If true, the findings of significant direct links from any of the KMP or OTS constructs to OE or OP are in fact not direct causal relationships but measuring correlation in the data. If true, they may be interpreted as an indication of measurement weaknesses in the intermediate QKPs and SOC construct measures. Further research would be needed to explore this possibility.

\subsection{Recommendations for Future Research}

The following are the specific opportunities and recommendations for further research that arise from the findings and additional questions posed from this study:

- There is definitely keen interest by NPP managers in further research on KM. It is quite possible the IAEA will repeat this survey in future. This will create more much needed NPP data on the global fleet that is needed to benchmark NPP performance. Such data could be produced and published for NPPs in future to enable self-assessment. Longitudinal data could be used to further examine and strengthen understanding of the relationships and track changes over time. This research could be used as a basis for further research into NPPspecific KM maturity models towards this end. This would aid industry 
managers to focus on identifying and filling gaps in the internal $\mathrm{KM}$ planning and implementation process.

- Research on additional organizational performance determinants would be useful to further fine-tune the model and more accurately explain the variances of operational performance. For example, organizational effectiveness could be broken down into sub-constructs that measure additional dimensions such as safety, production reliability, and effectiveness of the maintenance function. Direct linkages between these dimensions and the sub-constructs of operational performance could be investigated in future analyses. In addition, the impact of physical plant factors such as age, number of units, plant type, and plant size on these yet unexplored relationships is needed.

- Research to investigate the generalizability of these findings to other (similar) industries could be conducted. In particular, to confirm or refute the notion that quality of knowledge process inter-relationships found in this study are specific to NPPs or are applicable to other industries, or perhaps only to those with similar characteristics. The influence of support for knowledge management practices and supportive organizational culture on organizational performance is of great interest in all industries. Further research is needed to determine the applicability of the Knowledge Management Performance Model (KMPM) in other sectors. 
- If the survey is repeated by the LAEA or others, it is recommended that a glossary of standard KM terminology be available (e.g. provided via an Internet link) to ensure consistent interpretation of survey questions. It is also recommended that the survey design include multiple (repeated) measure responses from a single station to minimize response bias. If NPP's were to conduct self-assessments against the survey findings to benchmark themselves against the industry averages, greater awareness of the measures and their consistent interpretation would result. This would reduce common method error and likely greatly improve the predictive accuracy of future repetitions of this empirical study.

- Further research is recommended to better understand the combined intermediary and moderation effects of the level of supportive organizational culture on the links between KMPs, QKPs and OE. Specific dimensions of organizational culture could be identified and their specific role in the KMPM better understood. This topic is of great interest to managers in many industries and ripe for future research.

- It has been shown that the relative importance of the various relationships defined in this research will vary for a given NPP organization based on the level of support for specific KMPs and a number of other factors such as the level of supporting organizational culture. Further research is recommended to better understand these differences for example between stations with KM 
systems/programs that are mature versus in their infancy. It is also suspected (based on anecdotal evidence from IAEA assist visits) that self-report bias is highest amongst NPP organizations with low KMP maturity.

- Due to sample size limitations, it was not possible to develop an acceptable model fit using structural equation model (SEM) methods. The analytical benefits of this methodology would likely improve the fidelity of the analysis and may yield additional findings or further clarify some of the issues raised. If the survey is repeated in future, additional effort to increase response rates and quality is recommended to enable SEM methods be applied.

- Finally, possible improvements to the construct measures could be explored. For example, wording changes could be made to recalibrate some survey question wordings to reduce responses skewed in one direction. The use of 7 or 9 -point Likert scale could be considered to improve response discrimination. Questions with a higher number of missing values or outliers could be reviewed for possible wording improvements. 


\section{References}

[1.] Agresti, A., and Finlay, B. (1986). Statistical Methods for the Social Sciences. $2^{\text {nd }}$ Ed. Macmillan Inc.

[2.] Ahituv, N., Neumann, S. and Riley, H.N. (1994). Principles of Info Systems for Management (4th Ed.), IA, Brown Communication.

[3.] Ahmed, P.K., Lim, K.K. and Zairi, M. (1999). Measurement practice for knowledge management, Journal of Workplace Learning, 11 (8), p. 304-12.

[4.] Ahn, J.H. and Chang, S.G. (2004). Assessing the contribution of knowledge to business performance: the KP3 methodology, Decision Support Systems 36.

[5.] Akgin, A.E., Lynn, G.S. and Byrne, J.C. (2003, July). Organizational Learning: A Socio-cognitive Framework, Human Relations: 56, 7, p. 839.

[6.] Akgun, A.E., Lynn, G.S., and Reilly, R. (2002). Multi-dimensionality of Learning in New Product Development Teams. European Journal of Innovation Management. V5 (2), p. 57-72.

[7.] Smith, A.D. (2008). Malcolm Baldridge award as strategic leverage for educational and other service-related institutions. International Journal of Management in Education. V2(1). P60-76.

[8.] Albaum, G. (1999). The Likert Scale Revisited: An Alternate Version. Journal of the Market Research Society. V39 (2). p. 331.

[9.] Alikhan, S. (1991). Performance Assessment of Point Lepreau Generating Station, Tech. Meeting on Exchange of Operational Safety Experience of Pressurized Heavy Water Reactors.

[10.] Allee, V. (1997). Organizations and Environments. Englewood Cliffs, NJ, Prentice Hall.

[11.] American Productivity and Quality Center (2000).

[12.] Anderson, J.C. and Gerbing, D.W. (1988). Structural equation modeling in practice: A review and recommended two-step approach, Psychological Bulletin, 103, p. 411- 423.

[13.] Anderson, J.C. and Narus, J.A. (1990). A Model of Distributor Firm and Manufacturing Firm Working Relationships. Journal of Marketing, Vol. 54 (1), p. $42-58$.

[14.] Anderson, L.W., Krathwohl, D.R., Airaslan, P.W., Cruikshank, K.A., Mayer, R.E., Pintrich, P.R., Raths, J.D. and Wittrock, M.C. (1998). Taxonomy for Learning, Teaching and Assessing: A Revision of Bloom's Taxonomy of Educational Objectives, Longman, New York, NY.

[15.] Andriessen, D. and Tissen, R. (2000). Weightless Wealth; Find Your Real Value in a Future of Intangible Assets, Financial Times and Prentice-Hall, London. 
[16.] Angerson, R. and Boulanger, A. (2004, Nov). Columbia Univ., NY; Knowledge Management and Computational Learning for Lean Energy Mgt., Oil \& Gas Journal; p. 36.

[17.] Aramburu, N., Saenz, J. and Rivera, O. (2006). Fostering innovation and knowledge creation: the role of management context, Journal of Knowledge Management.

[18.] Arbuckle, J.L. (2009). Amos 18 User's Guide. Amos Development Corporation. Crawfordville, FL., USA.

[19.] Ardern, C.M., (1998, Jan). CRM; Change is Here! What are We Doing About It? ARMA Records Management Quarterly; 32, 1; p. 10.

[20.] Argyris, C. and Schon, D.A. (1978). Organizational Learning: A Theory of Action Perspective. Reading USA, Addison Wesley.

[21.] Arora, R. (2002). Implementing KM - a balanced score card approach, Journal of Knowledge Management.

[22.] Assudani, R.H. (2005). Catching the chameleon: understanding the elusive term "knowledge", Journal of Knowledge Management, 9 (2), p. 31-44.

[23.] Bach, C. (2004). Increase of Organizational Performance in Complex Knowledge Intensive Industries through the Employment of IT Applications, School of Information Science and Policy.

[24.] Backlund, F. and Hannu, J. (2002). Can We Make Maintenance Decisions on Risk Analysis Results?, Lulea Univ. of Tech., Lulea, Sweden, p. 77.

[25.] Badaracco, J.L. (1991). The Knowledge Link: Harvard Business School Press, Boston M.A.

[26.] Bahr, C. and Bier, V. (2000, Jul). The Human Element, Deregulation and Safety, Vol. 5, No. 3, University of Wisconsin-Madison.

[27.] Bailey, J.E. and Pearson, S.W. (1983, May). Development of a Tool for Measuring and Analyzing Computer User Satisfaction. Management Science, 29(5), p. 530-545.

[28.] Bailey, K.D. (1982). Methods of Social Research. $2^{\text {nd }}$ Ed. MacMillan Pub., New York.

[29.] Balderston, J., Birnbaum, P., Goodman, R. and Stahl, M. (1984). Modern Management Techniques in Engineering and R\&D, Van Nostrand Reinhold Co: New York, NY.

[30.] Baldwin, R. (2001). Determinants on the Use of Information Technology, and How Its' Use Impacts Performance; Nova Southeastern Univ.

[31.] Ballay, J.F. and Vannieuwenhuyse, J.P. (2004, September). Nuclear Knowledge management overview at EDF. International Conference on Nuclear Knowledge Management, Saclay, France IAEA-I1-CN-123.

[32.] Balthazard, P.A. and Cooke, R.A. (2004). Organizational Culture and Knowledge Management Success; Assessing The Behavior - Performance Continuum, Arizona State Univ. \& Center for Applied Research, Inc.

[33.] Barney, J. (1986). Organizational culture: can it be a source of competitive advantage?, Academy of Management Review, V11, No. 3, p. 656-65. 
[34.] Barney, J. (1991). Firm Resources and Competitive Advantage. Journal of Management. V17(1): p. 99-120.

[35.] Baron, R.M. and Kenny, D.A. (1986). The moderator-mediator variable distinction in social psychology research: Conceptual, strategic, and statistical considerations. Journal of Personality and Social Psychology, 51, 1173-1182.

[36.] Belardo, S., Karwan, K.R. and Wallace, W.A. (1982, Dec). DSS Component Design Through Field Experimentation. Proceedings of the 3rd Int'l Conf on Info Systems, p. 121-135.

[37.] Beraha, D., Heigl, T. and Westerheide P.P. (2005). Nuclear Knowledge management: the GRS realization, Int. J. Nuclear Knowledge Management, Vol. 1, No. 4.

[38.] Bergman, J., Jantunen, A. and Saksa, J.M. (2004). Managing knowledge creation and sharing-scenarios and dynamic capabilities in inter-industrial knowledge networks, Journal of Knowledge Management.

[39.] Bhattacharya, A.S. (2005). Experience with Diagnosis of Root Causes of Human Performance Problems in Indian Nuclear Power Plants, Nuclear Power Corp. of India Ltd.

[40.] Bin Syed-Ikhsan, S.O. and Rowland, F. (2004). Benchmarking Knowledge Management in a Public Organization in Malaysia. Benchmarking, Vol. 11, No. 3.

[41.] Birchall, D.W. and Tovstiga, G. (2002). Assessing the firm's strategic knowledge portfolio: a framework and methodology, International Journal of Technology Management, 24 (4), p. 419-434.

[42.] Bititci, U.S., Carrie, A.S. and Mcdevitt, L. (1997). Integrated performance measurement systems: a development guide, International Journal of Operations \& Production Management, 17 (5/6), p. 522-34.

[43.] Bontis, N. (2001). Assessing knowledge assets: a review of the models used to measure intellectual capital, International Journal of Management Reviews, 3 (1), 41-60.

[44.] Bontis, N., Crossan, M. and Hulland, J. (2002, Jun). Managing an Organizational Learning System by Aligning Stocks and Flows, Journal of Management Studies 39:4, 0022-2380 p. 438-469.

[45.] Bornemann, M. and Sammer, M. (2003). Assessment methodology to prioritize knowledge management related activities to support organizational excellence, Measuring Business Excellence 7, 2.

[46.] Bou-Llusar, J.C. and Segarra-Cipres, M. (2006). Strategic knowledge transfer and its implications for competitive advantage; an integrative conceptual framework, Journal of Knowledge Management.

[47.] Brauner, E. and Becker, A. (2006). Beyond knowledge sharing: the Management of transactive knowledge systems, Knowledge and Process Management 13, 1.

[48.] Brockman, B.K. and Morgan, R.M. (2003). The Role of Existing Knowledge in New Product Innovativeness and Performance. Decision Sciences. V 34(2), p. 385-420. 
[49.] Brooking, A. (1996a). Intellectual Capital: Core Assets for the Third Millennium Enterprise, London: Thomson Business Press.

[50.] Brooking, A. (1996b). Introduction to Intellectual Capital. Cambridge, England. The Knowledge Broker Ltd.

[51.] Brooking, A., Board, P. and Jones, S. (1998). The predictive potential of intellectual capital, International Journal of Technology Management, 16 (1/2/3), p. 115-125.

[52.] Bruner, J.S. (1973). Beyond the Information Given. J.M. Anglin, Ed.

[53.] Burkhart, L.A. (1987, Dec 10). Power Plant Performance Standards and Efficiency Incentives: An Overview, 120, 12; ABI/INFORM Global p. 47.

[54.] Burnett, S., Illingworth, L. and Webster, L. (2004). Knowledge Auditing and mapping: a pragmatic approach, Knowledge and Process Management.

[55.] Burrel, D.N. and Safi, A. (2008). How mentoring programmes can address the emerging knowledge management crisis at US federal regulatory agencies as the US faces a nuclear renaissance. International Journal of Nuclear Knowledge Management. V3(1).

[56.] Cabrera, A., Collins, W.C., and Salgado, J.F. (2006). Determinants of Organizational Engagement in Knowledge Sharing. International Journal of Human Resource Management. V(17), p.245-264.

[57.] Cai, J. (2006, Jan-Mar). Knowledge Management Within Collaboration Processes: A Perspective Modeling and Analyzing Methodology, Peking University, Beijing, P.R. China.

[58.] Calantone, R.J., Cavusgil, S.T. and Zhao, Y. (2002). Learning orientation, firm innovation capability, and firm performance, Industrial Marketing Management, Vol. 31, No. 6, p. 515-24.

[59.] Carlin, S., Lee, J., Lemons, D., O'Dell, C., and Swift, G. (2007). The Role of Evolving Technologies: Accelerating Collaboration and Knowledge Transfer. An APQC Consortium Benchmarking Study. APQC, Houston, Texas

[60.] Carlucci1, D. and Schiuma, G. (2006). Knowledge Asset Value Spiral: Linking Knowledge Assets to Company's Performance. Knowledge and Process Management Volume 13 Number 1 pp 35-46.

[61.] Carneiro, A. (2000). How does knowledge management influence innovation and competitiveness?, Journal of Knowledge Management. 4, 2.

[62.] Carpenter, S. and Rudge, S. (2003). A Self-help Approach to Knowledge Management Benchmarking, Journal of Knowledge Management; 7,5; ABI/INFORM Global p. 82.

[63.] Carroll, J.S. (1995). Knowledge Management in High-Hazard Industries, Accident Precursors as Practice.

[64.] Caulkins, D.D. (2004, Dec). Identifying Culture as a Threshold of Shared Knowledge - A Consensus Analysis Method, International Journal of Cross Cultural Management, CCM; 4, 3; p. 317.

[65.] Celani, A. and Weber, C.L. (1997). Pay-for-Knowledge Systems: Guidelines for Practice. IRC Press, Industrial Relations Centre, Queen's University. Kingston. 
[66.] CEN (2004). The European Guide to Good Practice in Knowledge Management (CEN CWA 14924-1 2004). European Committee for Standardization. Brussels.

[67.] Chang L.K., Lee, S. and Kang, I.W. (2005). KMPI: measuring knowledge management performance, Information and Management, Vol. 42 No. 3, p. 469-82.

[68.] Chang, S.G. and Ahn, J.H. (2005). Product and process knowledge in the performance-oriented knowledge management approach, Journal of Knowledge Management.

[69.] Charles, S. (2002, Jan). Lessons Learned from the Document Management Trenches. The Leading Magazine for Information Professionals.

[70.] Chatman, J. and Jehn, K. (1994). Assessing the relationship between industry characteristics and organisational culture: how different can you be? Academy of Management Journal, Vol. 37, No. 3, p. 522-53.

[71.] Chauvel, D. and Despres, C. (2002). A Review of Survey Research in Knowledge Management: 1997-2001, Journal of Knowledge Management; 6,3: ABI/INFORM Global, p. 207.

[72.] Chavez-Mercado, C., Zayas-Perez, B., Valdes-Parra, J. and Ocampo-Mansilla, H. (1997, Jun). The classroom analysis simulator: a training and research tool for nuclear power plant operations. Human Factors and Power Plants, 1997. apos; Global Perspectives of Human Factors in Power Generation apos;., Proceedings of the 1997 IEEE Sixth Conference on Human Factors and Power Plants. Volume, Issue , 8-13, Page(s):17/11 - 17/16.

[73.] Chen Liu, S. (2003). A Study of Factors that Facilitates Use of Knowledge Management Systems and the Impact of Use on Individual Learning, The Claremont Graduate Univ.

[74.] Chen, L. and Mohamed, S. (2007). Empirical study of interactions between knowledge management activities. Engineering, Construction and Architectural Management. Vol. 14, No. 3, p. 242-260.

[75.] Choi, Y.S. (2000, Dec). An Empirical Study of Factors Affecting Successful Implementation of Knowledge Management. Pub Unknown.

[76.] Choo, C.W. (1989). The Knowing Organization: How Organizations Use Information to Construct Meaning, Create knowledge, and Make Decisions. New York. Oxford University Press.

[77.] Chou, T.C., Chang, P.L., Tsai, C.T. and Cheng, T.P. (2005). International learning climate, knowledge management process and perceived knowledge management satisfaction, Journal of Information Science, Vol. 31, No. 4, p. 283-96.

[78.] Clare, M. and Detore, A. (2000). Knowledge Assets: (C), KM Toolbox: Inventory of Tools and Techniques.

[79.] Clark, K.B, and Fujimoto, T. (1991). Product Development Performance: Strategy, Organization, and Management in the World Auto Industry, Harvard Business School Press: Boston, Mass. 
[80.] Clayton, M.F. and Pett, M.A. (2008). AMOS versus LISREL: one data set, two analyses. University of Utah, Salt Lake City, USA.

[81.] CMA (Canadian Management Accountants) (1999). Focus Group Draft: Measuring Knowledge Assets, CMA, Mississauga. April 16.

[82.] CMBG (2005, Feb). Impact of the Nuclear Industry.

[83.] CMBG (2004). Nuclear Facility Configuration Management Survival Guide, Configuration Management Benchmarking Group, 2004 Conference.

[84.] CMBG (2006). Nuclear Industry Configuration Management Benchmarking Group - Principles and Practices.

[85.] Coes, S.H. (1990). Performance Measurement for Nuclear Maintenance Activities, American Association of Cost Engineers. Transaction of the American Assoc., p. C.2.1

[86.] Coffey, J.W. and Hoffman, R.R. (2003). Knowledge modeling for the preservation of institutional memory. Journal of Knowledge Management. V7 (3) p. $38-52$.

[87.] Coffey, J.W., Eskridge, T.C. and Sanchez, D.P. (2004). A Case Study in Knowledge Elicitation For Institutional Memory Preservation Using Concept Maps, Institute for Human and Machine Cognition, The University of West Florida, Pensacola, Fl, USA.

[88.] Cohen, D. (1998). Toward a knowledge context: report on the first annual UC Berkeley forum on knowledge and the firm". California Management Review, Vol. 40 No.3.

[89.] Cohen, M. and Levinthal, D. (1990). Absorptive Capacity: A New Perspective on Learning and Innovation. Administrative Science Quarterly. V35, p. 128152.

[90.] Concannon, K.H., Jardine, A.K.S., P. Eng. and McPhee, J., P. Eng. (1990, Jan). Balance Maintenance Costs Against Plant Reliability With Simulation Modeling, p. 22.

[91.] Condu, M., de Mella, J., Spiegelberg-Planer, R. (2005). IAEA - Improving Economic Performance, Nuclear Plant Journal; 23, 1; ABI/INFORM Global, p. 25.

[92.] Contractor, F.J. (2000). Valuing Corporate Knowledge and Intangible Assets: Some General Principles, Knowledge and Process Management; School of Management, Rutgers Univ., USA, 7,4; ABI/INFORM Global, p. 242.

[93.] Converse, J.M. and Presser, S. (1986). Survey Questions: Handcrafting the Standardized Questionnaire. Sage University Paper Series on Quantitative Applications in the Social Sciences, 07-063, Sage Publications, California.

[94.] Coombs, R. and Hull, R. (1998). Knowledge Management Practices and PathDependency in Innovation. Research Policy. V27(3), p. 237-253.

[95.] Cordero, R. (1989). The measurement of innovation performance in the firm: an overview, Research Policy, 19 (2) p. 185-92.

[96.] Corso, M., Martini, A., Pellegrini, L. and Paolucci, E. (2003). Technological and Organizational Tools for Knowledge Management: In Search of Configurations, Small Business Economics 21, 4. 
[97.] Crossan, M., Lane, H., and Roderick, E. (1999). An Organizational Learning Framework: From Intuition to Institution. Academy of Management Review. V24 (3).

[98.] Culnan, M.J. (1983, April). Environmental Scanning: The Effects of Task Complexity and Source Accessibility on Information Gathering Behavior. Decision Science, 14(2), p. 194-206.

[99.] Cummings, T. and Worley, C. (1997). Organizational Development and Change. 6th Ed., Southwestern College Pub., Cincinnati Ohio.

[100.] Daft, R. and Huber, G. (1987). How Organizations Learn: A Communication Framework. Research in the Sociology of Organizations, V5, p. 1-36.

[101.] Daft, R.L. and Weick, K.E. (1984). Toward a Model of Organizations as Interpretation Systems. Academy of Management Review. V9(2). p. 284-295.

[102.] Dalkir, K. (2005). Knowledge Management in Theory and Practice, Elsevier Butterworth-Heinemann, London.

[103.] Darroch, J. (2005). Knowledge management, innovation and firm performance, Journal of Knowledge Management.

[104.] Davenport, T.H., and Prusak, L. (1998). Working Knowledge: How Organizations Manage What They Know. Harvard Business School Press. Boston.

[105.] Davenport, T.H., De Long, D., and Beers, M.C. (1998). Successful Knowledge Management Projects. Massachusetts Institute of Tech.

[106.] De Gooijer, J. (2000). Designing a knowledge management performance framework, Journal of Knowledge Management, 4 (4), p. 303-310.

[107.] De Jager, M. (1999). The KMAT; Benchmarking Knowledge Management. Library Management. Bradford: Vol. 20, Iss. 7; p. 367-372.

[108.] De Tienne, K.B., Dr., Dyer, G., Dr., Hoopes, C. and Harris, S. (2004). Toward a Model of Effective Knowledge Management and Directions for Future Research: Culture, Leadership, and CKOs, Journal of Leadership \& Organizational Studies; 10,4; p. 26.

[109.] del-Rey-Chamorro, F.M., Roy, R., Wegan, B. and Steele, A. (2003), "A framework to create key performance indicators for knowledge management solutions", Journal of Knowledge Management, Vol. 7 No. 2, p. 46-62.

[110.] DeMaio, T.J. (ed.) (1983). Approaches to developing questionnaires. Statistical Policy Working Paper 10, United States Office of Management and Budget, Washington, D.C.

[111.] DeSanctis, G. (1982, Dec). An Examination of an Expectancy Theory Model of Decision Support System Use. Proceedings of the 3rd Int'l Conf. on Information Systems, p. 121-135.

[112.] Desouza, K.C. and Vanapalli, G.K. (2005). Securing Knowledge Assets and Processes: Lessons from the Defense and Intelligence Sectors, Univ. of Illinois, Chicago \& Indian Navy, New Delhi, India.

[113.] Detert, J.R., Schroeder, R.G., and Mauriel, J.J. (2000). A framework for linking culture and improvement initiatives in organizations, Academy of Management Review. V25 (4), p. 850- 863. 
[114.] Dewitz, S.D. (1996). Systems Analysis and Design and the Transition to Objects. New York: McGraw-Hill.

[115.] Diakoulakis, I.E., Georgopoulos, N.B., Koulouriotis, D.E. and Emiris, D.M. (2004). Towards a holistic knowledge management model. Journal of Knowledge Management, Vol. 8, No. 1, p. 32-46.

[116.] Dixon, N.M. (1999). The Organizational Learning Cycle: How We Can Learn Collectively. 2nd Ed. Brookfield, USA, Grower.

[117.] Djerdjouri, M. (2005, May). Assessing and Benchmarking Maintenance Performance in a Manufacturing Facility: A Data Envelopment Analysis Approach, Sate Univ. of New York, Plattsburgh, 43, 2 p. 121.

[118.] Drucker, P. (1994). The Age of Social Transformation. The Atlantic Monthly. p. 53-80.

[119.] Dwight, R.A. (1995). Concepts for measuring maintenance performance, in: Martin, H.H., New Developments in Maintenance: An International View, Moret Ernst and Young.

[120.] Edvinsson, L. and Malone, M.S. (1997). Intellectual Capital: Realizing Your Company's True Value by Finding Its Hidden Brainpower, Harper Business, New York, NY.

[121.] Edwards, J.S., Shaw, D., and Collier, P.M. (2005). Knowledge management systems: finding a way with technology, Journal of Knowledge Management. 9, 1.

[122.] Eldridge, M., Kohler, J. and Van Gulik, J. (1994, Aug). RCG/Hagler Baily Peer Plant Comparisons Can Help Managers Enhance Performance and Reduce Operating and Maintenance Expenses, Power Eng'g.; 98,8; ABI/INFORM Global, p. 32.

[123.] Eppler, M.J. (2001). Making Knowledge Visible Through Intranet Knowledge Maps: Concepts, Elements, Cases. Proceedings of the 34th Annual Hawaii International Conference on System Sciences, p. 1530-1539.

[124.] EPRI (1993, June): Technology Transfer and Innovation in the Utility Organization. Technical Report TR-102445. Electrical Power Research Institute.

[125.] EPRI (2006). Enterprise Knowledge Management System for Nuclear Power Plants. Electrical Power Research Institute. Report 1013108. Palo Alto, CA.

[126.] ERWG, 2007. Industry Equipment Reliability Index Estimator. Equipment Reliability Working Group. May 2007 (draft).

[127.] Fahey, L and Prusak, L. (1998). The Eleven Deadliest Sins of Knowledge Management. California Management Review. V40(3) (Special Issue on Knowledge and the Firm).

[128.] Feldman, S.P. (2004, June). The Culture of Objectivity: Quantification, Uncertainty, and the Evaluation of Risk at NASA, Human Relations, V57, 6: p. 691.

[129.] Feltham, G. and Xie, J. (1994). Performance Measure Congruity and Diversity in Multi-task Principal-Agent Relations. The Accounting Review. 69(3): p. 42953. 
[130.] Feng, K., Chen, E.T. and Liou, W. (2004-05, Winter). Implementation of Knowledge Management Systems and Firm Performance: An Empirical Investigation, Univ. of Massachusetts, National ChengChi Univ., Taiwan, ROC.

[131.] Fiol, C. and Lyles, M. (1985). Organizational Learning. Academy of Management Review. V10(4). p. 803-813.

[132.] Firestone, J.M. and McElroy, M.W. (2004). Organizational Learning and Knowledge Management: The Relationship. The Learning Organization. V11(2). p. 177-184.

[133.] Fitzgerald, L., Johnston, R., Brignall, S., Silvestro, R. and Voss, C. (1991). Performance Measurement in Service Business. CIMA, London.

[134.] Foos, T., Schum, G. and Rothenberg, S. (2006). Tacit knowledge transfer and the knowledge disconnect, Journal of Knowledge Management,

[135.] Foss, N. (1996). Knowledge-based Approaches to the Theory of the Firm: Some Critical Comments. Organizational Science. V7 (5).

[136.] Fowler, F.J. Jr. (1995). Improving Survey Questions: Design and Evaluation. Applied Social Research Methods Series, 38, Sage Publications, Thousand Oaks, California.

[137.] Fox, M.R. (1999, March 15). Nuke Plant Staffing: How Low Can You Go? Public Utilities Reports.

[138.] Fox, M.R. and Maidment, J.P.M. (1996, June 15). Numbers That Make Sense: Gauging Nuclear Cost Performance, Public Utilities Reports.

[139.] Fox, M.R. and Maidment, J.P.M. (1996, June 15). Numbers That Make Sense: Gauging Nuclear Cost Performance, Public Utilities Fortnightly; 134, 12; ABI/INFORM Global, p. 37.

[140.] Gagnon, S. (1999). Resource-based competition and the new operations strategy, International Journal of Operations and Production Management 19, 2 .

[141.] Gaines, B.R. (1989). "Integration Issues in Knowledge Support Systems," Int'l J. of Man-Machine Studies, Vol. 3 I, No. 5, p. 495-515.

[142.] Gallivan, M. J., Eynon, J. and Rai, A. (2003). The challenge of knowledge management systems - Analyzing the dynamic processes underlying performance improvement initiatives, Information Technology \& People 16, 3.

[143.] GAO/RCED-00-29 (2000). NRC Staff Have Not Fully Accepted Planned Changes. Nuclear Regulation.

[144.] Garvin, D. (1993, Jul-Aug). Building a Learning Organization. Harvard Business Review. p. 78-91.

[145.] Genov, S. (2001, Feb). Communication with Nuclear Operators Worldwide has been Vital for the Continual Safety Improvement of Kozloduy NPP.

[146.] Gharib, M. (2005). Knowledge degradation within routing operation practices in TRR: lessons learned, Int. J. Nuclear Knowledge Management, Vol 1, No. 4.

[147.] Glazer, R. (1991). Marketing in an information-intensive environment: strategic implications of knowledge as an asset". Journal of Marketing, Vol. 55 pp.1-19. 
[148.] Gleason, J.M. and Barnum, D.T. (1986). Toward Valid Measures of Public Sector Productivity: Performance Measures in Urban Transit, Management Science, 28, 4, p. 379-86.

[149.] Glisby, M. and Holden, N. (2004, Jan-Mar). Contextual Constraints in Knowledge Management Theory: The Cultural Embeddedness of Nonaka's Knowledge-creating Company; Knowledge and Process Management; 10, 1; p. 29.

[150.] Gloet, M. and Terziovski, M. (2004). Exploring the relationship between knowledge management practices and innovation performance, Journal of Manufacturing Technology Management, 15, 5.

[151.] Gordon, G. (1985). The relationship of corporate culture to industry sector and corporate performance, Gaining Control of the Corporate Culture, Jossey-Bass, San Francisco, CA, Kilmann, R.H., Saxton, M.J., Serpa, R. and Associates.

[152.] Gorelick, C. and Tantawy-Monsou, B. (2005). For performance through learning, knowledge management is the critical practice, Management Services, New York.

[153.] Gower, A.R. (1994). Questionnaire design for business surveys, Survey Methodology, 20, p. 125-136.

[154.] Grant, R.M. (1995). Contemporary Strategy Analysis: Concepts, Techniques, and Applications. Cambridge, Mass. Blackwell.

[155.] Grant, R.M. (1996). Towards A Knowledge-based Theory of the Firm. Strategic Management Journal. V17 (Winter Special Issue).

[156.] Griffith, T.L., Sawyer, J.E. and Neale, MA. (2003, June). Virtualness and Knowledge in Teams: Managing the Love Triangle of Organizations, Individuals and Information Technology, MIS Quarterly.

[157.] Gross, M., Hanes, L. and Ayres, T. (2002). Capturing Undocumented WorkerJob-Knowledge at Electric Utilities: The EPRI Strategic Project, New Century, New Trends, Proc. 2002 IEEE 7th Conf. Human Factors and Power Plants, IEEE Press, p. 6-20-6-24.

[158.] Grossman, M. Dr. (2006). An Overview of Knowledge Management Assessment Approaches, The Journal of American Academy of Business.

[159.] Gupta, N., Schweizer, T.P. and Jenkins, G.D. (1987, Oct). Pay for Knowledge Compensation Plan: Hypothesis and Survey Results. Monthly Labor Review.

[160.] Guthrie, J. (2001). The management, measurement and the reporting of intellectual capital, Journal of Intellectual Capital 2, 1.

[161.] Haber, S. B., O'Brien, J. N., Metlay, D. S. and Crouch, D. A. (1991). Influence of organizational factors on performance reliability. (Report to the Division of Systems Research, U.S. Nuclear Regularly Commission). Upton NY: Brookhaven National Laboratory.

[162.] Haines, G.H. Jr. (1978). The Effect of Information on Market Behavior. American Marketing Association. Proceeding Series.

[163.] Hair, J., Anderson, R., Tatham, R., and Black, W. (1995). Multivariate Data Analysis with Readings, Englewood Cliffs, NJ: Prentice Hall. 
[164.] Hall, D.J. (2002, August). Testing Performance and Perspective in an Integrated Knowledge Management System, The University of Texas at Austin.

[165.] Hall, M. (2006). Knowledge Management and the Limits of Knowledge Codification. Journal of Knowledge Management, Volume 10.

[166.] Hamel, G. (1991). Competition for Competence and Inter-partner Learning within International Alliances. Strategic Management Journal. V12 (special issue), p. 83.

[167.] Hamel, G. and Prahalad, C. (1990). The Core Competence of the Corporation. Harvard Business Review. 68(3).

[168.] Hancock, L.R. (2005, February 19). A Nuclear Power CM Historical Perspective, Configuration Management Benchmarking Group Papers.

[169.] Hanes, L.F., and Gross, M.M. (2002). Capturing Valuable Undocumented Knowledge: Lessons Learned at Electric Utility Sites," New Century, New Trends, Proc. IEEE 7th Conf. Human Factors and Power Plants, IEEE Press, p. 6-25-6-29.

[170.] Hanley, S.S. (1999). A Culture Built on Sharing. Information Week, 731, p. 16-18.

[171.] Harazim, M.L. and Ferguson, B.J. (2004). Equipment Reliability Process Based on the Institute of Nuclear Power Operations (INPO) AP-913, Guidelines in Conjunction with a Preventive Maintenance Living Program, Framatome ANP.

[172.] Harris, R. (2004). Nuclear Facility Configuration Management Survival Guide 2004, Configuration Management Benchmarking Group.

[173.] Haugh, J., Naser, J. and Parkinson, W. (2004). Enterprise Knowledge Management System Utility Requirements Document for Nuclear Power Plants, Knowledge Conservation and Transfer. EPRI.

[174.] Hedlund, G. (1994). A Model of Knowledge Management and the N-Form Corporation. Strategic Management Journal, Vol. 15, p. 73-90.

[175.] Heinrichs, J.H. (2001, May). Interaction Effects of Strategic Decision Models and Business Intelligence Tools on Knowledge Generation in Manufacturing Firms, The University of Toledo.

[176.] Heisig, P., Mertins, K. and Vorbeck J. (2000) Knowledge Management. Concepts and Best Practices in Europe. Journal of Knowledge Management, Vol.4, No. 2, pp 87-98

[177.] Hendriks, P. (1999). Why share knowledge? The Influence of ICT on the Motivation for Knowledge Sharing, John Wiley \& Sons, Ltd.

[178.] Hendriks, P.H.J. (2000). Many rivers to cross: from ICT to knowledge management systems, Journal of Information Technology.

[179.] Herschel, R.T., Nemati, H., Steiger, D. and Iyer, L. (2000, Jan-Feb). Using SOAP - How Knowledge Exchange Protocols Improve KM Performance, St. Joseph's University, Issue 12.

[180.] Hildebrand, C., (2001). Making KM pay off. In: CIO; In: Knowledge and Process Management, 2000, 8(2), April/June 2001

[181.] Hill, J. (2001). Management Systems: The Driver of Work Systems Performance, Nuclear Management. Co., p. 607. 
[182.] Hitt, M. and Ireland, D. (1995). Corporate Distinctive Competence, Strategy, Industry and Performance. Strategic Management Journal, 6.

[183.] Hoffman, J.J., Hoelscher, M.L. and Sherif, K. (2005). Social capital, knowledge management, and sustained superior performance, Journal of Knowledge Management, 9, 3.

[184.] Hoffman, R. and Hanes, L.F. (2002). The Boiled Frog Problem. IEEE Computer Society.

[185.] Hoffman, R.R. et al., (1992). Eliciting Knowledge from Experts: A Methodological Analysis. Organizational Behavior and Human Decision Processes, V62, p. 129-158.

[186.] Holsapple, C.W. (2005). The inseparability of modern knowledge management and computer-based technology, Journal of Knowledge Management.

[187.] Hoyle, R.M. [Ed.]. (1995). Structural Equation Models: Concepts, Issues and Applications, Thousand Oaks, CA: Sage Publications.

[188.] Huber, G.P. (1991). Organizational Learning: The Contributing Processes and Literatures. Organizational Science, 2(1), p. 88-115.

[189.] Huber, G.P. (1999). Facilitating Project Team Learning and Contributions to Organizational Knowledge, Creativity \& Innovation Management.

[190.] Huber, G. and Daft, R. (1987). The Information Environment of Organizations. In F. Jablin, Ed., Handbook of Organizational Communication, Newbury Park, CA. Sage Pub.

[191.] Hull, R., Coombs, R. and Peltu, M. (2000). Knowledge management practices for innovation: an audit for improvement, International Journal of Technology Management, 20 (5/6/7/8), p. 633-656.

[192.] Hwang, Y. (2005). Investigating enterprise systems adoption; uncertainty avoidance, intrinsic motivation, and the technology acceptance mode, European Journal of Information Systems.

[193.] Hyoung K.M. and Moon S.P. (2002. Jan-Feb). Effective Reward Systems for Knowledge Sharing, Knowledge Management Review.

[194.] IAEA (1999). Evaluating and Improving Nuclear Power Plant Operating Performance. TECDOC-1098. Vienna.

[195.] IAEA (2004). Managing Nuclear Knowledge: Strategies and Human Resource Development. Summary of International Conference, Saclay, September.

[196.] IAEA (2006a). Economic Performance Indicators for Nuclear Power Plants. Technical Series Report 437. Vienna.

[197.] IAEA (2006c). The Management System for Facilities and Activities, IAEA Safety Standards Series No. GS-R-3, IAEA, Vienna.

[198.] IAEA (2006d). Application of the Management System for Facilities and Activities, IAEA Safety Standards Series No. GS-G-3.1, IAEA, Vienna.

[199.] IAEA (2008). Planning and Execution of Knowledge Management Assist Missions for Nuclear Organizations. May 2008. (co-authored by J. de Grosbois).

[200.] IAEA and OECD/Nuclear Energy Agency (2008) - Using Operational Experience to Improve Safety, The IAEA/NEA Incident Reporting System. 
Download Aug. 2008 from IAEA at: http://www.iaea.org/Publications/Booklets/IaeaNea/iaeanea-irs.html

[201.] IAEA Guideline (2006). Competency Assessments for Nuclear Industry Personnel. Vienna.

[202.] IAEA Nuclear Economic Performance International System (NEPIS)

[203.] IAEA Safety Guide No. NS-G-2.4. (2001). The Operating Organization for Nuclear Power Plants. Vienna.

[204.] IAEA Safety Standards. (2006, July). A System for the Feedback of Experience from Events in Nuclear Installations.

[205.] IAEA TECDOC (2005, Nov-Dec). Nuclear Knowledge Loss Risk Assessment.

[206.] IAEA TECDOC 1098 (1999, July). Evaluating and Improving Nuclear Power Plant Operating Performance.

[207.] IAEA TECDOC 1204. (2001, March) A Systematic Approach to Human Performance Improvement in Nuclear Power Plants: Training Solutions.

[208.] IAEA TECDOC 1470. (2005b, October). Assessment and Management of Ageing of Major Nuclear Power Plant Components Important to Safety: BWR Pressure Vessels.

[209.] IAEA TECDOC 1490. (2006). Indicators for Management of Planned Outages in Nuclear Power Plants. Vienna.

[210.] IAEA TECDOC 1510 (2006b). Knowledge Management for Nuclear Industry Operating Organizations. IAEA, Vienna.

[211.] IAEA TECDOC-1209. (2001b, April). Risk Management: A Tool for Improving Nuclear Power Plant Performance, IAEA.

[212.] IAEA TECDOC-1393. (2004, May). International Outage Coding System for Nuclear Power Plants.

[213.] IAEA Technical Report Series No. 406. (2002). Developing and Economic Performance System to Enhance Nuclear Power Plant Competitiveness.

[214.] IAEA-TECDOC-1305. (2002b, August). Safe and Effective Nuclear Power Plant Life Cycle Management Towards Decommissioning.

[215.] INPO (Institute of Nuclear Power Operators) (2007, April). Featured Topic Engineering Fundamentals. Engineering Digest.

[216.] INPO AP-913. (2001, November). Equipment Reliability Process Description.

[217.] Ittner, C.D. and Larcher, D.F. (1998). Innovations in Performance Measurement: Trend and Research Implications. Journal of Management Accounting Research. Vol. 10.

[218.] Jantunen, A. (2005). Knowledge-processing capabilities and innovative performance: an empirical study, European Journal of Innovation Management, Vol. 8, No. 3.

[219.] Janz, B. and Prasarnphanich, P. (2003, Spring). Understanding the Antecedents of Effective Knowledge Management: The Importance of a KnowledgeCentered Culture, Decision Sciences.

[220.] Jennex, M. and Olfman, L. (2002). Organizational Memory/knowledge Effects on Productivity, A Longitudinal Study. Proceedings of the 35th Hawaii International Conference on System Sciences, p. 1-10. 
[221.] Jennex, M.E. and Olfman, L. (2002). Organizational Memory/Knowledge Effects on Productivity, a Longitudinal Study, San Diego State Univ. \& Claremont Graduate Univ.

[222.] Jennex, M.E. and Olfman, L. (2002). Organizational Memory/Knowledge Effects on Productivity, a Longitudinal Study, San Diego State Univ. \& Claremont Graduate Univ.

[223.] Jennex, M.E., Smolnik, S. and Croasdell, D. (2008). Towards Measuring Knowledge Management Success. Proceedings of the 41st Hawaii International Conference on System Sciences. IEEE. pp.1530-1605/08

[224.] Jie, Y. (2001, June). The Impact of Knowledge Management on the Performance of New Product Development, The Chinese University of Hongkong.

[225.] Jobson, J.D. (1999). Applied Multivariate Data Analysis. Vol. 1. Regression and Experimental Design. Springer.

[226.] Johannessen, J.A., Olaisen, J., and Olsen, B. (1999). Strategic Use of Information Technology for Increased Innovation and Performance. Info Mgmt and Computer Security, 7(1), p5-22.

[227.] Johnson, W.H.A. (2000, July). Technological Innovation and Knowledge Creation: A Study of the Enabling Conditions and Processes of Knowledge Creation in Collaborative R\&D Projects, Graduate Program at the Schulich School of Business, York Univ.

[228.] Jones, B. (2003, August). Learning Organizations for Nuclear Safety Theoretical Approaches to Organizational Learning. EC 5th Euratom Framework Programme. LearnSafe Project.

[229.] Jones, M.C., Cline, M. and Ryan, S. (2003, January). Exploring Knowledge Sharing in ERP Implementation: an Organizational Culture Framework, University of Texas.

[230.] Jones, M.C., Cline, M., and Ryan, S. (2006). Exploring knowledge sharing in ERP implementation: an organizational culture framework. V41, p. 411-434.

[231.] Junnarkar, B. and Brown, C.V. (1997). Re-Assessing the Enabling Role of Information Technology in KM, Journal of Knowledge Management.

[232.] Kailing, T. (2003). Knowledge management and the occasional links with performance, Journal of Knowledge Management.

[233.] Kandemir, D. and Hult, G.T.M. (2005). A conceptualization of an organizational learning culture in international joint ventures, Science Direct.

[234.] Kaner, M. and Karni, R. (2003). A Capability Maturity Model for KnowledgeBased Decision making, Faculty of Industrial Engineering and Management Technion, Haifa 32000, Israel, Information Knowledge Systems Management 4, 225-252 IOS Press.

[235.] Kankanhalli, A. and Tan, B.C.Y. (2004). A Review of Metrics for Knowledge Management Systems and Knowledge Management Initiatives, Dept. of Information Systems, National University of Singapore.

[236.] Kanter, R.M. (1983). The Change Masters. Simon and Schuster, New York. 
[237.] Kaplan, R. and Norton, D. (1996). The Balanced Scorecard, Harvard Business School Press, Boston, MA.

[238.] Kaplan, R.S. and Norton, D.P. (1992). The Balanced Scorecard - measures that drive performance, Harvard Business Review, 70, p. 71-9.

[239.] Karns, P. (2005, Jan-Feb). MRO Software - A Model for Improving Performance, Nuclear Plant Journal; 23, 1; ABI/INFORM Global, p. 30.

[240.] Ke, W. and Wei K. (2006, Jan-Mar). Organizational Learning Process: Its Antecedents and Consequences in Enterprise System Implementation, Journal of Global Information Management.

[241.] Ke, W. and Wei K. (2006, Jan-Mar). Organizational Learning Process: Its Antecedents and Consequences in Enterprise System Implementation, Journal of Global Information Management.

[242.] Kerlinger, F.N. and Lee, H.B. (2000). Foundations of Behavioral Research, Orlando, FL: Harcourt College Publisher.

[243.] Kern, D.R. and Anderson, J.A. (1988). Project Management For Operating Facilities, American Association of Cost Engineers, p. H.5.1

[244.] Kerssens-van Drongelen, I.C., and Cook, A. (1997). Design Principles for the Development of Measurement Systems for Research and Development Processes. R\&D Management. V27(4). p. 345-357.

[245.] Keskin, H. (2005, Sep). The Relationship between Explicit and Tacit Oriented KM Strategy, and Firm Performance, Journal of American Academy of Business, Cambridge.

[246.] Kiehl, J.K. (2004, May). Learning to Change: Organizational Learning and Knowledge Transfer, Case Western Reserve University.

[247.] Kim, D. (1993). The Link Between Individual and Organizational Learning. Sloan Management Review. 35(1), p. 37-50.

[248.] Kim, J. and King. (2004). John-Managing knowledge work: specialization and collaboration of engineering problem-solving, Journal of Knowledge Management.

[249.] Kim, S. and Lee, H. (2006, May-June). The Impact of Organizational Context and Information Technology on Employee Knowledge-Sharing Capabilities, Public Administration Review.

[250.] King, W.R. (2006, Jan-Mar). The Critical Role of Information Processing in Creating an Effective Knowledge Organization; University of Pittsburgh, USA, 17, 1; p. 1.

[251.] King, W.R. (2006, Winter). Maybe A “Knowledge Culture" Isn't Always So Important After All; Information Systems Management; 23, 1; p. 88.

[252.] Klein, D. and Prusak, L. (1994). Characterizing Intellectual Capital. CBI Working Paper. Ernst and Young. Boston.

[253.] Klein, G. (1992). Using Knowledge Engineering to Preserve Corporate Memory, The Psychology of Expertise: Cognitive Research and Empirical AI, R.R. Hoffman, ed., Erlbaum, p. 170-190. 
[254.] Klimecki, R. and Lassleben, H. (1998, Dec). Modes of Organizational Learning: Indications from an Empirical Study. Management Learning. 29(4), p. 405-430.

[255.] Knapp (1998, Jul-Sep). Knowledge Management. Business and Economic Review. p. 3-6.

[256.] Kock, N. Jr., McQueen, R. and Baker, M. (1996). Learning and process improvement in knowledge organizations: a critical analysis of four contemporary myths, The Learning Organization, Vol. 3, No. 1.

[257.] Komanoff, C. and Roelofs, C. (1994, Dec). Predicting Nuclear Plant, Public Utilities Fortnightly; 132,22; ABI/INFORM Global, p. 28.

[258.] Koskinen, K.U. (2003). Evaluation of tacit knowledge utilization in work units Journal of Knowledge Management; Volume: 7 Issue: 5.

[259.] Koskinen, K.U., Pihlanto, P. and Vanharanta, H. (2003). Tacit Knowledge Acquisition and sharing in a project work context, International Journal of Project Management 21.

[260.] Kotlarsky, J. and Oshri, I. (2005). Social ties, knowledge sharing and successful collaboration in globally distributed system development projects, European Journal of Information Systems.

[261.] Kotter, J. and Heskett, J. (1992). Corporate Culture and Performance, Free Press, New York, NY.

[262.] Kuehn, S.E. (1992, Aug). Reliability-Centered Maintenance Trims Nuclear Plant Costs, Power Engineering; 96,8; ABI/INFORM Global, p. 23.

[263.] Kuethe, J.L. (1959). The positive response set as related to task performance. Journal of Personality. The Johns Hopkins University. V27(1), pg 87-94

[264.] Kwan, M. and Cheung, P. (2006, Jan-Mar). The Knowledge Transfer Process: From Field Studies to Technology Development, Journal of Database Management.

[265.] Kwok, J.S.H. and Gao, S. (2004). Knowledge sharing community in P2P network: a study of motivational perspective. Journal of Knowledge Management, Vol. 8, No.1, p. 94-102.

[266.] Laitinen, E.K.A. (2002). Dynamic performance measurement system: evidence from small Finnish technology companies. Scandinavian Journal of Management Volume: 18, Issue: 1, p. 65-99.

[267.] Landry, J.R. (1997). If an Organization has a Memory Will It Need A Therapist? Metropolitan State College of Denver, Colorado.

[268.] Lank, E. (1997). Leveraging Invisible Assets: The Human Factors. Long Range Planning, 30, p. 406-412.

[269.] Lebas, M.J. (1995). Performance measurement and performance management, International Journal of Production Economics, 41, 1/3, p. 23-35.

[270.] Lee Klein, R., Bigley, G.A. and Roberts, K.H. (1995, July). Organizational Culture in High Reliability Organizations: An Extension; Human Relations; 48, $7 ;$ p. 771 .

[271.] Lee, K.C., Lee, S. and Kange, I.W. (2005). KMPI: measuring knowledge management performance, Information \& Management, 42. 
[272.] Lee, T-C and OuYang, Y-C (2010). The Knowledge Process Chain - How Knowledge Management Capability Impacts on Business Performance. Dept. of Industrial Technology Education, National Kaohsiung Normal University, Taiwan.

[273.] Leo, C., Sicard, Y. and Bartak, J. (2000). Simulation in Professional Industrial e-Training Applications. CORYS T.E.E.S. Grenoble France.

[274.] Leong, G.K., Snyder, D.L., and Ward, P.T. (1990). Research in the Process and Content of Manufacturing Strategy". OMEGA International Journal of Management Science. V.18 (2). p. 109-122.

[275.] Leseure, M.J. and Brookes, N.J. (2004). Knowledge Management Benchmarks for Project Management, Journal of Knowledge Management; 8, 1; ABI/INFORM Global, p. 103.

[276.] Lester, R.K. and McCabe, M. (1993). The Effect of Industrial Structure on Learning By Doing in Nuclear Power Plant Operation, The Rand Journal of Economics, p. 418.

[277.] Levine, S. (2006). High performance organizations: creating a culture of agreement. Handbook of Business Strategy. V7(1) p. 375 - 380.

[278.] Levinthal, D.A. and Claus R. (2004, Aug). Crossing an Apparent Chasm: Bridging Mindful and Less-Mindful Perspectives on Organizational Learning.

[279.] Levitt, B. and March, J. (1988). Organizational Learning. Scott and Blake, Eds. In Annual Review of Sociology, 14, p. 319-340.

[280.] Lewis, K. (2004, Nov). Knowledge and Performance in Knowledge-Worker Teams: A Longitudinal Study of Transactive Memory Systems, Management Science.

[281.] Li, X., Shi, Y., Lui, Y., Li, J., and Li, A. (2006). A Knowledge Management Platform for Optimization-based Data Mining. Sixth IEEE International Conference on Data Mining - Workshops (ICDMW'06), p. 833-837.

[282.] Liebowitz, J. et al. (2000). The Knowledge Audit, Knowledge and Process Management. Vol. 7, Issue 1, p. 3-10.

[283.] Lim, K.K. (1990). Managing for Quality Through Knowledge Management. Total Quality Management, 10(4), p. 615-622.

[284.] Liu, S.C. (2003). A Study of Factors that Facilitate Use of Knowledge Management Systems and the Impact of Use on Individual Learning, ProQuest Information and Learning Company.

[285.] Loermans, F. (2002). Synergizing the learning organization and knowledge management, Journal of Knowledge Management.

[286.] Lohr, S. (1999). Determining the Desired Sample Size, Sampling: Design \& Analysis, Chapter 2.

[287.] Lopez Perez, S., Montes P., Jose M., Vasques, O. and Camilio J. (2004). Managing Knowledge: The Link Between Culture and Organizational Learning; Univ. of Oviedo, Spain; 8,6; p. 93.

[288.] Lopez, S.P., Peon, J.M.M.P. and Ordas, C.J.V. (2004). Managing knowledge: the link between culture and organizational learning, Journal of Knowledge Management. 
[289.] Lowthert, W.H. (1995, Oct 30-Nov 9). Systematic Approach to Training Programs for Operations Personnel in the United States of America. Presented at the National Seminar on the Systematic Approach to Personnel Training and Qualifications for Safe Operations of Nuclear Power Plants. IAEA Workshop. Daya Bay Nuclear Station, People's Republic of China.

[290.] Lowthert, W.H.I.I.I. (1996, May). The Relationship between the Implementation of High Performance Work Practices and Nuclear Power Plant Performance, the Pennsylvania State Univ.

[291.] Lusignan, R.P. (2002). Enhancing the implementation and use of Groupware technologies and knowledge management practices: The integration of a technology-enables change process through the examination of cognitive frames, Boston Univ., School of Education.

[292.] Lynn, B.E. (1998). Performance evaluation in the new economy: bringing the measurement and evaluation of intellectual capital into the management planning and control system, International Journal of Technology Management, $16(1 / 2 / 3)$, p. 162-176.

[293.] Lytras, M. and Pouloudi, A. (2003). Project Management as a knowledge management primer: the learning infrastructure in knowledge-intensive organizations: projects as knowledge transformations and beyond, The Learning Organization.

[294.] MacCallum, R.C., Widaman, K.F., Preacher, K.J., and Hong, S. (2001). Sample Size in Factor Analysis: The Role of Model Error. Mutlivariate Behavioral Research. 36(4), pp. 611-637.

[295.] Maier R. and Remus, U. (2002). Defining Process-oriented Knowledge Management Strategies. Knowledge and Process Management, 9(2): p. 103118.

[296.] Maier, R., and Remus, U. (2003). Implementing Process Oriented Knowledge Management Strategies. Journal of Knowledge Management. V7(4).

[297.] Makis, V., Jiang, X. and Cheng, K. (2000, Feb). Optimal Preventive Replacement Under Minimal Repair and Random Repair Cost, Mathematics of Operations Research, Vol. 25, No. 1.

[298.] Malhotra, A. (1997). The Measurement of Organizational Knowledge: A Review of Approaches Taken in Management Research. Conference Proceedings of the Academy of Management. Boston, MA.

[299.] Malhotra, Y. (1998). Knowledge Management, Knowledge Organizations and Knowledge Workers: A View from the Front Lines. Mail Business Newspaper.

[300.] Malhotra, Y. (2005). Integrating knowledge management technologies in organizational business processes: getting real time enterprises to deliver real business performance, Journal of Knowledge Management.

[301.] Malhotra, Y. (2005). Integrating knowledge management technologies in organizational business processes: getting real time enterprises to deliver real business performance, Journal of Knowledge Management.

[302.] March, J. (1991). Exploration of Exploitation in Organizational Learning. Organization Science, 2. 
[303.] Marques, D.P. and Simon, F.J.G. (2006). The effect of knowledge management practices on firm performance, Journal of knowledge Management.

[304.] Marr, B., Gray, D. and Neely, A. (2003). Why do firms measure their intellectual capital? Journal of Intellectual Capital, 4 (4), p. 441-464.

[305.] Marshall, M. (1987). Web-based Management Launched. Computer Reseller News, Sept., (754), p. 88-89.

[306.] Marsick, V.J. and Watkins, K.E. (2003). Demonstrating the Value of an Organization's Learning Culture: The Dimensions of the Learning Organization Questionnaire, Advances in Developing Human Resources.

[307.] Marti, J.M.V. (2001). ICBS - intellectual capital benchmarking system, Journal of Intellectual Capital.

[308.] Marti, J.M.V. (2001). ICBS - Intellectual Capital Benchmarking System, Journal of Intellectual Capital; 2,2; ABI/INFORM Global, p. 148.

[309.] Marti, J.M.V. (2004). Strategic knowledge benchmarking system (SKBS): a knowledge-based strategic management information system for firms, Journal of Knowledge Management.

[310.] Marti, J.M.V. (2004). Strategic knowledge benchmarking system (SKBS): a knowledge-based strategic management information system for firms, Journal of Knowledge Management.

[311.] Martin, B. (2000). Knowledge Management Within the Context of Management: An Evolving Relationship, Singapore Management Review; 22, 2; ABI/INFORM Global, p. 17.

[312.] Mason, D. and Pauleen, D.J. (2003). Perceptions of Knowledge Management: A Qualitative Analysis; Victoria Univ. of Wellington, New Zealand; 7,4; p. 38.

[313.] Mason, R.M. (2003, Oct-Dec). Culture-Free or Culture-Bound? A Boundary Spanning Perspective on Learning in Knowledge Management Systems; Florida State Univ., p. 20-36.

[314.] McCann, J.E. and Buckner, M. (2004). Strategically integrating knowledge management initiatives, Journal of Knowledge Management.

[315.] McDermott, R. (1999). Why information technology inspired but cannot deliver knowledge management", California Management Review, Vol. 41, p. 103-17.

[316.] McDougall, G. and Hinks, J. (2000). Identifying priority issues in facilities management benchmarking, Facilities.

[317.] McElroy, M. (2003). The New Knowledge Management, Complexity, Learning, and Sustainable Innovation. Butterworth-Heinemann, Burlington, MA. VOL. 10 NO.

[318.] McElroy, M.W. (2002). Complexity, Learning, and Sustainable Innovation. Knowledge Management Consortium International (KMCI), Windsor, VT.

[319.] McGee, J. and Prusak, L. (1993). Managing Information Strategically. New York. Wiley \& Sons.

[320.] Meador, R.J. Maintaining the Solution to Operations and Maintenance Efficiency Improvement, Pacific Northwest Laboratory, Safety \& Analysis Dept., Washington, Contract No. DE-AC06-76RLO 1830. 
[321.] Meckler, M. (2001). Influences on the Performance of Organization Knowledge Transfer. Florida Atlantic University, UMI Proquest.

[322.] Mengshoel, O.J. and Delab, S. (1993). Knowledge validation: principles and practice. IEEE Expert, June. p. 62-68.

[323.] Meyer, M.W. and Gupta, V. (1994), The performance paradox, Research in Organizational Behaviour, Vol. 16, p. 309-69.

[324.] Mierzejewska, B. (2004). The second generation KM. Interview with Prof. Mark W. McElroy. Downloaded from Internet website August 2008 at http://www.e-mentor.edu.pl/_xml/wydania/7/93.pdf.

[325.] Mikesell, E.M. (2001). Organizational Intelligence and Vitality: A Systemic Framework for Organizational Renewal. Union Institute. UMI ProQuest.

[326.] Mitchell, H. (2000). Knowledge Sharing: The Value of Storey-telling. International Journal of Organisational Behaviour, Volume 9(5), 632-641 ISSN 1440-5377.

[327.] Moffet, S., McAdam, R. and Parkinson, S. (2003). An Empirical Analysis of Knowledge Management Applications; Univ. of Ulster at Magee, Northern Ireland; 7,3; p. 6.

[328.] Mohamed, M., Stankosky, M. and Arthur, M. (2004). Applying knowledge management principles to enhance cross-functional team performance, Journal of Knowledge Management.

[329.] Moore, G.C. and Benbasat, I. (1991). Development of an Instrument to Measure Perceptions of Adopting an IT Innovation, Information System Research. V2 (3), p. 192-222.

[330.] Moore, R. W. (2002, April). Preservation of Data, Information and Knowledge R. Moore, Proceedings of the World Library Summit, Singapore.

[331.] Moseng, B. and Bredrup, H. (1993). A methodology for industrial studies of productivity performance, Production Planning and Control, Vol. 4 No. 3.

[332.] Murray, A.J., and Greenes, K.A. (2006). Building the enterprise of the future. VINE. V36(1). p38-44

[333.] Murray, P. and Blackman, D. (2006). Managing Innovation through Social Architecture, Learning, and Competencies: A New Conceptual Approach, Knowledge and Process Management.

[334.] Mykytyn, P.P., Mykytyn, K. and Raja, M.K. (1994). Knowledge acquisition skills and traits: a self-assessment of knowledge engineers, Information and Management, Vol. 26, p. 95-104.

[335.] Naughton, R.J., Captain, U.S. Navy. (1993). A Nuclear Energy Strategy to Preserve the Industrial Base Into the Twenty-First Century, Executive Research Project F2.

[336.] Naylor, J.B., Griffiths, J. and Naim, M.M. (2001). Knowledge-Based System for Estimating Steel Plant Performance. International J. of Operations and Production Management. V21, N7.

[337.] Neely, A., Gregory, M. and Platts, K. (1995). Performance measurement system design: a literature review and research agenda, International Journal of Production \& Operations Management, Vol. 15, No. 4, p. 80-116. 
[338.] NEI (2003). The Standard Nuclear Performance Model - A Process Management Approach - Revision 4. Nuclear Energy Institute. Washington DC.

[339.] NEI (2003, Dec). Nuclear Asset Management Community of Practice Report. The Standard Nuclear Performance Model - A Process Management Approach. Revision 4. Electrical Utility Cost Group.

[340.] Nelson K. and Cooprider, J. (1996, Dec). The Contribution of Shared Knowledge to IS Group Performance, MIS Quarterly, p. 409.

[341.] Neuman, W. and Robson, K. (2008). Basics of Social Research - Qualitative and Quantitative Approaches. Pearson Canada. Toronto.

[342.] Nilakanta, S., Miller, L.L. and Zhu, D. (2006, Jan-Mar). Organizational Memory Management: Technological and Research Issues, Iowa State University; 17, 1; p. 85.

[343.] Noble, C.H., Sinha, R.K. and Kumar, A. (2002, October). Market Orientation and Alternative Strategic Orientations: A Longitudinal Assessment of Performance Implications; Univ. of Mississippi, 25-39 Journal of Marketing.

[344.] Nonaka, I. (1991). The knowledge creating company. Harvard Business Review, 69(6).

[345.] Nonaka, I. (1994). A dynamic theory of organizational knowledge creation. Organizational Science, 5(1).

[346.] Nonaka, I. and Konno, N. (1998). The concept of "Ba': Building foundation for knowledge creation. California Management Review, 40(3).

[347.] Nonaka, I. and Takeuchi, H. (1995). The Knowledge-creating Company: How Japanese Companies Create the Dynamics of Innovation. Oxford University Press.

[348.] Nonaka, I. and Takeuchi, H. (1995). The knowledge creating company: How Japanese companies create the dynamics of innovation. Oxford: Oxford University Press.

[349.] Nonaka, I. and Takeuchi, H. (1995). The Knowledge-Creating Company, Oxford University Press, New York, NY.

[350.] Nonaka, I. and Takeuchi, H. (1995). The Knowledge-Creation Company: How Japanese Companies Create the Dynamics of Innovation. New York/Oxford: Oxford University Press.

[351.] Nonaka, I., Takeuchi, H. and Umemoto, K. (1996). A Theory of Organizational Knowledge Creation. International Journal of Technology Management. V11(7-8): p. 833-845.

[352.] Novak, J.D. (1995). Concept Mapping: A Strategy for Organizing Knowledge, p. 229-245 in Glynn, S.M. and Duit, R. (eds.), Learning Science in the Schools: Research Reforming Practice, Lawrence Erlbaum Associates, (Mahwah).

[353.] O'Dell, C., Wig, K. and Odem, P. (1999). Benchmarking Unveils Emerging Knowledge Management Strategies. Bradford. V6, N3. p. 202.

[354.] O'Reilly, C., Chatman, J. and Caldwell, D. (1991). People and Organizational Culture: A Profile Comparison Approach to Assessing Person-Organization Fit. Academy of Management Journal, 34: p. 487-516. 
[355.] O'Donnell, F.J. and Duffy, A.H.B. (2002). Modeling design development performance, International Journal of Operations \& Production Management, $22,11,1198-222$.

[356.] OEDC (2000). Nuclear Education and Training - Cause for Concern? A Summary Report Nuclear Energy Agency Organization for Economic Cooperation and Development.

[357.] Olmstead, R. and Didsbury, R. (2002). Slide Presentation: AECL's Engineering Tools. Atomic Energy Canada Ltd., Chalk River Ontario.

[358.] Panayides, P. (2004). The Impact of organizational learning on relationship orientation, logistics service effectiveness and performance, Cyprus International Institute of Management.

[359.] Park, H., Ribiere, V. and Schulte, W.D., Jr. (2004). Critical Attributes of Organizational Culture that Promote Knowledge Management Technology Implementation Success.

[360.] Parker, K. and Chao, J.T. (2007). Wiki as a Teaching Tool. Interdisciplinary Journal of Knowledge and Learning Objects. V3.

[361.] Paulzen, O., Perc, P. (2002). “A Maturity Model for Quality Improvement in Knowledge Management", in: Wenn, A.; McGrath, M.; Burstein, F. (Hrsg.): Enabling Organisations and Society through Information Systems, Proceedings of the 13th Australasian Conference on Information Systems (ACIS 2002), S. 243-253, Melbourne, 2002.

[362.] Pedroni, M. (2007). E-learning and Knowledge Management: Context Structuration. Proceedings of the 2007 Informing Science and IT Education Joint Conference. CARID University of Ferrara, Italy.

[363.] Pemberton, J., Stonehouse, G.H. and Yarrow, D.J. (2001, Apr-Jun). Benchmarking and the Role of Organizational Learning in Developing Competitive Advantage. Knowledge and Process Management. V8, N2, p. 123-135.

[364.] Peteraf, M.A. (1993). The Cornerstones of Competitive Advantage: A Resource-Based View. Journal of Strategic Management, 14(3), p. 179-192.

[365.] Peterson, B.S. (2003). The Future of Asset Management, Strategic Asset Management.

[366.] Petty, R. and Guthrie, J. (2000). Intellectual capital literature review, Measurement, reporting and management, Journal of Intellectual Capital.

[367.] Pham, N.T. and Swierczek. (2006). F.W. Facilitators of organizational learning in design. The Learning Organization, Vol. 13, No. 2, p. 186-201.

[368.] Philip, R.J. et al. (1997, March). Process Cost Reduction Through Proactive Operations and Maintenance, State of the Art Report, Food Mfg. Coalition for Innovation \& Technology Transfer.

[369.] Pinto, M.B. and Pinto, J.K. (1990). Project Team Communication and Crossfunctional Cooperation in New Program Development. J. of Product Innovation Mgmt. 7, p200-212. 
[370.] Pinto, M.B., Pinto, J.K. and Prescott, J.E. (1993). Antecedents and Consequences of Project Team Cross-Functional Cooperation, Management Science.

[371.] Platek, R., Pierre-Pierre, F.K. and Stevens, P. (1985). Development and Design of Survey Questionnaires. Statistics Canada Catalogue, No. 12-519E.

[372.] Polanyi, K. (1967). The Great Transformation, Boston, Beacon Press.

[373.] Politis, J.D. (2003). The connection between trust and knowledge management: what are its implications for team performance, Higher Colleges of Tech, Dubai, United Arab Emirates.

[374.] Politis, J.D. (2005). The influence of managerial power and credibility on knowledge acquisition attributes. Leadership \& Organization Development Journal. V26, No. 3, p. 197-214.

[375.] Ponzio, F. (1989). Operating Plant Planning and Scheduling - Back to Basics, Amer. Assoc. of Cost Engineers, p. D.2.1.

[376.] Porter, M.E. (1980). Industry Structure and Competitive Strategy: Keys to Profitability. Financial Analysts Journal, 36(4), p. 30.

[377.] Predicting Nuclear Plant Capacity Factors, Public Utilities Reports, Dec. 01, 1994

[378.] Probst, G. (2002). Managing Knowledge, Building Blocks for Success. ISBN 0471-99768-4. Wiley. West Sussex, England.

[379.] Pyoria, P. (2005). Information technology, human relations and knowledge work teams, Team Performance Management.

[380.] Quick, J.C. (1992). Crafting an organizational culture: Herb's hand at Southwest Airlines, Organizational Dynamics, V21, No.2, p. 45-56.

[381.] Quinn, J.B. (1992). Intelligent Enterprise: A Knowledge and Service Based Paradigm for Industry. New York: The Free Press.

[382.] Quinn, J.B., Anderson, P. and Finkelstein, S. (1996). Leveraging Intellect. Academy of Management Executive. V10(3): p. 7-27.

[383.] Rastogi, P.N. (2000). Knowledge Management and Intellectual Capital - The new virtuous reality of competitiveness, Human Systems Management.

[384.] Reed, K.K. (2000). The Dynamics of Intellectual Capital. University of Connecticut. UMI Proquest.

[385.] Rempel, J., Holmes J. and Zanna, M. (1985). Trust in Close Relationships. Journal of Personality and Social Psychology. V49(1), p. 95.

[386.] Rolstadas, A. (1998). Enterprise performance measurement, International Journal of Operations \& Production Management, V18, No. 9/10, p. 989-99.

[387.] Roos, J., Roos, G., Dragonetti, N.C. and Edvinsson, L. (1997). Intellectual capital: navigating in the new business landscape, London: Macmillan.

[388.] Rothwell, G. and Maidment, J. (1998, April). All Nuclear Power Plants Are Not Created Equal, Public Utilities Reports.

[389.] Sackett, P. and Larson, J. Jr. (1992). Research Strategies and Tactics in Industrial and Organizational Psychology. In M.D. Dunnette and L.M. Hough, eds, Handbook of Industrial Organizational Psychology. P 419-489. Palo Alto CA. Consulting Psychologists Press. 
[390.] Sanchez, R. (1997). Managing Articulated Knowledge in Competence-based Competition. In Strategic Learning and Knowledge Management. Sanchez and Heene (Eds). Wiley and Sons, NY.

[391.] Sanchez, R. and Heene, A. (1997). A Competence Perspective on Strategic Learning and Knowledge Management. In Strategic Learning and Knowledge Management. Sanchez and Heene (Eds). Wiley and Sons, NY.

[392.] Sanchez, R., Heene, A. and Thomas, H. (1996). Dynamics of Competencebased Competition: Theory and Practice in the New Strategic Management. Oxford. Elsevier.

[393.] Sasson, J.R. and Douglas, I. (2006). A conceptual integration of performance analysis, knowledge management, and technology: from concept to prototype, Journal of Knowledge Management.

[394.] Satorra, A. and Bentler, P.M. (2001). A scaled difference chi-square test statistic for moment structure analysis, Psychometrika, 66, 507-514.

[395.] Schack, T. (2004). Knowledge and performance in action, Journal of Knowledge Management.

[396.] Schaffert, S. (2008). IkeWiki: A Semantic Wiki for Collaborative Knowledge Management. Salzburg Research, Forschungsgesellschaft / Salzburg New Media Lab, Salzburg, Austria.

[397.] Schein, E. (1990). Organizational Culture. American Psychologist, V45(2), p. 109.

[398.] Schinzel, G. (2002, March). Fine Tuning Maintenance Priorities Boosts South Texas Project to Record Performance Levels. Power Engineering. p. 43.

[399.] Schuman, H. and Presser, S. (1996). Questions and Answers in Attitude Surveys: Experiments on Question Form, Wording and Context. Sage Publications, Thousand Oaks, California.

[400.] Schutt, R.K. (2001). Investigating Social World. (Third Ed.). Thousand Oaks, Ca.: Pine Force Press.

[401.] Selamat, H.M. and Choudrie J. (2004). The Diffusion of Tacit Knowledge and its Implications on Information Systems: The Role of Meta-Abilities; Brunel Univ.

[402.] Senge, P. (1999). The dance of change: Mastering the twelve challenges to change in a learning organization. New York: Doubleday.

[403.] Sewell, R.T., Kuritzky, A.S., and Khatib-Rahbar, M. (1997). Guidance on the Implementation of a Risk-based Safety Performance Monitoring System for Nuclear Power Plants.

[404.] Shankar, R. and Gupta, A. (2005). Towards Framework for Knowledge Management Implementation. Knowledge and Process Management, Volume 12, Number 4.

[405.] Sharrat, M., and Usoro, A. (2003). Understanding Knowledge-Sharing in Online Communities of Practice. University of Paisley, Scotland. Academic, Conferences Limited.

[406.] Shrivastava, P. (1983). A Typology of Organizational Learning Systems. Journal of Management Studies. V20(1). 
[407.] Simo, A. and Nyobe, J.B. (2004). Human Resource Development Progress to Sustain Nuclear Science and Technology Applications. IAEA-CN-123/04/P/22. Energy Research Laboratory.

[408.] Sinclair, D. and Zairi, M. (1995). Effective process management through performance measurement: Part 3 - in integrated model of total quality-based performance measurement, Business Process Re-engineering and Management Journal, V1, No. 3, p. 50-65.

[409.] Sink, D.S. (1985). Productivity Management: Planning, Measurement, and Evaluation, Control, and Improvement, John Wiley \& Sons, New York, NY.

[410.] Sink, D.S., Tuttle, T.C. and DeVries, S.J. (1984). Productivity Measurement and Evaluation: What Is Available?, National Productivity Review, 4, 3, 265387.

[411.] Sink, S. and Tuttle, T. (1989). Planning and Measurement in your Organization of the Future, Industrial Engineering and Management Press, Norcross, GA.

[412.] Siu, L.H. (2007). Shared vision: a development tool for organizational learning. Development and Learning Organizations, V21, No. 4, p. 12-13.

[413.] Skyrme, D.J. and Amidon, D.M. (1997). Creating the Knowledge-Based Business. London: Business Intelligence.

[414.] Small, C.T. (2005). An Enterprise Knowledge-Sharing Model: A Complex Adaptive Systems Perspective on Improvement in Knowledge Sharing, ProQuest Information \& Learning Company.

[415.] Snyman, R. and Kruger, C.J. (2004). The interdependency between strategic management and strategic knowledge management, Journal of Knowledge Management, V8, No. 1.

[416.] Soliman, F. and Youssef, M. (2003). The role of critical information in enterprise knowledge management, Industrial Management \& Data Systems, 103 (7), 484-90.

[417.] Sørensen, C. and Snis, U. (2001). Innovation through Knowledge Codification. Special issue on Knowledge Management of Journal of Information Technology, V16(2): p83-97.

[418.] Staab, S., Studer, R., Schnurr, H.P. and Sure, Y. (2001, Jan-Feb). Knowledge Processes and Ontologies. IEEE Intelligent Systems.

[419.] Stainer, A. and Nixon, B. (1997). Productivity and Performance Measurement in R\&D. Technology Management. V13, No. 5/6.

[420.] Stake, R. (1995). The art of case research. Newbury Park, CA: Sage Publications.

[421.] Starns, J. and Odom, C. (2006). Using knowledge management principles to solve organizational performance problems; The Journal of information and knowledge management systems.

[422.] Stata, R. (1989). Organizational Learning - the Key to Management Innovation. Sloan Management Review. V17(1), p. 63.

[423.] Stein, E.W. and Zwass, V. (1995). Actualizing Organizational Memory with Information Systems. Information Systems Research, 6(2), p. 85-117. 
[424.] Stein, P.K. (1964). Measurement Engineering: Volume 1: Basic Principles. 5th Ed. Imperial Litho, Phoenix AR.

[425.] Stevens, J.M. and McGowan, R.P. (1985). Information Systems and Public Management, published by Praeger Scientific, New York.

[426.] Stewart, T.A. (1997). Intellectual Capital; The New Wealth of Organizations, Doubleday/Currency, New York, NY.

[427.] Stricker, L. (2003). Safety and Competitiveness. Nuclear Plant Journal, V21, No. 1.

[428.] Sussman, S.W. and Siegal, W.S. (2003). Informational Influence in Organizations: An Integrated Approach to Knowledge Adoption, Information Systems Research.

[429.] Sveiby, K.E. (1997). The New Organizational Wealth: Managing \& Measuring Knowledge-Based Assets, Berret-Koehler Publishers, San Francisco, CA.

[430.] Syed-Ikhsan O.S. and Rowland, F. (2004). Knowledge Management in a Public Organization; A Study on the Relationship Between Organizational Elements and the Performance of Knowledge Transfer.

[431.] Syed-Ikhsan, O.S. and Rowland, F. (2004). Knowledge management in a public organization: a study on the relationship between organizational elements and the performance of knowledge transfer, Journal of Knowledge Management.

[432.] Szulanski, G. (1996). Exploring Internal Stickiness: Impediments to the Transfer of Best Practice within the Firm. Strategic Management Journal, V17, p. $27-43$.

[433.] Tannenbaum S.I., and Alliger, G.M. (2000). Knowledge Management : Clarifying the Key Issues. ISBN 0967923913. IHRIM.

[434.] Thomas, D. and Keithley, T. (2000). Knowledge Management Improves Performance. AACE International Transactions.

[435.] Thompson, R.L., Higgins, C.A. and Howell, J.M. (1991). Personal Computing: Toward A Conceptual Model of Utilization. MIS Quarterly, 15(1), p. 125-143.

[436.] Tippins, M.J. and Sohi, R.S. (2003, August). IT Competency and Firm Performance: IS Organizational Learning a Missing Link? Strategic Management Journal.

[437.] Totalkm.com website - Corporate Knowledge Management, Human Resource Development for Effective Knowledge Management.

[438.] Treasury Board of Canada Secretariat (2002). Succession Planning for Corporate Knowledge Transfer - A Guide for Managers and Human Resource Specialists. Available online at: http://dsp-psd.pwgsc.gc.ca/Collection/BT2284-2002E.pdf

[439.] Uebel, M.F., Dangelmaier, W., Brinker, D. and Helmke, S. (2001). Using CBT for organizational knowledge management. Management of Engineering and Technology, 2001. PICMET apos;01. Portland International Conference on Volume 1, Page(s):112, vol.1.

[440.] Van Beveren, J. (2002). A Model of Knowledge Acquisition that Refocuses Knowledge Management, Journal of Knowledge Management, 6(1), (2002) p. 18-22. 
[441.] Van Beveren, J. (2002). A model of knowledge acquisition that refocuses knowledge management. Journal of Knowledge Management. V6(1), p. 18-22.

[442.] Van den Hooff, B. and de Ridder, J.A. (2004). Knowledge sharing in context: the influence of organizational commitment, communication climate and CMC (computer mediated communication) use on knowledge sharing, Journal of Knowledge Management.

[443.] Van der Vegt, G.S. and Bunderson, J. (2005). Stuart-Learning and Performance in Multidisciplinary Teams: The Importance of collective team identification, Academy of Management Journal.

[444.] Verkasalo, M. and Lappalainen, P. (1998, November). A method of measuring the efficiency of the knowledge utilization process. IEEE Transactions on Engineering Management, V45, No. 4, p. 414-23.

[445.] Verma, K., Mitnick, B.M. and Marcus, A.A. (1999, July). Making Incentive Systems Work: Incentive Regulation in the Nuclear Power Industry, Journal of Public Adm. Research \& Theory; 9,3: p. 395.

[446.] Wahlstrom, B. and Rollenhagen, C. (2004, September). Issues of Safety Culture; Reflections from the Learnsafe Project, VTT Industrial Systems \& SwedPower $A B$.

[447.] Wang, H. and Pham H. (1999). Some Maintenance Models and Availability with Imperfect Maintenance in Production Systems, Annals of Operations Research; 91, p. 305.

[448.] WANO (World Association of Nuclear Operators) (2005). 2005 Performance Indicators. WANO Coordinator Centre, London, UK.

[449.] WANO (2006). Principles for a Strong Nuclear Safety Culture. World Assoc. of Nuclear Operators.

[450.] WANO (2010). WANO Performance Indicator Programme Reference Manual. World Assoc. of Nuclear Operators.

[451.] Watkins, P.N. (2001). The Persistence of Learning and Acquisition Strategies. Acquisition Review Qtrly.

[452.] Webb, E.J., Campbell, D.T., Schwartz, R.D. and Sechrest, L. (1996). Unobtrusive Measures: Non-reactive Research in the social Sciences. Chicago. Rand McNally.

[453.] Wenger, E. (1998). Communities of Practice: Learning, Meaning, and Identity. New York. Cambridge Univ. Press.

[454.] Wenger, E., McDermott, R. and Snyder, W. (2002). Cultivating Communities of Practice. Boston. Harvard Business School Press.

[455.] Wenger, E.C. and Snyder, W.M. (2000, Jan-Feb). Communities of Practice: The Organizational Frontier. Harvard Business Review.

[456.] Wernerfelt, B. (1984). A Resource-based View of the Firm. Strategic Management Journal. V5; p. 171-180.

[457.] Wheatley, M. (2002). Supporting Pioneering Leaders as Communities of Practice. The Berkana Institute. 
[458.] Wicker, K. (2004). FitzPatrick Nuclear Plant's "Journey to Excellence" Has Reached its Destination: The Top Tier of Performance. Power. V148, No.7 p. 56.

[459.] Wiig, K.M. (1993). Knowledge Management Foundations. Arlington, Schema Press.

[460.] Wiig, K.M. (2000). Application of Knowledge Management in Public Administration, Knowledge Research Institute, Inc., Arlington, Texas, USA

[461.] Wiig, K.M. (1997). Knowledge Management: An Introduction and Perspective. Journal of Knowledge Management, vol. 1, no. 1, pp. 6-14

[462.] Wiig, K.M. (1999). What Future Knowledge Management Users May Expect' Journal of Knowledge Management, vol. 3, no. 2, pp. 155-165

[463.] Willis, R.A. (2004). Empowerment, Control and the Representations of Technology in Organizations. University of Calgary. UMI Proquest.

[464.] Wills, G., Alani, H., Ashri, R., Crowder, R., Kalfoglou, Y. and Kim, S. (2002, Dec). Design Issues for Agent-based Resource Locator Systems. In: Practical Aspects of Knowledge Management, Vienna.

[465.] World Association of Nuclear Operators - 2004 Industry Performance Indicators

[466.] World Association of Nuclear Operators - Performance Indicators, 2003

[467.] Yin, R. (1989). Case study research: Design and methods (Rev. ed.). Newbury Park, CA: Sage Publishing.

[468.] Young, T. (2006, Nov-Dec). Implementing a Knowledge Retention Strategy. Knoco.

[469.] Zack, M.H. (1999, Summer). Managing Codified Knowledge. Sloan Management Review.

[470.] Zakaria, N., Amelinckx, A. and Wilemon, D. (2004). Working Together Apart? Building a Knowledge - Sharing Culture for Global Virtual Teams. Blackwell Publishing Ltd.

[471.] Zhang, L. Dr., Tian, Y. and Qi, Z. (2006). Impact of Organizational Memory on Organizational Performance: An Empirical Study, The Business Review, Cambridge 5, 1.

[472.] Zheng, W. (2005). The Impact of Organizational Culture, Structure and Strategy on Knowledge Management Effectiveness and Organizational Effectiveness, ProQuest Information and Learning Co.

[473.] Zucker, L. (1986). Production of Trust: Institutional Source of Economic Structure, in Research in Organizational Behaviour, Staw B., Cummings, L.L. p. 53-111. 


\section{APPENDIX A. ENGLISH VERSION OF SURVEY INSTRUMENT}

\section{Carleton}

Canada's Capital University

\section{Cover Letter for NPP KM Survey}

\section{Dear Sir/Madam:}

I am a doctoral student at Eric Sprott School of Business at Carleton University in Ottawa, Canada. I am currently working towards completing my Ph.D. thesis on the role of knowledge management in improving organizational performance in nuclear power plant organizations.

Specifically this research will explore the impact of knowledge management practices and the level of organizational support for information technology systems have on knowledge processes and ultimately organizational effectiveness and performance. This research will offer theoretical and practical contributions to the area of nuclear power plant management by verifying and explaining the role of knowledge management and information technology in contributing to nuclear power plant performance.

This letter is to kindly request you to participate in this study by completing the enclosed questionnaire and returning it in the envelope provided. Your responses will be kept confidential and will not be disclosed to any outside parties. The survey is being distributed with the cooperation of the International Atomic Energy Agency (IAEA) to all operating nuclear power plant organizations. Anonymity will be ensured by aggregating the results of individual questionnaires when reporting the research findings.

There are no foreseen risks to the participants of this study. The research findings from this study will be presented in the final Ph.D. thesis report and in a series of papers to be submitted to conferences and academic journals. The data will be stored both in hard copy (filled out questionnaires) and electronic copy (file with all the responses) with only the primary researcher (i.e. myself) having access to it. It is not going to be destroyed and might be used for future analysis related to the topic of the present study.

Should you require additional information, please do not hesitate to contact me at 613-520-2600 or by email: jdegrosb@connect.carleton.ca. You are also welcome to contact my thesis supervisor: Professor Vinod Kumar at 613-520-2379 (mailto:Vinod Kumar@carleton.ca) for further information. A summary of the results of this study will be made available to participating NPPs on request. This research project has been reviewed and approved by the Carleton University Research Ethics Committee. If you have any questions or concerns you may contact the committee chair, Prof. Antonio Gualtieri at 613-520-2517 or ethics@carleton.ca

Thank you very much for your help.

John de Grosbois 


\title{
IAEA Global Nuclear Power Plant Survey: "Investigating the Link Between Knowledge Management Practices and Organizational Performance"
}

\author{
(Prepared by J. de Grosbois, Carleton University)
}

As a nuclear power plant (NPP) organization, your station is being formally requested to participate in this global survey on the topic of knowledge management (KM). The survey is being sent to operating NPP organizations around the world. The objective of this survey is to investigate the impact of the various knowledge management practices used in nuclear power plants on organizational performance. The survey should be completed by a senior plant manager at the station (e.g. Chief Nuclear Officer, Plant Manager, Vice-President of Operations or similar position, or his/her designate) who has a broad understanding of organizational practices and operational performance, and who can solicit input from various department managers as needed to complete particular questions.

Your responses will be kept confidential. Only aggregate data will be reported in the research findings to ensure anonymity. The questionnaire should take between 1 to 2 hours in total to complete. Input from various parts of the organization may be required to complete the survey. It has been designed as an electronic form in Microsoft Word (.doc file) and may be completed on your computer electronically ${ }^{1}$. If your organization does not use Microsoft Word, an Adobe Acrobat (.pdf file) version of the survey is attached. If you cannot open ".pdf" files, you will need to download and install the Adobe Reader at: http://get.adobe.com/reader/otherversions/. This software is free from Adobe and will allow you to print the pdf file version of the survey and complete it in hardcopy form. A version of Adobe Reader is available for most operating systems and languages. When completing the questionnaire, if you are not able to answer a given question, you are asked to try to get input from others in your organization who can help. If it is not possible to answer the question, it is preferred that you check the "unable to rate" box rather than guess at the answer. Please try to answer all questions objectively and without bias.

This survey will provide a useful benchmark for NPP organization self-assessment. Your participation in the survey is greatly appreciated. A summary of the findings will be made available to participants upon request.

\footnotetext{
${ }^{1}$ Note that you will need to have "macros" enabled in Microsoft Word to be able to use the check-box and text-box form input fields to complete the survey electronically. To enable macros in the Office 2003 version of Microsoft Word, go to the "Tools" menu, select "Macros", and select "Security". Then set security to "medium" and each time you open a Microsoft Word file containing macros you will be prompted to enable or disable macros. In the Office 2007 version of Microsoft Word, the survey will open in "compatibility mode". A task bar message "Security warning - macros have been disabled" will appear at the bottom of the main task menu at the top of the screen. In this task bar, click on the "OPTIONS" button and a pop-up message "security alert - macro" will appear. Select the option "enable this content" and click "OK" to enable form fields to be filled out electronically. You must enable macros to complete this survey electronically. If you have problems, please use the PDF version of the survey. It can be completed by hand.
} 


\section{PART A: KNOWLEDGE MANAGEMENT PRACTICES}

Please indicate your level of agreement with each of the following statements about your station organization, according to the following rankings:
1. Strongly disagree
2. Somewhat disagree
3. Neither agree nor disagree (neutral)
4. Somewhat agree
5. Strongly agree

\section{Knowledge Management Strategy and Plan}

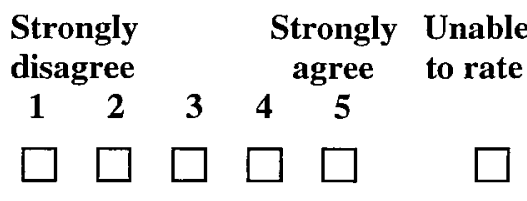

a. The organization has clear, documented high level knowledge management plan and goals.

b. Implementation of the knowledge management strategy and plan is openly and actively supported by management.

c. Knowledge management roles and responsibilities are clearly defined and understood by managers and employees.

d. Other management strategies (e.g. human resources, information systems, operations, communications and maintenance plans) are closely aligned with the knowledge management strategy and plan.

e. The needs and gaps in the organizational knowledge base are periodically reviewed and the knowledge management strategy and plan is revised to address them.

\section{Support for Organizational Learning}

$\begin{array}{ccccc}\begin{array}{c}\text { Strongly } \\ \text { disagree }\end{array} & & \begin{array}{c}\text { Strongly } \\ \text { agree }\end{array} & \begin{array}{l}\text { Unable } \\ \text { to rate }\end{array} \\ 1 & 2 & 3 & 4 & 5\end{array}$

a. Knowledge creation and application (e.g., finding better methods, technology innovation) is encouraged, recognized and rewarded.

b. Sharing of knowledge is promoted and rewarded (e.g., experts are encouraged and rewarded to coach or mentor other employees).

c. Open communication and a no-blame approach to reporting problems and sharing lessons learned are promoted (e.g., regular communication is encouraged between maintenance and operations personnel).

d. Learning opportunities are encouraged (e.g., joining specialist groups or attending training seminars). 


$\begin{array}{ccccc}\begin{array}{c}\text { Strongly } \\ \text { disagree }\end{array} & & \begin{array}{c}\text { Strongly } \\ \text { agree }\end{array} & \begin{array}{c}\text { Unable } \\ \text { to rate }\end{array} \\ \mathbf{1} & \mathbf{2} & 3 & 4 & 5\end{array}$

a. For all processes and procedures, priority is placed on ensuring the requirements, methods, inputs, outputs, interfaces, responsibilities, and workflow are documented correctly and maintained up to date.

b. Consideration of hazards and risk is built into all work and decision processes to ensure safety is not adversely impacted.

c. Procedures are aligned to knowledge and information requirements of both work tasks and decision processes.

d. A process to measure and improve the quality and control of all business, work, and decision processes is defined and followed.

e. Comprehensive knowledge management procedures (e.g. for knowledge loss risk assessment) are documented and in use.

f. Knowledge management processes and procedures are extended to suppliers and technical support organizations.

\section{Information Management Practices}

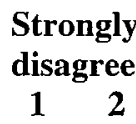

\section{Strongly Unable agree to rate}

a. Licensing documents, design basis documents, procedures, specifications, drawings, and training materials are updated promptly to address plant changes and are maintained under configuration management.

b. Records, data, and logs are required to be complete, meaningful, accurate and accessible (e.g., logs, minutes, test results).

c. Data standards, metadata, document codes, subject indexes and filing systems are widely used to enable efficient information correlation, storage and retrieval.

d. Procedures ensure the needs for data and information safety, security, maintainability, accessibility, quality and preservation.

\section{Organizational Performance Management Practices}

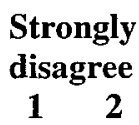

Strongly Unable agree to rate

a. Independent external peer review assessments are conducted regularly (e.g. WANO, INPO, or IAEA-OSART reviews).

b. Self-assessments are widely used to stimulate learning and improve performance (e.g. benchmarking against best practices).

c. Performance objectives are established and monitored for all levels and areas of the organization (including for knowledge processes).

d. Performance objectives for operations, maintenance, and safety are based on objectives established by industry best practice. 
e. The effectiveness of the management system (including

knowledge management aspects) is regularly reviewed.

f. Ongoing processes for operational experience capture, review, analysis and corrective action are defined and followed.

\section{Training Related Practices}

a. The organization incorporates principles of the "systematic approach to training" (SAT) in training programs.

$\begin{array}{ccccc}\begin{array}{c}\text { Strongly } \\ \text { disagree }\end{array} & & \begin{array}{c}\text { Strongly } \\ \text { agree }\end{array} & \begin{array}{l}\text { Unable } \\ \text { to rate }\end{array} \\ 1 & 2 & 3 & 4 & 5\end{array}$

b. Sufficient training is provided to achieve and maintain the required level of competence for all job positions.

c. Training material is reviewed to ensure it reflects lessons learned from operating experience and agrees with plant documentation.

d. Collaboration with universities and colleges ensures an appropriate supply of new graduates.

e. Other techniques are used for training (e.g. story-telling, concept mapping, pre-job briefings, informal seminars, mentoring programs etc.). Please specify:
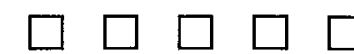

\section{Human Resource Related Practices}

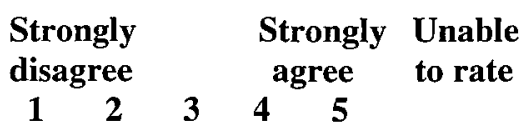

a. Expected retirements and unexpected departures are regularly tracked and the resulting need for and availability of critical knowledge and job skills is acted upon.

b. New hiring is done long before experts depart to facilitate knowledge transfer and ensure the competency of replacements is developed in time.

c. Interviews with departing employees are routinely carried out well in advance to identify critical knowledge and experience and $\square$ to facilitate knowledge capture and transfer.

d. Competency, training and knowledge sharing or transfer goals are identified, evaluated and rewarded in employee performance assessment.

e. Work assignments promote learning (e.g., job-rotations, team selections and staff assignments consider learning opportunities). 


\title{
PART B: TECHNOLOGY SUPPORT
}

Please indicate how effectively each of the following technologies is used in your station organization according to the following rankings:

\author{
1. Very effectively \\ 2. Effectively \\ 3. Somewhat effectively \\ 4. Not effectively \\ 5. Not used (at all)
}

\section{Information Systems and Technology Support}

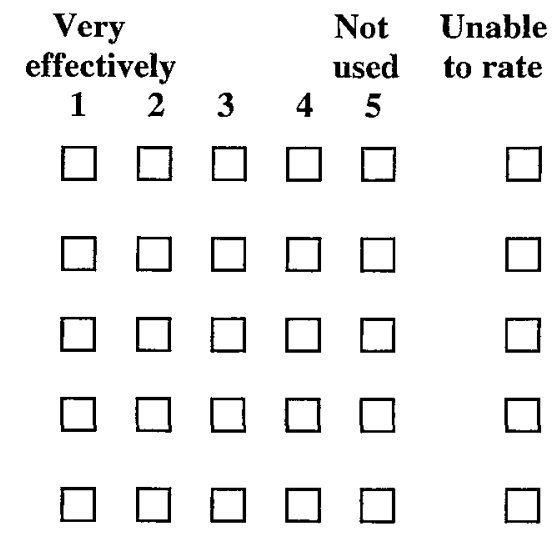

f. Enterprise application software (e.g. for financials, procurement, parts inventory management, work and outage management, etc.)

g. Intranet web portal with search/retrieval access to frequently used resources (e.g. documents, bulletins, contact lists, etc.)

h. Three-dimensional (3D) computer aided design (CAD) plant models and editable electronic drawings.

\section{Advanced Operational Support Systems}

$\begin{array}{cccccc}\begin{array}{c}\text { Very } \\ \text { effectively }\end{array} & & \begin{array}{c}\text { Not } \\ \text { used }\end{array} & \begin{array}{c}\text { Unable } \\ \text { to rate }\end{array} \\ 1 & 2 & 3 & 4 & 5 & \end{array}$

a. Operational decision support systems (e.g. refuelling software)

b. Regularly updated (i.e. "living") probabilistic risk models of equipment reliability for maintenance and outage planning.

c. Real-time probabilistic risk models for operator evaluation and awareness of plant safety (i.e. "a safety monitor").

d. System health monitors (e.g. predictive maintenance tools such as vibration, acoustic, thermal, or other monitors). 
e. Advanced model-based monitoring and diagnostics (e.g. physics, chemistry, boiler, feed water and thermal hydraulics models).

f. Advanced information exchange (e.g. hand-held computers, plant-wide equipment status monitoring, wireless communications).

g. Electronic (i.e. graphical) road-maps of business and decision processes or work-flows (e.g. operational flow-sheets) with links to supporting procedures, related resources or documents.

h. Automated field data collection (i.e., smart instruments, fieldbus, radio frequency identification (RFID) tagging, data logging, equipment monitors).

i. Other (please specify):

\section{PART C: QUALITY OF KNOWLEDGE PROCESSES}

Please indicate your level of agreement with each of the following statements about your station organization, according to the following rankings:
1. Strongly disagree
2. Somewhat disagree
3. Neither agree nor disagree (neutral)
4. Somewhat agree
5. Strongly agree

\section{Knowledge Acquisition}

$\begin{array}{ccccc}\begin{array}{c}\text { Strongly } \\ \text { disagree }\end{array} & & \begin{array}{c}\text { Strongly } \\ \text { agree }\end{array} & \begin{array}{l}\text { Unable } \\ \text { to rate }\end{array} \\ \mathbf{1} & 2 & 3 & 4 & 5\end{array}$

a. The organization has difficulty finding and hiring appropriately qualified graduates.

b. The organization excels at identifying and acquiring external technical information needed to operate and maintain the plant.

c. External information acquired is often not organized or stored in a maintainable and accessible way to facilitate use and reuse.

d. The organization is effective at acquiring knowledge from external (e.g. peer-plant) operating experiences.

e. The organization is highly effective at adopting external best practices. 
f. The organization is good at capturing technical know-how and relevant design information related to services or products received from outside organizations.

\section{Knowledge Creation}

a. NPP staff learn from operating experience and new and better ways of running the plant are seldom overlooked.

b. Independent review processes are effective at validating proposed operational or design changes that may impact safety or production.

c. Employees lack the questioning attitude needed to challenge assumptions and investigate anomalies or uncertainties.

d. Employees regularly create innovative solutions by combining or adapting existing and/or acquired knowledge.

e. The organization excels at generating, transforming, and presenting plant data as meaningful information.

f. Engineers have to spend too much time gathering and compiling data from many sources.

\section{Knowledge Transfer}

a. Findings, information, data, reports, or files generated in one area of the company are readily accessible to other areas.

b. Employees often do not know where in the organization to find specialized knowledge and information.

c. The problem of hoarding (keeping) knowledge does not exist and employees willingly share their knowledge with co-workers.

d. Expertise and skills are not effectively transferred to junior staff from more experienced employees.

e. Employees routinely and voluntarily share relevant information with other parts of the organization where it may be needed.

\section{Knowledge Utilization}

a. Lessons learned from operating experience are incorporated in work practices, manuals, procedures and decision-making.

b. The organization is often not able to apply its knowledge effectively to solve difficult technical problems.
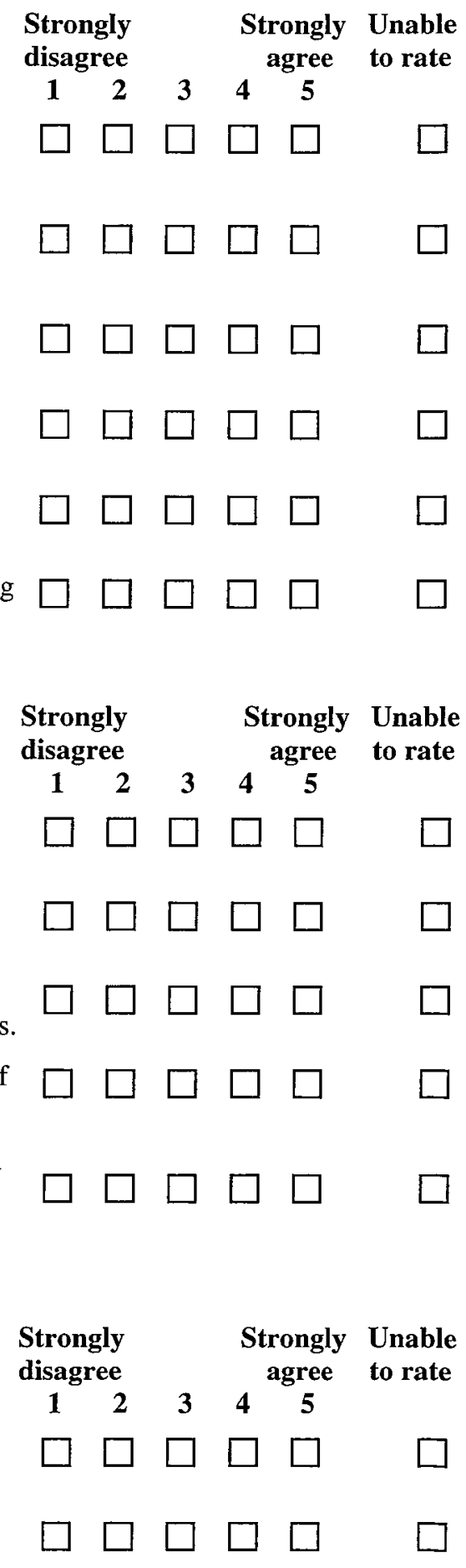
c. Employees are consistently able to make important technical decisions correctly.

d. Employees are not always aware of and do not always make effective use of each other's skills and expertise.

e. Equipment replacement and design change decisions are based on a risk-informed decision process.

\section{Knowledge Retention}

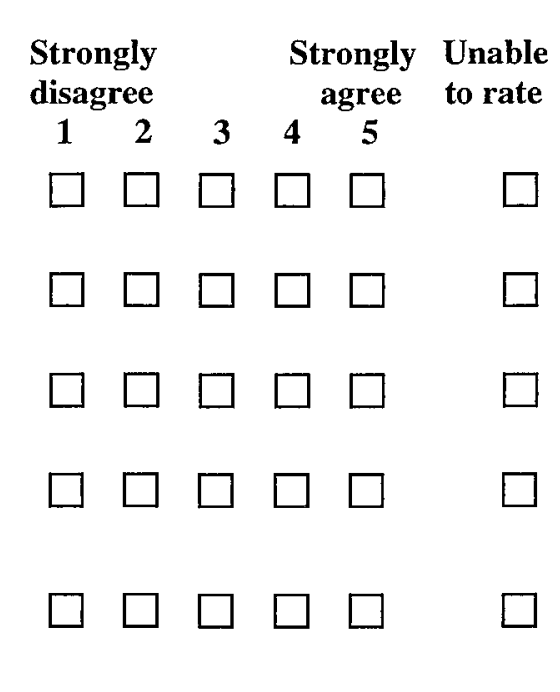

a. Employees often lack an appropriate knowledge of the reactor and power plant fundamentals.

b. Employees have adequate knowledge/understanding of work processes (e.g. industrial and radiation safety work practices).

c. There is often a shortage of critical skills and experience due to unexpected departures and retirements.

d. Plant design basis documents are easily located and are up-todate and accurate.

e. Maintenance, operations, or technical support specialists lack adequate knowledge of specific systems and technologies to enable them to work effectively and safely.

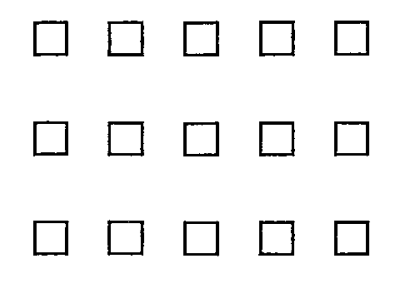

\section{PART D: ORGANIZATIONAL CULTURE}

Please indicate your level of agreement with each of the following statements about your station organization, according to the following rankings:

1. Strongly disagree

2. Somewhat disagree

3. Neither agree nor disagree (neutral)

4. Somewhat agree

5. Strongly agree

\section{Organizational Culture}

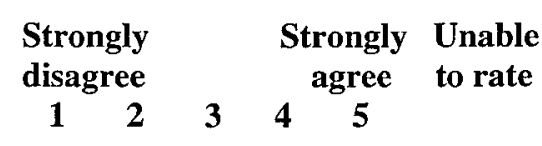

a. Managers and employees often do not see learning, innovation, and improvement as a part of their jobs.

b. Employees who innovate-feel recognized and rewarded.

c. There is a prevailing attitude and commitment to follow defined processes and fully comply with procedures. 


\section{Organizational Culture}

d. Employees often do not feel empowered to make decisions appropriate to their job duties.

e. There is shared vision, purpose, and expectations among employees and they see all their problems as mutual.

f. People are seen as the organisation's most valued asset.

g. Employees and managers are open-minded and respect each other's opinions and contributions.

h. There is a team-oriented approach throughout the station (e.g., employees trust, cooperate, and help each other).

i. Employees often do not feel responsible for plant performance and fail to demonstrate their commitment to it.

j. Consideration of safety is clearly evident in employee and management actions and decisions.

k. Improvements are mostly driven by externally imposed requirements (e.g. regulatory rulings, owner influences).

1. A questioning attitude is cultivated (i.e. information, approaches and decisions are carefully scrutinized).

m. The organization is focused primarily on short-term goals.

\section{Strongly Strongly Unable \\ disagree agree to rate \\ $\begin{array}{lllll}1 & 2 & 3 & 4 & 5\end{array}$}
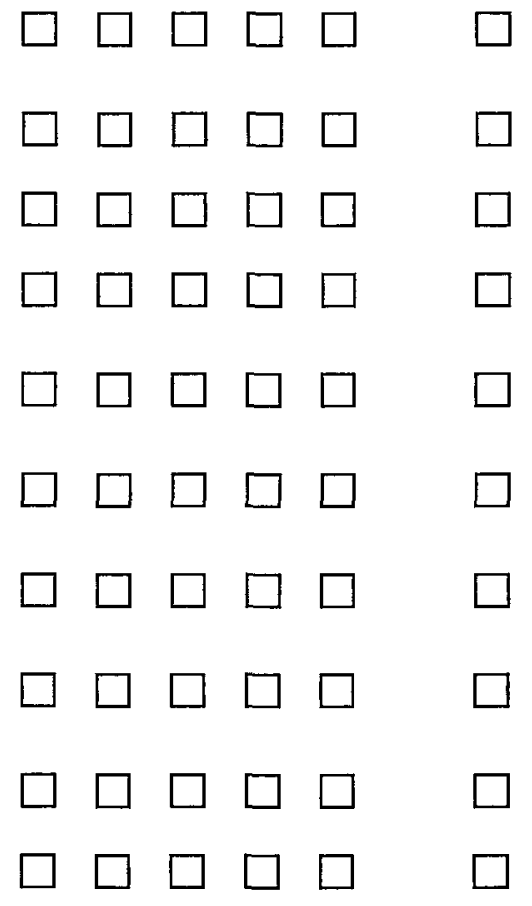

\section{PART E: ORGANIZATIONAL EFFECTIVENESS}

Please indicate your level of agreement with each of the following statements about your stationorganization, according to the following rankings:
1. Strongly disagree
2. Somewhat disagree
3. Neither agree nor disagree (neutral)
4. Somewhat agree
5. Strongly agree

\section{Organizational Effectiveness}

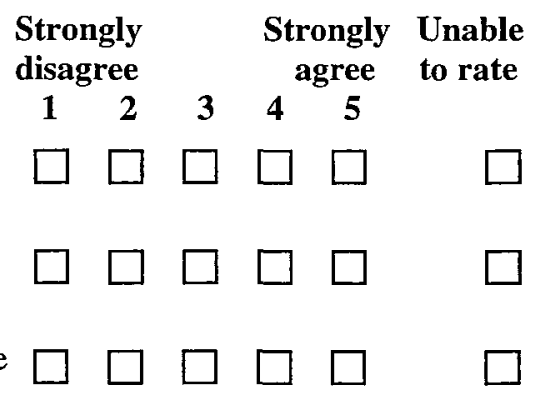

a. The organization has difficulty making operational changes smoothly and in a timely manner.

b. Maintenance technicians consistently conduct high-quality corrective and preventive maintenance.

c. The ratio of corrective to preventive maintenance is high relative to best performing NPPs of similar design. 


\section{Organizational Effectiveness}

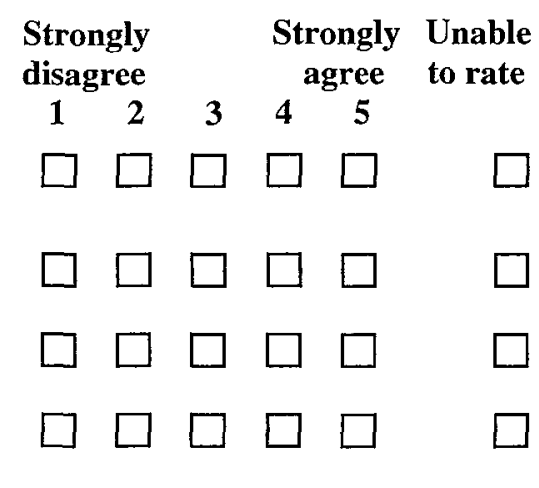

h. Radiological conditions are effectively controlled (i.e. field levels are as low as reasonably achievable and dose control is effective).

i. Quality of documentation (i.e. design, work-process and procedural documentation) needs to improve.

j. Operators effectively act on changing plant conditions to ensure ongoing safe and reliable plant operation.

k. Weekly operations objectives are regularly not met.

1. Work planning and management is effective (e.g. planned workscope is stable, little time is wasted waiting on approvals or parts)

$\mathrm{m}$. The average number of critical component failures per year is low relative to other similar plants.

n. Recurrence of known and avoidable operational problems is not always prevented.

o. The organization is effective at managing its external interfaces (i.e. the regulator, public, suppliers, contractors)

p. Environmental objectives are sometimes not met.

q. Maintenance objectives (e.g. level of corrective and preventive maintenance backlog) based on industry best practice are consistently met or exceeded.

r. Financial objectives are often not met.

s. Regulatory objectives are consistently met or exceeded.

t. System health improvement initiatives are effective.

u. Corrective and preventive maintenance and outage work is completed on schedule and in a timely manner.
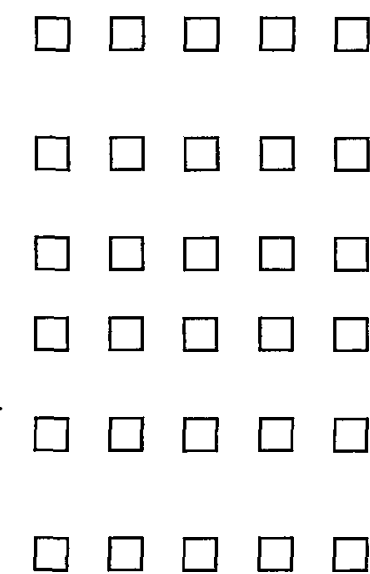

. Financial resources (budgets) are adequate and allocated wisely. 


\section{PART F: OPERATIONAL PERFORMANCE}

1. If you provide the name of your station (optional), the operational performance indicator ratings data for your station can be taken from published sources (a preferred approach). Your responses will remain confidential and only aggregate findings will be reported.

Name of your station (optional):

2. If you provided the name of your station above you do not need to complete the rest of Part $F$ and may then go directly to Part G. If you did not provide the name of your station, you are asked to provide the 2009 operational performance indicator ratings for each unit at your station. Ignore any columns for units that do not exist.

\begin{tabular}{|l|l|l|l|l|l|l|l|l|}
\hline $\begin{array}{l}\text { Unit Performance } \\
\text { Indicator }\end{array}$ & Unit 1 & Unit 2 & Unit 3 & Unit 4 & Unit 5 & Unit 6 & Unit 7 & Unit 8 \\
\hline \begin{tabular}{l} 
1. $\begin{array}{l}\text { Capability Factor } \\
\text { (annual \%) }\end{array}$ \\
\hline $\begin{array}{l}\text { 2. Forced Loss Rate } \\
\text { (annual \%) }\end{array}$
\end{tabular}$-$ - & - & - & - & - & - & - & - & - \\
\hline $\begin{array}{l}\text { 3. Unplanned Capability } \\
\text { Loss Factor (annual \%) }\end{array}$ & - & - & - & - & - & - & - & - \\
\hline
\end{tabular}

\begin{tabular}{|c|c|c|c|c|c|c|c|c|}
\hline $\begin{array}{l}\text { Unit Performance } \\
\text { Indicator }\end{array}$ & Unit 1 & Unit 2 & Unit 3 & Unit 4 & Unit 5 & Unit 6 & Unit 7 & Unit 8 \\
\hline \multirow{2}{*}{$\begin{array}{l}\text { 4. Collective Radiation } \\
\text { Exposure (annual) }\end{array}$} & 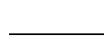 & {[} & — & {[} & 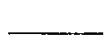 & $\overline{-}$ & $\longrightarrow$ & — \\
\hline & \multicolumn{8}{|c|}{ Specify Man-Sieverts per Unit $\square$ or Annual Man-rem per Unit $\square$} \\
\hline \multicolumn{9}{|l|}{$\begin{array}{l}\text { 5. Number of Unplanned } \\
\text { Automatic Scrams per } \\
7,000 \text { Hours Critical }\end{array}$} \\
\hline \multirow{2}{*}{$\begin{array}{l}\text { 6. Industrial Safety } \\
\text { Accident Rate }\end{array}$} & $\underline{-}$ & $\longrightarrow$ & $\longrightarrow$ & - & - & 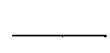 & - & {[} \\
\hline & \multicolumn{8}{|c|}{ Specify per 200,000 person-hours $\square$ or $1,000,000$ person-hours $\square$ worked } \\
\hline
\end{tabular}




\begin{tabular}{|l|l|l|l|l|l|l|l|l|l|l|}
\hline \multirow{2}{*}{ Unit Performance Indicator } & Unit 1 & Unit 2 & Unit 3 & Unit 4 & Unit 5 & Unit 6 & Unit 7 & Unit 8 \\
\cline { 2 - 9 } & \multicolumn{2}{|l|}{$\begin{array}{l}\text { Please indicate the quartile (i.e. } 1 \text { to 4) relative to similar plant types (e.g. a rating of } \\
\text { is a unit in the top } 25 \% \text { of similar units, see footnote }\end{array}$ for details) } \\
\hline $\begin{array}{l}\text { 7. Annual Safety System } \\
\text { Performance }\end{array}$ & - & - & - & - & - & - & - & - \\
\hline 8. Annual Fuel Reliability & - & - & - & - & - & - & - & - \\
\hline $\begin{array}{l}\text { 9. Annual Chemistry } \\
\text { Performance }\end{array}$ & - & - & - & - & - & - & - & - \\
\hline
\end{tabular}

\section{Notes: WANO definitions (for reference if needed to answer the questions $2 \mathrm{a}$ to $2 \mathrm{i}$ above):}

1. Unit Capability Factor: the annual percentage of maximum energy generation that a plant is capable of supplying to the electrical grid, limited only by factors within control of plant management.

2. Unplanned Capability Loss Factor: the annual percentage of maximum energy generation that a plant is not capable of supplying to the electrical grid because of unplanned energy losses, such as unplanned shutdowns or outage extensions.

3. Forced Loss Rate: is the annual percentage of energy generation during non-outage periods that a plant is not capable of supplying to the electrical grid because of unplanned energy losses, such as unplanned shutdown or load reductions.

4. Collective Radiation Exposure: the effectiveness of personnel radiation exposure controls for boiling water reactors (BWRs), pressurized water reactors (PWRs), pressurized heavy water reactors (PHWRs), light-water cooled graphite reactors (LWCGRs), and gas-cooled reactors (GCRs). Measured in Man-Sieverts per unit or Man-rem per unit annually.

5. Unplanned Automatic Scrams per 7,000 Hours Critical: the mean scram (i.e. automatic shutdown) rate for approximately one year (i.e. 7000 hours) of operation. Unplanned automatic scrams result in thermal and hydraulic transients that affect plant systems.

6. Industrial Safety Accident Rate: the number of accidents that result in lost work time, restricted work, or fatalities per 200,000 work-hours.

7. Safety System Performance: The annual safety system performance indicator monitors the availability of three important standby safety systems at each plant. Safety systems that are maintained in a high state of readiness have a high probability of being capable of mitigating offnormal events.

8. Fuel Reliability: The annual fuel reliability indicator monitors progress in preventing defects in the metal cladding that surrounds fuel. Maintenance of fuel cladding integrity reduces radiological impact on plant operations and maintenance activities.

9. Chemistry Performance: The annual chemistry performance indicator provides an indication of progress in controlling chemical parameters to retard deterioration of key plant materials and components. These parameters are already being maintained within strict guidance developed by the industry.

\footnotetext{
${ }^{3}$ Quartiles are defined as follows: Quartile 4 (bottom performance) is from $1 \%$ to $25 \%$; Quartile 3 is from $26 \%$ to $50 \%$; Quartile 2 is from $51 \%$ to $75 \%$; Quartile 1 (top performance) is from $76 \%$ to $100 \%$.
} 


\section{PART G: DEMOGRAPHIC AND OTHER DATA}

1. Please indicate the number of employees (excluding contractors) at your station:

2. Please indicate the typical number of full-time equivalent contractors during outages:

3. Please indicate the typical number of full-time equivalent contractors while at power:

4. Please indicate the percentage of employees with university degrees at your station:

5. Please indicate the country your station is located in:

6. Please indicate the number of operational units (i.e. power reactors) at your station:

7. Please indicate the type of reactor (e.g. PWR, BWR, PHWR, LWCGR, or GCR etc.):

8. Please indicate the plant model (i.e. product) name (e.g. EPR, AP1000, VVER 440, etc.):

9. Please check the appropriate row to indicate the approximate age of each unit at your station (measured in years from completion of construction) (ignore columns for any non-existent units):

\begin{tabular}{|l|l|l|l|l|l|l|l|l|}
\hline Age (years) & Unit 1 & Unit 2 & Unit 3 & Unit 4 & Unit 5 & Unit 6 & Unit 7 & Unit 8 \\
\hline $1-10$ & $\square$ & $\square$ & $\square$ & $\square$ & $\square$ & $\square$ & $\square$ & $\square$ \\
\hline $11-20$ & $\square$ & $\square$ & $\square$ & $\square$ & $\square$ & $\square$ & $\square$ & $\square$ \\
\hline $21-30$ & $\square$ & $\square$ & $\square$ & $\square$ & $\square$ & $\square$ & $\square$ & $\square$ \\
\hline $31-40$ & $\square$ & $\square$ & $\square$ & $\square$ & $\square$ & $\square$ & $\square$ & $\square$ \\
\hline $41+$ & $\square$ & $\square$ & $\square$ & $\square$ & $\square$ & $\square$ & $\square$ & $\square$ \\
\hline
\end{tabular}

10. Please indicate which communities of practice (COP) your station organization participates in and whether regular self-assessment is done against the performance indicators or benchmarks from that COP group.

Name or topic of COP Work Group

a.) Equipment Reliability

b.) Materials and Services (supply chain)

c.) Information Technology

d.) Business Services / Nuclear Asset Management

e.) Information Management

f.) Licensing / Regulatory Issues

g.) Human Resources

h.) Radiation Protection

i.) Nuclear Fuel

j.) Performance Monitoring / Improvement

k.) Plant Operations

l.) Chemistry Management
Indicate if a regular participant

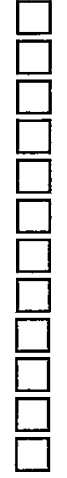

Indicate if doing benchmarking 
Name or topic of COP

Work Group

m.) Work Management

n.) Simulators

o.) Training

p.) Cost Estimation and Management

q.) Configuration Management

r.) Fire Protection

s.) Other (specify:
Indicate if a regular participant
Indicate if doing benchmarking

Please also indicate whether COP participations above include: local (e.g. national) $\square$, regional (e.g. European) $\square$, international (e.g. IAEA, EPRI, INPO or NEI) $\square$, or Owner's Group based COPs $\square$.

11. Please indicate the number of operations managers who helped complete this survey response:

12. Please make any additional comments on, or clarifications of your responses in the space provided below.

13. If you wish to have an electronic copy of the report summarizing the findings of this study once it is available, please provide your name, title and e-mail address (optional).

Name:

Title:

E-mail address:

Thank you for your valuable time in completing this questionnaire! 


\section{APPENDIX B. RUSSIAN TRANSLATION SURVEY INSTRUMENT}

\section{ЧАСТЬ А. IРАКТИЧЕСКИЕ МЕТОДЫ УТРРАВЛЕНИЯ ЗНАНИЯМИ}

Просьба обозначить свой уровень согласия с предложенными ответами относительно вашей АЭС, используя следуюшую шкалу ощенок:

1. решительно не согласен

2. частично не согласен

3. частично согласен, частично не согласен (нейтральное мнение)

4. частично согласен

5. полностью согласен

\section{1. Стратегия и план управления знаниями}

а. В организации существует четкие, документально зафиксированные план и цели управления знаниями.

b. Внедрение стратегии управления знаниями и план соответствуюшей работы открыто и активно поддерживаются руководством.

c. Роли и обязанности в сфере управления знаниями четко определены и понятны руководителям и сотрудникам.

d. Другие стратегии управления (например, планы в сфере кадровых ресурсов, информационных систем, эксплуатации, связи и ремонтных и обслуживающих работ) тесно соприкасаются со стратегией и планом управления знаниями.

e. Потребности и пробелы в базе знаний организации периодически рассматриваются, и в целях решения выявленных проблем пересматриваются стратегия и план управления знаниями.

\section{2. Поддержка усилий организации по накоплению} опыта

a. Создание и применение новых баз знаний (т. е. поиск более совершенных методов, технологических нововведений) приветствуется, признается и вознаграждается.

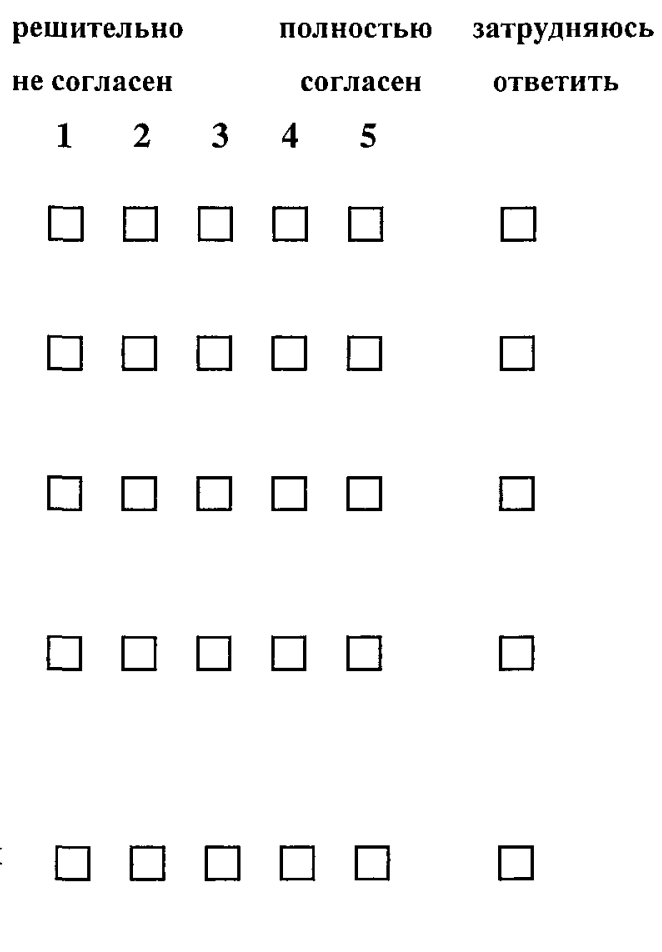

\begin{tabular}{ccccc} 
решительно & & полностью & затрудняюсь \\
не согласен & & \multicolumn{2}{c}{ согласен } & ответить \\
1 & 2 & 3 & 4 & 5
\end{tabular}


2. Поддержка усилий организации по накоплению опыта

$\begin{array}{cccc}\begin{array}{c}\text { решительно } \\ \text { не согласен }\end{array} & \begin{array}{c}\text { полностью } \\ \text { согласен }\end{array} & \begin{array}{c}\text { затрудняюсь } \\ \text { ответить }\end{array} \\ 1 \quad 2 & 3 & 4 & 5\end{array}$

b. Обмен знаниями приветствуется и вознаграждается (например, наставничество и обучение экспертами других сотрудников).

c. Оказывается содействие подходу неприменения наказаний за открытое признание ошибок, а также усвоению извлеченных уроков (т. е. приветствуется регулярный открытый обмен опытом между ремонтным и эксплуатационным персоналом).

d. Приветствуются любые возможности обучения (например, участие в дискуссиях групп специалистов или в учебных семинарах)

\section{3. Практические методы управления процессами}

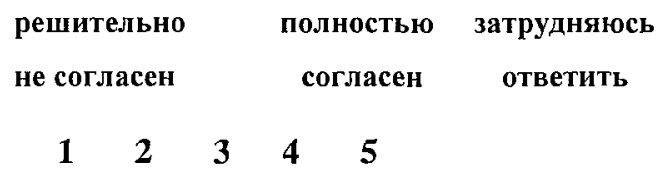

a. При реализации всех процессов и процедур приоритет отдается обеспечению того, чтобы требования, методы, исходные условия, способы взаимодействия, обязанности и рабочие процессы были правильно зафиксированы в документах и эти документы регулярно обновлялись.

b. Потенщиальные опасности и риски учитываются в ходе всех работ на станции, в том числе в процессах принятия решений, для обеспечения того, чтобы не было неблагоприятного воздействия на безопасность.

c. Процедуры соответствуют требованиям в отношени уровня знаний и информированности как при выполнении всех рабочих заданий, так и в процесса принятия решений.

d. Определен и соблюдается процесс измерения и улучшения качества и контроля всех действий, работ и процессов принятия решений.

е. Всеобъемлющие процедуры управления знаниями (например, оценка риска утери знаний) зафиксированы в документах и действуют в организации.

f. Процессы и процедуры управления знаниями распространяются на поставщиков и организации, обеспечивающие техническую поддержку. 


\section{4. Практические методы управления информацией}

$\begin{array}{lcc}\text { решительно } & \text { полностью } & \text { затрудняюсь } \\ \text { не согласен } & \text { согласен } & \text { ответить }\end{array}$

а. Документы, относящиеся к лицензированию, проектная документация, процедуры, спецификации, чертежи и учебные материалы обновляются достаточно быстро, с тем чтобы отразить изменения на станции, и ведутся в рамках процесса управления конфигурацией.

b. Учетные записи и данные в регистрационных журналах должны быть полными, отражающими сушество вопроса, точными и доступными (например, журналы, протоколы, результаты проверок).

c. Широко используются стандартные формы, реестры, учетные номера, каталожные индексы и системы регистрации документов в целях обеспечения эффективности сопоставления, хранения и поиска информации.

d. Процедуры обеспечивают потребности в данных, информационной безопасности, физической безопасности, обслуживании и ремонте, доступности, отвечают стандартам качества и сохранности информации.

\section{5. Практические методы управления} эффективностью функционирования организации

$\begin{array}{ccccc}\begin{array}{c}\text { решительно } \\ \text { не согласен }\end{array} & \begin{array}{c}\text { полностью } \\ \text { согласен }\end{array} & \begin{array}{c}\text { затрудняюсь } \\ \text { ответить }\end{array} \\ 1 & 2 & 3 & 4 & 5\end{array}$

a. Регулярно проводятся независкмые авторитетные внешние оценки (например, рассмотрения со стороны ВАО АЭС, ИНПО или ОСАРТ МАГАТЭ).

b. Для стимулирования накопления опыта и улучшения работы широко используется самооценка (например, сравнение с лучшими примерами передовой практики).

c. Задачи и цели в отношении эффективности работы поставлены и контролируются на всех уровнях и во всех подразделениях организации (включая процессы управления знаниями). 
d. Цели эффективности функционирования в отношении эксплуатации, обслуживания и обеспечения безопасности отвечают установленным в отрасли требованиям на основе лучшей передовой практики.

е. Эффективность системы управления (включая систему управления знаниями) регулярно рассматривается.

f. Определены и соблюдаются текущие процессы фиксации, рассмотрения и анализа опыта эксплуатации и осуществления корректирующих действий.

\section{6. Практические методы обучения}

$\begin{array}{lll}\text { решительно } & \text { полностью } & \text { затрудняюсь } \\ \text { не согласен } & \text { согласен } & \text { ответить }\end{array}$

a. Организация внедряет в учебные программы принципы "системного подхода к обучению" (СПО).

b. Обеспечивается обучение, достаточное для достижения и поддержания требуемого уровня компетенции для всех должностей.

c. Учебные материалы пересматриваются для обеспечения того, чтобы они отражали уроки, извлеченные из опыта эксплуатации и были увязаны с документацией станции.

d. Сотрудничество с университетами и колледжами обеспечивает соответствующий приток новых выпускников.

\section{$\begin{array}{lllll}1 & 2 & 3 & 4 & 5\end{array}$}

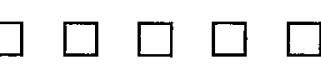

. Для обучения используются другие методики (например, деловая игра; концептуальное отображение; инструктажи перед началом работы; семинары; программы наставничества и т.д.).

Просьба указать 
7. Практические методы управления кадровыми

ресурсами

\section{решительно полностью затрудняюсь \\ не согласен согласен ответить}

$\begin{array}{lllll}1 & 2 & 3 & 4 & 5\end{array}$

а. Принимаются соответствующие меры в отношении ожидаемых выходов на пенсию и неожидаемых уходов сотрудников из организации, а также в отношении вытекающей из этого необходимости компенсации поддержания критических знаний и рабочих навыков.

b. Наем новой рабочей силы производится задолго до ухода опытных специалистов с целью содействия передаче их знаний и обеспечения постепенного роста компетентности заменяющих их сотрудников.

c. Проводятся регулярные собеседования с уходящими работниками для определения критических знаний и опыта с целью содействия их сохранению и передаче.

d. В оценках результатов деятельности сотрудников определяются, оцениваются и соответствующим образом вознаграждается их компетентность, уровень подготовки и желаниеобмена знаниями.

е. Порученные новые рабочие задания способствуют обучению персонала (т. е. возможности обучения учитываются при ротации персонала, подборе смен и назначениях сотрудников).

\section{ЧАСТЬ В: ПОДДЕРЖКА ТЕХНОЛОГИЙ}

Просьба указать, насколько эффективно применяется на Вашей АЭС каждая из приведенных ниже технологий, используя следующую шкалу оценок:

1. весьма эффективно

2. эффективно

3. частично эффективно

4. не эффективно

5. не используется (вообще) 


\section{1. Информационные системы и поддержка} технологий

a. Трехмерная (3D) виртуальная среда обучения (компьютерные 3D-тренажеры).

b. Обучение с использованием компьютера и/или на основе wеb-технологии.

c. Тренажеры (например, станции) на основе настольных ПК.

d. Полномасштабные тренажеры БЩУ.

е. Электронные архивы и базы данных (например, для документооборота, регистрации событий, записей о ремонтных работах и т.д.).

f. Общестанционные прикладные программы (например, для финансового учета, снабжения, управления процедурой инвентаризации, управления периодами эксплуатации и остановов и Т.Д.).

g. Веб-портал интранета с доступом к поиску/получению часто используемых ресурсов (например, документов, бюллетеней, выборок, списков контактных лиц и т.д.).

h. Трехмерное (3D) компьютерное проектирование (CAD) моделей станции и станционного оборудования, а также редактируемые электронные чертежи.

\section{2. Усовершенствованные системы поддержки} эксплуатации

a. Системы поддержки решений оператора (например, программное обеспечение для управления перегрузкой топлива).

b. Регулярно обновляемые (т.е. интерактивные) вероятностные модели риска для определения надежности оборудования и планирования ППР.

c. Работающие в реальном режиме времени вероятностные модели риска для оценки оператором состояния безопасности станции (т.е. "мониторы безопасности").
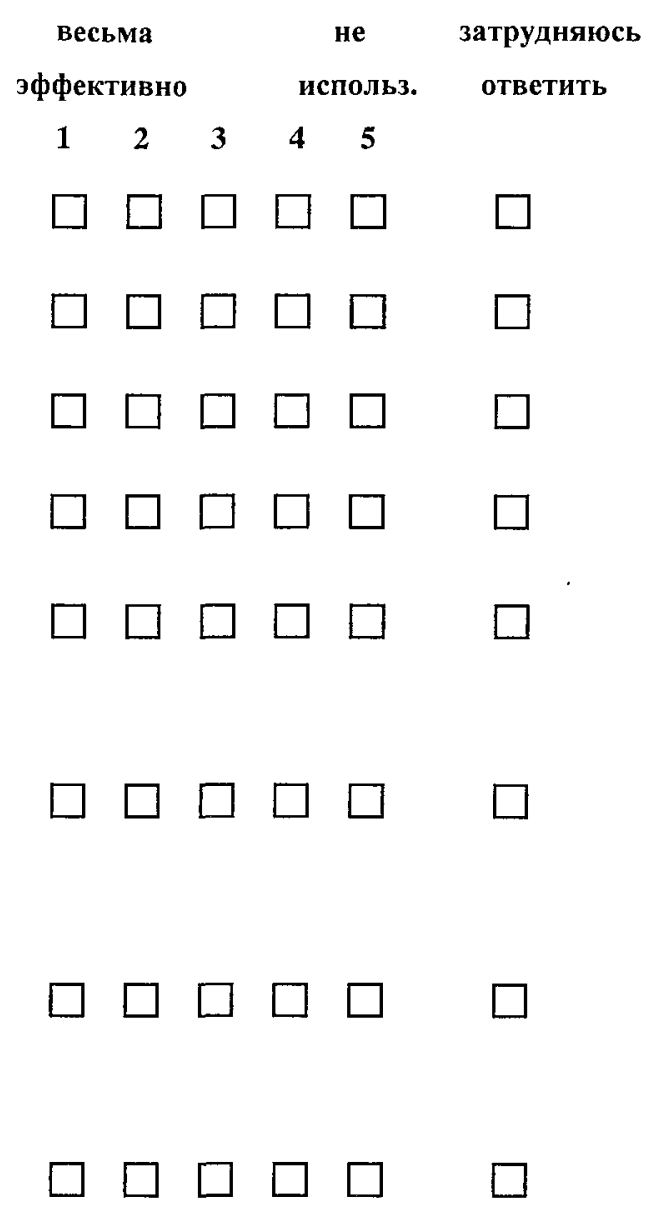

эффктиво использ. ответить


d. Мониторы состояния систем станции (например, средства профилактического техобслуживания и контроля, такие, как вибрационные, акустические, тепловые мониторы или другие приборы).

е. Усовершенствованные системы контроля и диагностики на основе моделирования (например, модели физики реактора, водно-химического режима, кипения, системы питательной воды и теплогидравлики).

f. Усовершенствованные методы обмена информацией (например, использование карманных ПК, дистанционный мониторинг оборудования станции, беспроводные средства передачи информации).

g. Электронные (т.е. графические) сетевые графики производства работ или системы документооборота (например, технологические схемы эксплуатации) со ссылками на вспомогательные процедуры, соответствующие ресурсы или документы.

h. Автоматический сбор данных (то есть, используются смарт-карты, сетевые (магистральные) линии связи, идентификационные карточки с радиометрами, компьютерные системы регистрации данных, мониторы параметров оборудования).

i. Другие (просьба укаэать):

ЧАСТЬ С: КАЧЕСТВО ПРОЦЕССОВ УПРАВЛЕНИЯ ЗНАНИЯМИ

Просьба указать степень вашего согласия с предложенными ответами относительно вашей АЭС, используя предложенную шкалу оценки:

1. решительно не согласен

2. частично не согласен

3. частично согласен, частично не согласен (нейтральное мнение)

4. частично согласен

5. полностью согласен 


\section{1. Приобретение знаний}

$\begin{array}{lrr}\text { решительно } & \text { полностью } & \text { затрудняюсь } \\ \text { не согласен } & \text { согласен } & \text { ответить } \\ 1 & & \end{array}$

а. Организация испытывает трудности в поиске и найме на работу выпускников вузов, имеющих соответствующую квалификацию.

b. Организация специально определяет и собирает техническую информацию из внешних источников, необходимую для эксплуатации и поддержания работоспособности станции.

c. Полученная из внешних источников информация часто организована или сохранена не в легкодоступном и редактируемом формате, что препятствует еe дальнейшему или повторному использованию.

d. Организация эффективно приобретает знания на основе данных об опыте эксплуатации других станций (например, результаты взаимопроверок).

е. Организация с большой эффективностью внедряет у себя на станции лучпие передовые методы, применяемые на других станциях.

f. Организация хорошо перенимает полученные из внешних организаций технические ноу-хау и соответствующую проектную информацию.

\section{2. Создание знаний}

$\begin{array}{lrr}\text { решительно } & \text { полностью } & \text { затрудняюсь } \\ \text { не согласен } & \text { согласен } & \text { ответить }\end{array}$

a. Работники АЭС извлекают уроки из эксплуатационного опыта, и поэтому новые и более рациональные способы ведения эксплуатации редко остаются без внимания.

b. Процессы независимых рассмотрений доказывают свою эффективность при проверке обоснованности предлагаемых изменений регламентов эксплуатации или изменений конструкции, которые могут влиять на безопасность или производство.

$\begin{array}{lllll}1 & 2 & 3 & 4 & 5\end{array}$

$\square$

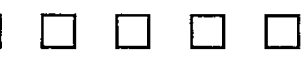

\section{$\begin{array}{lllll}1 & 2 & 3 & 4 & 5\end{array}$}
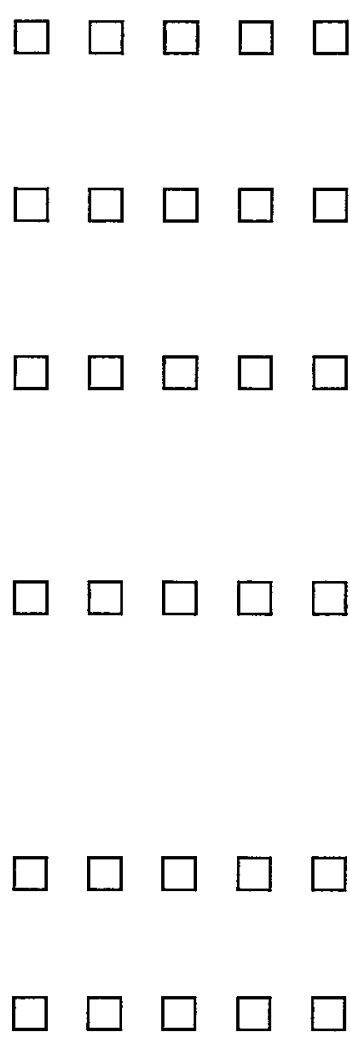
2. Создание знаний

\begin{tabular}{ccccc} 
решительно & полностью & затрудняюсь \\
не согласен & \multicolumn{2}{c}{ согласен } & ответить \\
1 & 2 & 3 & 4 & 5
\end{tabular}

c. У работников нет критической позиции, необходимой для тщательной проверки предположений и расследования причин возникновения отклонений от нормы или неопределенностей.

d. Работники регулярно предлагают новые решения на основе существующих и/или приобретенных знаний.

е. В процессе сбора, обработки и представления данных о работе станции организация относится к этой информации как к особо важной.

f. Инженеры должны тратить слишком много времени на сбор и компилирование данных из многих источников.

\section{3. Передача знаний}

$\begin{array}{ccccc}\begin{array}{c}\text { решительно } \\ \text { не согласен }\end{array} & \begin{array}{r}\text { полностью } \\ \text { согласен }\end{array} & \begin{array}{c}\text { затрудняюсь } \\ \text { ответить }\end{array} \\ 1 & 2 & 3 & 4 & 5\end{array}$

а. Результаты работ, различного рода информация, данные, отчеты или файлы, созданные в одном подразделении организации, легкодоступны для использования в других подразделениях.

b. Работники часто не знают, где в организации хранятся специализированные знания или информация.

c. Проблемы искусственного удержания знаний не существует, и работники охотно делятся своими знаниями с коллегами.

d. В организации не существует эффективного способа передачи экспертных знаний и навыков от более опытных работников младшим сотрудникам.

е. Сотрудники постоянно и добровольно передают актуальную информацию в другие подразделения организации, где она может потребоваться. 


\section{4. Использование знаний}

$\begin{array}{lrc}\text { решительно } & \text { полностью } & \text { затрудняюсь } \\ \text { не согласен } & \text { согласен } & \text { ответить }\end{array}$

$\begin{array}{lllll}1 & 2 & 3 & 4 & 5\end{array}$

a. Уроки, извлеченные из опыта эксплуатации, вводятся в рабочую практику, руководства, процедуры и используются при принятии решений.

b. Организация часто не в состоянии решать трудные технические проблемы эффективным образом.

c. Работники почти всегда могут принимать правильные решения по важным техническим вопросам.

d. Работники не всегда осведомлены о рабочих навыках и опыте коллег и поэтому не всегда эффективно используют их.

е. Решения о замене оборудования и изменениях в конструкции принимаются с учетом оценки рисков.

\section{5. Сохранение знаний}

$\begin{array}{lrc}\text { решительно } & \text { полностью } & \text { затрудняюсь } \\ \text { не согласен } & \text { согласен } & \text { ответить }\end{array}$

\section{$\begin{array}{lllll}1 & 2 & 3 & 4 & 5\end{array}$}

a. Работникам часто не хватает фундаментальных знаний по реакторной установке и основам АЭС.

b. У работников имеется достаточное знание/понимание рабочих процессов (то есть, рабочей практики с обеспечением промышленной и радиационной безопасности).

c. Часто наблюдается дефицит критических навыков и опыта из-за неожидаемых уходов работников и выходов на пенсию.

d. Проектная документация легкодоступна, точна и регулярно обновляется.

е. Специалистам в области технического обслуживания, эксплуатации или технической поддержки не хватает соответствующих знаний о конструкции конкретных систем и о применяемых технологиях, поэтому они не могут обеспечить их эффективную и безопасную работу. 


\section{ЧАСТЬ D: КУЛЬТУРА ОРГАНИЗАДИИ}

Просьба указать свой уровень согласия с предложенными ответами относительно вашей АЭС, используя следующую шкалу оценок:

1. решительно не согласен

2. частично не согласен

3. частично согласен, частично не согласен (нейтральное мнение)

4. частично согласен

5. полностью согласен

1. Культура организации

$\begin{array}{cccccc}\begin{array}{c}\text { решительно } \\ \text { не согласен }\end{array} & \begin{array}{r}\text { полностью } \\ \text { согласен }\end{array} & \begin{array}{c}\text { затрудняюсь } \\ \text { ответить }\end{array} \\ \begin{array}{ccccc}1 & 2 & 3 & 4 & 5\end{array}\end{array}$

a. Руководители и работники часто не рассматривают обучение, новаторство и внедрение усовершенствований в качестве части своих рабочих обязанностей.

b. Работники, которые предлагают какие-либо нововведенкя, чувствуют, что их отмечают и поошряют.

c. Существует общее позитивное отношение и приверженность в отношении выполнения четко определенных процессов и полного соблюдения предписанных процедур.

d. Работники часто не чувствуют то, что они уполномодены принимать решення, связанные с их функциональными обязанностями.

е. Работники разделяют перспективы, цели и ожидания организации, они рассматривают все свои проблемы в качестве общих проблем.

f. В организации сотрудники считаются самым ценным ее достоянием.

g. Руководители и подчиненные открыто относятся друг к другу и уважают чужие мнения и вклады в общее дело.

h. Во всей организации принят подход, ориентированный на коллективное выполнение задач (т. е. работники доверяют друг другу, взаимодействуют между собой и помогают друг другу).

i. Работники часто не чувствуют ответственности за работу станции и не проявляют приверженности этому. 
1. Культура организации

$\begin{array}{lrc}\text { решительно } & \text { полностью } & \text { затрудняюсь } \\ \text { не согласен } & \text { согласен } & \text { ответить }\end{array}$

$\begin{array}{lllll}1 & 2 & 3 & 4 & 5\end{array}$

j. Надлежащий учет аспектов безопасности явно просматривается в действиях и решениях работников и руководства.

k. Усовершенствования в основном инициируются под воздействием внешних факторов (регулирующих нормативных документов, требований владельцев).

1. Поощряется критическая позиция (т. е. тщательное рассмотрение поступающей информации, принимаемых подходов и решений).

m. Основное внимание в организации уделяется достижению краткосрочных целей.

\section{ЧАСТЬ Е: ЭФФЕКТИВНОСТЬ ОРГАНИЗАЦИИ}

Просьба указать свой уровень согласия с предложенными ответами относительно вашей АЭС, используя следующую шкалу оценок:

1. решительно не согласен

2. частично не согласен

3. частично согласен, частично не согласен (нейтральное мнение)

4. частично согласен

5. полностью согласен

1. Эффективность организации

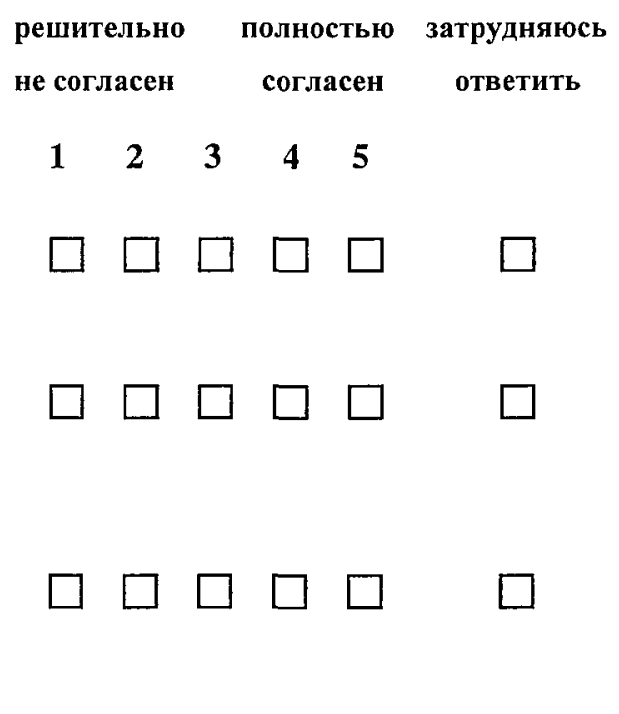




$\begin{array}{lcc}\text { решительно } & \text { полностью } & \text { затрудняюсь } \\ \text { не согласен } & \text { согласен } & \text { ответить }\end{array}$

\section{$\begin{array}{lllll}1 & 2 & 3 & 4 & 5\end{array}$}

d. Программа воднохимического режима станции обеспечивает постоянную работу станции в пределах установленных химических параметров.

е. Проекты, реализация которых требует участия многих подразделений, обычно отстают от графика, превышают запланированную смету расходов и плохо координируются.

f. Цели безопасности обычно достигаются или превышаются.

g. Система анализа рабочих показателей и/или инженеры, управляющие этой системой, не эффективны в деле решения проблем, которые влияют на безопасность или рабочие показатели станции.

h. Радиологические условия эффективно контролируются (т.е. уровни излучения поддерживаются на разумно достижимом низком уровне и существует эффективная система контроля дозовых нагрузок).

i. Качество документации (т.е. проектной, рабочей, регламентной документации) необходимо улучшить.

j. Операторы эффективно реагируют на изменения условий работы станции в целях постоянного обеспечения безопасной и надежной эксплуатации.

k. Устанавливаемые еженедельно цели эксплуатации часто не достигаются.

1. Система планирования работ и управления ими эффективна (например, запланированный объем работ выполняется без задержек, немного времени уходит на утверждение различных нарядов или доставку запчастей).

m. Среднее число отказов критических компонентов в год является низким по сравнению с другими аналогичными АЭС.

n. Не всегда предотвращается повторное возникновение известных проблем эксплуатации, а также проблем, возникновение которых можно было бы избежать.

o. Организация эффективно взаимодействует с внешними структурами (т.е. с регулирующими органами, населением, поставщиками, подрядчиками). 
1. Эффективность организации

$\begin{array}{lcc}\text { решительно } & \text { полностью } & \text { затрудняюсь } \\ \text { не согласен } & \text { согласен } & \text { ответить }\end{array}$

$\begin{array}{lllll}1 & 2 & 3 & 4 & 5\end{array}$

p. Цели охраны окружающей среды иногда не достигаются.

q. Цели ремонтных и профилактических работ (например, уровень невыполненных ремонтных и профилактических работ), поставленные на основе лучшей существующей в отрасли практики, как правило, достигаются или превышаются.

r. Финансовые цели часто не достигаются.

s. Цели надзора достигаются или превышаются.

t. Инициативы в отношении улучшения состояния систем эффективны.

u. Работы по профилактическому и внеплановому техобслуживанию и планово-предупредительные ремонты выполняются по графику и своевременно.

v. Финансовые ресурсы (бюджет) достаточны и используются эффективно.

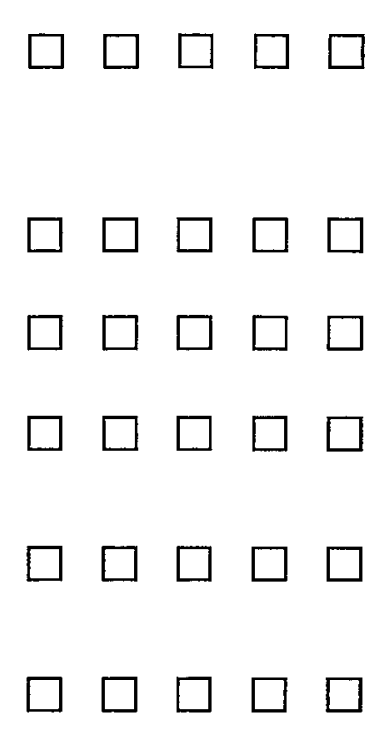

ЧАСТЬ F : ЭКСПЛУАТАЦИОННЫЕ ПОКАЗАТЕЛИ

1. Если вы указываете название вашей атомной станции (не является обязательным), то эксплуатационные показатели за 2009 будут указаны из опубликованных источников (предпочтительный вариант). Ваши ответы останутся конфиденциальными, и только суммирующие сведения будут включены в отчет.

Пожалуйста, напишите название вашей атомной станции (необязательно): 
2. Если вы указали выше название вапей атомной станции, то для вас нет необходимости заполнять оставтуюся часть $F$, и вы можете проследовать к части G. Если вы не указали название вашей атомной станции, то просим вас предоставить следующие эксплуатационные показатели для каждого блока вашей АЭС. Пожалуйста, приведите данные за последний год и обозначьте этот год: Если [требуемая] для колонок информация отсутствует на блоках вашей АЭС, то оставьте их пустыми.

\begin{tabular}{|l|c|c|c|c|c|c|c|c|}
\hline $\begin{array}{l}\text { Показатель эффективности } \\
\text { блока }\end{array}$ & $\begin{array}{c}\text { Блок } \\
1\end{array}$ & $\begin{array}{c}\text { Блок } \\
2\end{array}$ & $\begin{array}{c}\text { Блок } \\
3\end{array}$ & $\begin{array}{c}\text { Блок } \\
4\end{array}$ & $\begin{array}{c}\text { Блок } \\
5\end{array}$ & $\begin{array}{c}\text { Блок } \\
6\end{array}$ & $\begin{array}{c}\text { Блок } \\
7\end{array}$ & $\begin{array}{c}\text { Блок } \\
8\end{array}$ \\
\hline а. КИУМ (годовой \%) & - & - & - & - & & - & - & - \\
\hline $\begin{array}{l}\text { b. Коэффициент вынужденны } \\
\text { х остановов (годовой \%) }\end{array}$ & - & - & - & - & - & - & - & - \\
\hline $\begin{array}{l}\text { с. Коэффициент } \\
\text { незапланированных потерь } \\
\text { мощности (годов й \%) }\end{array}$ & - & - & - & - & - & - & - & - \\
\hline
\end{tabular}

\begin{tabular}{|c|c|c|c|c|c|c|c|c|}
\hline $\begin{array}{l}\text { Показатель } \\
\text { эффективности блока }\end{array}$ & $\begin{array}{c}\text { Блок } \\
1\end{array}$ & $\begin{array}{c}\text { Блок } \\
2\end{array}$ & $\begin{array}{c}\text { Блок } \\
3\end{array}$ & $\begin{array}{c}\text { Блок } \\
4\end{array}$ & $\begin{array}{c}\text { Блок } \\
5\end{array}$ & $\begin{array}{c}\text { Блок } \\
6\end{array}$ & $\begin{array}{c}\text { Блок } \\
7\end{array}$ & $\begin{array}{c}\text { Блок } \\
8\end{array}$ \\
\hline \multirow{2}{*}{$\begin{array}{l}\text { d. Коллективная доза } \\
\text { облучения (годовая) }\end{array}$} & - & - & $\longrightarrow$ & {[} & $\longrightarrow$ & 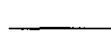 & - & \\
\hline & \multicolumn{8}{|c|}{ Человеко-зиверт на блок $\square$ или человеко-бэр на блок $\square$} \\
\hline \multicolumn{7}{|l|}{$\begin{array}{l}\text { е. Число } \\
\text { незапланированных } \\
\text { автоматических }\end{array}$} & & \\
\hline \multirow{2}{*}{$\begin{array}{l}\text { f. Интенсивность } \\
\text { нарушений техники } \\
\text { безопасности }\end{array}$} & - - & - & $\longrightarrow$ & - & $\ldots$ & $\longrightarrow$ & 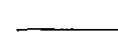 & $\underline{-2}$ \\
\hline & \multicolumn{8}{|c|}{ На 200000 часов работы $\square$ или } \\
\hline
\end{tabular}




\begin{tabular}{|c|c|c|c|c|c|c|c|c|}
\hline \multirow{2}{*}{$\begin{array}{l}\text { Показатель } \\
\text { эффективности блока }\end{array}$} & $\begin{array}{c}\text { Блок } \\
1\end{array}$ & $\begin{array}{c}\text { Блок } \\
2\end{array}$ & $\begin{array}{c}\text { Блок } \\
3\end{array}$ & $\begin{array}{c}\text { Блок } \\
4\end{array}$ & $\begin{array}{c}\text { Блок } \\
5\end{array}$ & $\begin{array}{c}\text { Блок } \\
6\end{array}$ & $\begin{array}{c}\text { Блок } \\
7\end{array}$ & $\begin{array}{c}\text { Блок } \\
8\end{array}$ \\
\hline & \multicolumn{8}{|c|}{$\begin{array}{c}\text { Пожалуйста, укажите квартиль (от } 1 \text { до 4) соотносящийся с } \\
\text { подобным типом станщии. (Подробное описание смотрите в сноске }{ }^{4} \text { ) }\end{array}$} \\
\hline $\begin{array}{l}\text { g. Годовой } \\
\text { эксплуатационный } \\
\text { показатель систем } \\
\text { безопасности }\end{array}$ & - & - & - & - & - & - & - & - \\
\hline $\begin{array}{l}\text { h. Годовой показатель } \\
\text { надежнос и топлива }\end{array}$ & - & - & - & - & - & - & - & - \\
\hline $\begin{array}{l}\text { i. Годовой показатель } \\
\text { водно-химического } \\
\text { режима }\end{array}$ & - & - & - & - & - & - & - & - \\
\hline
\end{tabular}

\section{Примечание:}

Определения ВАО (если надо в помощь при ответе на вышеприведенные вопросы 2a-2i)

1. кИУМ: отношение (в процентах за год) фактической энерговыработки реакторной установки за период эксплуатации к энерговыработке при работе без остановок на номинальной мощности.

2. Коэффициент незапланированных потерь мощности: доля (в процентах за год) максимально возможной произведенной энергии, которую станция не смогла передать в сеть потребления вследствие внеплановых потерь, таких, как незапланированные остановы или потери из-за вынужденных удлинений плановых остановов.

3. Коэффициент (интенсивность) вынужденных остановов: доля (в процентах за год) генерируемой энергии без учета плановых остановов, когда станщия не способна передавать энергию в сеть потребления вследствие незапланированных потерь, таких, как сброс АЗ или снижение мощности.

4. Коллективная доза облучения: сумма индивидуальных доз излучения различных категорий облучаемых лиц за определенный промежуток времени для реакторов различного типа: кипящих (BWRs), с водой под давлением (PWRs), с тяжелой водой под давлением (PHWRs), легководных графитовых (LWCGRs), и газоохлаждаемых (GCRs). Измеряется в человеко-зивертах на блок или в человекобэр на блок.

${ }^{4}$ Квартили определены следующим образом: квартиль 4 (нижний) - от $1 \%$ до 25\%; квартиль 3 - от $26 \%$ до 50\%; квартиль 2 - от $51 \%$ до $75 \%$; квартиль 1 (верхний) - от $76 \%$ до $100 \%$. 
5. Число незапланированных автоматических сбросов АЗ за 7000 часов работы: среднее число незапланированных сбросов АЗ (т.е. автоматический останов реактора) примерно за один год (т.е. 7000 часов) эксплуатации вследствие возникновения тепловых или гидравлических переходных режимов, которые влияют на работу систем станции.

6. Интенсивность нарушений техники безопасности: число инцидентов, которые в результате привели к потере рабочего времени, изпользование работника с ограничениями или к смертельным случаям за 200000 часов работы.

7. Эксплуатационный показатель системы безопасности: годовой показатель работы систем безопасности АЭС, указывающий на степень готовности трех основных систем безопасности в режиме ожидания на каждой станции. Системы безопасности, которые поддерживаются в высокой степени готовности, с высокой степенью вероятности способны минимизировать возникновение или последствия нештатных событий.

8. Надежность ТВС: годовой показатель надежности топлива, указывающий на прогресс в области предупреждения дефектов металлических оболочек твэлов. Сохранение целостности оболочек твэлов уменьшает радиологическое воздействие на работы во время эксплуатации и проведения техобслуживания и ремонта.

9. Показатель водно-химического режима: годовой показатель, указывающий на прогресс в области контроля химических параметров, замедляющих ухудшение состояния основных материалов и компонентов на станции. Эти параметры уже поддерживаются в рамках значений, изложенных в разработанных для использования в промышленности руководствах.

\section{ЧАСТЬ G: ДЕМОГРАФИЧЕСКИЕ И ДРУГИЕ ДАННЫЕ (ДЛЯ АЭС)}

1. Просьба указать число работников (не считая подрядчиков) на вашей станции:

2. Просьба указать типичное число подрядчиков (в эквиваленте полного рабочего дня) на вашей станции во время остановов:

3. Просьба указать типичное число подрядчиков (в эквиваленте полного рабочего дня) на вашей станции во время работы на мощности:

4. Просьба указать процент работников вашей станции, имеющих высшее образование:

5. Просьба указать страну, в которой находится ваша станция:

6. Просьба указать число действующих энергоблоков (т.е. энергетических реакторов) на вашей станции:

7. Просьба указать тип реакторов на станции (например, PWR, BWR, PHWR, , LWCGR или GCR и т.п.): 
8. Просьба указать название проекта реакторной установки (например, EPR, AP1000, BBЭP-440 и т.п.):

9. Пожалуйста найдите соответствующую ячейку и отметьте примерный возраст каждого энергоблока вашей станции (измеренный в годах от завершения сооружения). Колонки не имеющихся на вашей станции блоков просто пропустите:

\begin{tabular}{|c|c|c|c|c|c|c|c|c|}
\hline $\begin{array}{c}\text { Возраст } \\
\text { (сколько лет) }\end{array}$ & Блок 1 & Блок 2 & Блок 3 & Блок 4 & Блок 5 & Блок 6 & Блок 7 & Блок 8 \\
\hline $1-10$ & $\square$ & $\square$ & $\square$ & $\square$ & $\square$ & $\square$ & $\square$ & $\square$ \\
\hline $11-20$ & $\square$ & $\square$ & $\square$ & $\square$ & $\square$ & $\square$ & $\square$ & $\square$ \\
\hline $21-30$ & $\square$ & $\square$ & $\square$ & $\square$ & $\square$ & $\square$ & $\square$ & $\square$ \\
\hline $31-40$ & $\square$ & $\square$ & $\square$ & $\square$ & $\square$ & $\square$ & $\square$ & $\square$ \\
\hline $41+$ & $\square$ & $\square$ & $\square$ & $\square$ & $\square$ & $\square$ & $\square$ & $\square$ \\
\hline
\end{tabular}

10. Просьба указать, в каких сообществах практики (рабочих группах) участвует ваша АЭС, и сравниваются ли результаты вашей регулярной самооценки с эксплуатационными показателями или эталонными данными (бенчмаркинг) этих рабочих групп.

\section{Название и тематика сообщества практики}

\section{Рабочая группа}

a.) Надежность оборудования

b.) Материалы и услуги (цепочка снабжения)

c.) Информационные технологии

d.) Администрация/У правление ядерными активами

e.) Управление информацией

f.) Вопросы лицензирования/регулирования

g.) Людские ресурсы

h.) Радиационная защита

i.) Ядерное топливо

j.) Контроль исполнения/ввода усовершенствований

k.) Эксплуатация АЭС

l.) Управление химическим режимом

m.) Управление работами

n.) Тренажеры

о.) Обучение

p.) Управление финансами (сметы и затраты)

q.) Управление конфигурацией

r.) Пожарная безопасность

s.) Другая (укажите:

$$
\begin{gathered}
\text { укажите, ссли } \\
\text { регулярно участвуете в } \\
\text { работах по данному } \\
\text { направлению }
\end{gathered}
$$

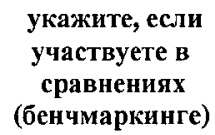

укажите, если участвуете в

сравнениях (бенчмаркинге)

Просьба указать также, в каком качестве выражалось ваше участие в вышеназванных РГ: местные группы (например, национальные) $\square$, региональные (например, европейские) $\square$, международные (например, МАГАТЭ, ЭПРИ, ИНПО, ИЯЭ) $\square$, либо группы владельцев атомных объектов $\square$. 
11. Просьба указать число руководящих работников по вопросам эксплуатации, которые оказывали помощь в заполнении данного вопросника:

12. Просьба ниже добавить любые дополнительные комментарии или разъяснения по поводу ваших ответов.

13. Если вы хотите, чтобы прислало вам окончательный отчет по данному исследованию, пожалуйста, сообщите свое имя и адрес электронной почты (необязательно):

Имя:

Должность (звание):

E mail aдpec:

благодарит Вас за время, затраченное на заполнение данного Вопросника! 


\section{APPENDIX C. CHINESE TRANSLATION OF SURVEY INSTRUMENT}

单元 A: 知识管理实践

PARTA: KNOWLEDGE MANA GEMENT PRACTICES

请根据下列评分标准说明您对本电厂组织以下每一状况的认可程度：

Please indicate your level of agreement with each of the following statements about your station organization, according to the following rankings:

1. 完全不同意

Strongly disagree

2. 部分不同意

Somewhat disagree

3. 既不同意也不反对 (中性)

Neither agree nor disagree (neutral)

4. 部分同意

Somewhat agree

5. 完全同意

Strongly agree

1. 知识管理战略和计划

完全不同意＼cjkstart完全同意 无法评估

Knowledge Management Strategy and Plan

a. 本组织拥有明确的、以文件记录的高水平的知 识管理计划和目标。

The organization has clear, documented high level knowledge management plan and goals.

b. 知识管理战略和计划的实施得到管理部门公 开和积极的支持。

Implementation of the knowledge management strategy and plan is openly and actively supported by management. 
1. 知识管理战略和计划

Knowledge Management Strategy and Plan

$\begin{array}{lcr}\text { 完全不同意 } & \text { 完全同意 } & \text { 无法评估 } \\ \text { Strongly } & \text { Strongly } & \text { Unable } \\ \text { disagree } & \text { agree } & \text { to rate }\end{array}$

$\begin{array}{lllll}1 & 2 & 3 & 4 & 5\end{array}$

c. 员工与管理人员在知识管理中的作用和责任

已明确确定并且各自都清楚地了解。

Knowledge management roles and

responsibilities are clearly defined and

understood by managers and employees.

d. 其他管理战略 (如人力资源、信息系统、运行、 通讯及维修计划等) 与知识管理战略和计划保 持高度一致。

Other management strategies (e.g. human resources, information systems, operations, communications and maintenance plans) are closely aligned with the knowledge management strategy and plan.

e. 定期审查对组织知识基础的需要和其中存在 的差距, 并及时修订知识管理战略和计划以解 决这些问题。

The needs and gaps in the organizational knowledge base are periodically reviewed and the knowledge management strategy and plan is revised to address them.

2. 对组织内业务学习的支持 Support for Organizational Learning

完全不同意 完全同意 无法评估 Strongly Strongly Unable disagree agree to rate

$\begin{array}{lllll}1 & 2 & 3 & 4 & 5\end{array}$

a. 知识的创造和应用 (例如, 找到更好的方法, 技术创新) 得到鼓励, 承认和奖励。

Knowledge creation and application (e.g., finding better methods, technology innovation) is encouraged, recognized and rewarded. 
2. 对组织内业务学习的支持

Support for Organizational Learning

$\begin{array}{lcr}\text { 完全不同意 } & \text { 完全同意 } & \text { 无法评估 } \\ \text { Strongly } & \text { Strongly } & \text { Unable } \\ \text { disagree } & \text { agree } & \text { to rate }\end{array}$

$\begin{array}{lllll}1 & 2 & 3 & 4 & 5\end{array}$

b. 提倡和奖励知识共享 (如鼓励和奖励专家对其 他员工进行业务指导和知识传授）。

Sharing of knowledge is promoted and rewarded

(e.g., experts are encouraged and rewarded to coach or mentor other employees).

c. 促进公开交流和以无问责方式揭示问题并共

同吸取教训(例如, 鼓励维修和操作人员之间 的定期沟通)。

Open communication and a no-blame approach to reporting problems and sharing lessons

learned are promoted (e.g., regular

communication is encouraged between

maintenance and operations personnel).

d. 鼓励各种学习机会（例如, 加入专业团体或参 加培训研讨会)。

Learning opportunities are encouraged (e.g., joining specialist groups or attending training seminars).

3. 过程管理实践

Process Management Practices

$\begin{array}{lcr}\text { 完全不同意 } & \text { 完全同意 } & \text { 无法评估 } \\ \text { Strongly } & \text { Strongly } & \text { Unable } \\ \text { disagree } & \text { agree } & \text { to rate }\end{array}$

$\begin{array}{lllll}1 & 2 & 3 & 4 & 5\end{array}$

a. 在所有过程和程序中, 工作重点是确保各类要 求、方法、输入、输出、接口、职责和工作流 程都被正确记录和及时更新。

For all processes and procedures, priority is placed on ensuring the requirements, methods, inputs, outputs, interfaces, responsibilities, and workflow are documented correctly and maintained up to date. 
3. 过程管理实践

Process Management Practices
完全不同意 完全同意 无法评估

Strongly

disagree

Strongly

Unable

agree

to rate

b. 在所有工作与决策过程中考虑到了危害与风

险, 以确保安全不受到任何负面影响。

Consideration of hazards and risk is built into all

work and decision processes to ensure safety is

not adversely impacted.

c. 各项程序与工作任务和决策过程的知识和信

息需求保持一致。

Procedures are aligned to knowledge and

information requirements of both work tasks and decision processes.

d. 制订并遵循了用以衡量和提高所有业务、工作 和决策过程的质量控制程序。

A process to measure and improve the quality and control of all business, work, and decision processes is defined and followed.

e. 全面的知识管理程序 (例如知识损失风险评

估) 都被记录, 并在使用中。

Comprehensive knowledge management

procedures (e.g. for knowledge loss risk

assessment) are documented and in use.

\section{$\begin{array}{lllll}1 & 2 & 3 & 4 & 5\end{array}$}

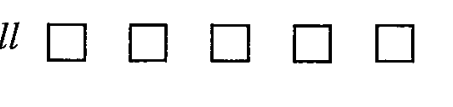


4. 信息管理实践

Information Management Practices

$\begin{array}{lcr}\text { 完全不同意 } & \text { 完全同意 } & \text { 无法评估 } \\ \text { Strongly } & \text { Strongly } & \text { Unable } \\ \text { disagree } & \text { agree } & \text { to rate }\end{array}$

$\begin{array}{lllll}1 & 2 & 3 & 4 & 5\end{array}$

a. 营运许可文件、设计基础文件、程序、技术规 范、图纸以及培训资料能及时更新以反映核电 厂的变更, 并被纳入配置管理。

Licensing documents, design basis documents, procedures, specifications, drawings, and training materials are updated promptly to address plant changes and are maintained under configuration management.

b. 要求各类记录、数据和日志都必须完整、有意 义、准确且易获取 (例如日志、会议纪要和测 试结果)。

Records, data, and logs are required to be complete, meaningful, accurate and accessible (e.g., logs, minutes, test results).

c. 数据标准、元数据、文件编码、主题索引和文 件归档系统得到了广泛使用, 以确保有效信息 的相关性、存储和检索。

Data standards, metadata, document codes, subject indexes and filing systems are widely used to enable efficient information correlation, storage and retrieval.

d. 有关程序确保了对数据和信息的安全性、保安 性、可维护性、可获取性、质量和可保存性的 需求。

Procedures ensure the needs for data and information safety, security, maintainability, accessibility, quality and preservation. 
5. 组织实绩的管理实践

Organizational Performance Management

Practices

$\begin{array}{lcr}\text { 完全不同意 } & \text { 完全同意 } & \text { 无法评估 } \\ \text { Strongly } & \text { Strongly } & \text { Unable } \\ \text { disagree } & \text { agree } & \text { to rate }\end{array}$

$\begin{array}{lllll}1 & 2 & 3 & 4 & 5\end{array}$

a. 定期进行独立的外部同行审查和评定 (如核电 营运者联合会（WANO）、核电运行研究所

（INPO）或原子能机构运行安全评审组的审 查)。

Independent external peer review assessments are conducted regularly (e.g. WANO, INPO, or IAEA-OSART reviews).

b. 广泛使用自评定, 以促进业务学习和改进工作 实绩 (如以最佳实践作为评审基准)。 Self-assessments are widely used to stimulate learning and improve performance (e.g. benchmarking against best practices).

c. 对组织内部所有各级部门和领域 (包括对知识 过程) 均确定了实绩目标并得到监测。 Performance objectives are established and monitored for all levels and areas of the organization (including for knowledge processes).

d. 以行业最佳实践确定的目标为基准制订本组 织运行、维护和安全实绩目标。

Performance objectives for operations, maintenance, and safety are based on objectives established by industry best practice.

e. 定期审查管理系统（包括知识管理系统）的有 效性。

The effectiveness of the management system (including the knowledge management system) is regularly reviewed.

f. 建立并遵循了运行经验的积累、评审、分析和 纠正行动的持续过程。

Ongoing processes for operational experience capture, review, analysis and corrective action are defined and followed. 
6. 培训相关实践

\section{Training Related Practices}

a. 本组织在培训大纲中纳入了 “系统培训法” 原则。

The organization incorporates principles of the "systematic approach to training" (SAT) in training programs.

b. 为所有工作岗位提供了充分的培训, 使员工 具备和保持所需水平的工作能力。

Sufficient training is provided to achieve and maintain the required level of competence for all job positions.

c. 培训材料得到了审查, 以确保其反映了从运 行经验中获得的教训并与核电厂文件保持一 致。

Training material is reviewed to ensure it reflects lessons learned from operating experience and agrees with plant documentation.

d. 与大学和院校保持协作关系, 以确保得到新 毕业生的适当补充。

Collaboration with universities and colleges ensures an appropriate supply of new graduates.

e. 其他培训技术的使用情况 (如实例讲述、概念 图、工作前简况介绍、非正式研讨会、辅导计 划等）。请说明：

Other techniques are used for training (e.g. story-telling, concept mapping, pre-job briefings, informal seminars, mentoring programs etc.). Please specify:

$\begin{array}{lcr}\text { 完全不同意 } & \text { 完全同意 } & \text { 无法评估 } \\ \text { Strongly } & \text { Strongly } & \text { Unable } \\ \text { disagree } & \text { agree } & \text { to rate }\end{array}$

$\begin{array}{lllll}1 & 2 & 3 & 4 & 5\end{array}$
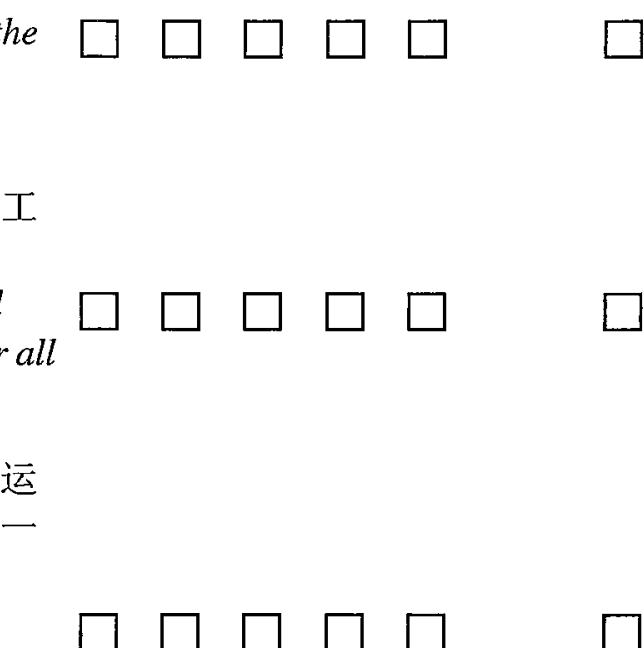

.

.


7. 人力资源相关实践

Human Resource Related Practices

$\begin{array}{lcc}\text { 完全不同意 } & \text { 完全同意 } & \text { 无法评估 } \\ \text { Strongly } & \text { Strongly } & \text { Unable } \\ \text { disagree } & \text { agree } & \text { to rate }\end{array}$

$\begin{array}{lllll}1 & 2 & 3 & 4 & 5\end{array}$

a. 定期跟踪预期退休和意外离职, 并对由此所致 关键知识和工作技能方面的需求和提供问题 采取行动。

Expected retirements and unexpected departures are regularly tracked and the resulting need for and availability of critical knowledge and job skills is acted upon.

b. 为促进知识传承, 在专家离职前及早做好了新 员工的招聘工作, 并确保新员工的能力得到及 时发展。

New hiring is done long before experts depart to facilitate knowledge transfer and ensure the competency of replacements is developed in time.

c. 提前开展离职员工的例行交谈, 以确定关键知 识和经验并促进知识保留和传承。

Interviews with departing employees are routinely carried out well in advance to identify critical knowledge and experience and to facilitate knowledge capture and transfer.

d. 在员工业绩考核中对其工作能力、培训情况、 知识共享或传授目标予以确认, 评价并作出奖 励。

Competency, training and knowledge sharing or transfer goals are identified, evaluated and rewarded in employee performance assessment.

e. 工作安排促进了业务学习的提高 (如在岗位轮 换、团队选择以及工作人员的岗位分配中慎重 考虑了各种学习机会）。

Work assignments promote learning (e.g., job-rotations, team selections and staff assignments consider learning opportunities). 
单元 B：技术支持

PART B: TECHNOLOGY SUPPORT

请根据下列评分标准对本电厂组织如何有效地应用下列每项技术作出说明:

Please indicate how effectively each of the following technologies is used in your station organization according to the following rankings:

1. 非常有效

Very effectively

2. 有效

Effectively

3. 部分有效

Somewhat effectively

4. 无效

Not effectively

5. 没有使用这些技术

Not used at all

1. 信息系统和技术支持
非常有效

Very

Information Systems and Technology Support
末使用

Not

used

$\begin{array}{lllll}1 & 2 & 3 & 4 & 5\end{array}$

a. 三维 (3D) 虚拟环境用于培训。

Three dimensional $(3 D)$ virtual reality environments for training.

b. 计算机和（或）基于网络的培训。 Computer and/or web-based training.

c. 台式计算机 (如工厂) 培训模拟器。 Desktop (e.g. plant) training simulators.

d. 全尺寸主控制室培训模拟机。

Full scope main control room training simulators.

\section{Effectively}

5
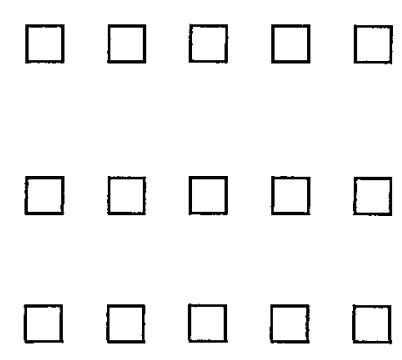

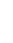


1. 信息系统和技术支持

Information Systems and Technology Support
非常有效

Very

Effectively

1

23

未使用

无法评估

Unable

Not

used

to rate

e. 电子档案和数据库 (如文件管理、事件报告、 维护记录等)。

Electronic archives and databases (e.g. for

document management, event reporting,

maintenance records, etc.)

f. 企业应用软件 (如财务、采购、零部件库存管 理、工作和故障管理等)。

Enterprise application software (e.g. for financials, procurement, parts inventory

management, work and outage management, etc.)

g. 局域网的门户网站能够搜索/检索经常使用的

资源（如文件、公告、联系人名单等）。

Intranet web portal with search/retrieval access

to frequently used resources (e.g. documents,

bulletins, contact lists, etc.)

h. 三维计算机辅助设计工厂模型和可编辑的电 子图纸。

Three-dimensional (3D) computer aided design (CAD) plant models and editable electronic drawings.

\section{2. 先进的运行支持系统}

Advanced Operational Support Systems

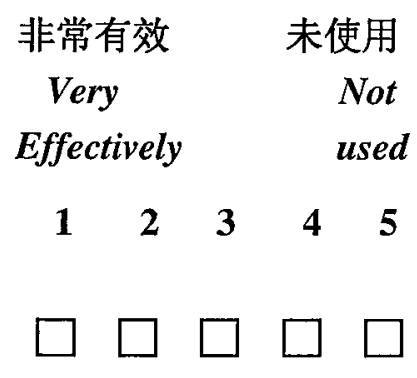

a. 运行决策支持系统 (如换料软件)。

Operational decision support systems (e.g. refuelling software) 
2. 先进的运行支持系统 Advanced Operational Support Systems

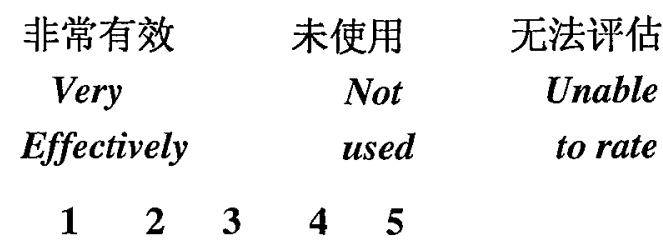

b. 定期更新（即 “实时”）用于维修和停机规划 的设备可靠性概率风险模型。

Regularly updated (i.e. "living") probabilistic risk models of equipment reliability for maintenance and outage planning.

c. 操纵员使用实时概率风险模型进行核电厂安 全状态评价和认知 (即 “安全监测器”)。 Real-time probabilistic risk models for operator evaluation and awareness of plant safety (i.e. "a safety monitor").

d. 系统正常状态监测器 (例如预测维修工具, 诸 如振动、声、热或其它监测器）。

System health monitors (e.g. predictive maintenance tools such as vibration, acoustic, thermal, or other monitors).

e. 以先进模型为基础的监测与诊断系统 (如物 理、化学、热交换器、给水和热工水力学模型 等)。

Advanced model-based monitoring and diagnostics (e.g. physics, chemistry, boiler, feed water and thermal hydraulics models).

f. 先进的信息交流 (如手提计算机、全厂范围内 设备运行状态的监测、无线通讯)。 Advanced information exchange (e.g. hand-held computers, plant-wide equipment status monitoring, wireless communications).

g. 电子 (图表式) 业务和决策过程路线图或工作 流程 (如运行流程图) 与支持程序、相关资源 或文件的链接。

Electronic (i.e. graphical) road-maps of business and decision processes or work-flows (e.g. operational flow-sheets) with links to supporting procedures, related resources or documents. 
2. 先进的运行支持系统

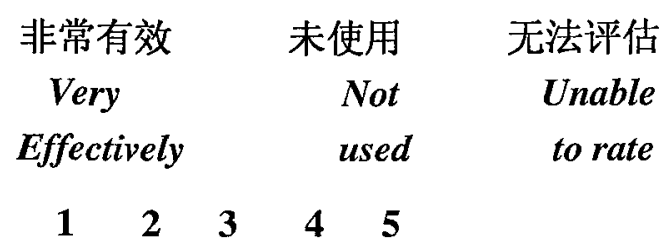

h. 数据的现场自动化采集 (如智能仪器、现场总 线、射频识别 (RFID) 标记、数据记录、设备 监测器)。

Automated field data collection (i.e., smart instruments, field-bus, radio frequency identification (RFID) tagging, data logging, equipment monitors).

i. 其他（请注明）:

Other (please specify):

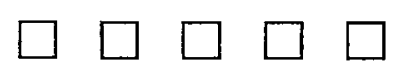




\section{单元 C: 知识管理过程的质量}

\section{PART C: QUALITY OF KNOWLEDGE PROCESSES}

请根据下列评分标准说明您对本电厂组织以下每一状况的认可程度:

Please indicate your level of agreement with each of the following statements about your station organization, according to the following rankings:

1. 完全不同意

Strongly disagree

2. 部分不同意

Somewhat disagree

3. 既不同意也不反对 (中性)

Neither agree nor disagree (neutral)

4. 部分同意

Somewhat agree

5. 完全同意

Strongly agree

1. 知识获取

\section{Knowledge Acquisition}

$\begin{array}{lcr}\text { 完全不同意 } & \text { 完全同意 } & \text { 无法评估 } \\ \text { Strongly } & \text { Strongly } & \text { Unable } \\ \text { disagree } & \text { agree } & \text { to rate }\end{array}$

\section{$\begin{array}{lllll}1 & 2 & 3 & 4 & 5\end{array}$}

a. 本组织难于发现和录用适当合格的毕业生。 The organization has difficulty finding and hiring appropriately qualified graduates.

b. 本组织善于查明和获取核电厂运行和维护所 需的外部技术信息。

The organization excels at identifying and acquiring external technical information needed to operate and maintain the plant.

c. 获取的外部信息往往没有以易于维护及存取 的方式得到整理或储存, 以便使用和再使用。 External information acquired is often not organized or stored in a maintainable and accessible way to facilitate use and re-use. 


\section{1. 知识获取}

Knowledge Acquisition

d. 本组织能够有效地从外部 (如同行核电厂) 运 行经验中获取知识。

The organization is effective at acquiring

knowledge from external (e.g. peer-plant)

operating experiences.

e. 本组织能够非常有效地采纳外部最佳实践。

The organization is highly effective at adopting external best practices.

f. 本组织善于捕捉技术诀空和与来自外部组织 服务或产品有关的设计信息。

The organization is good at capturing technical know-how and relevant design information related to services or products received from outside organizations.

\section{2. 知识创新}

\section{Knowledge Creation}

a. 员工能够从运行经验中学习并吸取教训, 新 的、更好的运行核电厂的方式很少被忽视。 NPP staff learn from operating experience and new and better ways of running the plant are seldom overlooked.

b. 独立评审过程对于验证可能影响安全或生产 的运行或设计变更提案是有效的。

Independent review processes are effective at validating proposed operational or design changes that may impact safety or production.

$\begin{array}{lcr}\text { 完全不同意 } & \text { 完全同意 } & \text { 无法评估 } \\ \text { Strongly } & \text { Strongly } & \text { Unable } \\ \text { disagree } & \text { agree } & \text { to rate }\end{array}$

\section{$\begin{array}{lllll}1 & 2 & 3 & 4 & 5\end{array}$}
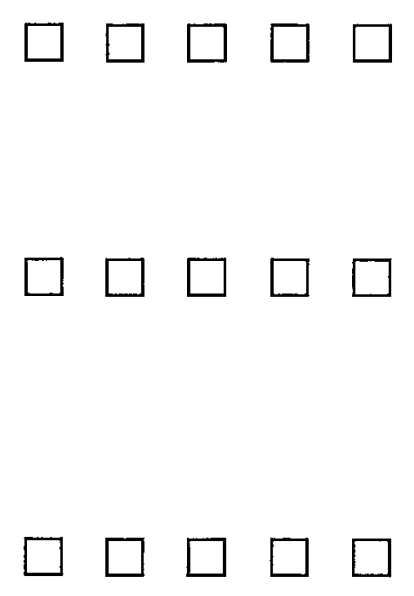

$\begin{array}{lcr}\text { 完全不同意 } & \text { 完全同意 } & \text { 无法评估 } \\ \text { Strongly } & \text { Strongly } & \text { Unable } \\ \text { disagree } & \text { agree } & \text { to rate }\end{array}$

$\begin{array}{lllll}1 & 2 & 3 & 4 & 5\end{array}$

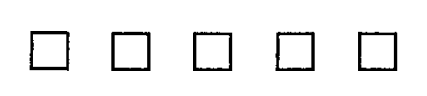




\section{2. 知识创新}

\section{Knowledge Creation}

$\begin{array}{lcr}\text { 完全不同意 } & \text { 完全同意 } & \text { 无法评估 } \\ \begin{array}{l}\text { Strongly } \\ \text { disagree }\end{array} & \text { Strongly } & \text { Unable } \\ \text { agree } & \text { to rate }\end{array}$

\section{$\begin{array}{lllll}1 & 2 & 3 & 4 & 5\end{array}$}

c. 员工缺乏挑战假设和调查异常或不确定情况所 需的质疑态度。

Employees lack the questioning attitude needed to challenge assumptions and investigate anomalies or uncertainties.

d. 员工结合或采用现有和/或获得的知识, 定期 提出有创新意义的解决方案。

Employees regularly create innovative solutions by combining or adapting existing and/or acquired knowledge.

e. 本组织善于生成、转化和提供核电厂的数据并 将其作为有意义的信息。

The organization excels at generating, transforming, and presenting plant data as meaningful information.

f. 工程师们需花费过多的时间从多种来源收集 和汇编数据。

Engineers have to spend too much time gathering and compiling data from many sources.

\section{3. 知识传承}

\section{Knowledge Transfer}

a. 公司一个部门能够容易获取其他部门的结果、

信息、数据、报告或文件档案。

Findings, information, data, reports, or files generated in one area of the company are readily accessible to other areas.

$\begin{array}{lcr}\text { 完全不同意 } & \text { 完全同意 } & \text { 无法评估 } \\ \text { Strongly } & \text { Strongly } & \text { Unable } \\ \text { disagree } & \text { agree } & \text { to rate }\end{array}$

$\begin{array}{lllll}1 & 2 & 3 & 4 & 5\end{array}$ 


\section{3. 知识传承}

Knowledge Transfer

b. 员工往往不知道该从本组织何处找到所需的 专业知识和信息。

Employees often do not know where in the organization to find specialized knowledge and information.

c. 本组织内部不存在封锁知识的问题, 员工愿意 分享彼此的知识。

The problem of hoarding (keeping) knowledge does not exist and employees willingly share their knowledge with co-workers.

d. 资深雇员的专门知识和技能不能有效地传承 给初级雇员。

Expertise and skills are not effectively transferred to junior staff from more experienced employees.

e. 员工们定期和自愿地与该组织其他部们分享 有关或许是必要的信息。 Employees routinely and voluntarily share relevant information with other parts of the organization where it may be needed.

4. 知识利用 Knowledge Utilization

a. 从运行实践中吸取的经验教训被纳入了工作 实践、手册、程序和决策过程。 Lessons learned from operating experience are incorporated in work practices, manuals, procedures and decision-making.

b. 本组织往往不能高效地解决技术难题。 The organization is often not able to solve difficult technical problems efficiently.

$\begin{array}{lcr}\text { 完全不同意 } & \text { 完全同意 } & \text { 无法评估 } \\ \text { Strongly } & \text { Strongly } & \text { Unable } \\ \text { disagree } & \text { agree } & \text { to rate }\end{array}$

$\begin{array}{lllll}1 & 2 & 3 & 4 & 5\end{array}$
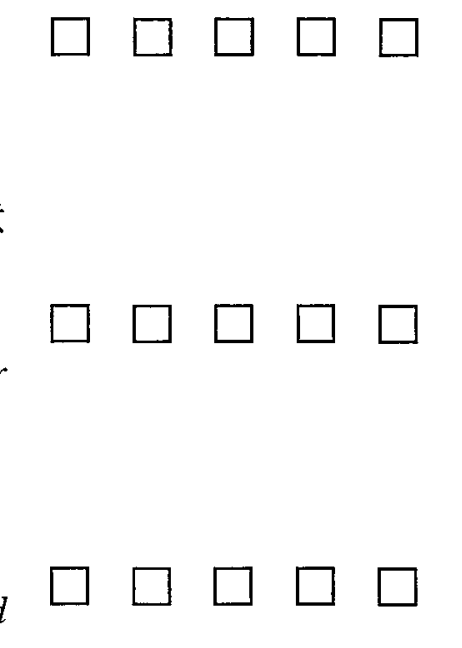
c. 员工能够始终正确地作出重要的技术决定。

Employees are consistently able to make important technical decisions correctly.

d. 员工并不总能意识到并有效地利用彼此之间 的技能和专门知识。

Employees are not always aware of and do not always make effective use of each other's skills and expertise.

e. 设备更换和设计变更决策基于风险知情决策 过程。

Equipment replacement and design change decisions are based on a risk-informed decision process.

5. 知识保存

\section{Knowledge Retention}

$\begin{array}{lcr}\text { 完全不同意 } & \text { 完全同意 } & \text { 无法评估 } \\ \text { Strongly } & \text { Strongly } & \text { Unable } \\ \text { disagree } & \text { agree } & \text { to rate }\end{array}$

a. 员工往往缺乏反应堆和核电厂的适当基本知 识。

Employees often lack an appropriate knowledge of the reactor and power plant fundamentals.

b. 员工对工作过程（如工业和辐射安全工作实

践）有充分的认识和了解。

Employees have adequate

knowledge/understanding of work processes (e.g. industrial and radiation safety work practices).

c. 由于员工退休和意外离职, 本组织往往缺乏关 键技能和经验。

There is often a shortage of critical skills and experience due to unexpected departures and retirements.

d. 核电厂设计的基础文件很容易找到，而且能够 得到更新, 内容准确。

Plant design basis documents are easily located

$\begin{array}{lllll}1 & 2 & 3 & 4 & 5\end{array}$
and are up-to-date and accurate. 
e. 维护，运营或技术支持专家对特定系统缺乏能 使其有效和安全开展工作所需的足够的知识 和技术。

Maintenance, operations, or technical support specialists lack adequate knowledge of specific systems and technologies to enable them to work effectively and safely. 
单元 D: 组织文化

PART D: ORGANIZATIONAL CULTURE

请根据下列评分标准说明您对本电厂组织以下每一状况的认可程度:

Please indicate your level of agreement with each of the following statements about your station organization, according to the following rankings:

1. 完全不同意

Strongly disagree

2. 部分不同意

Somewhat disagree

3. 既不同意也不反对 (中性)

Neither agree nor disagree (neutral)

4. 部分同意

Somewhat agree

5. 完全同意

Strongly agree

1. 组织文化

Organizational Culture

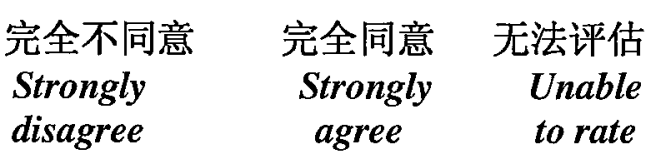

$\begin{array}{lllll}1 & 2 & 3 & 4 & 5\end{array}$

a. 管理人员和员工往往没有把学习、创新和改 进作为其工作的一部分。

Managers and employees often do not see learning, innovation, and improvement as a part of their jobs.

b. 具有创新作为的员工得到认可和奖励。 Employees who innovate feel recognized and rewarded.

c. 普遍存在遵循已确定的各项过程和充分执行 各项程序的态度和承若。

There is a prevailing attitude and commitment to follow defined processes and fully comply with procedures. 
1. 组织文化

Organizational Culture

d. 员工往往并不认为能够有权作出适合其工作 职责的决定。

Employees often do not feel empowered to make decisions appropriate to their job duties.

e. 员工拥有共同的理念、目标和期望, 他们将 出现的所有问题都视为大家共同的问题。

There is shared vision, purpose, and expectations among employees and they see all their problems as mutual.

f. 员工被视为本组织中最有价值的财富。

People are seen as the organisation's most valued asset.

g. 员工和管理人员思想开放, 并且彼此尊重对 方的意见和贡献。

Employees and managers are open-minded and respect each other's opinions and contributions.

h. 团队精神贯穿于整个电厂中 (如员工彼此信 任、合作和互助)。

There is a team-oriented approach throughout the station (e.g., employees trust, cooperate, and help each other).

i. 员工对核电厂实绩缺乏责任感, 未能做到恪 尽职守。

Employees often do not feel responsible for plant performance and fail to demonstrate their commitment to it.

j. 对安全的考虑明确贯穿于员工和管理人员的 行动和决定之中。 Consideration of safety is clearly evident in employee and management actions and decisions.

$\begin{array}{lcr}\text { 完全不同意 } & \text { 完全同意 } & \text { 无法评估 } \\ \text { Strongly } & \text { Strongly } & \text { Unable } \\ \text { disagree } & \text { agree } & \text { to rate }\end{array}$

$\begin{array}{lllll}1 & 2 & 3 & 4 & 5\end{array}$

$\square$
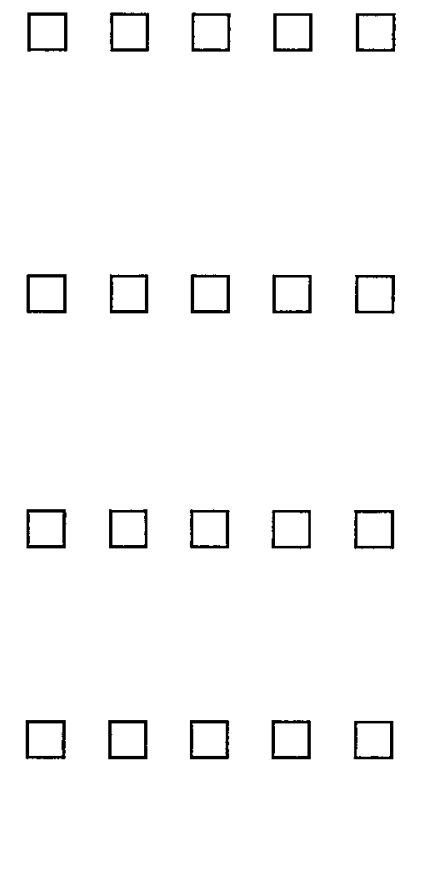
1. 组织文化

Organizational Culture

k. 改进工作大多始然于外部提出的要求 (如监 管部门的裁定、业主的影响）。

Improvements are mostly driven by externally imposed requirements (e.g. regulatory rulings, owner influences).

1. 工作中表现出质疑的态度 (如信息、方案和 决定得到仔细检查)。

A questioning attitude is cultivated (i.e. information, approaches and decisions are carefully scrutinized).

m. 本组织的工作重点主要集中于短期目标。 The organization is focused primarily on short-term goals.

$\begin{array}{lcr}\text { 完全不同意 } & \text { 完全同意 } & \text { 无法评估 } \\ \text { Strongly } & \text { Strongly } & \text { Unable } \\ \text { disagree } & \text { agree } & \text { to rate }\end{array}$

$\begin{array}{lllll}1 & 2 & 3 & 4 & 5\end{array}$

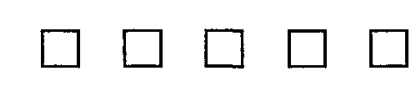

\section{(1)}




\section{里元 $\mathrm{E}:$ 组织效能}

\section{PARTE: ORGANIZATIONAL EFFECTIVENESS}

请根据下列评分标准说明您对本电厂组织以下每一状况的认可程度:

Please indicate your level of agreement with each of the following statements about your station organization, according to the following rankings:

1. 完全不同意

Strongly disagree

2. 部分不同意

Somewhat disagree

3. 既不同意也不反对 (中性)

Neither agree nor disagree (neutral)

4. 部分同意

Somewhat agree

5. 完全同意

Strongly agree

1. 组织效能

Organizational Effectiveness

$\begin{array}{lcr}\text { 完全不同意 } & \text { 完全同意 } & \text { 无法评估 } \\ \text { Strongly } & \text { Strongly } & \text { Unable } \\ \text { disagree } & \text { agree } & \text { to rate }\end{array}$

$\begin{array}{lllll}1 & 2 & 3 & 4 & 5\end{array}$

a. 本组织难以顺利和及时地对业务工作做出改 革。

The organization has difficulty making operational changes smoothly and in a timely manner.

b. 维护技师一贯地进行高质量的纠正和预防性 维护。

Maintenance technicians consistently conduct high-quality corrective and preventive maintenance.

c. 纠正性维护与预防性维护的比例相对而言高 于设计相似且实绩最佳的核电厂。

The ratio of corrective to preventive maintenance is high relative to best performing NPPs of similar design. 
1. 组织效能

Organizational Effectiveness

$\begin{array}{lcr}\text { 完全不同意 } & \text { 完全同意 } & \text { 无法评估 } \\ \text { Strongly } & \text { Strongly } & \text { Unable } \\ \text { disagree } & \text { agree } & \text { to rate }\end{array}$

$\begin{array}{lllll}1 & 2 & 3 & 4 & 5\end{array}$

d. 电厂化学程序确保电厂始终在化学相关规定 范围之内运行。

The plant chemistry program ensures the plant consistently operates within the chemistry specifications.

e. 涉及多个部门的项目通常会拖延, 超出预算, 而且缺乏良好的协调。

Projects involving multiple departments are typically behind schedule, over-budget, and not well coordinated.

f. 始终达到或超过既定的安全目标。

Safety objectives are consistently met or exceeded.

g. 系统和/或实绩分析工程师们不能有效地解决 影响核电厂安全或实绩的问题。

System and/or performance analysis engineers are not effective at resolving problems that affect plant safety or performance.

h. 辐射条件得到有效地控制 (即现场保持合理可 行尽量低的辐射水平, 且剂量控制是有效的)。 Radiological conditions are effectively controlled (i.e. field levels are as low as reasonably achievable and dose control is effective).

i. 文件的质量 (即设计、工作过程和程序文件) 需要改进。 Quality of documentation (i.e. design, work-process and procedural documentation) needs to improve. 
1. 组织效能

Organizational Effectiveness

$\begin{array}{lcr}\text { 完全不同意 } & \text { 完全同意 } & \text { 无法评估 } \\ \text { Strongly } & \text { Strongly } & \text { Unable } \\ \text { disagree } & \text { agree } & \text { to rate }\end{array}$

$\begin{array}{lllll}1 & 2 & 3 & 4 & 5\end{array}$

j. 操纵员能有效地采取行动, 应对核电厂状况变 化，以确保核电厂的持续安全和可靠的运行。 Operators effectively act on changing plant conditions to ensure ongoing safe and reliable plant operation.

k. 每周的各项运行目标通常不能达到。

Weekly operations objectives are regularly not met.

1. 工作规划和管理是有效的（即计划的工作范围 是稳定的，没有将时间浪费在等待审批或零部 件供应上）。

Work planning and management is effective (e.g. planned work-scope is stable, little time is wasted waiting on approvals or parts)

m. 关键部件的年平均故障率低于其它类似的核 电厂。

The average number of critical component failures per year is low relative to other similar plants.

n. 已知和可避免的运行问题的复发并非总能防 止。

Recurrence of known and avoidable operational problems is not always prevented.

o. 本组织能有效地处理好与外部的接口关系 (如 监管部门、公众、供应商、承包商)。 The organization is effective at managing its external interfaces (i.e. the regulator, public, suppliers, contractors)

p. 环境目标有时不能达到。

Environmental objectives are sometimes not met.

\section{$\square$}

$\begin{array}{lll}3 & 4 & 5\end{array}$

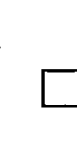

$\square$
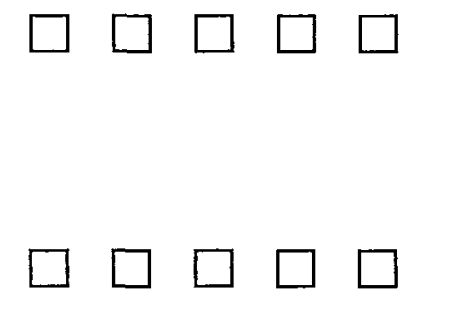

\section{$\square$} 事 
1. 组织效能

$\begin{array}{lcr}\text { 完全不同意 } & \text { 完全同意 } & \text { 无法评估 } \\ \text { Strongly } & \text { Strongly } & \text { Unable } \\ \text { disagree } & \text { agree } & \text { to rate }\end{array}$

Organizational Effectiveness

$\begin{array}{lllll}1 & 2 & 3 & 4 & 5\end{array}$

q. 基于行业最佳实践所制订的维护目标（例如纠

正性和预防性维修积压水平) 始终能够达到或

超过。

Maintenance objectives (e.g. level of corrective and preventive maintenance backlog) based on industry best practice are consistently met or exceeded.

r. 财务目标通常不能达到。

Financial objectives are often not met.

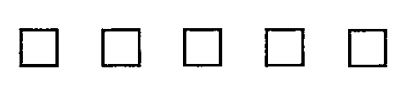

s. 监管目标始终能够达到或超过。

Regulatory objectives are consistently met or exceeded.

t. 系统的健康改善措施是有效的。

System health improvement initiatives are effective.

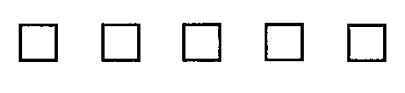

u. 纠正和预防性维护以及停机检修能如期及时 完成。

Corrective and preventive maintenance and outage work is completed on schedule and in a timely manner.

v. 财政资源 (预算) 充足, 分配明智。

Financial resources (budgets) are adequate and allocated wisely. 
1. 如果您提供贵电厂 (可选) 的名称, 贵电厂 2009 年运行实绩指标评定数据可以取自 公布的资料来源（首选方法）。您的回答将保密，只将报告综合结论。

If you provide the name of your station (optional), the 2009 operational performance indicator ratings data for your station can be taken from published sources (a preferred approach). Your responses will remain confidential and only aggregate findings will be reported.

贵电厂名称 (任选) :

Name of your station (optional):

2. 如果您提供了贵电厂名称, 您不必完成单元 $\mathbf{F}$ 的剩余部分, 便可直接进入单元 $\mathbf{G}$. If you have provided the name of your station, you do not need to complete the remainder of Part $F$ and may then go directly to Part $G$.

如果您没有提供贵电厂名称, 您被要求根据以下运行实绩指标对贵电厂每个机组评 分。请使用最近一年提供的评级并请注明年份: . 请忽略不存在的机组列。 If you did not provide the name of your station, you are asked to provide the following operational performance indicator ratings for each unit at your station. Please use the latest year for which data is available and indicate the year: . Ignore any columns for units that do not exist.

\begin{tabular}{|c|c|c|c|c|c|c|c|c|}
\hline $\begin{array}{l}\text { 机组实绩指标 } \\
\text { Unit Performance } \\
\text { Indicator }\end{array}$ & $\begin{array}{c}\text { 机组 } \\
1 \\
\text { Unit 1 }\end{array}$ & $\begin{array}{l}\text { 机组 } \\
2 \\
\text { Unit } 2\end{array}$ & $\begin{array}{c}\text { 机组 } \\
3 \\
\text { Unit } 3\end{array}$ & $\begin{array}{c}\text { 机组 } \\
4 \\
\text { Unit } 4\end{array}$ & $\begin{array}{l}\text { 机组 } \\
5 \\
\text { Unit } 5\end{array}$ & $\begin{array}{l}\text { 机组 } \\
6 \\
\text { Unit } 6\end{array}$ & $\begin{array}{l}\text { 机组 } \\
7 \\
\text { Unit } 7\end{array}$ & $\begin{array}{c}\text { 机组 } \\
8 \\
\text { Unit } 8\end{array}$ \\
\hline 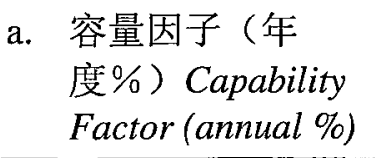 & & & & & & & & \\
\hline $\begin{array}{l}\text { b. 强迫损失率 (年 } \\
\text { 度\%) Forced Loss } \\
\text { Rate (annual \%) }\end{array}$ & & & & & & & & \\
\hline
\end{tabular}




\begin{tabular}{|l|c|c|c|c|c|c|c|c|}
\hline 机组实绩指标 & 机组 & 机组 & 机组 & 机组 & 机组 & 机组 & 机组 & $\begin{array}{c}\text { 机组 } \\
\text { Unit Performance }\end{array}$ \\
$\begin{array}{l}\text { Indicator } \\
\text { Unit 1 }\end{array}$ & $\begin{array}{c}2 \\
\text { Unit 2 }\end{array}$ & $\begin{array}{c}3 \\
\text { Unit 3 }\end{array}$ & $\begin{array}{c}4 \\
\text { Unit 4 }\end{array}$ & $\begin{array}{c}\text { Unit 5 } \\
\text { Unit 6 }\end{array}$ & $\begin{array}{c}\text { Unit 7 } \\
\text { Unit 8 }\end{array}$ \\
\hline $\begin{array}{l}\text { c. 非计划的容量损 } \\
\text { 失因子 (年度\%) } \\
\begin{array}{l}\text { Unplanned } \\
\text { Capability Loss } \\
\text { Factor (annual \%) }\end{array}\end{array}$ & & & & & & & & \\
\hline
\end{tabular}

\begin{tabular}{|c|c|c|c|c|c|c|c|c|}
\hline $\begin{array}{l}\text { 机组实绩指标 } \\
\text { Unit Performance } \\
\text { Indicator }\end{array}$ & $\begin{array}{c}\text { 机组 } \\
1 \\
\text { Unit 1 }\end{array}$ & $\begin{array}{l}\text { 机组 } \\
2 \\
\text { Unit } 2\end{array}$ & $\begin{array}{c}\text { 机组 } \\
3 \\
\text { Unit } 3\end{array}$ & $\begin{array}{c}\text { 机组 } \\
4 \\
\text { Unit } 4\end{array}$ & $\begin{array}{l}\text { 机组 } \\
\quad 5 \\
\text { Unit } 5\end{array}$ & $\begin{array}{l}\text { 机组 } \\
6 \\
\text { Unit } 6\end{array}$ & $\begin{array}{l}\text { 机组 } \\
7 \\
\text { Unit } 7\end{array}$ & $\begin{array}{l}\text { 机组 } \\
\quad 8 \\
\text { Unit } 8\end{array}$ \\
\hline \multirow{2}{*}{$\begin{array}{l}\text { d. 集体辐射照射 (年 } \\
\text { 度量) } \\
\text { Collective } \\
\text { Radiation } \\
\text { Exposure (annual) }\end{array}$} & 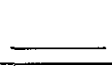 & 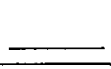 & $=$ & 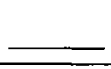 & $=$ & $=$ & 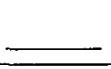 & \\
\hline & \multicolumn{8}{|c|}{$\begin{array}{c}\text { 请说明是用人-希沃特 } \square \text { 或人-雷姆 } \square \text { 为测量单位。 } \\
\text { Specify Man-Sieverts per Unit } \square \text { or Annual Man-rem per Unit } \square\end{array}$} \\
\hline \multicolumn{9}{|l|}{$\begin{array}{l}\text { e. 每 } 7000 \text { 临界小时 } \\
\text { 的非计划自动紧 } \\
\text { 急停堆次数 } \\
\text { Number of } \\
\text { Unplanned } \\
\text { Automatic Scrams } \\
\text { per 7,000 Hours } \\
\text { Critical }\end{array}$} \\
\hline \multirow[b]{2}{*}{$\begin{array}{l}\text { f. 工业安全事故率 } \\
\text { Industrial Safety } \\
\text { Accident Rate }\end{array}$} & & & & & & & & \\
\hline & \multicolumn{8}{|c|}{$\begin{array}{c}\text { 请说明是每 } 20 \text { 万人工时 } \square \text { 或每一百万人工时 } \square \\
\text { fy per } 200,000 \text { person-hours } \square \text { or } 1,000,000 \text { person-hour } \\
\text { worked }\end{array}$} \\
\hline
\end{tabular}




\begin{tabular}{|c|c|c|c|c|c|c|c|c|}
\hline \multirow[b]{2}{*}{$\begin{array}{l}\text { 机组实绩指标 } \\
\text { Unit Performance } \\
\text { Indicator }\end{array}$} & $\begin{array}{l}\text { 机组 } 1 \\
\text { Unit } 1\end{array}$ & $\begin{array}{l}\text { 机组 } 2 \\
\text { Unit } 2\end{array}$ & $\begin{array}{l}\text { 机组 } 3 \\
\text { Unit } 3\end{array}$ & $\begin{array}{l}\text { 机组 } 4 \\
\text { Unit } 4\end{array}$ & $\begin{array}{l}\text { 机组 } 5 \\
\text { Unit } 5\end{array}$ & $\begin{array}{l}\text { 机组 } 6 \\
\text { Unit } 6\end{array}$ & $\begin{array}{l}\text { 机组 } 7 \\
\text { Unit } 7\end{array}$ & $\begin{array}{l}\text { 机组 } 8 \\
\text { Unit } 8\end{array}$ \\
\hline & \multicolumn{8}{|c|}{$\begin{array}{c}\text { 请说明四分位数（即 } 1 \text { 至 4) 相对于同类型的核电厂（例如, 评 } \\
\text { 分 } 1 \text { 是指该机组排列在前 } 25 \% \text { 的同类型机组中, 详见 见注 } 3 \text { ) } \\
\text { Please indicate the quartile (i.e. } 1 \text { to 4) relative to similar plant types } \\
\text { (e.g. a rating of } 1 \text { is a unit in the top 25\% of similar units, see footnote } \\
\text { for details) }\end{array}$} \\
\hline $\begin{array}{l}\text { g. 年度安全系统性 } \\
\text { 能 Annual Safety } \\
\text { System } \\
\text { Performance }\end{array}$ & - & - & - & - & - & - & - & - \\
\hline $\begin{array}{l}\text { h. 年度燃料可靠性 } \\
\text { Annual Fuel } \\
\text { Reliability } \\
\end{array}$ & - & - & - & - & - & - & - & - \\
\hline $\begin{array}{l}\text { i. } \quad \text { 年度化学性能 } \\
\text { Annual Chemistry } \\
\text { Performance }\end{array}$ & - & - & - & - & - & - & - & - \\
\hline
\end{tabular}

注: WANO 定义 (供在需要回答上文 $2 \mathrm{a}$ 至 $2 \mathrm{i}$ 问题时参考) :

Notes: WANO definitions (for reference if needed to answer the questions $2 a$ to $2 i$ above):

1. 机组容量因子: 核电厂能够给电网提供最大发电量的年度百分比, 其完全取决于对 核电厂的管理控制因素。

Unit Capability Factor: the annual percentage of maximum energy generation that a plant is capable of supplying to the electrical grid, limited only by factors within control of plant management.

各注 3 四分位数的定义如下: 四分四 $4^{\text {th }}$ (底部) 是从 $1 \%$ 至 $25 \%$; 四分三 $3^{\text {rd }}$ 是从 $26 \%$ 到 $50 \%$; 四分二 $2^{\text {nd }}$ 是从 $51 \%$ 到 $75 \%$; 四分一 $1^{\text {st }}$ (最高) 是由 $76 \%$ 至 $10 \%$ 。

1 Quartiles are defined as follows: Quartile 4 (bottom performance) is from $1 \%$ to $25 \%$; Quartile 3 is from $26 \%$ to $50 \%$; Quartile 2 is from $51 \%$ to $75 \%$; Quartile 1 (top performance) is from $76 \%$ to $100 \%$. 
2. 非计划的容量损失因子: 由于非计划的发电量损失, 如非计划停堆或计划停机检修 工期延长, 核电厂不能给电网提供最大发电量的年度百分比。

Unplanned Capability Loss Factor: the annual percentage of maximum energy generation that a plant is not capable of supplying to the electrical grid because of unplanned energy losses, such as unplanned shutdowns or outage extensions.

3. 强迫损失率: 由于非计划的发电量损失, 如非计划停堆或降功率运行, 核电厂在非 停机期间不能给电网提供发电量的年度百分比。

Forced Loss Rate: is the annual percentage of energy generation during non-outage periods that a plant is not capable of supplying to the electrical grid because of unplanned energy losses, such as unplanned shutdown or load reductions.

4. 集体辐射照射: 控制沸水堆、压水堆、加压重水堆、轻水冷却石墨慢化堆和气冷堆 对工作人员辐射照射的有效性，以人-希沃特或人-雷姆为测量单位 (年度量)。

Collective Radiation Exposure: the effectiveness of personnel radiation exposure controls for boiling water reactors ( $B W R$ s), pressurized water reactors ( $P W R s)$, pressurized heavy water reactors (PHWRs), light-water cooled graphite reactors (LWCGRs), and gas-cooled reactors (GCRs). Measured in Man-Sieverts per unit or Man-rem per unit annually.

5. 每 7000 临界小时的非计划自动紧急停堆次数: 大约一年 (即 7000 小时) 运行中的 平均紧急停堆 (即自动停机) 率。非计划自动紧急停堆导致热工水力瞬态变化而影 响到核电厂各系统。

Unplanned Automatic Scrams per 7,000 Hours Critical: the mean scram (i.e. automatic shutdown) rate for approximately one year (i.e. 7000 hours) of operation. Unplanned automatic scrams result in thermal and hydraulic transients that affect plant systems.

6. 工业安全事故率：导致工作时间损失、工作限制或每 20 万工作小时的人员死亡率的 事故数。

Industrial Safety Accident Rate: the number of accidents that result in lost work time, restricted work, or fatalities per 200,000 work-hours.

7. 安全系统性能: 安全系统性能年度指标监测每座核电厂的三套重要的备用安全系统。 被保持在高度戒备状态的安全系统具有很高的减轻非正常事件的概率。

Safety System Performance: The annual safety system performance indicator monitors the availability of three important standby safety systems at each plant. Safety systems that are maintained in a high state of readiness have a high probability of being capable of mitigating off-normal events.

8. 燃料可靠性: 燃料可靠性年度指标监测防止燃料金属包壳周围发生的缺陷变化过程。 保持燃料包壳的完整性会降低辐射对核电厂运行和维护活动的影响。

Fuel Reliability: The annual fuel reliability indicator monitors progress in preventing defects in the metal cladding that surrounds fuel. Maintenance of fuel cladding integrity reduces radiological impact on plant operations and maintenance activities. 
9. 化学性能：化学性能年度指标监测化学参数控制以延缓核电厂关键材料和部件退化 的变化过程。已经按照行业的严格导则对这些参数实施了控制。

Chemistry Performance: The annual chemistry performance indicator provides an indication of progress in controlling chemical parameters to retard deterioration of key plant materials and components. These parameters are already being maintained within strict guidance developed by the industry.

\section{单元 G：人力资源数据和其他数据}

\section{PART G: DEMOGRAPHIC AND OTHER DATA}

1. 请说明贵电厂员工人数 (不包括合同工) :

Please indicate the number of employees (excluding contractors) at your station.

2. 请说明贵电厂在计划停机检修期间相当于全日制的合同工人数:

Please indicate the typical number of full-time equivalent contractors during outages

3. 请说明贵电厂在功率运行期间相当于全日制的合同工人数:

Please indicate the typical number of full-time equivalent contractors while at power

4. 请说明贵电厂拥有大学学历的员工比例:

Please indicate the percentage of employees with university degrees at your station.

5. 请说明贵电厂所在国家:

Please indicate the country your station is located in.

6. 请说明贵电厂在运机组数 (即动力堆) :

Please indicate the number of operational units (i.e. power reactors) at your station.

7. 请说明反应堆的类型（如压水堆、沸水堆、加压重水堆、轻水冷却石墨堆、气冷堆 等) :

Please indicate the type of reactor (e.g. PWR, BWR, PHWR, LWCGR, or GCR etc.)

8. 请说明电厂模式 (即产品) 名称 (如大型欧洲压水堆 EPR、美国的 AP1000 先进压水 堆、俄罗斯的 VVER440 先进压水堆等):

Please indicate the plant model (i.e. product) name (e.g. EPR, AP1000, VVER 440, etc.):

9. 请在适当的行中指出贵电厂反应堆每个机组的大约堆龄 (以年份来衡量从竣工算起)

(忽略不存在的机组列) :

Please check the appropriate row to indicate the approximate age of each unit at your station (measured in years from completion of construction) (ignore columns for any non-existent units): 


\begin{tabular}{|c|c|c|c|c|c|c|c|c|}
\hline $\begin{array}{c}\text { 堆龄 (年) } \\
\text { Age (years) }\end{array}$ & $\begin{array}{c}\text { 机组 1 } \\
\text { Unit 1 }\end{array}$ & $\begin{array}{c}\text { 机组 2 } \\
\text { Unit 2 }\end{array}$ & $\begin{array}{c}\text { 机组 3 } \\
\text { Unit 3 }\end{array}$ & $\begin{array}{c}\text { 机组 4 } \\
\text { Unit 4 }\end{array}$ & $\begin{array}{c}\text { 机组 5 } \\
\text { Unit 5 }\end{array}$ & $\begin{array}{c}\text { 机组 6 } \\
\text { Unit 6 }\end{array}$ & $\begin{array}{c}\text { 机组 7 } \\
\text { Unit 7 }\end{array}$ & $\begin{array}{c}\text { 机组 8 } \\
\text { Unit 8 }\end{array}$ \\
\hline $1-10$ & $\square$ & $\square$ & $\square$ & $\square$ & $\square$ & $\square$ & $\square$ & $\square$ \\
\hline $11-20$ & $\square$ & $\square$ & $\square$ & $\square$ & $\square$ & $\square$ & $\square$ & $\square$ \\
\hline $21-30$ & $\square$ & $\square$ & $\square$ & $\square$ & $\square$ & $\square$ & $\square$ & $\square$ \\
\hline $31-40$ & $\square$ & $\square$ & $\square$ & $\square$ & $\square$ & $\square$ & $\square$ & $\square$ \\
\hline $41+$ & $\square$ & $\square$ & $\square$ & $\square$ & $\square$ & $\square$ & $\square$ & $\square$ \\
\hline
\end{tabular}

10. 请指明贵电厂组织所加入的实践团体, 以及是否定期参照该团体的实绩指标或基准 进行自评定。

Please indicate which communities of practice (COP) your station organization participates in and whether regular self-assessment is done against the performance indicators or benchmarks from that COP group:

实践团体名称或专题 是否定期参与 是 否进行评定

Name or topic of COP Work Group Indicate if a regular

Indicate if doing benchmarking

$$
\text { participant }
$$

a.) 设备可靠性

Equipment Reliability

b.) 材料和服务 (供应链)

Materials and Services (Supply Chain)

c.) 信息技术

Information Technology

d.) 业务服务/核资产管理

Business Services / Nuclear Asset Management

e.) 信息管理

Information Management

f.) 许可证/监管问题

Licensing / Regulatory Issues

g.) 人力资源

Human Resources

h.) 辐射防护

Radiation Protection 
i.) 核燃料

Nuclear Fuel

j.) 实绩监测/改进

Performance Monitoring / Improvement

k.) 核电厂运行

Plant Operations

1.) 化学管理

Chemistry Management

m.) 工作管理

Work Management

n.) 模拟机

Simulators

o.) 培训

Training

p.) 成本估算和管理

Cost Estimation and Management

q.) 配置管理

Configuration Management

r.) 防火

Fire Protection

s.) 其它（请说明:

)

Other (specify:)

还请说明参与上述实践团体是否包括：国内（如全国性） $\square$, 地区（如欧洲） $\square$, 国际 (例如原子能机构、电力研究机构 (EPRI)、核电运行研究所 (INPO) 或核能 研究所 (NEI) ) $\square$, 或贵电厂组织内部实践团体 $\square$ 。

Please also indicate whether COP participations above include: local (e.g. national) regional (e.g. European) $\square$, international (e.g. IAEA, EPRI, INPO or NEI) $\square$, or Owner's Group based COPs $\square$.

11. 请说明帮助完成这项问卷调查表的业务经理的人数:

Please indicate the number of operations managers who helped complete this survey response.

12. 请在以下空白处对您的答复意见提出补充说明或澄清。

Please make any additional comments on, or clarifications of any of your responses in the space provided below. 
13. 如果您希望向您发送一份本问卷调查结果报告的电子副本当本报告发表后, 请提供 您的姓名, 职务和电子邮件地址（可选）。

If you wish to have an electronic copy of the report summarizing the findings of this study once it is available, please provide your name, title and e-mail address (optional).

姓名 Name:

职务 Title:

电子邮件地址 E-mail address:

感谢您在百忙之中完成本问卷调查表的填写!

Thank you for your valuable time in completing this questionnaire! 


\section{APPENDIX D. FRENCH TRANSLATION OF SURVEY INSTRUMENT}

\section{PARTIE A : PRATIQUES DE GESTION DES CONNAISSANCES}

Veuillez indiquer votre position par rapport aux affirmations ci-après à l'aide des appréciations suivantes :
1. absolument pas d'accord
2. pas vraiment d'accord
3. sans opinion (neutre)
4. plutôt d'accord
5. absolument d'accord

1. Stratégie et plan de gestion des connaissances

a. L'entreprise a un plan et des objectifs de gestion des connaissances importantes clairs et documentés.

b. La mise en ouvre de la stratégie et du plan de gestion des connaissances fait l'objet d'un soutien explicite et actif de la direction.

c. Les rôles et les responsabilités des responsables et des employés en matière de gestion des connaissances sont bien définis et bien compris de tous.

d. Les autres stratégies de gestion (par ex. des ressources humaines, des systèmes d'information, de l'exploitation, des communications et de la maintenance) sont alignées de près sur la stratégie et le plan de gestion des connaissances.

e. Les besoins et les lacunes dans la base des connaissances de l'entreprise sont périodiquement examinés et la stratégie et le plan de gestion des connaissances sont révisés en conséquence.

$\begin{array}{ccccc}\begin{array}{c}\text { Absolument } \\ \text { pas d'accord }\end{array} & \begin{array}{c}\text { Absolument } \\ \text { d'accord }\end{array} & \begin{array}{c}\text { Pas en mesure } \\ \text { d'évaluer }\end{array} \\ 1 & 2 & 3 & 4 & 5\end{array}$
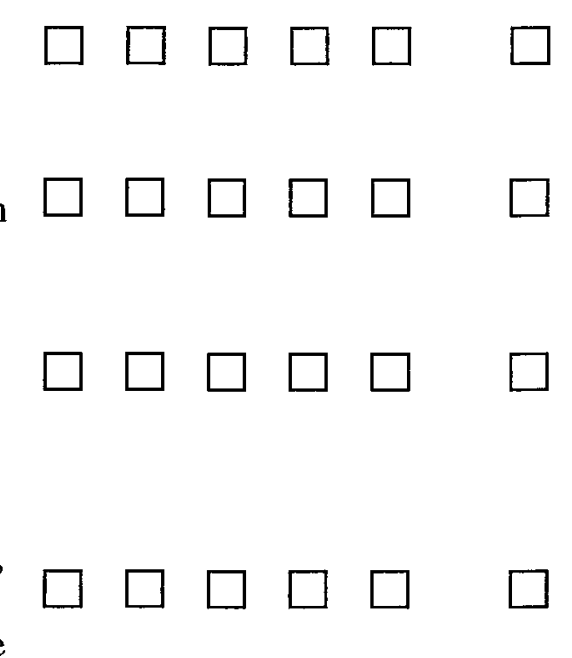


\section{Soutien à l'apprentissage en entreprise}

a. La création de connaissances et leur application (par ex., la recherche de meilleures méthodes, innovations technologiques) sont encouragées, reconnues et récompensées.

b. Le partage des connaissances est encouragé et récompensé (par ex., des experts sont incités à encadrer ou à parrainer d'autres employés et en sont récompensés).

c. Le dialogue et une approche exempte de critiques sont encouragés pour la communication des problèmes et des enseignements (par ex., des communications régulières entre le personnel de maintenance et le personnel d'exploitation sont encouragées).

d. Les possibilités d'apprentissage sont encouragées (par ex., participation à des groupes de spécialistes ou à des colloques de formation).

\section{Pratiques de gestion des processus}

a. S'assurer en priorité, pour l'ensemble des processus et procédures, que les prescriptions, les méthodes, les entrées, les sorties, les interfaces, les responsabilités et l'organisation du travail sont documentés correctement et tenus à jour.

b. La prise en compte des dangers et des risques est intégrée dans toutes les méthodes de travail et tous les processus de prises de décisions pour que la sûreté ne soit pas affectée.

c. Les procédures sont alignées sur les exigences en matière de connaissances et d'informations, aussi bien au niveau des tâches que des processus de prises de décisions.

d. Une marche à suivre visant à mesurer et à améliorer la qualité et le contrôle de l'ensemble des processus au niveau de l'entreprise, du travail et des décisions est définie et appliquée.

\section{Absolument pas d'accord \\ Absolument d'accord \\ $\begin{array}{lllll}1 & 2 & 3 & 4 & 5\end{array}$ \\ Pas en mesure \\ d'évaluer}
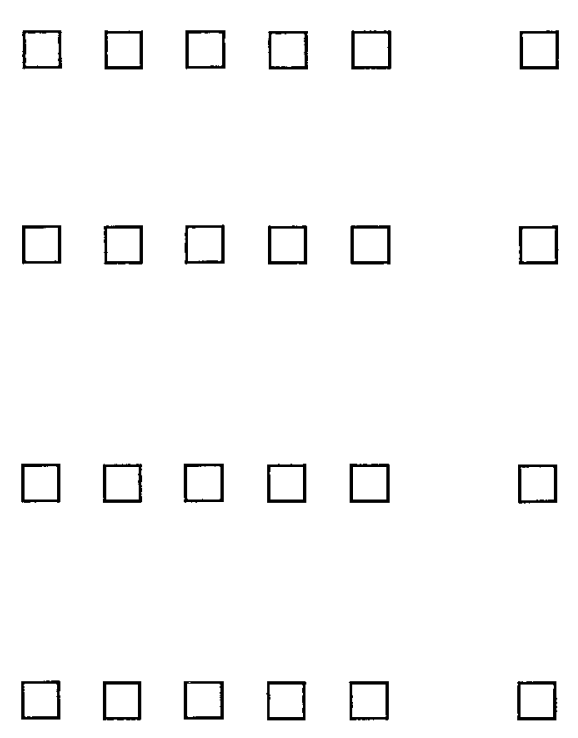

Pas en mesure d'évaluer $\begin{array}{llll}\text { pas d'accord } & & \text { d'accord } \\ 1 & 2 & 3 & 5\end{array}$ 
e. Des procédures exhaustives de gestion des connaissances (par ex., pour l'évaluation du risque de perte de connaissances) sont documentées et appliquées.

f. Les processus et procédures de gestion des connaissances s'appliquent aussi aux fournisseurs et aux organismes d'appui.

\section{Pratiques de gestion de l'information}

a. Les documents d'autorisation, les documents sur la base de conception, les procédures, les spécifications, les plans et le matériel de formation sont promptement mis à jour pour prendre en compte les modifications apportées à la centrale et sont soumis à la gestion de la configuration.

b. Les archives, données et registres doivent être complets, utiles, précis et accessibles (par ex., enregistrements, procès verbaux, résultats d'essais).

c. Des données normalisées, métadonnées, codes de documents, indexation par sujet et systèmes d'archivage sont largement utilisés pour corréler, stocker et extraire les informations de manière efficace.

d. Des procédures permettent de répondre aux exigences de sûreté, de sécurité, de maintenabilité, d'accessibilité, de qualité et de préservation des données et de l'information.

\section{Pratiques de gestion de la performance organisationnelle}

\begin{tabular}{|c|c|}
\hline $\begin{array}{l}\text { Absolument } \\
\text { pas d'accord }\end{array}$ & $\begin{array}{l}\text { Absolument } \\
\text { d'accord }\end{array}$ \\
\hline 12 & 45 \\
\hline
\end{tabular}

Pas en mesure d'évaluer

a. Des évaluations externes indépendantes sont effectuées régulièrement par des pairs (par ex. missions WANO, INPO ou OSART-AIEA).

\begin{tabular}{|c|c|}
\hline $\begin{array}{l}\text { Absolument } \\
\text { pas d'accord }\end{array}$ & $\begin{array}{l}\text { Absolument } \\
\text { d'accord }\end{array}$ \\
\hline 12 & 45 \\
\hline
\end{tabular}

\section{oava}
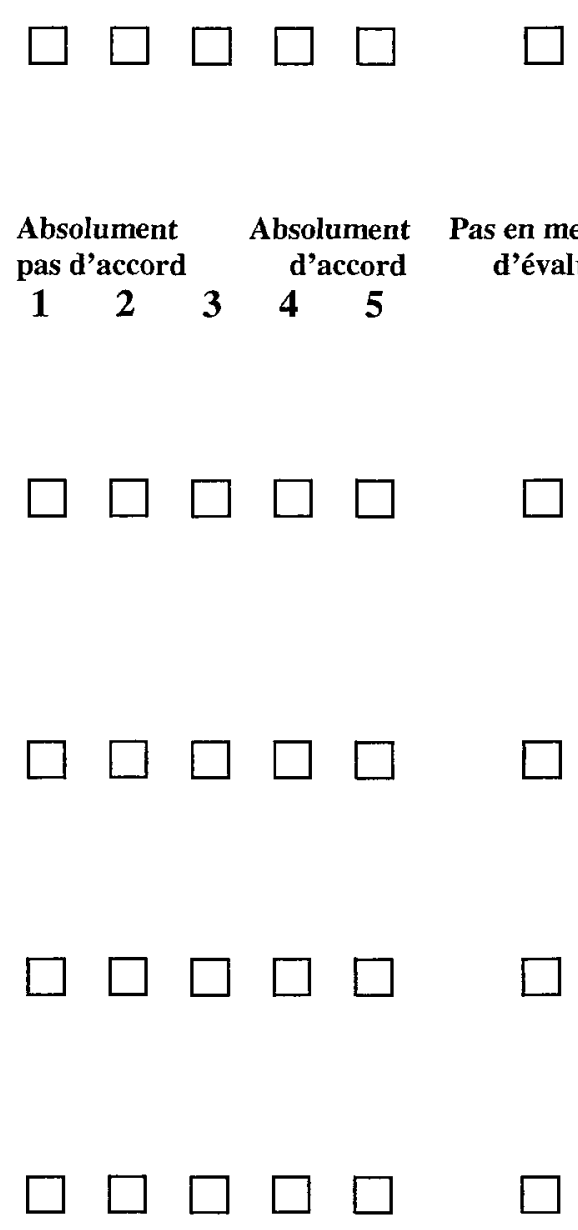
b. Les auto-évaluations sont courantes pour stimuler l'apprentissage et améliorer la performance (par ex., comparaisons avec les meilleures pratiques).

c. Des objectifs de performance sont établis et leur évolution est suivie à tous les niveaux et dans tous les services de l'organisme (y compris pour les processus concernant les connaissances).

d. Les objectifs de performance pour l'exploitation, la maintenance et la sûreté sont basés sur les objectifs fixés en fonction des meilleures pratiques de l'industrie.

e. L'efficacité du système de gestion (y compris le système de gestion des connaissances) est évaluée périodiquement.

f. Des processus continus de collecte, d'examen et d'analyse des données sur l'expérience d'exploitation ainsi que de mesures correctives sont définis et appliqués.

\section{Pratiques liées à la formation}

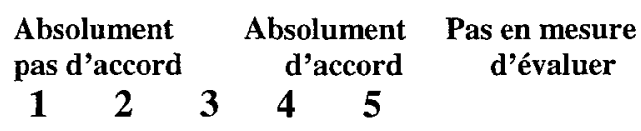

a. L'organisme incorpore dans ses programmes de formation les principes dits de l'approche systémique de la formation (ASF).

b. Une formation suffisante est assurée pour atteindre et maintenir le niveau de compétences requis à tous les postes.

c. Le matériel de formation est passé en revue de sorte qu'il reflète les enseignements tirés de l'expérience d'exploitation et qu'il soit conforme à la documentation de la centrale.

d. La collaboration avec des universités et des établissements d'enseignement supérieur permet de constituer un vivier de nouveaux diplômés. 
e. D'autres techniques sont employées pour la formation (par ex. récit d'expériences présentées sous forme d'histoires), schématisation conceptuelle, discussions avant un travail, colloques informels, programmes de parrainage, etc.). Prière de préciser :

\section{Pratiques liées aux ressources humaines}

$\begin{array}{ccccc}\begin{array}{c}\text { Absolument } \\ \text { pas d'accord }\end{array} & \begin{array}{c}\text { Absolument } \\ \text { d'accord }\end{array} & \begin{array}{c}\text { Pas en mesure } \\ \text { d'évaluer }\end{array} \\ 1 & 2 & 3 & 4 & 5\end{array}$

a. Les retraites prévues et les départs imprévus font l'objet d'un suivi régulier et le besoin et la disponibilité de connaissances et de compétences professionnelles indispensables sont pris en considération.

b. Le recrutement pour assurer la relève se fait bien avant que les experts ne partent à la retraite de manière à faciliter le transfert des connaissances et à former à temps une relève compétente.

c. Des entretiens avec les employés qui partent sont effectués régulièrement bien avant leur départ pour recueillir et transférer la somme d'expériences et de connaissances indispensables acquises.

d. Des objectifs en matière de partage ou de transfert des compétences, de la formation et des connaissances sont fixés, et les résultats sont évalués et notés au cours des évaluations du comportement du personnel.

e. L'organisation du travail favorise l'apprentissage (par ex., il est tenu compte des possibilités d'acquisition de connaissances pour le roulement des tâches, la composition des équipes et l'affectation du personnel). 


\section{PARTIE B : APPUI TECHNOLOGIQUE}

Veuillez indiquer le degré d'efficacité avec lequel les techniques suivantes sont employées dans votre organisation à la centrale:
1. très efficace
2. efficace
3. plutôt efficace
4. inefficace
5. néant (pas employé)

\section{Systèmes d'information et appui technologique}

$\begin{array}{ccccc}\begin{array}{c}\text { Très } \\ \text { efficace }\end{array} & & \begin{array}{c}\text { Pas } \\ \text { employé }\end{array} & \begin{array}{c}\text { Pas en mesure } \\ \text { d'évaluer }\end{array} \\ 1 & 2 & 3 & 4 & 5\end{array}$

a. Environnements virtuels de formation en trois dimensions (3D).

b. Formation sur ordinateur et/ou le web.

c. Simulateurs de formation (modèle de la centrale) sur ordinateur de bureau.

d. Simulateurs intégraux de salle de commande principale pour la formation.

e. Archives électroniques et bases de données (par ex. pour la gestion de documents, la notification des événements, les dossiers de maintenance, etc.).

f. Logiciels d'applications d'entreprise (par ex. pour les finances, les achats, la gestion des stocks de pièces détachées, la gestion des opérations et arrêts, etc.).

g. Portail web intranet avec accès pour la recherche de ressources fréquemment utilisées (par ex. documents, bulletins, listes de contacts, etc.).

h. Modèles de centrales tridimensionnels (3D) avec conception assistée par ordinateur (CAO) et dessins électroniques modifiables.

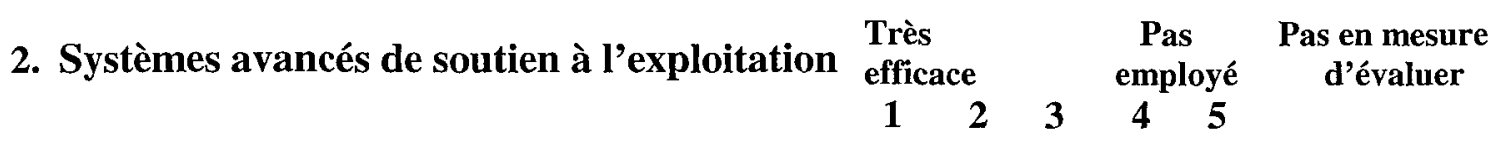


2. Systèmes avancés de soutien à l'exploitation

a. Systèmes de soutien aux décisions d'exploitation (par ex. logiciel pour le rechargement en combustible).

b. Modèles probabilistes régulièrement mis à jour («vivants ») d'évaluation de la fiabilité du matériel utilisés pour la maintenance et la planification des arrêts.

c. Modèles probabilistes utilisés pour l'évaluation en temps réel des opérateurs et pour la sensibilisation à la sûreté de la centrale (par ex. un moniteur de sûreté).

d. Moniteurs de surveillance de l'état des systèmes (par ex., outils de maintenance prédictive, comme des moniteurs de niveaux de vibrations, moniteurs acoustiques, moniteurs thermiques ou autres).

e. Outils avancés de diagnostic et de surveillance basés sur des modèles (par ex. pour la physique, la chimie, le générateur de vapeur, l'eau d'alimentation et la thermohydraulique).

f. Systèmes avancés d'échange d'information (par ex. ordinateurs portatifs, surveillance de l'état de l'équipement dans toute la centrale, communications sans fil).

g. Tableaux électroniques des processus opérationnels et décisionnels internes ou de gestion des flux (par ex. schémas de fonctionnement) avec des liens vers des procédures d'appui, des ressources ou des documents connexes.

h. Collecte automatisée de données de terrain (instruments 'intelligents', 'fieldbus', marquage d'identification par radiofréquence (RFID), enregistrement de données, moniteurs de matériel).

i. Autres (À préciser)

\section{Très $\quad$ Pas $\quad$ Pas en mesure \\ efficace employé d'évaluer}
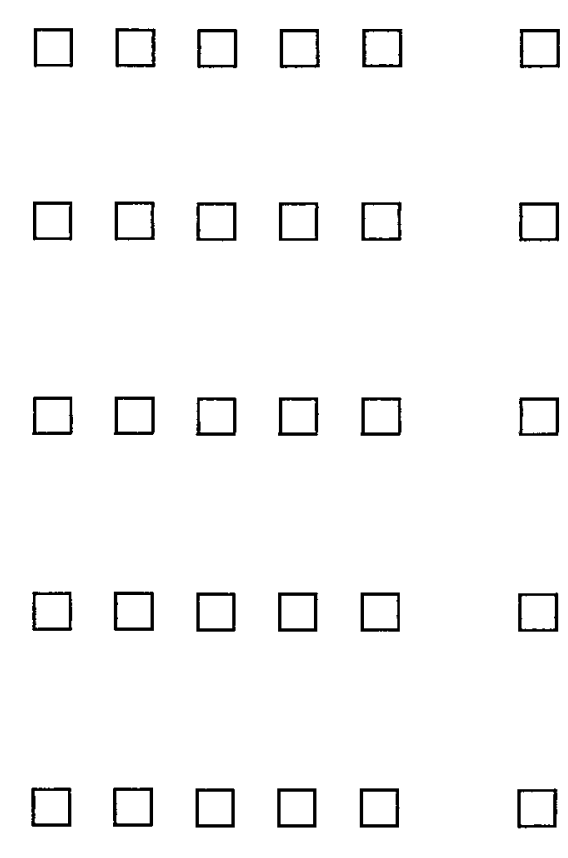


\title{
PARTIE C : QUALITÉ DES PROCESSUS LIÉS AUX CONNAISSANCES
}

\author{
Veuillez indiquer votre position par rapport aux affirmations ci-après à l'aide des \\ appréciations suivantes :
}

\section{1. absolument pas d'accord}

2. pas vraiment d'accord

3. sans opinion (neutre)

4. plutôt d'accord

5. absolument d'accord

\section{Acquisition des connaissances}

$\begin{array}{lllll}\begin{array}{c}\text { Absolument } \\ \text { pas d'accord }\end{array} & \begin{array}{r}\text { Absolument } \\ \text { d'accord }\end{array} & \begin{array}{r}\text { Pas en mesure } \\ \text { d'évaluer }\end{array} \\ 1 & 2 & 3 & 4 & 5\end{array}$

a. L'organisme a du mal à recruter des étudiants diplômés possédant les qualifications requises.

b. L'organisme excelle à sélectionner et à acquérir à l'extérieur de l'organisme des informations techniques nécessaires à l'exploitation et à la maintenance de la centrale.

c. Les informations acquises à l'extérieur souvent ne sont pas organisées ou stockées de manière systématique et accessible pour pouvoir être utilisées et ré-utilisées.

d. L'organisme sait efficacement acquérir des connaissances externes sur l'expérience d'exploitation (par ex. auprès de centrales analogues).

e. L'organisme sait adopter avec succès des pratiques optimales externes.
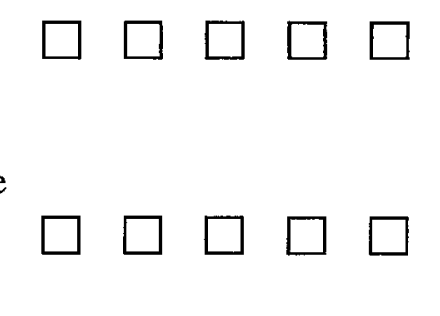

L'organisme sait bien prendre en compte les connaissances techniques et les renseignements descriptifs relatifs aux services ou produits reçus d'organismes externes. 
Absolument

pas d'accord
Absolument

d'accord

Pas en mesure

d'évaluer

a. Le personnel de la centrale profite des enseignements tirés de l'expérience d'exploitation, et il est rare que l'on ne tienne pas compte de nouveaux et meilleurs moyens d'exploiter la centrale.

b. Les procédures d'examen indépendant sont efficaces pour valider les modifications proposées au niveau de l'exploitation ou de la conception qui sont susceptibles d'influer sur la sûreté ou la production.

c. Le personnel ne fait pas preuve d'un esprit critique suffisant pour mettre en doute la validité des théories et enquêter sur les anomalies ou incertitudes.

d. Le personnel trouve régulièrement des solutions innovantes en associant ou en adaptant des éléments de connaissance existants et/ou acquis.

e. L'organisme excelle à produire, à traiter les données concernant la centrale et à les présenter comme dignes d'intérêt.

f. Les ingénieurs doivent passer trop de temps à recueillir et à compiler des données provenant de sources multiples.

\section{Transfert des connaissances}

$\begin{array}{lllllr}\begin{array}{l}\text { Absolument } \\ \text { pas d'accord }\end{array} & \begin{array}{r}\text { Absolument } \\ \text { d'accord }\end{array} & \begin{array}{r}\text { Pas en mesure } \\ \text { d'évaluer }\end{array} \\ 1 & 2 & 3 & 4 & 5 & \end{array}$

a. Les conclusions, informations, données, rapports ou fichiers qui sont établis dans un service de l'organisme sont aisément accessibles à d'autres services.

b. Il est fréquent que le personnel ne sache pas où trouver dans l'organisme le savoir et les informations spécialisés dont il a besoin.

c. Il n'y a pas de problème pour thésauriser les connaissances, et les employés n'hésitent pas à partager leurs connaissances avec des collègues.
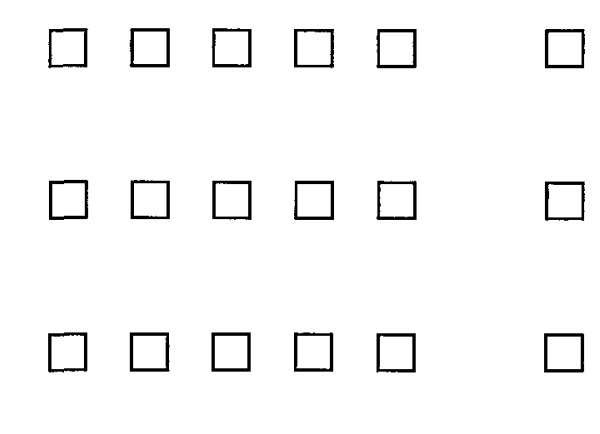


\section{Transfert des connaissances}

$\begin{array}{cccccc}\text { Absolument } & \begin{array}{r}\text { Absolument } \\ \text { pas d'accord }\end{array} & \begin{array}{r}\text { Pas en mesure } \\ \text { d'accord } \\ \text { d'évaluer }\end{array} \\ 1 & 2 & 3 & 4 & 5 & \end{array}$

d. L'expertise et les compétences ne sont pas transmises efficacement par les aînés plus expérimentés aux plus jeunes qui prennent la relève.

e. Les employés partagent régulièrement et volontairement des informations pertinentes avec les autres services de l'organisme qui peuvent en avoir besoin.

\section{Utilisation des connaissances}

$\begin{array}{cccccc}\text { Absolument } & \begin{array}{r}\text { Absolument } \\ \text { pas d'accord }\end{array} & \begin{array}{r}\text { Pas en mesure } \\ \text { d'accord } \\ \text { d'évaluer }\end{array} \\ 1 & 2 & 3 & 4 & 5 & \end{array}$

a. Les enseignements tirés de l'expérience d'exploitation sont incorporés dans les pratiques de travail, les manuels, les procédures et la prise de décisions.

b. Il est fréquent que l'organisme ne soit pas à même de résoudre efficacement des problèmes techniques difficiles.

c. Le personnel est toujours capable de prendre à bon escient d'importantes décisions techniques.

d. Les employés ne sont pas toujours conscients des compétences et de l'expertise internes dont ils pourraient mutuellement profiter.

e. Les décisions concernant le remplacement d'équipements et les modifications de la conception de la centrale s'appuient sur un processus de prise de décisions en fonction des risques. 


\section{Conservation des connaissances}

$$
\begin{aligned}
& \text { Absolument Absolument Pas en mesure } \\
& \text { pas d'accord d'accord d'évaluer } \\
& \begin{array}{lllll}
1 & 2 & 3 & 4 & 5
\end{array}
\end{aligned}
$$

a. Il est fréquent que le personnel n'ait pas des connaissances suffisantes sur les fondamentaux des réacteurs et des centrales.

b. Le personnel a une connaissance/compréhension satisfaisante des procédures de travail (par ex. pratiques de sûreté industrielle et de sûreté radiologique).

c. Il y a souvent pénurie de compétences et d'expériences précieuses due aux départs à la retraite et aux départs imprévus.

d. Les documents relatifs à la base de conception de la centrale sont facilement consultables et sont tenus à jour avec rigueur.

e. Les spécialistes de la maintenance, de l'exploitation ou de l'appui technique, connaissent mal certains systèmes qui leur permettraient de travailler efficacement et en toute sûreté.

\section{PARTIE D : CULTURE D'ENTREPRISE}

Veuillez indiquer votre position par rapport aux affirmations ci-après à l'aide des appréciations suivantes :
1. absolument pas d'accord
2. pas vraiment d'accord
3. sans opinion (neutre)
4. plutôt d'accord
5. absolument d'accord

\section{Culture organisationnelle}

$$
\begin{aligned}
& \text { Absolument Absolument Pas en mesure } \\
& \begin{array}{lllll}
1 & 2 & 3 & 4 & 5
\end{array}
\end{aligned}
$$

a. Il est fréquent que les directeurs et les employés ne reconnaissent pas que l'apprentissage, l'innovation et les améliorations font partie de leur travail.

b. Les employés qui font preuve d'innovation se sentent reconnus et récompensés.

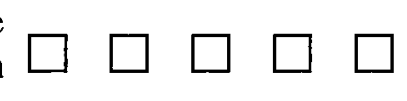




\section{Culture organisationnelle}

\begin{tabular}{|c|c|}
\hline $\begin{array}{l}\text { Absolument } \\
\text { pas d'accord }\end{array}$ & $\begin{array}{l}\text { Absolument } \\
\text { d'accord }\end{array}$ \\
\hline 12 & 45 \\
\hline
\end{tabular}

c. L'attitude et le souci dominants sont de suivre les processus définis et de se conformer intégralement aux procédures.

d. Il est fréquent que les employés ne se sentent pas habilités à prendre des décisions en rapport avec les tâches qui leur sont confiées.

e. Les employés partagent une même vision, un même objectif et les mêmes attentes et ils sont solidaires en cas de problèmes.

f. Les individus sont perçus comme l'atout le plus précieux de l'organisme.

g. Les employés et les directeurs sont ouverts d'esprit et respectent mutuellement leurs opinions et leurs contributions.

h. L'esprit d'équipe prévaut dans toute la centrale (confiance, coopération et entraide parmi les employés).

i. Il est fréquent que les employés ne se sentent pas responsables de la performance de la centrale et qu'ils ne se montrent pas impliqués.

j. Il ressort clairement des actions et des décisions prises par la direction et les employés que la sûreté est prise en considération.

k. Les améliorations sont essentiellement imposées de l'extérieur (par ex., arrêtés de l'organisme de réglementation, pressions du propriétaire).

1. L'esprit critique est entretenu (par ex., les informations, les approches et les décisions sont étudiées avec le plus grand soin).

m. L'organisme se concentre avant tout sur les objectifs à court terme.
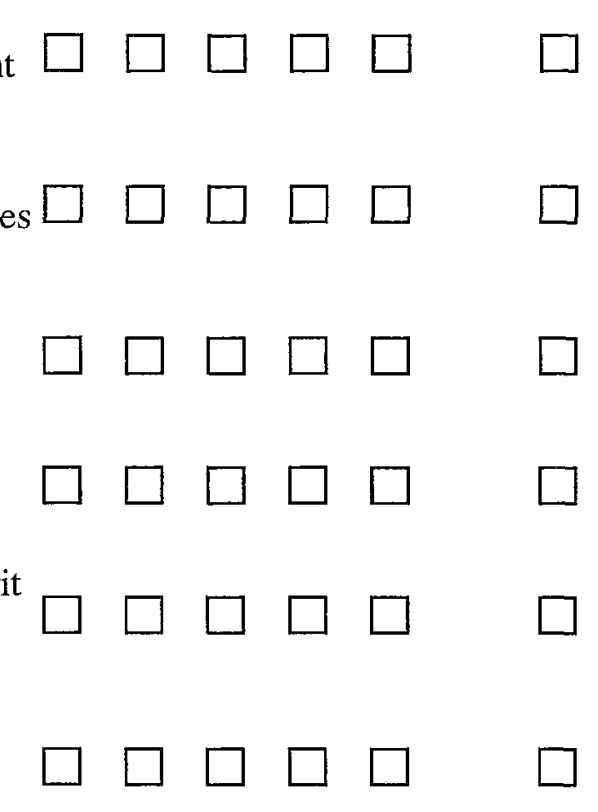


\section{PARTIE E : EFFICACITÉ ORGANISATIONNELLE}

Veuillez indiquer votre position par rapport aux affirmations ci-après à l'aide des appréciations suivantes :
1. absolument pas d'accord
2. pas vraiment d'accord
3. sans opinion (neutre)
4. plutôt d'accord
5. absolument d'accord

\section{Efficacité organisationnelle}

$\begin{array}{cccccc}\begin{array}{c}\text { Absolument } \\ \text { pas d'accord }\end{array} & \begin{array}{r}\text { Absolument } \\ \text { d'accord }\end{array} & \begin{array}{r}\text { Pas en mesure } \\ \text { d'évaluer }\end{array} \\ 1 & 2 & 3 & 4 & 5 & \end{array}$

a. L'organisme a du mal à apporter graduellement et en temps voulu des modifications à l'exploitation.

b. Les agents de maintenance exécutent systématiquement des travaux correctifs et préventifs de qualité.

c. La proportion de maintenance corrective par rapport à la maintenance préventive est élevée comparativement aux meilleures centrales nucléaires de conception similaire.

d. Le programme de chimie dans la centrale garantit que la centrale fonctionne dans les limites des spécifications chimiques.

e. Les projets faisant intervenir plusieurs services ne respectent généralement ni les délais, ni les budgets et sont mal coordonnés.

f. Les objectifs de sûreté sont régulièrement atteints ou dépassés.

g. Les ingénieurs systèmes et/ou en analyse de la performance ne résolvent pas efficacement les problèmes affectant la sûreté ou la performance de la centrale.

h. La situation radiologique est contrôlée efficacement (les niveaux de rayonnement sont aussi bas que raisonnablement possible et le contrôle des doses est efficace). 


\section{Efficacité organisationnelle}

$\begin{array}{lrr}\text { Absolument } & \text { Absolument } & \text { Pas en mesure } \\ \text { pas d'accord } & \text { d'accord } & \text { d'évaluer }\end{array}$

$\begin{array}{llllll}1 & 2 & 3 & 4 & 5\end{array}$

i. Il faut améliorer la qualité de la documentation (par ex., celle concernant la conception, les méthodes de travail et les procédures).

j. Les opérateurs réagissent efficacement aux variations des conditions de la centrale pour en assurer en continu l'exploitation sûre et fiable.

k. Il est fréquent que les objectifs d'exploitation hebdomadaires ne soient pas atteints.

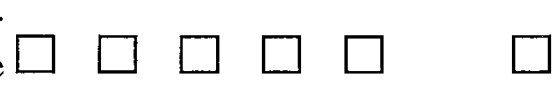

La planification et la gestion du travail sont efficaces (par ex., calendrier des tâches stable, peu de temps perdu à attendre des approbations ou des pièces de rechange).

$\mathrm{m}$. Le nombre annuel moyen de défaillances de composantes critiques est peu élevé comparativement à d'autres centrales similaires.

n. La répétition de problèmes d'exploitation connus et évitables n'est pas toujours évitée.

o. L'organisme gère avec efficacité les différents contacts externes (organisme de réglementation, public, fournisseurs, sous-traitants).

p. Il arrive que les objectifs environnementaux ne soient pas atteints.
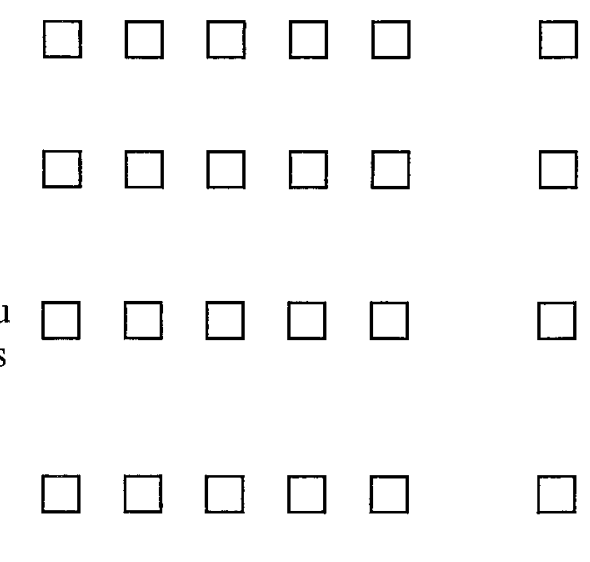

q. Les objectifs de maintenance (par ex., quantité de travaux de maintenance corrective et préventive en souffrance) basés sur les meilleures pratiques de l'industrie sont régulièrement atteints ou dépassés.

r. Il est fréquent que les objectifs financiers ne soient pas atteints.

s. Les objectifs de réglementation sont régulièrement atteints ou dépassés.

t. Les mesures destinées à améliorer l'état des systèmes sont efficaces.

u. Les travaux de maintenance et de mise à l'arrêt à titre correctif ou préventif sont effectués comme prévu et dans les délais.

v. Les ressources financières (budgétaires) sont adéquates et allouées de manière judicieuse.
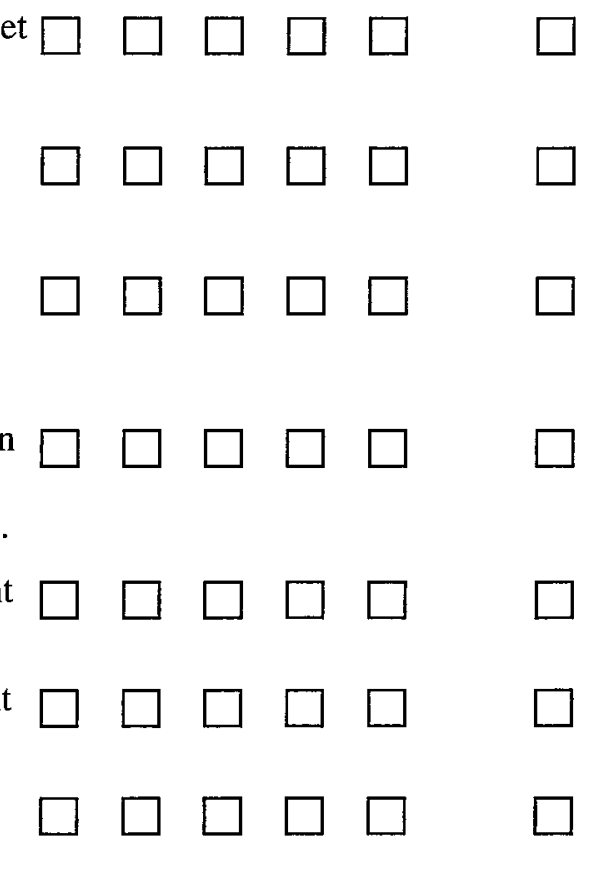


\section{PARTIE F : PERFORMANCE D'EXPLOITATION}

1. Si vous fournissez le nom de votre centrale (facultatif), les évaluations des indicateurs de performance pour 2009 de votre centrale peuvent être obtenues de sources publiées (l'approche préférée). Vos réponses demeureront confidentielles et seulement des données regroupées seront publiées.

Nom de votre centrale (facultatif):

2. Si vous avez fournis le nom de votre centrale vous n'avez pas besoin de compléter le reste de la partie $\mathrm{F}$ et vous pouvez allez directement a la partie $\mathrm{G}$. Si vous n'avez pas fournis le nom de votre centrale, nous vous demandons de fournir les évaluations d'indicateurs de performance opérationnelles pour chacune des tranches de votre centrale. S'il vous plait utilisez la dernière année pour laquelle les données sont disponibles et indiquez l'année: . Ignorez les colonnes pour lesquelles ces tranches n'existent pas à votre centrale.

\begin{tabular}{|c|c|c|c|c|c|c|c|c|}
\hline $\begin{array}{l}\text { Indicateur de } \\
\text { performance de la } \\
\text { tranche }\end{array}$ & $\begin{array}{c}\text { Tranche } \\
1\end{array}$ & $\begin{array}{l}\text { Tranche } \\
\quad 2\end{array}$ & $\begin{array}{l}\text { Tranche } \\
3\end{array}$ & $\begin{array}{c}\text { Tranche } \\
4\end{array}$ & $\begin{array}{l}\text { Tranche } \\
5\end{array}$ & $\begin{array}{l}\text { Tranche } \\
6\end{array}$ & $\begin{array}{l}\text { Tranche } \\
7\end{array}$ & $\begin{array}{c}\text { Tranche } \\
8\end{array}$ \\
\hline $\begin{array}{l}\text { a. Indice de } \\
\text { capacité de la } \\
\text { tranche (\% } \\
\text { annuel) } \\
\end{array}$ & & & & & & & & \\
\hline $\begin{array}{l}\text { b. Indice de perte } \\
\text { de capacité non } \\
\text { programmée (\% } \\
\text { annuel) }\end{array}$ & & & & & & & & \\
\hline $\begin{array}{l}\text { c. Taux de perte } \\
\text { forcé (\% } \\
\text { annuel) }\end{array}$ & & & & & - & & $\ldots$ & \\
\hline $\begin{array}{l}\text { d. Radio } \\
\text { expositions }\end{array}$ & & & 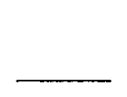 & & 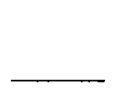 & $\longrightarrow$ & 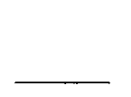 & \\
\hline (annuel) & \multicolumn{8}{|c|}{ Spécifié Homme-Sieverts par Tranche $\square$ ou Homme-rem par Tranche $\square$} \\
\hline $\begin{array}{l}\text { e. Nombre d'arrêts } \\
\text { d'urgence } \\
\text { automatiques } \\
\text { non programmés } \\
\text { pour } 7000 \\
\text { heures de } \\
\text { criticité }\end{array}$ & & & & & & - & 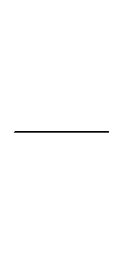 & $\longrightarrow$ \\
\hline
\end{tabular}




\begin{tabular}{|c|c|c|c|c|c|c|c|c|}
\hline $\begin{array}{l}\text { Indicateur de } \\
\text { performance de la } \\
\text { tranche }\end{array}$ & $\begin{array}{c}\text { Tranche } \\
1\end{array}$ & $\begin{array}{l}\text { Tranche } \\
2\end{array}$ & $\begin{array}{c}\text { Tranche } \\
3\end{array}$ & $\begin{array}{c}\text { Tranche } \\
4\end{array}$ & $\begin{array}{c}\text { Tranche } \\
5\end{array}$ & $\begin{array}{c}\text { Tranche } \\
6\end{array}$ & $\begin{array}{c}\text { Tranche } \\
7\end{array}$ & $\begin{array}{c}\text { Tranche } \\
8\end{array}$ \\
\hline \multirow{2}{*}{$\begin{array}{l}\text { f. Taux d'accidents } \\
\text { relevant de la } \\
\text { sûreté } \\
\text { industrielle }\end{array}$} & & 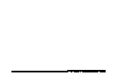 & $\square$ & & 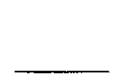 & 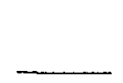 & & \\
\hline & \multicolumn{8}{|c|}{ Spécifié pour 200,000 personne-heures $\square$ or $1,000,000$ personne-heures $\square$} \\
\hline $\begin{array}{l}\text { g. Performance des } \\
\text { systèmes de } \\
\text { sûreté }\end{array}$ & - & - & - & - & - & - & - & - \\
\hline $\begin{array}{l}\text { h. Fiabilité du } \\
\text { combustible } \\
\text { (annuel) }\end{array}$ & - & - & - & - & - & - & - & - \\
\hline $\begin{array}{l}\text { i. Performance } \\
\text { chimique } \\
\text { (annuel) }\end{array}$ & - & - & - & - & - & - & - & - \\
\hline
\end{tabular}

Notes : définitions WANO (comme référence si nécessaire pour répondre aux questions 2a

à 2i)

1. Indice de capacité de la tranche : pourcentage annuel de la production maximale d'énergie qu'une centrale est capable de fournir au réseau électrique, limité seulement par des facteurs sous le contrôle de la direction de la centrale.

2. Indice de perte de capacité non programmée : pourcentage annuel de la production maximale d'énergie qu'une centrale est incapable de fournir au réseau électrique en raison de pertes d'énergie non programmées, telles que des mises à l'arrêt non programmées ou des arrêts prolongés.

3. Taux de perte forcée : pourcentage annuel de la production d'énergie, pendant une période en dehors des arrêts, qu'une centrale est incapable de fournir au réseau électrique à cause de pertes d'énergie non programmées, telles que les mises à l'arrêt non programmées ou les réductions de charge.

4. Radio expositions collectives : efficacité du contrôle de la radio exposition du personnel pour les réacteurs à eau bouillante (REB), les réacteurs à eau sous pression (REP), les réacteurs à eau lourde sous pression (RELP), les réacteurs refroidis par eau ordinaire et modérés par graphite et les réacteurs refroidis par gaz (RRG). Mesure : homme-sieverts par tranche par année, homme-rem par tranche par année.

5. Arrêts d'urgence automatiques non programmés pour $\mathbf{7 0 0 0}$ heures de criticité : taux moyen d'arrêts automatiques pendant environ une année (soit 7000 heures) d'exploitation. Les arrêts d'urgence automatiques non programmés provoquant des transitoires thermiques et hydrauliques qui affectent les systèmes de la centrale.

6. Taux d'accidents relevant de la sûreté industrielle : nombre d'accidents se traduisant en temps de travail perdu, en travail restreint ou en décès, pour 200000 heures ouvrées.

7. Performance des systèmes de sûreté : l'indicateur annuel de performance des systèmes de sûreté surveille la disponibilité de trois systèmes de sûreté de secours importants dans chaque centrale. Les systèmes de sûreté qui sont prêts à toute éventualité sont très probablement capables d'atténuer des événements anormaux. 
8. Fiabilité du combustible : l'indicateur annuel de fiabilité du combustible surveille les progrès en matière de prévention des défauts de la gaine métallique qui entoure le combustible. La maintenance de l'intégrité de la gaine du combustible réduit l'impact radiologique sur l'exploitation de la centrale et les activités de maintenance.

9. Performance chimique: l'indicateur annuel de performance chimique fournit une indication sur des progrès dans le contrôle des paramètres chimiques destinés à retarder la détérioration des matériaux et des composants clés de la centrale. Ces paramètres sont déjà maintenus en stricte conformité avec les orientations établies par l'industrie.

\section{PARTIE G : DONNÉES DÉMOGRAPHIQUES ET AUTRES DONNÉES}

1. Nombre type d'employés (à l'exclusion des surnuméraires) dans votre centrale :

2. Nombre représentant de surnuméraires équivalents à plein temps durant les arrêts programmés de votre centrale :

3. Nombre représentant de surnuméraires équivalents à plein temps durant l'opération en puissance de votre centrale :

4. Pourcentage du personnel de votre centrale possédant un diplôme universitaire :

5. Nom du pays dans lequel se trouve votre centrale :

6. Nombre de tranches en exploitation (réacteurs de puissance) dans votre centrale :

7. Type de réacteur (REP, REB, RELP, REO modérés par graphite, RRG, etc .) :

8. Modèle de centrale (nom commercial) (EPR, AP1000, VVER 440, etc.)

9. S'il vous plait cochez la rangée appropriée pour indiquer l'âge approximative de chaque tranche de votre centrale (déterminé à partir de la fin de la construction) (ignorez les colonnes pour les tranches qui n'existent pas à votre centrale)

\begin{tabular}{|c|c|c|c|c|c|c|c|c|}
\hline $\begin{array}{c}\text { Age } \\
\text { (années) }\end{array}$ & $\begin{array}{c}\text { Tranche } \\
1\end{array}$ & $\begin{array}{c}\text { Tranche } \\
2\end{array}$ & $\begin{array}{c}\text { Tranche } \\
3\end{array}$ & $\begin{array}{c}\text { Tranche } \\
4\end{array}$ & $\begin{array}{c}\text { Tranche } \\
5\end{array}$ & $\begin{array}{c}\text { Tranche } \\
6\end{array}$ & $\begin{array}{c}\text { Tranche } \\
7\end{array}$ & $\begin{array}{c}\text { Tranche } \\
8\end{array}$ \\
\hline $1-10$ & $\square$ & $\square$ & $\square$ & $\square$ & $\square$ & $\square$ & $\square$ & $\square$ \\
\hline $11-20$ & $\square$ & $\square$ & $\square$ & $\square$ & $\square$ & $\square$ & $\square$ & $\square$ \\
\hline $21-30$ & $\square$ & $\square$ & $\square$ & $\square$ & $\square$ & $\square$ & $\square$ & $\square$ \\
\hline $31-40$ & $\square$ & $\square$ & $\square$ & $\square$ & $\square$ & $\square$ & $\square$ & $\square$ \\
\hline $41+$ & $\square$ & $\square$ & $\square$ & $\square$ & $\square$ & $\square$ & $\square$ & $\square$ \\
\hline
\end{tabular}

10. Indiquez les communautés de pratiques (COP) auxquelles votre centrale participe et précisez si des auto-évaluations sont effectuées régulièrement à l'aune des indicateurs de performance ou des données de référence des COP : 


\begin{tabular}{|c|c|c|}
\hline $\begin{array}{l}\text { Nom ou thème du groupe } \\
\text { de travail COP }\end{array}$ & $\begin{array}{r}\text { Vous y participez } \\
\text { régulièrement }\end{array}$ & $\begin{array}{c}\text { Vous recourez } \\
\text { à la référenciation }\end{array}$ \\
\hline
\end{tabular}

a. Fiabilité du matériel

b. Matières et services (chaîne d'approvisionnement)

c. Technologie de l'information

d. Services aux entreprises/gestion des actifs nucleaires

e. Gestion de l'information

f. Délivrance de licences/Réglementation

g. Ressources humaines

h. Radioprotection

i. Combustible nucléaire

j. Surveillance/amélioration de la performance

k. Exploitation d'une centrale

1. Gestion de la chimie

m. Gestion du travail

n. Simulateurs

o. Formation

p. Estimation et gestion des coûts

q. Gestion de la configuration

r. Protection contre l'incendie

s. Autre (précisez) :

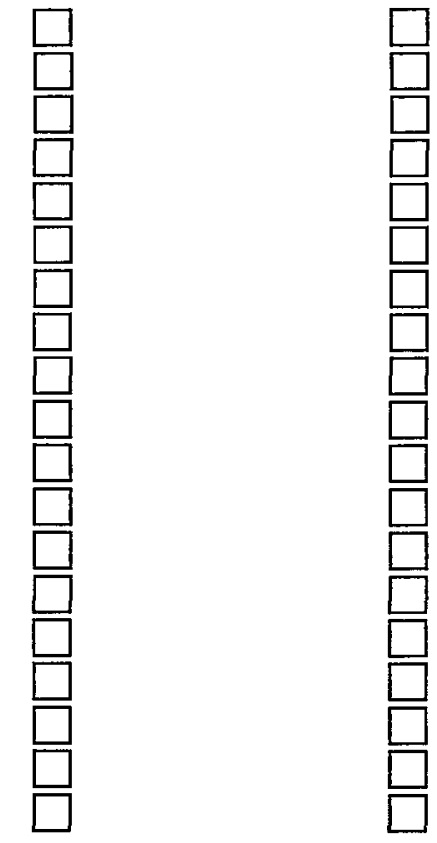

Indiquez aussi la (les) COP en question est (sont) notamment : locale(s) (par ex., nationale(s)) $\square$, régionale(s) (Europe) $\square$, internationale(s) (AIEA, EPRI, INPO ou NEI) $\square$ ou une (des) COP de groupe de propriétaires $\square$.

11. Indiquez le nombre de responsables des opérations qui ont contribué à remplir ce questionnaire.

12. Pour toute informations que vous souhaiteriez ajouter ou clarifications que vous souhaiteriez apporter à vos réponses, veuillez utiliser l'espace ci-dessous :

13. Si vous désirez une copie électronique du rapport qui résumera les résultats de cette étude, une fois disponible, s'il vous plait fournissez votre nom, position et adresse de courriel (facultatif)

Nom:

Position:

Adresse de courriel:

Vous remercie d'avoir pris le temps de remplir ce questionnaire. 


\section{APPENDIX E. OPERATIONAL PERFORMANCE INDICATORS}

This appendix is provided to explain the operational performance indicators in detail. Note that there are several other WANO indicators available, including the following:

- Grid-Related Loss Factor

- Safety System Performance

- Fuel Reliability

- Chemistry Performance

- Collective Radiation Exposure

- Industrial Safety Accident Rate

- Contractor Industrial Safety Accident Rate

However, they were deemed not appropriate for the purposes of this study and are not explained here. For example, several are calculated differently depending on the type of NPP design (e.g. BWR vs. PHWR) and this involved more complicated interpretation and questions about their reliability when used statistically for performance measures. Consider for example, it is quite possible an indicator used for one type of reactors could introduce a bias into the data relative to other NPP designs.

In this study, unit capability factor (UCF), unit capability loss (UCL), forced loss rate (FLR) and unplanned actuations (UA7) are used. Planned energy losses (PEL) and outage extension losses (OEL) are underlying measures used to calculate these indicators and are explained further below. Figure 120 illustrates graphically how they 
inter-relate and shows typical values. A more detailed explanation of the derivation of each indicator is provide below (from WANO, 2010). Reference back to this figure may be useful to visualize how the indicators are calculated.

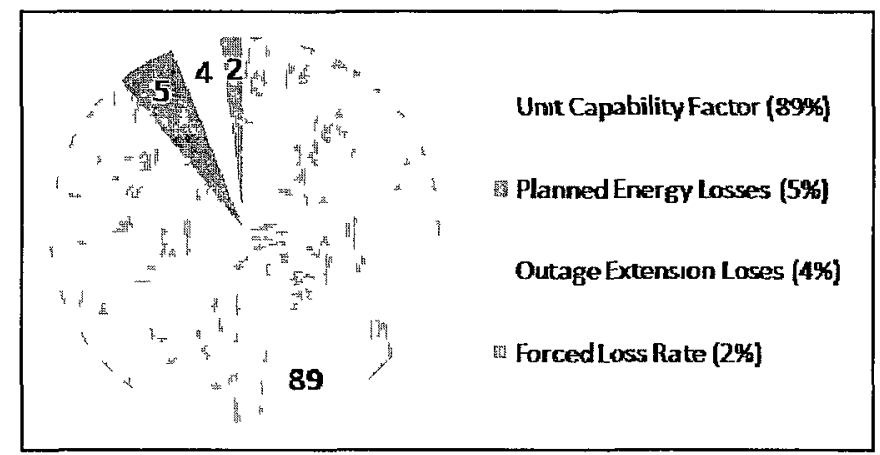

Figure 120. Operational Performance Indicators (Typical Values in \%)

The following is an summary excerpt of the indicator definitions from the "WANO Performance Indicator Programme Reference Manual” (WANO, 2010).

\section{Unit Capability Factor (UCF)}

Unit capability factor is defined as the ratio of the available energy generation over a given time period to the reference energy generation over the same time period, expressed as a percentage. Both of these energy generation terms are determined relative to reference ambient conditions. Available energy generation is the energy that could have been produced under reference ambient conditions considering only limitations within control of plant management, i.e., plant equipment and personnel performance, and work control. Reference energy generation is the energy that could be produced if the unit were operated continuously at full power under reference ambient conditions. Reference ambient conditions are environmental conditions representative of the annual mean (or typical) ambient conditions for the unit.

The unit capability factor is determined for each period as shown below:

- value for a unit $=[(R E G-P E L-U E L) \times 100 \%] / R E G$ 
where:

$R E G=$ reference energy generation for the period

$P E L=$ total planned energy losses for the period

$U E L=$ total unplanned energy losses for the period

- planned energy loss: $P E L=\Sigma(P P L \times H R P)$

where:

$P P L=$ planned power loss: the power decrease in megawatts due to a planned event

$H R P=$ hours operated at reduced power (or shutdown) due to the planned event

Note: The total planned energy loss for the period is the sum of the losses from all planned events.

- unplanned energy loss: $U E L=\Sigma(U P L \times H R U)$

where:

$U P L=$ unplanned power loss: the power decrease in megawatts due to an unplanned event

$H R U=$ hours operated at reduced power (or shutdown) due to the unplanned event Note: The total unplanned energy loss for the period is the sum of the losses from all unplanned events.

- $\quad$ value for the industry = median of the unit values

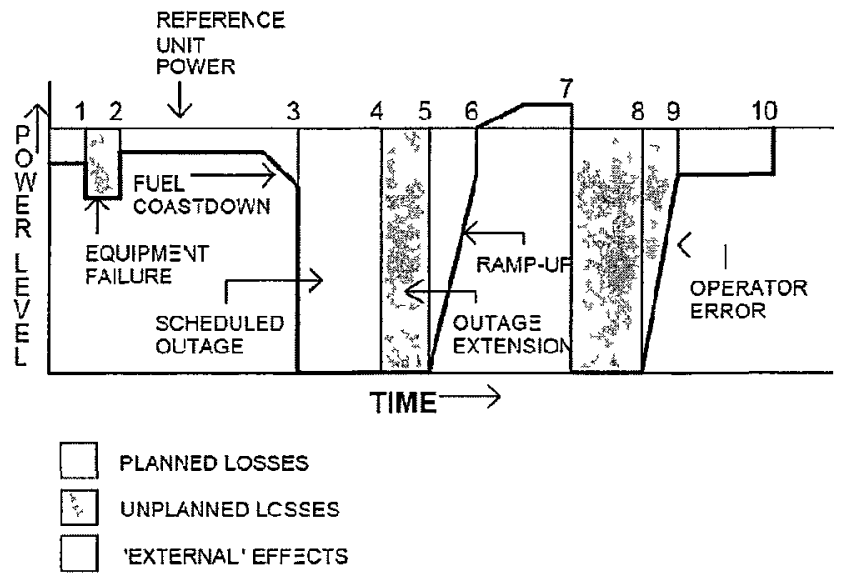

Notes to Figure X· Point-to-point power level explanations.

$0-1$ reduced power due to load following

$1-2$ reduced power due to equipment fallure 
2 - 3 reduced power due to ambient conditions and fuel coast-down

3 - 6 unit shutdown (outage) and subsequent ramp-up

6 - 7 increased power due to very cold water

7 - 9 unit shutdown (operator error) and subsequent ramp-up

9 - 10 reduced power due to environmental limitations not under management control

\section{Unplanned Capability Loss Factor (UCF)}

Unplanned capability loss factor is defined as the ratio of the unplanned energy losses during a given period of time, to the reference energy generation, expressed as a percentage. Unplanned energy loss is energy that was not produced during the period because of unplanned shutdowns, outage extensions, or unplanned load reductions due to causes under plant management control. Causes of energy losses are considered to be unplanned if they are not scheduled at least four weeks in advance. Causes considered to be under plant management control are further defined in the clarifying notes. Reference energy generation is the energy that could be produced if the unit were operated continuously at full power under reference ambient conditions throughout the period. Reference ambient conditions are environmental conditions representative of the annual mean (or typical) ambient conditions for the unit.

The unplanned capability loss factor is determined for each period as shown below:

- value for a unit $=[$ UEL $\times 100 \%] / R E G$

where:

$U E L=$ total unplanned energy losses for the period

$R E G=$ reference energy generation for the period

- unplanned energy loss: $U E L=\Sigma(U P L x H R U)$ where:

$U P L=$ unplanned power loss: the power decrease in megawatts due to an unplanned event

$H R U=$ hours operated at reduced power (or shutdown) due to the unplanned event Note: The total unplanned energy loss for the period is the sum of the losses from all unplanned events.

- value for the industry = median of the unit values 
- unplanned capability loss factors for individual units will be presented for a three year period to maintain consistency with the three year Unit Capability Factor.

\section{Forced Loss Rate (FLR)}

The forced loss rate (FLR) is defined as the ratio of all unplanned forced energy losses during a given period of time to the reference energy generation minus energy generation losses corresponding to planned outages and any unplanned outage extensions of planned outages, during the same period, expressed as a percentage. Unplanned energy losses are either unplanned forced energy losses (unplanned energy generation losses not resulting from an outage extension) or unplanned outage extension of planned outage energy losses. Unplanned forced energy loss is energy that was not produced because of unplanned shutdowns or unplanned load reductions due to causes under plant management control when the unit is considered to be at the disposal of the grid dispatcher. Causes of forced energy losses are considered to be unplanned if they are not scheduled at least four weeks in advance. Causes considered to be under plant management control are further defined in the clarifying notes. Unplanned outage extension energy loss is energy that was not produced because of an extension of a planned outage beyond the original planned end date due to originally scheduled work not being completed, or because newly scheduled work was added (planned and scheduled) to the outage less than 4 weeks before the scheduled end of the planned outage. Planned energy losses are those corresponding to outages or power reductions which were planned and scheduled at least 4 weeks in advance (see clarifying notes for exceptions). Reference energy generation is the energy that could be produced if the unit were operated continuously at full power under reference ambient conditions throughout the given period. Reference ambient conditions are environmental conditions representative of the annual mean (or typical) ambient conditions for the unit.

The forced loss rate is calculated for a period as shown below. value for a unit $(\%)=F E L /[R E G-(P E L+O E L)] \times 100 \%$ where:

$$
F E L=\text { unplanned forced energy losses }
$$


$R E G=$ reference energy generation

$P E L=$ planned energy losses

$O E L=$ unplanned outage extension energy losses

value for the industry $=$ median of the unit values
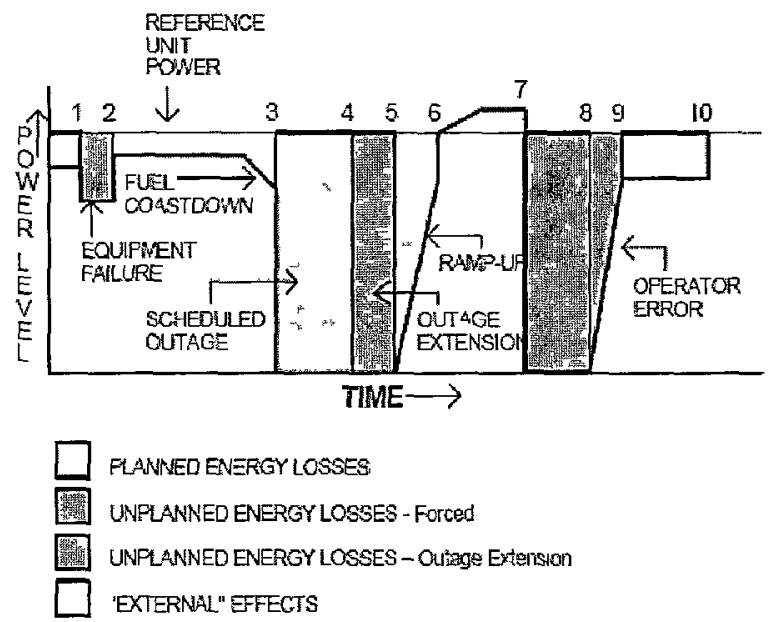

Point-to-point power level explanations:

- $\quad 0-1$ reduced power due to load following

- 1 - 2 reduced power due to equipment fallure

- 2 - 3 reduced power due to ambient conditions and fuel coast down

- 3 - 4 unit shutdown (planned outage duration)

- 4 - 5 unit remains shutdown for outage extension due to originally work not being completed during the

- planned/scheduled outage

- 5-6 subsequent planned ramp-up

- 6-7 increased power due to very cold water

- $\quad$ - 9 unit shutdown (operator error) and subsequent ramp-up

- 9-10 reduced power due to environmental limitations not under management control

\section{Unplanned Automatic SCRAMS per 7000 Hours Critical (UA7)}

The indicator is defined as the number of unplanned automatic scrams (reactor protection system actuations) that occur per 7000 hours of critical operation. The indicator is defined as follows:

- Unplanned means that the scram was not an anticipated part of a planned test.

- Scram means the automatic shutdown of the reactor by a rapid insertion of negative reactivity (e.g., by control rods, liquid injection shutdown system, etc.) that is caused by actuation of the reactor protection system. The scram signal may have resulted from exceeding a setpoint or may have been spurious.

- Automatic means that the initial signal that caused actuation of the reactor protection system logic was provided from one of the sensors monitoring plant 
parameters and conditions, rather than the manual scram switches or, in certain cases described in the clarifying notes, manual turbine trip switches (or pushbuttons) provided in the main control room.

- Critical means that during the steady-state condition of the reactor prior to the scram, the effective multiplication factor $\left(k_{e f f}\right)$ was essentially equal to one.

- The value of 7000 hours is representative of the critical hours of operation during a year for most plants, and provides an indicator value that typically approximates the actual number of scrams occurring during the year.

The unit and industry values for this indicator are determined for a period as shown below:

- $\quad$ value for a unit $=($ total unplanned automatic scrams while critical $) \times 7000$ (total number of hours critical)

- $\quad$ worldwide value = median of the unit values 


\section{APPENDIX F. SUMMARY OF STATIONS PARTICIPATING}

Table 175 lists the NPPs that participated in the study and indicates the country of

location, reactor type, first year in-service, and MWe power output rating.

Table 175. Summary of Participating Reactor Units

\begin{tabular}{|c|c|c|c|c|}
\hline NPP Name & Country & Reactor Type & In-Service & MWe Rating \\
\hline Almaraz Unit 1 & Spain & PWR & 1983 & 1050 \\
\hline Almaraz Unit 2 & Spain & PWR & 1984 & 983 \\
\hline Angra NPP 1- Unit 1 & Brazil & PWR & 1985 & 640 \\
\hline Angra NPP 2- Unit 1 & Brazil & PWR & 2000 & 1350 \\
\hline ANO Unit 1 & USA & PWR & 1974 & 845 \\
\hline ANO Unit 2 & USA & PWR & 1980 & 1012 \\
\hline Asco NPP 1 & Spain & PWR & 1984 & 1033 \\
\hline Asco NPP 2 & Spain & $\begin{array}{l}\text { PWR } \\
\end{array}$ & 1985 & 1027 \\
\hline Beznau Unit 1 & Switzerland & PWR & 1969 & 380 \\
\hline Beznau Unit 2 & Switzerland & PWR & 1972 & 380 \\
\hline Biblis NPP A & Germany & PWR & 1975 & 1225 \\
\hline Biblis NPP B & Germany & $\overline{P W R}$ & 1977 & 1300 \\
\hline Bohunice Unit 3 & Slovakia & PWR & 1984 & 436 \\
\hline Bohunice Unit 4 & Slovakia & PWR & 1985 & 436 \\
\hline Borssele Unit 1 & Netherlands & PWR & 1973 & 478 \\
\hline Braidwood Unit 1 & USA & PWR & 1988 & 1194 \\
\hline Braidwood Unit 2 & USA & PWR & 1988 & 1166 \\
\hline Brokdorf Unit 1 & Germany & PWR & 1986 & 1440 \\
\hline Bruce Nuclear A, Unit 3 & Canada & PHWR & 1978 & 825 \\
\hline Bruce Nuclear A, Unit 4 & Canada & PHWR & 1979 & 825 \\
\hline Bruce Nuclear B, Unit 5 & Canada & PHWR & 1985 & 840 \\
\hline Bruce Nuclear B, Unit 6 & Canada & PHWR & 1984 & 866 \\
\hline Bruce Nuclear B, Unit 7 & Canada & PHWR & 1986 & 840 \\
\hline Bruce Nuclear B, Unit 8 & Canada & PHWR & 1987 & 840 \\
\hline Brunsbuettel Unit 1 & Germany & BWR & 1976 & 806 \\
\hline Bugey Unit 2 & France & PWR & 1979 & 920 \\
\hline Bugey Unit 3 & France & PWR & 1979 & 920 \\
\hline Bugey Unit 4 & France & PWR & 1979 & 880 \\
\hline Bugey Unit 5 & France & PWR & 1980 & 880 \\
\hline Byron Unit 1 & $\overline{\text { USA }}$ & PWR & 1985 & 1183 \\
\hline Byron Unit 2 & USA & PWR & 1987 & 1153 \\
\hline Callaway Unit 1 & USA & PWR & 1985 & 1284 \\
\hline Catawba Unit 1 & USA & PWR & 1985 & 1153 \\
\hline Catawba Unit 2 & USA & PWR & 1986 & 1305 \\
\hline Cernavoda Unit 1 & Romania & PHWR & 1996 & 706 \\
\hline Cernavoda Unit 2 & Romania & PHWR & 2007 & 704 \\
\hline Chinshan Unit 1 & Taiwan & BWR & 1978 & 629 \\
\hline Chinshan Unit 2 & Taiwan & BWR & 1979 & 629 \\
\hline Civaux Unit 1 & France & PWR & 2002 & 1495 \\
\hline
\end{tabular}




\begin{tabular}{|c|c|c|c|c|}
\hline NPP Name & Country & Reactor Type & In-Service & MWe Rating \\
\hline Civaux Unit 2 & France & PWR & 2002 & 1495 \\
\hline Clinton Unit 1 & USA & BWR & 1987 & 1067 \\
\hline Comanche Peak Unit 1 & USA & PWR & 1990 & 1166 \\
\hline Comanche Peak Unit 2 & USA & PWR & 1993 & 1166 \\
\hline Cook Unit 1 & USA & PWR & 1975 & 1056 \\
\hline Cook Unit 2 & USA & PWR & .1978 & 1133 \\
\hline Cooper Unit 1 & USA & BWR & 1974 & 787 \\
\hline Cruas Unit 1 & France & PWR & 1984 & 880 \\
\hline Cruas Unit 2 & France & PWR & 1985 & 915 \\
\hline Cruas Unit 3 & France & PWR & 1984 & 915 \\
\hline Cruas Unit 4 & France & PWR & 1985 & 880 \\
\hline Darlington Unit 1 & Canada & PHWR & 1992 & 934 \\
\hline Darlington Unit 2 & Canada & PHWR & 1990 & 934 \\
\hline Darlington Unit 3 & Canada & PHWR & 1993 & 934 \\
\hline Darlington Unit 4 & Canada & PHWR & 1993 & 934 \\
\hline Daya Bay Unit 1 & China & PWR & 1994 & 984 \\
\hline Daya Bay Unit 2 & China & PWR & 1994 & 984 \\
\hline Diablo Canyon Unit 1 & USA & PWR & 1985 & 1153 \\
\hline Diablo Canyon Unit 2 & USA & PWR & 1986 & 1149 \\
\hline Doel Unit 1 & Belgium & PWR & 1975 & 392 \\
\hline Doel Unit 2 & Belgium & PWR & 1975 & 433 \\
\hline Doel Unit 3 & Belgium & PWR & 1982 & 1006 \\
\hline Doel Unit 4 & Belgium & PWR & 1985 & 1008 \\
\hline Dresden Unit 2 & USA & BWR & 1970 & 869 \\
\hline Dresden Unit 3 & USA & BWR & 1971 & 871 \\
\hline Duane Arnold Unit 1 & USA & BWR & 1975 & 647 \\
\hline Dungeness B Unit 3 & UK & AGR & 1985 & 555 \\
\hline Dungeness B Unit 4 & UK & AGR & 1985 & 555 \\
\hline Farley Unit 1 & USA & PWR & 1977 & 851 \\
\hline Farley Unit 2 & USA & PWR & 1981 & 860 \\
\hline Fermi Unit 2 & USA & BWR & 1988 & 1179 \\
\hline FitzPatrick Unit 1 & USA & BWR & 1975 & 862 \\
\hline Fort Calhoun Unit 1 & USA & PWR & 1973 & 499 \\
\hline Fukushima Daini Unit 1 & Japan & BWR & 1982 & 1100 \\
\hline Fukushima Daini Unit 2 & Japan & BWR & 1984 & 1100 \\
\hline Fukushima Daini Unit 3 & Japan & BWR & 1985 & 1100 \\
\hline Fukushima Daini Unit 4 & Japan & BWR & 1987 & 1100 \\
\hline Ginna Unit 1 & USA & PWR & 1970 & 602 \\
\hline Goesgen Unit 1 & Switzerland & PWR & 1979 & 1035 \\
\hline Golfech Unit 1 & France & PWR & 1991 & 1345 \\
\hline Golfech Unit 2 & France & PWR & 1994 & 1345 \\
\hline Grafenrheinfeld Unit 1 & Germany & PWR & 1982 & 1345 \\
\hline Grand Gulf Unit 1 & USA & BWR & 1985 & 1288 \\
\hline Gravelines B Unit 1 & France & PWR & 1980 & 910 \\
\hline Gravelines B Unit 2 & France & PWR & 1980 & 910 \\
\hline Gravelines B Unit 3 & France & PWR & 1981 & 910 \\
\hline Gravelines B Unit 4 & France & PWR & 1981 & 910 \\
\hline Gravelines C Unit 5 & France & PWR & 1985 & 910 \\
\hline Gravelines C Unit 6 & France & PWR & 1985 & 910 \\
\hline
\end{tabular}




\begin{tabular}{|c|c|c|c|c|}
\hline NPP Name & Country & Reactor Type & In-Service & MWe Rating \\
\hline Grohnde NPP 1 & Germany & PWR & 1985 & 1430 \\
\hline Gundremmingen NPP B & Germany & BWR & 1984 & 1344 \\
\hline Gundremmingen NPP $\bar{C}$ & Germany & BWR & 1985 & 1344 \\
\hline Hartlepool Unit 1 & UK & AGR & 1983 & 605 \\
\hline Hartlepool Unit 2 & UK & $\overline{\mathrm{AGR}}$ & 1984 & 605 \\
\hline Hatch Unit 1 & $\overline{\text { USA }}$ & BWR & 1976 & 876 \\
\hline Hatch Unit 2 & USA & BWR & 1979 & 883 \\
\hline Heysham A Unit 1 & UK & AGR & 1983 & 575 \\
\hline Heysham A Unit 2 & UK & AGR & 1983 & 575 \\
\hline Heysham B Unit 1 & UK & $\overline{A G R}$ & 1988 & 625 \\
\hline Heysham B Unit 2 & UK & AGR & 1988 & 625 \\
\hline Hinkley Point B Unit 1 & UK & AGR & 1978 & 610 \\
\hline Hinkley Point B Unit 2 & UK & $\overline{\mathrm{AGR}}$ & 1976 & 610 \\
\hline Hunterston B Unit 1 & UK & $\overline{\mathrm{AGR}}$ & 1976 & 595 \\
\hline Hunterston B Unit 2 & UK & AGR & 1977 & 595 \\
\hline Ignalina Unit 2 & Lithuania & LWCGR & 1987 & 1500 \\
\hline Indian Point Unit 2 & USA & PWR & 1974 & 1062 \\
\hline Indian Point Unit 3 & USA & PWR & 1976 & 1079 \\
\hline Isar 1 Unit 1 & Germany & BWR & 1979 & 912 \\
\hline Un-named Unit 1 & Russia & PWR & 1984 & 1000 \\
\hline Un-named Unit 2 & Russia & PWR & & \\
\hline Un-named Unit 3 & Russia & PWR & & \\
\hline Kashiwazaki Kariwa Unit 1 & Japan & BWR & 1985 & 1100 \\
\hline Kashiwazaki Kariwa Unit 2 & Japan & BWR & 1990 & 1100 \\
\hline Kashiwazaki Kariwa Unit 3 & Japan & BWR & 1993 & 1100 \\
\hline Kashiwazaki Kariwa Unit 4 & Japan & BWR & 1994 & 1100 \\
\hline Kashiwazaki Kariwa Unit 5 & Japan & BWR & 1990 & 1100 \\
\hline Kashiwazaki Kariwa Unit 6 & Japan & BWR & 1996 & 1356 \\
\hline Kashiwazaki Kariwa Unit 7 & Japan & BWR & 1997 & 1356 \\
\hline Koeberg Unit 1 & S. Africa & PWR & 1984 & 900 \\
\hline Koeberg Unit 2 & S. Africa & PWR & 1985 & 900 \\
\hline Kori A Unit 1 & Korea & PWR & 1978 & 603 \\
\hline Kori A Unit 2 & Korea & PWR & 1983 & 675 \\
\hline Kori B Unit 3 & Korea & PWR & 1986 & 1035 \\
\hline Kori B Unit 4 & Korea & PWR & 1986 & 1035 \\
\hline Kozloduy Unit 5 & Bulgaria & PWR & 1988 & 1000 \\
\hline Kozloduy Unit 6 & Bulgaria & PWR & 1993 & 1039 \\
\hline Krsko Unit 1 & Slovenia & PWR & 1983 & 666 \\
\hline Kuosheng Unit 1 & Taiwan & BWR & 1981 & 950 \\
\hline Kuosheng Unit 2 & Taiwan & BWR & 1983 & 970 \\
\hline LaSalle Unit 1 & USA & BWR & 1984 & 1138 \\
\hline LaSalle Unit 2 & USA & BWR & 1985 & 1150 \\
\hline Leibstadt Unit 1 & Switzerland & BWR & 1984 & 1220 \\
\hline Limerick Unit 1 & USA & BWR & 1986 & 1199 \\
\hline Limerick Unit 2 & USA & BWR & 1990 & 1204 \\
\hline Lingao Unit 1 & China & PWR & 2002 & 990 \\
\hline Lingao Unit 2 & China & PWR & 2003 & 990 \\
\hline Loviisa Unit 1 & Finland & PWR & 1977 & 510 \\
\hline Loviisa Unit 2 & Finland & PWR & 1981 & 510 \\
\hline
\end{tabular}




\begin{tabular}{|c|c|c|c|c|}
\hline NPP Name & Country & Reactor Type & In-Service & MWe Rating \\
\hline Maanshan Unit 1 & Taiwan & PWR & 1984 & 936 \\
\hline Maanshan Unit 2 & Taiwan & PWR & 1985 & 936 \\
\hline McGuire Unit 1 & USA & PWR & 1981 & $11 \overline{40}$ \\
\hline McGuire Unit 2 & USA & PWR & 1984 & 1149 \\
\hline Mochovce Unit 1 & Slovakia & PWR & 1998 & 470 \\
\hline Mochovce Unit 2 & Slovakia & PWR & 2000 & 470 \\
\hline Muehleberg Unit 1 & Switzerland & BWR & 1972 & 372 \\
\hline Nine Mile Point Unit 1 & USA & BWR & 1969 & 628 \\
\hline Nine Mile Point Unit 2 & USA & BWR & 1988 & 1163 \\
\hline Oconee Unit 1 & USA & PWR & 1973 & 934 \\
\hline Oconee Unit 2 & USA & PWR & 1974 & 934 \\
\hline Oconee Unit 3 & USA & PWR & 1974 & 934 \\
\hline OHI Unit 1 & Japan & PWR & 1979 & 1175 \\
\hline OHI Unit 2 & Japan & PWR & 1979 & 1175 \\
\hline OHI Unit 3 & Japan & PWR & 1991 & 1180 \\
\hline OHI Unit 4 & Japan & PWR & 1993 & 1180 \\
\hline Oldbury Unit 1 & UK & GCR & 1967 & 217 \\
\hline Oldbury Unit 2 & UK & GCR & 1968 & 217 \\
\hline Olkiluoto Unit 1 & Finland & BWR & 1979 & 878 \\
\hline Olkiluoto Unit 2 & Finland & BWR & 1982 & 878 \\
\hline Oskarshamn Unit 1 & Sweeden & BWR & 1972 & 487 \\
\hline Oskarshamn Unit 2 & Sweeden & BWR & 1975 & 623 \\
\hline Oskarshamn Unit 3 & Sweeden & BWR & 1985 & 1197 \\
\hline Oyster Creek Unit 1 & USA & BWR & 1969 & 650 \\
\hline Paks Unit 1 & Hungary & PWR & 1983 & 500 \\
\hline Paks Unit 2 & Hungary & PWR & 1984 & 500 \\
\hline Paks Unit 3 & Hungary & PWR & 1986 & 500 \\
\hline Paks Unit 4 & Hungary & PWR & 1987 & 500 \\
\hline Palisades Unit 1 & USA & PWR & 1971 & 842 \\
\hline Palo Verde Unit 1 & USA & PWR & 1986 & 1402 \\
\hline Palo Verde Unit 2 & USA & PWR & 1986 & 1406 \\
\hline Palo Verde Unit 3 & USA & PWR & 1988 & 1405 \\
\hline Peach Bottom Unit 2 & USA & BWR & 1974 & 1172 \\
\hline Peach Bottom Unit 3 & USA & BWR & 1974 & 1172 \\
\hline Pickering A Unit 1 & Canada & PHWR & 1971 & 542 \\
\hline Pickering A Unit 4 & Canada & PHWR & 1973 & 542 \\
\hline Pickering B Unit 5 & $\begin{array}{l}\text { Canada } \\
\end{array}$ & PHWR & 1983 & 540 \\
\hline Pickering B Unit 6 & Canada & PHWR & 1984 & 540 \\
\hline Pickering B Unit 7 & Canada & PHWR & 1985 & 540 \\
\hline Pickering B Unit 8 & Canada & PHWR & 1986 & 540 \\
\hline Pilgrim Unit 1 & USA & BWR & 1972 & 711 \\
\hline Point Beach Unit 1 & USA & PWR & 1970 & 524 \\
\hline Point Beach Unit 2 & USA & PWR & 1972 & 524 \\
\hline Point Lepreau Unit 1 & Canada & PHWR & 1983 & 638 \\
\hline Qinshan 1- Unit 1 & China & PWR & 1994 & 310 \\
\hline Qinshan 3-Unit 1 & China & PHWR & 2002 & 650 \\
\hline Qinshan 3- Unit 2 & China & PHWR & 2003 & 700 \\
\hline Quad Cities Unit 1 & $\overline{\text { USA }}$ & BWR & 1973 & 866 \\
\hline Quad Cities Unit 2 & USA & BWR & 1973 & 871 \\
\hline
\end{tabular}




\begin{tabular}{|c|c|c|c|c|}
\hline NPP Name & Country & Reactor Type & In-Service & MWe Rating \\
\hline Ringhals Unit 1 & Sweeden & BWR & 1976 & 848 \\
\hline Ringhals Unit 2 & Sweeden & PWR & 1975 & 875 \\
\hline Ringhals Unit 3 & Sweeden & PWR & 1981 & 1045 \\
\hline Ringhals Unit 4 & Sweeden & PWR & 1983 & 913 \\
\hline River Bend Unit 1 & USA & BWR & 1986 & 1055 \\
\hline San Onofre Unit 2 & USA & PWR & 1983 & 1127 \\
\hline San Onofre Unit 3 & USA & PWR & 1984 & 1127 \\
\hline Santa Maria De Garona Unit 1 & Spain & BWR & 1971 & 466 \\
\hline Seabrook Unit 1 & USA & PWR & 1990 & 1296 \\
\hline Shimane Unit 1 & Japan & BWR & 1974 & 460 \\
\hline Shimane Unit 2 & Japan & BWR & 1989 & 820 \\
\hline Sizewell B Unit 1 & UK & PWR & 1995 & 1188 \\
\hline South Ukraine Unit 1 & Ukraine & PWR & 1982 & 1000 \\
\hline South Ukraine Unit 2 & Ukraine & PWR & 1985 & 1000 \\
\hline South Ukraine Unit 3 & Ukraine & PWR & 1989 & 1000 \\
\hline St. Lucie Unit 1 & USA & PWR & 1976 & 839 \\
\hline St. Lucie Unit 2 & USA & PWR & 1983 & 839 \\
\hline Susquehanna Unit 1 & USA & BWR & 1983 & 1199 \\
\hline Susquehanna Unit 2 & USA & BWR & 1985 & 1204 \\
\hline Tarapur Unit 3 & India & PHWR & 2006 & 540 \\
\hline Tarapur Unit 4 & India & PHWR & 2005 & 540 \\
\hline Temelin Unit 1 & Czech Republic & PWR & 2002 & 1000 \\
\hline Temelin Unit 2 & Czech Republic & PWR & 2003 & 1000 \\
\hline Three Mile Island Unit 1 & USA & PWR & 1974 & 890 \\
\hline Tianwan Unit 1 & China & PWR & 2007 & 1000 \\
\hline Tianwan Unit 2 & China & PWR & 2007 & 1000 \\
\hline Tihange Unit 1 & Belgium & PWR & 1975 & 962 \\
\hline Tihange Unit 2 & Belgium & PWR & 1983 & 1008 \\
\hline Tihange Unit 3 & Belgium & PWR & 1985 & 1054 \\
\hline Tomari Unit 1 & Japan & PWR & 1989 & 579 \\
\hline Tomari Unit 2 & Japan & PWR & 1991 & 579 \\
\hline Tomari Unit 3 & Japan & PWR & 2009 & 912 \\
\hline Torness Unit 1 & UK & AGR & 1988 & 625 \\
\hline Torness Unit 2 & UK & AGR & 1989 & 625 \\
\hline Trillo Unit 1 & Spain & PWR & 1988 & 1066 \\
\hline Turkey Point Unit 3 & USA & PWR & 1972 & 693 \\
\hline Turkey Point Unit 4 & USA & PWR & 1973 & 693 \\
\hline Ulchin A Unit 1 & Korea & PWR & 1988 & 985 \\
\hline Ulchin A Unit 2 & Korea & PWR & 1989 & 984 \\
\hline Ulchin C Unit 5 & Korea & PWR & 2004 & 1048 \\
\hline Ulchin C Unit 6 & Korea & PWR & 2005 & 1048 \\
\hline Unterweser Unit 1 & Germany & PWR & 1979 & 1410 \\
\hline Vandellos NPP 2 & Spain & PWR & 1988 & 1087 \\
\hline Vermont Yankee Unit 1 & USA & BWR & 1972 & 515 \\
\hline Vogtle Unit 1 & USA & PWR & 1987 & 1109 \\
\hline Vogtle Unit 2 & USA & PWR & 1989 & 1127 \\
\hline Waterford Unit 3 & USA & PWR & 1985 & 1075 \\
\hline Watts Bar Unit 1 & USA & PWR & 1996 & 1202 \\
\hline Wolf Creek Unit 1 & USA & PWR & 1985 & 1226 \\
\hline
\end{tabular}




\begin{tabular}{|l|l|c|c|c|}
\hline NPP Name & Country & Reactor Type & In-Service & MWe Rating \\
\hline Wolsong A Unit 1 & Korea & PHWR & 1983 & 622 \\
\hline Wolsong A Unit 2 & Korea & PHWR & 1997 & 730 \\
\hline Wolsong B Unit 3 & Korea & PHWR & 1998 & 729 \\
\hline Wolsong B Unit 4 & Korea & PHWR & 1999 & 730 \\
\hline Wylfa Unit 1 & UK & GCR & 1971 & 475 \\
\hline Wylfa Unit 2 & UK & PWR & 1972 & 475 \\
\hline Yonggwang A Unit 1 & Korea & 1986 & 985 \\
\hline Yonggwang A Unit 2 & Korea & PWR & 1987 & 978 \\
\hline Yonggwang B Unit 3 & Korea & PWR & 1996 & 1039 \\
\hline Yonggwang B Unit 4 & Korea & PWR & 2002 & 1039 \\
\hline Yonggwang C Unit 5 & Korea & PWR & 2002 & 1046 \\
\hline Yonggwang C Unit 6 & Korea & PWR & 1984 & 1050 \\
\hline Zaporozhye Unit 1 & Ukraine & PWR & 1985 & 1000 \\
\hline Zaporozhye Unit 2 & Ukraine & PWR & 1986 & 1000 \\
\hline Zaporozhye Unit 3 & Ukraine & PWR & 1987 & 1000 \\
\hline Zaporozhye Unit 4 & Ukraine & PWR & 1989 & 1000 \\
\hline Zaporozhye Unit 5 & Ukraine & PWR & 1995 & 1000 \\
\hline Zaporozhye Unit 6 & Ukraine & & 1000 \\
\hline
\end{tabular}




\section{APPENDIX G. BIVARIATE SCATTERPLOTS - ALL MEASURES}

Table 176 is a legend to the variable names used on the scatterplots that follow:

Table 176. Index of Variable (and Construct) Names Used in Scatter-plots

\begin{tabular}{|l|l|l|}
\hline Variable Name & Description \\
\hline 1. & $\begin{array}{l}\text { KMSTRATAVG } \\
\text { (KMS) }\end{array}$ & KM Strategy and Plan - Average response from Questions A1 a-e. \\
\hline 2. & $\begin{array}{l}\text { SOLEARNAVG } \\
\text { (SOL) }\end{array}$ & Support for Organizational Learning - Average response from Questions A2 a-d. \\
\hline 3. & $\begin{array}{l}\text { PMPRACTAVG } \\
\text { (PMP) }\end{array}$ & Process Management Practices - Average response from Questions A3 a-f. \\
\hline 4. & $\begin{array}{l}\text { IMPRACTAVG } \\
\text { (IMP) }\end{array}$ & Information Management Practices - Average response from Questions A4 a-d. \\
\hline 5. & $\begin{array}{l}\text { OPMPRACTAVG } \\
\text { (OPM) }\end{array}$ & Organizational Performance Management Practices - Average response from Questions A5 a-f. \\
\hline 6. & $\begin{array}{l}\text { TRPRACTAVG } \\
\text { (TRP) }\end{array}$ & Training Related Practices - Average response from Questions A6 a-e. \\
\hline 7. & $\begin{array}{l}\text { HRPRACTAVG } \\
\text { (HRP) }\end{array}$ & Human Resource Related Practices - Average response from Questions A7 a-e. \\
\hline 8. & ISTSAVG (IST) & Information Systems and Technology Support - Average response from Questions B1 a-h. \\
\hline 9. & AOSSAVG (OSS) & Advanced Operational Support Systems - Average response from Questions B2 a-i. \\
\hline 10. & KACQAVG (KA) & Quality of Knowledge Acquisition Processes - Average response from Questions C1 a-f. \\
\hline 11. & KGAVAVG (KG) & Quality of Knowledge Generation \& Validation Processes - Average response from Questions C2 a-f. \\
\hline 12. & KSATAVG (KS) & Quality of Knowledge Sharing and Transfer Processes - Average response from Questions C3 a-e. \\
\hline 13. & KAAUAVG (KU) & $\begin{array}{l}\text { Quality of Knowledge Utilization and Application Processes - Average response from Questions C4 a- } \\
\text { e. }\end{array}$ \\
\hline 14. & KRAVG (KR) & Quality of Knowledge Retention Processes - Average response from Questions C5 a-e. \\
\hline 15. & ORCULAVG (SOC) & Supportive Organizational Culture - Average response from Questions D1 a-m. \\
\hline 16. & ORGEFFAVG (OE) & Organizational Effectiveness - Average response from Questions E1 a-v. \\
\hline 17. & $\begin{array}{l}\text { CPERFNormBins } \\
\text { (OP) }\end{array}$ & \begin{tabular}{l} 
Transformation into normalized bins of CperfINDEXccopy \\
\hline
\end{tabular} \\
\hline
\end{tabular}



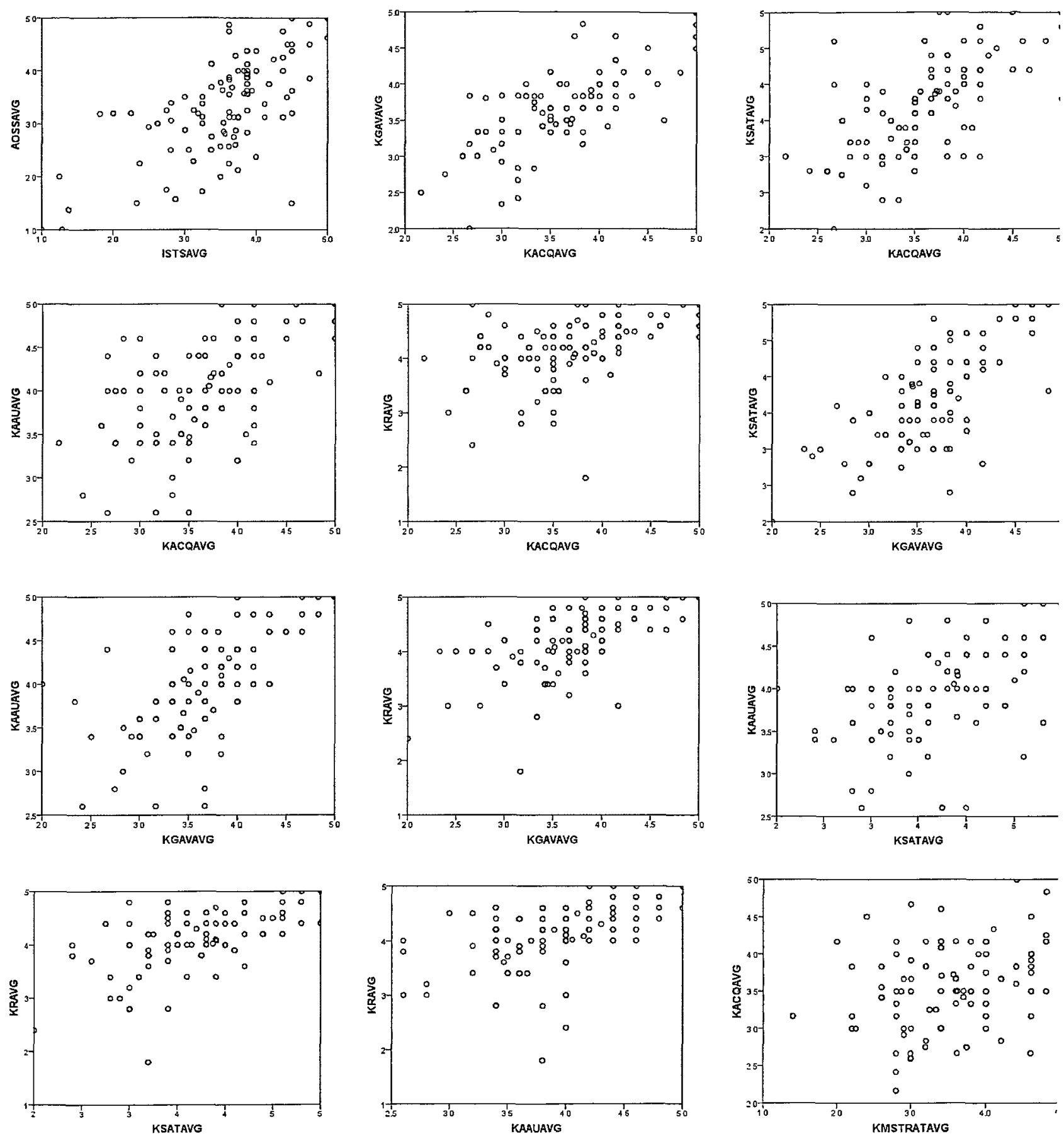

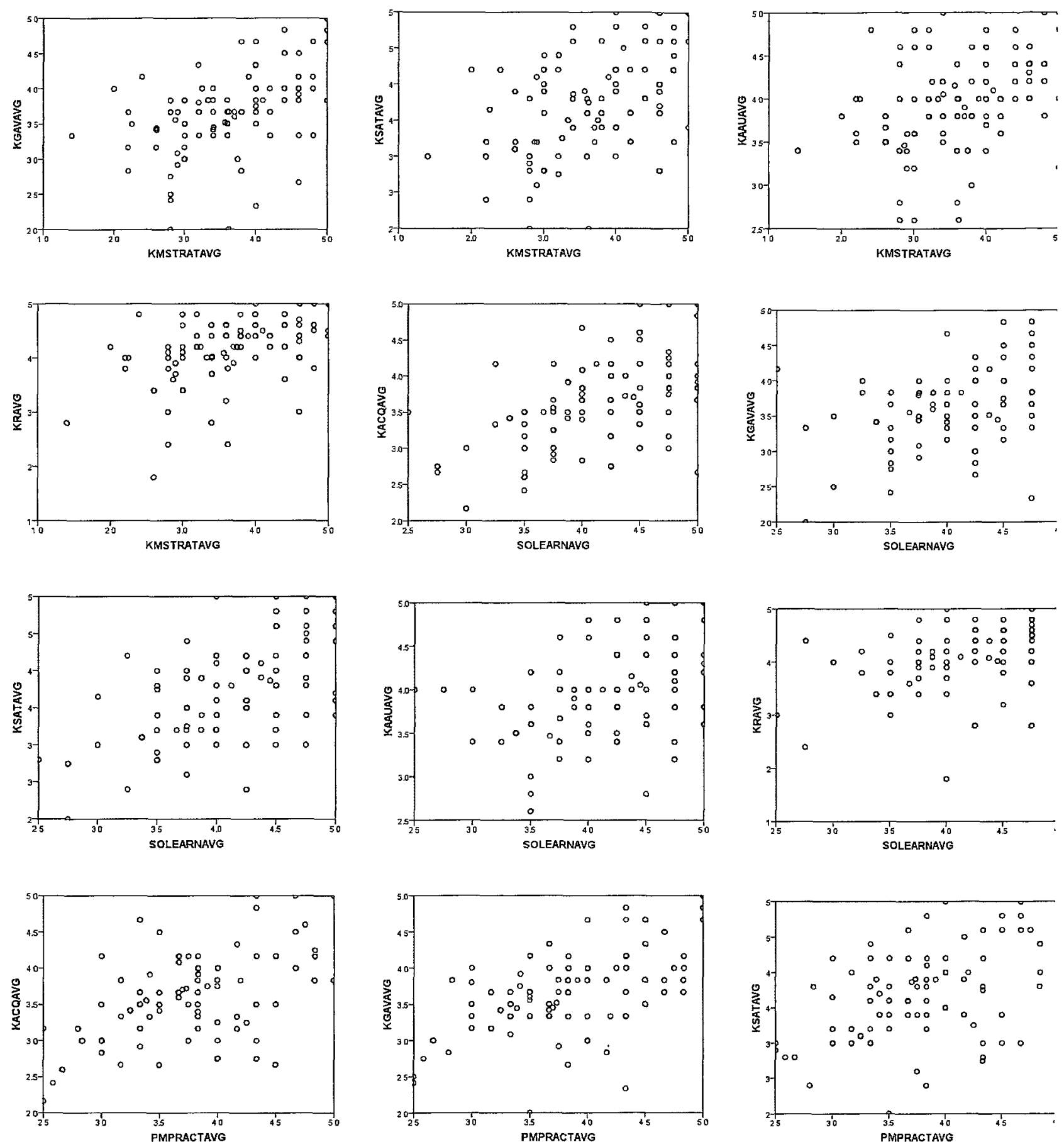

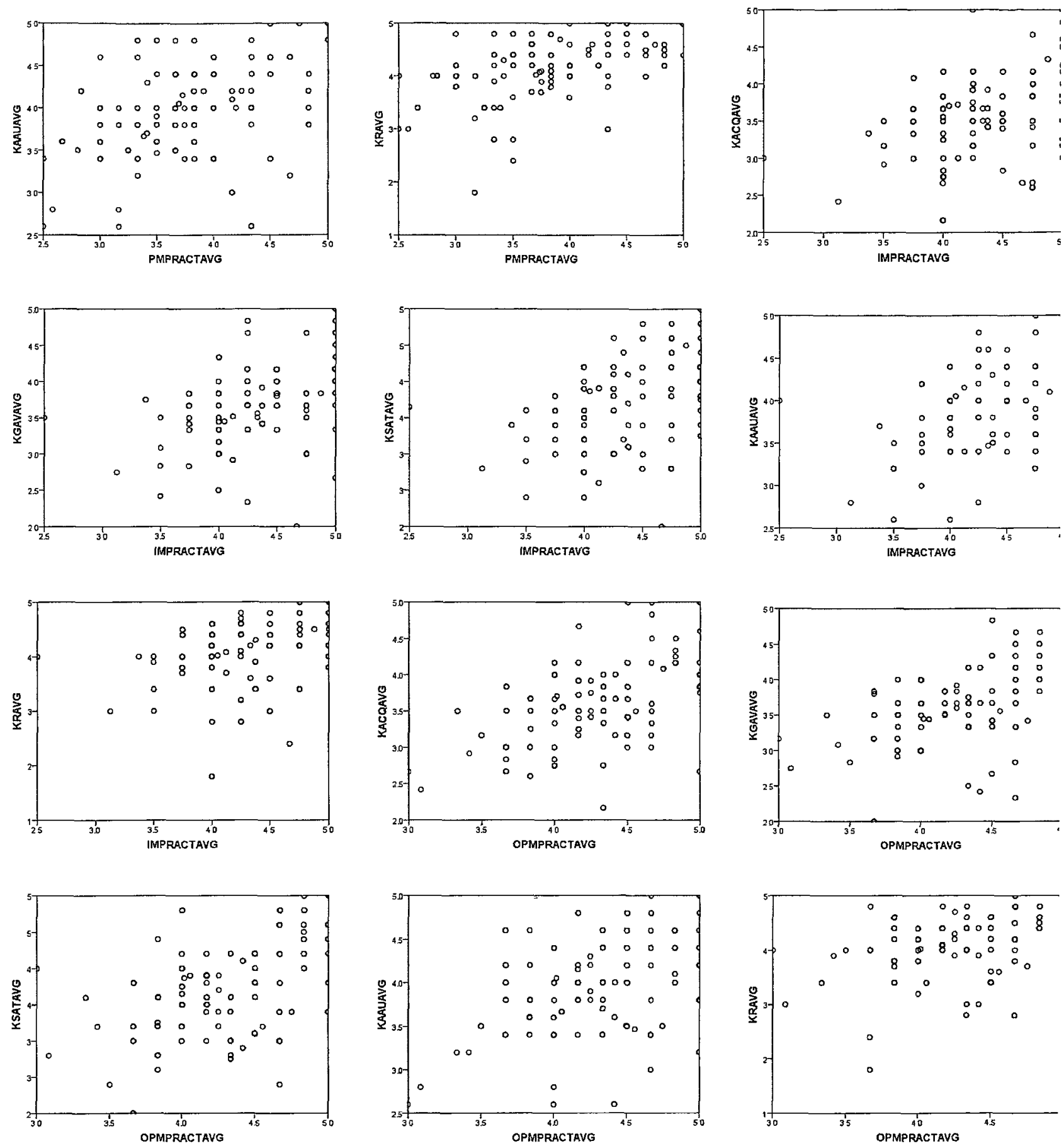

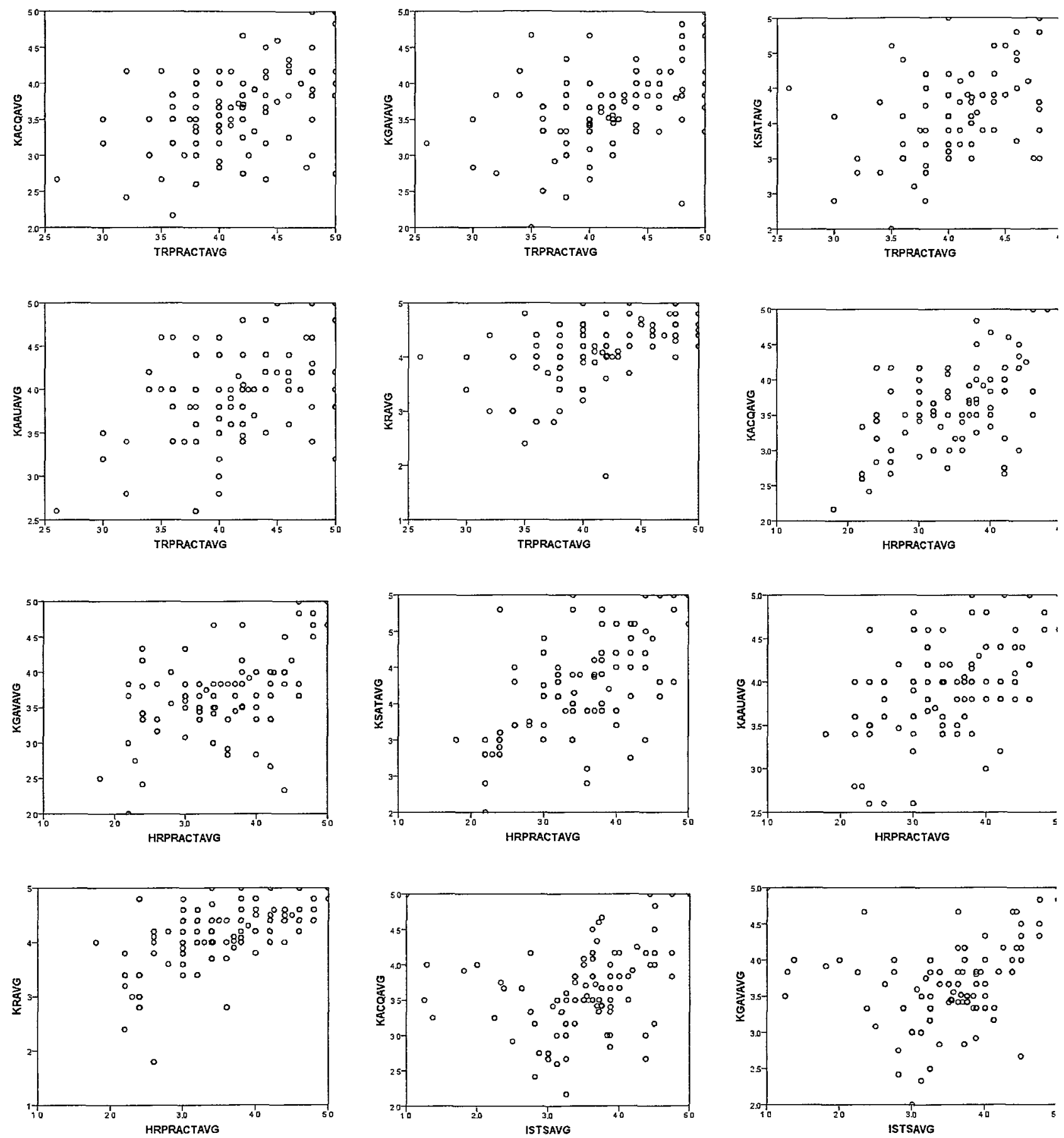

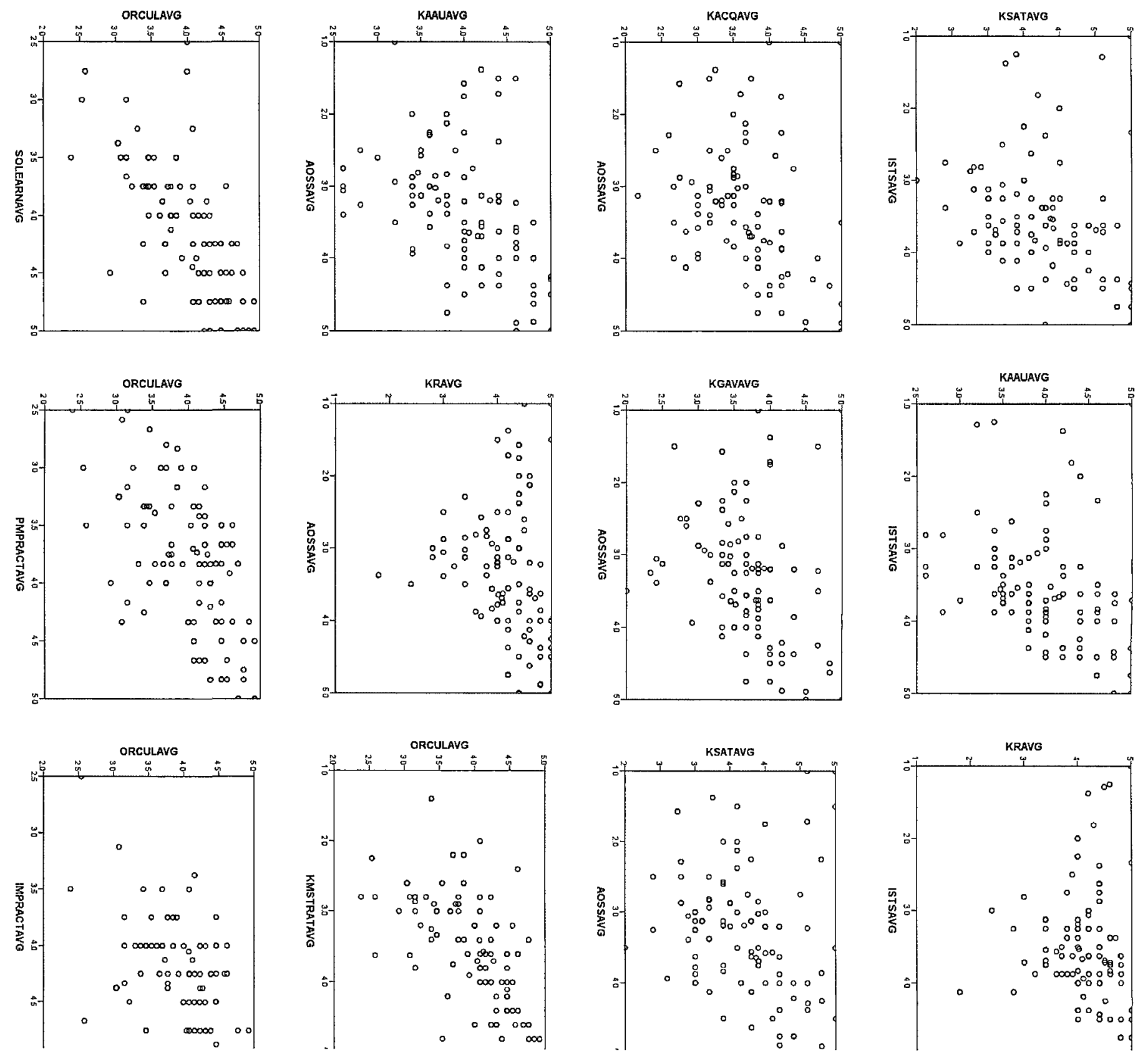

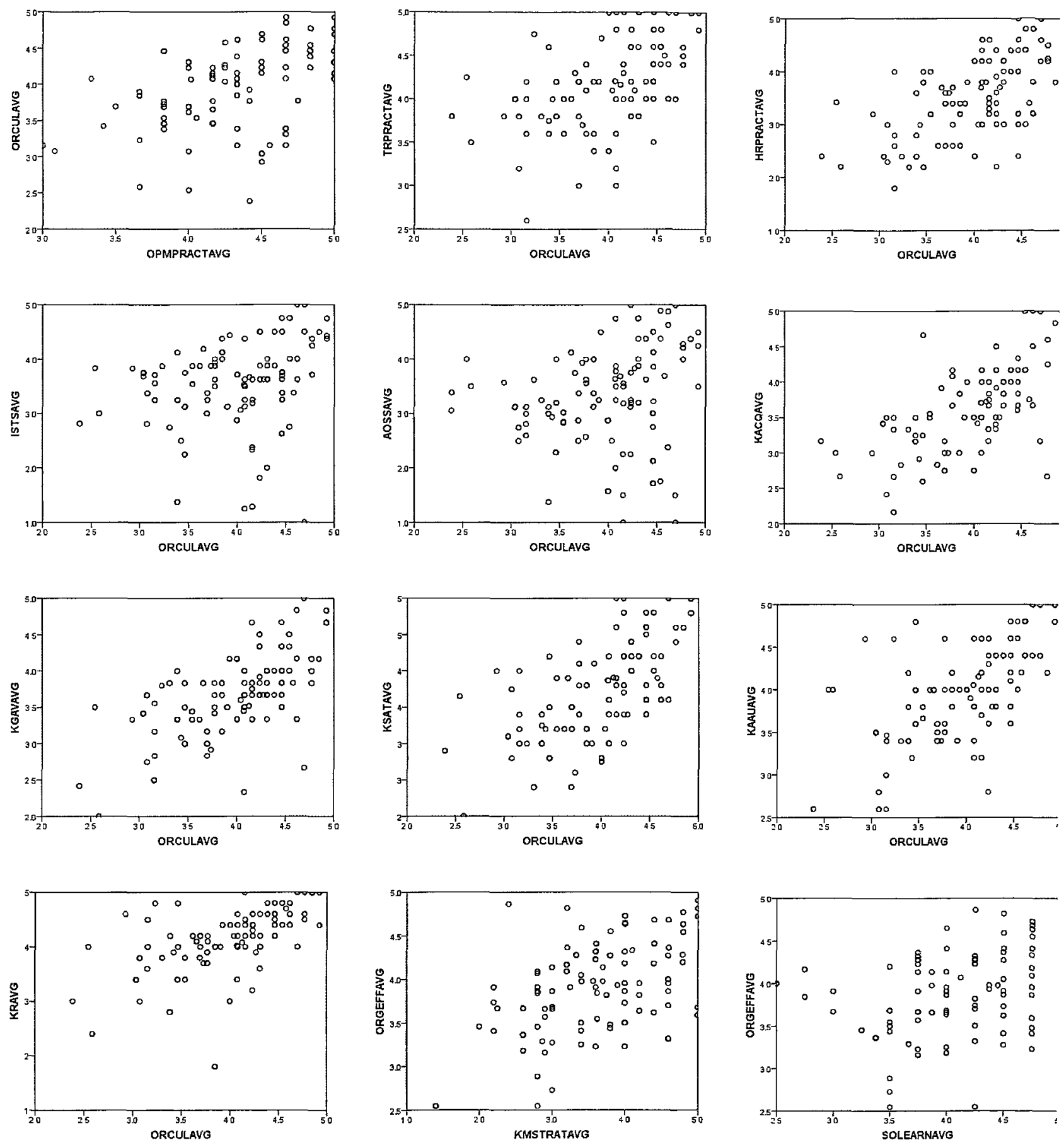

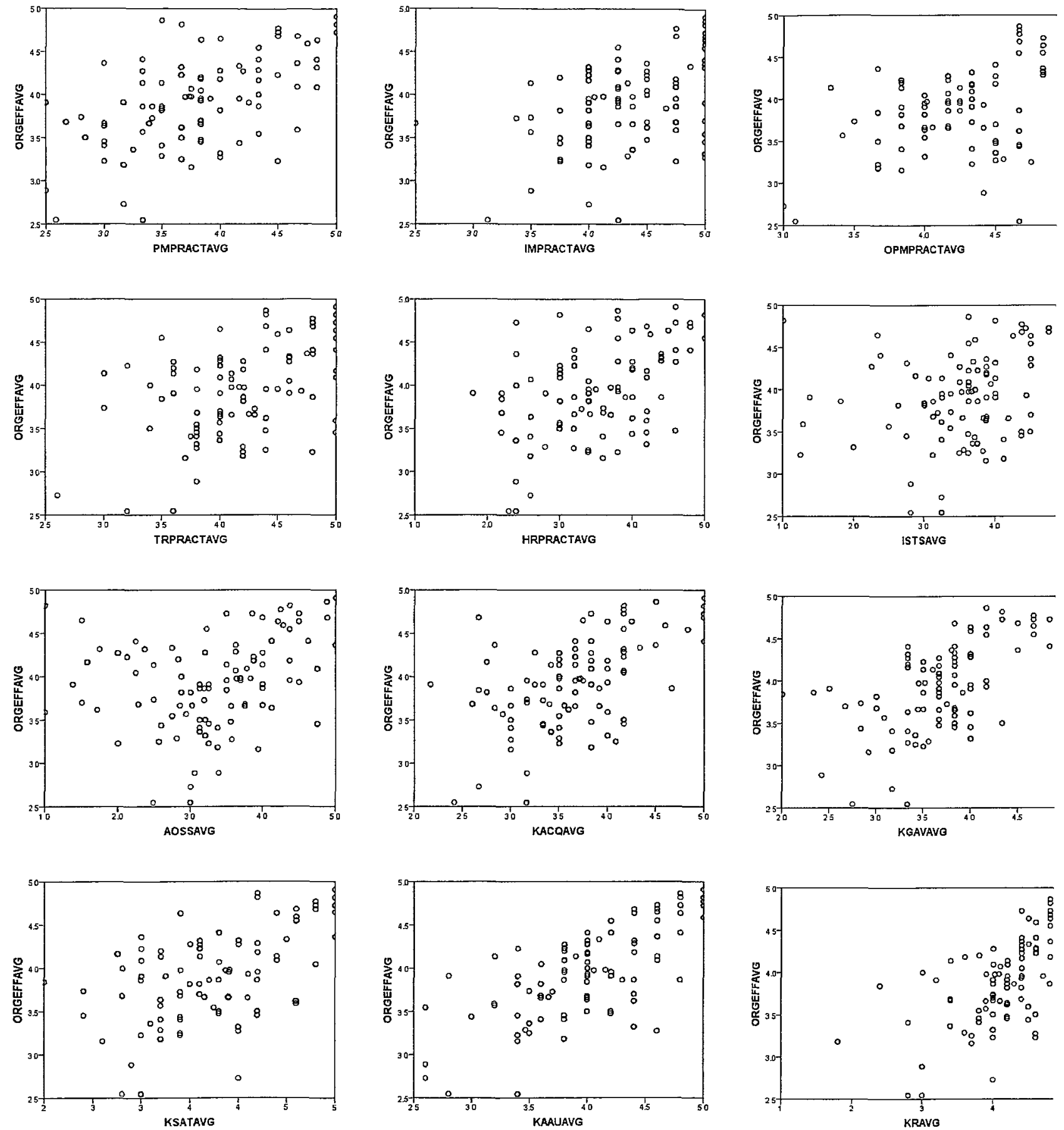

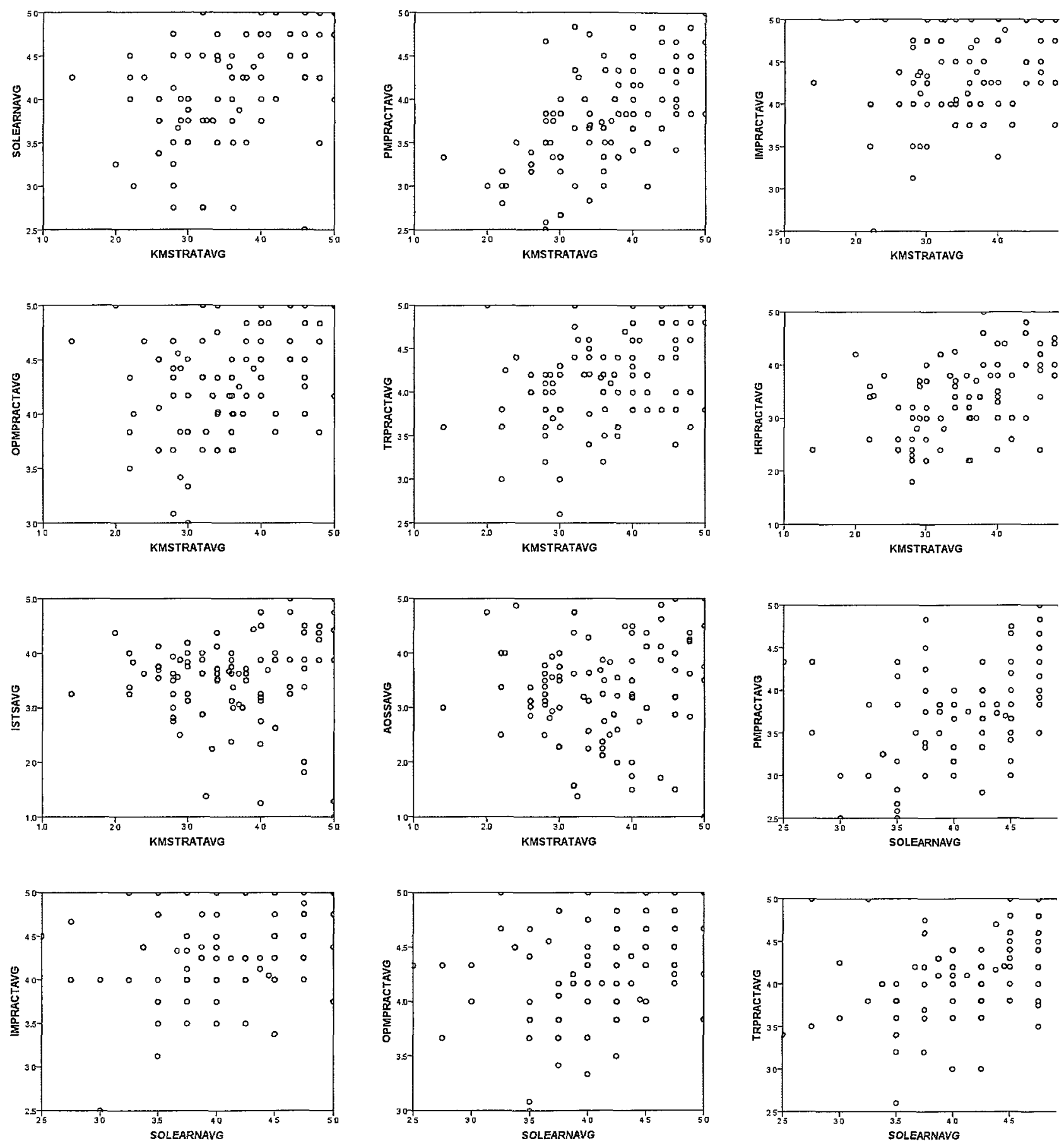

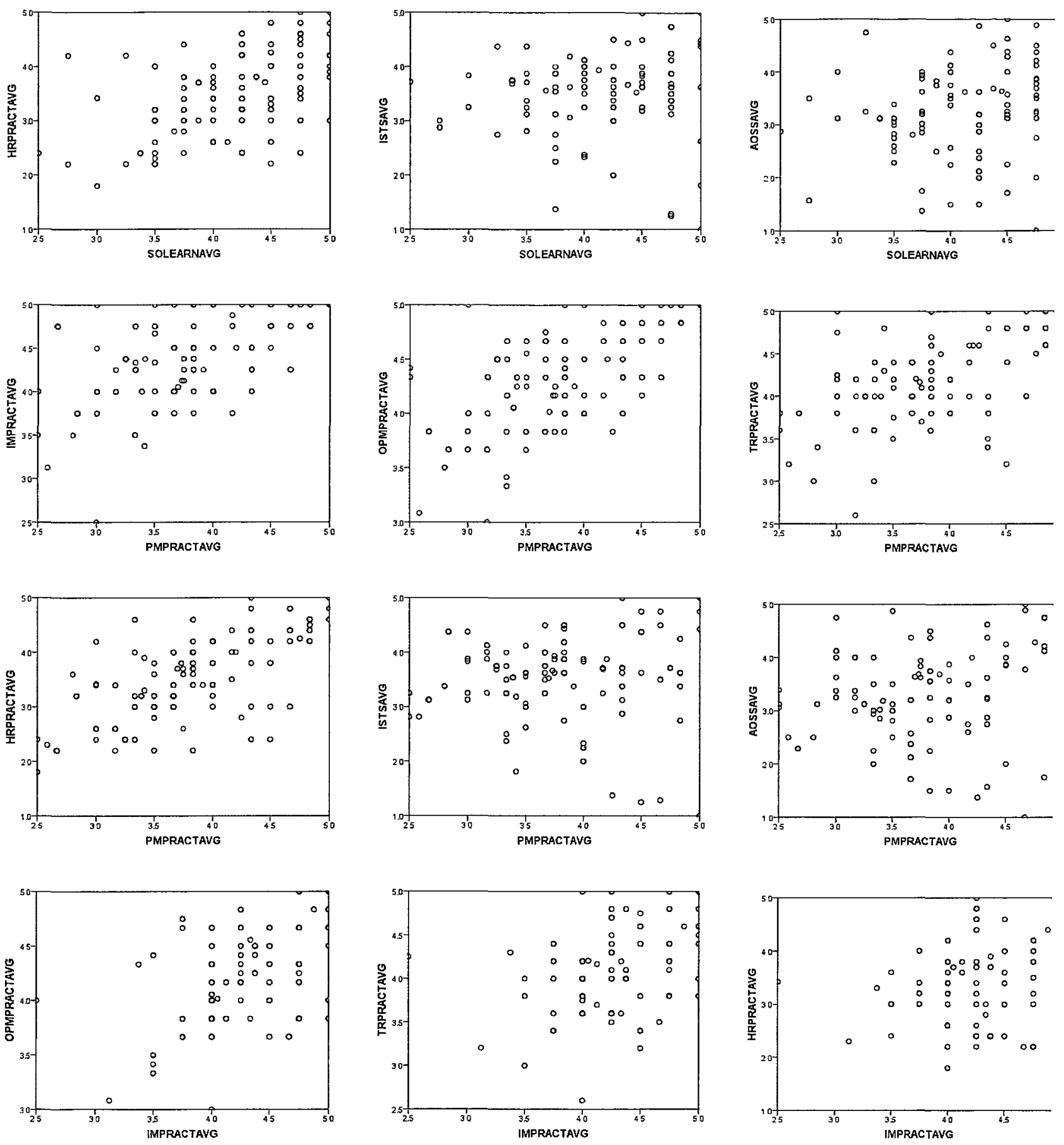

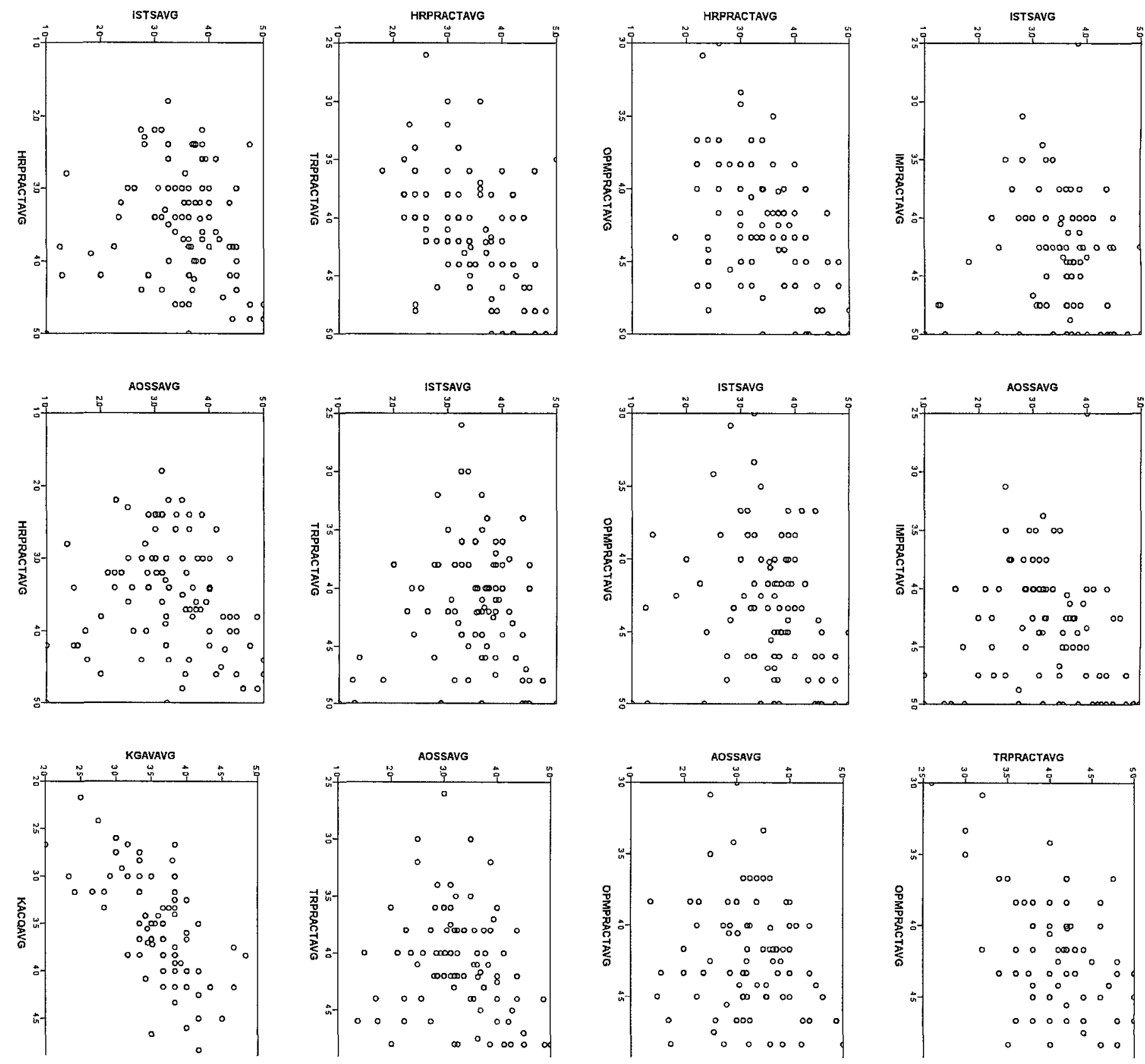

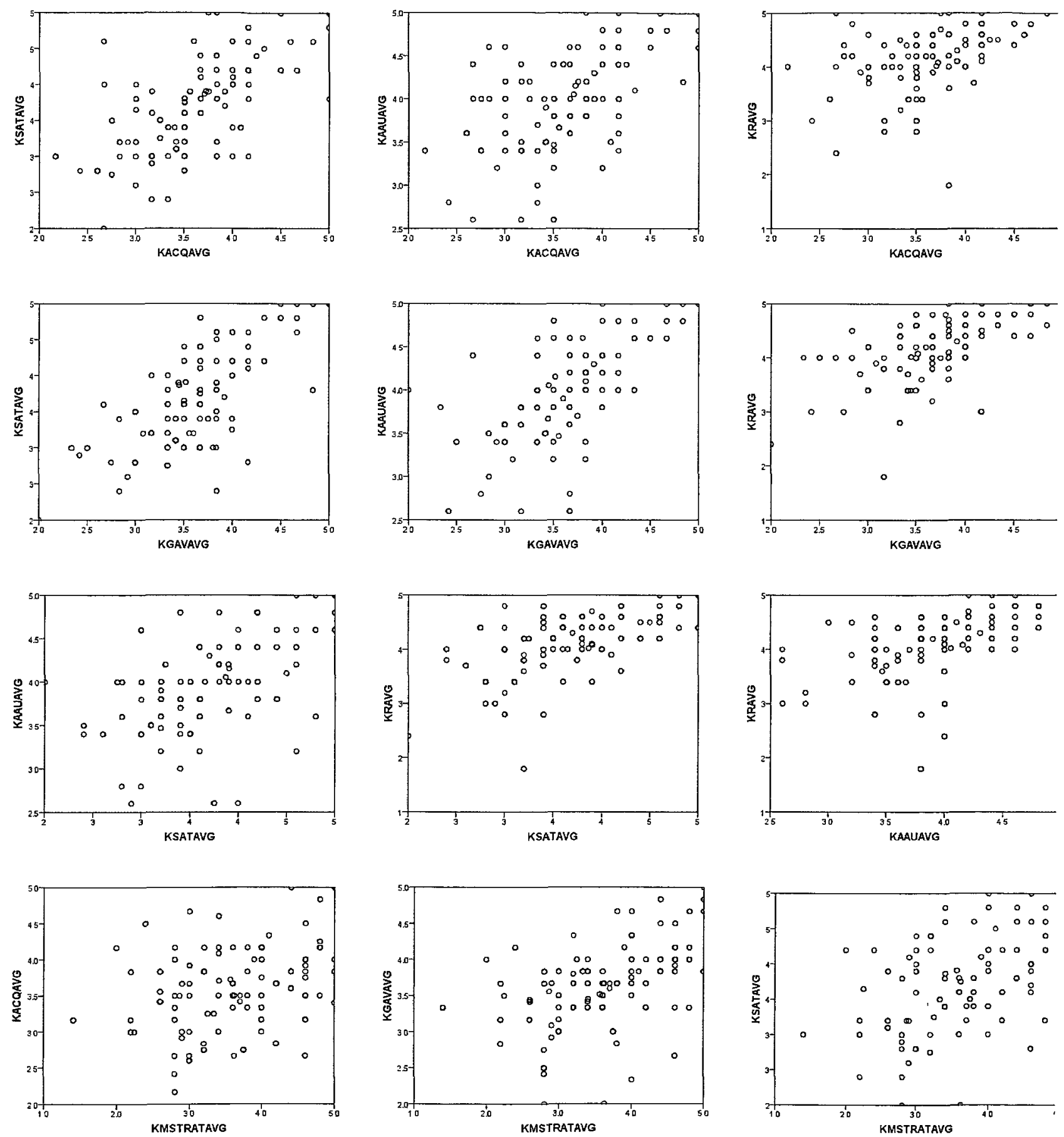

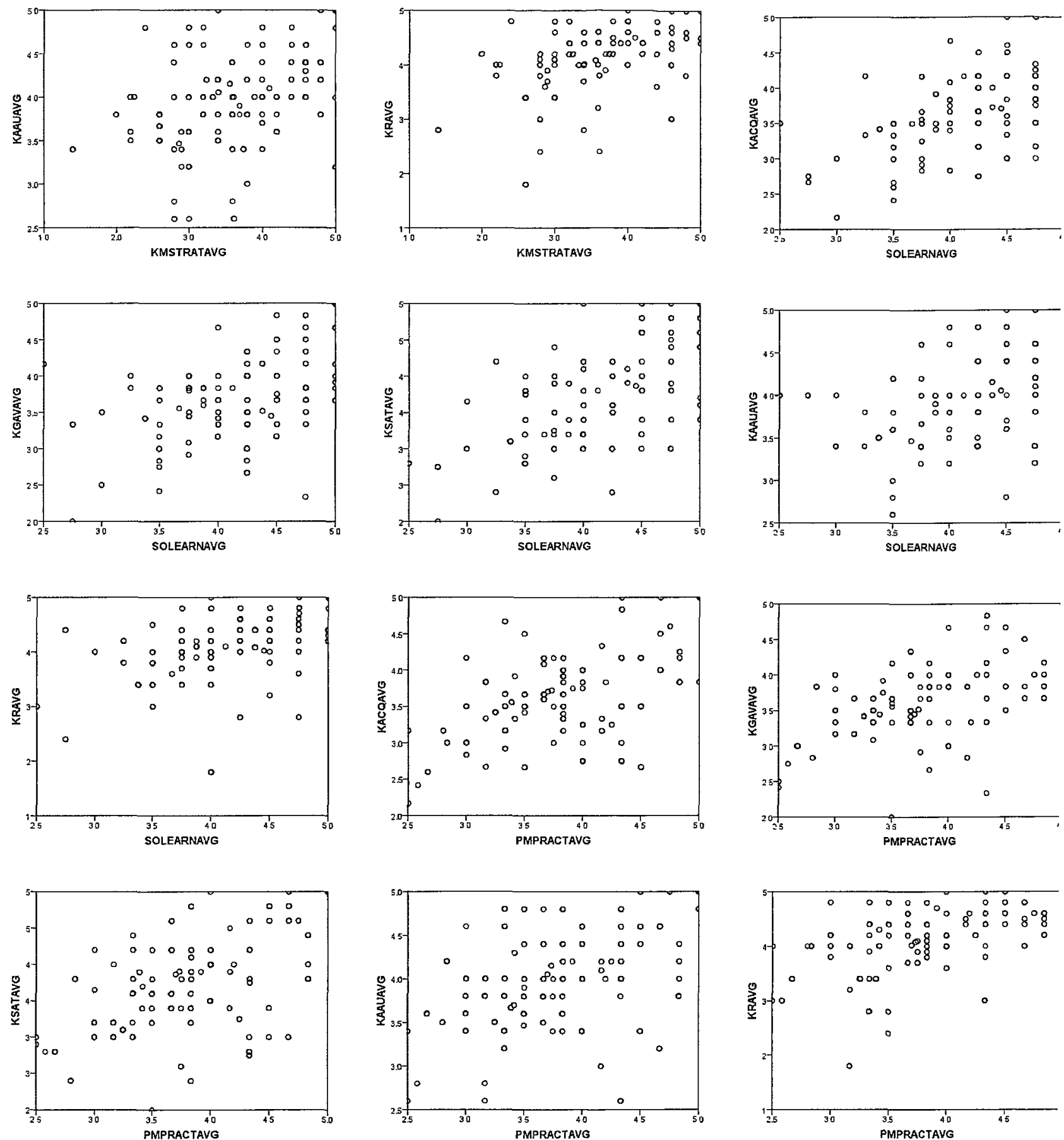

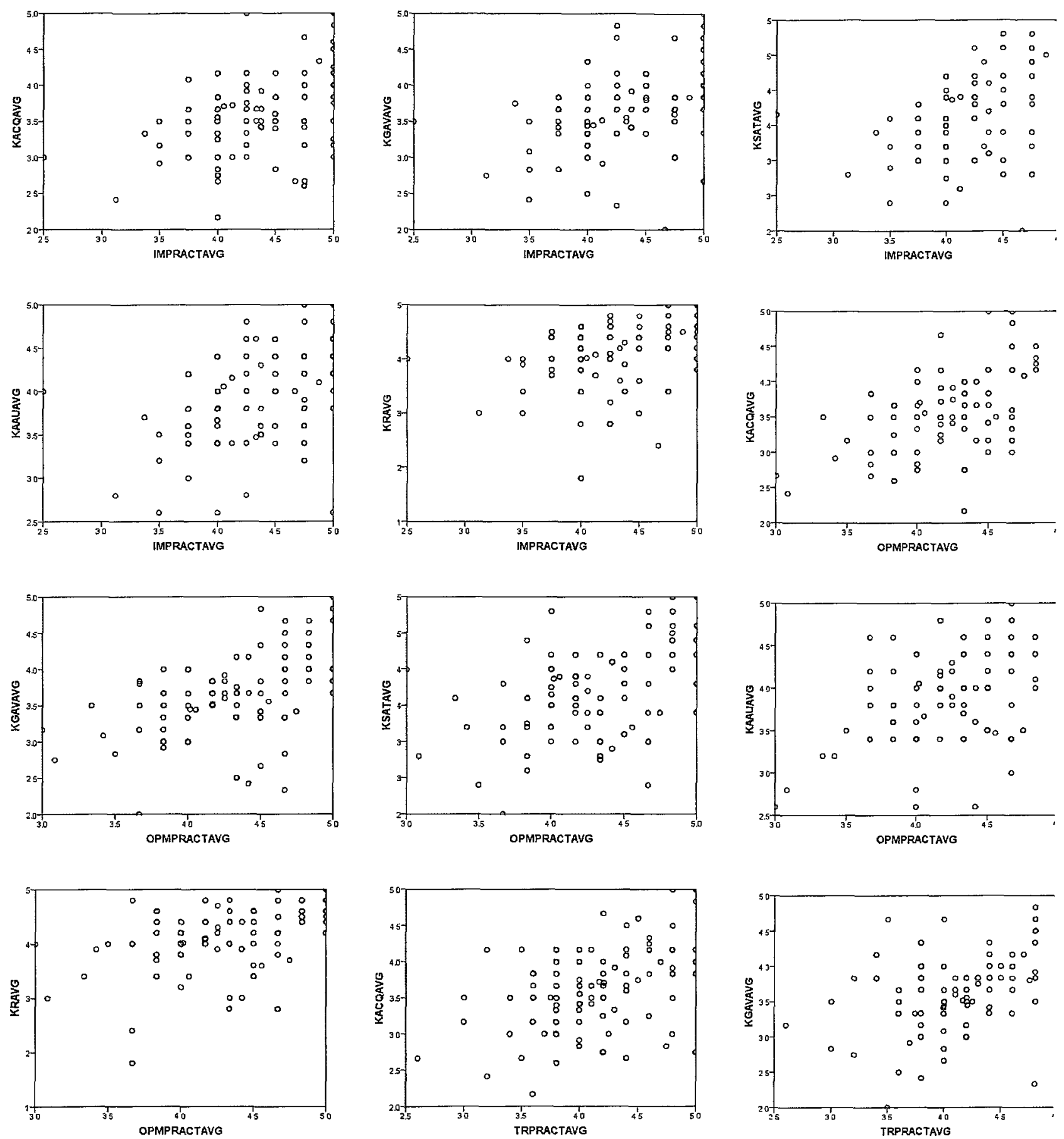

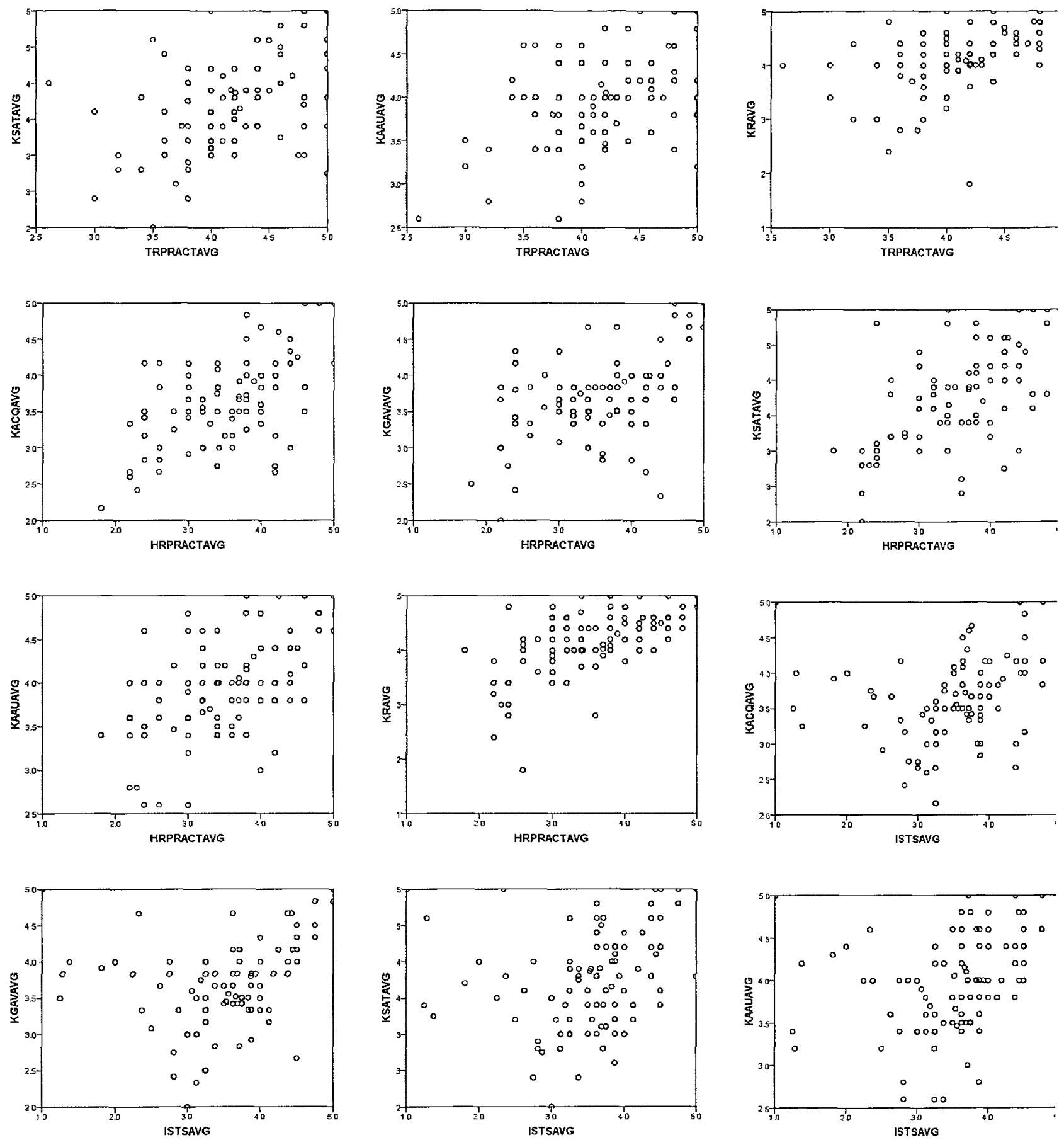

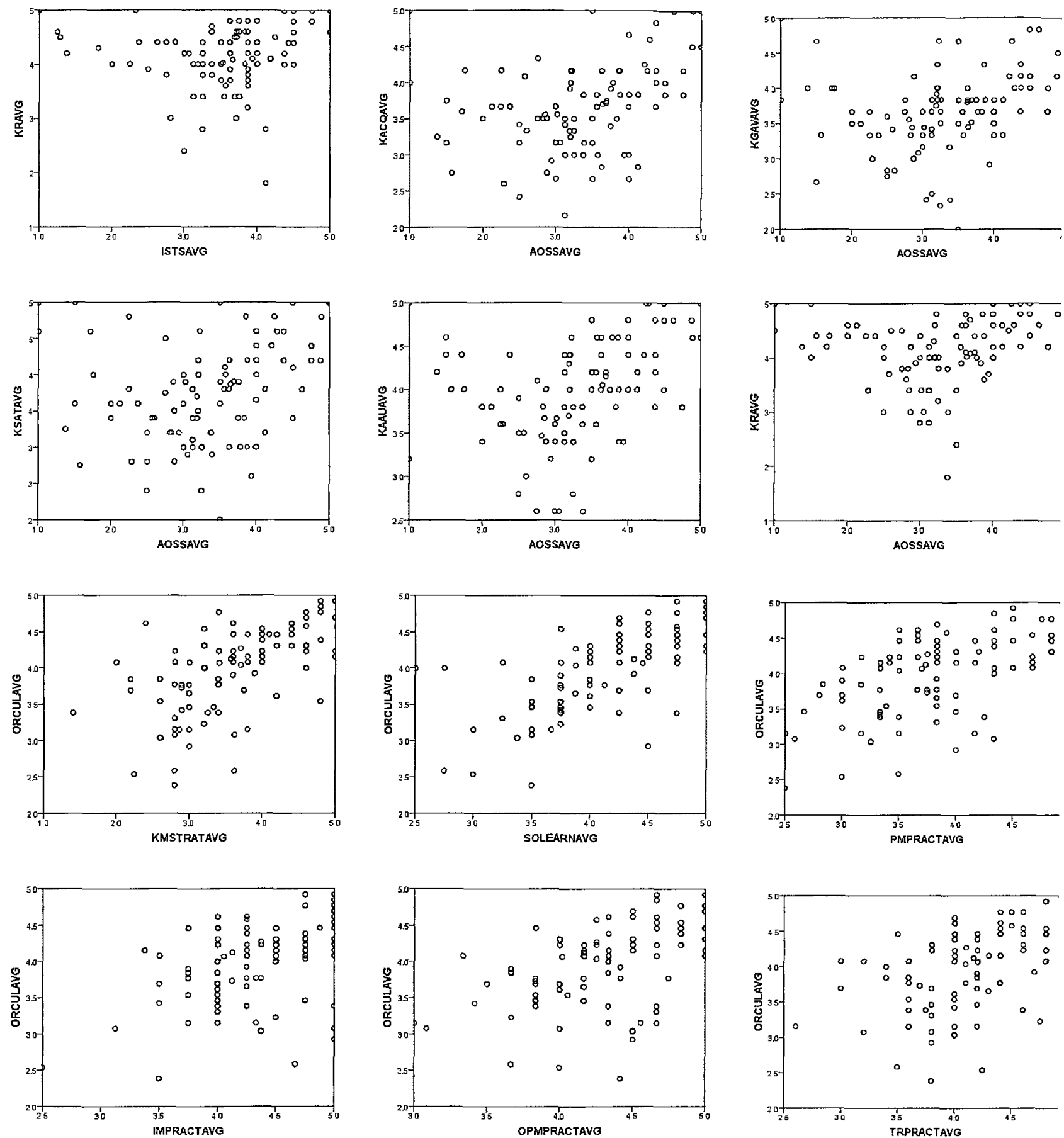

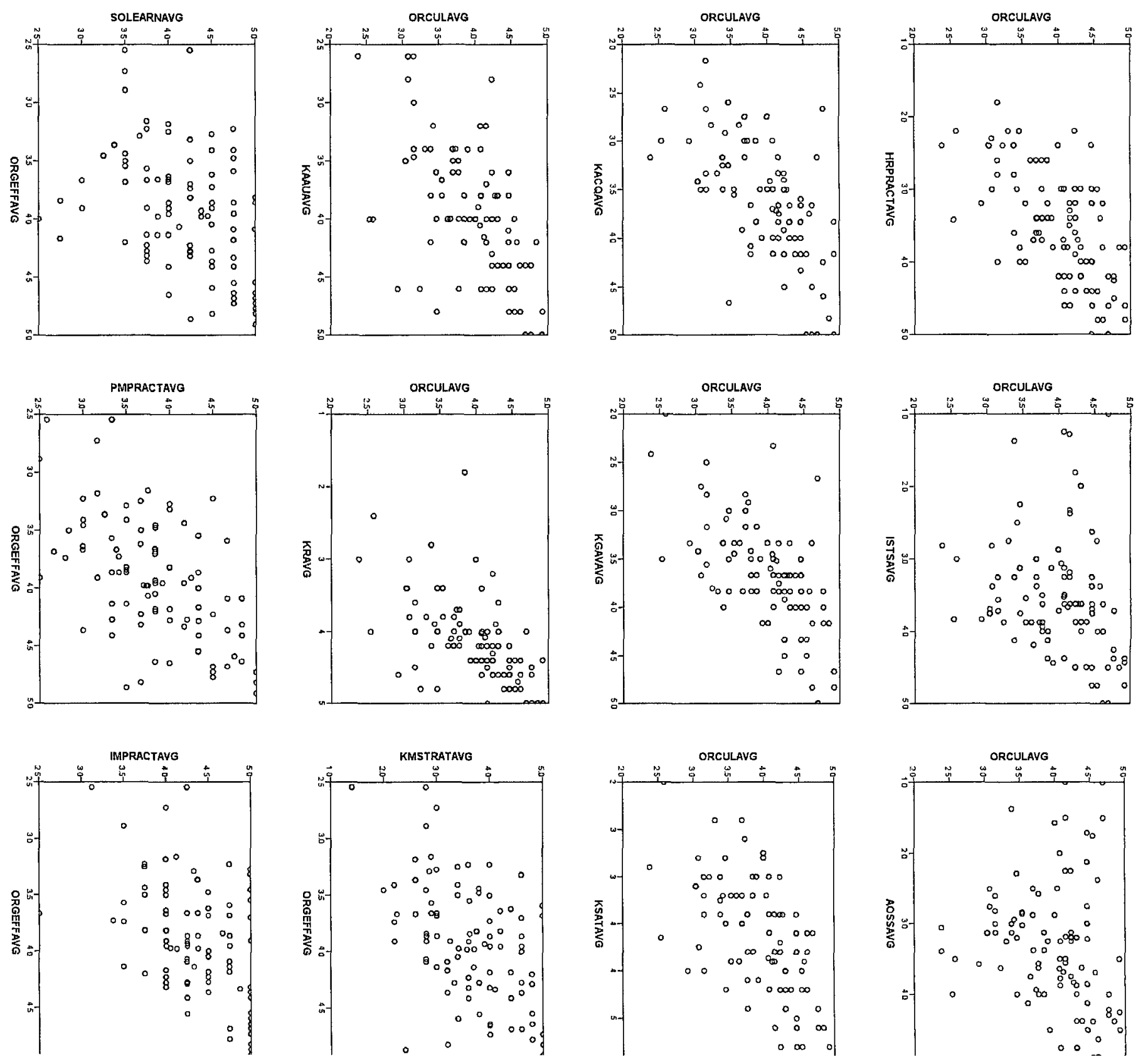

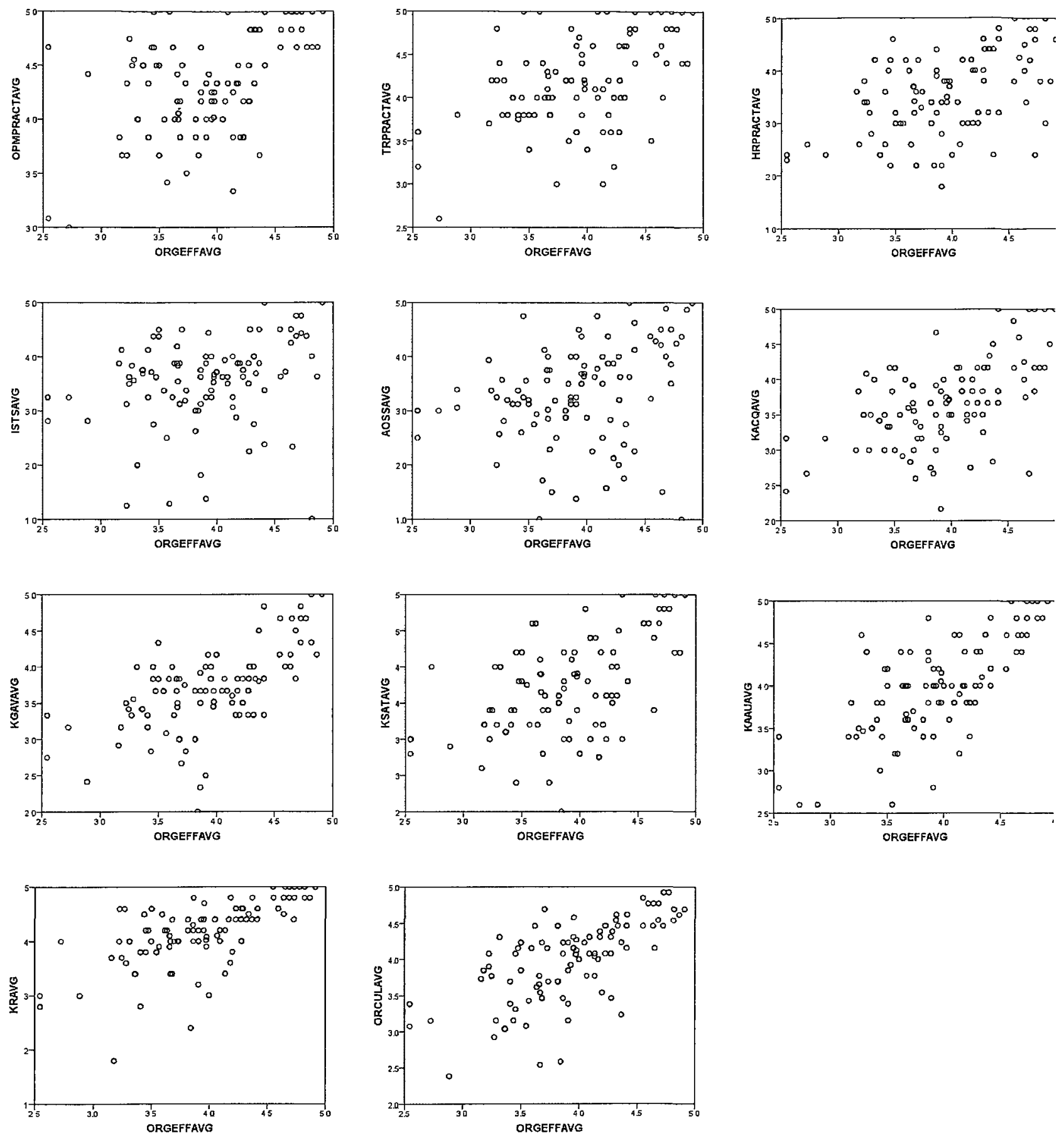